\title{
A Mechanistic Investigation of the Relationship Between Extramural Vascular Invasion (EMVI) and CpG Island Methylator Phenotype (CIMP) in Rectal Cancer
}

\author{
Rory Kokelaar \\ B.A. M.A. MBBS. M.Ed. MRCS
}

A thesis submitted in fulfilment of the requirements for the degree of Doctor of Philosophy

DNA Damage and Cancer Research Group Institute of Life Sciences

College of Medicine

Swansea University 
Immunohistochemistry images from an EMVI-positive tumour (a. \& b.) and an EMVI-negative tumour (c. \& d.) stained for metalloproteinase 2 (MMP2).
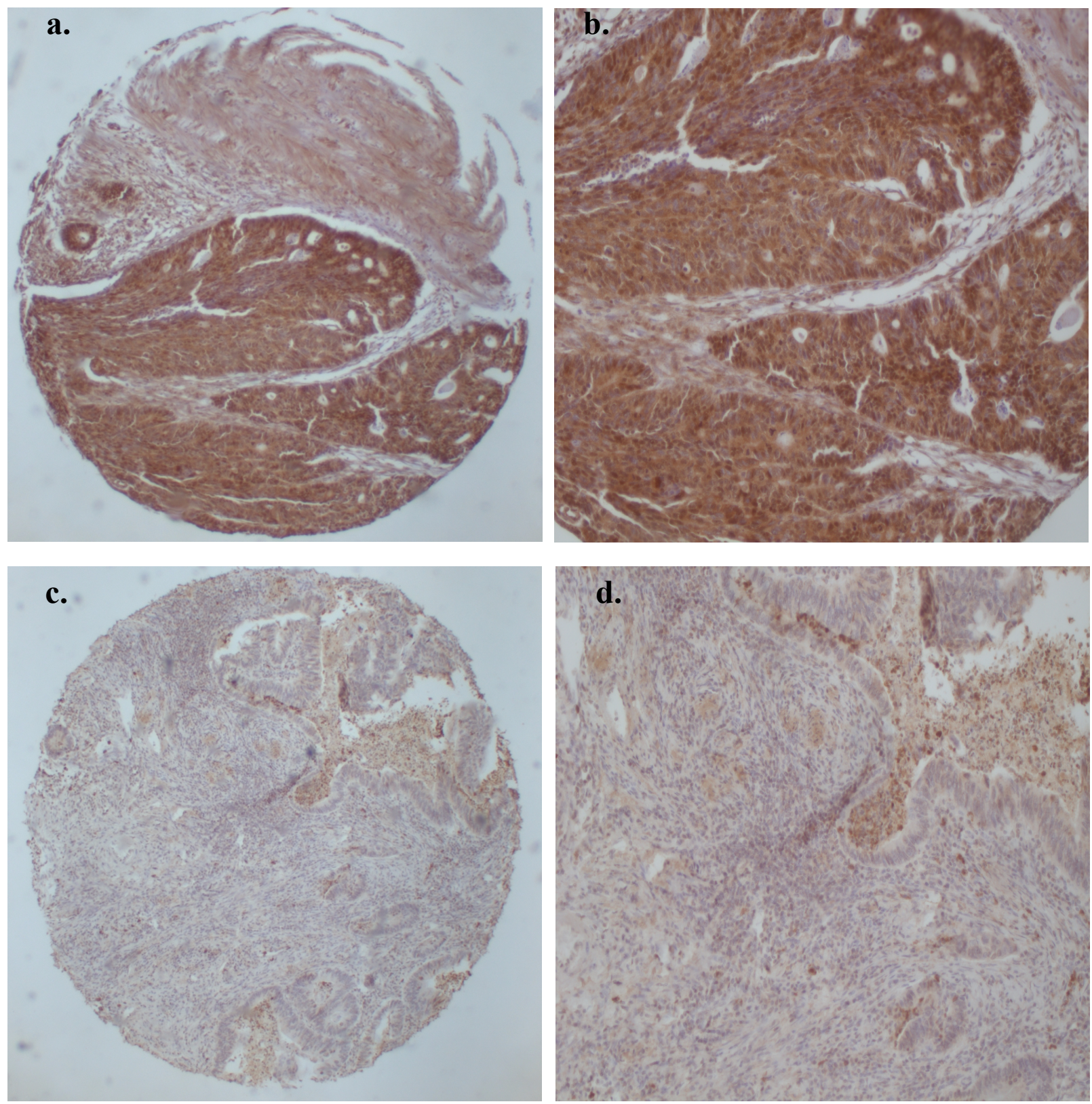

MMP2 was demonstrated to be associated with EMVI-status in resected rectal cancers $(p<0.0001)$, was an accurate predictor of EMVI-status (Test Accuracy 0.90, AUC $>0.95)$, and was superior to methylation status as an EMVI-status discriminator. Furthermore, MMP2 was associated with worse disease-free and overall survival in rectal cancers ( $p=0.030$ and 0.049 , respectively). Full results in Chapter 7. 


\section{Summary}

Colorectal cancer (CRC) is the third most frequent cancer and the second leading cause of cancer death worldwide. Each year, one million people will develop CRC, and 40-50\% will die within five years. Furthermore, rectal and distal sigmoid cancers are known to present at a later stage and have a poorer prognosis than other colonic cancers. Rectal cancers that demonstrate pathological extramural vascular invasion (EMVI-positive) are known to have a poorer prognosis than those that do not (EMIV-negative), and EMVI has is acknowledged as an important risk factor for systemic recurrence, local recurrence and death. Additionally, EMVI status influences the need for pre- and post-operative chemoradiation (CRT); which may influence survival outcomes.

Aberrant DNA methylation is emerging as a carcinogenic mechanism and potential biomarker in colorectal cancer. This study investigates the association between hypermethylation and EMVI in vivo and in vitro. Firstly, the in vivo associations between hypermethylation, EMVI, and clinical and histopathological outcomes are examined. Secondly, an investigation of the effects of demethylation on invasive colorectal cell lines in vitro aims to illuminate the genetic and cellular mechanisms that underlie methylation-dependent pathological cellular behaviour. Finally, highlighted biologic mechanisms are investigated in vivo to discover if there is an association with EMVI and survival outcomes. By these means the axis of association between hypermethylation, EMVI, and clinical outcomes is investigated. The investigation is conducted within the framework of consensus molecular subtyping in colorectal cancer, and in concordance with current methodologies of assessing DNA methylation status.

The primary findings demonstrate that EMVI is associated with hypermethylation in vivo, but that there is no direct correlation between hypermethylation and disease-free (DFS) or overall survival (OS). In vitro, demethylation of hypermethylated colorectal cancer cells, by means of established demethylating agent 5-azacytidine and putative demethylator RRx-001, reduces their propensity to migrate and invade. Demethylation in vitro is also associated with changes in the expression of the metalloproteinases involved in the metabolism of the basement membrane and the epithelial-to-mesenchymal transition and tumour metastasis, notably MMP2 and TIMP2. Changes in expression were confirmed at transcriptomal and proteomic levels in response to demethylation. In vivo, MMP2 expression was shown to be statistically significantly associated with EMVI, DFS, and OS, and was also independently predictive of EMVI, raising the possibility that it could act as a diagnostic and predictive biomarker in rectal cancers.

These findings indicate a mechanistic association between hypermethylation and EMVI, mediated by methylation-dependent expression of metalloproteinases. Metalloproteinase expression, specifically MMP2, may act as a potential biomarker for EMVI and correlates to survival outcomes in rectal cancer. 


\section{Hypothesis Cycle \& Chapters}

A Mechanistic Investigation of the Relationship Between Extramural Vascular Invasion (EMVI) and CpG Island Methylator Phenotype (CIMP) in

Rectal Cancer

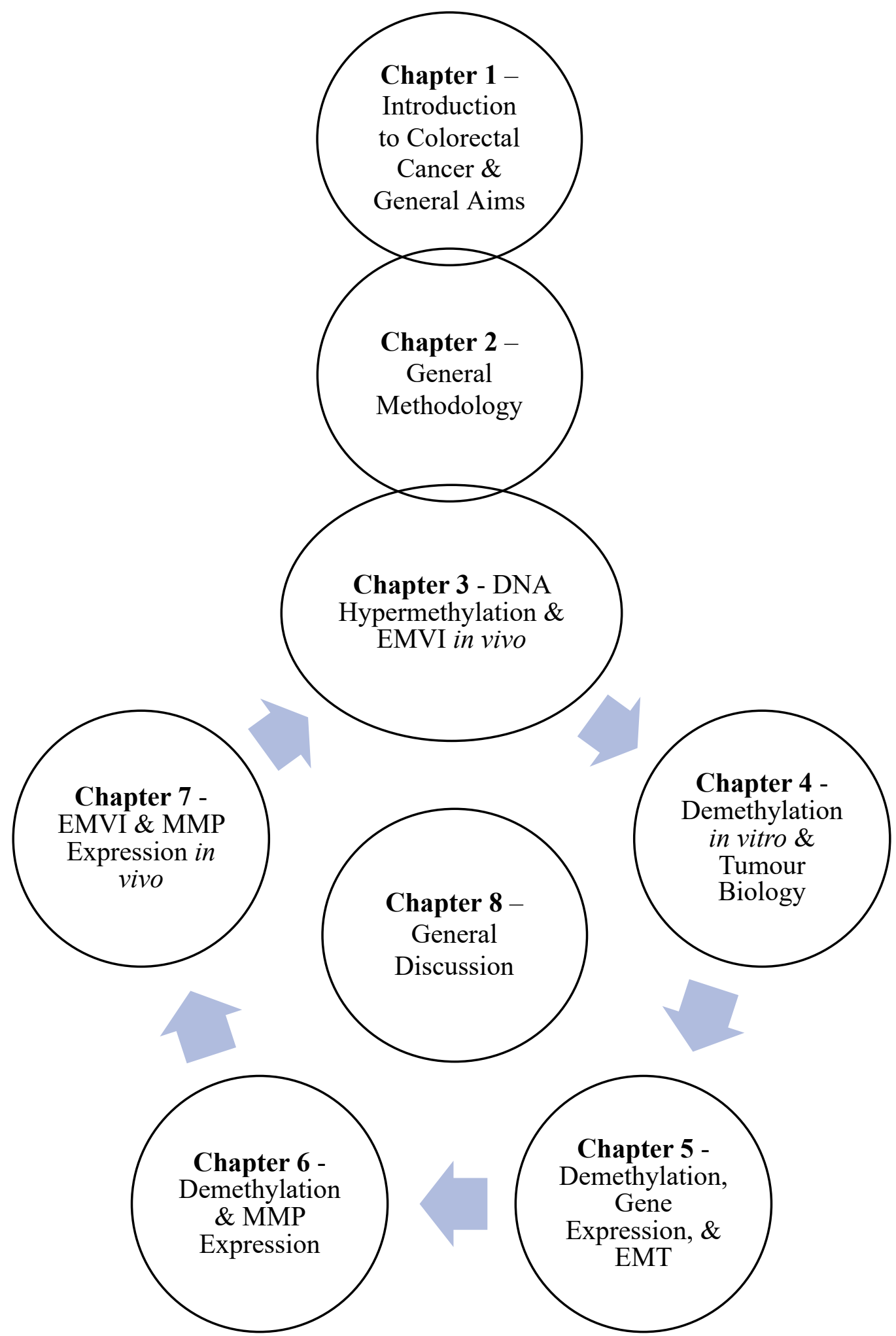




\section{Declarations}

\section{DECLARATION}

This work has not previously been accepted in substance for any degree and is not being concurrently submitted in candidature for any degree.

Signed (candidate)

Date

\section{STATEMENT 1}

This thesis is the result of my own investigations, except where otherwise stated. Where correction services have been used, the extent and nature of the correction is clearly marked in a footnote(s).

Other sources are acknowledged by footnotes giving explicit references. A bibliography is appended.

Signed (candidate)

Date

\section{STATEMENT 2}

I hereby give consent for my thesis, if accepted, to be available for photocopying and for inter-library loan, and for the title and summary to be made available to outside organisations.

Signed (candidate)

Date

NB: $\quad$ Candidates on whose behalf a bar on access has been approved by the University (see Note 7), should use the following version of Statement 2:

I hereby give consent for my thesis, if accepted, to be available for photocopying and for inter-library loans after expiry of a bar on access approved by the Swansea University.

Signed (candidate)

Date 


\section{Contents}

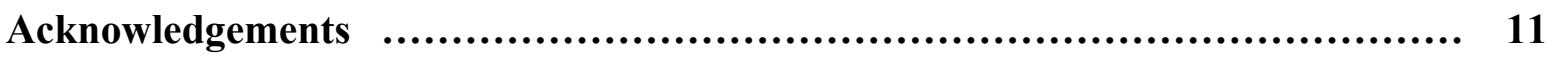

Publications Associated with this Thesis $\quad$........................................... 12

Abbreviations $\quad$................................................................. 13

Chapter 1 - General Introduction ........................................... 15

$\begin{array}{lr}\text { 1.1 Colorectal Cancer } & 15\end{array}$

1.1.1 Structure \& Function of the Colon \& Rectum 15

1.1.2 Epidemiology of Colorectal Cancer $\quad 20$

1.1.3 Aetiology of Colorectal Cancer 22

1.1.4 Staging and Histopathological Standards in Colorectal Cancer $\quad 22$

1.1.5 Survivorship and Outcomes in Colorectal Cancer 23

1.2 Treatment for Colorectal Cancer 25

1.2.1 Surgery for Colorectal Cancer $\quad 25$

1.2.2 Emergent Surgical Strategies for Rectal Cancer 26

1.2.3 Chemotherapy for Colorectal Cancer 28

1.2.4 Biological Therapies for Colorectal Cancer $\quad 30$

1.2.5 Radiotherapy for Colorectal Cancer $\quad 30$

1.2.6 Associated Therapies in Colorectal Cancer $\quad 31$

1.3 The Genetic \& Genomic Basis of Colorectal Cancer 32

1.3.1 The Vogelstein Model $\quad 32$

1.3.2 Key Signal Transduction Pathways in CRC 33

1.3.3 Tumour Suppressors - TP53 \& 18q 36

1.3.4 Microsatellite Instability \& Mismatch Repair $\quad 37$

1.3.5 Chromosomal Instability (CIN) 38

1.3.6 DNA Methylation \& CpG Island Methylator Phenotype 41

1.3.7 Other Epigenetic Factors 41

1.3.8 Consensus Molecular Subtyping in Colorectal Cancer 43

1.4 General Aims of this Thesis $\quad 46$

Chapter 2 - General Methodology ............................................... 47

$\begin{array}{ll}\text { 2.1 Overarching Considerations \& Ethics } & 47\end{array}$

2.1.1 Patient Selection $\quad 47$

2.1.2 Database Maintenance \& Anonymisation $\quad 48$

2.1.3 Ethics 48

2.2 Materials \& General Methods $\quad 49$

2.2.1 Laboratory Equipment, Consumables, \& Reagents 49 
2.2.2 Programmable Equipment

2.2.3 5-Azacytadine $\quad 52$

2.2.4 RRx-001 55

$\begin{array}{ll}2.2 .5 \text { Primers } & 57\end{array}$

$\begin{array}{ll}2.2 .6 \text { Kits } & 59\end{array}$

2.2.7 Antibodies \& Immunofluorescence $\quad 60$

2.2.8 Software $\quad 60$

2.3 Cell Lines \& Culture $\quad \mathbf{6 1}$

2.3.1 Cell Lines $\quad 61$

2.3.1.1 DLD-1 61

2.3.1.2 HFL-1 61

2.3.2 Cryopreservation $\quad 61$

2.3.3 Cell Seeding \& Maintenance 62

\section{Chapter 3 - DNA Hypermethylation as a Predictor of Extramural Vascular}

Invasion (EMVI) in Rectal Cancer .................................... 63

$\begin{array}{lr}3.1 \text { Introduction } & 63\end{array}$

3.1.1 Epidemiology of Rectal Cancer 63

3.1.2 Extramural Vascular Invasion in Rectal Cancer 64

3.1.3 Epigenetic Biomarkers in Colon \& Rectal Cancer 69

3.1.3.1 Introduction 69

$\begin{array}{ll}\text { 3.1.3.2 Diagnosis } & 70\end{array}$

3.1.3.3 Staging \& Prognosis 71

3.1.3.4 Response to Therapies $\quad 75$

3.1.3.5 Summary 76

$\begin{array}{ll}3.2 & \text { Aims \& Objectives }\end{array}$

$\begin{array}{ll}\text { 3.3 Material \& Methods } & \mathbf{7 8}\end{array}$

3.3.1 Patient Selection, Data Collection, \& Storage 78

$\begin{array}{ll}\text { 3.3.2 Tissue Collection } & 79\end{array}$

$\begin{array}{ll}\text { 3.3.3 DNA Extraction } & 79\end{array}$

$\begin{array}{ll}\text { 3.3.4 Bisulfite Conversion } & 80\end{array}$

3.3.5 Methylation Specific PCR (msPCR) $\quad 82$

3.3.6 Visualisation of PCR Products $\quad 83$

$\begin{array}{ll}\text { 3.3.7 CIMP Classification } & 83\end{array}$

3.3.8 Statistical Analysis $\quad 84$

$\begin{array}{ll}3.4 \text { Results } & \mathbf{8 5}\end{array}$

3.4.1 Patient and Tumour Characteristics $\quad 86$

3.4.2 CIMP Classification \& EMVI $\quad 86$

3.4.3 Survival Analysis $\quad 92$

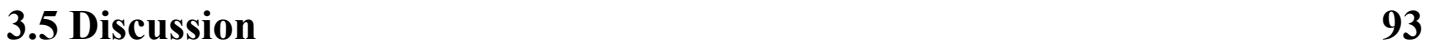

$\begin{array}{lr}\text { 3.6 Conclusion } & 96\end{array}$ 
Chapter 4 - The Biological Effects of Demethylation on Colorectal Cancer

Cells In Vitro ........................................................... 97

$\begin{array}{lr}4.1 \text { Introduction } & 97\end{array}$

4.1.1 In Vitro Studies of Colorectal Cancer 97

4.1.2 Migration \& Invasion Assays $\quad 99$

4.1.2.1 Introduction 100

4.1.2.2 Wound Healing (Scratch) Assay 100

4.1.2.3 Cell Exclusion Assay 100

4.1.2.4 Boyden Chambers \& Transwell ${ }^{\circledR}$ Systems 101

4.1.2.5. Other Migration \& Invasion Models 102

4.1.3 In Vitro Demethylation in Colorectal Cancer Models 103

4.1.4 In Vivo Demethylation in Colorectal Cancer Trials 103

4.2 Aims and Objectives $\quad 104$

$\begin{array}{ll}4.3 \text { Methods } & 105\end{array}$

4.3.1 Cell Culture, Treatment, \& Cytotoxicity 105

4.3.1.1 Cell Culture 105

4.3.1.2 Treatment with Demethylating Agents 105

4.3.1.3. Cytotoxicity 106

4.3.2 Wound Healing Assay 107

4.3.3 Extracellular Matrix Invasion 109

4.3.4 Assessment of Methylation Status 110

4.3.4.1 CIMP Status 110

4.3.4.2 Global DNA Methylation $\quad 110$

$\begin{array}{lr}4.4 \text { Results } & 113\end{array}$

4.4.1 Cytotoxicity 113

4.4.1.1 Azacytidine $\quad 113$

4.4.1.2 RRx-001 114

4.4.2 Two-Dimensional Migration (Scratch) Assay 115

4.4.3 Three-Dimensional Invasion Assay 119

4.4.4. Demethylating Effects of Azacytidine and RRx-001 122

4.4.4.1 CIMP Status 122

4.4.4.2 Global Methylation $\quad 123$

$\begin{array}{ll}\text { 4.5 Discussion } & 126\end{array}$

4.5.1 Cytotoxicity of Azacytidine and RRx-001 126

4.5.2. The in vitro biologic effects of AZA and RRx-001 127

4.5.3. Assessment of Methylation Status 129

$\begin{array}{ll}\text { 4.6 Conclusions } & 131\end{array}$ 
Chapter 5 - Methylation Dependent Gene Expression and the Epithelial-Mesenchymal \& Mesenchymal-Epithelial Transition $\quad$...................................... 132

5.1 Introduction 132

5.1.1 The Epithelial-Mesenchymal \& Mesenchymal-Epithelial Transitions 132

5.1.2 Metalloproteinases \& Cancer 138

5.1.3 Differential Gene Expression $\quad 141$

5.2 Aims \& Objectives $\quad 145$

$\begin{array}{ll}5.3 \text { Methods } & 146\end{array}$

5.3.1 RT R Profiler PRC Array Gene Expression $^{146}$

5.3.1.1 RNA Extraction \& Purification 146

$\begin{array}{ll}\text { 5.3.1.2 cDNA Synthesis } & 147\end{array}$

5.3.1.3 RT RTofiler Array $^{2} \quad 147$

5.3.1.4 Data Analysis 148

5.3.2 qRT-PCR of Metalloproteinases 149

5.4 Results 151

5.4.1 RT2 Profiler PRC Array Gene Expression 151

5.4.2 qPCR of Metalloproteinases 155

5.4.2.1 MMP2 155

5.4.2.2 MMP9 155

5.4.2.3 MMP11 156

5.4.2.4 MMP13 156

5.4.2.5 TIMP2 156

5.4.2.5 TIMP4 157

$\begin{array}{lr}\text { 5.5 Discussion } & 158\end{array}$

5.5.1 RT $\mathrm{R}^{2}$ Profiler PRC Array 158

5.5.1.1 Downregulated Genes $\quad 159$

5.5.1.1 Upregulated Genes $\quad 161$

5.5.1.3 Unchanged Genes 163

5.5.1.4 Summary 164

5.5.2. Metalloproteinases \& qRT-PCR 165

$\begin{array}{ll}\text { 5.6 Conclusions } & 167\end{array}$

Chapter 6 - Metalloproteinase Homeostasis and Demethylation $\ldots . . . . . . . . . . . . . . \quad 168$

$\begin{array}{lr}\text { 6.1 Introduction } & 168\end{array}$

6.1.1 Metalloproteinase Homeostasis 168

6.1.2 siRNA Knockdowns 171

6.1.3 Immunocytochemistry \& Protein Analysis 173

6.2 Aims \& Objectives $\quad 174$

$\begin{array}{lr}\text { 6.3 Methods } & 175\end{array}$

6.3.1 siRNA Knockdown \& AZA Exposure 175

6.3.2 Immunocytochemistry \& Immunofluorescence 177

$\begin{array}{ll}\text { 6.3.3 Western Blotting } & 178\end{array}$

6.3.4 Statistics and Data Presentation 180 
$\begin{array}{lc}\text { 6.4 Results } & 181\end{array}$

6.4.1 Immunocytochemistry and Immunofluorescence $\quad 181$

6.4.2 Western Blotting 185

$\begin{array}{lr}6.5 \text { Discussion } & 188\end{array}$

$\begin{array}{lr}6.5 \text { Conclusions } & 193\end{array}$

Chapter 7 - Extramural Vascular Invasion (EMVI) in Rectal Cancer and the

In Vivo Expression of Metalloproteinases \& their Inhibitors $\quad$............. 194

$\begin{array}{ll}7.1 \text { Introduction } & 194\end{array}$

7.1.1 Metalloproteinases as Biomarkers 195

7.1.2 Tissue Microarrays 197

$\begin{array}{ll}\text { 7.2 Aims \& Objectives } & 198\end{array}$

$\begin{array}{lr}7.3 \text { Methods } & 199\end{array}$

7.3.1 Patient Selection, Data Collection, \& Storage 199

7.3.2 Tissue Microarrays \& IHC 199

7.3.3 Statistical \& Test Analysis 201

$\begin{array}{ll}7.4 \text { Results } & 204\end{array}$

7.4.1 Patient and Tumour Characteristics 204

7.4.2 Staining Characteristics and EMVI 204

7.4.3 Proportional Hazards in relation to EMVI and

$\begin{array}{ll}\text { 7.4.4 Survival Analysis } & 208\end{array}$

7.4.5 MMP2 Biomarker Test Optimisation 210

7.4.6 CIMP, MMP2, \& EMVI 212

$\begin{array}{ll}7.5 \text { Discussion } & 213\end{array}$

$\begin{array}{lr}\text { 7.6 Conclusions } & 216\end{array}$

Chapter 8 - General Discussion $\quad$................................................ 217

$\begin{array}{ll}\text { 8.1 Introduction } & 217\end{array}$

8.2 The CpG Methylator Phenotype and Extramural Vascular Invasion 219

8.3 Demethylating Agents in Colorectal Cell Lines 220

8.4 Mechanisms Underlying the Response to Demethylation 222

8.4 Metalloproteinases in Rectal Cancers \& EMVI 224

8.5 Concluding Remarks $\quad 225$

Appendices $\quad$..................................................................... 226

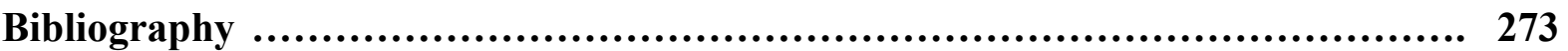




\section{Acknowledgements}

I would first like to acknowledge and thank those who have been most central to supporting me in this research in both a personal and professional capacity, whether through supervision, funding, good advice, or moral support. Foremost in this list are Professor Dean Harris and Professor Gareth Jenkins, without whom I could not have completed this work. The support and mentorship of Professor John Beynon and Mr Martyn Evans has also been invaluable to me through these past years. This work could also not have been completed with the continued support of members of the Swansea Colorectal MDT, namely; Mr Mark Davies, Professor Khot, Mr Chandrasekaran, Mr Roger Morgan, Mr Greg Taylor, Professor Paul Griffiths, Dr Namor Davies, and others. The wider support of a variety of departments within Abertawe Bro Morgannwg University Health Board (ABMU) at Singleton \& Morriston Hospitals has also been invaluable, including Mrs Christine Davies and Miss Kate Murphy in Histopathology.

At Swansea University, many members of the DNA Damage Group have helped me through this research, and particular thanks must be given to Dr Kate Chapman, who has guided me through many challenging protocols! I also wish to thank Mrs Margaret Clatworthy and Mrs Sally James for their help and patience.

Lastly, I would like to thank my wife, Sanam, for her enduring love and unquestioning support. 


\section{Published Papers \& Presentations Associated with this Thesis}

\section{Papers}

- Kokelaar, R.F., et al., Locally advanced rectal cancer: management challenges. Onco-Targets Therapy, 2016. 9: p. 6265-6272.

- Jones, H., Kokelaar, R.F., et al., Perineal reconstruction and surgical complication delay adjuvant chemotherapy after surgery for rectal cancer but do not impact upon survival. Diseases of the Colon \& Rectum, 2017. 60(6): p. e47-e593

- Kokelaar, R.F., et al., DNA hypermethylation as a predictor of extramural vascular invasion (EMVI) in rectal cancer. Cancer Biology \& Therapy, 2017: p. 1-8.

- Kokelaar, R.F., et al., The role of hypermethylation in radiosensitivity of colorectal cancer - an in vitro model. British Journal of Surgery, 2017. 104: p. 7-57.

- Kokelaar, R.F., et al., Meta-analysis of the prognostic value of $\mathrm{CpG}$ island methylator phenotype in rectal cancer. International Journal of Colorectal Disease, 2018. 33(8): p. 995-1000.

\section{Presentatins (oral international / national only)}

- Kokelaar, R.F., et al. Epigenetic and biological effects of 5-azacytidine and RRX001 on DLD-1 colorectal cancer cell lines. American Association for Cancer Research; 2017. April 04-06, 2017. Washington DC, USA.

- Kokelaar, R.F., et al., Demethylation inhibits migration and invasion of DLD-1 colorectal cancer cells in vitro. Diseases of the Colon \& Rectum, 2017. 60(6): p. e47e593. The American Society of Colon and Rectal Surgeons; June 10-14, 2017 Washington State Convention Centre and Sheraton Seattle Hotel, Seattle, WA, USA.

- Kokelaar, R.F., et al., Demethylation of DLD-1 colorectal cancer cell lines reduces tumour cell invasion in vitro, correlating with modulation of metalloproteinase expression. Colorectal Disease, 2018. 20(S7): p. 13-58. ACPGBI Annual Meeting, Birmingham, UK 


\section{Abbreviations}

5-FU

ACPGBI

AJCC

APC

AZA

BMI

bp

cDNA

CEA

CI

CIN

CIMP

cm

CMS

CRC

CRT

CT

DFS

DMSO

DNMT

ECM

EGFR

EMT

ERAS

FAP

Gy

HR

IBD

(K)RAS

LARC

LOH

LRRC
5-Fluorouracil

Association of Coloproctology of Great Britain \& Ireland

American Joint Committee on Cancer

Adenomatous Polyposis Coli gene

5-Azacytidine

Body Mass Index $\left(\mathrm{kg} / \mathrm{m}^{2}\right)$

Base pairs

Complimentary DNA

Carcinoembryonic Antigen

Confidence Interval (95\%, unless specified otherwise)

Chromosomal Instability

CpG Island Methylator Phenotype

Centimetres

Consensus Molecular Subtype

Colorectal Cancer

Chemoradiotherapy

Computed Tomography

Disease-Free Survival (months)

Dimethyl sulfoxide

DNA methyltransferase

Extracellular Matrix

Epidermal Growth Factor Receptor

Endothelial - Mesenchymal Transition

Enhanced Recovery After Surgery

Familial Adenomatous Polyposis

Gray $\left(\mathrm{J} \mathrm{Kg}^{-1}\right)$

Hazard Ratio

Inflammatory Bowel Disease (Crohn's \& Ulcerative Colitis)

(Kirsten) RAt Sarcoma oncogene

Locally Advanced Rectal Cancer

Loss of Heterozygosity

Locally Recurrent Rectal Cancer 


\begin{tabular}{|c|c|}
\hline $\mathbf{m A b}$ & Monoclonal Antibody \\
\hline MMP & Matrix Metalloproteinase \\
\hline MMR & Mismatch Repair (Genes) \\
\hline mr- & MRI-based assessment of tumour characteristic (e.g. mrEMVI) \\
\hline MRI & Magnetic Resonance Imaging \\
\hline mRNA & Messenger Ribonucleic Acid \\
\hline MSI & Microsatellite Instability \\
\hline msPCR & Methylation-Specific PCR \\
\hline nCRT & Neoadjuvant Chemo-Radiotherapy \\
\hline p- & $\begin{array}{l}\text { Histopathology-based assessment of tumour characteristic (e.g. } \\
\text { pEMVI) }\end{array}$ \\
\hline pCR & Pathologically Complete Response \\
\hline PCR & Polymerase Chain Reaction \\
\hline qRT-PCR & Quantitative Real-Time PCR \\
\hline $\mathbf{R 0}$ & Complete Macro- and Microscopic Tumour Excision \\
\hline RFS & Relapse-Free Survival (months) \\
\hline $\mathbf{R R}$ & Relative Risk \\
\hline rtPCR & Real-Time PCR \\
\hline SAM & S-Adenosyl Methionine \\
\hline siRNA & Short Interfering RNA \\
\hline TAMIS & Transanal Minimally Invasive Surgery \\
\hline TaTME & Transanal Total Mesorectal Excision \\
\hline TEMS & Transanal Endoscopic Microsurgery \\
\hline TIMP & Tissue Inhibitor of Metalloproteinase \\
\hline TME & Total Mesorectal Excision \\
\hline TMA & Tissue Microaray \\
\hline VEGF & Vascular Endothelial Growth Factor receptor \\
\hline VTE & Venous Thromboembolism \\
\hline WHO & World Health Organisation \\
\hline
\end{tabular}




\section{Chapter 1}

\section{General Introduction}

\subsection{Colorectal Cancer}

\subsubsection{Structure \& Function of the Colon \& Rectum}

The colon and rectum constitute the large bowel/intestine, and along with the anal canal, form the final portion of the alimentary tract. Together, the colon and rectum are approximately $160 \mathrm{~cm}$ in length, extending from the ileocaecal junction with the small bowel (marked by the ileocaecal valve) to the anal verge and skin of the perineum. The primary function of the colon and rectum is to absorb water and salts form the bowel content before it is expelled, the rectum also acting as a reservoir for stool prior to evacuation. The constituent portions of the colon are the caecum, right or ascending colon, transverse colon, left or descending colon, and sigmoid colon. The colon and rectum are comprised of concentric layers with different structure and function, the innermost of which is the mucosa (predominantly columnar epithelium with mucous producing goblet cells), followed by the neurovascular submucosa, then the muscularis propria (two layers of smooth muscle, the innermost circumferential and the outer in discontinuous longitudinal bands called taeniae coli), and finally the serosa or adventitia (depending on relationship to the peritoneum). At the distal end of the sigmoid colon the taeniae coli converge as they pass over the sacral promontory to form the rectum as a distinct anatomical portion of the large bowel.

The rectum itself is approximately $15 \mathrm{~cm}$ in length and is divided into thirds dependent upon its peritoneal relations; the upper rectum having a peritoneal covering on three sides (anterior and bilateral), the mid rectum having only an anterior covering of peritoneum, and the low rectum being entirely below the peritoneal reflection completely enveloped in its mesentery. The classical boundary of the rectum and its supporting mesentery below the peritoneal reflections is described by the plane of complete mesorectal excision (CME), originally described in Heald's seminal work ${ }^{1}$. The rectum is also different from the colon in its relation to the abdominal and pelvic peritoneal cavity; being entirely located within the confines of the bony pelvis. This is of particular importance when considering both the relative limitations of 
surgical access to the rectum compared to the colon, and its relationship to significant visceral, neurovascular, bony, and other structures located in the pelvis.

As the rectum reaches the pelvic floor the smooth muscular fibres constituting its outer wall condense, and with the contribution of the skeletal muscles of the adjacent pelvic floor, the anal sphincter complex is formed, investing within it the anal canal. The canal itself is $2-4 \mathrm{~cm}$ in length, and as well as its role as a sphincter, is also marked by the change from the columnar epithelium of the colon and rectum to the squamous epithelium of the skin at the pectineal line. Due the different nature of its epithelium, the anus possesses pathology that is specific to itself and quite different to that of the colon and rectum, especially when considering carcinogenesis, and thus is outside the remit of this thesis.

Figure 1.1 The Colon (A) and Rectum (B).

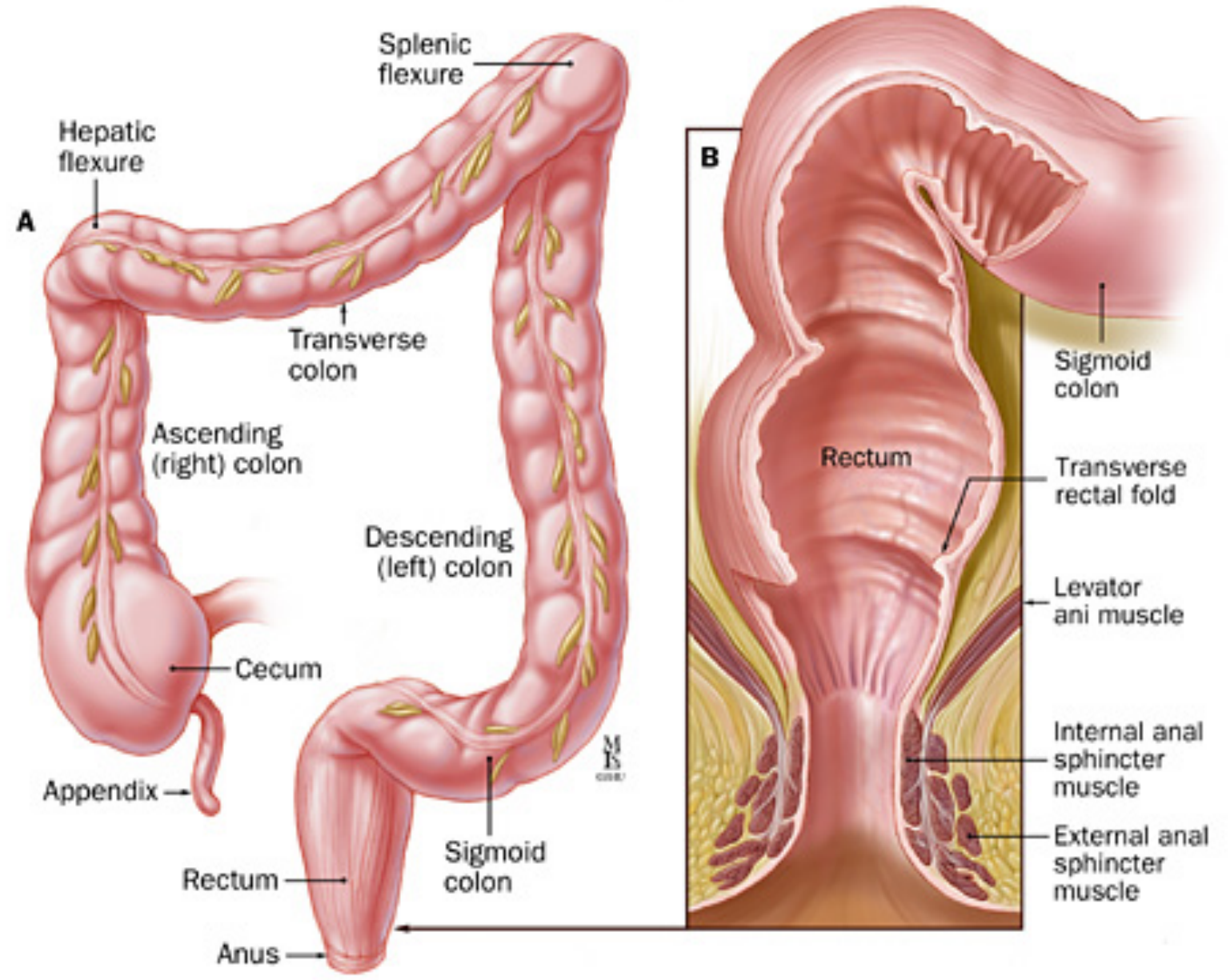


The blood supply to the colon and rectum is primarily comprised of terminal branches of the superior mesenteric artery (which also supplies the majority of the small bowel) and the inferior mesenteric artery, both of which originate from the abdominal aorta. Classically, the watershed between these two arterial systems is defined as the transition from mid-gut to hind-gut, two thirds of the way across the transverse colon where the marginal artery forms an arcade connecting the two. A third arterial supply is also provided by the middle and inferior rectal arteries, which are terminal branches of intermediate arteries originating from the internal iliac artery. The venous drainage of the colon and rectum reflects the arterial in its peripheral distribution, but instead of returning blood to the systemic circulation directly, blood is diverted through the liver first via the portal venous system. The exception to this is in the low rectum where the haemorrhoidal veins return venous blood to the systemic circulation directly via the internal iliac veins. The lymphatic drainage mirrors the arterial system and comprises the superior and inferior mesenteric lymph node chains, which themselves drain to the pre-aortic nodes at levels corresponding to the origin of the arteries.

As well as absorbing water and forming and storing stool, the colon and rectum allow the bacterial fermentation of residual organic matter and act as a reservoir of bowel flora; the colorectal microbiome. Although the colon does not play an active role in absorption of nutrients, it does however absorb some of the products of bacterial fermentation, importantly fat-soluble vitamin $\mathrm{K}$, a key factor in blood coagulation and calcium metabolism. Beyond fermentation, the colorectal microbiome is attracting significant interest as a potential factor in cancer and other (particularly inflammatory) processes in the large intestine, both from a pathogenic and potentially therapeutic perspective ${ }^{2,3}$. 


\subsubsection{Epidemiology of Colorectal Cancer}

Colorectal cancer (CRC) is a highly prevalent cancer in Western societies, with approximately 140,000 new cases and 52,000 deaths (estimated) in the United States in 2018 $8^{4}$ In the UK the figures are 42,000 and 16,000, respectively ${ }^{5}$. Globally CRC is the third most common cancer in men $(10.0 \%$ of total cancer) and the second most common cancer in women $(9.2 \%$ of total cancer). Approximately $45 \%$ of those diagnosed die of the disease despite treatment ${ }^{6}$. The overall lifetime risk of developing colorectal cancer is 1 in $22(5.5 \%)$ for men and 1 in 24 $(5.15 \%)$ for women in the United States, although incidence is highly variable internationally. The incidence of CRC per 100,000 ranges from 4.1 (India) to 49.1 (Czech Republic) in men, and from 3.9 (India) to 39.5 (New Zealand) in women, with broadly higher incidence in North America, Europe (including ex-Soviet nations), and Oceania, and lower incidence in South America, Africa, and Southern Asia ${ }^{7}$. Although incidence had generally remained consistent in most developed nations, mortality is slowly declining, although this is primarily observed in long-industrialised nations with developed healthcare systems (Figure 1.1 a-d).

Although the incidence of CRC is highest in older populations (median age at diagnosis is 70 years), it may affect any age, although is uncommon under the age of 50 years. The increased risk of CRC in later life is the primary factor in the design of the national bowel screening programme in the UK, which offers screening in the form of faecal occult blood testing to both men and women over the age of 60 years, or a one-off colonoscopy to those of 55 years (regional variation dictating). The age at which screening begins is however under current review and is likely to decrease to 50 years in the near future. Screening ceases at 74 years of age. By 2012, the UK screening programme had identified almost 15,000 cancers, of which a significantly higher proportion were found at an earlier stage than in the un-screened population $^{8}$. Uptake of screening is generally good (approximately 55\%), and an up to $18 \%$ reduction in mortality has been reported ${ }^{9}$. 
Figure. 1.2 Incidence (a \& b) and mortality (c \& d) of CRC (male and female) - selected countries. WHO International Agency for Research on Cancer: Global Cancer Observatory (http://gco.iarc.fr ) (accessed 28 ${ }^{\text {th }}$ August 2018).

a.

Colon, rectum and anus

Age Standardised Incidence Rate (World), Female age [0-85+]

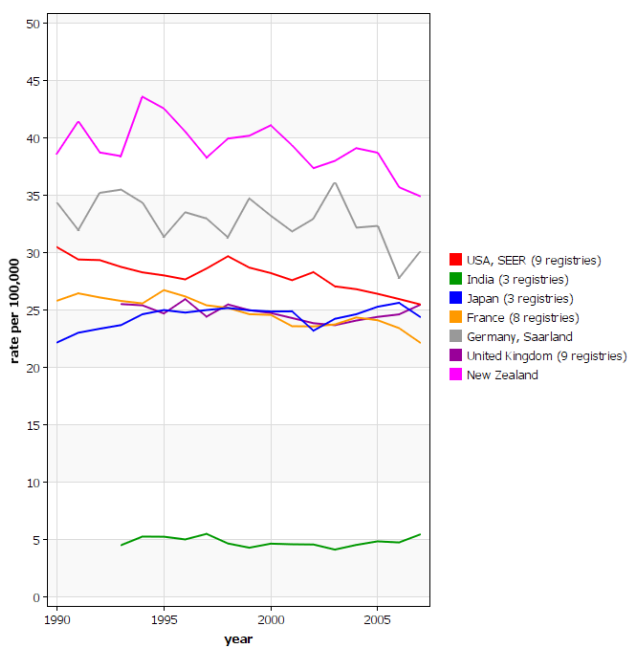

c.

Mortality from Colon, rectum and anus cancers Age-standardised rate (World): Female, all ages

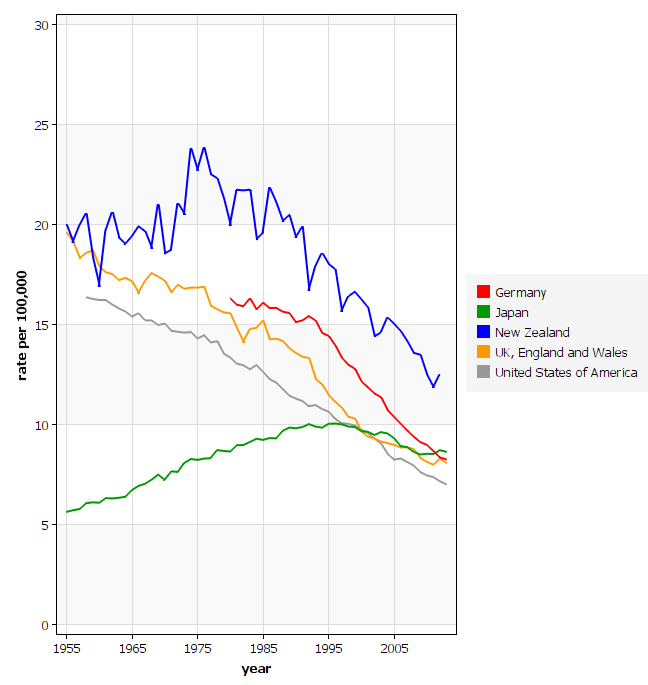

b.

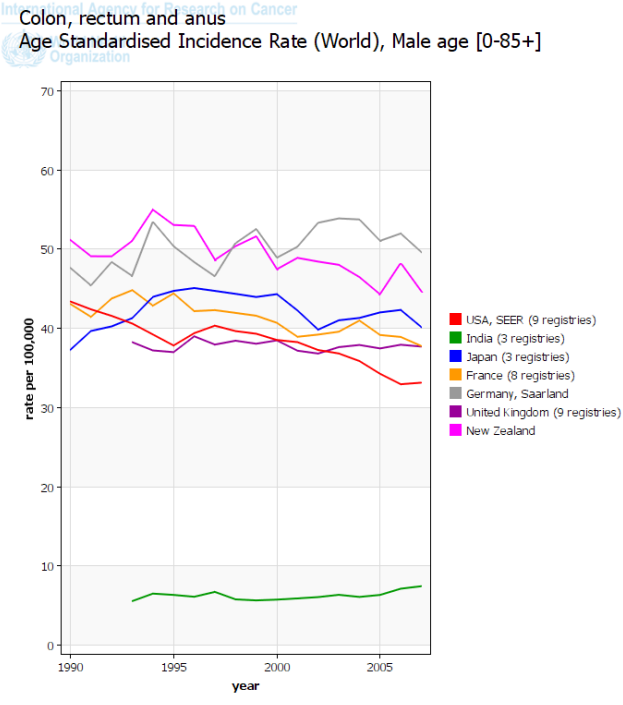

d.

Mortality from Colon, rectum and anus cancers Age-standardised rate (World): Male, all ages

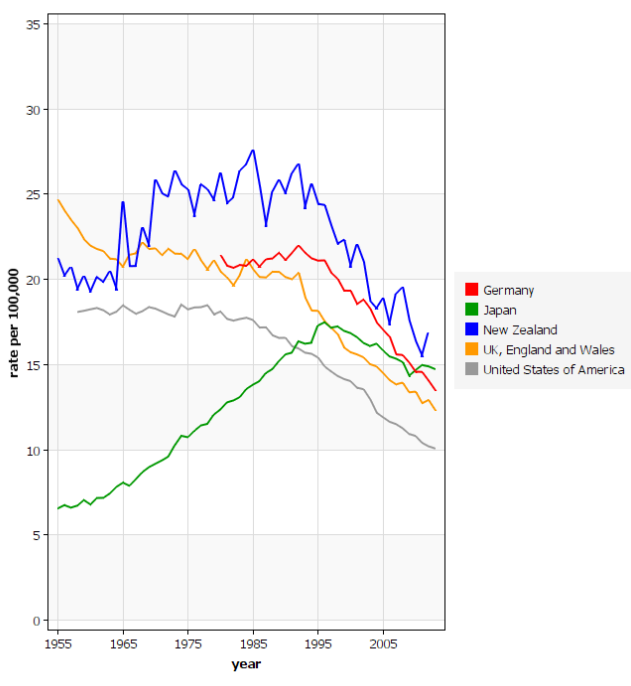




\subsubsection{Aetiology of Colorectal Cancer}

The primary unmodifiable risk factor in CRC is age; the median age at diagnosis being 70 years in Western societies ${ }^{10}$. The association with increased age is due to cumulative generalised DNA and cell damage that occurs during ageing, although specific aberrations at the genomic, epigenomic, transcriptomic, and other mechanistic levels are constantly being hypothesised, tested, and proposed. Colorectal cancers affecting younger patients are more frequently encountered in the hereditary cancer syndromes, which account for $2-5 \%$ of all $\mathrm{CRC}^{11}$. These syndromes are frequently characterised by the presence of polyps and are associated with highly penetrant inherited mutations and clinical syndromes, such as Lynch syndrome, familial adenomatous polyposis, MUTYH-associated polyposis, and hamartomatous polyposis. The detection of such syndromes through familial screening, given the high risk of CRC, frequently prompts prophylactic colectomy, although a significant proportion present sporadically ${ }^{12}$. As well as the small percentage of hereditary CRC associated with specific syndromes, it is estimated that a further $20-30 \%$ of CRC is linked to heritable mutation(s), although the loci are not characterised ${ }^{13}$.

A further significant non-modifiable risk factor for the development of CRC is inflammatory bowel disease (IBD: Crohn's and ulcerative colitis), although the incidence has been reducing in recent years. The pathogenesis of CRC in IBD is due to chronic inflammation of the colon and rectum, and thus anti-inflammatory therapies for IBD are effective at reducing the lifetime risk of developing cancer. Correspondingly, the severity of inflammation, length of exposure, and extent of colitis are all positively correlated with risk of $\mathrm{CRC}^{14}$. For ulcerative colitis the incidence is approximately 3 cases of CRC per 1000 patient-years ( $0.3 \%$ annually), corresponding to approximate risk of $2 \%, 8 \%$, and $18 \%$ at 10,20 , and 30 years diseaseexposure, respectively ${ }^{15}$. The corresponding risk in Crohn's disease is $3 \%, 5.5 \%$, and $8 \%$, respectively ${ }^{16}$.

Beyond non-modifiable risk factors, it is estimated that $54.3 \%$ of CRC is preventable due to population-level exposure to numerous modifiable risk-factors ${ }^{17}$. Foremost amongst these factors are smoking, obesity, lack of dietary fibre, and consumption of red/processed meat; contributing $6.8 \%, 11.5 \%, 28.0 \%$, and $12.5 \%$ to the excess cancer burden, respectively, based upon a population attributable fractions analysis. Alcohol (dose dependent ${ }^{18}$ ), ionising radiation, and insufficient exercise contributed smaller but measurable effects. 
The risk of developing CRC from smoking is directly proportionate to the number of cigarettes smoked per day, the number of years an individual has smoked, and the pack-year metric; and is measurable as a relative risk (RR) based on meta-analysis as 1.20 (95\% confidence interval (CI): 1.10-1.30), compared to never-smokers. The effect is greater in men than women (RR 1.38 vs 1.06$)$ and is persistent following cessation of smoking ${ }^{19}$. Smokers (RR 1.26) and former-smokers (RR 1.11) also suffer a significantly higher all-cause mortality following a diagnosis of CRC compared to never-smokers, based on a random effects hazard ratio model ${ }^{20}$.

Obesity (BMI $\geq 30$ ), and to a lesser extend overweight (BMI $\geq 25.0-29.9)$, are independent risk factors for CRC, especially in men. Of particular concern is visceral/abdominal obesity, where each increase in BMI of $1 \mathrm{~kg} / \mathrm{m}^{2}$ infers an increased risk (RR 1.03) $)^{21}$. Various mechanisms are suggested, including metabolic syndrome and the effects of insulin resistance, and dysregulating of adipocytokines. Hand-in-hand with obesity, type-2 diabetes has also been demonstrated to show an increased risk of CRC (Hazard Ratio (HR) 1.26, CI 1.18-1.33), with risk increasing with duration of risk-exposure ${ }^{22}$.

The role of dietary fibre in reducing the risk of colorectal cancer has long been the subject of speculation, and a number of mechanisms have been postulated, including bulking and dilution of luminal toxins, decreased transit time, and short-chain fatty acid metabolism of fibre by the microbiome, amongst others ${ }^{23}$. A meta-analysis of dietary fibre intake and risk of developing CRC demonstrated that there was an inverse correlation between increased fibre intake and risk, and that this correlation was dose dependent $(10 \% \mathrm{RR}$ reduction per $10 \mathrm{~g} /$ day total dietary fibre $)^{24}$. The effects were observable for total dietary fibre, wholegrain fibre, and cereal fibre, but no association was discernible with fibre sourced from fruit, vegetables, or legumes. The authors of this interesting analysis suggest that the bulking and decreased transit time associated with cereal fibre may be causal in this relationship, although they do admit that there is likely to be an element of confounding due to the insulin-sensitising and excess-weight preventing effects of a high-fibre diet.

Consumption of red (fresh) and processed meat is associated with an increased risk of developing $\mathrm{CRC}$, although the data is somewhat heterogeneous ${ }^{25}$. In a recent meta-analysis, consumption of one portion of processed red meat per day was associated with a pooled increase in CRC risk (HR 1.15, CI 1.01-1.32), but this increased for tumours located in the distal colon and rectum (HR 1.36, CI 1.09-1.69) ${ }^{26}$. Interestingly, the data for fresh red meat 
suggested a reduced risk of distal cancers (HR 0.75, CI 0.69-0.82), but a possible increased risk for proximal cancers (statistically non-significant). Although the mechanism for these effects remains contentious, the addition of nitrates and nitrites in processed meat, and the increase in mutagenic heterocyclic amines caused by cooking (and especially burning) meat are proposed as the most likely mechanisms ${ }^{27,28}$.

\subsubsection{Staging and Histopathological Standards in Colorectal Cancer}

Current standards for the staging of colorectal cancer are set by the American Joint Committee on Cancer (AJCC), $8^{\text {th }}$ Edition ${ }^{29,30}$, and were formally adopted for practice in the UK from January 2018 (the previous 2 editions having been bypassed due to lack of supporting evidence $)^{31}$. Broadly, tumours are staged dependent on their depth of invasion through the bowel wall ( $\mathrm{T}$ stage), by lymph node spread ( $\mathrm{N}$ stage), and metastatic spread (M stage). A composite of these three scores (TNM) is then used to provide an overall Stage (I-IV), which is intended to stratify survival related to the disease. The gold-standard for staging is histopathological examination of the resected tumour, although at different stages of the patient's treatment staging may be performed using other modalities (such as MRI), or following therapies (such as neoadjuvant chemo-radiotherapy). To aid in differentiating how staging has been performed a prefix may be added to the TNM system to indicate modality (e.g. $p$ : pathological staging; $c$ : clinical staging), or to indicate whether therapies have been applied (e.g. yp: pathological staging post chemo-/radiotherapy).

Historically, the Dukes' and Bussey Classification (Stages A-C) has been used to characterise the local and nodal extent of colorectal cancer, with the later addition of Stage D by Turnbull to denote metastatic spread ${ }^{32,33}$. This system is still advocated for use in parallel with the AJCC classification by the Association of Coloproctology of Great Britain and Ireland (ACPGBI) in their current (2017) guidelines for the management of cancer of the colon, rectum, and anus ${ }^{34}$. To aid in the classification of colorectal cancers it is important that strict standards are set in the reporting of histopathological specimens. This is crucial not just in determining the extent of an individual's disease, and therefore their management, but also for audit, research, and strategy for the local, national, and international management of colorectal cancer. To this end a minimum-reported dataset is published by the Royal College of Pathologists (latest edition 2017), setting standards for histopathological examination of resected specimens ${ }^{35}$. As well as a macroscopic description of the tumour and the basic tumour characteristics that comprise the TNM classification system, the core dataset also includes information that may aid in 
prognostic stratification, such as the degree of tumour differentiation, the presence of mucin, and extra-mural and peri-neural invasion. Estimation of tumour regression following neoadjuvant therapy is also included in the minimum dataset (where appropriate) and is based upon the four-tier system (0-3) proposed by Ryan ${ }^{36}$. Increasingly, genetic and molecular biomarkers are also employed in the characterisation of colorectal cancers, and routine testing for mismatch repair (MMR) status (see 1.3.4) (and subsequently BRAF V600 E and/or MLH1 analysis) to distinguish sporadic cancers from Lynch Syndrome is now considered part of the core dataset for reporting colorectal cancers ${ }^{37}$. Additionally, testing targeted at genes associated with response to EGFR therapies (KRAS and NRAS codons) may be performed in patients with metastatic colorectal cancers to identify those who may benefit from treatment ${ }^{38}$.

\subsubsection{Survivorship and Outcomes in Colorectal Cancer}

Survival following diagnosis and treatment for colorectal cancer is primarily dependent on the stage at which the cancer was diagnosed, with earlier stage cancers translating into improved survivorship; and is the premise of colorectal cancer screening programmes. Approximately $80 \%$ of individuals diagnosed with colorectal cancer undergo surgery ${ }^{39}$. Data form the American Cancer Society suggest that the 5-year survival from Stage I colon and rectal cancer is approximately $92 \%$ and $88 \%$, respectively, falling to $12 \%$ and $13 \%$ respectively for Stage IV cancer ${ }^{40}$. This data is summarised in Table 1.1. Patients who are elderly, present as an emergency, or have a high number of comorbidities are at an increased risk of dying compared to the background risk ${ }^{10,41}$. For patients with Stage III or high-risk Stage II disease, the addition of adjuvant chemotherapy to surgery increases 5 -year survival across the population by $5 \%$, although we currently lack the ability to predict which individuals will benefit. Unfortunately, recurrence following treatment with curative intent in colorectal cancer is approximately $45 \%$, of which only $5-10 \%$ of patients may be eligible for further "curative" surgery ${ }^{42}$.

Beyond staging, the factors affecting the risk of recurrence are not well understood. Follow-up after treatment with curative intent is aimed at identifying recurrence early (especially lung and liver metastasis), and thus increasing the chances of surgical management or eliciting a better response to chemo- or immunotherapy. The detection of metachronous tumours and polyps is also facilitated by follow-up, as well as providing psychological and social support to potentially vulnerable patients ${ }^{43}$. There is some variation in the protocols for following-up patients, but a combination of CEA and CT-scanning at 6-monthly intervals initially, and then declining over time, is the mainstay of assessment, augmented with colonoscopy or other 
imaging modalities where necessary ${ }^{44}$. Recent meta-analysis has demonstrated that more intensive follow-up regimes have shortened the time to detection of recurrence by up to 10 months, although this has so far failed to translate into statistically significant population survival benefit ${ }^{45}$. Effective follow-up is also important for MDT and audit purposes, is key to delivery of many of these services in the Colorectal Clinical Nurse Specialist, and is reassuring for patients ${ }^{46}$.

Table 1.1. 5-year survival following diagnosis of colon or rectal cancer, by AJCC Stage ${ }^{40}$.

\begin{tabular}{|l|l|l|l|l|l|l|l|}
\hline AJCC Stage & I & IIA & IIB & IIIA & IIIB & IIIC & IV \\
\hline Colon & $92 \%$ & $87 \%$ & $65 \%$ & $90 \%$ & $72 \%$ & $53 \%$ & $12 \%$ \\
\hline Rectum & $88 \%$ & $81 \%$ & $50 \%$ & $83 \%$ & $72 \%$ & $58 \%$ & $13 \%$ \\
\hline
\end{tabular}




\subsection{Treatment for Colorectal Cancer}

\subsubsection{Surgery for Colorectal Cancer}

The primary therapy for colorectal cancer is surgery, with approximately $80 \%$ of patients diagnosed with CRC undergoing surgery with curative intent. The surgical strategy employed is based upon the anatomical location of the tumour(s) and the associated lymphovascular package. Segmental resection of the affected colon or rectum in association with its draining lymph nodes and vasculature reduces the local recurrence by excising the primary tumour plus any local macro- or micro-metastasis. Broadly, tumours of the right colon require right hemicolectomy, tumours of the left colon a left hemicolectomy or sigmoid colectomy, and tumours of the rectum an anterior or abdominoperineal resection. For metachronous tumours affecting the left and right hemicolons, or where a heritable or sporadic pro-carcinogenic fieldchange in the colonic mucosa is suspected or proved, excision of larger portions of or the whole colon (with or without the rectum) may be performed as a subtotal or total colectomy (or panproctocolectomy). If a tumour has invaded structures/organs beyond the normal plane of excision (T4), these may be amenable to resection en bloc. In the majority of cases, following resection of the affected colon or rectum, an anastomosis may be formed between the remaining proximal and distal bowel in order to restore gastrointestinal continuity, although a temporary or permanent ostomy may be constructed depending on a number of technical or patient factors.

Although the enduring principles of surgery for colonic cancer were laid down by Cuthbert Duke and Lockhart-Mummery at St Marks Hospital in the 1950's, and standards for rectal cancer surgery by Heald in 1982 in Basingstoke, recent advances in technique and perioperative management have improved outcomes for patients. Enhanced recovery after surgery (ERAS) programmes have been demonstrated to be safe and effective in reducing perioperative morbidity and reducing the length of hospital stay following surgery ${ }^{47}$. The administration of carbohydrate-rich drinks immediately prior to surgery has also been shown to reduce insulin resistance and facilitates gastrointestinal recovery ${ }^{48}$. The role of mechanical bowel preparation is still controversial, but a growing body of evidence suggests that it is unnecessary in the majority of situations, with rectal resection and the formation of a (low) colo-rectal anastomosis being the possible exception ${ }^{49}, 50$. However, as the role of the colorectal microbiome becomes better understood, and intraoperative fluorescence technologies become more widely adopted, the role and nature of mechanical (or microbiomal) bowel preparation will likely change $\mathrm{e}^{51,52}$. 
Laparoscopic techniques now have an accepted role in surgery for CRC. Laparoscopic resection of colonic cancers has been demonstrated to be equivalent to open resection in terms of R0 resection rate and nymph node harvest, but superior in terms of length of hospital stay, post-operative pain, wound healing, wound infection, blood loss, and risk of incisional hernia. The Association of Coloproctology of Great Britain and Ireland (ACPGBI) guidelines for the surgical management of colorectal cancer support the implementation of laparoscopic techniques in all suitable cases (recommendation grade $\mathrm{A})^{53}$. The case for laparoscopic surgery in rectal resection is less well evidenced. Although many of the same short-term advantages of laparoscopy may be apparent as for colonic resection, the ALaCaRT and ACOSOG Z5061 trials failed to demonstrate non-inferiority of laparoscopic surgery compared to open for core pathological criteria ${ }^{54,55}$. Despite these rather disappointing findings, the data is deemed insufficiently significant to prevent the application of laparoscopic techniques to rectal resection, and the ACPGBI support its implementation (recommendation grade B) ${ }^{53}$. As yet, there is no substantial evidence to support the use of robotic surgery in either routine colorectal cancer surgery or selected challenging rectal cancer cases, although long-term outcome data still awaited ${ }^{56}$.

\subsubsection{Emergent Surgical Strategies for Rectal Cancer}

Surgery for rectal cancer is increasingly being considered as a highly sub-specialised practice within general colorectal surgical practice. The challenges of operating on the rectum due to the confines of the bony pelvis and close proximity of significant neurovascular structures (e.g. iliac vessels and major branches, sciatic and obturator nerves) and other organ systems (e.g. male or female urogenital systems) requires specialist expertise and increasingly specialised techniques.

For large polyps and early cancers (T1 and early $\mathrm{T} 2)$ transanal techniques such as transanal endoscopic microscopic surgery (TEMS) and transanal minimally invasive surgery (TAMIS) have increased the options available for surgical excision and organ preservation ${ }^{57,58}$. Currently there is no consensus regarding whether one technique is superior to the other due to a lack of controlled trials and limited adoption, but early outcomes suggest that either technique is oncologically acceptable compared to traditional rectal excision ${ }^{59,60}$. For patients with more advanced disease that require formal rectal resection, but who may be predicted to be operatively challenging due to a low tumour position, narrow (usually male) pelvis, or obesity, a transanal total mesorectal excision (TaTME) may be performed with laparoscopic abdominal 
assistance. The aim of this technique is to perform the most challenging component of the procedure transanally, thus improving oncological resection and facilitating laparoscopic resection with the benefits of a faster return to GI function, shortening length of hospital stay, and lower postoperative morbidity ${ }^{61,62}$. Currently the evidence base for an advantage in performing TaTME is poor, although data submission to the centralised international registry is designed to alleviate this issue ${ }^{63,64}$.

Locally advanced rectal cancers (LARC) and locally recurrent rectal cancers (LRRC) pose additional challenges to surgeons as they extend beyond the planes of classical TME. As successful surgery is dependent upon an R0 resection it is frequently necessary to resect adjacent structures that are not typically included in a standard rectal resection, either as partial composite resections (e.g. portions of the posterior wall of the vagina, sections of sacral nerve, or anterior table of sacral vertebrae), or as whole organs (e.g. total pelvic exenteration) ${ }^{65,66}$. Evolving techniques in surgery (resectional and reconstructive), as well as improved perioperative care (physiotherapy, clinical psychology, etc) are driving advances to increasingly extensive resections ${ }^{67,68}$. Again, due to limited numbers of patients undergoing beyond-TME excisions for LARC and LRRC the evidence base is not strong, and thus international collaborative work is aimed resolving this deficiency ${ }^{69}$.

The converse side of the management paradigm from exenteration is the possibility for organ preservation and watch-and-wait strategies, largely brought about due to advances in neoadjuvant therapies ${ }^{70,71}$. Up to $30 \%$ of patients treated with nCRT may experience a complete pathological response (pCR) and benefit from a watch-and-wait strategy of nosurgery and close observation, and for those who do not undergo pCR but do undergo downsizing/down-staging an organ preserving surgery such as TEMS or TAMIS may be appropriate $^{72-74}$. Currently, however, there is no reliable method of predicting which patients will respond to $\mathrm{nCRT}$ before it is administered and there is no level I evidence supporting a watch-and-wait strategy. Many patients will not respond or progress during nCRT treatment, some becoming technically inoperable or medically unfit for surgery, and for those who do have a good response there is much debate as to the best interval between therapy and surgery ${ }^{75}$. The current basis for predication and assessment of response is MRI, although PET-CT and endorectal ultrasound (ERUS) also have a role ${ }^{36}$. Additional tumour and patient factors including new molecular means of stratification of risk and response are being sought ${ }^{76,77}$. 


\subsubsection{Chemotherapy for Colorectal Cancer}

Chemotherapy has three roles in the management of colorectal cancer; prior to surgery to increase the operability of tumours and outcomes following surgery (neoadjuvant chemotherapy), following surgery with curative intent to reduce the chance of recurrence (adjuvant chemotherapy), and in the metastatic or palliative setting.

The role of neoadjuvant chemo-radio therapy (nCRT) in rectal tumours where the resection margin is threatened or breached is established ${ }^{78-80}$. Data from the FFCD 9203 trial demonstrated that local recurrence rates were halved from $16.5 \%$ to $8.1 \%(\mathrm{p}<0.05)$ and $\mathrm{pCR}$ improved from $3.4 \%$ to $11.6 \%(\mathrm{p}<0.05)$ with the addition of neoadjuvant chemotherapy to radiotherapy, although overall 5-year survival was not improved ${ }^{79}$. However, the NSABP R03 trial demonstrated that an improvement in DFS was achievable, with trend towards increased $\mathrm{OS}^{81}$. There is currently a degree of variation between guidelines for administration of nCRT, although long-course 5-fluorouracil (5-FU) or capecitabine neoadjuvant chemotherapy combined with radiotherapy (45-50.4 Gy) is the basis of most protocols. Patients deemed at lower risk or unsuitable for long-course therapy may be offered short-course protocols based on local guidance, although PCR rates are lower in this group ${ }^{82}$. The role of adjuvant CRT following nCRT is contentious, especially given the EORTC 22921 trial of over one thousand patients, where no DFS or OS advantage was observed with the addition of CRT following neoadjuvant therapy ${ }^{83}$. Currently there is no role for neoadjuvant chemotherapy for the management of colon cancer, although this is the subject of ongoing clinical trials led by the FOxTROT Collaborative ${ }^{84}$. Early outcomes from this study suggest that for locally advanced but operable colon cancers (T3/4), neoadjuvant chemotherapy and sensitivityspecific immunotherapy improves 2-year recurrence rates (personal communication unpublished).

Adjuvant chemotherapy for colorectal cancer should be offered to all patients with high-risk Stage II and all Stage III disease who have undergone surgery with curative intent to reduce the chance of local and systemic recurrence ${ }^{85}$. The current guidance offers a choice of a sixmonth course of capecitabine as monotherapy, or folinic acid with 5-FU and oxaliplatin (FOLFOX), to begin within six weeks of the date of surgery ${ }^{86}$. A pooled analysis of randomised trial data for both regimes (including DFS as well as relapse-free survival (RFS)) indicates an increase in DFS at 5-years from $42 \%$ to $58 \%$, and an increase in OS from $51 \%$ to $64 \%$, compared for surgery alone for stage III colon cancer. The same regimes are employed 
for high-risk stage II and stage III cancers of both the colon and rectum, despite the majority of evidence pertaining to stage III colon cancers. The choice between regimes is determined between the patient, clinician, and local protocols, as there is not significant evidence for superiority between regimes on either a clinical effectiveness or cost effectiveness analysis, although the primary cited evidence is limited to only three trials (X-ACT, MOSAIC, and NSABP C-07) ${ }^{87-89}$. Capecitabine is a precursor of 5-FU and can be administered orally in tablet form or by intravenous infusion. Its primary mechanism of action is as an inhibitor of thymidylate synthase and thus preventing the synthesis of thymidine; a nucleotide required for DNA replication. As this action is not cancer-specific other rapidly regenerating tissues such as the normal gastrointestinal mucosa may be affected, resulting in common side effects such as diarrhoea, abdominal pain, nausea and vomiting, and mucositis. More rarely hand-foot syndrome and myelosuppression may occur. Each of these side-effects may be dose-limiting, and administration is contraindicated in myelosuppression and severe hepatic or renal impairment. Oxaliplatin is a platinum-based agent administered by intravenous infusion that prevents DNA replication by causing DNA cross-linking. As well as myelosuppression and gastrointestinal side-effects, the most common side-effect of oxaliplatin is peripheral neuropathy, which may have a cumulative effect over the course of multiple administrations.

The chemotherapy treatment options for patients with locally advanced and/or metastatic colorectal cancer, whether potentially operable or not, are multiple and therefore local protocols and patient preference determine practice ${ }^{85}$. First- and second-line regimes combine FOLFOX protocols with irinotecan as a single agent or in combination with 5-FU (FOLFIRI). Capecitabine and oxaliplatin in combination may also be used as a first-line therapy, followed by FOLFIRI. Irinotecan is a plant-derived cytotoxic alkaloid that inhibits both DNA replication and transcription by interference with the topoisomerase-1 enzyme, which is responsible for regulating DNA double-helix winding during these processes. For patients intolerant of 5-FU based regimes raltitrexed may also be considered, its action similarly focused on thymidylate synthase although by a different action and having a different side effect profile ${ }^{90}$. Tegafur (a 5-FU pro-drug) is also licensed for use as a first-line therapy in advanced colorectal cancer but is not normally preferred over FOLFOX or capecitabine regimes. 


\subsubsection{Biological Therapies for Colorectal Cancer}

Patients with metastatic colorectal cancer may benefit from monoclonal antibody $(\mathrm{mAb})$ therapy if they over-express epidermal growth factor receptor (EGFR) and are (K)RAS (Kirsten RAt Sarcoma oncogene) wild-type ${ }^{91}$. Both cetuximab (chimeric m-IgG1) and panitumumab (human recombinant $\mathrm{m}-\mathrm{IgG} 2$ ) act by specifically binding EGFRs and blocking the activating ligand, thus inhibiting activation of tyrosine kinase and a cascade of intracellular signalling pathways that promote DNA synthesis and cell proliferation. Both agents in combination therapy with FOLFOX have been shown in clinical trials (OPUS and PRIME) to be superior to FOLFOX alone in terms in improved PFS and OS, although the numbers of patients benefiting from therapy were more modest than anticipated based on RAS status ${ }^{92,93}$. One possible reason for mAb therapy response to correspond poorly to RAS status is the discovery of an increasing number of RAS mutations that are outside the standard analysis (RAS G13D and NRAS, for example), thus leading to unexpected EGFR therapy resistance. These findings have affected several recent trials and may steer management towards a smaller proportion of patients being eligible for $\mathrm{mAb}$ therapy, but with a higher response rate ${ }^{94}$. Two further $\mathrm{mAb}$, bevacizumab and aflibercept, which are targeted towards the vascular endothelial growth factor receptor (VEGF), have also reported outcomes that do not indicate superiority to more conventional regimes and are thus not recommended by NICE for patients with metastatic $\mathrm{CRC}$, although they remain licensed ${ }^{95-98}$.

\subsubsection{Radiotherapy for Colorectal Cancer}

Radiotherapy in the context of colorectal cancer is predominantly focused on the neoadjuvant treatment of locally advanced or locally recurrent rectal cancer ${ }^{65}$. Long-course neoadjuvant chemoradiotherapy (CRT) has been the established preference for T3/4 N1/2 rectal cancers for over 20 years, and in association with TME offers the best chance of complete excision and increases OS \& $\mathrm{DFS}^{81,99}$. Strategies aimed at increasing the dose and accuracy of pre-/intraoperative radiotherapy have been trialled, notably in the form of rectal brachytherapy and intraoperative radiotherapy, although results have not been conclusive and currently these practices are largely confined to trials ${ }^{100-102}$. There is however a shifting attitude towards radiotherapy for LARC with the advent of increasingly effective chemotherapy and immunotherapy regimes ${ }^{103}$. One possibility is that chemotherapy alone may be as effective as CRT, and thus negate the requirement for radiotherapy altogether, theoretically reducing the risk of poor wound healing, anastomotic breakdown, and more challenging operating planes, and is the subject of the ongoing PROSPECT trial ${ }^{104}$. Another perspective is that radiotherapy should be 
applied in the neoadjuvant setting only after neoadjuvant chemotherapy has been administered and had an opportunity to cause tumour involution; a methodology referred to as induction chemotherapy. This approach is currently undergoing investigation in a number of different trials, including the E-LARC Study and UK BACCHUS trial ${ }^{105,106}$. Radiotherapy may also be employed in the palliative setting, especially where symptoms such as per-rectum bleeding are distressing to patients, or for LRRC where the pelvis has not previously been irradiated.

\subsubsection{Associated Therapies in Colorectal Cancer}

In addition to surgery and clinical- and medical-oncological therapies, a range of other strategies may be employed to treat CRC. Colonic stents are becoming an increasingly versatile tool in combating colorectal cancer, especially in the frail and elderly, palliative, or emergency setting (up to $20 \%$ of presentations) as a bridge to definitive therapy. Early outcomes form the CReST trial found that mortality and length of hospital stay were no different between emergency surgery and stent groups, but that the rate of permanent stoma formation was significantly lower in the stent group $(45 \% \text { vs } 69 \%, \mathrm{p}<0.001)^{107}$. Oncological outcomes are awaited. The Enhanced Recovery After Surgery (ERAS) programme has also helped deliver improvement in perioperative care, especially with respect to facilitating a reduced time to normal gastrointestinal function, early return to mobility, and reduced length of inpatient admission. These improvements have been won by standardising care as much as possible, improving patient education, early enteric feeding, reducing unnecessary drain and nasogastric tube use, early mobilisation, optimising perioperative pain management, and auditing of practices ${ }^{47}$. Carbohydrate loading, optimisation of the use of blood products, advanced stoma care, and venous thromboembolism prophylaxis (VTE) are also important considerations in delivering high quality perioperative care and all are included in current management guidelines ${ }^{53}$. 


\subsection{The Genetic \& Genomic Basis of Colorectal Cancer}

\subsubsection{The Vogelstein Model}

The development of sporadic colorectal cancer has long been considered as a step-wise progression along the adenoma-to-carcinoma pathway, and the genetic processes underlying this sequence were formulated by Vogelstein ${ }^{108,109}$. The basis of this model is that sequential mutation in the adenomatous polyposis coli (APC) gene, and subsequent KRAS and TP53 mutations leads to increasing adenomatous changes in the colonic mucosa, resulting in polyps and eventually to dysplasia and invasion.

The first sequence in this pathway is brought about by APC mutation and subsequent dysregulation of the $\mathrm{Wnt} / \beta$-catenin canonical signalling pathway. APC mutation leads to inappropriate intracellular accumulation of $\beta$-catenin due to inactivation of the destruction complex, resulting in activation of transcription factors governing the cell cycle. Mutations in this pathway are detected in up to $90 \%$ of sporadic colorectal cancers, and specific autosomaldominant inherited mutations in APC are responsible for familial adenomatous polyposis $(\mathrm{FAP})^{110}$. Secondly, KRAS is a proto-oncogene that regulates the cell cycle by activating growth factors, cell signalling, and glucose transportation via the MAPK-pathway. When mutated, deactivation and subsequent negative-signalling is disrupted leading to increased signalling and uncontrolled cellular proliferation ${ }^{111}$. The important role of KRAS in CRC therapy is underscored by its impact on the efficacy of EGFR immunotherapies as discussed in Section 1.2.4, although the relatively high proportion of patients with advanced cancers who are KRAS wild-type demonstrates that it is not a necessary mutation in the classical pathway. Thirdly, the role of the heterozygosity in TP53 gene (and its related protein p53) is established in tumour suppression, where its acts to stimulate DNA repair, arrest the cell cycle, initiate apoptosis, and moderate telomere response. Loss heterozygosity is associated with a number of different cancers, including CRC (also breast, endometrial, and renal tumours), although the association with mutation and risk of cancer is not linear ${ }^{12,113}$. However, in the colorectal adenoma to carcinoma pathway, loss of TP53 function is detected in approximately $25 \%$ of adenomas with no adverse features and $50 \%$ of adenomas with foci of invasion, underlining its likely causative contribution to carcinogenesis ${ }^{114}$. Additional mutations in PIK3CA (a phosphorylation-related kinase oncogene) and allelic deletion of segments of chromosome $18 \mathrm{q}$ encoding the DCC (deleted in colorectal carcinoma) gene (tumour suppressor) cumulatively drive carcinogenesis as a core set of oncogenic mutations ${ }^{115}$. 
Figure 1.3 The multi-step genetic model of colorectal carcinogenesis (adapted from Pino, et. al.) ${ }^{115}$.

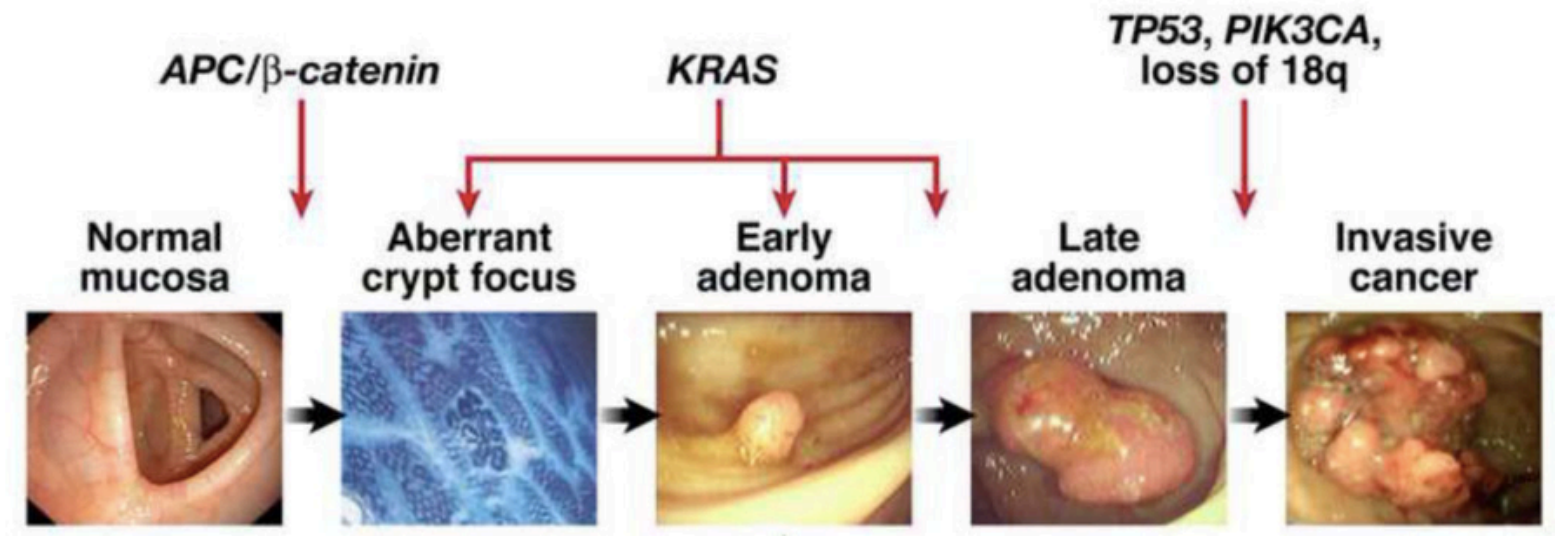

These cumulative genetic insults are thought to underlie many colorectal cancers, although due to the relatively short lifespan of individual colorectal mucosal cells and the low background rate of mutation, the likelihood of any single cell developing the multiple mutations required to undergo carcinogenic change is limited. What underlies the accelerated process of mutation, therefore opening the opportunity for multiple tumourigenic transformations, is loss of genomic stability ${ }^{116,117}$. There are several processes thought to underlie the development of genomic instability, each of which may contribute to malignant transformation, but in the context of CRC the primary factors are microsatellite instability (MSI) and chromosomal instability $(\mathrm{CIN})^{118}$.

\subsubsection{Key Signal Transduction Pathways in CRC}

Several key signal transduction pathways play an important role in CRC carcinogenesis, and are implicated in the Vogelstein and other models of cellular dysregulation. Although frequently presented as isolated pathways, these signal transduction pathways are in fact complex webs that govern cellular homeostasis; controlling vital processes such as cellular proliferation, apoptosis, cell-cell adhesion, and metabolism. The primary genes/proteins governing signal transduction pathways in CRC are APC/ $\beta$-catenin, EGFR, KRAS, and BRAF. A schematic representation of these pathways and their interdependent relationships is demonstrated in Figure 1.3.

Activation of Wnt signalling through APC mutation, typified by the germline mutations that define FAP, is an important initiator of CRC carcinogenesis ${ }^{108}$. APC dysfunction is identified in $70 \%$ of $\mathrm{CRC}$, and has widespread intracellular effects, including regulation of cellular proliferation, differentiation, apoptosis, and chromosomal segregation, although its primary 
pathological effect when dysfunctional is to increase cytoplasmic and nuclear $\beta$-catenin and thus drive cell proliferation and invasion through activation of T-cell factor/lymphoid enhancer factor (LEF) transcription factors ${ }^{119,120}$. Downstream tumourigenic effects of increased LEF activity are modulation of tissue remodelling via the urokinase receptor, plasminogen activation system, and tight-junction proteins ${ }^{120}$.

The epidermal growth factor receptor (EGFR) is a transmembrane protein which acts as a receptor for extracellular ligands, binding of which modulates intracellular signalling cascades that are intimately associated with cell proliferation, adhesion, and migration ${ }^{121}$. As discussed previously, EGFR has a role in the biologic therapy of colorectal cancers, the efficacy of which may be determined by KRAS and BRAF mutation. Modulation of EGFR activity, its downstream signalling pathway, and other associate genes is an active research area for novel chemotherapeutics $^{122-124}$.

The KRAS (Kirsten Rat Sarcoma Viral Oncogene Homolog) gene encodes a small GTPase that is primarily involved in regulating cell division, acting as an on-off switch for intracellular signal transduction via both vertical (nuclear-directed) and horizontal (cytoplasmic) pathways. The role of KRAS in CRC has long been established and forms a central tenet of the Vogelstein model of carcinogenesis ${ }^{109}$. When mutated, the negative (inhibitory) effects of the RAS are lost, allowing cell proliferation and inhibition of apoptosis. KRAS mutations are very common in colorectal cancers (35-40\%), especially those located in the proximal colon, and form a distinct morphological subset associated with polyps, mucin formation, and MSI ${ }^{125,126}$. As discussed in Section 1.2.4, KRAS mutations are important in determining the sensitivity of CRC to biologic therapies

$B R A F$ is a gene located on chromosome $7 \mathrm{q} 34$ that encodes a $94 \mathrm{kDa}$ protein called serine/threonine-protein kinase B-Raf, which acts as the common lead point for the RAS-RAFMEK-ERK-MAP kinase signalling pathway [modulated by PKC as well as RAS] ${ }^{127}$. As well as being associated with a poor prognosis in CRC, BRAF mutations are also associated with $15 \%$ of all human cancers, as well as some birth defects ${ }^{128}$. Activation of proto-oncogenes, particularly those associated with apoptosis and programmed cell death, underlies BRAF's role in carcinogenesis, and may be associated with CIMP, particularly in the frequently encountered BRAFV600E mutant ${ }^{129,130}$. BRAF-dependent signalling is also a potential therapeutic target in cancers, with successful biologic therapies having been developed for treatment of melanomas ${ }^{131}$. Currently, there are no specific therapies directed against this pathway for 
BRAF-mutant CRC, although the role of biologic agents for metastatic CRC is being explored $^{132,133}$.

Figure 1.4 Schematic representation of APC, EGRF, KRAS, and BRAF signal transduction pathways in relation to CRC gene regulation and apoptosis. (adapted from Lodish, et. al.) ${ }^{134}$

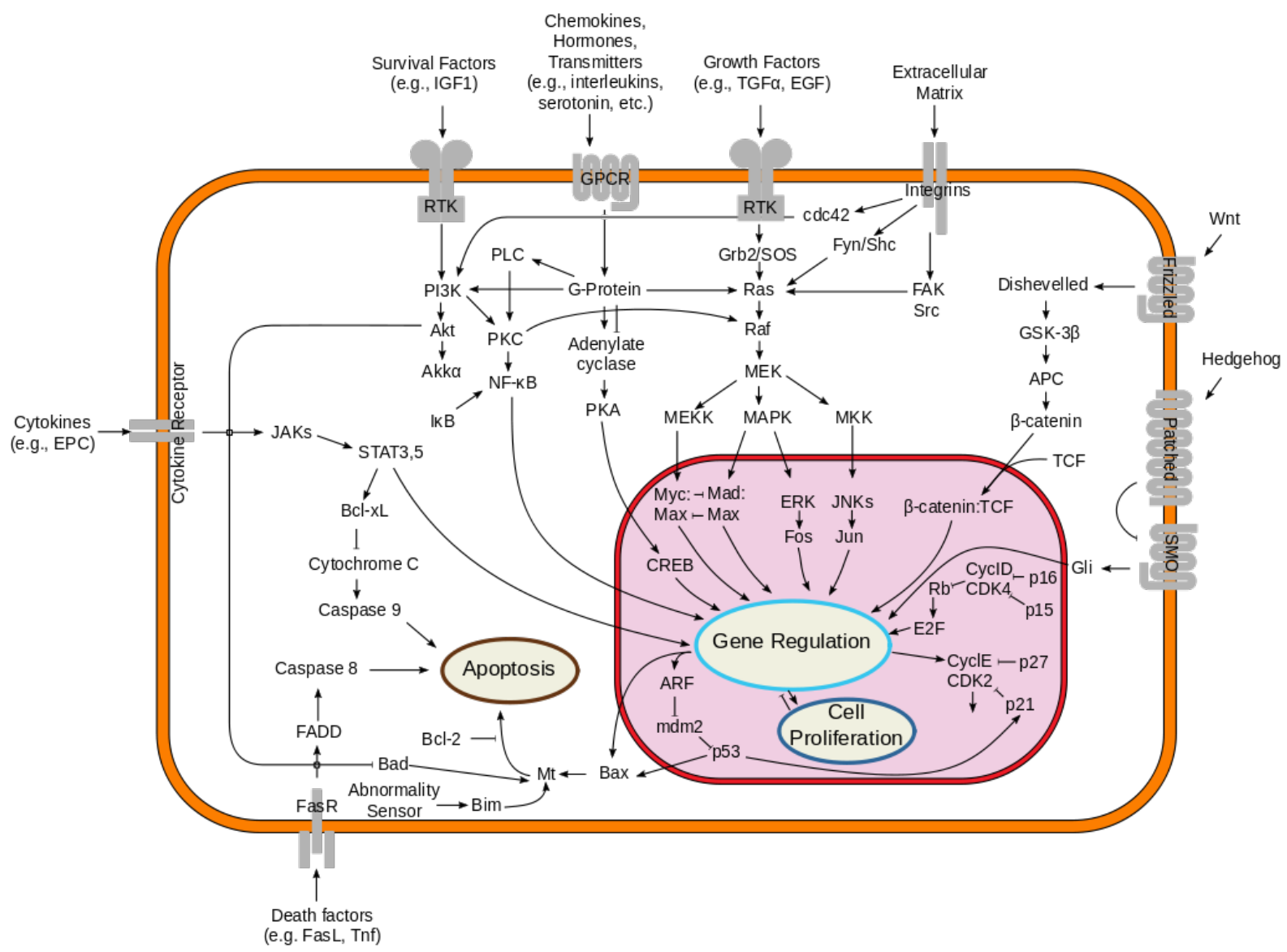




\subsubsection{Tumour Suppressors - TP53 \& 18q}

TP53 is a gene located on the short arm of chromosome 17 that codes for a corresponding protein (p53) which has a critical role in tumour suppression. The majority of mutations are missense mutations that occur in a number of common loci; leading to inactive and long-lived proteins. In response to a wide range of signals, including oxidative stress, DNA damage, and abnormal intracellular signalling (including RAS), p53 coordinates a multitude of cellular actions by initiating the transcription of genes associated with cellular metabolism, apoptosis (especially caspase regulation), cell cycle regulation, immunological signalling and response, and cell differentiation ${ }^{135}$. p53 mutations are widely reported in human cancers, and are present in at least $70 \%$ of invasive CRC, as well as $25 \%$ of adenomas and $50 \%$ of polyp cancers, indicating the early and likely necessary contribution to CRC formation ${ }^{114}$.

Approximately $70 \%$ of CRC demonstrate allelic loss at chromosome 18q, although the suspected tumour suppressor genes located here have not been demonstrated to be common in $\mathrm{CRC}^{136}$. Examples of such genes are the unfortunately named Deleted in Colorectal Cancer gene (DCC), which is only found in $6 \%$ or tumours, and the SMAD2 (20\% of tumours) and SMAD4 (10\% of tumours) genes involved in TGF- $\beta$ signalling ${ }^{137,138}$. Loss of heterozygosity of the Cables gene, which is located on chromosome 18q, has however been associated with colorectal cancer $(70 \% \mathrm{LOH})$, although its role in carcinogenesis is not well defined ${ }^{139}$.

\subsubsection{Microsatellite Instability \& Mismatch Repair}

Microsatellite Instability (MSI) describes a situation linked to defective mismatch repair (MMR) where repeating motifs of non-coding DNA are introduced during the S-phase of DNA replication, leading to cells demonstrating a high degree of mutability ${ }^{140}$. Mutations may take the form of single base substitutions or short insertions or deletions, which are accumulated over time to form multiple-repeated motifs known as microsatellites (typically 1-6bp, repeated up to 50 times). The most common microsatellite in human DNA is the dinucleotide repeat of cytosine and adenine (i.e. CACACACACACACA...), leading to a frame-shift mutation in the daughter strand of DNA and subsequent protein inactivity ${ }^{141}$. International standards for the diagnosis and classification of MSI were developed by international consensus and depend on a panel of five validated microsatellites for clinical and research use ${ }^{142}$. Tumours may be classified as MSI-high (MSI-H), $\geq 2 / 5$ microsatellites; MSI-low (MSI-L), 1/5 microsatellites; or MSI-stable (MSI-S), $\leq 1 / 5$ microsatellites but dependent of further characterisation. 
Approximately $15 \%$ of CRC express MSI (12\% sporadic, 3\% Lynch syndrome); predominantly due to defects in the mutL homologues (MLH1 \& MLH3) and mutS homologues (MSH2, MSH3, MSH6) involved in $\mathrm{MMR}^{143}$. CRCs expressing a high degree of MSI (MSI-H) have a slightly better prognosis compared to those with an MSI stable or low (MSI-S / MSI-L) phenotype ${ }^{144}$. Autosomal-dominant inherence of mutations in MMR genes (primarily MSH2, MLH1, and MSH6) characterises Lynch syndrome (Hereditary NonPolyposis Colorectal Cancer - HNPCC), a heritable CRC syndrome which carries an $80 \%$ lifetime risk of $\mathrm{CRC}^{145}$. MSI is also associated with hypermethylation, particularly $\mathrm{CpG}$ island methylations phenotype high (CIMP), often affecting MMR genes such as MLH1, leading to silencing ${ }^{146}$. This form of genomic instability is most commonly associated with the serrated pathway and BRAF mutations that are more commonly observed in the right colon, although many of the gene mutations are shared with the classical adenomatous pathway found throughout the colon and rectum. Where this sub-type of tumour is found within the rectum it may represent a less favourable molecular subtype ${ }^{125,147-150}$.

\subsubsection{Chromosomal Instability (CIN)}

CIN is a process where portions of chromosomes are either deleted or amplified asymmetrically, leading to an asymmetric distribution of DNA on mitosis and subsequent aneuploidy of daughter cells. It should be noted that errors in chromosomal distribution during mitosis are common in normal tissues (approximately 1\%), so for criteria to be met for CIN an aneuploidic mitosis must occur in at least $20 \%$ of divisions ${ }^{151,152}$. This must be assessed based on an evaluation of a population of cells in reference to a standard population, rather than observations of individual cells without reference. CIN results from mutations affecting several processes: Chromosome segregation defects, typified by anaphase promoting complex/C dysfunction as a result of MAD (Mitotic Arrest Deficient) and BUB (Budding Uninhibited by Benzimidazoles) mutation; telomere dysfunction resulting in a failure of cells to naturally undergo senescence; and loss of DNA damage response characterised by loss of TP53 function $^{115}$.

CIN is present in up to $70 \%$ of sporadic CRC, although the methods of determining CIN and the subsequent differences in classifying CIN has led to some discrepancy across the body of literature, although CIN is generally determined as a binary feature of tumours (CIN-positive or CIN-negative) $)^{153,154}$. However, the cellular processes that underlie CIN are not mutually exclusive of those that drive MSI and CIMP, and thus a proportion (up to 25\%) of CRC will 
demonstrate an overlapping picture of $\mathrm{CIN}$ and $\mathrm{MSI}^{155}$. CIN-positivity is associated with a worse outcome in CRC; meta-analysis demonstrating higher hazard-ratios for later stage and decreased progression-free survival for CIN-positive tumours ${ }^{156}$. The specific cellular pathways that lead to colorectal carcinogenesis are shared between the genomic aberrations; dysfunction in EGFR, BRAF, and KRAS are again implicated as the victims of CIN within the "mutator phenotype" population of cells that have developed intrinsic genomic instability ${ }^{157}$. The specific role of APC mutations in CIN is also significant; as APC is associated with microtubule and centrosome function in cytoskeletal regulation, and thus mitotic dysfunction, which itself leads to $\mathrm{CIN}^{158,159}$.

\subsubsection{DNA Methylation \& CpG Island Methylator Phenotype}

DNA hypermethylation and the $\mathrm{CpG}$ island methylator phenotype (CIMP) are another significant contributor to the genomic pathogenesis of colorectal cancer and is a key epigenetic mechanism for altering gene expression. As previously discussed, CIN is present in approximately $70 \%$ of CRC and MSI-H in $15 \%$, with an overlap of the two accounting for $25 \%$. There are however a significant proportion of sporadic tumours that do not demonstrate either CIN or MSI, and of the tumours where CIN or MSI is demonstrated many do not have the classical Vogelstein mutations in APC, KRAS, and TP53 ${ }^{160,161}$. The third genomic mechanism underlying CRC carcinogenesis is thought to be abnormal DNA methylation, and in particular CIMP.

DNA methylation refers to the addition of a methyl group to the 5-position of cytosine in DNA by DNA methyltransferase (DNMT), to produce 5-methylcytosine (Figure 1.4) ${ }^{162,163}$. Methylation is an active process and is performed by three DNMTs; DNMT1, DMNT3A, and DNMT3B, which are located on chromosomes 19, 2, and 20, respectively. The DNMT family are structurally similar, although $3 \mathrm{~A}$ and $3 \mathrm{~B}$ have a slightly lower molecular weight of 100$101 \mathrm{kDa}$, whilst DNMT1 is $183 \mathrm{kDa}$. Each shows a maximum efficiency for methylation of CpG dinucleotides, and especially islands that are hemi-methylated, although DNMT1 is the most efficient and acts as the primary de novo DNA methylator following DNA replication in cell division ${ }^{164}$. Each uses S-adenosyl methionine (SAM) as a methyl donor. An RNA methyltransferase (tRNA aspartic acid methyltransferase 1-TRDMT1) performs a similar role for RNA. 
The most frequent location for methylation of cytosine within DNA is at the CpG dinucleotide pairs that occur sporadically throughout the genome, of which $80-90 \%$ are normally methylated in humans. Beyond sporadic $\mathrm{CpG}$ pairs, dinucleotide $\mathrm{CpG}$ islands (repetition motifs of greater than $200 \mathrm{bp}$ ) also exist throughout the DNA (1-2\% of total) but are not methylated in healthy cells. These $\mathrm{CpG}$ islands are often found in the promotor regions of ubiquitously expressed proteins and are thought to facilitate efficient transcription. When these regions become (pathologically) methylated, especially in the context of MSI, they are referred to as hypermethylated $\mathrm{CpG}$ islands and affected cells are said to express CIMP, although this process also occurs with ageing and does not always indicate carcinogenesis ${ }^{165}$. Hypermethylation usually results in silencing of the downstream gene.

Figure 1.5 Methylation of the 5-position of cytosine by DNMT to form 5-methylcytosine (from Saini, et.al. $)^{163}$.<smiles>Nc1ccn([18O])c(=O)n1</smiles>

Cytosine<smiles>Cc1c(N)nc(=O)n([18O])c1[14CH2][14CH2][14CH2][14CH2][14CH3]</smiles>

5-Methylcytosine

Both hypo- and hypermethylation have a role in CRC carcinogenesis, although hypermethylation is understood to be the predominant process. Global demethylation has been observed in CRC in the context of specific locus hypermethylation and in association with chromosomal instability, and thus a concept of methylation-redistribution has been proposed, although the hypothesis is contentious ${ }^{166-169}$. The mechanisms that underlie hypermethylation gene silencing are not well understood, although interactions between aberrantly hypermethylated DNA and factors that inhibit transcription such as histone deacetylases and polycomb proteins are thought to dominate, as well as inhibiting pro-transcriptomic agents such as RNA polymerase $\mathrm{II}^{170}$. These effects may be mediated by a relatively newly characterised group of proteins that express a common domain called the methyl-CpG-binding domain (MBD), which acts to facilitate inhibitory chromatin restructuring by recruitment of histone deacetylases ${ }^{171}$. Irrespective of the mechanism, it is accepted that the hypermethylation of $\mathrm{CpG}$ islands in the promotor regions of a number of genes significantly associated with 
$\mathrm{CRC}$, including APC, p16INK4a, and TIMP3, is a genuine epigenetic event that contributes to carcinogenesis by gene silencing ${ }^{172-175}$.

Unfortunately, there is no standard definition of CIMP, even within studies focusing on CRC. Early attempts focused on assessing panels of gene promotor regions that were chosen by candidate gene analysis, leading to the 'classic' panel of five loci as described by Park, and including a number of methylated in tumours (MINT) sites ${ }^{176,177}$. An alternative panel was later proposed by Weisenberger based on cluster analysis, and a further panel by Ogino which discriminated between high- and low-level methylation ${ }^{178-180}$. As techniques have evolved further panels were proposed, although whole genome analysis has facilitated a wider understanding of the methylation status of CRC, and techniques in the field are still evolving ${ }^{181}$.

Table 1.2 summarises previously proposed panels for assessing CIMP, with a brief note on their attributes and contribution.

Table 1.2. Summary of CIMP panels and their differing attributes (adapted from Curtin) ${ }^{165}$.

\begin{tabular}{|c|c|c|}
\hline Study & CIMP Panel Markers & Note \\
\hline Toyota, et.al. ${ }^{177}$ & $\begin{array}{l}\text { CDKN2A (p16), MINT1, MINT2, MINT12, } \\
\text { MINT17, MINT25, MINT27, MINT31, } \\
\text { MLH1, THBS1. }\end{array}$ & $\begin{array}{l}\text { Pioneering work to identify } \\
\text { markers that distinguish CIMP } \\
\text { from age-related methylation. }\end{array}$ \\
\hline Park, et.al. ${ }^{176}$ & $\begin{array}{l}C D K N 2 A, \text { MINT1, MINT2, MINT31, } \\
\text { MLH1. }\end{array}$ & "Classic" or traditional panel. \\
\hline Weisenberger, et.al. ${ }^{178}$ & $\begin{array}{l}\text { CACNA1G, IGF2, NEUROG1, RUNX3, } \\
\text { SOCS1. }\end{array}$ & $\begin{array}{l}\text { "New" panel based on stepwise } \\
\text { screen of } 195 \text { markers. }\end{array}$ \\
\hline Ogino, et.al. ${ }^{162}$ & $\begin{array}{l}\text { CACNA1G, CDKN2A, CRABP1, MLH1, } \\
\text { NEUROG1. }\end{array}$ & $\begin{array}{l}\text { Selected markers to distinguish } \\
\text { high-level from low-level } \\
\text { methylation. }\end{array}$ \\
\hline Shen, et.al. ${ }^{\mathbf{1 8 2}}$ & $\begin{array}{l}\text { CIMP1: MINT1, MLH1, RIZ1, TIMP3, } \\
B R A F \text { mutation; CIMP2: MINT2, MINT27, } \\
\text { MINT31, Megalin, KRAS mutation. }\end{array}$ & $\begin{array}{l}\text { Examined } 27 \text { CpG sites, proposed } \\
\text { optimal epigenetic and genetic } \\
\text { markers to identify CIMP1, } \\
\text { CIMP2, or CIMP-. }\end{array}$ \\
\hline Tanaka, et.al. ${ }^{183}$ & $\begin{array}{l}C A C N A 1 G, C D K N 2 A, C H F R, C R A B P 1, \\
\text { HIC1, IGF2, IGFBP3, MGMT, MINT1, } \\
\text { MINT31, NEUROG1, p14, RUNX3, SOCS1, } \\
\text { WRN. }\end{array}$ & $\begin{array}{l}\text { Correlation structures of markers } \\
\text { and CIMP differ by } K R A S \text { and } \\
B R A F \text { status. }\end{array}$ \\
\hline Ang, et.al. ${ }^{184}$ & $\begin{array}{l}202 \mathrm{CpG} \text { sites differentially methylated } \\
\text { between tumour and normal. }\end{array}$ & $\begin{array}{l}\text { Comprehensive DNA methylation } \\
\text { profiling in } 807 \text { cancer genes. }\end{array}$ \\
\hline Kaneda \& Yagi ${ }^{185}$ & $\begin{array}{l}\text { Group 1: IGF2, LOX, MINT1, MINT2, } \\
\text { MINT31, MLH1, RUNX3, SOCS1; Group 2: } \\
\text { ADAMTS1, DUSP26, EDIL3, ELMO1, } \\
\text { FBN2, HAND1, IGFBP3, NEUROG1, } \\
\text { RASSF2, STOX2, THBD, UCHL1. }\end{array}$ & $\begin{array}{l}\text { Comprehensive DNA } \\
\text { epigenotyping of genome wide } \\
\text { regions identified two groups } \\
\text { (high and intermediate to low } \\
\text { methylation). }\end{array}$ \\
\hline
\end{tabular}


Of the studies examining methylation panels Kaneda and Yagi is the most exhaustive, as a whole-genome approach to assessing CIMP status was undertaken by employing a two-way unsupervised hierarchical clustering method and quantitative methylation techniques ${ }^{185}$. This study clearly demonstrated that CIMP status was clustered into three distinct groups (DNA methylation epigenotypes); CIMP-High(-H), CIMP-Intermediate(-I), and CIMP-Low(-L), with grouped methylation markers determining the classification of each epigenotype ${ }^{186}$. The CIMP-H group was found to correlate strongly with BRAF mutation and MSI-high, and CIMPI with KRAS mutation, but that classification into each of the epigenotypes was independent of mutational analysis. Of the three sub-classifications, CIMP-I with KRAS mutation demonstrated the poorest prognosis. The loci used for the Kaneda and Yagi classification are outlined in Table 1.3, divided into those strongly associated with CIMP-H (Group1) and those with CIMP-I and -L (Group 2). The epigenotyping system is described below.

- CIMP-High if $\geq 2 / 3$ of Group 1 markers are methylated, irrespective of Group 2 markers

- CIMP-Intermediate if 1/3 Group 1 markers and $\geq 3 / 5$ Group 2 markers is methylated

- CIMP-Low if $\leq 1$ Group 1 markers is methylated and $\leq 2$ Group 2 markers is methylated

\subsubsection{Other Epigenetic Factors}

Cytosine methylation is only one epigenetic factor influencing gene expression and physiological and pathological phenotype. Another key epigenetic process governing gene expression is histone modification by protein methylation, phosphorylation, or other covalent processes; thus effecting chromatin structure ${ }^{187,188}$. There is increasing evidence that histone modification may be important in the pathogenesis of colorectal cancer, and several histonemethylation enzymes (acetyltransferases and demethylases) have been associated with oncogenic or tumour-suppressor roles ${ }^{189}$. As the process of histone modification is dynamic and modifiable it presents an opportunity for therapeutic intervention, although this avenue of therapeutics is in its infancy ${ }^{190,191}$. 
Table 1.3 Group 1 and 2 methylation markers for Kaneda and Yagi epigenotype classification.

\begin{tabular}{|c|c|c|c|c|}
\hline & Gene & Alias & Location & Function \\
\hline \multirow{3}{*}{ 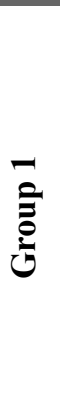 } & SOCS1 & $\begin{array}{l}\text { SSI1, } \\
\text { CISH1 }\end{array}$ & $16 \mathrm{p} 13$ & $\begin{array}{l}\text { STAT induced STAT-inhibitor (suppressor of cytokine } \\
\text { signalling - SOCS): negative feedback loop signalling in } \\
\text { inflammatory cytokine pathways. }\end{array}$ \\
\hline & MINT1 & $\begin{array}{l}\text { APBA-1, } \\
\text { X11, IN10 }\end{array}$ & $9 \mathrm{q} 21$ & $\begin{array}{l}\text { Member of the X11 protein family: believed to be } \\
\text { involved in signal transduction pathways. }\end{array}$ \\
\hline & hMLH & $\begin{array}{l}\text { COCA1, } \\
\text { HNPCC2 }\end{array}$ & $3 p 22$ & $\begin{array}{l}\text { DNA MMR gene. Part of the MutL-alpha complex. } \\
\text { Strong association with HNPCC. }\end{array}$ \\
\hline \multirow{5}{*}{ ڤัँ } & NEUROG-1 & $\begin{array}{l}\text { NEUROD } \\
3, \text { NGN-1 }\end{array}$ & $5 q 31$ & $\begin{array}{l}\text { Interacts with CREB-binding protein as a transcription } \\
\text { activating co-factor. Protein complex demonstrates } \\
\text { histone acetyltransferase activity. }\end{array}$ \\
\hline & THBD & $\begin{array}{l}\text { THRM, } \\
\text { CD141 }\end{array}$ & 20p11 & $\begin{array}{l}\text { Endothelial cell surface membrane protein. Co-factor for } \\
\text { thrombin activation. }\end{array}$ \\
\hline & HAND1 & $\begin{array}{l}\text { EHand, } \\
\text { Things1, } \\
\text { Hxt }\end{array}$ & $5 q 33$ & $\begin{array}{l}\text { Basic loop-helix-loop transcription protein found in } \\
\text { myocardium and associated with vasculogenesis. }\end{array}$ \\
\hline & ADAMTS1 & METH1 & $21 \mathrm{q} 21$ & $\begin{array}{l}\text { Multimodular proteins demonstrating a variety of active } \\
\text { domains including metalloproteinase activity, } \\
\text { disintegrin-like domain, and thrombospontin type } 1 \\
\text { motif. Inhibitor of tissue remodelling and anti- } \\
\text { angiogenesis. }\end{array}$ \\
\hline & IGFBP3 & $\begin{array}{l}\text { IBP-3, } \\
\text { BP-3 }\end{array}$ & $7 \mathrm{p} 12$ & $\begin{array}{l}\text { One of six insulin-like growth-factor binding proteins; } \\
\text { acts to transport IGFs and modulate interaction at cell } \\
\text { surface and at nucleus. High circulating levels associated } \\
\text { with worse outcomes in some cancers. }\end{array}$ \\
\hline
\end{tabular}




\subsubsection{Consensus Molecular Subtyping in Colorectal Cancer}

The process of carcinogenesis is now accepted to be an imbalance between the cell-cycle control mechanism and the development of mutations. Evolving from the Vogelstein model, three overlapping genomic and epigenomic pathways are implicated in genomic instability and the carcinogenesis process; chromosomal instability (CIN), microsatellite instability (MSI), and an epigenetic mechanism resulting from DNA hypermethylation called $\mathrm{CpG}$ island methylator phenotype $(\mathrm{CIMP})^{161}$. The relationship between these mechanisms and the influence of the tumour inflammatory and immunological microenvironment is complex; and as a greater understanding of the transcriptomic pathways that influence the cancer cell phenotype is developed, the true complexity of CRC tumorigenesis is emerging ${ }^{118}$.

One of the earlier molecular classifications for CRC that incorporated methylation status, as well as MSI, CIN, and histopathological characteristics was the Jass Classification ${ }^{161}$. This system separated CRC into five categories that demonstrate a degree of overlap; broadly dividing the serrated from the adenomatous pathways, and splitting the total population $60 \%$ 40\% between CIMP-negative and CIMP-positive, and CIMP-positive further into 20\% CIMP$\mathrm{H}$ and 20\% CIMP-L. Unfortunately, subsequent studies aiming to stratify cohorts of patients with CRC based on Jass' classification and then to examine if there is a prognostic significance to the Jass phenotypes have failed both to adequately stratify patients based on the classification and have not demonstrated any significant prognostic implications. The proportions of cancers defined by CIMP status in subsequent studies has also been variable, with Zlobec identifying an approximately $7 \%, 43 \%$, and 50\% split between CIMP-H, -I, and -L, and $41 \%$ of tumours not being able to be classified according to the Jass Classification ${ }^{192}$. What was important about this early work, however, was that it introduced the concepts of different molecular pathways and classifications to CRC and provided a framework for their investigation ${ }^{161,193}$.

Subsequent studies, many of which were based on whole-genome sequencing and global methylation characterisation, proposed various molecular classifications for CRC and attempted to validate them against large clinical and pathological datasets (Table 1.4) ${ }^{194-201}$. Most of these systems have subsequently been abandoned due to failures in external validation, although components of each have been incorporated into and/or informed the subsequent consensus position on molecular subtyping in CRC. 
Table 1.4 Molecular \& Phenotypical Subtyping in Colorectal Cancer

\begin{tabular}{|c|c|c|c|}
\hline Study & Classifications & Primary Differentiators & Note \\
\hline $\begin{array}{l}\text { Jass, et.al. }{ }^{\mathbf{1 6 1}} \\
2007\end{array}$ & Types I - V & $\begin{array}{l}\text { CIMP-H, -L, -Nil; } \\
\text { MSS/MSI-L, MSI-H }\end{array}$ & $\begin{array}{l}\text { Types I \& II associated with } \\
\text { serrated pathway, IV \& V with } \\
\text { Vogelstein, III with either }\end{array}$ \\
\hline $\begin{array}{l}\text { Perez-Villamil, et.al. }{ }^{197} \\
2012\end{array}$ & Clusters 1 - 4 & $\begin{array}{l}\text { MSS, MSI; } \\
\text { Histology (mucinous); } \\
\text { \% stromal content; } \\
\text { BRAF V600E }\end{array}$ & $\begin{array}{l}\text { Emphasis on the role of the } \\
\text { tumour microenvironment to } \\
\text { classification and "malignant } \\
\text { power" }\end{array}$ \\
\hline $\begin{array}{l}\text { Schlicker, et.al. }{ }^{\mathbf{2 0 0}} \\
2012\end{array}$ & $\begin{array}{l}\text { Types } 1 \& 2: \\
\text { subdivided into } \\
1.1,1.2,1.3,2.1 \\
\& 2.2\end{array}$ & $\begin{array}{l}\text { EMT (mesenchymal vs } \\
\text { epithelial); MSI, MSS; } \\
\text { Pathway upregulation }\end{array}$ & $\begin{array}{l}\text { Demonstrated stratification } \\
\text { across } 74 \text { immortalised cell lines } \\
\text { and differentiation in response to } \\
\text { in vitro therapies }\end{array}$ \\
\hline $\begin{array}{l}\text { Budinska, et.al. }{ }^{194} \\
2013\end{array}$ & Types I - V & $\begin{array}{l}\text { MSS, MSI; BRAF } \\
\text { V600E; p53; KRAS }\end{array}$ & $\begin{array}{l}\text { Division into typically crypt-like } \\
\text { (upper \& lower), CIMP, } \\
\text { mesenchymal, or } \\
\text { expressional clusters }\end{array}$ \\
\hline $\begin{array}{l}\text { De Sousa, et.al. }{ }^{195} \\
2013\end{array}$ & & $\begin{array}{l}\text { CIN+ve, -ve; MSS, MSI; } \\
\text { CIMP-H, -L }\end{array}$ & $\begin{array}{l}\text { Type } 3 \text { (MSS CIMP-H) } \\
\text { associated with sessile-serrated } \\
\text { pathway }\end{array}$ \\
\hline $\begin{array}{l}\text { Marisa, et.al. }{ }^{196} \\
2013\end{array}$ & Subtypes C1 - C6 & $\begin{array}{l}\text { Wnt; } \quad \text { MSS, MSI; } \\
\text { CIN+ve, -ve; KRAS }\end{array}$ & $\begin{array}{l}\text { Subtypes C4 (stem cell-like) \& } \\
\text { C6 associated with worse relapse- } \\
\text { free survival }\end{array}$ \\
\hline $\begin{array}{l}\text { Sadanandam, et.al. }{ }^{199} \\
2013\end{array}$ & Subtypes $1-6$ & $\begin{array}{lrr}\text { Wnt; Crypt } & \text { location; } \\
\text { MUC2 \& \& } & \text { TFF3; } \\
\text { "Inflammatory" } & \end{array}$ & $\begin{array}{l}\text { Subtypes reflect distinct normal } \\
\text { crypt cell types and exhibit } \\
\text { "stemness". Response to } \\
\text { chemotherapy differs between } \\
\text { subtypes }\end{array}$ \\
\hline $\begin{array}{l}\text { Roepman, et.al. }{ }^{\mathbf{1 9 8}} \\
2014\end{array}$ & Types A, B, \& C & $\begin{array}{l}\text { BRAF V600E; EMT } \\
\text { expression; MSS, MSI }\end{array}$ & $\begin{array}{l}\text { Subtypes may predict prognosis } \\
\text { and response to chemotherapy }\end{array}$ \\
\hline $\begin{array}{l}\text { Guinney, et.al. }{ }^{201} \\
2015\end{array}$ & $\begin{array}{l}\text { CMS 1 } 1-4 \\
\text { [Consensus } \\
\text { Molecular } \\
\text { Subtype] }\end{array}$ & $\begin{array}{l}\text { Consensus methodology } \\
\text { of previous molecular \& } \\
\text { phenotypic studies }\end{array}$ & $\begin{array}{l}\text { Four streams of subtyping with } \\
\text { distinct phenotypic and molecular } \\
\text { characteristics. }\end{array}$ \\
\hline
\end{tabular}


Current consensus suggests four identifiable molecular subtypes (CMS1-4) based on transcriptomic analysis, the first of which is based upon a high degree of underlying MSI and hypermethylation (including CIMP), as opposed to CIN (Figure 1.5) $)^{118,201,202}$. The further three pathways are based on chromosomal instability with APC dysfunction and the Vogelstein model of compound genetic insult, but subsequent canonical divergence between phenotypes displaying divergent metabolic or inflammatory phenotypes. This collaborative study, based on the large-scale pooling of cohorts and bioinformatic methodologies, has helped characterised CRCs and framed the ongoing discourse in precision medicine as it will apply to the disease. Although not clinically validated (except for KRAS wild-type anti-EGFR therapies), predictive biomarkers are emerging that may in future help guide therapies to facilitate treatment based on individual tumour biology; resulting in less wasteful and more effective care.

Figure 1.6 Consensus Molecular Subtypes 1 - 4 of CRC (adapted from Dienstmann ${ }^{118}$ ).
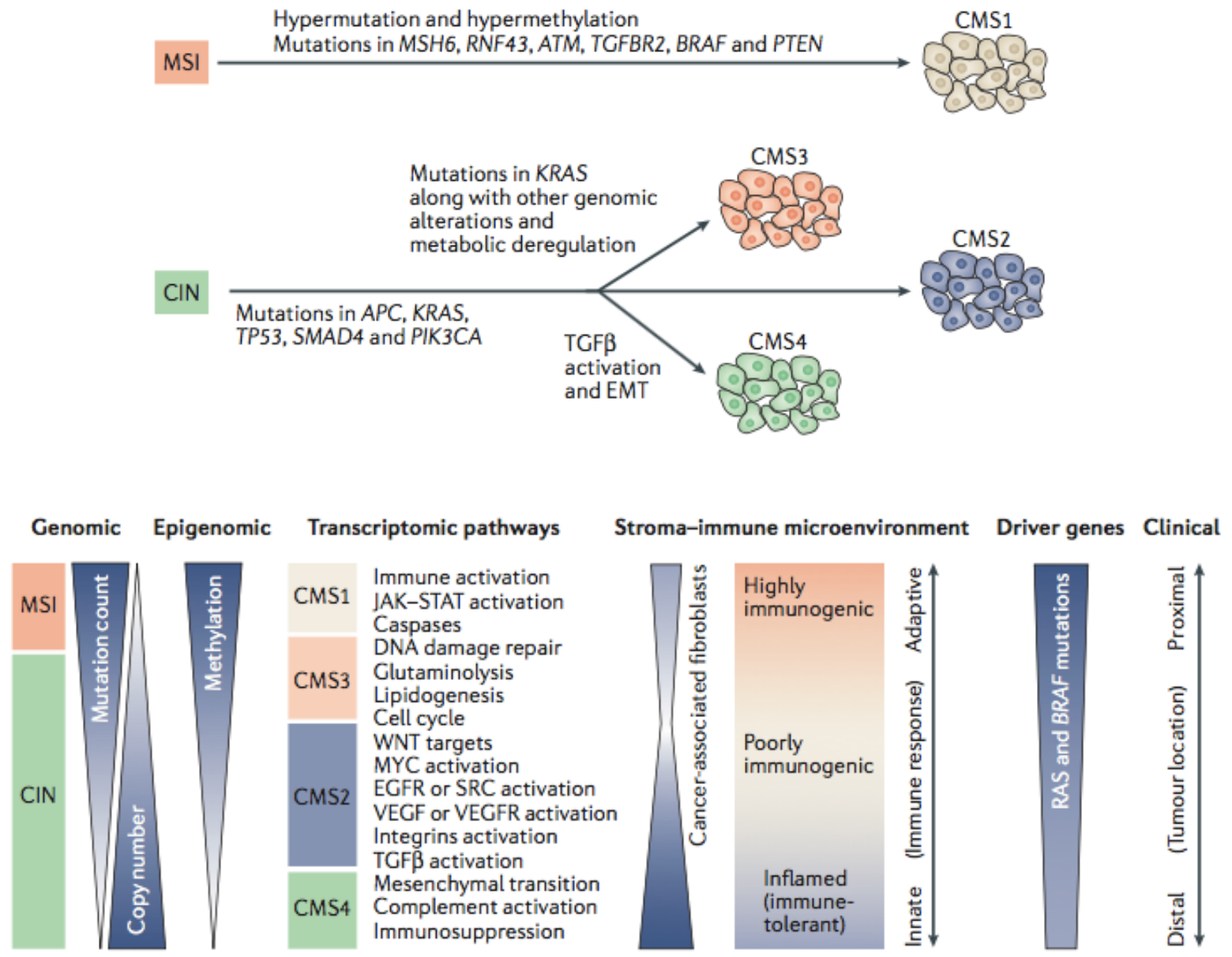


\subsection{General Aims of this Thesis}

Previous work has demonstrated that extramural vascular invasion (EMVI) has an inverse relationship to KRAS mutation, indicating a CIN dominated pathway in this tumour phenotype more typical of the classical adenoma-to-carcinoma pathway often found the distal colon and rectum $^{203}$. MSI-high tumours on the other hand have been associated with serrated polyps and a high prevalence of BRAF mutations in the proximal colon, although a significant percentage affects the rectum and may represent a pathologically worse molecular subtype ${ }^{125,147-149}$. There is however no current biomarker available to accurately predict the presence of EMVI, although it is suspected that MSI hypermutation and hypermethylation may be the predominating tumourigenic processes in this phenotype.

This thesis will investigate the relationship between methylation and EMVI in rectal cancers, its implications for prognosis, and seek to illuminate the biological processes that underlie any relationship. Each chapter will set out its own objectives, methodologies, results, and conclusions; although the body of work as a whole should constitute a unified enquiry based on this overarching aim. Three principle methodologies will be employed throughout this investigation; assessment of the methylation status of resected rectal cancers and the correlation to prospectively collected clinical and histopathological datasets; in vitro studies of methylation in colorectal cancer tissue models, the manipulation of methylation in such models, and biological consequence; and a mechanistic analysis of both tissue models and resected rectal cancer specimens to elucidate the cellular processes that may underlying observed effects. 


\section{Chapter 2}

\section{General Methodology}

\subsection{Overarching Considerations \& Ethics}

\subsubsection{Patient Selection}

The primary aim of this research was to investigate the relationships between DNA methylation, the pathophysiology of rectal adenocarcinoma (especially extramural vascular invasion), and the mechanisms that underlie any relationship. The key to linking cellular processes with clinical outcomes lies in the dual analysis of DNA extracted from patient tissue samples and the corresponding clinicopathological data. All patients included in this study were drawn from a prospectively maintained database of all patients undergoing treatment for rectal cancer at a single centre (Abertawe Bro Morgannwg University Health Board (ABMU) Singleton \& Morriston Hospitals - Swansea Colorectal MDT). Although primarily a local MDT, the Swansea Colorectal MDT has a supra-regional practice in the management of advanced pelvic malignancy requiring sub-specialised surgical and oncological expertise, and therefore captures a greater-than-normal frequency of advanced rectal cancers undergoing treatment with curative intent. Each of the patients included in this study have had surgical treatment for rectal cancer with curative intent, but patients receiving neoadjuvant therapy (nCRT) have been excluded.

Patients who have received neoadjuvant therapy have been excluded due to the likelihood that DNA extracted from tumours previously exposed to nCRT will have undergone epi-/genetic alterations that will serve as confounding factors in analysis. Currently, there is no substantive evidence to associate methylation status with response to nCRT, although there is some equivocation between single/multi locus and whole-epigenome studies, and neoadjuvant demethylation is being explored as a therapeutic augmentation to traditional nCRT in other organ systems ${ }^{148,204,205}$. Further exclusion criteria were patients with inflammatory bowel disease, known or suspected high-risk of familial cancer syndromes, recurrent cancers, nonadenocarcinoma tumour types (melanoma, squamous, GIST, etc), and tumours identified as rectosigmoid and beyond $15 \mathrm{~cm}$ from the anal verge. All patients received pre-operative diagnosis consistent with the prevailing guidelines (most recent; ACPGBI 2017206), and 
retrieved specimens were retrieved from Singleton Hospital Pathology Department and screened by consultant histopathologist for quality and appropriate tumour representation in accordance with the validated Royal College of Pathologists colorectal cancer minimum reporting datasheet ${ }^{207}$.

\subsubsection{Database Maintenance \& Anonymisation}

A prospective database of all patients undergoing treatment at the Swansea Colorectal MDT was maintained throughout and prior to the investigation. Data was primarily extracted directly from the MDT meetings themselves by use of a standard data proforma and real-time data input to the database but was also cross-referenced against a number of clinical and public health resources. Basic demographic information was captured from patient records presented at MDT, and radiological, histopathological, and further clinical data was harvested from respective hospital digitised services. Patient deaths were cross-checked against the NHS Wales Informatics Service (Myrddin), and cancer specific outcomes against the Cancer Information Network System Cymru (CaNSIC) database. Following identification of patients that met inclusion criteria and extraction of raw data from the database, investigators were blinded during the experimental phases of the data collection by use of a dual-labelling methodology (random number allocation) so that no patient-identifiable data was present during laboratory analysis. The two datasets were recombined for dual analysis. All data was stored in accordance with processes set out in the NIHR international Good Clinical Practice (GCP) framework ${ }^{208}$.

\subsubsection{Ethics}

Ethical approval was granted by South West Wales REC (Project Ref No.:11/WA/0256), and sponsored by the R\&D Department, Abertawe Bro Morgannwg University LHB, Morriston Hospital, Swansea. Consent was not required in accordance with the Human Tissue Act (2004). All data was handled in accordance with the Data Protection Act (1988 \& 2018) and GCP guidelines, and all investigators held current GCP certification ${ }^{208}$. 


\subsection{Materials \& General Methods}

\subsubsection{Laboratory Equipment, Consumables, \& Reagents}

Throughout this research a great variety of laboratory equipment and consumables was employed during specific processes, each of which will be described in detail in the methods section of each chapter. Described here are the materials and general methods that are common to multiple sections of the research and which will not be described in detail again later. Where these consumables constitute a bought-in kit they will be listed in section 2.2.6, but thereafter be referred to by their kit name, except where kit modification or process optimisation has taken place. The exceptions to the above are listed below (Table 2.1). Items of occasional use are not listed. A laboratory safety folder was maintained throughout.

Table 2.1. Laboratory Equipment \& Consumables

\begin{tabular}{|c|c|}
\hline Item & Supplier \\
\hline 100bp DNA Ladder & Promega, USA \\
\hline 2-well Tissue Culture / Wound Healing Inserts $(500 \mu \mathrm{m})$ & Ibidi GmbH, Germany \\
\hline $30 \%$ Acrylamide- 1 Bis Sol ${ }^{\mathrm{n}}(37.5: 1)$ & Sigma-Aldrich (Merck), USA \\
\hline Albumin (bovine) Serum & Sigma-Aldrich (Merck), USA \\
\hline Ammonium Persulfate & Sigma-Aldrich (Merck), USA \\
\hline BICELL Cellular Cryopreservation Vessel & NIHON FREEZER, Japan \\
\hline Borate & Fisher Scientific, USA \\
\hline Centrifuges (various) & Various \\
\hline $\begin{array}{l}\text { Corning } \AA \text { Matrigel } \AA \text { Invasion Chamber } \\
\text { (6-well plate, } 8.0 \text { micron pore) }\end{array}$ & Corning (Merck), USA \\
\hline Cryovial $1.8 \mathrm{ml}$ & SPL Life Sciences, USA \\
\hline EpiTect $^{\circledR}$ PCR Control DNA & QIAGEN, Germany \\
\hline Ethanol & Fisher Scientific, USA \\
\hline Ethylenediaminetetraacetic acid (EDTA) & Fisher Scientific, USA \\
\hline Interlukin-6 (IL-6) Human (recombinant) & Sigma-Aldrich (Merck), USA \\
\hline L-Glutamine & Fisher Scientific, USA \\
\hline $\begin{array}{l}\text { Glassware, including microscope slides and cover slips } \\
\text { (reusable \& single use) }\end{array}$ & Various \\
\hline GoTaq ${ }^{\circledR}$ Hot Start Green Master Mix & Promega, USA \\
\hline Grant Dry Block Heater (variable temperature) & Fisher Scientific, USA \\
\hline Falcon Tubes $15 \mathrm{ml} \&$ 50ml (Collar / Conical) & Fisher Scientific, USA \\
\hline Freezers: $-20^{\circ} \mathrm{C} \&-80^{\circ} \mathrm{C}$ & Various \& New Brunswick Scientific \\
\hline Fume Hood & Clean Air, Ltd \\
\hline Horse Serum & Gibco (Fisher Scientific), USA \\
\hline
\end{tabular}




\begin{tabular}{|c|c|}
\hline Ice Machine (crushed) & Hoshizaki, Japan \\
\hline Isopropanol & Fisher Scientific, USA \\
\hline L-Glutamine & Gibco (Fisher Scientific), USA \\
\hline Light Microscopes x5, x10, x20, x40 (+/- oil) & Various \\
\hline Methanol & Fisher Scientific, USA \\
\hline Microcentrifuge Tubes & Eppendorf, Germany \\
\hline $\begin{array}{l}\text { Milli-Q Water Purification System for Type } 1 \text { Ultrapure } \\
\text { Water }\end{array}$ & Millipore (Merck), USA \\
\hline Multi-well Cell Culture Plates $(6,12,24)$ & Corning (Merck), USA \\
\hline Nuclease-Free Water (Ambion) & Sigma-Aldrich (Merck), USA \\
\hline Paraformaldehyde & Sigma-Aldrich (Merck), USA \\
\hline PCR Plates (96 well) & Bio-Rad, USA \\
\hline PCR Biofilm & Bio-Rad, USA \\
\hline $\mathrm{pH}$ Meter & Mettler Toledo, USA \\
\hline pH Buffers for Calibration & Sigma-Aldrich (Merck), USA \\
\hline Phosphate Buffered Saline (PBS) & Gibco (Fisher Scientific), USA \\
\hline Pipette Tips (Tip One System) - various volumes & StarLAB, UK \\
\hline Pipette Tips (Aspiration) - various volumes & Corning (Merck), USA \\
\hline Scalpels (disposable) & Swann-Morton, UK \\
\hline ScanLaf Tissue Culture Hood (Class 2) & BioGene, Denmark \\
\hline Silver Nitrate & Sigma-Aldrich (Merck), USA \\
\hline Sodium Hydroxide & Sigma-Aldrich (Merck), USA \\
\hline Tetramethylethlenediamene (TEMED) & Sigma-Aldrich (Merck), USA \\
\hline Thermometers (immersion; digital \& liquid) & Various \\
\hline $\begin{array}{l}\text { Thinpipette Focus Fixed Volume } 5 \mu \mathrm{l}, 50 \mu \mathrm{l}, 300 \mu \mathrm{l}, 1000 \mu \mathrm{l} \\
\text { (+ various single \& multi-channel) }\end{array}$ & Fisher Scientific, USA \\
\hline Tris (hydroxylmethyl) aminomethane & Sigma-Aldrich (Merck), USA \\
\hline Trypan Blue Solution (0.4\%) & Sigma-Aldrich (Merck), USA \\
\hline Tissue Culture Flasks $25 \mathrm{~cm}^{2}$ and $75 \mathrm{~cm}^{2}$ (vented) & Corning (Merck), USA \\
\hline Vectashield Mounting Medium with DAPI & Vector Labs (Maravai LS), USA \\
\hline Vortex & Various \\
\hline Water Baths $\left(37^{\circ} \mathrm{C}\right)$ & Various \\
\hline Xylene & Fisher Scientific, USA \\
\hline Z1 Coulter Counter & Beckman Coulter, USA \\
\hline
\end{tabular}




\subsubsection{Programmable Equipment}

A variety of programmable laboratory equipment was employed at various stages of this research; including but not limited to automated and real-time PCR equipment, microscopes, and tissue-bloc processors. Each item of equipment, its set-up, programming, and analysis will be discussed in the methods section of the relevant chapter; but are listed in Table 2.2.

Table 2.2. Programmable Laboratory Equipment.

\begin{tabular}{|c|c|}
\hline Item \& Description & Supplier \\
\hline $\begin{array}{l}\text { ChemiDoc XRS+ System } \\
\text { - } \quad \text { Gel imaging \& product analysis }\end{array}$ & Bio-Rad, USA \\
\hline $\begin{array}{l}\text { etaluma } 560 \text { Inverted Live-Cell Microscope } \\
\text { - } \quad \text { Time-lapse wide-field microscopy }\end{array}$ & etaluma, USA \\
\hline $\begin{array}{l}\text { IN Cell Analyser } 2000 \\
\text { - } \quad \text { Automated wide-field cell analysis }\end{array}$ & GE Life Sciences, USA \\
\hline $\begin{array}{l}\text { IQ-5 }^{\mathrm{TM}} \text { Real-Time PCR System } \\
-\quad \text { RT PCR \& product analysis system }\end{array}$ & Bio-Rad, USA \\
\hline $\begin{array}{l}\text { Metafer Automated Slide-Scanning Platform } \\
\text { - } \quad \text { Automated fluorescence slide-scanning \& image capture }\end{array}$ & MetaSystems Group, Germany \& USA \\
\hline $\begin{array}{l}\text { NanoDrop }{ }^{\circledR} 1000 \text { UV Vis Spectrophotometer } \\
\text { - } \quad \text { DNA, RNA, and Protein yield \& purity analysis }\end{array}$ & Labtech (Fisher Scientific), USA \\
\hline $\begin{array}{l}\text { Olympus BX51 Fluorescence Microscope } \\
\text { - } \quad \text { Multi-filter programmable fluorescence microscopy }\end{array}$ & Olympus, Japan \\
\hline $\begin{array}{l}\text { T100'M Thermal Cycler } \\
\text { - } \quad \text { PCR \& Microcentrifuge heater block }\end{array}$ & Bio-Rad, USA \\
\hline $\begin{array}{l}\text { Ventana Benchmark ULTRA } \\
\text { - } \quad \text { Automated slide processing and IHC }\end{array}$ & Ventana (Roche), Switzerland \\
\hline
\end{tabular}




\subsubsection{5-Azacytadine}

5-azacytidine (AZA) is a pyrimidine ring analogue of the DNA and RNA nucleotide cytidine. After transportation into cells, 5-AZA undergoes de-oxy metabolism to form 5-aza-2'deoxycytidine, which can then be incorporated into DNA in place of cytidine. Once in C-G dinucleotide formation, DNA methyltransferases (DNMT) bind AZA to catalyse methylation but are inhibited from being released (normally by beta-elimination of the covalent bond at the 5 -carbon position) by the presence of a nitrogen ${ }^{209,210}$. By this mechanism, DNMT activity is inhibited by sequestration of the DNMT enzyme and subsequent failure of de-novo methylation during DNA replication and repair. AZA does not actively demethylate DNA by competitive inhibition of the DNMT's active site, by direct removal of methyl groups from methylated cytosine, or by upregulation of the base excision repair enzyme OGG1 in response to oxidative stress $^{211}$. Due to the necessity of DNA replication to effect demethylation, the cellular and clinical effects of AZA are only evident following multiple cell cycles ${ }^{212}$. DNA demethylation by AZA has been shown to activate previously silent genes, initiate decondensation of chromatin, and induce cellular differentiation; ultimately leading to significant changes in cellular phenotype ${ }^{213-215}$.

AZA was supplied by Sigma-Aldrich (now Merck), Germany [A2385]. AZA has a molecular formula of $\mathrm{C}_{8} \mathrm{H}_{12} \mathrm{~N}_{4} \mathrm{O}_{2}$, a molecular weight of 244.20 , and its structure is shown in Figure 2.1 ${ }^{216}$. AZA was stored at $-20^{\circ} \mathrm{C}$ in its dehydrated form (as shipped), and was dissolved in $\mathrm{DMSO} / \mathrm{H}_{2} \mathrm{O}$ prior to use, where after it was stored at $-80^{\circ} \mathrm{C}$ for a maximum of one week.

Figure 2.1. Molecular structure of 5-azacytidine.

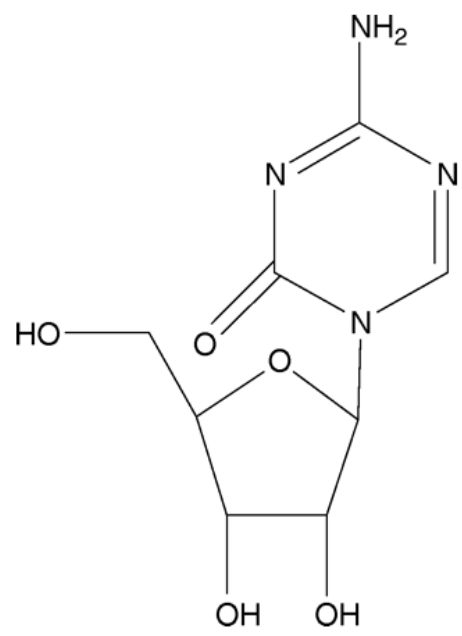


The effect of demethylation with AZA on DNA are global, and pan-demethylation (hypomethylation) has been demonstrated to contribute to chromosomal instability (CIN) and even to induce malignant transformation in vivo; patterns of global demethylation have been observed in a variety of malignant conditions, including T-cell lymphomas (murine model), and human prostate and hepatocellular carcinoma ${ }^{168,217,218}$. The consequence of these findings is that AZA may itself be mutagenic due to whole-genome demethylation, and thus some authors have suggested that this may limit its use as a therapeutic agent ${ }^{168}$. The global demethylating effects of AZA at therapeutic doses have however been challenged, with parallel studies examining the demethylating effects of AZA on preserved Alu elements in patients receiving treatment for myelodysplastic disorders indicating that, at therapeutic doses, AZA has only a moderate demethylating effect ${ }^{169}$. The authors of this study speculate that the doses of AZA employed in previous in vitro and murine models were not representative of normal physiological conditions (the authors report "extreme modelling"), but they do concede that LINE-1 hypomethylation was observed in their own study of colorectal cancers, although to a less significant degree. The association between CIN and hypomethylation was also investigated in colorectal cell-lines with differing expression of CIN as part of the same investigation, although only a minor, non-statistically significant decrease in methylation between $\mathrm{CIN}+$ and $\mathrm{CIN}$ - cell lines were discovered $(\mathrm{p}=0.48)^{169}$. Other cell-line work has however demonstrated AZA (and its de-oxy derivatives) to be an effective demethylator of oncogenic hypermethylated promotor regions, such as the C/EBP $\delta$ tumour suppressor in acute myeloid leukaemia (AML) ${ }^{219}$. In this study, microarray analysis of U937 cells treated with AZA derivatives demonstrated that 274 transcripts were significantly upregulated, including the entire C/EBP family, although $\mathrm{C} / \mathrm{EBP} \delta$ showed a 10-fold expressional increase following demethylation. These findings correlated to the clinical finding that AML patients demonstrated hypermethylation at the $\mathrm{C} / \mathrm{EBP} \delta$ promotor region.

AZA was initially licenced in 2004 by the Federal Drug Administration in the USA for use in all types of myelodysplastic syndrome, including AML, based on one randomised control study and two single-arm studies ${ }^{212,220,221}$. Unfortunately, the complete response rate in the treatment arms of these studies was low (16\%), although a return to normal blood counts and bone marrow morphology was observed. A partial response was observed in a greater number of patients $(19 \%)$, but individuals receiving the treatment remained therapy-dependent, with relapse of bone marrow failure following treatment withdrawal. Side-effects were observed in a significant proportion of patients, including $16 \%$ who developed severe liver dysfunction, 
although minor gastrointestinal and haematological side effects were more common. Despite these poor beginnings, more recent studies employing decitabine (a de-oxy derivative of AZA) showed a greater response rate without increased toxicity or safety concerns, although overall survival was not statistically better between treatment arms, although trend was demonstrated in older ( $>70 \mathrm{yrs}$ ) patient groups, especially for de novo AML rather than secondary disease $\mathrm{e}^{222}$.

There is currently no role for AZA in the treatment of solid-organ tumours, including colorectal cancer (CRC). Early trials of demethylating agents for a variety of solid-organ tumours, based on the success of therapy for myelodysplastic disorders, were both speculative and disappointing; with very little if any clinical response observed in a mixed cohort of end-stage tumours ${ }^{223,224}$. When identified as a separate cohort, the results specifically for CRC were especially poor; decitabine monotherapy producing no tumour regression whatsoever in any patient, and a less than $10 \%$ response (defined in this study as regression or stable disease) when used in combination therapy. Further studies on patients with end-stage solid-tumour disease also showed no clinical effectiveness when decitabine was combined with a histone deacetylase inhibitor (sodium phenylbutyrate), although toxicity was limited and there were no adverse drug-related events ${ }^{225}$.

More recently, the potential role of AZA as a chemo-sensitizer in CIMP-high solid tumours has been suggested, based on the observation that CIMP-high has been associated with chemoresistance in colorectal and other tumours 226,227 . In vitro and xenograft studies have also indicated that demethylation may restore sensitivity to 5-FU therapy in colorectal and other cancer models ${ }^{228,229}$. Early clinical outcomes have suggested that AZA derivatives may restore partial chemotherapy sensitivity to treatment-refractory non-small cell lung cancers and ovarian cancers, although the numbers in these studies are small and the benefits (in terms of PFS) were marginal ${ }^{230,231}$. Unfortunately, these results have not been observed in early clinical trials in CRC. In one trial, twenty-six treatment-refractory patients (fourteen CIMP-high) were treated with AZA in addition to standard chemotherapy (CAPOX) to determine if chemosensitivity could be restored; no patient responded and there was no correlation between CIMPstatus and $\mathrm{PFS}^{232}$. A similar study of forty-seven patients with advanced treatment-refractory liver metastasis of colorectal origin also showed no clinical response (by RECIST standards) to combined AZA and entinostat therapy, although tumours demonstrating demethylation had a slight improvement in PFS $^{233,234}$. 


\subsubsection{RRx-001}

RRx-001 (formerly ABDNAZ) is a novel compound produced by modification of the byproducts of solid rocket propellant manufacture and has shown some activity suggesting it may have chemotherapeutic or chemo-sensitising properties ${ }^{235-237}$. RRx-001 acts as an alkylating agent, covalently binding nucleophilic targets following liberation of a bromine (the leaving group) in a non-selective and non-reversible manner. This action is similar to that observed with traditional non-selective alkylating agents such as nitrogen mustard (mustard gas) and nitrosoureas, each of which can cause potentially devastating DNA, RNA, and protein damage leading to significant toxicity or death ${ }^{238}$. At a cellular level RRx-001 has been shown to increase reactive nitrogen and oxygen species; resulting in direct DNA damage, increased $\gamma \mathrm{H} 2 \mathrm{AX}$ expression reflecting breakage in dsDNA, and apoptosis ${ }^{237}$. The chemotherapeutic mechanisms of RRx-001 are however not well understood, although on in vivo modelling, action on the $\beta$-cysteinyl residue of haemoglobin and other circulating thiols (glutathione and cysteine) by nucleophilic substitution is believed to underlie the production of circulating nitrogen oxides; thus modulating redux reactions in the circulation and modifying tumour microvasculature through nitrogen dependent pathways. In early trials, RRx-001 did not demonstrate significant "off-target" toxicity, possibly due to the rapid alkylation of haemcomponents on administration, thus limiting damage to tissues not reached until after the compound has given up its leaving group 235,239 .

RRx-001 was supplied by EpicentRx under experimental licence. RRx-001 is a cyclic nitro compound with molecular formula $\mathrm{C}_{5} \mathrm{H}_{6} \mathrm{BrN}_{3} \mathrm{O}_{5}$, a molecular mass of 268.02, and a molecular structure as shown in Figure 2.2. RRx-001 was stored at room temperature in its dehydrated form (as shipped), and was dissolved in $\mathrm{DMSO} / \mathrm{H}_{2} \mathrm{O}$ prior to use, where after it was stored at $80^{\circ} \mathrm{C}$ for a maximum of one week.

Figure 2.2. Molecular structure of RRx-001.

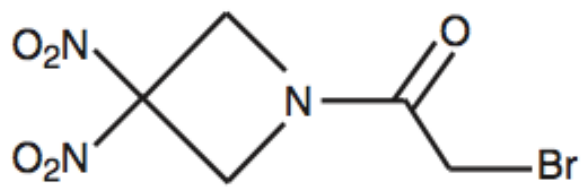


RRx-001 was initially investigated as a potential tumour chemo/radio re-sensitiser in the context of end-stage metastatic disease and was trialled against a number of solid organ tumours, including colorectal cancer, and has demonstrated favourable outcomes ${ }^{236,237,240 .}$ The role and mechanisms of small molecule agents in re-establishing tumour sensitivity is not well understood, but some emerging theories suggest epigenetic mechanisms underlie this phenomenon. Supporting this theory are the observations that demethylating agents (including AZA) and histone deacetylating inhibitors have all been shown to have some ability to induce tumour re-sensitisation in previously refractory disease, raising the possibility of "rewriting the epigenetic code" as a means of modulating a tumour's response to therapy ${ }^{241,242}$. RRx-001 has been demonstrated to cause global DNA demethylation in squamous-cell cancer cell lines in vitro (SCC VII), and reduce DNMT1 and DNMT3a expression in a time-and dose-dependent manner ${ }^{243}$. In this study, global DNA demethylation was comparable to that observed with equivalent doses of AZA, although at an individual gene level there was a high degree of variance in the effects; some loci demonstrating hyper-and some hypo-methylation.

In clinical trials RRx-001 has been shown to be biologically active at safe doses in target populations (those with advanced metastatic solid organ tumours), and to be a potent generator of reactive nitrogen and oxygen species in vivo ${ }^{244,245}$. A number of case reports and Phase I trials against metastatic or end-stage Non-Small and Small Cell Lung Cancers, squamous tumours, prostate cancers, and melanomas have indicated that RRx-001 may have beneficial effects when combined with previously exhausted chemo- or radio-therapeutic strategies ${ }^{246-251}$. A current Phase III trial is examining the role of RRx-001 in platinum-treatment-refractory Small Cell Cancer (lung \& non-lung), with progression-free survival of 12 months the primary outcome, estimated to be complete by $2020^{252}$. RRx-001 has also been shown to have immunomodulating effects in the tumour microenvironment in neuroendocrine tumours, and has demonstrated the ability to enhance tumour-related macrophage polarisation and $\mathrm{T}$ lymphocyte infiltration ${ }^{253-256}$. Further studies have suggested a link between RRx-001 induced demethylation and activation of interferon responsive genes in colorectal cell lines (HCT 116); inhibiting tumour cell growth and activating cellular anti-viral defence by "viral mimicry"257.

Despite a growing body of research into the cellular and tumour-related effects of RRx-001, the exact mechanisms of its action are not well understood, and it is likely that it acts via number of different mechanisms that contribute to its effect. There is however reliable evidence to suggest that at least some of these effects are mediated by a demethylating action. 


\subsubsection{Primers}

Methylation specific PCR primers were selected, designed, and optimised by myself and other members of the research group based on previous enquiry ${ }^{204,258}$. Metalloproteinase pathway cDNA primers were selected from open-source references by the author and optimised inhouse. Each primer was ordered through Sigma-Aldrich; and is summarised in Table 2.3. PCR conditions will be described in relevant chapters.

Table 2.3 Primers. U, Un-Methylated; M, Methylated; FWD, Forward; REV, Reverse; MW, Molecular Weight; DM, Dimerization; $2^{\text {nd }}$, Secondary (VW - very weak; W - Weak; M Moderate; S - Strong).

\begin{tabular}{|l|l|l|l|l|}
\hline Name & MW & DM & $\mathbf{2}^{\text {nd }}$ & Sequence 5'-3' \\
\hline hMLH U FWD & 7477 & No & None & AGAGTGGATAGTGATTTTTAATGT \\
\hline hMLH U REV & 7534 & No & None & ACTCTATAAATTACTAAATCTCTTC \\
\hline MINT1 U FWD & 8603 & No & None & AATTTTTTATATATATTTTTGAAGTGT \\
\hline MINT1 U REV & 6603 & No & None & AACAAAAAACCTCAACCCCACA \\
\hline SOCS1 U FWD & 9332 & No & None & TTTTTTGGTGTTGTTTGGAGGTTGGATTTT \\
\hline SOCS1 U REV & 10681 & No & None & AAAACAAAACAATAAACTAAAACACTACAAAACCA \\
\hline HAND1 U FWD & 6233 & No & None & AATAGTTTAGGGTGTTGGTT \\
\hline HAND1 U REV & 5700 & No & None & AAATTTTACACTCAACCCA \\
\hline ADAMTS1 U FWD & 7831 & No & None & GTGAGTAATATTGTAGTTAAGGTGG \\
\hline ADAMTS1 U REV & 7582 & No & None & AAAACAAAAAAACACTCTAAAACTCC \\
\hline NEUROG U FWD & 7482 & No & None & TTGTTGGTTAATTGGTGGTGTTGT \\
\hline NEUROG U REV & 7459 & No & VW & CATTACCTCAACCACTAATCACCCA \\
\hline IGFBP3 U FWD & 7704 & No & None & TTATTTTGGTTTTTATATAGTGGTT \\
\hline IGFBP3 U REV & 7573 & No & None & AACAAAAAACAACTAATCCTCAACA \\
\hline THBD U FWD & 7407 & No & None & ATGTGTTTGTTTTTATTTGGTGTT \\
\hline THBD U REV & 6618 & No & None & CATAACTAACCAAAAACCCACA \\
\hline hMLH M FWD & 5514 & No & M & GATAGCGATTTTTAACGC \\
\hline hMLH M REV & 7261 & No & None & TCTATAAATTACTAAATCTCTTCG \\
\hline MINT1 M FWD & 7939 & No & None & AATTTTTTTATATATATTTTCGAAGC \\
\hline MINT1 M REV & 5407 & Yes & None & AAAAACCTCAACCCCGCG \\
\hline SOCS1 M FWD & 5881 & No & None & TTGTTCGGAGGTCGGTTT \\
\hline SOCS1 M REV & 6088 & No & None & ACTAAAACGCTACGAAACCG \\
\hline HAND1 M FWD & 6203 & No & None & AATAGTTTAGGGCGTTGGTC \\
\hline HAND1 M REV & 5419 & No & None & AATTTTACGCTCAACCCG \\
\hline ADAMTS1 M FWD & 7891 & No & None & GTGAGTAATATCGTAGTTAAGGCGG \\
\hline
\end{tabular}




\begin{tabular}{|l|l|l|l|l|}
\hline ADAMTS1 M REV & 7629 & No & None & CTAAAACAAAAAACGCTCTAAAACG \\
\hline NEUROG1 M FWD & 6492 & No & None & AATTTATGTTCGCGGGAGGTC \\
\hline NEUROG1 M REV & 6024 & No & None & ACCAACTTAACCCGAACCGA \\
\hline IGFBP3 M FWD & 6737 & No & None & TTTCGGTTTTAATATAGCGGTC \\
\hline IGFBP3 M REV & 6689 & No & None & AAAAAACGACTAATCCTCAACG \\
\hline THBD M FWD & 6385 & No & None & CGTTCGTTTTTATTCGGCGTC \\
\hline THBD M REV & 5678 & No & None & GCCAAACCCCATCTCATCG \\
\hline MMP2 cDNA FWD & 6926 & No & None & TTTCCATTCCGCTTCCAGGGCAC \\
\hline MMP2 cDNA REV & 7473 & No & None & TCGCACACCACATCTTTCCGTCACT \\
\hline MMP9 cDNA FWD & 5979 & No & None & CCTGCCAGTTTCCATTCATC \\
\hline MMP9 cDNA REV & 6019 & No & None & GCCATTCACGTCGTCCTTAT \\
\hline MMP11 cDNA FWD & 6133 & No & W & GGGGATGTCCACTTCGACTA \\
\hline MMP11 cDNA REV & 6262 & No & None & CAGTGGGTAGCGAAAGGTGT \\
\hline MMP13 cDNA FWD & 6057 & No & None & AACATCCAAAAACGCCAGAC \\
\hline MMP13 cDNA REV & 6109 & No & None & GGAAGTTCTGGCCAAAATGA \\
\hline TIMP2 cDNA FWD & 7135 & No & W & GGCGTTTTGCAATGCAGATGTAG \\
\hline TIMP2 cDNA REV & 6975 & No & W & CACAGGAGCCGTCACTTCTCTTG \\
\hline TIMP4 cDNA FWD & 6087 & No & W & AGACCTCACAGGCTCAGTCG \\
\hline TIMP4 cDNA REV & 6029 & No & W & CATTCCTGCCAGTCAGCCTG \\
\hline bACT FWD & 5492 & No & None & GATGGCCACGGCTGCTTC \\
\hline bACT REV & 5550 & No & S & TGCCTCAGGGCAGCGGAA \\
\hline
\end{tabular}




\subsubsection{Kits}

To facilitate the reproducibility and maintenance of standards across replications of experimental cycles, and to provide literature-referenced and evidenced methodologies, a number of bought-in experimental kits were employed. Kits reduced the necessity for in-house method design and development and were also a convenience. Deviations or optimisation of kit protocols will be discussed in each relevant chapter methodology. Kits are listed in Table

2.3.

Table 2.3 Kits

\begin{tabular}{|c|c|c|}
\hline Name & Use & Supplier \\
\hline DNeasy ${ }^{\circledR}$ Blood \& Tissue Kit & DNA extraction \& purification. & QIAGEN, Germany \\
\hline $\begin{array}{l}\text { EpiQuik }^{\mathrm{TM}} \text { DNMT Activity/ } \\
\text { Inhibition Assay Ultra Kit } \\
\text { (colourimetric) }\end{array}$ & $\begin{array}{l}\text { Measurement of DNMT activity or } \\
\text { inhibition based on nuclear } \\
\text { extracts. }\end{array}$ & Epigenetek, USA \\
\hline EpiQuik $^{\mathrm{TM}}$ Nuclear Extraction Kit & Nuclear protein extraction. & Epigenetek, USA \\
\hline Imprint ${ }^{\circledR}$ DNA Modification Kit & $\begin{array}{l}\text { One-step } \quad \text { DNA bisulfite } \\
\text { conversion \& post-modification } \\
\text { clean up. }\end{array}$ & Sigma-Aldrich (Merck), USA \\
\hline $\begin{array}{l}\text { MethylFlash }^{\mathrm{TM}} \text { Global DNA } \\
\text { Methylation (5-mC) ELISA Easy } \\
\text { Kit (colorimetric) }\end{array}$ & $\begin{array}{l}\text { Detection of global DNA } \\
\text { methylation. }\end{array}$ & Epigenetek, USA \\
\hline $\begin{array}{l}\text { QuantiFast }^{\circledR} \text { SYBR }^{\circledR} \text { Green RT- } \\
\text { PCR Kit }\end{array}$ & Quantitative one-step PCR. & QIAGEN, Germany \\
\hline $\begin{array}{l}\text { QuantiTect }{ }^{\circledR} \text { Reverse } \\
\text { Transcription Kit }\end{array}$ & $\begin{array}{l}\text { Reverse transcription of RNA to } \\
\text { cDNA with gDNA wipe-out }\end{array}$ & QIAGEN, Germany \\
\hline RNeasy ${ }^{\circledR}$ Mini Kit & RNA extraction \& purification. & QIAGEN, Germany \\
\hline $\mathrm{RT}^{2}$ First Strand Kit & cDNA synthesis for RT PCR & QIAGEN, Germany \\
\hline $\begin{array}{l}\mathrm{RT}^{2} \text { Profiler }{ }^{\mathrm{TM}} \text { PCR Array - } \\
\text { Human Tumour Metastasis }\end{array}$ & $\begin{array}{l}\text { Pathway \& process specific } \\
\text { quantitative RT PCR }\end{array}$ & QIAGEN, Germany \\
\hline
\end{tabular}




\subsubsection{Antibodies \& Immunofluorescence}

Antibodies were employed at two stages during this research; firstly during expressional analysis and assessment of knock-down by indirect immunofluorescence (IIF) in cell culture experiments, and secondly during immunohistochemistry (IHC) of tissue microarrays. Each process will be discussed in detail in the relevant chapter, but antibodies employed are listed in Table 2.4. Appropriate horse-radish peroxidase conjugated secondary antibodies were used for Western Blotting.

Table 2.4 Antibodies

\begin{tabular}{|c|c|c|}
\hline & Name & Supplier \\
\hline \multirow{3}{*}{ 刍 } & MMP-2 Monoclonal Antibody [sc-13594] (mouse, IgG) & Santa Cruz Biotech, USA \\
\hline & TIMP-2 Monoclonal Antibody [sc-365671] (mouse, IgG) & Santa Cruz Biotech, USA \\
\hline & TIMP-4 Polyclonal Antibody [PA5-30228] (rabbit, IgG) & Fisher Scientific, USA \\
\hline \multirow{5}{*}{$\stackrel{U}{\Theta}$} & MMP-2 Monoclonal Antibody [EPR1184] (rabbit, IgG) & Abcam, USA \\
\hline & MMP-9 Polyclonal Antibody [ab38898] (rabbit, IgG) & Abcam, USA \\
\hline & MMP-11 Polyclonal Antibody [ab119284] (rabbit, IgG) & Abcam, USA \\
\hline & TIMP-2 Monoclonal Antibody 3A4 [ab1828] (mouse, IgG) & Abcam, USA \\
\hline & TIMP-4 Polyclonal Antibody [ab58425] (rabbit, IgG) & Abcam, USA \\
\hline \multirow{3}{*}{$\begin{array}{l}\vec{E} \\
\vec{\Xi} \\
\vec{Z}\end{array}$} & Goat Anti-Rabbit IgG H\&L Alexa Fluor ${ }^{\circledR} 488$ [ab150077] & Abcam, USA \\
\hline & Goat Anti-Mouse IgG H\&L Alexa Fluor ${ }^{\circledR} 488$ [ab_2534060] & Fisher Scientific, USA \\
\hline & OV HQ Universal Linker + HPR Multimer & Ventana (Roche), Switzerland \\
\hline
\end{tabular}

\subsubsection{Software}

Table 2.5 summarises the primary computer software employed during this research (not including benchtop equipment with integrated software - see section 2.2.2), including plugins, but excluding web browsing software and operating systems.

Table 2.5 Software

\begin{tabular}{|l|l|}
\hline Name \& Edition & Primary Role \\
\hline Image J (v.1.51) & Image analysis \\
\hline Image Lab 6.0.1 & Gel analysis \\
\hline Lumaview v.17.11.04 & Time-lapse microscopy \\
\hline Metafer 4 v. 3.8.5 (MSearch) & Slide handling and microscopy \\
\hline Microsoft Office (Word v.16.12, Excel v.16.12) & Word processing, data handling \& analysis \\
\hline XLStat, Addinsoft (v.2017) & Plug-in statistics package for MS Excel \\
\hline
\end{tabular}




\subsection{Cell Lines \& Culture}

\subsubsection{Cell Lines}

\subsubsection{DLD-1}

The primary cell line used as a model for rectal cancer throughout this research is DLD-1, originally sourced from the European Collection of Animal Cell Cultures (ECACC 90102540), and maintained in-house (average passage 7$)^{259}$. DLD-1 is a human colon epithelial cell line derived from an adenocarcinoma in a male patient (also the source of colorectal cell lines HCT15, HCT-8, and HRT-18) ${ }^{260}$. DLD-1 have a $2 \mathrm{n}=46$ pseudodiploid karyotype. DLD-1 has an adherent growth mode and is best cultured in RMPI $1640+2 \mathrm{mM}$ glutamine $+10 \%$ foetal bovine serum (FBS). DLD-1 was selected as it is a widely used cell line for the investigation of colorectal cancer in vitro and has been used extensively in the proceeding and preparatory studies for this research ${ }^{204}$. DLD-1 demonstrates a high level of microsatellite instability and is CIMP-positive across two epigenomes commonly examined in tumour DNA methylation research, in addition to our own methylation panel, and thus serves as a good model for investigating consensus molecular subtype I tumours. DLD-1 is also CIN-negative and BRAF $w t$, but demonstrates KRAS G13D amino acid substitution relating to underlying mutation ${ }^{261}$, 262. Unfortunately, there is no commercially available rectum-specific human adenocarcinoma cell line.

\subsubsection{HFL-1}

Human foetal lung fibroblasts, HFL-1, were used as positive controls for immunocytochemistry of metalloproteinase expression in knockdown experiments, originally sourced from the European Collection of Animal Cell Cultures (ECACC 89071902), and maintained in-house (average passage 5) ${ }^{263}$. HFL-1 have a $2 \mathrm{n}=46$ diploid karyotype. HFL-1 has an adherent growth mode and is best cultured in Ham's F12 $+2 \mathrm{mM}$ glutamine $+1 \%$ NEAA $+10 \%$ FBS.

\subsubsection{Cryopreservation}

Cells were grown to a maximum of $70 \%$ confluency and then washed twice with sterile phosphate buffered saline (PBS), lifted with trypsin, and then washed a further two times with PBS by serial centrifugation and re-suspension. Cells were then diluted to a concentration of $1 \times 10^{6}$ cells $\mathrm{ml}^{-1}$, spun to pellet, and resuspended in FBS and DMSO at a ratio of 9:1 maintaining the starting concentration. Aliquots of $1 \mathrm{ml}$ were then transferred to cryovials and frozen for 
$24 \mathrm{hrs}$ at $-80^{\circ} \mathrm{C}$ in a BICELL Cellular Cryopreservation Vessel. Frozen cryovials were then transferred to liquid nitrogen at $-196^{\circ} \mathrm{C}$ for long-term storage.

\subsubsection{Cell Seeding \& Maintenance}

Standard laboratory personal protective equipment (lab coat and gloves) were employed to maintain personal protection and prevent cross-contamination. Surfaces were washed with $70 \%$ ethanol and allowed to air dry. All culture took place in ScanLaf Class II Tissue Culture Hoods. Frozen cells were incubated at $37^{\circ} \mathrm{C}$ with $5 \% \mathrm{CO}_{2}$ prior to seeding, and all culture media \& solutions (PBS, trypsin, etc) were pre-warmed to $37^{\circ} \mathrm{C}$. Active, adherent cell lines were detached from their growing environment by incubating in warmed trypsin for 5-10 minutes after washing.

Cell lines were maintained in their growth medium of preference (see section 2.3.1) at a maximum of $70 \%$ confluency. Cells were initially seeded at a concentration of $3 \times 10^{4}$ cell ml${ }^{1}$ based on published cell growth rate / doubling times in $75 \mathrm{~cm}^{2}$ flasks (working volume typically $25 \mathrm{mls}$ ) and allowed to proliferate. Cells were maintained in flasks for a maximum of four days between splitting, and concentrations were determined using a Beckman Coulter Counter. Cells were removed from maintenance culture and split to experimental flasks (typically $25 \mathrm{~cm}^{2}$, working volume $5 \mathrm{mls}$ ) or other culture environments according to protocol; each of which will be discussed in the relevant chapter. 


\section{Chapter 3}

\section{DNA Hypermethylation as a Predictor of Extramural Vascular Invasion (EMVI) in Rectal Cancer.}

\subsection{Introduction}

\subsubsection{Epidemiology of Rectal Cancer}

Colorectal cancer (CRC) is the third most frequent cancer and the second leading cause of cancer death worldwide ${ }^{264}$. Each year, one million people will develop CRC, and 40-50\% will die within five years ${ }^{265}$. Furthermore, rectal and distal sigmoid cancers are known to present at a later stage, and have a poorer prognosis than other colonic cancers ${ }^{266}$. Data from the Surveillance, Epidemiology, and End Results programme (SEER) indicates that between 1995 and 2008 there were 110,607 cases of rectal cancer recorded in the US (estimated population capture $25 \%$ ), representing approximately $30 \%$ of all colorectal cancers ${ }^{267}$. Patients presenting were on average five years younger than those with colonic cancer (median age 67 vs 72 years; $\mathrm{p}=<0.001)$ and were more likely to be male $(56.8 \% ; \mathrm{p}=<0.001)$. This data is in keeping with the most recent National Bowel Cancer Audit from the UK (NBOCA 2018), where of the 30,541 patients diagnosed with colorectal cancer between April 2016 and March 2017, 8,514 (27.9\%) had a rectal cancer, and of whom 53\% progressed to major resection and $7 \%$ to local excision $^{268}$. A recent European population-based study of 139,457 patients with colorectal cancer from England, Denmark, Norway, and Sweden demonstrated that the pooled (all stages) 3-year survival from rectal cancer in England was actually better than that for colon cancer (69.7\% vs 63.9\%), but that survival for both cancer locations was worse in England compared to the other Northern European countries ${ }^{269}$. Survival in England was notably worse in stage II and III rectal cancers (and stage IV colon cancer), as demonstrated in Figure 3.1, with the number of patients undergoing surgery for rectal cancer lowest in England (59.9\%) when compared to other nations (highest: Sweden, 70.8\%). A systematic review of surgery for locally advanced or locally recurrent rectal cancer (1016 patients) demonstrated a mean overall survival of 31 months and median 5-year survival of $32 \%$. 
Figure 3.1 Northern European age-standardised survival for rectal adenocarcinoma diagnosed between 2010 and 2012 (adapted from Benites Majano et.al.) ${ }^{269}$.
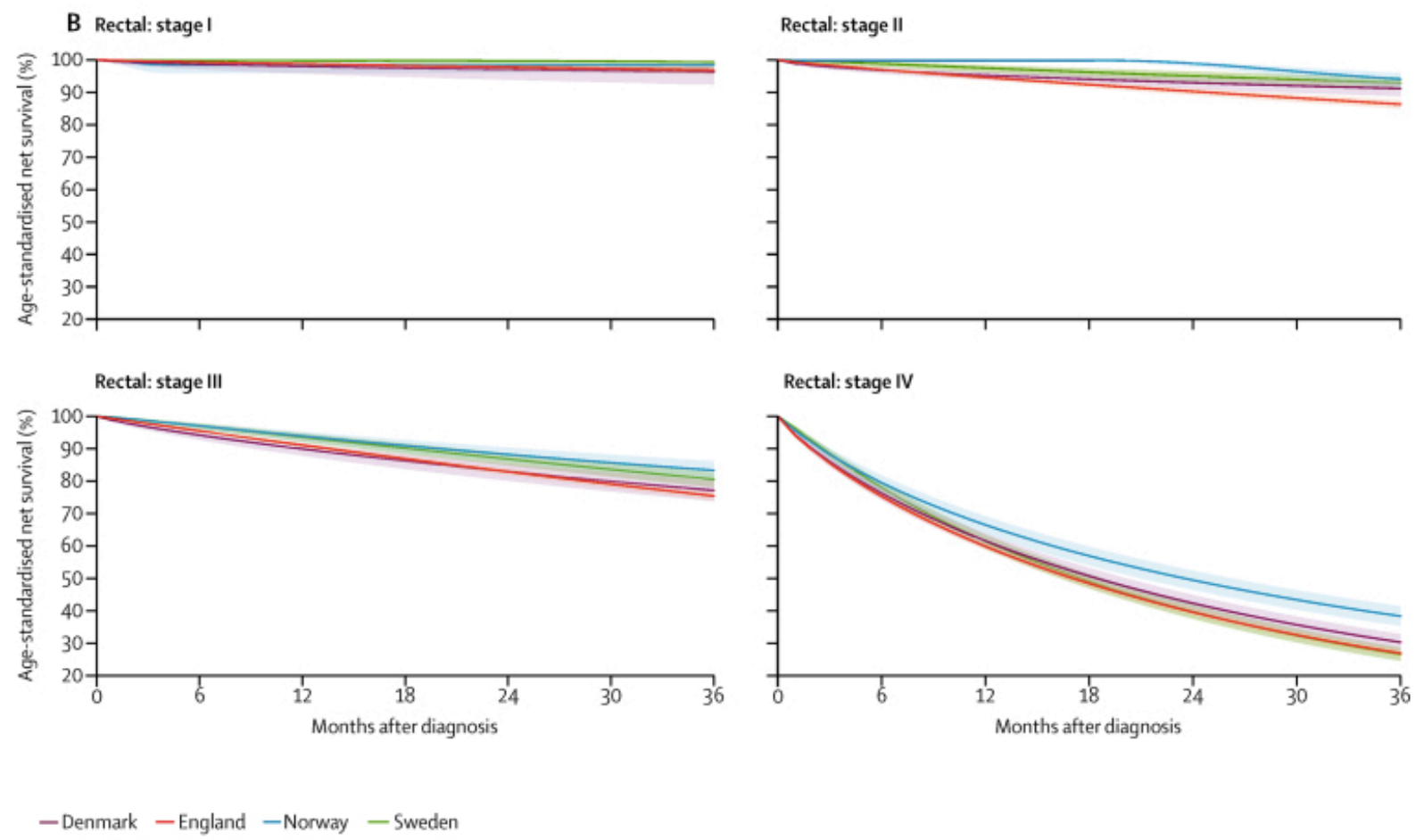

-Denmark - England - Norway - Sweden

\subsubsection{Extramural Vascular Invasion in Rectal Cancer}

Extramural vascular invasion (EMVI) is characterised by the presence of organised tumour in the vessels adjacent to the primary tumour beyond the muscularis propria and has been reported in approximately $25 \%$ of resected rectal tumours, although the reported incidence has varied between $17 \%$ to $70 \%{ }^{271-273}$. Despite its recognition within pathological specimens for some time, defining and recording its presence as a separate entity from other T3 tumours has not been consistent, although the current reporting criteria issued by the Royal College of Pathologists stipulates that it is a mandatory reporting criterum ${ }^{207,274,275}$. EMVI has been demonstrated to be an important risk factor for systemic recurrence, local recurrence and death, independent of $\mathrm{T}$ stage $272,273,276-279$. Additionally, EMVI status influences the need for neoadjuvant chemoradiotherapy and adjuvant chemotherapy, as it has been demonstrated that chemoradiation (CRT) can cause vessel fibrosis in EMVI-positive tumours; which may influence survival outcomes ${ }^{85,280}$. 
Although magnetic resonance imaging (MRI) has an undisputed role in defining involvement in the circumferential resection margin $(\mathrm{CRM})$ in rectal cancers, as demonstrated in the MERCURY trials, it's role in assessing EMVI not threatening the CRM is not as well defined $^{281-284}$. The outcome of the MERCURY II trial (2016) demonstrated the diagnostic accuracy of MRI in predicting CRM involvement in low rectal cancers, and in stratifying them into "safe" and "unsafe" groups that would or would not benefit from neoadjuvant radiotherapy and/or sphincter saving surgery ${ }^{285}$. This prospective validation also demonstrated the utility of MRI in assessing "safety" following neoadjuvant therapy, allowing the potential for sphinctersaving operations to be considered for those who had shown disease response.

However, the seminal study of MRI assessed EMVI led by G. Brown in Cardiff in 2003 suggested that there was a weighted agreement of $\kappa=0.64$ between pre-operative MRI and histopathology, although the total study population was only 98 patients, of whom 26 had histologically confirmed pEMVI and of these only 18 were visible to the naked eye with standard staining techniques ${ }^{284}$. The study suggested that the relatively poor concordance, even when compared to a naked-eye assessment of histology specimens, was the result of MRI not being sensitive enough to detect mrEMVI in vessels of less than $3 \mathrm{~mm}$. Concordance between mrEMVI against pEMVI identified at microscopy would therefore be even less favourable due to a higher denominator. In a subsequent study led by the same author, mrEMVI (as defined by a 0-5 point scoring system, later dichotomised into mrEMVI-positive or -negative), has a sensitivity and specificity of only 68 and 88 percent, respectively, compared to histology ${ }^{280}$. However, despite poor sensitivity and specificity, univariable analysis of 3-year relapse-free survival for each group was highly significant $(35 \% \mathrm{vs} 75 \%, \mathrm{p}=<0.001)$ and corresponded well with outcomes based on histology.

The predictive prognostic significance of MRI detected EMVI has been further evaluated in larger cohort studies, such as that by Bugg in 2014; this study demonstrated that within a population of 788 patients with rectal adenocarcinoma diagnosed between 2007 and 2012, $26.2 \%$ had an mrEMVI-positive tumour and of this proportion approximately $25 \%$ went on to develop liver metastasis within 1 year, compared to $6.7 \%$ of mrEMVI-negative tumours $(p=<0.001, R R=3.7)^{286}$. These findings have been corroborated further by meta-analysis of 1262 patients with rectal cancer (403 mrEMVI-positive), where EMVI was found to be associated with a higher chance of presenting with or developing metastasis $(\mathrm{OR}=5.68$ \& $\mathrm{OR}=3.91$, respectively, both $\mathrm{p}=<0.001)^{287}$. 
However, the lack of preoperative MRI diagnostic certainty for staging beyond CRM clearance was clearly demonstrated by a large meta-analysis of 21 studies by Al-Sukhni published in 2012 288 . MRI specificity for CRM involvement was 94\% (95\% CI 88-97) despite a high degree of inter-study heterogeneity, but specificity fell to $75 \%(95 \%$ CI $68-80)$ and $71 \%$ (95\% CI 59-81) for $\mathrm{T}$ and $\mathrm{N}$ stage, respectively.

The presence of mrEMVI is therefore considered an important component of pre-operative staging but is largely limited to its impact on the CRM. The presence or absence of EMVI is included in several international reporting proformas for preoperative radiological disease staging, including the European Society for Medical Oncology guidelines for the diagnosis and management of rectal cancer and the Royal College of Radiologists guidelines for crosssectional imaging in cancer management ${ }^{289-292}$. mrEMVI does not however constitute a specific item within of the preoperative TNM system advocated by the Union for International Cancer Control (UICC), which does not discriminate between a T3 primary tumour mass and serpiginous and/or distant vascular tumour extension as described by Brown in $2003^{280}$. Interestingly, the ACPGBI 2017 Guidelines for the Management of Colorectal Cancer (Investigation \& Staging section) does not mention mrEMVI in the context of preoperative staging; only that threat to CRM should be assessed by MRI [Recommendation grade B] (see section 2.2.1 of the 2017 guidelines, p12) ${ }^{206}$. Again, this suggests that mrEMVI is regarded as only significant when threatening the CRM and is not regarded as a separate pathological entity within the broader context of T3 disease, although conflictingly, mrEMVI is discussed in the same 2017 guidelines (Multidisciplinary Management section, see section 4.1.2, p28) where it is highlighted as a risk factor for local and distant recurrence independent of T stage (Figure 3.2) ${ }^{293}$. However, despite discrepancies in detection and classification, pre-operative mrEMVI has been shown to be a poor prognostic feature irrespective of subsequent management strategy; mrEMVI being associated with a four-fold increase in risk of distant metastasis (52\% vs $12 \%)$ and reduced 3-year relapse free survival at three years $(35 \%$ vs $74 \%)(\text { Figure 3.3 })^{294}$. 
Figure 3.2 Risk of local recurrence following resection of rectal cancer with curative intent based on preoperative MRI findings (adapted from Gollins, et.al.) ${ }^{293}$. Note that EMVI is listed as an independent risk factor for a moderate increased risk of local recurrence.

\begin{tabular}{|c|c|}
\hline Risk of pelvic local recurrence & Characteristics of rectal tumours predicted by MRI \\
\hline Low (resectable) & $\begin{array}{l}\text { cTl or } \mathrm{cT} 2 \text { or } \mathrm{cT} 3 \mathrm{a} \text { and } \\
\text { no lymph node involvement }\end{array}$ \\
\hline Moderate (resectable) & $\begin{array}{l}\text { any } \mathrm{cT} 3 \mathrm{~b} \text { or greater, in which the potential surgical margin is not threatened or } \\
\text { any suspicious lymph node not threatening the surgical resection margin or } \\
\text { the presence of extramural vascular invasion }\end{array}$ \\
\hline $\begin{array}{l}\text { High (borderline resectable } \\
\text { or unresectable i.e. threatened } \\
\text { or involved CRM) }\end{array}$ & $\begin{array}{l}\text { a threatened }(<1 \mathrm{~mm}) \text { or breached resection margin or } \\
\text { low tumours encroaching onto the inter-sphincteric plane or with levator involvement }\end{array}$ \\
\hline
\end{tabular}

Figure 3.3 EMVI is associated with worse 3-year relapse-free survival (RFS) irrespective of whether it is detected preoperatively (mrEMVI) or at histopathology, irrespective of management strategy (adapted from Smith et.al) ${ }^{280}$.
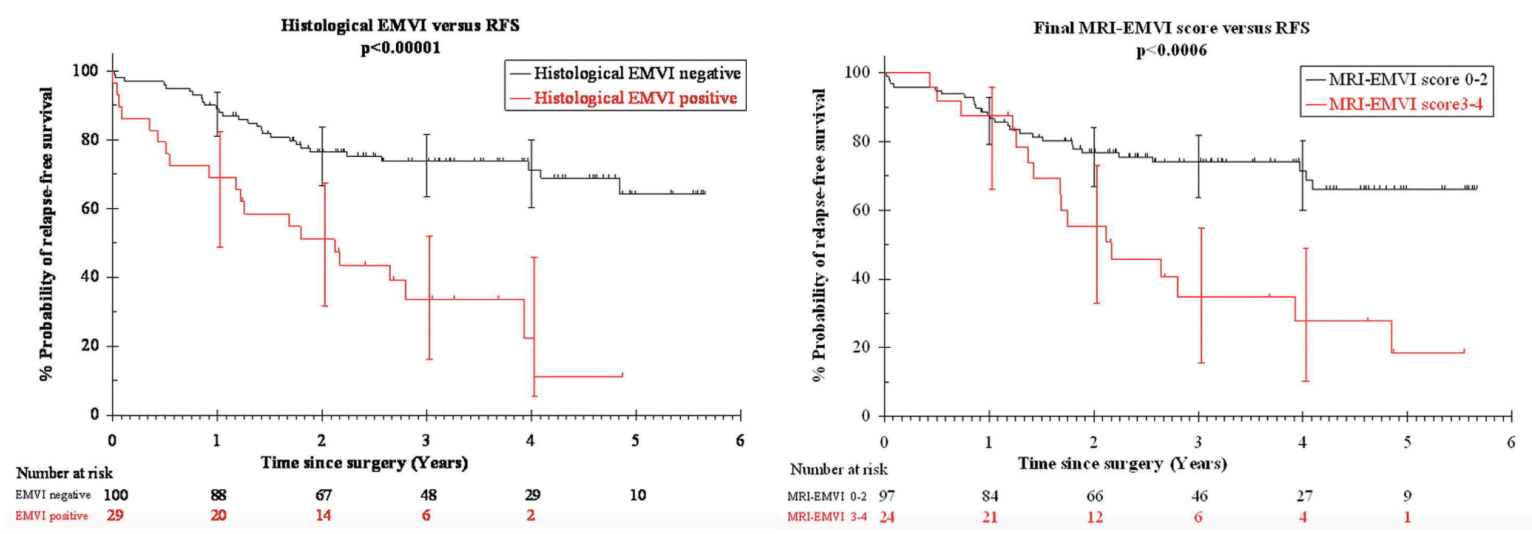

It is clear therefore that the presence of EMVI is a marker of poor prognosis, but it is not clear how this should affect management strategy. Even in the absence of a threatened CRM neoadjuvant short-course radiotherapy (SCPRT) and long-course chemoradiotherapy (LCCRT) have been shown to reduce local recurrence by approximately $50 \%$, although the absolute benefit is small (5-6\%) and the numbers needed to treat are high (17-20), resulting in a high proportion of futile overtreatment. Both SCPRT and LC-CRT have no effect on the risk of systemic recurrence or overall survival. Despite lacklustre benefits, the most recent ACPGBI guidelines suggest that all patients with high-risk CRM clear disease (including mrEMVI positive) should be considered for either SCPRT or LC-CRT to reduce the chance of local recurrence [Recommendation grade A], although as shown by the NBOCA data, clinical practice is highly variable across MDTs ${ }^{268,293}$. 
The role for neoadjuvant therapy in locally advanced and CRM-threatened rectal cancer is well evidenced; based on multicentre international randomised-controlled trials (the Dutch trials and UK MRC CR07 trial) ${ }^{295-297}$. Evidence for treatment is irrespective of whether it is EMVI or primary tumour extension that is threatening the CRM, and the primary benefit is in local disease control (LR). The current standard of care in this situation is LC-CRT with a singleagent fluoropyrimidine and radiotherapy, followed by surgery 6-12 weeks after treatment to allow tumour regression; based on large trials demonstrating the superiority of combined chemo- and radiotherapy (EORTC 22921 and FFCD 9203) 79, 80, 99. Neoadjuvant CRT was further demonstrated to be superior to adjuvant CRT, in terms of LR and reduced toxicity, in the German GAO/ARO/AIO-94 trial, although again, no DFS or OS benefit was evident ${ }^{78,298}$. Interestingly, long-term outcomes from the German study indicate that perioperative complications, including anastomotic leak and wound complications were associated with poor overall and disease-specific survival (recurrence and metastasis), and that surgical complication was predictive of reduced OS based on Cox regression analysis $(\mathrm{p}=0.008)$. Shortcourse protocols were found to be inferior regarding pCR rates (Polish trials), although LR, DFS, and OS were not significantly affected (TROG trial) ${ }^{299-301}$.

However, as discussed in Section 1.2.2, a significant proportion of patients will undergo a PCR following neoadjuvant CRT, although a further significant proportion will progress during therapy; including the development of EMVI, progression of EMVI, local tumour extension, and development of metastatic disease ${ }^{72-75}$. The response to therapy ultimately dictates surgical strategy, presenting the opportunity for both organ and sphincter preserving techniques, but also for progression and palliation. Currently there is no reliable pre-treatment test to aid in defining which patients will benefit from neoadjuvant therapy for either high-risk CRM-clear or CRM-threatened/involved disease, although biomarkers, including CIMP, are being examined as potential aids to direct therapy ${ }^{76,77}$. 


\subsubsection{Epigenetic Biomarkers in Colon \& Rectal Cancer}

\subsubsection{Introduction}

Hypermethylation and hypomethylation in colorectal cancer were first observed by Feinberg and Vogelstein in 1983, occurring at $\mathrm{CpG}$ islands and resulting in transcriptomic silencing of tumour suppressor genes and genes involved in DNA mismatch repair ${ }^{302,303}$. As associations are made between the development of colorectal cancer and epigenetic aberrations there is a potential for epigenetic analysis to help guide our diagnosis and management of colorectal cancer $^{304}$. Gaining an insight into tumour behaviour, including diagnosis, prognosis, and response to therapy by analysing epigenetic changes may potentially improve outcomes if they can effectively guide therapy ${ }^{305,306}$. Important pathways in cell regulation have already been implicated in the epigenetic pathogenesis of CRC; including those of the Wnt pathway (APC, AXIN2, DKK1, SFRP1, SFRP2, WNT5A), the CDKN2A cell-cycle genes, RAS signalling, and the DNA repair genes MGMT and hMLH1 \& hMLH2 $2^{307-309}$. However, despite epigenetic silencing of classical tumour suppressor genes and dysregulation of other cancerrelated pathways having been demonstrated in CRC, epigenetic profiling has only been implemented in guiding personalised therapies in a limited number of clinical scenarios. Specific examples are in the management of some gliomas and prostate cancers, where in the case of gliomas, the hypermethylation-related silencing of $\mathrm{O}^{6}$-methylguanine-DNA methyltransferase (MGMT) is predictive of response to treatment by alkylating agents, although the beneficial response in the hypermethylated group is eventually lost and all patients relapse $^{310-312}$. The reason for limited implementation may lie in the complexity of the epigenetic factors potentially involved in tumourigenesis; thus epigenome-wide studies such as the BLUEPRINT and NIH Roadmap projects are being performed to map the human DNA methylome to provide reference for investigators seeking disease-specific factors ${ }^{313-315}$. It is hoped that these projects will provide the groundwork for further insight and prove as important for epigenetics as the Human Genome Project was for genetics. Epigenetic techniques do not however have to be considered in parallel to classical genetic methods, and the combination of the two can usefully be applied in screening and other tests, as has already been demonstrated in small studies of stool DNA analysis for adenoma and carcinoma ${ }^{316}$. It is worth noting at this point that much of the research into epigenetic aberrations in lower gastrointestinal cancers fails to distinguish colonic from rectal cancers, and thus much of the literature refers to colorectal cancer in general, although a small sub-population do focus on individual tumour sites. This issue is highlighted by Jia in a meta-analysis of CIMP and its relationship to 
outcomes in colorectal cancer, where only $1 / 30$ included papers were specific to rectal cancer $^{317,318 .}$.

\subsubsection{Diagnosis}

Identifying and validating the consistency of aberrant methylation in CRC opens the possibility that DNA detected in serum or stool may provide a non-invasive means of diagnosing patients with CRC, and even detecting adenomas ${ }^{319}$. Currently there are three commercially available serum tests for colorectal cancer, each of which is based up detecting hypermethylated (m)SEPT9 tumour DNA (Epi proColon ${ }^{\circledR} 2.0$; ColoVantage $\AA$; Real Time mS9 $\left.{ }^{3}\right)^{320-323}$. Each test employs a qualitative PCR methodology to detect the presence of mSEPT9 (normally an unmethylated gene coding a protein involved in pseudopod protrusion and cell migration), but individually reported sensitivities range from approximately $40 \%-95 \%$ and specificities of $80 \%-90 \%$ based on training and test cohorts of 250-1544 patients (typically 1:2 ratio of cancers to controls) $)^{320,324}$. However, a recent meta-analysis by Song identified 25 investigations including 8643 participants (2613 CRC cases) examining the diagnostic accuracy of mSEPT9 serum tests and reports that in the asymptomatic population mSEPT9 is inferior to FIT testing, although it may be superior in the symptomatic group ${ }^{325}$. The diagnostic accuracy described in this analysis was widely variable, with pooled analysis indicating the overall sensitivity for mSEPT9 ranged between $36.4 \%$ and $93.4 \%$, dependent on the test algorithm employed and disease stage. The utility of mSEPT9 is further limited by heterogeneity in the assays and testmodels employed in binarizing or stratifying at-risk groups, its projected poor costeffectiveness in population-based screening, and limited utility in detecting adenomas ${ }^{326}$. These findings are corroborated by the American College of Gastroenterology led MultiSociety Taskforce on Colorectal Cancer, who highlight the $48 \%$ crude sensitivity for cancer detection of mSEPT9 reported by Church (sensitivity ranged 35\%-78\%, increasing with disease stage), and thus do not recommend it as a diagnostic or screening test ${ }^{327,328}$.

Beyond mSEPT9, a number of other serum methylation-based diagnostic tests have been trialled, although in a limited manner. A study examining the methylation status of frizzledrelated protein 2 gene demonstrated a sensitivity of $67 \%$ across serum, blood, and tumour samples, and a serum-based screening methodology identified methylated THBD as a potential biomarker for CRC, although it was only sensitive and specific (75\% and $80 \%$, respectively) for stage II and III disease ${ }^{329,330}$. Compound tests employing multiple methylation markers have also been trialled with a degree of success. In a study of 243 patients, a test model (M- 
score) analysing hypermethylation at a set of four genes (APC, MGMT, RASSF2A, and Wif1) was sensitive (86.5\%) and specific (92.1\%) for the presence of sporadic colorectal cancer and large adenomas, translating to a positive- and negative-predictive value of $90.6 \%$ and $88.8 \%$, respectively (test cut-off 1.6$)^{331}$. A further study based on a candidate-epigene methodology (restriction analysis) and subsequent microarray and RT-PCR analysis identified three hypermethylated markers (TMEFF2, NGFR, and SEPT9) with acceptable test binarization based on circulating DNA analysis between patients with colorectal cancer and controls, although not at a high enough level to provide a utilisable clinical test ${ }^{332}$. Further studies of circulating tumour DNA methylation have proposed other epigenetic targets, although none has yet been translated into an implementable screening tool ${ }^{333,334}$.

Methylation-based stool tests have also been trialled, and currently a methylation analysis of the vimentin gene is under development in the USA, boasting a sensitivity of $83 \%$ and specificity of $82 \%$ irrespective of tumour location, stage, or patient age $\mathrm{e}^{335}$. However, two recent metanalyses of stool DNA tests, one specifically examining methylation in stool DNA, have demonstrated that in pooled cohorts of 2,356 and 5,876 individuals, no marker was superior to current screening tools in unselected populations, although methylation markers performed better than mutation markers (sensitivity $75 \%$ versus $67 \%$, specificity $91 \%$ versus 94\%, respectively), all be it across different effects models (methylation fixed model; mutation random model) ${ }^{336,337}$.

Currently, there are no methylation-based serum or stool DNA tests that are used in the screening or diagnosis of CRC, including mSEPT9, and most guidelines do not directly address DNA based testing of any sort ${ }^{338}$. Future evaluation of methylation-based markers such as hypermethylated GFRA1 and GSTM2, which were recently identified as potential diagnostic biomarkers in a genome-wide expressional analysis by Wei, may eventually prove fruitful if they can be translated into clinically utilisable tools ${ }^{339}$.

\subsubsection{Staging \& Prognosis}

As previously discussed, there are multiple genes and other loci where aberrations in methylation have been associated with the development of colorectal cancer, and a growing body of evidence suggesting specific hypermethylation events occurring on the classical adenoma-to-carcinoma pathway, as well as the development of tumour metastasis ${ }^{307}$. Examples of genes that are frequently found to be methylated in the early stages of dysplasia, 
including in aberrant crypt foci, are ITGA4, MGMT, SCL5A8, FFRP2, and MINT1 ${ }^{340-343}$. Interesting, the study by Kim and later confirmed by Oster, suggests that hypermethylation at specific genes is predominantly an early feature in the adenoma-to-carcinoma pathway, as many of the hypermethylation events that are present in cancers are already present in early

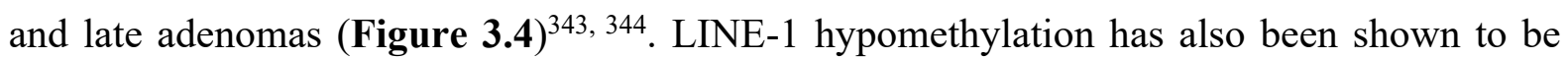
associated with early adenomatous changes in the colorectal mucosa, and increased with a linear relationship to disease progression (adenoma-carcinoma-metastasis) ${ }^{345}$. As well as individually hypermethylated genes or loci, the CIMP phenotype is also detectable in late adenomas, particularly of the serrated subtype, but is generally not present in earlier lesions, leading to the speculation that this is a gateway hypermethylation event in the development of more advanced disease ${ }^{346}$. Despite the identification of hypermethylated genes and application of the CIMP phenotype, no reliable method of discriminating between hyperplastic polyps and sessile serrated adenomas has been reliably developed, partially because of the heterogeneity in CIMP studies already discussed in section 1.3.6. As dysplastic polyps transform to invasive cancers further hypermethylation occurs at conserved sites, specifically CXLC12, TIMP3, ID4, and IRF8, although these markers have not yet proved adequate for risk-stratifying and guiding the subsequent management of resected polpys ${ }^{347}$.

Figure 3.4 Aberrant Hypermethylation Events in the Adenoma-to-Carcinoma Pathway (adapted from Lao \& Grady) ${ }^{307}$.

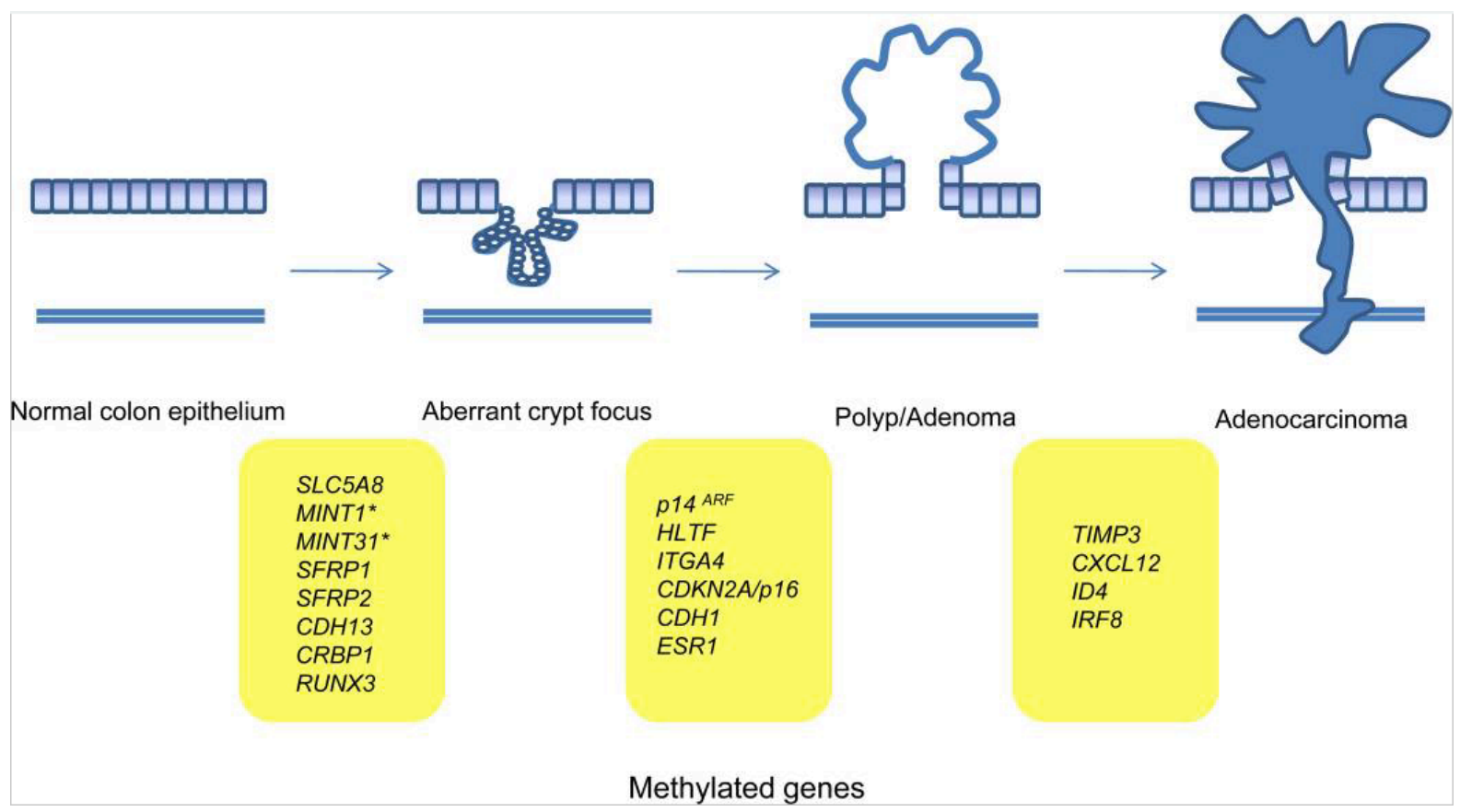


From a prognostic perspective, some aberrant methylation events have been associated with inferring an improved survival, such as MGMT and MLH1 hypermethylation, although hypermethylation of APC and p14 may infer a survival disadvantage 348,349 . Additionally, meta-analysis of $3440 \mathrm{CRC}$ patients found that hypermethylation of $\mathrm{p} 16$ gene CDKN2A to be associated with a worse OS (HR 1.49, CI 1.28-1.74), as well as with lymphovascular invasion (HR 1.68, CI 1.15-2.47) and lymph node metastasis (HR 1.68, CI 1.09-2.59) ${ }^{350}$. Hypermethylation of HOPX- $\beta$, IGFBP3, EVL, CD109, and FLNC has also been associated with a worse prognosis, although the association to disease stage is less well defined in these cases $^{306,351}$.

Since Toyota identified and first described CIMP, much of the focus relating to outcomes in CRC has been focused on methylator phenotypes rather than specific loci ${ }^{177,317}$. CIMP is known to be associated with differing clinical and molecular characteristics in colorectal cancer and has previously been linked to outcomes when associated with MSI status and BRAF mutation $^{178,352}$. These studies have not, however, demonstrated an independent relationship between CIMP and outcomes for a number of reasons; firstly, due to lack of statistical power and the necessity of compound modelling, but more importantly due to the heterogeneous methodologies employed in determining CIMP. In Dahlin's study of 604 patients from two Swedish databases, CIMP-L was found to be associated with poor survival compared to CIMPnegative irrespective of microsatellite status, but inconsistently across cohorts in MSS groups. CIMP-H was also found to have a worse prognosis in the MSS groups, but the effect was lost in pooled analysis ${ }^{352}$. No relationship was evident between CIMP and cancer specific survival in the MSI pools. In this study the proportion of CIMP-negative patients was approximately 50\%, with CIMP-L and CIMP-H contributing 35\% and 15\%, respectively. MSI was associated with CIMP-H, although statistical significance for RR was not achieved due to the small numbers involved (HR not interpretable on multivariate analysis). Although the datasets and the methodology of assessing methylation at each gene promotor region (bisulfite conversion and MethlyLight Q-RT-PCR) in this study are robust, the categorisation into CIMP status is essentially arbitrary; based on a simple and non-validated system of categorised cumulative numbers of methylated genes (CIMP-negative $=0$ genes methylated; CIMP-L $=1-5$ genes methylated; CIMP-H = 6-8 genes methylated). 
To further investigate the relationship between CIMP and survival outcomes Juo performed a meta-analysis of 33 studies including 10,635 patients which demonstrated that CIMP is significantly associated with shorter DFS (pooled HR 1.45, CI 1.07-1.97) and OS (pooled HR 1.43, CI 1.18-1.73) irrespective of microsatellite instability ${ }^{353}$. The same study also examined survival following 5FU therapy based on CIMP status and found directly contradicting evidence for both survival advantage and disadvantage in different studies that lead to nonsignificance in meta-analysis. Although a strong indicator for CIMP being associated with poor outcomes in CRC, Juo's meta-analysis demonstrated further that there is a high degree of heterogeneity in the patient cohorts included in analysis, and in the methods by which CIMP is classified. Of the 33 studies included, 21 employed a dichotomised CIMP classification while the remainder employed a trichotomized methodology, and there was an even greater range of genes within each panel (ranging from 3 to 13 individual genes/loci), as well as methods for determining methylation status at each locus (described by Juo as, "often chosen arbitrarily"). A pooled analysis of the different methodologies used to determine CIMP was performed by Jia and Guo in an attempt to discern if one panel was more associated with outcomes than another, but unfortunately no significant relationship between any one panel and clinical outcomes was discovered, although a consensus was reached that CIMP-positive as determined by any panel was likely to confer a worse survival outcome ${ }^{317}$. The authors speculate that the lack of superiority in one panel over another is due to the large number of and high degree of overlap in the genes used in each panel, although CACN1G, IGF2, NEUROG1, RUNX3, hMLH1, p16, MINT1, MINT2, and MINT31 were most commonly used.

Few studies specifically address the survival of rectal cancer patients in relation to CIMP status as most present mixed cohorts of colon and rectal cancers. Samowitz examines a population of 864 cancers where 103 were found to be CIMP-positive based on a dichotomized panel of five genes (MHL1, MINT1, MINT2, MINT31, and CDKN2A) where $\geq 2$ loci being hypermethylated indicating CIMP-positive $\mathrm{e}^{318}$. The authors chose this panel and system based on their previous use of the method in previous publications but do not offer further justification beyond stating that there is currently no consensus in determining CIMP ${ }^{175,354}$. This study reports that the 5-year survival for CIMP-negative and CIMP-positive is $72.0 \%$ and $63.2 \%$, respectively ( $\mathrm{p}=0.04)$ with an unadjusted hazard rate ratio (HRR) of 1.43 (CI $1.01-2.01$ ), although the HRR fell to 1.32 and $95 \%$ CI crossed $1.0(0.88-1.97)$ on multivariate analysis. 
In another study by Jo, 150 patients with locally advanced rectal cancer enrolled in two phase III clinical trials (CAO/ARO/AIO-94 and -04) were analysed for dichotomized CIMP status based on a panel of five genes (RUNX3, SOCS1, NEUROG1, IGF2, and CAGNA2G) ${ }^{149}$. CIMP status was associated with a worse 3- and 5-year disease-free survival for patients identified as CIMP-positive versus -negative (56\% vs $0 \%$, and $85 \%$ vs $75 \%$, respectively; HR 5.5 (CI 2.1 - 13.9). In this study, CIMP-positive was based on $\geq 3 / 5$ loci being hypermethylated and accounted for only $10 \%$ of the study population.

\subsubsection{Response to Therapies}

There are conflicting reports of the impact of epigenetic events in the response of CRC to chemo- and radiotherapy, and the field is plagued by the same inconsistencies in methodology that limit the analysis of methylation in diagnosis and outcomes. Williamson reviews the development of CIMP as a marker for outcomes in rectal cancer, specifically in response to neoadjuvant therapy, summarising the findings that both hypermethylation and hypomethylation may have important prognostic roles in defining the response of lower gastrointestinal cancers to CRT. However, as study cohorts are frequently a mix of colon and rectal cancers there is inconsistency in CIMP-determining methodologies and in definitions of response to therapy (such as tumour regression grades - TRG) there is no consensus ${ }^{148}$.

Specific examples of aberrant methylation affecting response to therapies have however been reported, including hypermethylated MGMT which is found in approximately $40 \%$ of CRC. In a phase II study of 68 patients with metastatic disease who have exhausted other therapies, methylated MGMT has been associated with a response to dacarbazine, where no response was detected in patients with the non-methylated gene ${ }^{355,356}$. Furthermore, Sun reports that a greater reduction of methylated MGMT in circulating tumour DNA was observed in patients demonstrating a good response to CRT (assessed by TRG) compared with those that did not respond well, although the number of participants in this study was extremely limited ${ }^{357}$. Molinari identified that methylation of the TIMP3 tumour suppressor gene was found to be different between tumours categorised on the basis of their TRG (ANOVA, $\mathrm{p}<0.05$ ), although it was not differentially methylated between tumour and healthy tissues prior to treatment. The case series was also restricted to 74 patients, limiting its predictive value, and the only markers found to be differentially methylated between cancerous and healthy tissue were ESR1, CDH13, RARB, IGSF4, and APC, although none of these genes was predictive of response to 
therapy or changed in methylation status following therapy ${ }^{358}$. In a study of 155 stage II and III tumour samples, Kawakami showed that LINE-1 hypomethylation was associated with a better response to adjuvant oral fluoropyrimidine therapy and overall survival compared to patients with high LINE-1 methylation ${ }^{359}$.

As well as potential predictors of favourable response, methylation has also been identified as a predictor of poor response. In a study by Ebert, hypermethylation of the TFAP2E gene was associated with poor response to CRT irrespective of regime, demonstrating a less that $10 \%$ tumour-regression rate compared with $82 \%$ of tumours with hypomethylated gene $(\mathrm{p}<0.001)^{360}$. Williamson also showed that CIMP assessed on the two-panel approach advocated by Kaneda and Yagi was significantly associated with EMVI, which itself was found to be an independent predictor of poor disease-related outcomes, in line with the wider literature ${ }^{185,186,204}$. However, in the previously discussed study by Jo, no association was found between CIMP status and tumour regression based on histological examination following neoadjuvant CRT and resection $^{149}$.

\subsubsection{Summary}

Methylation is emerging as an important biomarker in colorectal cancer. Its limitations are partly in the complexity of epigenetic events that are detectable in the carcinogenic pathway and discriminating between what is significant and what may be incidental. Additionally, divergence in methodologies for assessing individual gene promotor hypermethylation and in classifying CIMP has led to a lack of robustness in the evidence supporting its role CRC. This problem is not limited to CRC but is also a factor in defining the role of epigenetics in other tumour types, including gastric, breast, and many others ${ }^{361-363}$. The problems are however more acute in rectal cancer as it is most commonly included in broader cohorts of colorectal cancer; limiting the insight into what is increasingly becoming regarded as a separate disease entity and one that certainly has different management pathways. 


\subsection{Aims \& Objectives}

Rectal cancer is a disease that has a unique natural history and poses specific challenges to patients, clinicians, and scientists. Disease-specific outcomes for rectal cancer are worse than those for colonic cancers, as demonstrated by worse disease-free and overall survival. Rectal cancer also presents operative challenges that are generally not applicable to colonic tumours, especially when considering operating within the bony pelvis and constructing a "low" anastomosis. From a patients' perspective, pelvic surgery is frequently morbid and associated with the risk of significant side-effects and even death. Locally-advanced and locally-recurrent rectal tumours also frequently require neoadjuvant chemoradiotherapy (nCRT) to improve resection rates and reduce local recurrence, the administration of which is largely based on radiological staging. The presence of extramural vascular invasion (EMVI) is an important but inconsistently assessed component of this evaluation, meaning a proportion of patients with EMVI who may benefit from nCRT do not receive it. However, not all patients that are eligible for neoadjuvant therapy will benefit from it, and some may be better treated by primary surgery. Currently there is no method of predicting which tumours will respond to neoadjuvant therapy.

The role of epigenetic aberrations in colorectal cancer is not well defined. Methylation analysis, including CpG Island Methylator Phenotype (CIMP) classification, has been suggested as important in defining the pathogenesis, diagnosis, prognosis, and response to therapy of colorectal cancer; although currently there is no defining evidence of a clinically utilisable methylation-based investigation that is significant in managing individual patients with rectal cancer.

The general aims of this chapter are to investigate the methylation status of rectal cancers based upon CIMP and determine if there is any association to clinicopathological variables that may impact outcomes. 


\subsection{Material \& Methods}

\subsubsection{Patient Selection, Data Collection, \& Storage}

One hundred consecutive rectal cancer patients were extracted from the prospectively maintained database as described in section 2.1.1 \& 2.1.2, and in accordance with the ethics laid out in section 2.1.3. As previously stated, patients were neoadjuvant therapy naïve, and exclusion criteria also included patients with hereditary or other identifiable predispositions to carcinogenesis, such as inflammatory bowel disease. Patients who had received locoregional radiotherapy or prior chemotherapy within 10 years for other malignant diseases (such as radiotherapy for prostate cancer or previous chemotherapy for breast cancer) were also excluded. All patients underwent index surgery between January 2010 and May 2013 at a single centre (ABMU, Morriston Hospital, Swansea, SA6 6NL, UK) by a team of surgeons participating in the Swansea Pelvic Oncology MDT.

Tumour height was defined as low rectal (within $5 \mathrm{~cm}$ of the anus), mid rectal $(5-10 \mathrm{~cm}$ form the anus), or high ( $\geq 10 \mathrm{~cm}$ from the anus) dependent on the lowest extent of the tumour on initial MRI. Tumours at the rectosigmoid junction where the bulk of the tumour was located in the sigmoid, negating a TME excision, were excluded. Gender was dichotomised. Tumour characteristics were defined as per the ACPGBI or Royal College of Pathology guidelines with respective relation to pre-operative $(\mathrm{mTNM})$ or postoperative (pTNM) staging and other tumour characteristics (CRM, differentiation, EMVI) ${ }^{35,206}$. All patients underwent full preoperative staging as described by the ACPGBI guidelines; including computed tomography of thorax, abdomen, and pelvis; high-resolution magnetic resonance imaging of the pelvis; and in selected cases, endorectal ultrasound ${ }^{206}$. All cases were confirmed by biopsy taken preoperatively at endoscopy and cases other than adenocarcinoma (squamous, melanoma, sarcoma, gastrointestinal stromal tumour) were excluded. Surgical treatment included anterior resection, abdomino-peroneal resection, and beyond-TME procedures in keeping with guidelines, patient choice, and expertise (see section 1.2). Adjuvant chemotherapy was administered based on current guidelines and best-practice.

Investigators were blinded to individual and identifying patient data during sample processing and analysis by a process of anonymisation. After the core data clinical was collected and samples extracted from archive, information linking samples to patient information was randomised and only re-associated following completion of data collection to prevent bias. Patient identifiable data was stored on an encrypted and password protected spreadsheet, and 
information linking specimens for laboratory analysis to individuals was kept in a locked filing cabinet away from the laboratory, in keeping with the principles of NIHR Good Clinical Practice (GCP) and the UK Policy Framework for Health and Social Care Research ${ }^{364}$.

\subsubsection{Tissue Collection}

Tissue was extracted from the archives of ABMU Singleton Hospital Department of Pathology (Sketty Lane, Swansea, SA2 8QA, UK). Tissues were supplied in triplicate as fixed-informalin paraffin-embedded (FFPE) slides at $5 \mu \mathrm{m}$ thickness. Slides were stained with haematoxylin and eosin (H\&E) and checked by consultant histopathologist to ensure that each contained a minimum of $60 \%$ tumour. Where tumour was less than $60 \%$ of slide tissue it was marked so it could be selectively dissected from the slide to provide a more representative sample of tumour for analysis. Three slides were used for each patient to ensure adequate tumour DNA extraction.

\subsubsection{DNA Extraction}

Slides underwent de-waxing by submersion in $100 \%$ xylene for 5 minutes at room temperature, repeated twice in the laboratory fume hood. Slides were then submerged in $100 \%$ ethanol for a further 5 minutes and allowed to air dry at room temperature. Tumour cells were then removed using a clean scalpel blade by scraping into a $1.5 \mathrm{ml}$ eppendorf microcentrifuge tube.

DNA from tissues was obtained using the MasterPure Complete DNA and RNA purification kit (Epicentre, Illumina, Wisconsin, USA). A mixture of $2 \mu \mathrm{l}$ proteinase $\mathrm{K}$ and $300 \mu \mathrm{l}$ of Tissue and Cell Lysis Solution was applied to each sample and vortexed thoroughly. This mixture was then incubated at $65^{\circ} \mathrm{C}$ overnight (minimum $12 \mathrm{hrs}$ ) in a heater block and then re-vortexed the following day. This protocol is a slight deviation from the manufacturers protocol (longer incubation), as a prolonged period at $65^{\circ} \mathrm{C}$ was shown to increase DNA yield. Samples were then cooled to $37^{\circ} \mathrm{C}$ and $1 \mu \mathrm{l}$ of RNase A was added to the sample and vortexed. This was incubated at $37^{\circ} \mathrm{C}$ for 60 minutes. The samples were placed on ice for 3-5 minutes before proceeding with DNA extraction.

DNA extraction was performed by adding $175 \mu$ of MPC Protein Precipitation Reagent to 300 $\mu \mathrm{l}$ of lysed sample and vortexed vigorously for 10 seconds. The debris was pelleted by centrifugation at $4^{\circ} \mathrm{C}$ for 10 minutes at $\geq 10,000 \mathrm{x}$ g in a microcentrifuge. The supernatant was transferred to a clean microcentrifuge tube, the pellet was discarded, and $500 \mu \mathrm{l}$ of isopropanol 
was added to the recovered supernatant. The eppendorf containing the supernatant was then inverted 40 times to mix contents and precipitate DNA.

The mixture was then centrifuged again $4^{\circ} \mathrm{C}$ for 10 minutes at $\geq 10,000 \times \mathrm{g}$ to pellet the DNA. The isopropanol was carefully poured off without dislodging the DNA pellet. The pellet was then rinsed gently twice with $70 \%$ ethanol. Residual ethanol was removed with a pipette and the samples were left to air dry for 10 minutes. The DNA was re-suspended in $35 \mu 1$ of TE Buffer.

The quantity and quality of DNA was measured at absorbance between $230 \mathrm{~nm}$ and $320 \mathrm{~nm}$ using spectrophotometry (Nanodrop ND-1000, Software v 3.1.2, Thermoscientific, Delaware, USA). DNA quantity was calculated by multiplying the measured concentration following spectrophotometry at $260 \mathrm{~nm}$ with the dilution factor. DNA was diluted to a working concentration of $20 \mathrm{ng} \mathrm{l}^{-1}$. Purity was further assured by calculating the absorbance at $260 \mathrm{~nm}$ to absorbance at $280 \mathrm{~nm}$ ratio. Extracted DNA was stored at $-20^{\circ} \mathrm{C}$ during active experimentation and archived at $-80^{\circ} \mathrm{C}$ when not in use.

\subsubsection{Bisulfite Conversion}

Methylation specific PCR (msPCR) was carried out as a two-stage process of bisulfiteconversion and then amplification by bisulfite-specific primers, as described by Herman (Figure 3.5) $^{365}$. The process of bisulfite-conversion first exchanges unmethylated cytosine in CpG islands for uracil, and then subjects the converted DNA to parallel PCR reactions with primers targeted at the $\mathrm{CpG}$ islands of the genes of interest; one primer amplifying $\mathrm{CpG}$ islands containing uracil, and the other amplifying native methylated cytosine. Genes with unmethylated $\mathrm{CpG}$ islands will amplify against the converted uracil primer, and those with methylated $\mathrm{CpG}$ islands will amplify against the cytosine primer.

Bisulfite conversion was performed using the Imprint DNA Modification Kit (Sigma Aldrich, St. Louis, USA). A two-step bisulfite-conversion protocol was employed as per the manufacturers recommendation as a means of producing high-quality DNA from a lowquantity input (100 pg to $10 \mathrm{ng}$ ); the first step performs bisulfite conversion and the second step is a post-modification clean-up to remove unconsumed reagents. 
Figure 3.5 Bisulfite conversion \& the nucleotides cytosine and uracil.
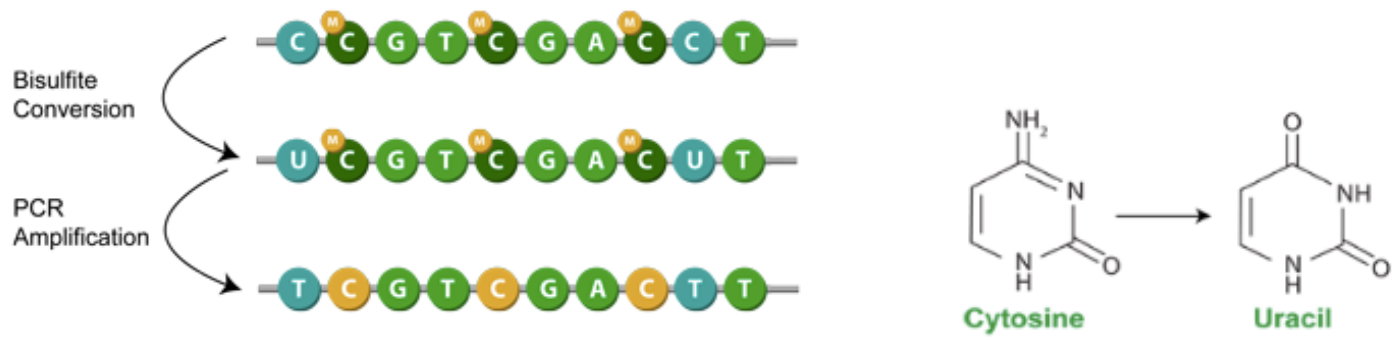

The DNA modification solution was first prepared by adding $1.1 \mathrm{ml}$ of DNA Modification Solution to 1 vial of DNA Modification Powder. This mixture was vortexed for 2 minutes at room temperature and then $40 \mu \mathrm{L}$ of Balance Solution was added, before a further brief vortex. The vial was examined for any undissolved particles was incubated at $65^{\circ} \mathrm{C}$ for 2 minutes if any were present, followed by further vortex. DNA was placed in a $1.5 \mathrm{ml}$ micro-centrifuge tube and $110 \mu \mathrm{L}$ of the prepared DNA modification solution was then added into the DNA and mixed by gentle pipetting. The mixture was the incubated at $99^{\circ} \mathrm{C}$ for 6 minutes and then at $65{ }^{\circ} \mathrm{C}$ for 90 minutes.

Clean-up was then performed by placing a spin column into a cap-less collection tube to which $300 \mu \mathrm{L}$ of Capture Solution had been added and allowed to sit on the column for 1 minute. The modified DNA solution was then placed into the spin column and centrifuged at $12,000 \mathrm{x} g$ for 20 seconds at $4^{\circ} \mathrm{C}$. The flow-through was then discarded. $200 \mu \mathrm{L}$ of an ethanol-diluted cleaning solution was the added to the spin column and centrifuged for 20 seconds. $50 \mu \mathrm{L}$ of a balance/ethanol wash solution was added to the bottom of the spin column and incubated for 8 minutes at room temperature. After incubation, further centrifugation for 20 seconds was performed and the flow-through discarded. $200 \mu \mathrm{L}$ of $90 \%$ ethanol solution was then added to the spin column and centrifuged for 20 seconds and the flow-through again discarded. The capless collection tube was then discarded, and the spin column placed into a $1.5 \mathrm{ml}$ collection tube. $20 \mu \mathrm{L}$ of elution solution was then added to the bottom of the spin column to extract the DNA from the column and return to solution. The spin column and elution solution were incubated for 1 minute at room temperature and then centrifuged for 20 seconds. The spin column was then discarded, and the eluted solution collected contained the purified bisulfite modified DNA. Modified DNA the proceeded directly to msPCR or was stored at $-20^{\circ} \mathrm{C}$ for up to 2 months. 


\subsubsection{Methylation Specific PCR (msPCR)}

All msPCR took place in the T100 ${ }^{\mathrm{TM}}$ Thermal Cycler (Bio-Rad, Berkeley, CA, USA). Oligos and PCR conditions had previously been sourced and optimised by colleagues in-house during preceding research and from previously published studies and had also been further interrogated against methBlast bioinformatic software for target specificity ${ }^{204,365}$. The genes targeted whose CpG islands are targeted are hMLH1, SOCS1, MINT1, THBD, IGFBP3, HAND1, ADAMTS1 and NEUROG1. Target genes were selected based on the Kaneda \& Yagi system for CIMP assessment (see section 1.3.6 and 3.3.7) ${ }^{185}$. For oligonucleotides refer to section 2.2.5. Desalted oligonucleotide PCR primers (Sigma Aldrich, UK) were diluted to a working concentration of $10 \mathrm{uM}$ and stored at $-20^{\circ} \mathrm{C}$ with all other PCR reagents. Conditions for each primer are set out in Appendix I. Preparation for each msPCR took place in laminar flow hoods cleaned with 70\% ethanol solution and allowed to air dry under UV light to reduce the risk of DNA cross contamination or fouling of reactions. PCR-grade filtered pipette tips were used for handling DNA and reagents, and reactions were performed in micro-eppendorfs. msPCR reactions were carried out in a volume of $25 \mu \mathrm{L}$ per sample as per Table 3.1.

Table 3.1 msPCR reagents.

\begin{tabular}{|l|l|}
\hline Constituent & Volume \\
\hline $\begin{array}{l}\text { GoTaq }{ }^{\circledR} \text { Hot Start Green Master Mix } \\
\text { (Promega, Madison, WI, USA) }\end{array}$ & $12.5 \mu \mathrm{L}$ \\
\hline Nuclease Free Water (Ambion) & $10.5 \mu \mathrm{L}$ \\
\hline Forward Primer (200 nM) & $0.5 \mu \mathrm{L}$ \\
\hline Reverse Primer (200nM) & $0.5 \mu \mathrm{L}$ \\
\hline Bisulfite Converted DNA (40ng/ $\mu \mathrm{L})$ & $2 \mu \mathrm{L}$ \\
\hline
\end{tabular}

msPCR utilised a hot-start protocol consisting of an 8 -minute hot start at $95^{\circ} \mathrm{C}$ followed by denaturing at $95^{\circ} \mathrm{C}$ for 30 seconds, then annealing at temperatures and durations specifically optimised to the primers (typically 30 to 37 seconds and $50-61{ }^{\circ} \mathrm{C}$ ). An extension phase at $72^{\circ} \mathrm{C}$ followed and the cycle repeated. Positive controls for both the methylated and unmethylated DNA (Promega, UK) and a negative control were used throughout. 


\subsubsection{Visualisation of PCR Products}

msPCR products were visualised by gel electrophoresis on $6 \%$ polyacrylamide gels against a $100 \mathrm{bp}$ DNA ladder containing 11 dsDNA fragments of 100-1000bp (in 100bp steps) \& 1500bp (Promega, Madison, WI, USA). Hand formed 15-lane $1.5 \mathrm{~mm}$ polyacrylamide gels were made in duplicate according to the following recipe: $16 \mathrm{ml} \mathrm{dH}_{2} \mathrm{O}, 2.25 \mathrm{ml} 10 \mathrm{x} \mathrm{TBE}, 4 \mathrm{ml}$ acrylamide, $110 \mu 1$ 10\% APS, $22 \mu 1$ TEMED. This mixture was mixed by pipetting and transferred to two gel casts and allowed to polymerise for 60 minutes. Gels were then submerged vertically in electrophoresis tanks containing $1 \mathrm{x}$ TBE buffer and $10 \mu \mathrm{L}$ PCR product was mixed with $2 \mu \mathrm{L}$ coloured loading buffer (RETROscript, Invitrogen) and then loaded into each well. Electrophoresis was performed at $170 \mathrm{~V}, 400 \mathrm{~mA}$ for 25 minutes or until the loading dye had reached the bottom of the plate.

Following electrophoresis, the gels were separated from their glass casting plates and stained by sequentially submerging into silver nitrate solution $(1 \mathrm{~g} / \mathrm{L})$ for 7 minutes and then submerging in formaldehyde \& sodium hydroxide solution for 2 minutes until visualisation of bands was achieved. Gels are then rinsed with $\mathrm{dH}_{2} \mathrm{O}$ and photographed in white light using Gel Doc XR (Bio-Rad) system and Quantity One software to adjust the brightness and size of the image (Bio-Rad). Gels were then discarded into a hazardous waste container.

\subsubsection{CIMP Classification}

CIMP status was classified according to the methodology described by Kaneda \& Yagi ${ }^{185}$. This method was chosen due to the approach taken to classifying CIMP status, as discussed in section 1.3.6. The epigenotyping system is again described below.

- CIMP-High if $\geq 2 / 3$ of Group 1 markers are methylated, irrespective of Group 2 markers

- CIMP-Intermediate if 1/3 Group 1 markers and $\geq 3 / 5$ Group 2 markers is methylated

- CIMP-Low if $\leq 1$ Group 1 markers is methylated and $\leq 2$ Group 2 markers is methylated 


\subsubsection{Statistical Analysis}

Data analysis was performed using the Microsoft Office Excel (v.16.12) and the XLStat Addinsoft (v.2017) plug-in (macro). Results were expressed as mean $+/$ - standard error of the mean (SEM) where appropriate. Normalcy of data distribution was determined using a Kolmogorov-Smirnov test. If data were normally distributed, statistical significance was determined using Student's t-test for single comparisons or one-way ANOVA where more than one comparison was made. If data were not normally distributed, Mann-Whitney's test was used to compare unpaired means and Wilcoxon test used for paired data. Log-Rank (MantelCox) tests were used where data was right-side skewed and censored. Comparison of expected frequencies was performed with two-tailed Chi Squared or Fishers exact test if observed events were less than 10. Survival curves were calculated using Kaplan-Meier estimate. The null hypothesis was rejected at the level of $5 \%(\mathrm{p}>0.05)$. 


\subsection{Results}

\subsubsection{Patient and Tumour Characteristics}

There were 100 patients included in this study (44 patients with an EMVI-positive tumour and 56 with no evidence of EMVI). Seventy patients were male, with a statistically significant proportion of male patients demonstrating EMVI positivity ( $82 \%$ positive vs $61 \%, \mathrm{p}=0.022$ ). The median age was 70 years (range 45 - 89 years), and patients were of a similar age in both groups. Nineteen patients had died at the time analysis was undertaken (01/09/2017). Median follow up was 54.2 months (IQR 25 - 68 months). Mean overall DFS and OS were 29 and 34 months, respectively. When differences between both groups were analysed (Table 3.2), patients with EMVI-positive tumours had more advanced pathological staging by $\mathrm{pT}, \mathrm{pN}$, and AJCC classifications $(p=0.002, p=<0.0001$, and $p-<0.0001$, respectively). There was a corresponding association between EMVI-positive tumours and the need for adjuvant chemotherapy $(\mathrm{p}=<0.0001)$. There was, however, no statistically significant difference in CRM positivity $(p=0.86)$, tumour perforation $(p=0.07)$ or tumour differentiation $(p=0.52)$.

Figure 3.6 Resolved polyacrylamide gel demonstrating tandem-PRC products for methylated (M) \& unmethylated (U) primers against samples (1-4) with controls \& DNA ladder. Samples $1 \& 2$ were methylated (strong $M$ bands as well as $U$ bands), samples $3 \& 4$ unmethylated (U bands only). Two negative control reactions (no template DNA) and a positive control lane (hypermethylated DNA) are also shown.

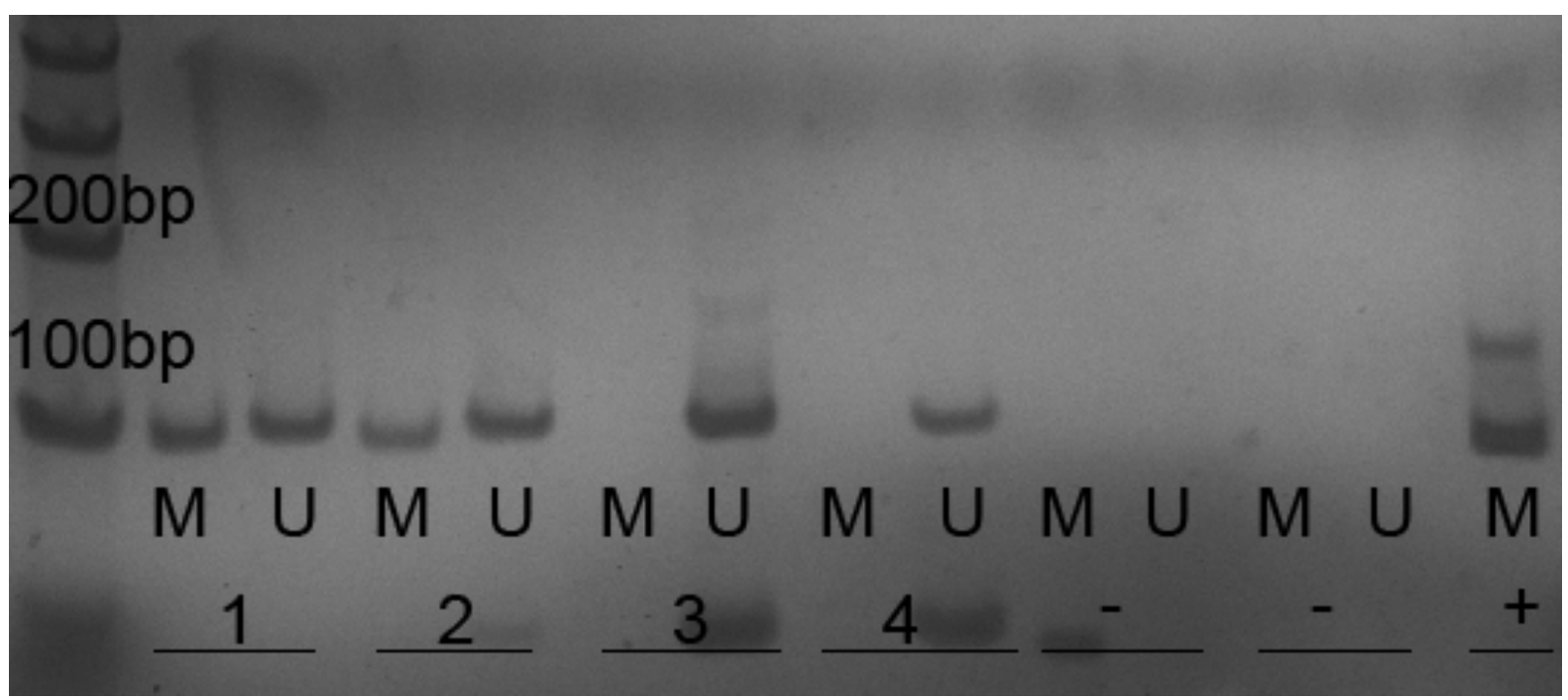




\subsubsection{CIMP Classification \& EMVI}

There were a total of 51 patients with CIMP-L, 48 patients with CIMP-I, and one patient with CIMP-H phenotype (Table 3.3). The assessment of 40 patients' CIMP status was conducted by a co-investigator, although all subsequent analysis was performed by the author. Chi-square (Pearson) demonstrated a positive correlation between EMVI-positive tumours and CIMPintermediate epigenotype $(\mathrm{p}=<0.001)$. This effect was preserved if the single CIMP-high sample was amalgamated with the CIMP-intermediate group $(\mathrm{p}=0.00014)$ and did not significantly affect any of the regression analysis performed henceforth.

CIMP-intermediate labelling was associated with worse tumour pAJCC stage $(p=0.03)$ and showed a trend towards association with the need for adjuvant chemotherapy $(p=0.055)$, but was not associated with $\mathrm{T}$ or $\mathrm{N}$ stage, tumour height, sex, or CRM involvement (Table 3.4). 
Table 3.2. Patient and Tumour Characteristics

\begin{tabular}{|c|c|c|c|}
\hline & $\begin{array}{l}\text { EMVI +ve } \\
(n=44)\end{array}$ & $\begin{array}{l}\text { EMVI-ve } \\
(\mathrm{n}=56)\end{array}$ & P-value ${ }^{1}$ \\
\hline Median Age (range) & $72(45-85)$ & $69(46-89)$ & $0.86^{2}$ \\
\hline Sex (male) & $36(82 \%)$ & $34(61 \%)$ & $0.022 *$ \\
\hline Tumour Height & & & 0.34 \\
\hline Upper $(10-15 \mathrm{~cm})$ & $8(30 \%)$ & $17(27 \%)$ & \\
\hline $\operatorname{Mid}(5-10 \mathrm{~cm})$ & $23(43 \%)$ & $23(47 \%)$ & \\
\hline Lower $(0-5 \mathrm{~cm})$ & $13(27 \%)$ & $16(27 \%)$ & \\
\hline pT & & & $0.002 *$ \\
\hline $\mathrm{T} 1$ & $0(0 \%)$ & $1(2 \%)$ & \\
\hline $\mathrm{T} 2$ & $2(5 \%)$ & $12(21 \%)$ & \\
\hline $\mathrm{T} 3$ & $31(75 \%)$ & $41(70 \%)$ & \\
\hline $\mathrm{T} 4$ & $11(20 \%)$ & $2(7 \%)$ & \\
\hline $\mathrm{pN}$ & & & $<0.0001 *$ \\
\hline No & $8(20 \%)$ & $42(73 \%)$ & \\
\hline N1 & $21(46 \%)$ & $7(14 \%)$ & \\
\hline $\mathrm{N} 2$ & $15(34 \%)$ & $7(13 \%)$ & \\
\hline pAJCC & & & $<0.0001^{*}$ \\
\hline 1 & $0(0 \%)$ & $8(27 \%)$ & \\
\hline 2 & $8(16 \%)$ & $37(41 \%)$ & \\
\hline 3 & $32(75 \%)$ & $9(27 \%)$ & \\
\hline 4 & $4(9 \%)$ & $2(5 \%)$ & \\
\hline $\mathrm{CRM}+\mathrm{ve}$ & $1(3 \%)$ & $1(3 \%)$ & 0.86 \\
\hline Tumour Perforation & $6(7 \%)$ & $2(9 \%)$ & 0.07 \\
\hline Tumour Differentiation & & & 0.52 \\
\hline Poor & $6(14 \%)$ & $5(9 \%)$ & \\
\hline Moderate & $38(87 \%)$ & $50(89 \%)$ & \\
\hline Well & $0(0 \%)$ & $1(2 \%)$ & \\
\hline Non-Restorative Operation & $18(27 \%)$ & $20(46 \%)$ & 0.60 \\
\hline Adjuvant Chemotherapy & $31(68 \%)$ & $10(16 \%)$ & $<0.0001^{*}$ \\
\hline Systemic Recurrence & $15(30 \%)$ & $11(13 \%)$ & 0.10 \\
\hline Local Recurrence & $3(7 \%)$ & $3(5 \%)$ & 0.76 \\
\hline
\end{tabular}

${ }^{1}$ Pearson's Chi Square. EMVI, Extramural Vascular Invasion; pAJCC, American Joint Cancer Committee pathological stage; CRM, circumferential resection margin; OS, Overall Survival; DFS; Disease Free Survival. 
Table 3.3 CIMP Classification and EMVI status

\begin{tabular}{|l|l|l|l|}
\hline Contingency Distribution & $\begin{array}{l}\text { EMVI +ve } \\
(\mathbf{n = 4 4 )}\end{array}$ & $\begin{array}{l}\text { EMVI-ve } \\
(\mathbf{n}=\mathbf{5 6})\end{array}$ & $\begin{array}{l}\text { Distribution } \\
\mathbf{p}=<\mathbf{0 . 0 0 1} \mathbf{1}^{\mathbf{1}}\end{array}$ \\
\hline CIMP-low & $13(30 \%)$ & $38(68 \%)$ & \\
\hline CIMP-intermediate & $31(70 \%)$ & $17(30 \%)$ & \\
\hline CIMP-high & $0(0 \%)$ & $1(2 \%)$ & \\
\hline
\end{tabular}

${ }^{1}$ Pearson Chi-Square. CIMP, CpG Island Methylator Epigenotype; EMVI, Extramural Vascular Invasion.

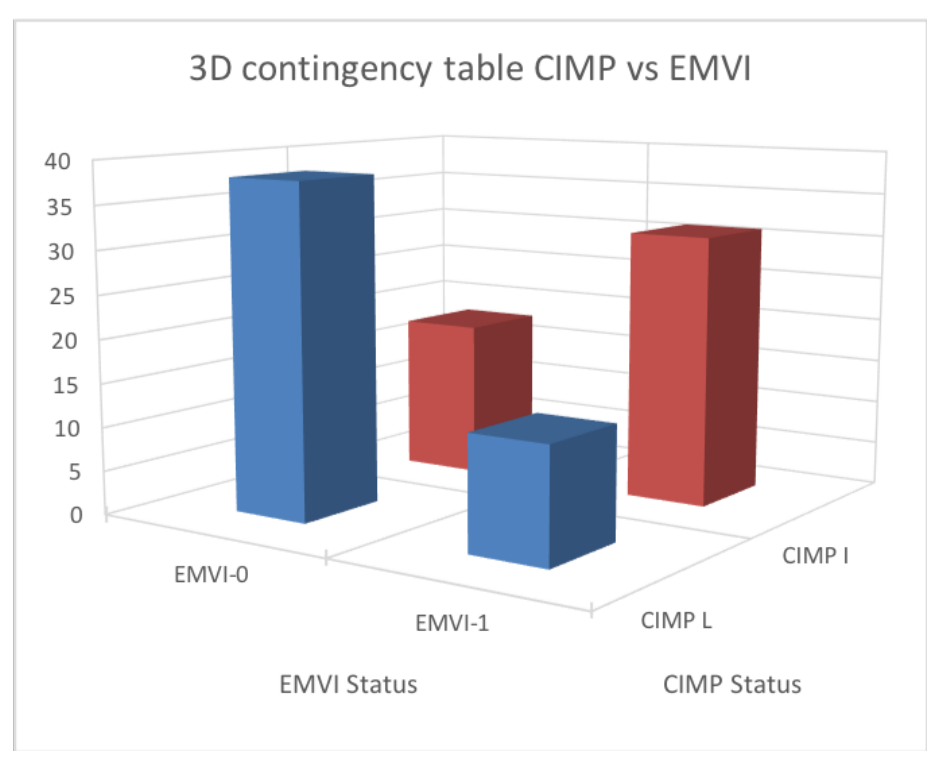


Table 3.4. CIMP status and association with patient and tumour factors.

\begin{tabular}{|c|c|c|c|}
\hline & $\begin{array}{l}\text { CIMP-I } \\
(n=48)\end{array}$ & $\begin{array}{l}\text { CIMP-L } \\
(n=51)\end{array}$ & P-value \\
\hline Median Age (range) & $67(45-89)$ & $70(47-89)$ & $0.86^{2}$ \\
\hline Sex (male) & $33(68.8 \%)$ & $36(70.6 \%)$ & 0.42 \\
\hline Tumour Height & & & 0.33 \\
\hline Upper $(10-15 \mathrm{~cm})$ & $13(27.1 \%)$ & $12(23.5 \%)$ & \\
\hline $\operatorname{Mid}(5-10 \mathrm{~cm})$ & $25(52.1 \%)$ & $21(41.2 \%)$ & \\
\hline Lower $(0-5 \mathrm{~cm})$ & $10(20.8 \%)$ & $18(35.3 \%)$ & \\
\hline $\mathrm{pT}$ & & & 0.17 \\
\hline $\mathrm{T} 1$ & $0(0.0 \%)$ & $1(2.0 \%)$ & \\
\hline $\mathrm{T} 2$ & $4(8.3 \%)$ & $9(17.6 \%)$ & \\
\hline $\mathrm{T} 3$ & $36(75.0 \%)$ & $36(70.6 \%)$ & \\
\hline $\mathrm{T} 4$ & $8(16.7 \%)$ & $5(9.8 \%)$ & \\
\hline $\mathrm{pN}$ & & & 0.28 \\
\hline No & $24(50 \%)$ & $26(51.0 \%)$ & \\
\hline N1 & $17(35.4 \%)$ & $11(21.6 \%)$ & \\
\hline $\mathrm{N} 2$ & $7(14.6 \%)$ & $14(27.5 \%)$ & \\
\hline pAJCC & & & $0.03 *$ \\
\hline 1 & $0(0.0 \%)$ & $8(15.7 \%)$ & \\
\hline 2 & $24(50.0 \%)$ & $21(41.2 \%)$ & \\
\hline 3 & $20(41.7 \%)$ & $20(39.2 \%)$ & \\
\hline 4 & $4(8.3 \%)$ & $2(3.9 \%)$ & \\
\hline CRM +ve & $2(4.2 \%)$ & $0(0.0 \%)$ & 0.15 \\
\hline Tumour Perforation & $4(8.3 \%)$ & $4(7.8 \%)$ & 0.98 \\
\hline Tumour Differentiation & & & 0.58 \\
\hline Poor & $5(10.4 \%)$ & $6(11.8 \%)$ & \\
\hline Moderate & $43(89.6 \%)$ & $44(86.3 \%)$ & \\
\hline Well & $0(0.0 \%)$ & $1(2.0 \%)$ & \\
\hline Non-Restorative Operation & $16(33.3 \%)$ & $22(43.1 \%)$ & 0.25 \\
\hline Adjuvant Chemotherapy & $25(52.1 \%)$ & $16(31.4 \%)$ & 0.055 \\
\hline Systemic Recurrence & $13(27.1 \%)$ & $7(13.7 \%)$ & 0.61 \\
\hline Local Recurrence & $3(6.3 \%)$ & $3(5.9 \%)$ & 0.98 \\
\hline
\end{tabular}

${ }^{1}$ Pearson's Chi Square, ${ }^{2}$ Students T-Test. EMVI, Extramural Vascular Invasion; pAJCC, American Joint Cancer Committee pathological stage; CRM, circumferential resection margin; OS, Overall Survival; DFS; Disease Free Survival. 
Using a multivariable logistic regression model (Table 3.5), CIMP status remained a highly significant predictor of EMVI status $(p=0.001)$, independent of pAJCC staging $(p=<0.001)$. When considering the development of systemic recurrence, only the presence of a perforated tumour was significantly associated $(\mathrm{p}=0.038)$. Only EMVI positivity was associated with poor DFS $(p=0.038)$, but not significantly associated with poor OS, although trend was observed $(\mathrm{p}=0.08)$ for both EMVI positivity and CIMP-I status $(\mathrm{p}=0.08)$ (Figure 3.6).

Table 3.5 Multivariable Logistic Regression analysis of factors associated with EMVI, Metastatic Disease, and Overall \& Disease-Free Survival: Standard Coefficients (SC) \& Hazard Ratios (HR).

\begin{tabular}{|l|l|l|l|}
\hline & Factor & SC or HR (95\% CI) & P value \\
\hline EMVI & CIMP Intermediate & $1.27(0.55-1.91)$ & $0.001^{* 1}$ \\
\hline & Male Sex & $-0.13(-0.59-0.34)$ & $0.59^{1}$ \\
\hline & Increasing pAJCC stage & $1.17(0.59-1.76)$ & $<0.001^{* 1}$ \\
\hline & CRM positivity & $0.09(-0.32-0.50)$ & $0.66^{1}$ \\
\hline & Perforated Tumour & $0.21(-0.26-0.68)$ & $0.38^{1}$ \\
\hline & Non-restorative Operation & $-0.27(-0.75-0.20)$ & $0.26^{1}$ \\
\hline Systemic Recurrence & CIMP Intermediate & $0.78(-0.20-1.76)$ & $0.12^{1}$ \\
\hline & Male Sex & $-0.79(-1.81-0.24)$ & $0.13^{1}$ \\
\hline & Increasing pAJCC stage & $-0.36(-1.46-0.73)$ & $0.51^{1}$ \\
\hline & CRM positivity & $0.14(-0.29-0.57)$ & $0.53^{1}$ \\
\hline & Perforated Tumour & $0.54(0.04-1.04)$ & $0.034^{* 1}$ \\
\hline & Non-restorative Operation & $0.56(-0.19-1.31)$ & $0.14^{1}$ \\
\hline Overall Survival (OS) & CIMP Intermediate & $0.36(0.12-1.12)$ & $0.08^{2}$ \\
\hline & EMVI Positive & $4.59(0.85-24.69)$ & $0.08^{2}$ \\
\hline & Increasing pAJCC stage & & $0.91^{2}$ \\
\hline & Perforated Tumour & & $0.99^{2}$ \\
\hline $\begin{array}{l}\text { Disease-Free Survival } \\
\text { (DFS) }\end{array}$ & CIMP Intermediate & $0.39(0.13-1.18)$ & $0.10^{2}$ \\
\hline & EMVI Positive & $5.98(1.10-32.50)$ & $0.038^{* 2}$ \\
\hline & Increasing pAJCC stage & & $1.0^{2}$ \\
\hline & Perforated Tumour & & $1.0^{2}$ \\
\hline & & & \\
\hline
\end{tabular}

${ }^{1}$ Binary Logistic Regression, ${ }^{2}$ Cox Proportional Hazard. CIMP, CpG Island Methylator Phenotype, EMVI, Extramural Vascular Invasion; pAJCC, American Joint Cancer Committee pathological stage; CRM, circumferential resection margin; OS, Overall Survival; DFS; Disease Free Survival. 
Figure 3.7 Cox $\log (-\log ($ survival)) analysis for EMVI versus DFS (a) and OS (b), demonstrating a significant association with poor DFS (left-shift, $\mathrm{p}=0.038$ ), but not OS (insignificant but trend towards left-shift, $\mathrm{p}=0.08$ ).

a.

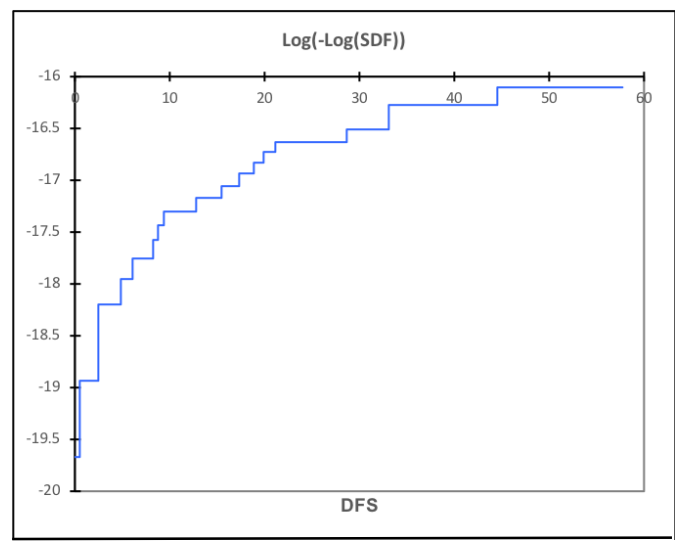

b.

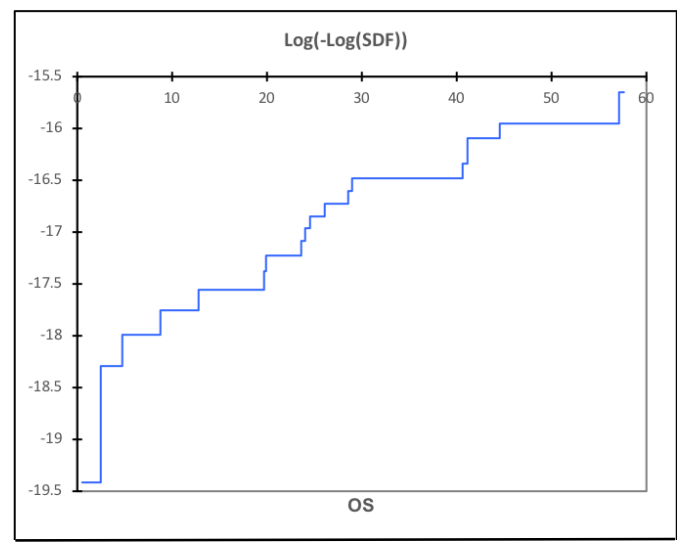




\subsubsection{Survival Analysis}

Kaplan-Meier analysis (Figure 3.7) demonstrated that overall survival (OS) and disease-free survival (DFS) were greater in the EMVI-negative group, but only reached statistical significance for DFS, although strong trend was observed for OS $(p=0.012$ and $p=0.52$ respectively, by log-rank). This effect was however overcome by Gehan-Breslow-Wilcoxon method (which weighs positively for early deaths $(\mathrm{p}=0.018$ and $\mathrm{p}=0.028$ for DFS and OS, respectively). CIMP status was not statistically related to DFS or OS, although CIMP and OS approached significance at $\mathrm{p}=0.08$. There was a total of 20 patients with systemic metastases (two of these had synchronous metastases) and six had local recurrence. Although a significantly greater number of patients with EMVI-positive tumours received adjuvant chemotherapy $(\mathrm{p}<0.0001)$, this relationship was not observed for CIMP-intermediate tumours, although trend was observed $(\mathrm{p}=0.055)$. Significance was not achieved through a re-sampling (bootstrapping) technique.

Figure 3.8 Kaplan-Meier overall and disease-free survival analysis for EMVI and CIMP.
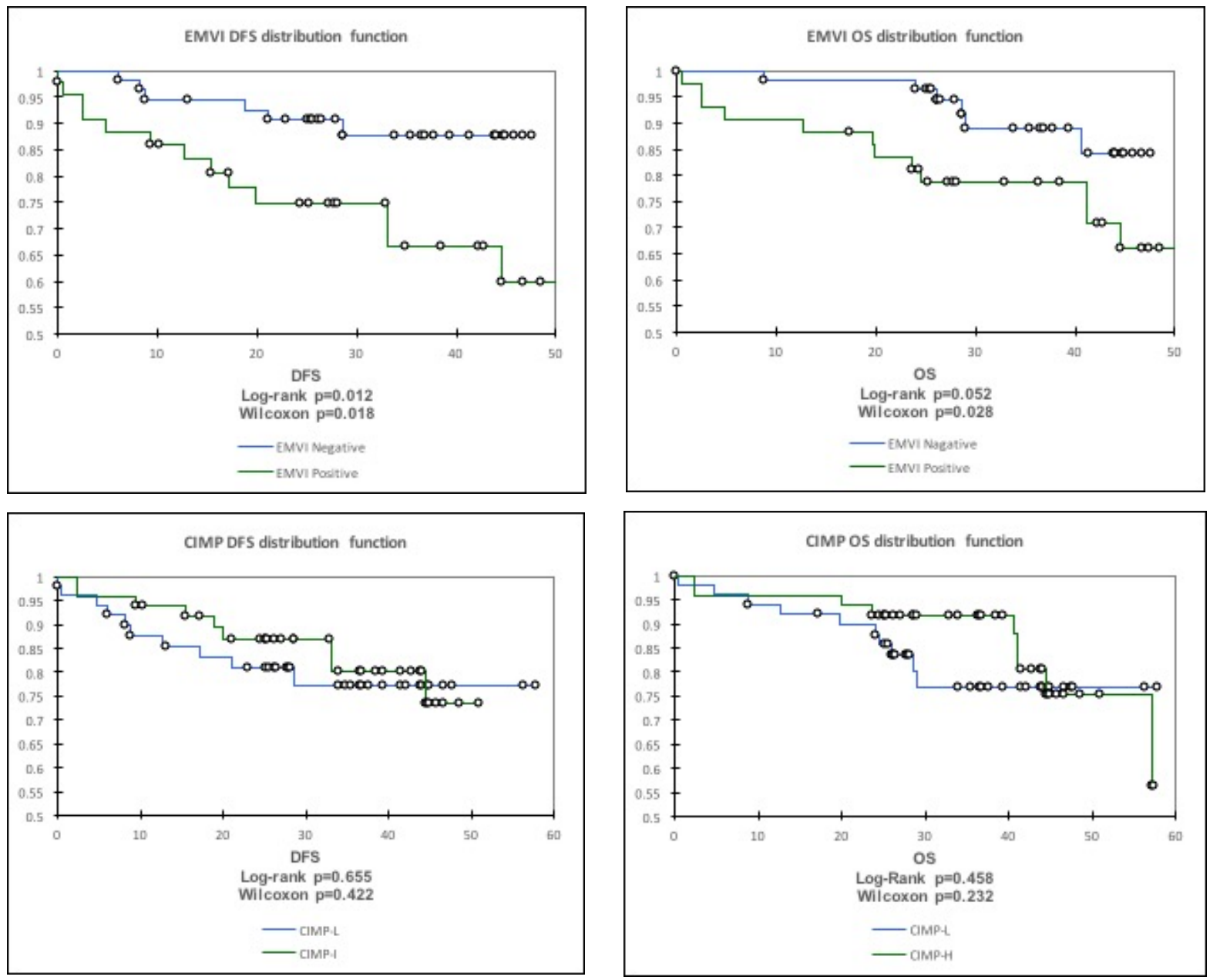


\subsection{Discussion}

The aim of this chapter was to investigate the methylation status of rectal cancers based upon CIMP and determine if there was any association with EMVI or other clinicopathological variables that may impact outcomes. The findings are considered in the context of the poorer outcomes from rectal cancer in terms of local and systemic recurrence, and survival.

A clear association was demonstrated between the presence of $\mathrm{pEMVI}$ and male sex, $\mathrm{T}$ and $\mathrm{N}$ stage, AJCC classification, and the requirement for adjuvant chemotherapy. EMVI has previously been shown to be an independent risk factor for systemic and local recurrence, and death; and is an indication for adjuvant chemotherapy ${ }^{276-278,293}$. This study confirms the association of EMVI with worse clinicopathological features, and on Kaplan-Meier analysis, these translated into statistically significant survival disadvantage, in keeping with the findings of Smith (Figure 3.3) ${ }^{280}$. Currently, neoadjuvant therapy from rectal cancers is advised on the basis of threatened circumferential resection margins as demonstrated on preoperative radiological imaging, but tumour genetic and epigenetic analysis is not routinely part of pretherapeutic assessment ${ }^{85}$. By associating CIMP epigenotype with EMVI, the possibility of utilising neoadjuvant therapies in patients who are not demonstrated to have locally advanced disease by radiological standards, but who may be judged to be at higher risk based on epigenetic tumour profiling, may improve outcomes. For this reason, it is important to understand the role that epigenetic phenotypes may have on tumour behaviour so that they may be harnessed to therapeutic effect and inform clinical decision making.

Although a significant relationship was demonstrated between CIMP-intermediate status and EMVI positivity, this was not translated in to a disease-free or overall survival disadvantage for CIMP-intermediate patients based on Kaplan-Meier analysis, despite EMVI-positivity being an independent risk-factor for disease recurrence (Cox Proportional Hazard r=5.98 (1.10$32.50), \mathrm{p}=0.038$ ). These findings are in keeping with prior results in a similar cohort from our own unit, which indicate a positive correlation between CIMP-high status and EMVI in a series of 160 rectal cancers undergoing neoadjuvant treatment; although this did not translate into a significant relationship to survival ${ }^{204}$. There was also no significant relationship between KRAS or BRAF and CIMP. More broadly, results published by Kim also demonstrated that a higher CIMP status was associated with worse DFS, but only for colonic tumours as opposed to rectal tumours based on a mixed cohort of 157 patients $^{366}$. In another study, high CIMP epigenotype was also found to be associated with worse overall- and progression-free survival 
in patients with metastatic colorectal cancer, although this study also included both colonic and rectal tumours ${ }^{367}$. Each of these studies are contributory to the hypothesis that CIMP positivity is associated with more locally advanced tumour types and poorer clinical outcomes, although the relationship is not straightforward. In a review of 20 heterogeneous studies, Gallois discusses the prognostic value of EMVI in mixed cohorts of colorectal cancers, concluding that currently there is not sufficient evidence to support EMVI as a prognostic indicator ${ }^{368}$. Despite the differences between the panels used and mixed cohorts, what is clear is that the relationship between CIMP status and clinical outcomes is not solely limited to the relationship to EMVI, else DFS and OS outcomes would be more closely aligned; pointing towards additional roles for methylation in processes such as response to CRT, cell death, and tumour-immunological interaction.

The consensus molecular subtyping (CMS) of colorectal cancers has identified 4 distinct tumour subtypes based on molecular analysis, with CMS1 being defined as typically hypermethylated and associated with the serrated pathway in the proximal large bowel, rather than in the rectum ${ }^{118,201}$. In the original CMS study, the frequency of CMS1 reported in the rectum was $3 \%$. Considering this, the dataset in this study of 100 consecutive patients with rectal cancers, given exclusions, demonstrated a higher than expected proportion of CIMPintermediate and -high (49\%) which may be considered as analogous to CMS1 tumours. Although the CIMP-I and CIMP-H groups were considered as one for the purposes of analysis (equivalent to CIMP-positive in other studies), this finding does question the assumption that hypermethylation is a predominantly right colonic finding associated with the serrated pathway and may therefore represent an under-reported subgroup of rectal cancers. These findings must be considered in the context of the methodology chosen to determine CIMP (Kaneda \& Yagi), although as previously discussed, there is no consensus on determining CIMP and the methodology employed in this study was chosen for its robustness and use throughout other contemporaneous investigations, although other methods may have returned a lower frequency $^{185,186}$.

One reason for the discrepancy is that the cohorts examined are not alike in several ways, especially when considering the relatively selective cohort used in this chapter (exclusion of patients who had received neoadjuvant therapy, for example) compared to the inclusive nature of the Guinney study which was based on eighteen public and proprietary colorectal cancer datasets comprising 4,151 patients ${ }^{201}$. However, although the Guinney dataset is much larger, 
only 966 patients $(23 \%)$ had extractable methylation data available for contribution to the subtyping algorithms, raising the possibility of this component being under-represented when compared to other molecular markers in the modelling. Furthermore, the assessment of CIMP employed by Guinney was also atypical; relying on comparative sub-group methylation analysis to determine which loci would be best at discriminating between the putative molecular subtypes, and then augmenting the effect within the model to facilitate discrimination, as opposed to a standardised and blinded assessment of methylation status at previously identified $\mathrm{CpG}$ islands and thus determine CIMP status.

Although patients receiving neoadjuvant therapy were excluded in our study, the relationship between methylation and response to CRT (neoadjuvant and adjuvant) is likely to be significant, as has been identified in previous studies ${ }^{204,369}$. The benefits of neoadjuvant therapy in rectal cancer are clear, but it is acknowledged that a tumour's response to neoadjuvant therapy is currently not predictable, and identifying significant factors that affect response may be beneficial in managing patients ${ }^{370}$. Currently, mucinous tumours and poorly differentiated tumours have all been associated with poor response to neoadjuvant therapy, but the prognostic values of these measures is limited and has no clinical utility in restricting access to pre-surgical therapies ${ }^{371}$. Unfortunately this means that a proportion of patients who undergo neoadjuvant therapy will gain no benefit, and may potentially come to harm as a result of systemic chemotherapy and/or local radiotherapy, and/or miss the opportunity to have a surgical intervention ${ }^{372}$. For these reasons there is an urgency in identifying reliable molecular markers of tumour response, including exploration of the methylome for significant relationships ${ }^{373-375}$. One successful example of this approach is the utilisation of a single nucleotide mutation in the KRAS gene in predicting the response of patients to adjuvant therapy using epidermal growth factor receptor (EGFR) inhibitors; which are increasingly utilised in order to increase the effectiveness of treatment ${ }^{376}$. With regards methylation, Yokoi reported that DNA methylation may play an important role in affecting response to radiotherapy in an in vitro colorectal cell line model ${ }^{377}$. This process was dependent on methylation-controlled expression of cellular retinol binding protein 1; cellular response to radiotherapy being strongly related to expression. In our study, CIMP-intermediate was associated with a trend towards requirement for adjuvant chemotherapy $(\mathrm{p}=0.055)$, although it did not reach significance and was not an independent risk factor for DFS or OS. Studies examining the correlation between CIMP status and response to CRT have shown some promise, although the results have been inconsistent and are plagued by methodological 
inconsistencies $^{378-380}$. Further studies have identified methylation to be important in the response of tumours to chemoradiotherapy in other organ systems, including lung, breast, glioma, and others; and is a field that warrants further investigation in the context of rectal cancers $^{381-386}$. The response of tumours to neoadjuvant therapy is especially important when considering the challenges of predicting EMVI on pre-operative imaging. MRI has been shown to be accurate in the local staging rectal tumours and indicating where circumferential resection margins are threatened, thus indicating the need for neoadjuvant therapy, but there is an acknowledged shortcoming in the sensitivity and specificity of MRI-detected EMVI, especially where EMVI is present in vessels smaller than 3mm or when EMVI volume is low $281,282,387$, If the prognostic validity of CIMP status could be established based on biopsy, it may open the opportunity to intervene on radiologically EMVI-negative but CIMP-intermediate/-high and pathologically unfavourable tumours before surgery.

\subsection{Conclusion}

A clear relationship was demonstrated between CIMP-positive (pooled CIMP-intermediate and -high) and EMVI. The CIMP-positive pool may be considered as analogous to CMS1, although the number of cases reported in our series was significantly higher than that expected given the frequency of CMS1 in the previously published data. EMVI-positive tumours demonstrated worse DFS and OS, and were associated with poorer clinicopathological features, although this disadvantage was not conferred to CIMP-positive tumours, suggesting a more complex relationship between EMVI, CIMP, and outcomes. A better understanding of the biological mechanisms underlying CIMP and EMVI may help illuminate the clinicopathological relationship between EMVI and CIMP and prove useful in stratifying and guiding disease management. This may be especially important for determining patients who may be at risk of developing early local or distant disease recurrence and therefore benefit from systemic therapy, either before or after surgery, and who may not have obvious evidence of EMVI on initial staging. 


\section{Chapter 4}

\section{The Biological Effects of Demethylation on Colorectal Cancer Cells In Vitro.}

\subsection{Introduction}

Methylation is emerging as an important factor in carcinogenesis of colorectal cancer, as well as other cancers ${ }^{309,388,389}$. Human tissue studies have identified specific epigenetic changes in colorectal cancers, many of which have putative cellular effects that may be directly contributory to the carcinogenic process; such as the hypermethylation events identified in the adenoma-to-carcinoma model and the Wnt pathway ${ }^{307,309}$. Other methylation markers identified in colorectal cancer are less obviously linked to carcinogenesis but may act as proxies for assessing the overall methylation status of DNA in healthy and diseased states. Assessment of methylation is important in understanding colorectal cancer pathogenesis and in classifying colorectal cancers; demonstrated by methylation being an integral part of the classification systems employed to characterise colorectal cancer ${ }^{118,161,185}$. As DNA methylation is an active and dynamic cellular process, important in the normal homeostasis of cells and phenotypic differentiation, it is open to modification in vitro and in vivo for experimental and, potentially, therapeutic purposes.

\subsubsection{In Vitro Studies of Colorectal Cancer}

The systematic investigation of colorectal cancer in the laboratory can be dated to at least 1940 , when mice were fed diets containing potential carcinogens and the effects on the gastrointestinal mucosa examined at vivisection ${ }^{390}$. This work was succeeded by further studies in animals until experimental techniques in in vitro cell culture began to be developed in the 1960's, based first on cells extracted from viable tumours, and then on immortalised cell lines ${ }^{391,392}$. Many of the themes of the current investigation of colorectal carcinogenesis were being explored in relatively early work during the 1970s, including the colonic microbiome, the role of dietary fibre and fats, and even the niche field of DNA alkylating agents derived from rocket propellants ${ }^{393,394}$ ! It wasn't until the 1980 s, however, that the culture of colorectal cell lines (healthy, adenomatous, \& cancerous) became a viable and widespread technique, 
partially due to the relative ease with which colorectal cancer cell lines may be propagated, compared to other cancer types ${ }^{395-397}$. The widespread adoption of cancer cell culture has greatly facilitated the mechanistic understanding of carcinogenesis, as well as drug discovery. For instance, the NCI-60 cancer cell line panel is a cohort of cell lines employed by the National Cancer Institute (USA) for high-volume screening of potential anti-cancer drugs against well established and characterised cell lines, including multiple colon cancers (original colon set: COLO 205, HCC-2998, HCT116, HCT-15, HT-29, KM12, SW-620; latter additions: DLD-1, KM20L2), and has aided in drug discovery (and arguably in early abandonment of futile investigation $)^{398}$.

Despite advances, there are however many shortcomings in in vitro modelling of colorectal cancer that limit its utility and possibly account for some of the failings in translating benchtop science into the clinic. New strategies are required to overcome these dilemmas. Specifically, regarding the investigation of rectal cancer as an individual disease entity separate from colorectal cancer, there is currently no commercially available rectal cancer-specific immortalised cell line available to investigators. This shortcoming, however, must be balanced against the consideration that within the cohort of rectal cancers are found many epigenotypes, genotypes, and phenotypes; as demonstrated by the consensus molecular subtyping systems, limiting the utility of a single rectum-derived cell line. Additionally, single-cell culture models of this manner do not account for the intratumoral heterogeneity displayed in rectal and other cancers $^{399}$. Monoculture and 2D-culture are also limited in their modelling of in vivo cancers in that they fail to provide the complex inter-cell interactions and tumour microenvironmental factors at play in in situ tumours. This is particularly important when considering the important emerging role of immune responses to colorectal cancer, and the hypoxic conditions involved in cellular redox reactions ${ }^{400}$. Latterly, these challenges have been met with developments in tissue organoid culture (3D culture) and co-culture techniques in tissue engineering 401,402 . Spheroid cell culture has been proposed as one model for better modelling tumour microenvironments and cellular interactions in colorectal cancer and has been performed with success in a number of cell lines. However, as a relatively novel method of tissue culture, new challenges such as divergent differentiation dependent on location within the spheroid and changes in cell behaviour when compared to $2 \mathrm{D}$ culture (e.g. propensity to invade) have emerged $^{403}$. Despite its limitations and technical challenges, cell culture, whether 2D or 3D, is still well placed to facilitate the understanding of colorectal carcinogenesis and aid in 
developing therapeutic strategies, especially when coupled to modern technologies in translational research ${ }^{404}$.

\subsubsection{Migration \& Invasion Assays}

\subsubsection{Introduction}

Cellular invasion and migration are two inter-related processes that are crucial in the pathogenesis of colorectal cancer, and are similarly rooted in the cellular events that facilitate epithelial-mesenchymal transition $(\mathrm{EMT})^{405}$. EMT describes the process by which epithelial cells lose their polarity and cell-to-cell adhesion and become migratory through the basement membrane, whereupon they may differentiate into other tissue types and move from location to location. Once migrated, cells having undergone EMT may reverse the process to terminally differentiate in a new location by a process of mesenchymal-epithelial transition (MET) ${ }^{406}$. This process is crucial during embryological development and has been demonstrated to be critically related to healthy organogenesis and to congenital malformation ${ }^{407}$. EMT is also important in wound healing, where epidermal keratinocytes undergo an EMT-like process to re-epithelialise damaged tissues ${ }^{408}$. Beyond normal tissue remodelling, EMT has a pathological role in carcinogenesis; where dysplastic epithelial cells undergo invasive transformation to become invasive cancers ${ }^{409}$. Indeed, the definition of colorectal carcinoma $(\mathrm{T} 1+)$, as separate from adenomas with dysplasia or carcinomas in situ (Tis: AJCC 0), is characterised by cellular invasion through the basement membrane and muscularis mucosae into the submucosa $\mathrm{a}^{30,410}$. Metastatic seeding may be considered as an analogous process to $\mathrm{MET}^{411}$. It is therefore important to gain an understanding of the processes that govern tumour cell migration and invasion so that the disease process may be better understood and combatted.

In in vitro studies of tumour behaviour, migration and invasion are considered as two separate entities defined and limited by the experimental design, although in vivo the two are considered to a large degree as part of the same EMT process ${ }^{412}$. Migration specifically refers to the ability of cells to move in two dimensions over a surface, which may be the inside of a plastic well or flask, an extracellular matrix (ECM) laid down in a plate, a glass slide, or any other flat substrate free of barriers. Invasion refers to the movement of cells through a matrix of substrate, necessitating remodelling of the substrate (such as an ECM) and modification of cellular architecture, cell-cell adhesion, polarity, and expression of proteins required for these processes. Invasion almost exclusively occurs in three dimensions, although there are some experiments that utilise a three-dimensional migration model where cells pass through pores in 
a substrate without the need for ECM remodelling, and should be considered as a separate process. Although different methods of cellular motility may be found throughout biology, well-differentiated cancer cells migrate and invade primarily by mesenchymal migration in the manner of fibroblasts; relying on a strong interaction with the ECM, cytoskeletal contraction, and extensive spindle-like remodelling of the cell body ${ }^{413}$. A smaller proportion of cancer cells may migrate or invade by amoeboid movement, although this tends to be limited to melanoma and other irregularly migratory cancer types ${ }^{414}$.

\subsubsection{Wound Healing (Scratch) Assay}

Scratch assays are a basic model of cell migration that utilises a "wounding" to a uniformly confluent sheet of cells growing on a two-dimensional substrate. The method can be as simple as performing a standard cell culture on a plastic well and scratching the confluent sheet with a pipette tip, although additional experimental complexity can be introduced by culturing cells on different substrates (collagen, ECM, etc), or by modifying other experimental variables. The time taken to restore confluence is usually taken as the primary end-point of the experiment, although as a measure of migration this is best estimated in short culture protocol $(<24 \mathrm{hrs})$ as longer culture times increase the proportion of confluence that is reached by proliferation rather than migration ${ }^{415}$. Generally, the technique is quick, cheap, and easily reproducible. Drawbacks include the unevenness of the scratch when performed with a pipette tip and the possibility that cells scratched from the sheet accumulate at the edge of the wound and may re-attach, artificially closing the wound. Investigators have attempted to control for these inaccuracies by electro-scratching the sheet of cells and assessing wound-closure by measuring electrical impedance, although these techniques have not been broadly adopted ${ }^{416}$, 417 .

\subsubsection{Cell Exclusion Assay}

Another method of performing a migration scratch-type wound healing assay is to construct a barrier to cell attachment at the time of seeding, a process referred to as micro-stencilling ${ }^{418}$. This may be performed by use of an electrical-fence, but more commonly by use of a gel-insert that is well-enough attached to the culture substrate that cells cannot adhere to the area below it following seeding. One of the more popular commercially available assays is the Ibidi Culture-Insert ${ }^{\circledR}$ system of re-usable silicone 2 -well inserts with a $500 \mu \mathrm{m}$ cell-free exclusion zone (Figure 4.1) ${ }^{419}$. This, and other similar systems, has the advantage of producing a sharp 
edged and reproducible cell-free zone, as well as minimising cell damage and reducing detached cells at the wound margin.

Figure 4.1 Ibidi Culture-Insert 2 Well System ${ }^{419}$.

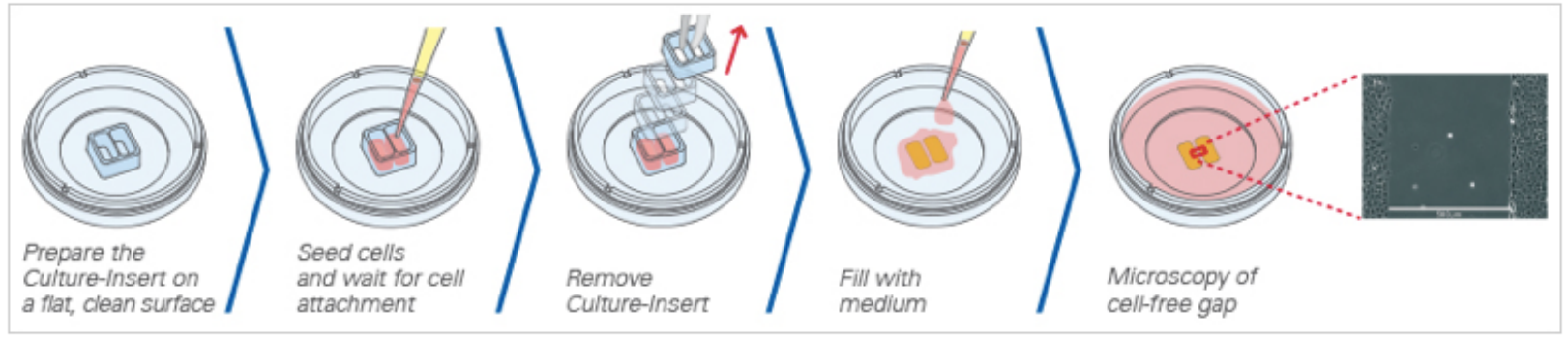

\subsubsection{Boyden Chambers \& Transwell@ Systems}

Both scratch and cell exclusion assays are only suitable for assessing cell migration as they are performed in two dimensions and do not involve active remodelling of the ECM. Boyden chambers, however, present the opportunity to assess cell migration and invasion in three dimensions by use of a suspended culture chamber with pores to allow cellular movement ${ }^{420}$. The base of the chamber can either be un-treated and inert to facilitate migration without invasion or be pre-treated with a layer of ECM through which cells must invade ${ }^{421,422}$. Typically, a gradient between the top and bottom chambers is provided to induce cellular migration or invasion (such as a serum gradient or chemoattractant), and pore size chosen dependent on the size and characteristics of the cell line being studies. Typically pore sizes are between $3-12 \mu \mathrm{m}$ but must be smaller than the cells being examined to prevent "dropping". It is also important to consider the seeding density, port density, and two-dimensional migration speed of seeded cells, as low-density slow-to-migrate cells will increase the overall threedimensional migration/invasion time during migration to a pore. Following culture, cells that have moved to the distant side of the membrane can either be fixed, stained, and counted directly (after removal of non-migrated cells from the top), or be detached and counted by a fluorescence counter following tagging. Boyden chamber systems have the advantages of being tailorable to different cell types and culture conditions, including co-culture, but require individual calibration for each cell type. They are generally more expensive than scratch or exclusion assays, but there are several commercially available models offering various potential benefits. 
Transwell@ systems exploit the physical set-up of the Boyden chamber but occlude the pores in the membrane by application of ECM, thus preventing the migration of non-invasive cells (Figure 4.2). Invading cells must degrade the ECM on the membrane before passing to the distant side of the membrane, induced by a gradient as previously discussed ${ }^{423}$. The most commonly used ECM is Matrigel ${ }^{\circledR}$ (Corning, New York, USA), a solubilised basement membrane extracted from Engbreth-Holm-Swarm (EHS) mouse sarcoma that has a high proportion of ECM proteins such as laminin, collagen IV, heparin sulfate proteoglycans, and others $^{424}$. Matrigel is a good model for invasion through the ECM as it has characteristics that are highly similar to the protein and growth factor profile found in naturally occurring basement membrane ${ }^{425-427}$. As previously discussed, invasion of the basement membrane is the defining feature of invasive cancer, including $\mathrm{CRC}$, and ECM remodelling has been observed in the invasive-front microenvironment ${ }^{410,428,429}$. Transwell ${ }^{\circledR}$ systems are available pre-treated with ECM in a variety of pore sizes, although modifications are feasible by purchasing individual components, and are very widely used in cancer research.

Figure 4.2. Transwell ${ }^{\circledR}$ System. An ECM such as Matrigel may be placed on top of the microporous membrane onto which cells are seeded ${ }^{423}$.

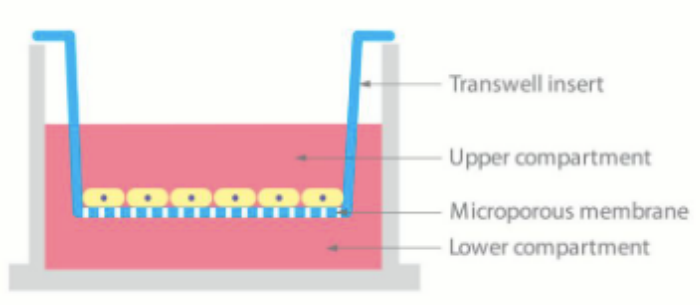

\subsubsection{Other Migration \& Invasion Models}

As well as the three models already described a number of other techniques for estimating cellular migration and invasion exist, although they are less popular. Fence assays utilise a reverse methodology to scratch and exclusion assays by introducing small areas of cells at confluence to larger areas of naïve substrate and observe outgrowth from the pool, rather than closure of a wound. Carrier-bead and spheroid migration assays take this principle further by introducing beads coated with confluent cells or spheroids to naïve substrate and observing outgrowth (akin to bacterial or fungal seeding on growth substrates for antimicrobial/fungal assessment). More complex invasion assays may be performed by constructing a platypus assay, where cells are sequentially sandwiched between two layers of ECM with an acellular 
void in a proportion of the plate (created by exclusion), into which cells might invade laterally rather than through a porous membrane. Similarly, cells and spheroid may be introduced to larger blocks of gelatinised ECM and their free migration through or outward extension into the matrix may be observed like veins running through a block of blue cheese. Co-culture models of spheroid invasion (spheroid confrontation assay) may also be constructed in threedimensional ECM blocks to introduce a further degree of three-dimensional and tissue microenvironmental complexity. Although the techniques and technology required for each of these assays is not advanced or expensive, they are time-consuming and prone to error, making them unsuitable for high-throughput screening or baseline biologic investigation, but may be appropriate in specific circumstances where a more elegant model is required ${ }^{412}$.

\subsubsection{In Vitro Demethylation in Colorectal Cancer Models}

Epigenetic aberrations, including DNA hypermethylation, have been established as important factors in the molecular classification of CRC, although the pathogenic role is less well understood $^{118,430}$. In vitro studies of methylation in colorectal cancer have demonstrated that 10-day treatment with AZA at sub-cytotoxic doses can reactivate previously transcriptionally silenced genes, although the patterns of demethylation at specific promotor regions did not uniformly match gene reactivation ${ }^{431}$. However, in another study based on array analysis of 14,000 gene promotors in HCT116 cells, AZA treatment was demonstrated to produce specific and reproducible patterns of demethylation as well as conserved loci that were never demethylated ${ }^{432}$. A more recent study has found that demethylation of SW480 cells by AZA reactivates tumour suppressor NDN and potentially down-regulates Wnt signalling ${ }^{433}$. A further study examined the effects of AZA derivatives and 5-FU co-therapy on cell lines SW48 and HT-29, finding that there was potentially a synergistic effect with dual therapy ${ }^{434}$.

\subsubsection{In Vivo Demethylation in Colorectal Cancer Trials}

The suggestion of synergy between AZA derivatives and other chemotherapy agents has led to a number of limited clinical trials in treatment-exhausted metastatic colorectal cancers, although results have so far been disappointing. A recently published phase II study of AZA with histone deacetylase inhibitor entinostat against multiply-treated metastatic colorectal cancer demonstrated no benefit ${ }^{233}$. A similarly disappointing outcome was found in a phase I/II trial of AZA with CAPOX in refractory CIMP-high metastatic colorectal cancer ${ }^{232}$. This study's methodology of determining CIMP-H was not well described and a tenuous focus on serum and stool vimentin hypermethylation as a proxy goes some way to undermining their 
classification of CIMP-H, although their clinical outcomes were robust. However, another in vitro co-therapy study demonstrated that AZA acted synergistically with irinotecan to improve survival and improve tumour response in HCT116 CRC cell xenografted into mice, and has led to Phase I/II trials examining the efficacy of another DNMT inhibitor (Guadecitabine, SGI110) with irinotecan in previously treated colorectal cancer, although results are yet to be reported ${ }^{435}$

\subsection{Aims and Objectives}

This chapter will examine the effects of demethylation on colorectal cell lines in vitro. Established methods of assessing locus-specific methylation (those already employed in Chapter 3) will be utilised for internal and external validity and consistency, as well as additional assessments of global methylation for broader perspective. Both a two-dimensional model of migration and three-dimensional models of invasion will be employed to determine the effects of demethylation in increasingly complex models that might provide an insight into and EMT/MET processes. Established (AZA) and putative (RRx-001) demethylating agents will be employed to investigate their comparative effects, following baseline assessments of their cellular cytotoxicity. 


\subsection{Methods}

\subsubsection{Cell Culture, Treatment, \& Cytotoxicity}

\subsubsection{Cell Culture}

Maintenance and control culture of DLD-1 cells was performed as outlined in Section 2.3 and modified as described below for experimental purposes.

\subsubsection{Treatment with Demethylating Agents}

Both AZA and RRx-001 are stable in powdered form at $-20^{\circ} \mathrm{C}$ and room temperature, respectively, but degrade when in solution. Although the rate of degradation for AZA is known (half-life dissolved in $\mathrm{dH}_{2} \mathrm{O}$ or DMSO at room temperature at treatment concentrations $(0.25$ $5.0 \mathrm{uM}$ ) is approximately $10-15 \mathrm{hrs}$ ), the degradation of RRx-001 in solution and subsequent loss of biological activity is not known, although in vitro studies indicate it to be rapidly reactive with blood components and biologically effectively consumed almost immediately following initial administration 235,239 . For the purposes of this study, a trial of the stability (in terms of biological efficacy) of each agent was undertaken by assessing cytotoxicity and demethylating ability of each agent following fresh preparation and storage at $-80^{\circ} \mathrm{C}$ in stock concentration of $100 \mathrm{uM}$. Stored reagents were trialled alongside fresh-preparations, negative controls, and solvent controls to assess biological activity (molecular chemistry \& pharmacokinetics of each compound was beyond the remit of this study). Both AZA and RRx001 were found to be soluble in $\mathrm{H}_{2} \mathrm{O} / \mathrm{DMSO}$ at stock and working concentrations and stable at $-80^{\circ} \mathrm{C}$ for at least one week, which was the maximum period these reagents were stored for when used in experimental models.

Each cell-culture experiment was conducted in multiple technical and biological replicates in order to assess and reduce sources of variation, and with negative, solvent, and where applicable positive controls (AZA acted as positive control for RRx-001) ${ }^{436}$. AZA and RRx001 were presented across a dose-curve of $0.10,0.25,0.5,1.0,2.0$, and $5.0 \mathrm{uM}$ for initial screening of cytotoxicity and biological activity, and then the dose-curve rationalised for further experiments based on results. Doses were calculated and solutions of drug created by a serial dilution technique. This was to ensure the adequate mixing of drug in solution, and the accuracy and control between replicates of doses, especially at lower concentrations (versus direct pipetting of small doses). 


\subsubsection{Cytotoxicity}

The number of cells observed in a population exposed to any particular treatment is the product of a number of factors, specifically; time, the starting population, rate of cell division (proliferation), and rate of cell death (natural apoptosis \& toxicity). Each of these factors must be accounted for when assessing the effects of a chemical on a biological process so that effects on outcomes such as cell migration and invasion are not confounded by co-factors related to toxicity. As such, a baseline assessment of cytotoxicity and cytostasis must be made prior to experimetation ${ }^{437}$. The OECD Guidelines for the Testing of Chemicals (In Vitro Mammalian Cell Micronucleus Test) sets out the standards for assessing the cytotoxicity of a compound whether or not the investigator is employing actin polymerisation inhibitor cytochalasin $\mathrm{B}$ (cytoB); recommending that Relative Population Doubling (RPD) or Relative Increase in Cell Count (RICC) be used to assess cytotoxicity and cytostasis when cytoB is not used ${ }^{438}$. Cytotoxicity should be assessed across a treatment gradient and in biological and technical replicate. The maximum cytotoxic effect used in biological modelling should be based upon a reduction of RPD of $55 \%+/-5 \%$ at the maximum dose, although care should be taken when assessing outcomes at doses approaching this threshold. Additional tests examining endpoints such as cell integrity, apoptosis, necrosis, etc, can also be employed but should not substitute RPD or RICC ${ }^{439,440}$. For the purposes of this research, a limit of $50 \%$ reduction in RPD will be set.

Assessment of RPD was made by culture of DLD-1 cells in control, solvent control, and treatment media and a dose-curve was employed to examine the cytotoxic effects of each compound. DLD-1 cells were grown until approximately $70 \%$ confluent according to standard culture conditions and then trypsinised and $5 \mathrm{mls}$ of cell suspension transferred to $25 \mathrm{~cm}$ plastic cell culture flasks at a concentration of $3 \times 10^{4}$ cells $\mathrm{ml}^{-1}$ (coulter counter assessment). Cells were allowed to adhere for a minimum of 8 and maximum of 12 hours (usually overnight) and then treated daily with AZA or RRx-001 (or control or solvent control) for a further $72 \mathrm{hrs}$ at $0.10,0.25,0.5,1.0,2.0$, and $5.0 \mathrm{uM}$ dose concentration. At the end of the $72 \mathrm{hr}$ period cells were trypsinised, washed, and re-suspended in $10 \mathrm{ml}$ PBS and counted on a haemocytometer or on the coulter counter. 
RPD was calculated using the formula below.

Population Doubling $(\mathrm{PD})=\underline{\log (\text { cell count after treatment } / \text { cell count in control })}$

$$
\log ^{2}
$$

Relative Population Doubling $($ RPD $)=$ Number of population doublings in treatment

Number of population doublings in control

In addition to RPD, trypan blue, a commonly used stain that penetrates the membranes of dead but not viable cells, will be used for an additional assessment of cytotoxicity ${ }^{441}$. DLD-1 cells were cultured and treated as per the protocol for RPD testing. At the point at which cells were re-suspended in PBS for counting, cells were instead treated with $0.4 \%$ Trypan Blue solution (Sigma-Aldrich (Merck), USA) and incubated at room temperature for 5 minutes. Cells were then counted on a haemocytometer and a crude ratio of viable (unstained) cells versus dead cells (stained) was calculated.

\subsubsection{Wound Healing Assay}

An exclusion-zone assay was used as this provide the most easily reproducible and controllable method of assessing two-dimensional cell migration. The system employed was the Ibidi Culture-Insert 2 Well System as outlined above ${ }^{419}$. This provided a consistent $500 \mu \mathrm{m}$ "scratch" across which cells migrate on the base of a standard plastic cell culture well.

DLD-1 cells were grown until approximately 70\% confluent according to standard culture conditions and then trypsinised and transferred to $25 \mathrm{~cm}$ plastic cell culture flasks at a concentration of $3 \times 10^{4}$ cells $\mathrm{ml}^{-1}$ (coulter counter assessment) and allowed to adhere for a minimum of 8 and maximum of 12 hours (usually overnight). Cells were then cultured for a further $72 \mathrm{hrs}$ in control, solvent control, or treatment (AZA or RRx-001) medium at a concentration based on cytotoxicity studies. Control or treatment media was refreshed daily.

After a full $72 \mathrm{hrs}$ of culture, treated (or control) cells were again trypsinised, washed, and diluted to a concentration of $5 \times 10^{5}$ cells $\mathrm{ml}^{-1}$ in fresh culture medium. Ibidi cell culture inserts were applied to the base of 12 -well culture plates and $70 \mu 1$ of suspended cells applied to each 
side of the insert (either side of the exclusion zone). $700 \mathrm{ml}$ of suspended cells were applied to the well outside of the insert. After a period of $24 \mathrm{hrs}$ during which cell adherence to the base of the plate occurred, the insert was removed with sterilised forceps leaving a cell-free $500 \mu \mathrm{m}$ "scratch" in the adherent cell monolayer. A further $700 \mathrm{ml}$ of fresh culture media was added at this point to prevent dehydration during undisturbed culture during time-lapse microscopy.

12-well plates were then monitored by time-lapse light wide-field microscopy (frame capture once every 10 minutes) using the etaluma 560 Inverted Live-Cell Microscope platform. Timeto-convergence was measured by the clock starting when the Ibidi cell culture insert was removed and stopped when "first contact" was made between cells from either side of the cellfree exclusion zone. First contact was chosen as an endpoint as it favours cell migration as the predominant biological process (over cell proliferation), although wound-width, given by the formula below, was also employed an alternative endpoint (using Image $J$ software) ${ }^{442,} 443$. Techniques to inhibit cell proliferation, such a mitomycin $C$, were not employed ${ }^{444}$.

Formula for calculating Rate of Cell Migration.

$$
\begin{gathered}
R_{M}=\frac{W_{i}-W_{f}}{t} \\
R_{M}=\text { Rate of cell migration }(\mathrm{nm} / \mathrm{h}) \\
W_{i}=\text { initial wound width }(\mathrm{nm}) \\
W_{f}=\text { final wound width }(\mathrm{nm}) \\
t=\text { duration of migration (hour) }
\end{gathered}
$$

In addition to the method set out above, an attempt was made to use the IN Cell Analyser (GE Healthcare) platform to facilitate high-throughput analysis of multiple wells in the 12-well plate, thus negating the limitation of the single-lens non-mobile platform of the etaluma system. Unfortunately, after several attempts, this was abandoned due to technical and physical constraints (programming/software errors, demand from other research groups performing time-sensitive experiments, physical location in another building thus requiring culture transfer). This did not affect the outcomes of the research, and in fact may have aided in increasing robustness through the necessity of performing multiple biological and technical replicates over an extended period. 


\subsubsection{Extracellular Matrix Invasion}

A model of three-dimensional cellular invasion was constructed utilising the Corning (New York, USA) BioCoat ${ }^{\mathrm{TM}}$ Transwell $^{\circledR}$ Boyden chamber system with a pre-loaded layer of Matrigel acting as the invasion matrix. This system provides a growth area of $0.3 \mathrm{~cm}^{2}$ of Matrigel ECM on top of a polyethylene terephthalate (PET) membrane perforated by 8-micron pores. Cells in the top chamber must therefore penetrate the EMC and translocate through the pores to the inferior aspect of the membrane in order to satisfy the definition for invasion. This system was chosen because of the likeness of the Matrigel ECM to the acellular components of the colorectal basement membrane and the ability of the DLD-1 cells to translocate through a pore of this size $\mathrm{s}^{425-427}$.

DLD-1 cells were cultures as per the protocol set out for wound healing assay (section 4.3.2) until the full period of $72 \mathrm{hrs}$ of treatment (or control) had been achieved. Invasion chambers were removed from storage at $-20^{\circ} \mathrm{C}$ and allowed to come to room temperature and were then rehydrated in $1000 \mu \mathrm{L}$ of serum-free growth medium $(500 \mu \mathrm{L}$ in each of the top and bottom of the wells/inserts) for 2 hours in standard incubator conditions. This rehydrating medium was then removed by pipetting, making sure not to damage the layer of Matrigel. A companion plate of invasion chambers consisting of PET membrane with 8micron pores but containing no ECM was also prepared.

Cells were trypsinised, washed, and made to a concentration of $3 \times 10^{5}$ cells $\mathrm{ml}^{-1}$ in serum-free medium. $750 \mu \mathrm{L}$ of fully composed medium (i.e. including serum) was added to the bottom compartment of each well and the invasion chambers (ECM \& companion) carefully placed inside. $500 \mu \mathrm{L}$ of suspended cells was then placed in the upper portion of the invasion chambers atop the ECM and incubated for 24 hours. A variation to this protocol was also performed where $200 \mu \mathrm{g} \mathrm{ml}{ }^{-1}$ fibronectin was added to the bottom chamber to provide an additional chemoattractant (invasion gradient), as it has been shown to be an important factor in stimulation and regulating ECM remodelling and cellular invasion in healthy and cancerous tissue $^{445-447}$.

Following incubation, invasion chambers were removed from incubation wells and inverted to remove growth medium. The seeding-surface of the invasion membrane (top) was then wiped twice with a cotton bud to remove ECM and non-invading cells, as per manufacturers protocol. Invading cells on the under-surface of the invasion membrane were then fixed and stained by 
sequential submersion in $100 \%$ methanol for two minutes and then crystal violet for two minutes, and then rinsed in $\mathrm{dH}_{2} \mathrm{O}$ and left to air dry. Invading cells were then counted on a haemocytometer under light microscopy following removal of the invasion membrane from the chamber with a sharp scalpel.

Invasion was calculated as a percentage and index using the following formulae:

Percentage Invasion $=($ Mean number of cells invading through ECM $/$ Mean number of cells invading through control membrane) x 100

Invasion Index $=(\%$ invasion for test (treated) cells $/ \%$ invasion for control cells $)$

\subsubsection{Assessment of Methylation Status}

\subsubsection{CIMP Status}

Methylations status of control, solvent control, and treated DLD-1 cells was assessed by the same methodology of bisulfite-conversion and msPCR as set out in Chapter 3 (Sections 3.3.3, 3.3.4, 3.3.5, and 3.3.6). The only difference to these protocols was that DNA was harvested from cultured cells, negating the steps involving DNA extraction from FFPE slides. Cells were first trypsinised, washed with PBS twice, and then vortexed to pellet and the supernatant discarded. Cell pellets were then resuspended in proteinase $\mathrm{K}$ and Tissue and Cell Lysis Solution and the previously described protocol of the MasterPure Complete DNA and RNA purification kit (Epicentre, Illumina, Wisconsin, USA) followed. The quantity and quality of DNA was again measured by spectrophotometry and extracted DNA was stored at $-20^{\circ} \mathrm{C}$ during active experimentation and archived at $-80^{\circ} \mathrm{C}$ when not in use.

\subsubsection{Global DNA Methylation}

In addition to assessment by msPCR, global DNA methylation was also assessed by means of the MethylFlash Global DNA Methylation (5-mC) ELISA Easy Kit (Colorimetric) kit (Epigentek, New York, USA). In this assay, DNA harvested from DLD-1 cells is bound to pre-prepared wells containing antibodies with a high affinity to DNA and then captureindicator antibodies specific to methylated DNA are added and the plate subjected to analysis by spectrophotometer, the proportion of methylated DNA being proportionate to the optical 
density (OD). This method was chosen as it is widely used across the methylation literature to provide a broad and quantitative assessment of global DNA methylation ${ }^{448,449}$.

DNA was extracted from DLD-1 cells as set out previously, its quantity and quality assessed

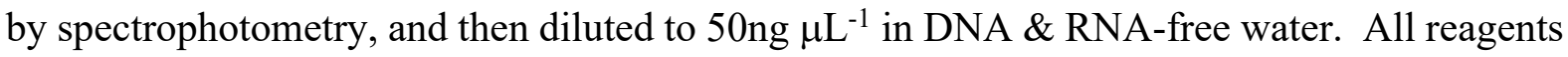
were prepared according to the manufacturers protocol (https://www.epigentek.com/docs/P1030.pdf) and 100ng of DNA added to each sample well of the plate (see Figure 4.3). Negative controls and positive controls (both provided in the kit) were applied to the appropriate wells of the plate, including the $0.1 \%$ to $5.0 \%$ concentration gradient for the positive control that generates the standard curve for quantitative assessment. DNA and control solutions were gently swirled in the test wells and then the whole plate was covered with parafilm and incubated at $37^{\circ} \mathrm{C}$ for 60 minutes.

Detection Complex Solution (DCS) was then mixed according to supplied protocol and, after washing each well three times with $150 \mu \mathrm{L}$ of supplied washing buffer (WB), 50 $\mu \mathrm{L}$ DCS was applied to each well. The plate was then re-covered with parafilm and left to incubate for 50 minutes at room temperature, after which the DCS was removed by pipetting and each well washed with $150 \mu \mathrm{L}$ WB a further five time. $100 \mu \mathrm{L}$ detection solution is then added to each well (vertical columns simultaneously) and the plate briefly agitated and then left for several minutes until the 5\% PC wells turn dark blue, whereupon the stop solution is added in a similar fashion. Wells with a positive detection will then turn from blue to yellow after several minutes and the plate is read by spectrophotometry at $450 \mathrm{~nm}$ within 15 minutes.

Percentage global DNA 5-mC can then be calculated using the formula shown below, following calculation of the slope from the standard curve. (OD, optical density; NC, negative control; S, amount of sample DNA in ng).

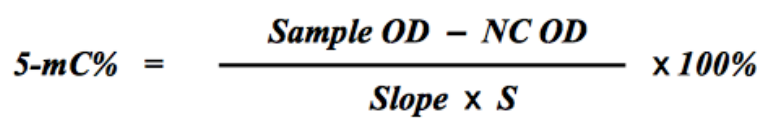


Figure 4.3. Schematic of 96-well plate for MethylFlash Global DNA Methylation (5-mC) ELISA Easy Kit. (NC, negative control; PC, positive control).

\begin{tabular}{|l|l|l|l|l|l|l|}
\hline Well \# & Strip 1 & Strip 2 & Strip 3 & Strip 4 & Strip 5 & Strip 6 \\
\hline A & NC & $1 \%$ PC & Sample 2 & Sample 6 & Sample 10 & Sample 14 \\
\hline B & NC & $1 \% P C$ & Sample 2 & Sample 6 & Sample 10 & Sample 14 \\
\hline C & $0.1 \%$ PC & $2 \% P C$ & Sample 3 & Sample 7 & Sample 11 & Sample 15 \\
\hline D & $0.1 \%$ PC & $2 \% P C$ & Sample 3 & Sample 7 & Sample 11 & Sample 15 \\
\hline E & $0.2 \%$ PC & $5 \% P C$ & Sample 4 & Sample 8 & Sample 12 & Sample 16 \\
\hline F & $0.2 \%$ PC & $5 \% P C$ & Sample 4 & Sample 8 & Sample 12 & Sample 16 \\
\hline G & $0.5 \%$ PC & Sample 1 & Sample 5 & Sample 9 & Sample 13 & Sample 17 \\
\hline H & $0.5 \%$ PC & Sample 1 & Sample 5 & Sample 9 & Sample 13 & Sample 17 \\
\hline
\end{tabular}




\subsection{Results}

\subsubsection{Cytotoxicity}

\subsubsection{Azacytidine}

AZA was applied to DLD-1 cells across a dose curve as described previously. At the end of the treatment period of $72 \mathrm{hrs}$, cells were harvested and counted both by coulter counter and on a haemocytometer. Percentage RPD is shown in Figure 4.4.1 across the dose curve, demonstrating a $50 \%$ reduction in RPD at between the $0.5 \mathrm{uM}$ and $1.0 \mathrm{uM}$ AZA doses. Averages of biologic replicates (typically 5) are represented by individual technical replicates RPD1-5, and an over-all average by the bold line (total replicates 25). Standard deviation (SD) at the $0.5 \mathrm{uM}$ dose is $\pm 7.3 \%$ from a baseline of $61.0 \%$ RPD, and at no point on this series is $\geq 10.0 \%$.

Figure 4.4.1 \%RPD Dose Curve for Azacytidine. (Doses, where stated in uM, are for azacytidine (AZA), error bars indicated standard deviation).

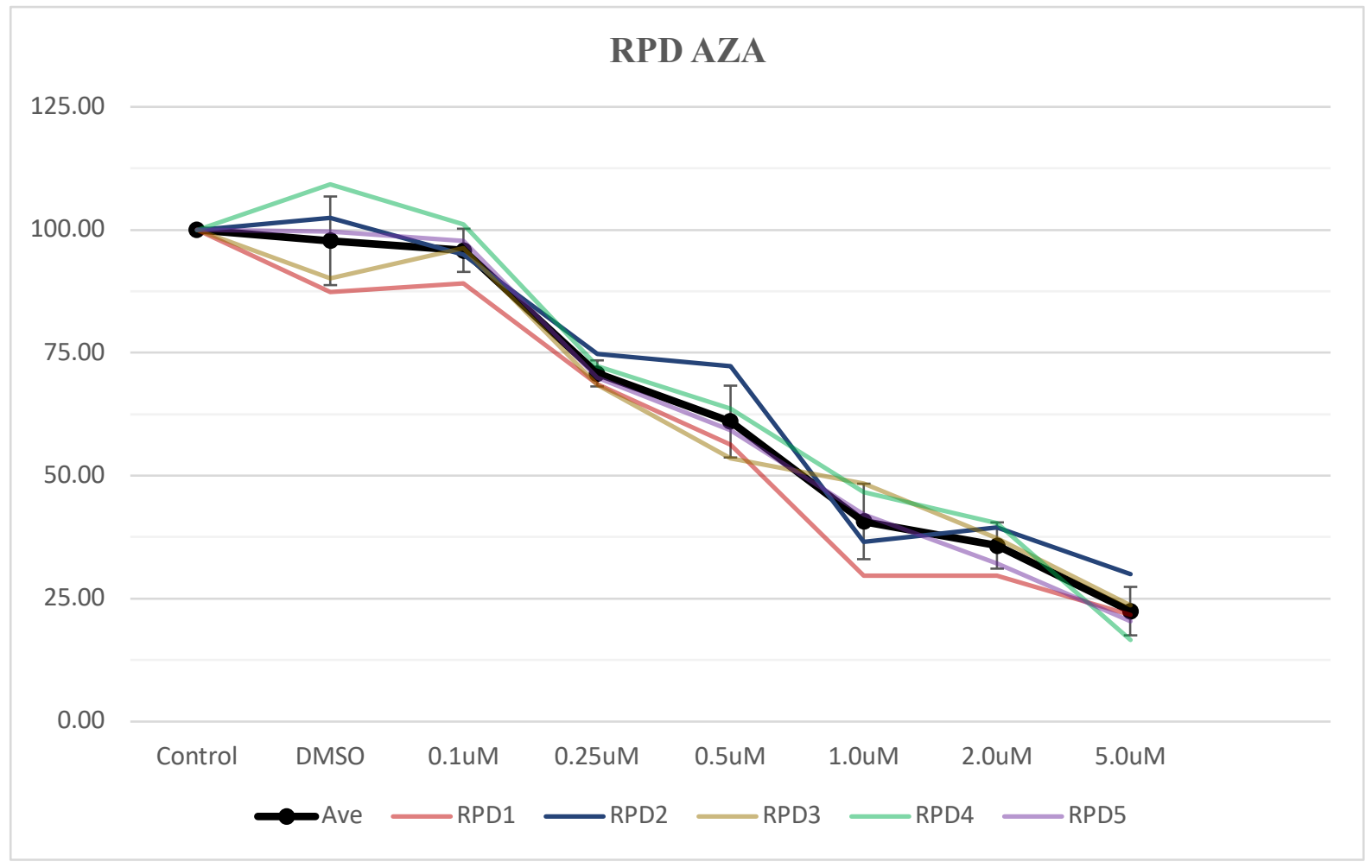

Trypan blue analysis demonstrated that there was an increase in non-viable cells at AZA concentrations of greater than $1.0 \mathrm{uM}(\geq 30 \%$ non-viable), whereas concentrations below this level did not significantly affect the ratio of non-viable cells. The discrepancy between a significant effect of AZA on RPD ( $\geq 50 \%$ reduction at over $0.5 \mathrm{uM})$ versus cell non-viability 
(significant effects at $\geq 1.0 \mathrm{uM}$ ) suggests biologic activity of AZA at lower concentrations that is not due to cell death.

\subsubsection{RRx-001}

RRx-001 was applied to DLD-1 cells across a dose curve as described previously. At the end of the $72 \mathrm{hr}$ treatment period, cells were harvested and counted both by coulter counter and on a haemocytometer. Percentage RPD is shown in Figure 4.4.2 across the dose curve. Averages of biologic replicates (typically 5) are represented by individual technical replicates RPD1-5, and an over-all average by the bold line (total replicates 25 ). A 50\% reduction in RPD at a dose of approximately the 1.0uM RRx-001 is demonstrated, however, standard deviation indicates that there is a high degree of variability of response to treatment at this dose ( $\mathrm{SD} \pm 35.6 \%$ from $50 \%$ RPD), and thus an experimental dose of $0.5 \mathrm{uM}$ would be more appropriate (SD $\pm 10.1 \%$ from $72.4 \%$ RPD).

Figure 4.4.2 \%RPD Dose Curve for RRx-001. (Doses, where stated in uM, are for RRx-001, error bars indicate standard deviation).

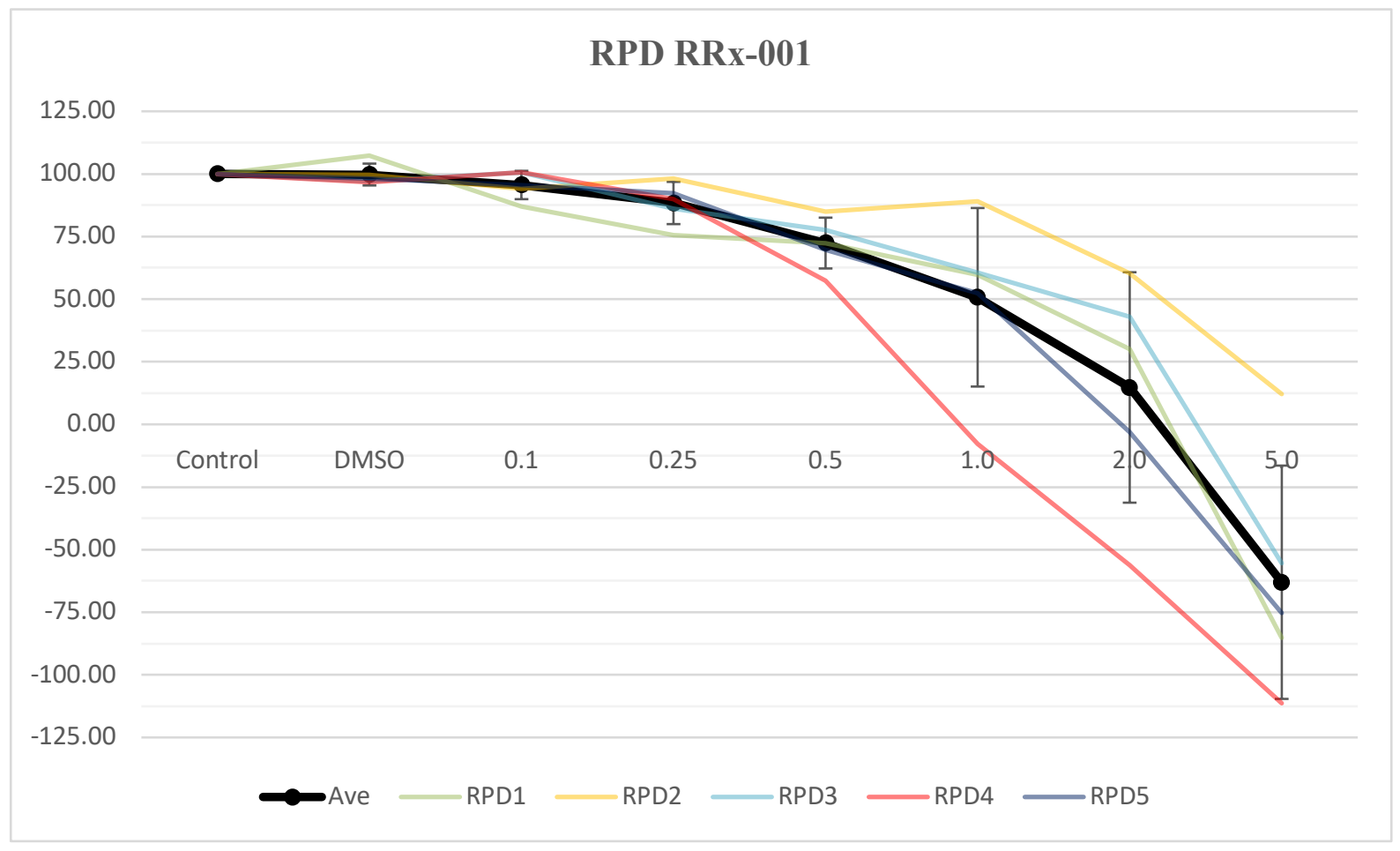


Figure 4.4.2 also indicates that at doses of $\geq 5.0 \mathrm{uM}$, and more variably at $2.0 \mathrm{uM}$, the RPD becomes negative, indicating a population decline compared to baseline seeding, and a highly cytotoxic effect of RRx-001. This was reflected in the trypan blue examination of RRx001 where there was a lower ratio of viable cells $(\leq 70 \%)$ at doses of $1 \mathrm{uM}$ and above $(<20 \%$ at 5.0uM). Viable cell ratios were however acceptable at doses up to $0.5 \mathrm{uM}$ RRx-001.

\subsubsection{Two-Dimensional Migration (Scratch) Assay}

Given the results of the cytotoxicity assays (RPD and trypan blue), a maximum experimental dose of $0.5 \mathrm{uM}$ over a $72 \mathrm{hr}$ exposure period (daily treatments) of both AZA and RRx-001 was employed as a baseline for wound healing and invasion assays.

Using the Ibidi culture system, untreated control and solvent control (DMSO) exposed DLD-1 cells took a mean time of 38 hours $(\mathrm{SD} \pm 1.5)$ and 41 hours $(\mathrm{SD} \pm 3.0)$, respectively, to converge (reach first contact) across the $500 \mu \mathrm{m}$ cell-free zone. Prior treatment for three days with either $0.25 \mathrm{uM}$ or $0.5 \mathrm{uM}$ AZA resulted in a mean convergence time of $37.5(\mathrm{SD} \pm 2.0)$ and 48.0 (SD \pm 4.5 ) hours, respectively. Prior treatment for three days with either $0.25 \mathrm{uM}$ or $0.5 \mathrm{uM} R R \mathrm{x}-001$ resulted in a mean convergence time of $38.5(\mathrm{SD} \pm 3.0)$ and $58.5(\mathrm{SD} \pm 4.5)$ hours, respectively. The difference of the means between the control and $0.5 \mathrm{uM}$ treatments with both AZA and RRx-001 were highly significant based on students t-test $(\mathrm{p}<0.001)$, but not for DMSO or the lower doses of 0.25uM of either compound. These results are shown in Figure 4.4.3.

Figure 4.4.4 demonstrates the process of cell migration across the void using the Ibidi cell system, and the measured endpoints for illustrative purposes. Image a. demonstrates the starting position of the cells in culture at timepoint 0 , with the gel insert only just removed. Image b. demonstrates the point of first convergence $\mathrm{PoC}$, where cells from either side of the void make physical contact and stop the clock (time to convergence, TtC). Image c. demonstrates a greater degree of convergence although complete exclusion of the void is yet to occur. Images a. and b. have also been marked-up to demonstrate the area that remains to be filled-in by cells that forms the final width metric that could be used to calculate the Rate of Cell Migration, although this was only applied when cells reached $\mathrm{PoC}$ as demonstrated in image $\mathbf{b}$ as this is the least subjective time-point for assessing convergence. Image d. illustrates almost complete occlusion of the void and proliferative overgrowth away from the original cell-free zone. 
Figure 4.4.3 Time-to-Convergence (first contact) for DLD-1 cells across a 500 $\mu \mathrm{m}$ cell-free zone.

\section{Time-to-Convergence}

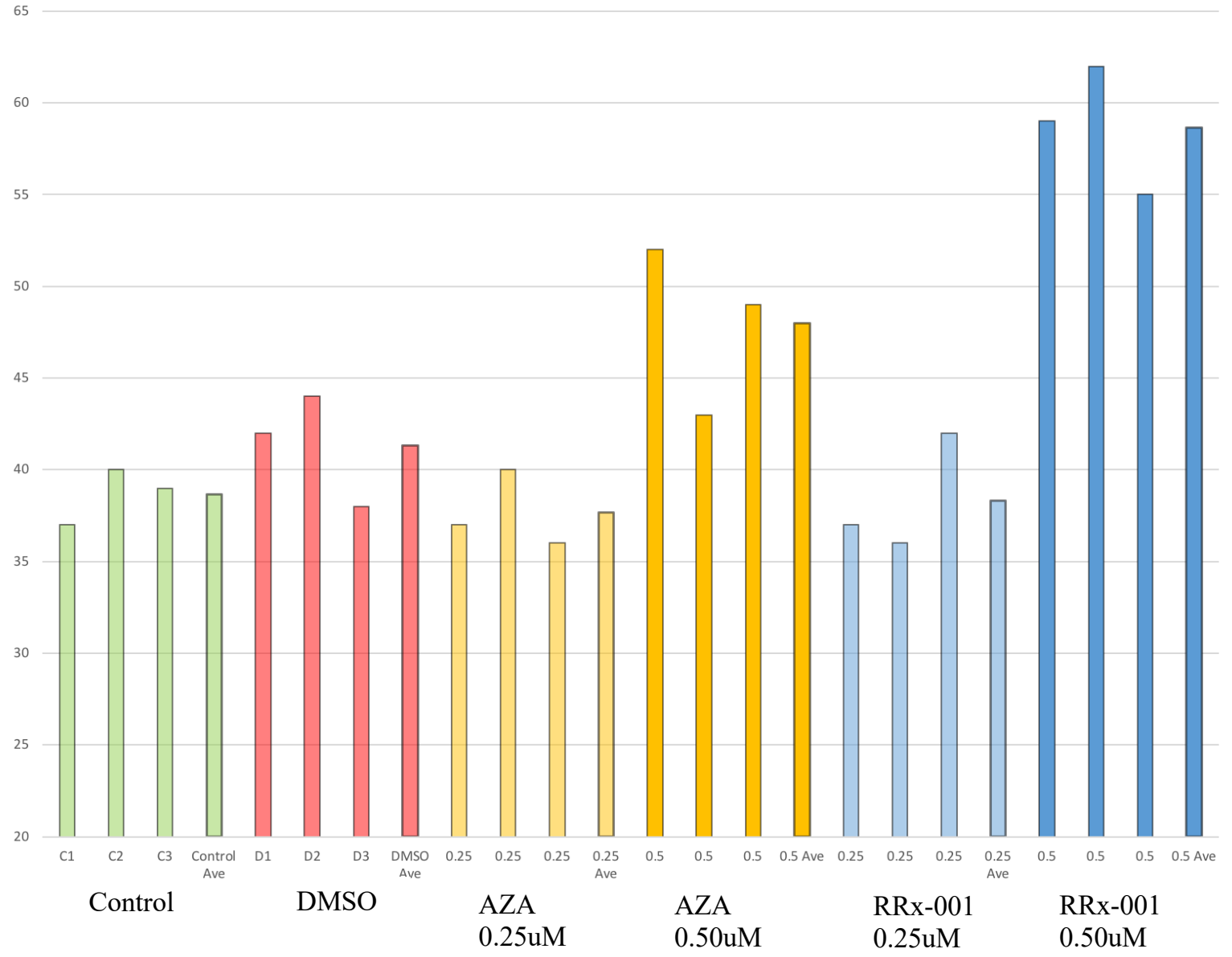

\begin{tabular}{|l|l|l|l|l|}
\hline & $\begin{array}{l}\text { Mean Time-to- } \\
\text { Convergence (TtC) }\end{array}$ & Range TtC & $\begin{array}{l}\text { Standard } \\
\text { Deviation }\end{array}$ & $\begin{array}{c}\text { p } \\
\text { (Students-t) }\end{array}$ \\
\hline Control & $38.5 \mathrm{hrs}$ & $37.0-40.0 \mathrm{hrs}$ & $\pm 1.5 \mathrm{hrs}$ & - \\
\hline Solvent Control (DMSO) & $41.5 \mathrm{hrs}$ & $38.0-44.0 \mathrm{hrs}$ & $\pm 3.0 \mathrm{hrs}$ & $\mathrm{NS}$ \\
\hline AZA 0.25uM & $37.5 \mathrm{hrs}$ & $36.5-40.5 \mathrm{hrs}$ & $\pm 2.0 \mathrm{hrs}$ & $\mathrm{NS}$ \\
\hline AZA 0.50uM & $48.0 \mathrm{hrs}$ & $43.0-52.0 \mathrm{hrs}$ & $\pm 4.5 \mathrm{hrs}$ & $<0.001$ \\
\hline RRx-001 0.25uM & $38.5 \mathrm{hrs}$ & $36.0-42.5 \mathrm{hrs}$ & $\pm 3.0 \mathrm{hrs}$ & $\mathrm{NS}$ \\
\hline RRx-001 0.50uM & $58.5 \mathrm{hrs}$ & $55.0-62.5 \mathrm{hrs}$ & $\pm 3.5 \mathrm{hrs}$ & $<0.001$ \\
\hline
\end{tabular}


Figure 4.4.4 Etaluma light microscopy images demonstrating stages in cell migration during the wound healing (scratch) assay.

b.

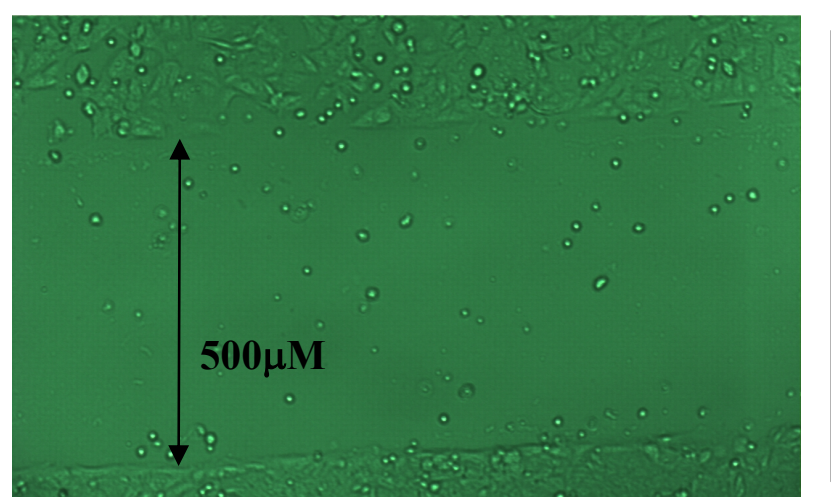

c.

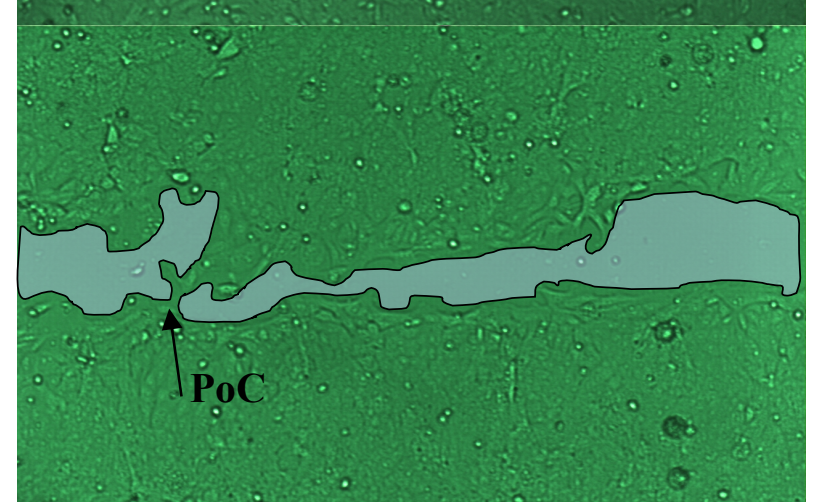

d.

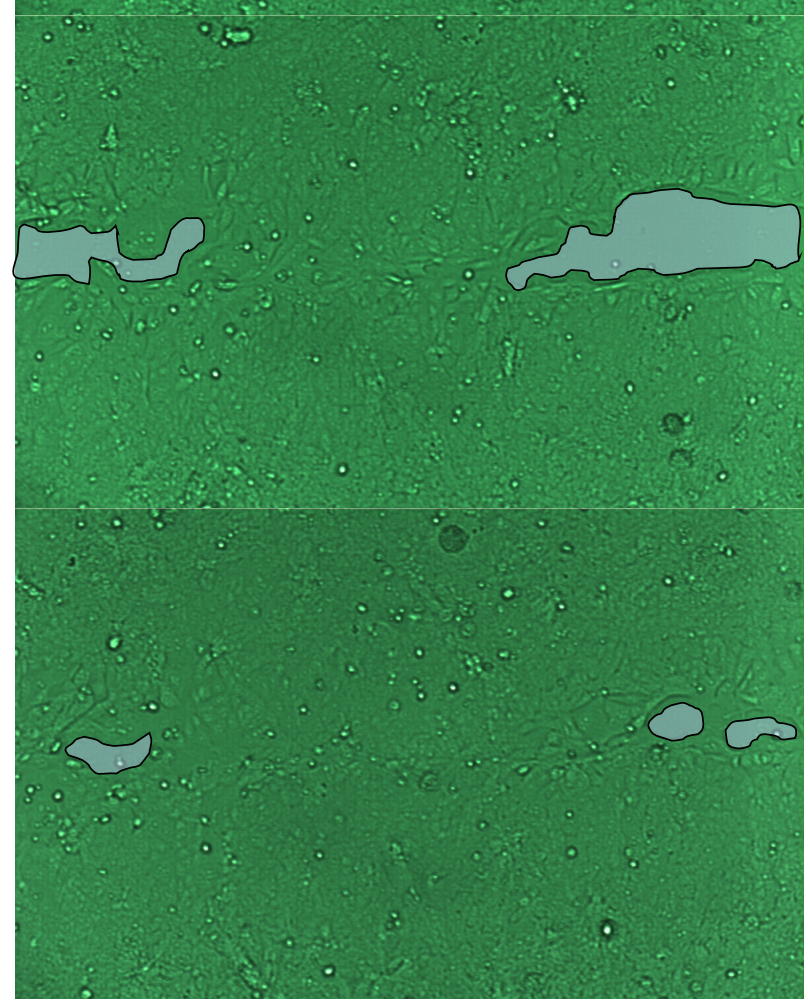

DLD-1 cells at time point 0 immediately after the gel insert has been removed. Note the $500 \mu \mathrm{M}$ cell-free void (scratch) between the relatively confluent cell fronts.

DLD-1 cells at the point of convergence (PoC), representing the 'clock-stop' for Time to Convergence. Note cell-free shaded area that forms the basis of the width (area) metric.

DLD-1 cells beyond the PoC but with some cell-free void remaining. Note the cell morphology along the convergent zone; elongated cells have bridged the void, suggesting a cell-migratory process, rather than proliferative.

DLD-1 cells at beyond PoC. There are still a few cell-free voids but $>70 \%$ confluence has been reached and the cells behind the convergent zone are crowded, demonstrating a proliferative process away from the original cell-free zone. 
The Rate of Cell Migration metric $R_{m}$ was found to be unreliable. Firstly, for the formula set out in Section 4.3.2, $R_{m}$ is defined as the initial width, minus the residual width, divided by time. Employing the PoC definition as described (the residual width being 0), the only independent variable is time, and therefore the metric $R_{m}=500 \mu \mathrm{M} / \mathrm{TtC}$ and is not a better discriminator over $\mathrm{TtC}$ alone, when examining the effects of $\mathrm{AZA}$ and $\mathrm{RRx}-001$ on migration. Secondly, if the metric $R_{m}$ is modified to replace initial width and residual width with initial area and residual area, as demonstrated by the shaded areas on Figure 4.4.5 a. - c., no advantage was found over $\mathrm{TtC}$ alone, nor was a statistically significant difference in mean cellfree area at PoC evident.

Figure 4.4.5 Examples of PoC and residual cell-free areas for Control, AZA, and RRx-001.

a.

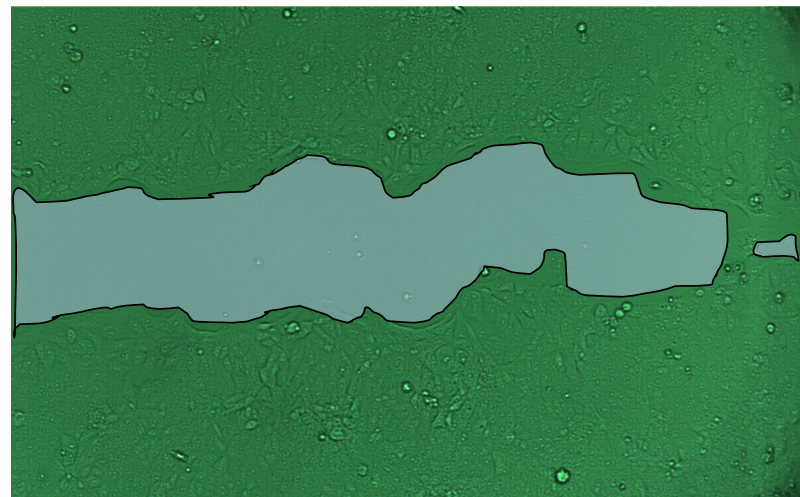

b.

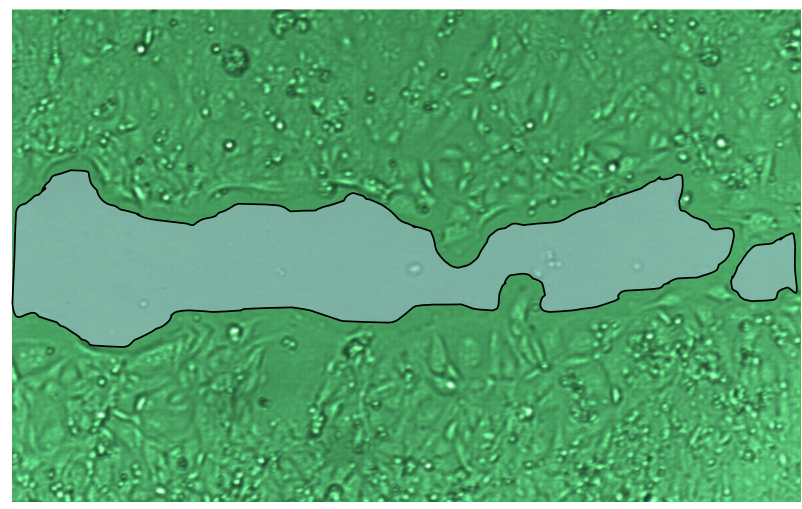

c.

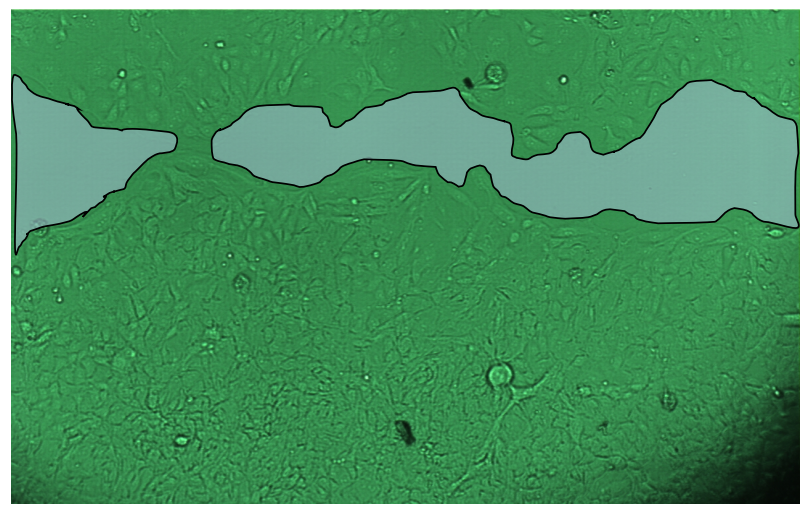

Control DLD-1 cells at PoC. The shaded cell-free area is measured at $154,000 \mu \mathrm{M}^{2}$.

DLD-1 cells exposed to $0.5 \mathrm{uM}$ AZA at PoC. The shaded cell-free area is measured at $149,000 \mu \mathrm{M}^{2}$. Students-t test for significant difference between mean area in treated vs control cells was nonsignificant.

DLD-1 cells exposed to $0.5 \mathrm{uM}$ RRx-001 at PoC. The shaded cell-free area is measured at $145,500 \mu \mathrm{M}^{2}$. Students-t test for significant difference between mean area in treated vs control cells was nonsignificant. 


\subsubsection{Three-Dimensional Invasion Assay}

Given the results of the cytotoxicity assay, a maximum experimental dose of $0.5 \mathrm{uM}$ of both AZA and RRx-001 was employed in invasion assays over a $72 \mathrm{hr}$ exposure period with a daily dosing regime.

After 24 hours incubation there was no difference in the mean percentage invasion (PI) between control and solvent control (DMSO) exposed DLD-1 cells (100\% and 97-98\%, respectively), with a mean invasion index (II) of 77 and 75 , respectively. Prior treatment for three days with either $0.25 \mathrm{uM}$ or $0.5 \mathrm{uM}$ AZA resulted in a mean PI of $50.0 \%$ (SD $\pm 3.4 \%$ ) and $31.1 \%$ (SD $\pm 3.1 \%$ ), and II of 42 and 27 , respectively. Prior treatment for three days with either $0.25 \mathrm{uM}$ or $0.5 \mathrm{uM} R \mathrm{Rx}-001$ resulted in a mean PI of $49.5 \%$ (SD $\pm 2.6 \%$ ) and $33.10 \%$ (SD $\pm 2.4 \%$ ), and II of 41 and 28.5, respectively. The difference between the mean PI of the control DLD-1 and DLD-1 exposed to either $0.25 \mathrm{uM}$ or $0.5 \mathrm{uM}$ treatments with either AZA and RRx-001 were highly significant based on students t-test ( $\mathrm{p}<0.001$ ), but not for DMSO. The addition of $200 \mu \mathrm{g}$ $\mathrm{ml}^{-1}$ fibronectin, an established chemoattractant, resulted in an increase in the baseline percentage invasion, PI, at control and solvent control (mean increase PI $=11$ ) and was maintained across the experimental arms with both compound at all doses. This increase did not however cause a significant change to the invasion index, II, as it affected controls and experimental arms equally. These results are shown in Figure 4.4.6 and Figure 4.4.7. 
Figure 4.4.6. Light microscopy demonstrating haemocytometer counts of invading DLD-1 cells on the underside of the invasion well. Note the 8micron pores in the film. Cells have been fixed with $100 \%$ methanol and crystal violet. Image $\mathbf{a}$ - control; $\mathbf{b}$-control + fibronectin; c-0.25uM AZA; d-0.50uM AZA.

a.

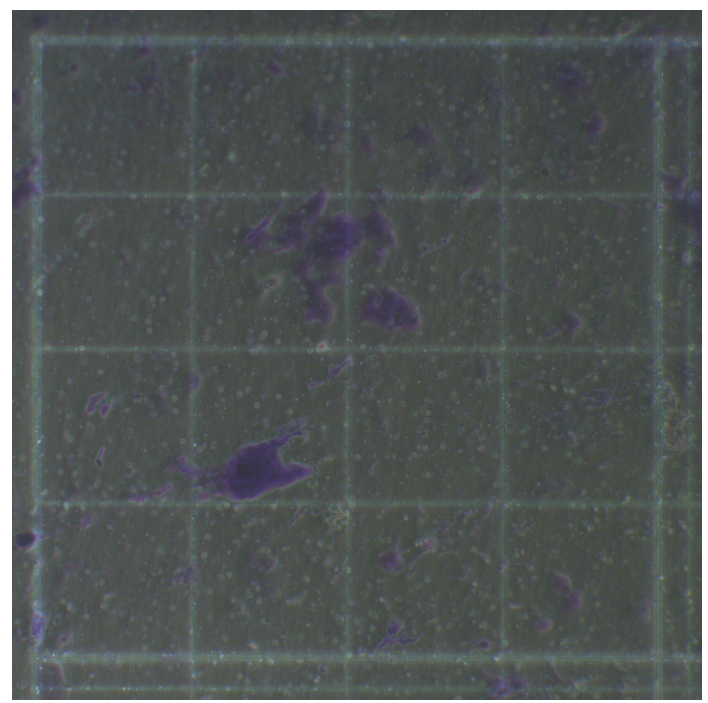

c.

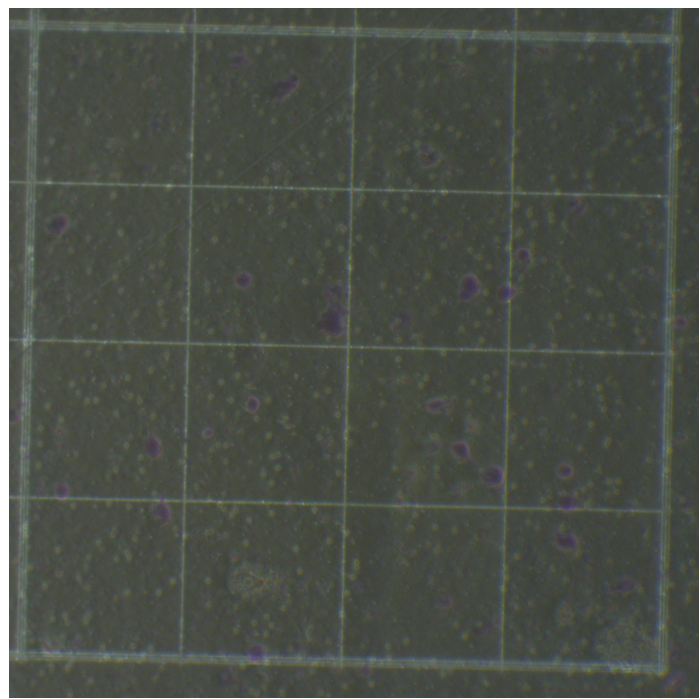

b.

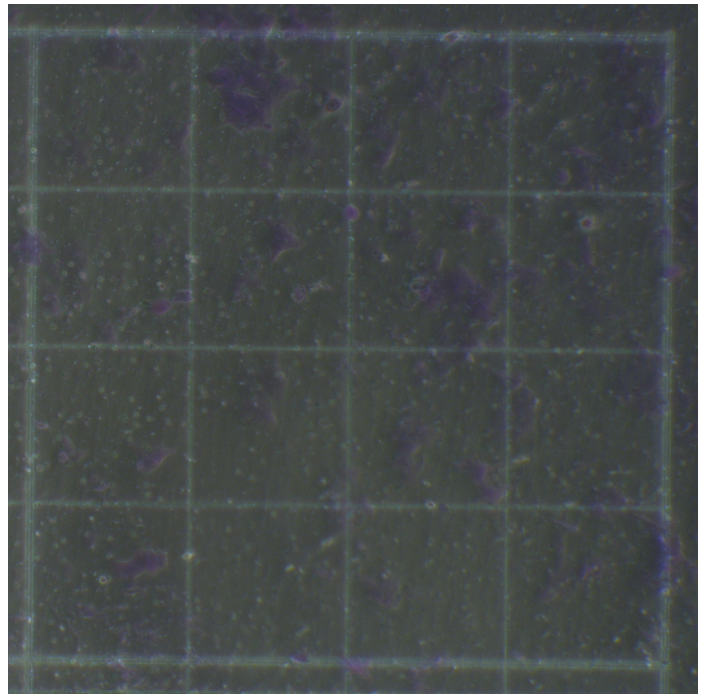

d.

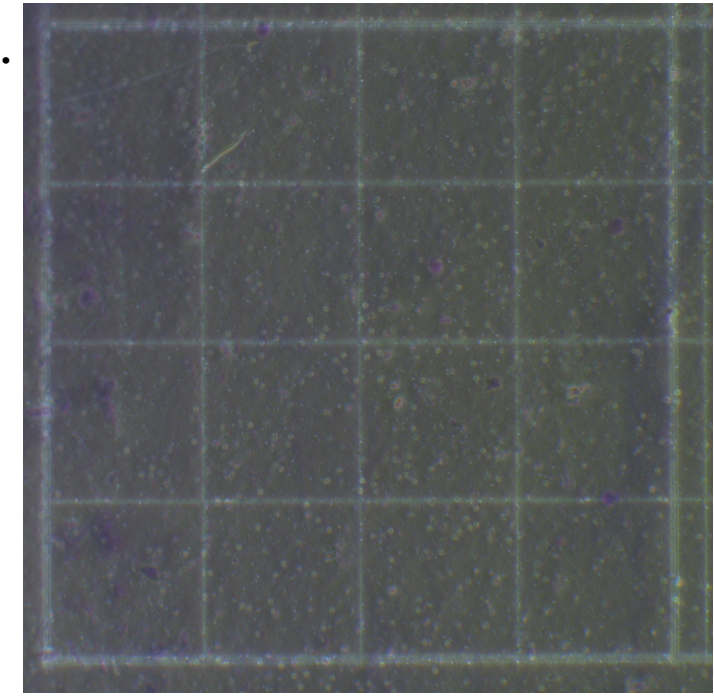


Figure 4.4.7 DLD-1 invasion through Matrigel ECM expressed as Percentage Invasion (PI) and Invasion Index (II).

a. DLD-1 PI \& II versus AZA

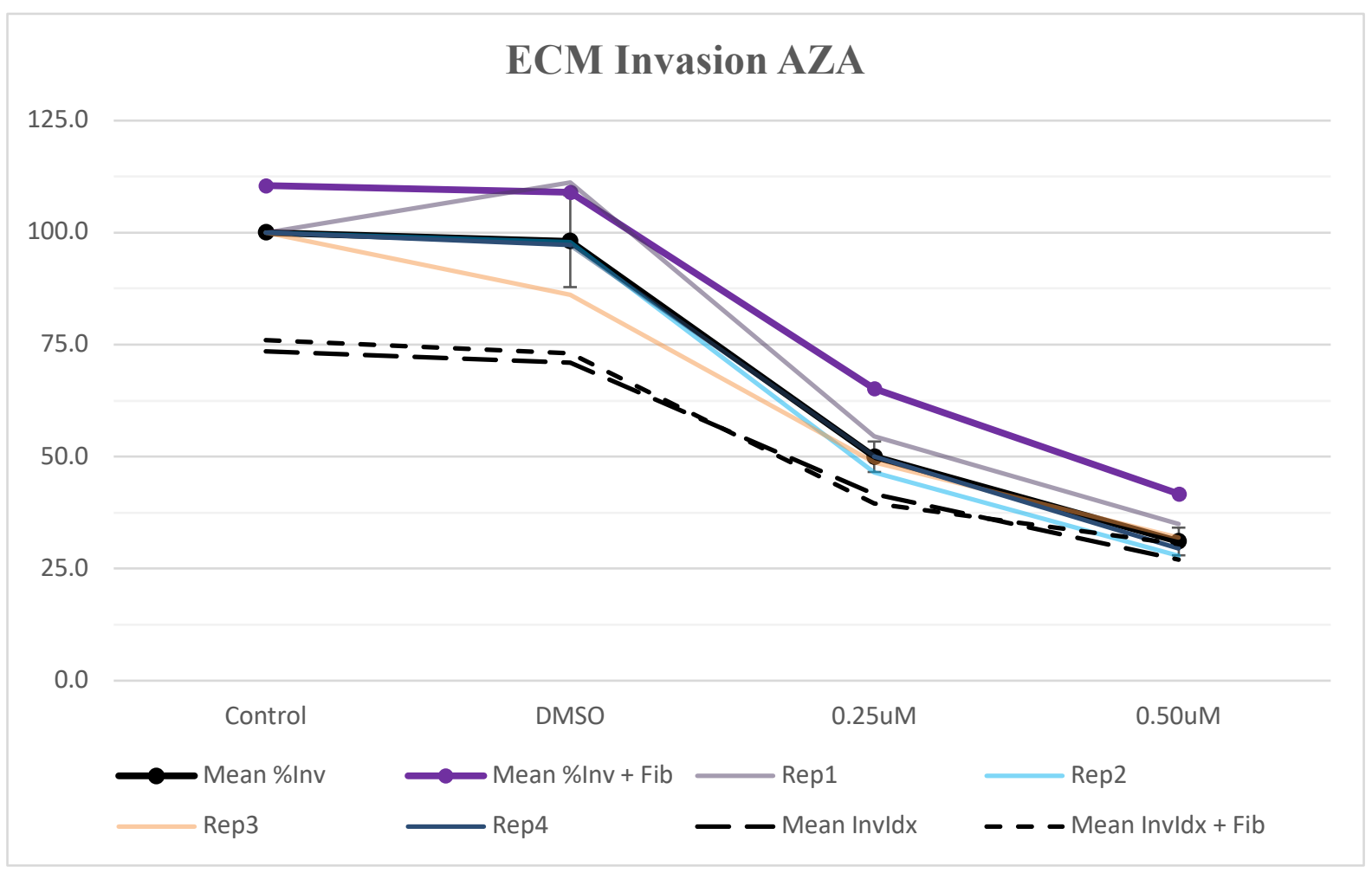

b. DLD-1 PI \& II versus RRx-001

\section{ECM Invasion RRx-001}

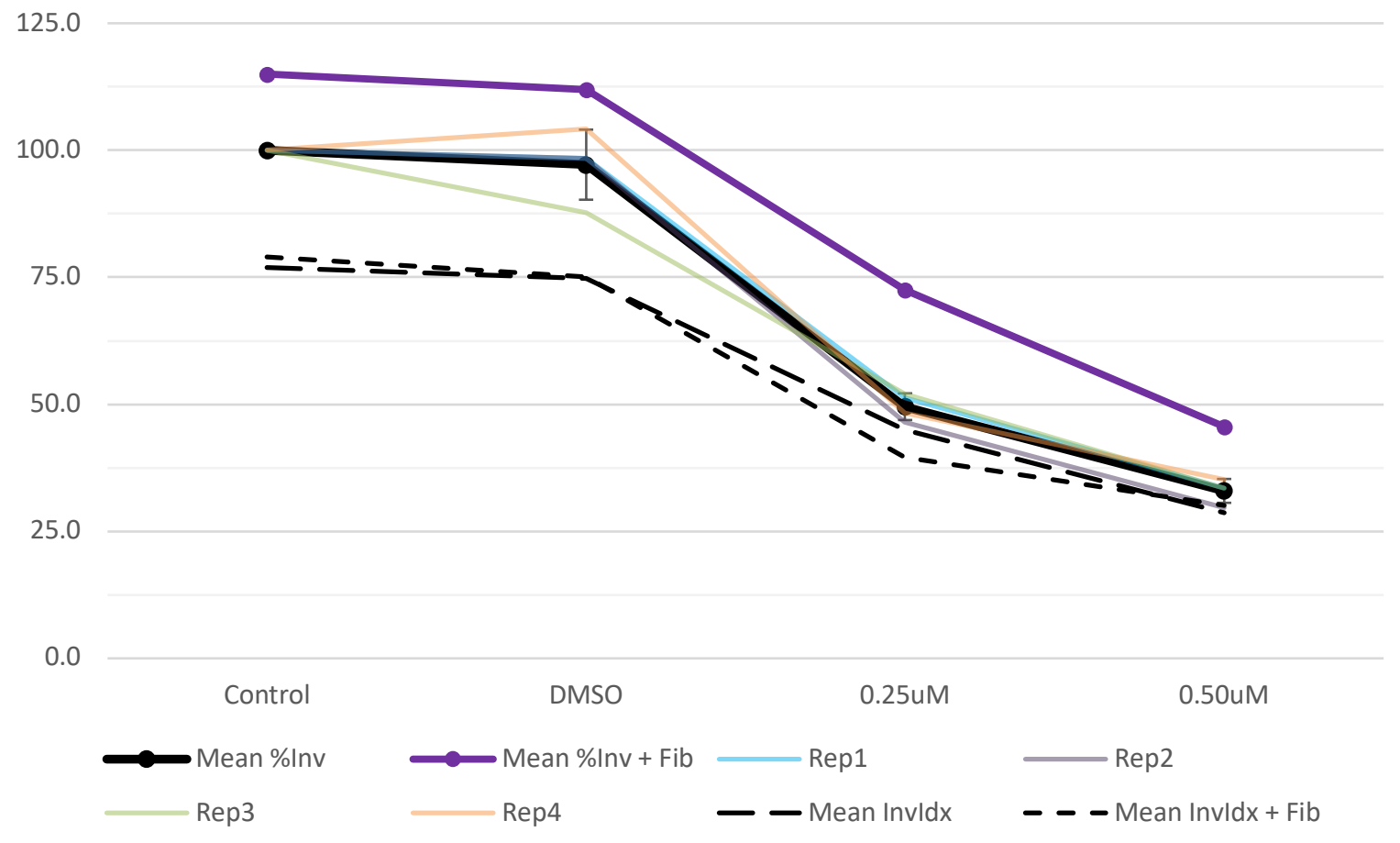


4.4.4. Demethylating Effects of Azacytidine and RRx-001

\subsubsection{CIMP Status}

Methylation status of control, solvent control, and treated DLD-1 cells was assessed by the same methodology of bisulfite-conversion and msPCR as set out in Chapter 3 (Sections 3.3.3, 3.3.4, 3.3.5, and 3.3.6). Cells were first cultured for three days and exposed to AZA 0.50uM or RRx-001 $0.50 \mathrm{uM}$ according to the protocol employed for cytotoxicity and migration / invasion assays in Section 4.3.1 onwards.

The baseline methylation status of DLD-1 cells is pan-methylated at each of the loci employed in the experimental panel, evidenced by strong amplification of DNA by the methylatedspecific primers in the control and solvent control datasets. There was, however, a minor degree of amplification of non-methylated DNA for both SOCS and IGFBP, although this did not reach the 10\% image density saturation (Gel Doc XR (Bio-Rad) system, Quantity One software) threshold for non-anomaly when analysed against the control bands.

Following exposure to $0.50 \mathrm{uM}$ AZA, DLD-1 cell DNA demonstrated significant de-novo demethylation at five out of eight loci; MINT, HAND, ADAMTS1, NEUROG, and THBD. This effect, however, was not universal across the entire cell population, as DNA was extracted which also amplified against the methylated primers, although non-methylated amplification was well above the $10 \%$ threshold, indicating substantial demethylation. The previous patterns of methylation for the other three loci (hMLH, SOCS, and IGFBP) remained unchanged.

Following exposure to $0.50 \mathrm{uM}$ RRx-001, DLD-1 cell DNA demonstrated significant de-novo demethylation at four out of eight loci; MINT, HAND, NEUROG, and THBD. Again, demethylation at these loci was incomplete across the extracted DNA and therefore the cell population, as DNA was also amplified against methylated primers at these loci. Subjectively, the bands representing amplification of unmethylated DNA at the HAND, NEUROG, and THBD loci were weaker than that observed following exposure to AZA, suggesting a lesser degree of demethylation. The methods used in the assay were not, however, quantitative, and thus this result should be considered as qualitative only. The previous patterns of methylation for the other three loci (hMLH, SOCS, ADAMTS1, and IGFBP) remained unchanged. 
According to the epigenotype classification described in Section 1.3.6, DLD-1 cells are CIMP$\mathrm{H}$ at baseline and remain so despite demethylation (with either AZA or RRx-001) of MINT and the Group 2 Markers ${ }^{185}$. The above results are summarised in Table 4.1.

Table 4.1 Methylation status of selected individual genes and CIMP Status of DLD-1 cells after exposure to AZA and RRx-001. $\mathrm{M}=$ methylated, $\mathrm{U}=$ unmethylated, $+=$ strong, $-=$ weak, $\mathrm{H}=$ CIMP High.

\begin{tabular}{|c|c|c|c|c|c|c|c|c|c|c|}
\hline & $\sum_{E}^{ \pm}$ & 点 & ర్ల & 空 & 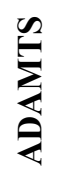 & - & 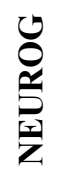 & 产 & $\underline{ }$ & 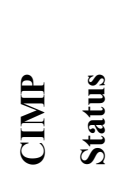 \\
\hline Control & $\mathrm{M}$ & M & M & M & $\mathrm{M}$ & & $\mathrm{M}$ & $\mathrm{M}$ & M & $\mathrm{H}$ \\
\hline AZA & M & $\mathrm{U}$ & M & U- & $\mathrm{U}$ & & $\mathrm{U}$ & M & $\mathrm{U}$ & $\mathrm{H}$ \\
\hline RRx-001 & M & U+ & M & U- & $\mathrm{M}$ & & U- & M & U- & $\mathrm{H}$ \\
\hline
\end{tabular}

\subsubsection{Global Methylation}

The MethylFlash Global DNA Methylation (5-mC) ELISA Easy Kit (Colorimetric) kit measured at 450nm was used to assess global DNA methylation (global 5-mC) in control DLD1 cells and in cells exposed to AZA and RRx-001. As this method allows global quantitative assessment of DNA methylation, a dose curve methodology was applied.

A standard curve for analysis of methylation at $\leq 1 \%$ global 5 -mC was constructed using the controls provided in the kit and a linear regression model, yielding an $\mathrm{R}^{2}=0.8136$, which was improved to $\mathrm{R}^{2}=0.9542$ if the upper limit was set at $\leq 0.5 \%$ global $5-\mathrm{mC}$, and delivering a slope of $b=0.414$ (Figure 4.4.8 a.). This is in accordance with the manufacturer's recommendation for the use of 4 data points $(0 \%, 0.1 \%, 0.2 \%$, and $0.5 \%)$ to attain a slope at the most linear section of the curve.

Both AZA and RRx-001 effectively demethylated DLD-1 cells, as demonstrated in Figure 4.4.8 b. The total percentage of methylated cytosines within DNA of control DLD-1 cells was $0.05676 \%(0.27 \%$ global cytosine - GC), which fell to $0.00008 \%(0.0004 \%$ GC) in cells treated with $0.5 \mathrm{uM}$ AZA and to $0.02093 \%(0.0996 \%$ GC) in cell treated with $0.50 \mathrm{uM} \mathrm{RRx}-001$. Treatment with AZA demonstrated a precipitous demethylation of cytosine, even at very low 
doses, whereas the demethylation demonstrated by RRx-001 was dose-dependent up to $1.0 \mathrm{uM}$. Above doses of $>1.0 \mathrm{uM}$ of both agents a paradoxical rebound in global 5-mC was observed.

Figure 4.4.9 also demonstrates the global demethylation produced by both AZA and RRx-001 at a macro level by showing the ELISA plate colorimetric output. Note the both the precipitous decline in the proportion of 5-mC with AZA, and the dose dependent relationship with RRx001 , as well as the paradoxical increase in $5-\mathrm{mC}$ at the $1.5 \mathrm{uM}$ dose of each agent.

Figure 4.4.8 a. Standard curve for analysis of global 5-mC ELISA \& b. global methylation in DLD-1 cells, expressed as percentage of methylated cytosine.

a.

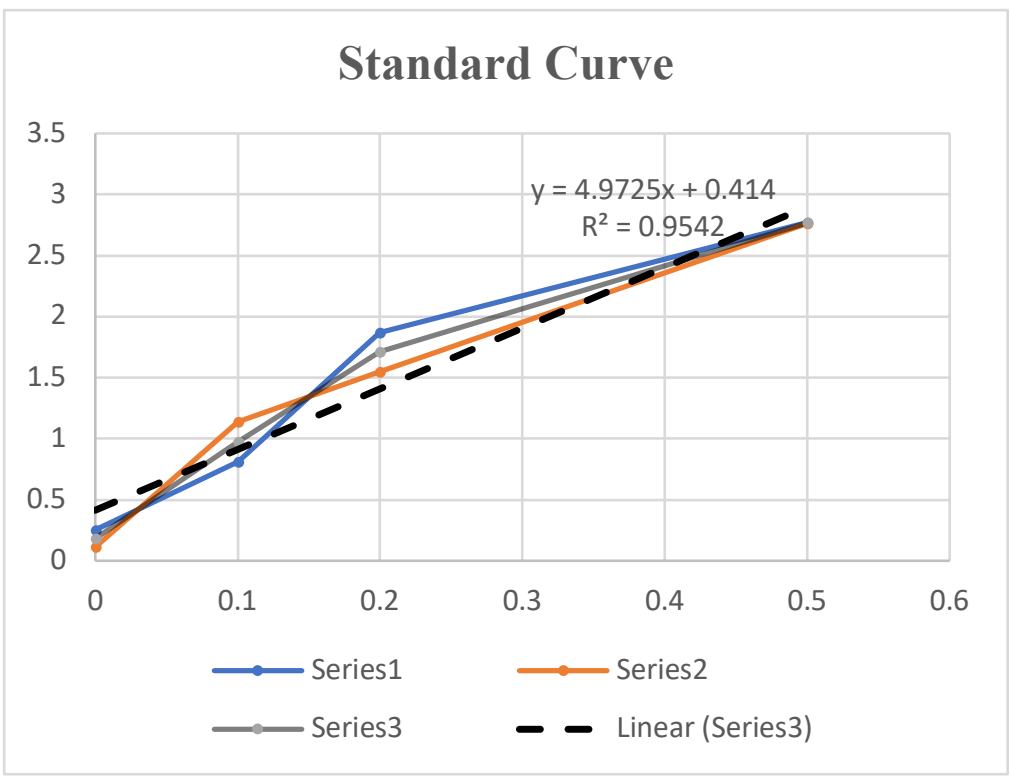

b.

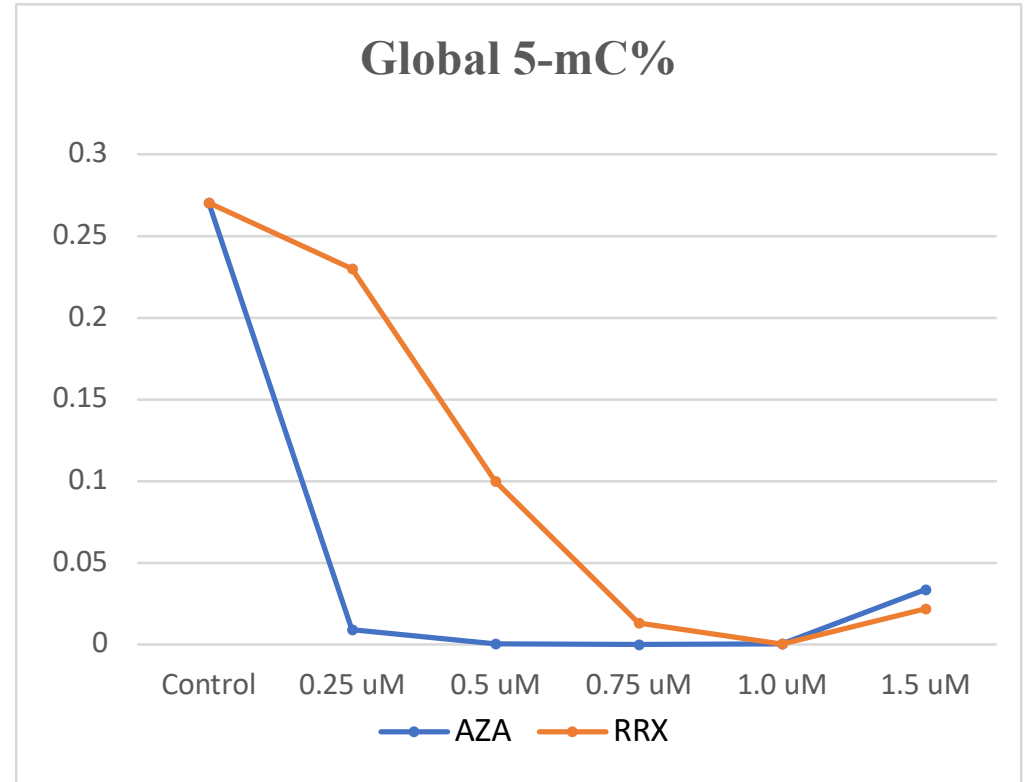


Figure 4.4.9 Resolved MethylFlash Global DNA Methylation (5-mC) ELISA plate demonstrating differential demethylation with AZA and RRx-001. Yellow = methylation.

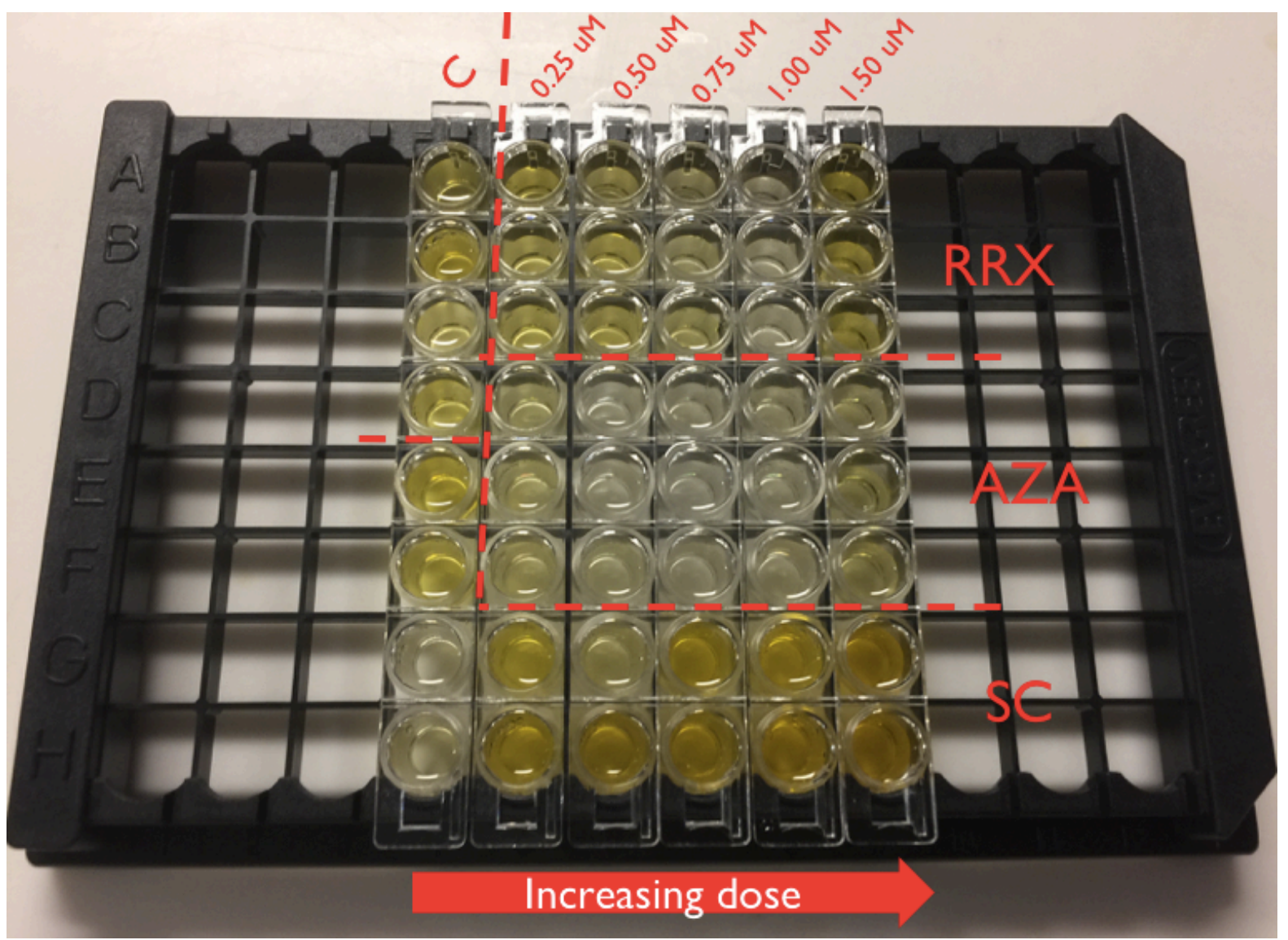




\subsection{Discussion}

This chapter's aims were to examine the effects of demethylation on a colorectal cell line in vitro, and to set parameters for the further investigation of demethylating agents in a model of colorectal cancer epithelial-mesenchymal and mesenchymal-epithelial transition (EMT/MET). To this end, the first element of this chapter was to establish the baseline cytotoxicity of AZA and RRx-001 in DLD-1 cells so that any effects observed in subsequent experiments cannot be confounded by toxicity. The second element of this chapter was to examine the in vitro biologic effects of AZA and RRx-001 at sub-cytotoxic doses on DLD-1 cells in models of EMT \& MET. The third element was to establish the demethylating effects of AZA and RRx001 at sub-cytotoxic and biologically effective doses, both in terms of the locus-specific methylation status employed by the Kaneda \& Yagi CIMP panel, and global methylation ${ }^{185}$. By these means the overarching aim was to establish a three-way-look at the investigation of an association between the methylation status of DLD-1 cells the biologic activity that is dependent on their methylation status.

\subsubsection{Cytotoxicity of Azacytidine and RRx-001}

An appropriate upper-limit for cytotoxicity is important when selecting doses of potentially biologically active or mutagenic compounds since excessive cytotoxicity is a well-established source of misleading positive results ${ }^{439}, 450$. Population-doubling (RPD) is advocated by many authors as a reliable method of excluding cytotoxicity as a result of disruption to off-target cellular physiological processes and is supported by the OECD and other regulatory authorities ${ }^{437,438,451}$. Based on this guidance an upper limit of 55 $\pm 5 \%$ RPD was set as a cutoff for both AZA and RRx-001; doses above this being considered cytotoxic and doses below being considered non-cytotoxic. Exposure times were informed partly on the basis of prior experimentation within our own department, and also in accordance with other authors' protocols ${ }^{452}$. For both compounds, $55 \pm 5 \%$ RPD was reached between $0.50 \mathrm{uM}$ and $1.00 \mathrm{uM}$, with $0.50 \mathrm{uM}$ of AZA and RRx-001 being considered as a reliably sub-cytotoxic dose (see Section 4.4.1 for full results). These finding are broadly in keeping with those previously published by other authors investigating the in vitro effects of AZA on a variety of cell lines, although both higher and lower concentration tolerances have been reported ${ }^{209}, 453$. Interestingly, Juttermann and colleagues suggest that the cytotoxic effects of AZA observed at higher concentrations ( $\geq 0.50 \mathrm{uM}$ in their investigation) are caused by an irreversible covalent trapping of DNMT to DNA, rather than the effects of demethylation itself, underlining the significance of establishing sub-cytotoxicity when investigating the specific effects of 
demethyltion ${ }^{213}$. As RRx-001 is a novel compound, there is little published evidence on its biologic effects and cytotoxicity in vitro, although doses of between $0.5 \mathrm{uM}$ and 5.0uM have been found to be sub-cytotoxic (and demethylating) in squamous cell carcinoma (SSC VII) cells $^{243}$. Furthermore, the same study examined the comparative global demethylating effects of RRx-001 against AZA at doses of between $0.5 \mathrm{uM}$ and $2.0 \mathrm{uM}$, finding a comparative epigenetic effect. This is consistent with another in vitro study, where RRx-001 was found to modulate DNMT1 and DNMT3a activity by increasing free oxygen and nitrogen radicals, reducing the abundance of methylation substrates and thus causing demethylation ${ }^{454}$. The establishment of sub-cytotoxic doses of both AZA and RRx-001 at $\leq 0.50 \mathrm{uM}$ concentrations was also supported by the favourable results of the trypan blue assays. Furthermore, as RPD is a more sensitive measure of early toxicity, effects observed inviable cells following protracted treatment are likely to represent biologic effects rather than toxicity.

\subsubsection{The in vitro biologic effects of AZA and RRx-001}

Wound healing (scratch) and Boyden chamber-type assays are established methods of investigating cell migration and invasion ${ }^{419,420}$. Increasing elements of complexity may be added to the basic experimental design to create cellular environments more comparable to those that are key in the EMT and MET processes ${ }^{422,423}$. The results of the scratch assay (Section 4.4.2) demonstrated that, at sub-cytotoxic doses, both AZA and RRx-001 significantly increased the time taken for DLD-1 cell to migrate across the cell-free zone $(p<0.001$ for both compounds). Similarly, AZA and RRx-001 at the same sub-cytotoxic doses reduced the ability of DLD-1 cells to invade through the ECM and migrate through the porous membrane of the Transwell ${ }^{\circledR}$ Boyden chamber system ( $\mathrm{p}<0.001$ for both compounds) (Section 4.4.3). These results suggest that both compounds reduce the migratory and invasive propensity of DLD-1 cells in in vitro models of EMT and MET, therefore suggesting that hyper-methylation could increase motility and invasion in the carcinogenic process.

The results of this section are in keeping with the findings of other investigators, who have reported that other colorectal cell lines (HCT-116) were less able to migrate across and invade through a control and ECM treated Transwell system, respectively, following treatment with $\mathrm{AZA}^{452}$. Another similar study, this time employing different colorectal cancer cell lines (SW480 and SW620) and decitabine (an AZA derivative), also demonstrated a reduced propensity for CRC cell to migrate and invade following treatment with a demethylating agent ${ }^{455}$. Despite the different cell types and demethylating agents used in these studies, the 
alignment with the findings from this investigation suggest a consistency in the application of demethylating agents to CRC cell lines and the reduction in the ability of cells to invade. This is particularly interesting when considering the EMT/MET process, especially in the case of SW620 which is a lymph node metastasis derived colorectal cancer cell line.

Similar findings have also been reported for other tumour types, including oesophageal squamous cell carcinomas and adenocarcinomas, renal cell carcinomas, gastric carcinomas, and laryngeal squamous carcinomas ${ }^{456-459}$. In a study of multiple oesophageal tumour cell types, treatment with AZA (and/or decitabine) resulted in a reduced ability of tumour cells to migrate and invade, but only when dual therapy with other epigenetic modifiers was applied; specifically, histone deacetylases (HDACs) ${ }^{456}$. These findings were echoed in another study examining the effects of dual treatment with the HDAC valproic acid in conjunction with AZA on renal cell carcinomas (786-O and 769-P) in vitro ${ }^{457}$. These studies suggest that there is a synergistic effect between demethylating agents such as AZA with other epigenetic modifiers, and that the biological effects may only be observed when the two are applied in tandem, counter to the findings in this chapter. However, as well as the previously discussed studies on colorectal cancer cell lines, other authors have also found that monotherapy with AZA effectively reduces the ability of cancer cell lines to migrate and invade. This is demonstrated by the study on gastric cancer (AGS cells) by Zuo and colleagues, who demonstrated reduced migration and invasion with AZA monotherapy ${ }^{458}$. Lui also found that AZA monotherapy reduced the ability of laryngeal squamous cells (HEp-2) to migrate and invade, based on scratch and Transwell assays ${ }^{459}$.

No previous study has examined the biologic effects of RRx-001 in terms of the propensity of treated cells to migrate or invade. The results in this chapter indicate that, at sub-cytotoxic doses, RRx-001 significantly reduces the ability of DLD-1 cells to migrate in two-dimensions in a wound healing assay, and to invade in three-dimensions through the ECM components of Matrigel in a Transwell Boyden-chamber assay. This was demonstrated by the reduced percentage invasion (PI) and invasion index (II) with increasing doses of RRx-001 when compared to control and was statistically significant $(\mathrm{p}<0.001)$. The reduction in invasion was comparable to that observed with the same doses of AZA. The addition of fibronectin to the bottom chamber increased the percentage invasion by $10-15 \%$ at each dose-point for both AZA and RRx-001 but did not increase the II as it also proportionately increased the denominator. 


\subsubsection{Assessment of Methylation Status}

At baseline, DLD-1 cell were found to be CIMP-high based upon the two-panel classification system described in by Kaneda and Yagi, and summarised in Section 1.3.6 ${ }^{185}$. Each of the eight loci was found to be methylated; each demonstrating strong amplification at the specific $\mathrm{CpG}$ islands against the methylation specific-primers. A minor degree of amplification was evident for both SOCS and IGFBP, although this did not reach the $10 \%$ threshold for significant heterogeneity.

Following treatment with AZA at $0.50 \mathrm{uM}$ concentration, DLD-1 demonstrated significant but incomplete demethylation of five out of eight loci; MINT, HAND, ADAMTS1, NEUROG, and THBD. Although incomplete, demethylation of these loci was significant as the demethylated bands for these sites was stronger than the methylated bands, which is converse to the baseline methylation status. The finding that AZA is an effective demethylator of human DNA is not unexpected, as it is a well-established demethylating agent with widespread laboratory and some clinical applications (see Section 2.2.3 of General Methodology). The previous patterns of methylation for the other three loci (hMLH, SOCS, and IGFBP) remained unchanged. Despite demethylation at five of the eight loci, the CIMP status of DLD-1 cells exposed to AZA remains CIMP-high, as two of the Group 1 markers remain methylated (hMLH and SOCS).

AZA also produced a dramatic decline in global $5-\mathrm{mC}$, as demonstrated by the ELISA. At $0.50 \mathrm{uM}$ dose of AZA, global 5-mC was reduced by a factor of over 700 , from $0.05676 \%$ of global DNA to $0.00008 \%$. The figure of just over a twentieth of $1 \%$ for baseline $5-\mathrm{mC}$ is objectively very low, with expected levels of methylated cytosine in human DNA typically being approximately $1 \%$, dependent on the tissue $e^{460,461}$. Indeed, Donoghue reports $62 \%$ methylation at CCGG sites throughout DLD-1 DNA, suggesting a much higher percentage of global 5-mC $\mathrm{m}^{462}$. These findings do not however take account of the stochastic variations in DNA methylation observed in cancer cells, or the acknowledged pan-hypomethylation across the entire epigenome despite the locus-specific hypermethylation observed in CIMP-high tumours ${ }^{463}$. Additionally, although there are multiple methods of determining global DNA methylation, the ELISA-based method employed in this chapter is considered reliable and validated against human and non-human DNA and in a variety of disease states, including cancers $^{448,464,465}$. Despite the low absolute figures, what is clear from the global 5-mC ELISA 
is that the relative proportion of methylated cytosine has fallen dramatically following AZA treatment, and was controlled against a well-fitting standard curve $\left(\mathrm{R}^{2}=0.9542\right)$.

Following treatment with $0.50 \mathrm{uM} \mathrm{RRx}-001$, four of eight loci were effectively demethylated; MINT, HAND, NEUROG, and THBD. Patterns of methylation at the remaining four loci remained unchanged. As was the case with demethylation with AZA, the CIMP status of DLD1 cells treated with RRx-001 did not change, remaining HIMP-high, as hMLH and SOCS were again resistant to demethylation. Although essentially a binary test, subjectively the demethylated bands observed for HAND, NEUROG, and THBD were weaker than they were for AZA, suggesting a less efficient demethylation at these loci at an equal dose. Note that the methodology employed is not quantitative, and thus this is a qualitative observation.

This finding of potentially less efficient demethylation fits with the findings of the ELISA, where although RRx-001 did produce a demonstrable global decline in the proportion of 5$\mathrm{mC}$, it was not such a precipitous drop as that observed with AZA. At 0.50uM RRx-001 demethylation was only approximately half that of observed with the same dose of AZA, and equivalence was only reached at a dose of $0.75 \mathrm{uM}$ RRx-001 (just above the cytotoxic threshold). This is consistent with a previous in vitro study where RRx-001 was found to modulate DNMT1 and DNMT3a activity by increasing free oxygen and nitrogen radicals, thus reducing the abundance of methylation substrates in a dose dependent but dynamic manner ${ }^{454}$. This is opposed to the permanent sequestration of DNMT1 and thus failure of de-novo methylation during cell division brought about by AZA.

The observation that high doses of either compound cause a paradoxical rebound in global methylation must disregarded as an anomaly, primarily because these doses ( $>1.0 \mathrm{uM}$ AZA and RRx-001) are well above the cytotoxic threshold. Additionally, because the level of methylation that might be expected if the agents are acting in a targeted manner would be so low, the efficiency and therefore results of the ELISA cannot be relied upon. Furthermore, there may also be direct chemical interference with the colorimetric assay at higher doses, particularly for free-radical generating RRx-001. 


\subsection{Conclusions}

The results of this chapter demonstrate that DLD-1 colorectal cancer cells are CIMP-high at baseline, despite the absolute proportion of 5-mC being lower than expected based on the ELISA employed. Both AZA and RRx-001 were found to be effective demethylators of DLD1 cells, both at locus specific CpG islands that form the CIMP classification system and at a global methylation level. AZA was a much more efficient demethylator than RRx-001 based on ELISA, but both agents effectively demethylated at least half of the loci associated with CIMP status; AZA demethylating 5/8 loci, RRx-001 4/8 loci, based on methylation-specific PCR. Despite locus specific demethylation, neither agent changed the CIMP status of DLD-1 cells as 2/3 Group 1 genes remained methylated (hMLH and SOCS).

When DLD-1 cells were exposed to the same doses of AZA and RRx-001 that resulted in effective demethylation, their ability to migrate in two-dimensions and invade in threedimensions through and ECM-like substrate were significantly restricted. These effects occurred at doses that had previously been demonstrated to be sub-cytotoxic. We can therefore infer that demethylation results in a reduced ability of DLD-1 cells to migrate and invade in a manner akin to the EMT and MET processes of in vivo colorectal cancers, and that this pathological process may be driven by aberrant locus specific hypermethylation. The next chapter of this thesis will therefore investigate locus specific hypermethylation as a potential epigenetic mechanism underlying the EMT/MET of colorectal cancer. 


\section{Chapter 5}

\section{Methylation Dependent Gene Expression and the Epithelial-Mesenchymal \& Mesenchymal-Epithelial Transition.}

\subsection{Introduction}

\subsubsection{The Epithelial-Mesenchymal \& Mesenchymal-Epithelial Transitions}

The epithelial to mesenchymal transition (EMT) is a normal physiological process whereby cells of the epithelium undergo multiple phenotypic changes to become mesenchymal-type cells; typically losing their polarity and normal cell-cell adhesions and thus allowing them to become migratory and invasive ${ }^{466,467}$. During embryological development this process is key to normal tissue differentiation and organ development, and malfunction in this process can lead to abortion or congenital abnormality ${ }^{407}$. Cells that have undergone EMT are also more resistant to apoptosis and senescence and have a role in localised immunosuppression. EMT is also an important process in wound healing and tissue fibrosis outside of embryological development, and dysfunction may underlie pathological healing (scarring) in adult tissues ${ }^{468}$. The reverse process, mesenchymal to epithelial transition (MET), is also a fundamental normal physiological process that describes the migration and terminal differentiation of mesenchymal cells to polarised epithelial cells ${ }^{469}$. Cells undergoing EMT or MET progress through a number of stages characterised by differential expression of intracellular proteins, cytoskeletal changes, and expression of cell-surface receptors and junctional complexes (Figure 5.1). These phenomena are also observed in carcinoma, and thus the normal processes of EMT and MET are thought to be integral to the development of cancers ${ }^{470,471}$. This is particularly important when considering the processes necessary for tumours of epithelial cell origin to first become invasive through the basement membrane, to penetrate vasculature and lymphatics, and then to seed as metastasis; as these stages of tumour progression share many similarities with the EMT and MET process ${ }^{467,472,473}$. 
Figure 5.1.1 The Epithelial-Mesenchymal Transition (adapted from Klymkowsky \& Savagner) ${ }^{474}$.

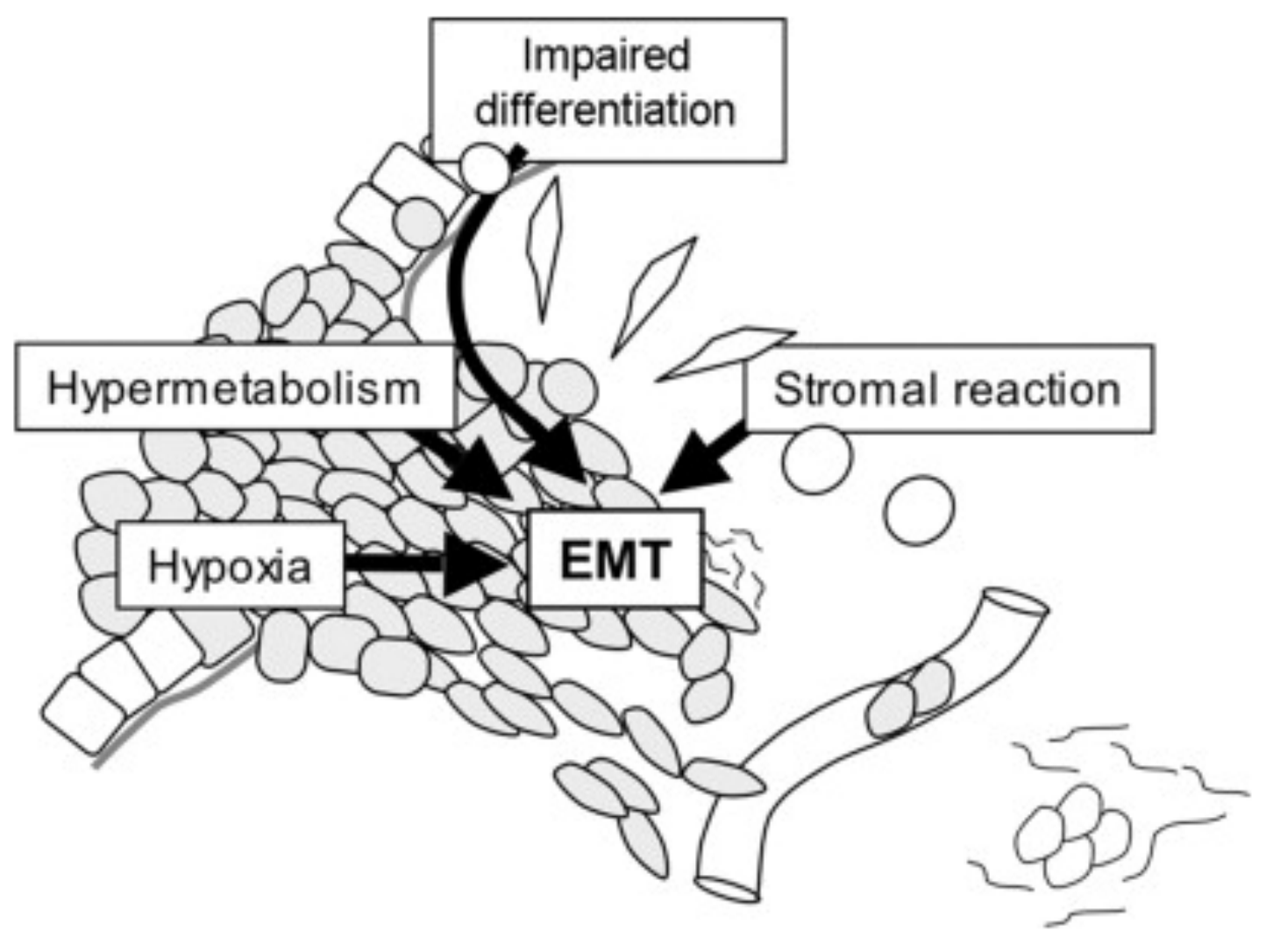

When considering EMT-like progression in early cancers, including CRC, it is important to recognise that the process of tumourigenesis shows plasticity from a cellular and heterogeneous from a population perspective ${ }^{470}$. Based on a model of early skin squamous cell carcinoma, Pastushenko and colleagues describe how induced EMT-like tumourigenesis resulted in a significant increase in the heterogeneity of expressed cell surface markers. By characterising these markers as either epithelial-associated of mesenchymal-associated the degree of EMTprogress was able to be characterised, revealing sub-populations of tumour cells displaying differing degrees of transition, described as degrees of stemness. This concept has been previously reported by authors when investigating the heterogeneity of early invasive tumours and the observation that some cells undergoing an EMT-like transformation are able to transit between different states ${ }^{475}$. Even when tumours have become well established (by size criteria), solid-type (non-diffuse) tumours, such as colorectal adenocarcinoma, display localised heterogeneity within the tumour mass; cells at the invasive front displaying phenotypic differentiation to those at the centre of the tumour mass ${ }^{476}$. This is also evident at a genetic and epigenetic level ${ }^{477}$. 
Due to the levels of observed heterogeneity, some authors have advocated a classification system for the degree of EMT-like phenotypes in carcinoma based on characteristic such as cell polarity, cell-cell adhesion, loss of keratin expression, and upregulation of vimentin ${ }^{474}$. This system is based on EMT-like phenotypic changes that are conserved across different tumour types (including colorectal adenocarcinoma, lobular breast carcinoma, melanoma, and sarcomas) and may serve as an exercise in classifying tumour from an academic perspective. However, there is currently no clinical application for this system, and no clinicopathological correlation has been attempted, although the conservation of some phenotypic features serves as a starting point for understanding some of the genetic and cellular events that underlie the process.

In healthy tissues, the adhesion between epithelial cells is predominated by E-cadherin, which is itself linked to the actin component of the cytoskeleton via its intracellular domain and associated proteins $\beta$ - \& $\alpha$-catenin. During physiological and cancer-related EMT, loss of Ecadherin cell-cell interaction is regarded as a critical step, and E-cadherin as a "master regulator" of the loss-of-adhesion component of the EMT process ${ }^{472}$. Normal regulation of Ecadherin relies on a highly complex set of interactions between canonical intracellular signalling pathways, although $\beta$-catenin, RAS, and src are thought to play a central role ${ }^{478}$. As previously discussed in Section 1.3.2, activation of the APC/ $\beta$-catenin pathway through mutation and pathological RAS signalling are regarded as key early steps in the classical adenoma-carcinoma pathway of $\mathrm{CRC}$, and may result in loss of E-cadherin function. Of note, very few cancers are thought to originate with a primary mutation in E-cadherin itself, although some diffuse-type cancers (notably lobular breast carcinoma) have been associated with heterogeneous methylation of $\mathrm{CpG}$ island associated with E-cadherin gene $\mathrm{CDH} 1$ on the long arm of chromosome $16^{479}$.

As well as loss of E-cadherin, EMT/invasive changes are induced in response to growth factor signalling, specifically at receptor tyrosine kinases in response to insulin-like growth factor (IGF) or transforming-growth factor $\beta$ (TGF- $\beta)^{480}$. Response to IGF is thought to dominate the normal EMT process by activation of the RAS/MAPK, PIK3AK/AKT, and src pathways, although pathological carcinoma invasion in response to IGF is primarily mediated through a $\beta$-catenin pathway. As well as disrupting cell-cell adhesion by effects on E-cadherin, IGF-II is able to induce EMT by upregulation of $\beta$-catenin/TCF-3 target genes including cyclin-D1 and c-myc; which themselves are a cell-cycle protein important at G1 (associated with VEGF 
mediated angiogenesis in tumours) and a transcription factor (proto-oncogene associated with cell proliferation in cancers), respectively ${ }^{481}$. TGF- $\beta$ s are regulatory growth factors secreted by stromal cells and are essential to healthy EMT (intrauterine global loss of TGF- $\beta$ s is fatal) and contribute to normal crypt architecture in the small bowel and colon ${ }^{482}$. In healthy colon the TGF- $\beta$ family of signalling ligands normally act on epithelial cells to suppress cell proliferation and tumour development via their effects on SMAD expression and reciprocal $\beta$ catenin suppression, and by the activation of cyclin-dependent kinase inhibitors ${ }^{483}$. However, in the context of early tumours undergoing and EMT-like progression, TGF- $\beta$ s can act conversely to suppress the stromal anti-tumoural inflammatory response mediated by immune cells, thus facilitating invasion ${ }^{484,485}$.

During EMT-like invasion, cancerous cells must change their cytoskeletal structure to facilitate migration through the ECM. Although also integral to the E-cadherin regulated cell-cell adhesion, cytoskeletal components such as cytokeratins (intermediate filaments: IF) are critical to cell division \& migration during EMT and are highly conserved across tissue and tumour types $^{486,487}$. The IFs themselves are a huge family of highly conserved proteins that are differentially expressed in different tissue types and are typically sub-divided into groups. Groups I and II are typically found in epithelial cells and the expression of specific cytokeratins in this group has aided in the characterisation of cancers, especially when identifying the source of metastasis ${ }^{488}$. However, group III IFs such as desmin and vimentin are more typically expressed in stromal cells such as fibroblasts but have been found to be over-expressed in a variety of epithelial-type cancers such as bladder and colorectal cancer, particularly following metastasis 489,490 . The increased expression of stromal cytoskeletal proteins is another indication that cells undergoing malignant transformation initially progress through an EMTlike process. In colorectal cancer, EMT-like induction of cytoskeletal changes is again primarily mediated via the TGF- $\beta$ pathway and is downstream effects on the SMAD3 and SMAD4 transcription factors, and the wnt/ $\beta$-catenin pathway and downstream effects on SLUG and SNAIL ${ }^{490}$. Despite increases in expression of group III IFs in epithelial cancers, the use of proteins such as vimentin as biomarkers in colorectal cancer had not been adopted, largely due to poor sensitivity during early disease stages and lack of superiority in stage IV disease over traditional markers such as $\mathrm{CEA}^{491,492}$. 
As well as loss of normal cell-cell adhesion and changes to the cytoskeleton, cells undergoing physiological and pathological EMT demonstrate loss of normal cell polarity. Cell polarity is normally maintained by polarity regulator complexes (PRCs) such as Bazooka, Crumbs, and Scribble, which are not only highly conserved across cell types but also between species ${ }^{493}$. As well as maintaining basal-apical polarity, these complexes also have an important role in cell migration and proliferation, both in EMT and in carcinogenesis. Under normal physiological conditions, polarity is maintained by the interaction of PRCs with components of the basement membrane and stroma such as laminin, collagens I-IV, and other integrins. Changes to the cellular microenvironment can, however, induce EMT in a number of epithelial call types in a process mirrored by early cancer transformation ${ }^{494}$. This is demonstrated by the observation that blockade of normal $\beta 1$ integrin binding stimulates expression of the malignant phenotype in some pre-malignant epithelial cancers ${ }^{495}$. In colorectal cancer, PRC apical protein kinase C (aPKC) may be found to be over-expressed as a result of RAS mutation, and has been found to be a critical factor in regulating EMT ${ }^{496,497}$. The relationship between aPKC over-expression and dysfunction of PRCs such as Scribble seem to be key to this element of early cancer development, and may act via a common pathway of ErbB2 transcription and subsequent HER2 expression, leading to downstream effects on MAPK and PIK3AK signal transduction pathways ${ }^{498}$. The central role of HER2 in CRC may be reflected in HER2 expression being a factor in predicting loss of treatment response to anti-EGFR therapies, and may itself be a future therapeutic target ${ }^{499}$.

The reverse process of MET is less well characterised than EMT, and the genetic and cellular events that underlie the observed phenotypic changes are not well understood. It should also be noted that when considering healthy physiological MET the original mesenchymal cells become terminally differentiated epithelial cells, whereas the cells of a tumour metastasis remain abnormally differentiated ${ }^{500}$. There is, however, an acceptance that tumour cells that have successfully metastasised have adhered to and penetrated the host tissue vascular or lymphatic wall, migrated to the parenchyma, and seeded in their metastatic niche, after which they must proliferate to form a metastasis. The limiting factor in this process is deemed to be the ability of the micrometastasis to induce angiogenesis (Figure 5.2) ${ }^{501}$. This phenotypic change may occur after a long period of tumour cell dormancy, sometimes many years after the primary tumour has been treated. The complexity of this process perhaps underlies the estimation that only $0.01 \%$ of circulating tumour cells form a secondary tumour ${ }^{502}$. 
Figure 5.1.2 The Metastatic Process (adapted from Chambers, et.al. $)^{502}$.

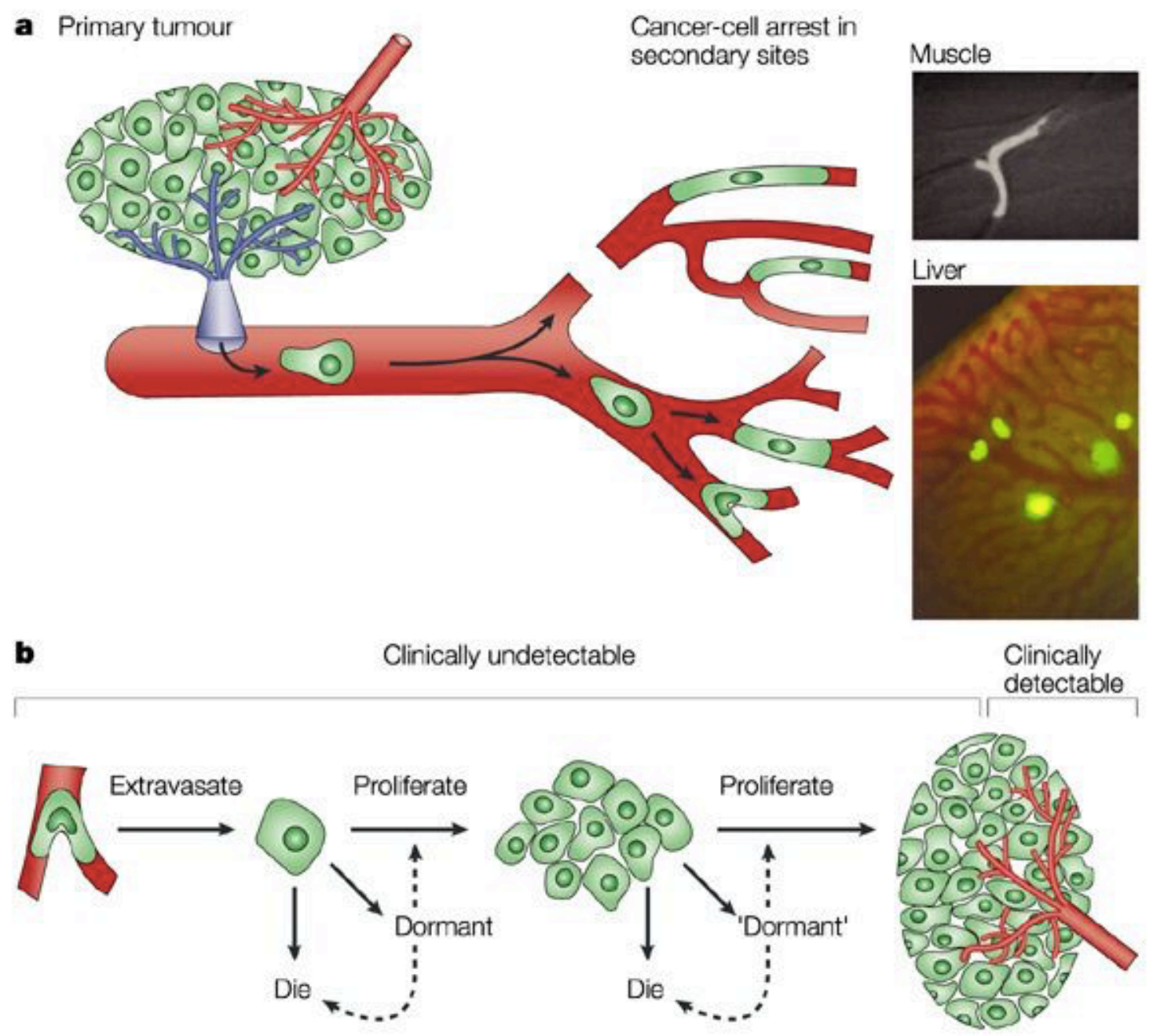

Once epithelial cells have undergone the EMT-like process of loss of normal cell-cell adhesion, loss of polarity, and changed their cytoskeletal architecture they are more able to negotiate the basement membrane and stromal layers. Fundamentally this is the process that facilitates colorectal cancers to progress form tumourous cells in situ (Tis) to more advanced tumours of the bowel wall (T1-4), to invade blood vessels and lymphatic, and eventually metastasise to local lymph nodes and more distant sites. Another requirement for tumours progress on this pathway is the ability to degrade the extracellular matrix. 


\subsubsection{Metalloproteinases \& Cancer}

The metalloproteinases (MMPs) are a large group of proteolytic enzymes capable of degrading all of the protein components of the extracellular matrix (ECM), as well as some growth factors. This large family is sub-divided into groups dependent on the preferred target substrate of each MMP, such as the collagenases (MMP1, $-8,-13$ ) or the gelatinases (MMP2, -9) ${ }^{503}$. Throughout the body of scientific literature, MMPs are referred to variably by their MMP assignment, their substrate assignment, or common names, leading to significant potential for confusion. One such example is collagen-IV-specific MMP2, which is variably also known as Gelatinase-A or $72 \mathrm{kDa}$ Type-IV collagenase. For the purpose of this thesis each MMP will be referred to by its MMP assignment (e.g. MMP2, MMP9, etc). MMPs are summarised in Figure 5.3. Most MMPs are released into the ECM, often in the form of an inactive pro-enzyme (zymogen), after which they are activated and free to act upon the surrounding substrate. However, some subgroups of MMP can be found attached to the cell surface (the GPI-anchored MMPs) or as transmembrane proteins (the transmembrane-type MMPs) ${ }^{504}$.

Figure 5.1.3 Matrix Metalloproteinases (MMPs) (adapted from Stamenkovic) ${ }^{505}$.

\begin{tabular}{|c|c|c|c|c|c|}
\hline MMP\# & Common name & $\operatorname{Mr}(\mathrm{kDa})$ & Substrates & Cell surface & Docking mechanism \\
\hline 1 & $\begin{array}{l}\text { interstitial collagenase, } \\
\text { collagenase-1 }\end{array}$ & $52 / 43$ & $\begin{array}{l}\text { collagen I, II, III, VII, } \\
\text { X, IGFBP }\end{array}$ & yes & EMMPRIN (CD147) \\
\hline 2 & gelatinase $\mathrm{A}$ & $72 / 62$ & $\begin{array}{l}\text { gelatin, collagen I, IV } \\
\mathrm{V}, \mathrm{X} \text {, laminin, IGFBP } \\
\text { latent TGF- } \beta\end{array}$ & yes & $\begin{array}{l}\text { MT1-MMP/TIMP-2 } \\
\alpha \nu \beta 3 \text { integrin }\end{array}$ \\
\hline 3 & stromelysin-1 & $52 / 43$ & $\begin{array}{l}\text { collagen III, IV, V, IX } \\
\text { X, gelatin, laminin proteoglycans, } \\
\text { fibronectin, elastin, E-cadherin, IGFBP, } \\
\text { HB-EGF, perlecan, proMMP-13 }\end{array}$ & not shown & \\
\hline 7 & matrilysin & $28 / 19$ & $\begin{array}{l}\text { gelatin, fibronectin, } \\
\text { laminin, collagen IV, } \\
\text { proteoglycans FasL, } \\
\text { HB-EGF, pro-MMP-1 }\end{array}$ & yes & $\begin{array}{l}\text { heparan sulfate proteo- } \\
\text { glycans, CD } 44 v 3\end{array}$ \\
\hline 8 & $\begin{array}{l}\text { neutrophil collagenase, } \\
\text { collagenase-2 }\end{array}$ & $51 / 42$ & collagen I, II, III, VII, X & not shown & \\
\hline 9 & gelatinase $\mathrm{B}$ & $92 / 83$ & $\begin{array}{l}\text { gelatin, collagen I, IV,V, } \\
\text { X, IGFBP, latent TGF- } \beta\end{array}$ & yes & $\begin{array}{l}\text { CD } 44 \text {, collagen IV, } \\
\text { others? }\end{array}$ \\
\hline 10 & stromelysin-2 & $52 / 43$ & $\begin{array}{l}\text { collagen III, IV, IX, X } \\
\text { gelatin, proteoglycans } \\
\text { laminin, pro-MMP-1 } \\
\text { pro-MMP-13 }\end{array}$ & not shown & \\
\hline 11 & stromelysin-3 & $51 / 44$ & $\alpha$-1-antiprotease, IGFBP & not shown & \\
\hline 12 & metalloelastase & $52 / 43$ & elastin, pro-MMP-13 & not shown & \\
\hline 13 & collagenase-3 & $52 / 42$ & $\begin{array}{l}\text { collagen I, II, III,IV,VII, } \\
\mathrm{X} \text {, XIV, tenascin, aggrecan, } \\
\text { fibronectin, pro-MMP-9 }\end{array}$ & not shown & \\
\hline 14 & MT1-MMP & $64 / 54$ & $\begin{array}{l}\text { gelatin, collagen I, fibrin, } \\
\text { fibronectin, proteoglycans, } \\
\text { laminin, pro-MMP- } 2 \text {. } \\
\text { Pro-MMP-13 }\end{array}$ & yes & transmembrane domain \\
\hline 15 & MT2-MMP & $71 / 61$ & $\begin{array}{l}\text { fibronectin, laminin, } \\
\text { tenascin, pro-MMP-2 } \\
\text { pro-MMP-13 }\end{array}$ & yes & transmembrane domain \\
\hline 16 & MT3-MMP & $66 / 56$ & $\begin{array}{l}\text { collagen III, gelatin } \\
\text { fibronectin, pro-MMP-2 }\end{array}$ & yes & transmembrane domain \\
\hline 17 & MT4-MMP & 54 & unknown & yes & transmembrane domain \\
\hline $18 / 19$ & RASI-1 & $55 / 47$ & unknown & not shown & \\
\hline 20 & Enamelysin & $52 / 43$ & amelogenin & not shown & \\
\hline
\end{tabular}


The role of MMPs is to modulate the structural components of the ECM; predominantly, collagens and gelatins. This is an important part of healthy embryological development and organogenesis, as well as tissue homeostasis, remodelling, and repair in mature tissues ${ }^{506}$. This is particularly pertinent when considering wound healing and the migration and invasion of mesenchymal cells through he ECM, although most cells in their base state do not express MMPs strongly. Transcriptomal control of MMPs is partially mediated by growth factors, hormones, cytokines, and cellular transformation, including soluble suppressive factors such as TGF- $\beta$, retinoic acid, and glucocorticoids. However, the primary induction of MMP expression is mediated by integrins or cell-cell interactions; particularly cells of the immune system $^{507,508}$. The extracellular action of MMPs further regulated by the balance of inhibitory factors; specifically, $\alpha$-macroglobulins and MMP-specific tissue-inhibitors of metalloproteinases (TIMPs) ${ }^{509}$.

In addition to their roles is healthy tissue maintenance, MMPs also have a long-established role in carcinogenesis ${ }^{505}$. In relation to epithelial-derived tumours, such as CRC, the first stage of tumour invasion is through the basement membrane, which itself is primarily composed of type-IV collagen, and thus much interest has been directed towards MMP2 and MMP9 ${ }^{510}$. The role of MMP2 has been investigated specifically in relation to colorectal cancer, where increased expression has previously been associated with worse Duke's stage ${ }^{511}$. Similarly, TIMP2, the specific inhibitor of MMP2, has been associated with increased likelihood of localised disease spread in colorectal cancer, although MMP2 and MMP9 were not found to be significant ${ }^{512}$. At best, the role and significance of increased MMP2 and MMP9 expression in solid cancers, or their respective inhibitors, is not consistent. Several tumour types have shown association between worsening stage and/or tumour biology associated with increased MMP2 expression, although MMP2 has failed to be reliable as a diagnostic tumour marker or in stratifying clinical outcomes ${ }^{513}$.

A more recent perspective on the role of collagen-IV specific MMPs in colorectal cancer has examined the failures to translate laboratory models of CRC and MMP activity to observations made in vivo ${ }^{514}$. Mook and colleagues consider that MMPs and early invasive CRC are interacting with a complex stromal environment in vivo, and that the previous modelling of CRC (specifically in the application of MMP-blocking agents as potential therapy) has been inadequate in accounting for the complex cell-cell and cell-stromal interactions that partially determine MMP activity. One hypothesis suggested is that MMP inhibition may actually 
contribute to carcinogenesis by preventing MMPs degrading angiogenic growth factors and cytokines. Furthermore, selective inhibition of MMP2 and MMP9 may also contribute to carcinogenesis in $\mathrm{CRC}$ by preventing macrophage and other inflammatory cell migration to early tumours, thus reducing anti-tumoural response. The failure of MMP inhibitors as anticancer therapies, and a growing appreciating that these failures may be due to a poor appreciation of the role of these proteinases in the immune response, have subsequently prompted investigators to examine whether MMP inhibitors may have a role in inflammatory diseases such as rheumatoid arthritis ${ }^{515}$.

Surprisingly, there has been little investigation of methylation of MMPs and TIMPs relating to any type of cancer, and almost none relating to colorectal cancer. A single study identified relative hypermethylation and subsequent silencing of MMP16 (a transmembrane MMP) in in vitro colorectal cancers and in colorectal cancer cell lines (SW480, DLD-1, and LoVo) $)^{516}$. Treatment with AZA restored expression of MMP16 following demonstrable locus-specific demethylation. Further studies have highlighted a potential role for epigenetic mechanisms (including methylation and chromatic restructuring) in the regulation if MMPs in other diseases, including ischaemic stroke, diabetic retinopathy, and rheumatoid arthritis ${ }^{517-519}$. 


\subsubsection{Differential Gene Expression}

Phenotypic changes in epithelial cells undergoing EMT-like progression to early invasive cancers will change the expression of genes required to effect this transformation. As this process defines a malignant transformation, the genetic factors that bring about the change in gene expression can thus be assumed to carcinogenic; whether chromosomal, mutational, or epigenetic. However, due to the high-level of interlinkage between canonical intracellular signalling pathways, care must be taken when interpreting expressional changes associated with signalling, and thus end-effect changes in expression (such as those that define EMT or the relationship to the ECM) may provide better indicators of invasive transformation ${ }^{520}$.

One method of examining differential gene expression is to examine messenger RNA (mRNA) profiles; relative increases or decreases in specific gene mRNA provide an insight into biologic mechanisms, and may be attributable to specific genetic events, exposure to genotoxic agents, or therapies. This methodology is widely applied in toxicogenomics, where cells are exposed to putative genotoxins and expressional changes associated with exposure can therefore indicate a mechanism of carcinogenic action ${ }^{521}$. The process may also be applied in reverse, where exposure to a potentially therapeutic agent may result in differential gene expression that may be regarded as effecting an anti-tumoural phenotypic change or cell death. The combination of these molecular techniques with chemo- and bioinformatics can also reveal expressional changes associated with therapeutic agents that are not evident at a phenotypic level ${ }^{522,523}$.

When screening for expressional changes, a high-throughput wide-net approach is usually employed in the first instance to capture a potentially relevant expressional event, which can then be focused-down upon by more targeted methods. One such method of initial screening is through microarrays ${ }^{524}$. Microarrays exploit the relative binding saturation of fluorescently labelled target RNA to specific pre-loaded oligonucleotide primers on rigid surfaces, causing differential fluorescence to indicate relative expression. In this format microarrays are commonly known as gene chips and have the ability to screen multiple genes (from the tens to the many hundreds, depending on the platform). Once differential expression has been examined, clustering techniques (supervised or unsupervised data analysis) may be used to identify genes with a similar response to stimulus, or which genes best indicate the application of specific stimuli ${ }^{525}$. 
This process has been refined further to incorporate quantitative real-time PCR (qRT-PCR) techniques to the basic array format and is now considered the gold-standard in quantitative assay screening for expressional change ${ }^{526,527}$. This process relies upon real-time observation of relative fluorescence during exponential amplification of complimentary cDNA, and thus both an internal and external control for expression must be employed, as well as a preamplification stage of cDNA synthesis from mRNA. Despite the additional complexity, qRTPCR has been shown to be an accurate and precise method of assessing gene expression ${ }^{528}$. If a biologic process is suspected, pathway-specific microarrays based upon genes known to be important to that processes may facilitate in the initial screening by narrowing the target spectrum of genes, increasing efficiency.

qRT-PCR can also be used to validate the array data once it has suggested specific target genes of interest and may also demonstrate dose-dependent expressional change when examining the effects of genotoxic of therapeutic agents. The selection of target genes for qRT-PCR is based upon array data, but also consideration of the biologic process being investigated and the complexity of cellular signalling; thus, differential extracellular enzyme expression may provide more illuminating to phenotypic changes than, for instance, a canonical signalling protein with multiple downstream effects. The expressional changes that may be observed in array and specific qRT-PCR may be confirmed further by standard quantitative protein techniques, such as Western blotting.

For the purposes of this study, the Qiagen $\mathrm{RT}^{2}$ Profiler PCR Array for Human Tumour Metastasis was chosen as the PCR array ${ }^{527}$. There were several factors influencing the choice of this array, especially considering there are EMT-specific arrays provided by the same manufacturer that might have provided a more specific insight into the expressional events that underlie EMT-like transformation of an early cancer. However, the overarching narrative of this thesis is to examine specifically the biological processes that underlie extramural vascular invasion in rectal cancer, particularly in the context of locally advanced disease. These processes depend not just on and EMT-like process enabling invasion of the submucosa, but also invasion of other tissues, including blood vessels and lymph nodes. A decision was made, therefore, to look more closely at the extracellular remodelling, locally invasive, and locally metastatic abilities of DLD-1 cells (derived from a Dukes C adenocarcinoma), as opposed to early EMT-like changes such as loss of cell polarity and cell-cell adhesion. Clearly there is a high degree of overlap between EMT and local invasion from the point of view of underlying 
biologic process, and thus some of the same genes are examined on the corresponding EMT and metastatic arrays. However, on balance, the coverage of likely genes of interest was more appropriate on the human tumour metastasis array than the EMT array. This was particularly the case for MMPs and TIMPs, with only MMP2, -3, \& -9, and TIMP1 being examined on the EMT array, whilst MMP7, $-10,-11, \&-13$, and TIMPs $-2,-3, \&-4$ are all examined on the metastasis array in addition to those examined on the EMT array. Additionally, the APC, KRAS, and SMAD genes are also examined on the metastasis array but are absent on the EMT panel. Cadherins and Type IV collagen are present on both. A full list of gene targets on the Qiagen $\mathrm{RT}^{2}$ Profiler PCR Array for Human Tumour Metastasis focused array is presented in

Figure 5.1.4.

Figure 5.1.4 Genes on the Qiagen $\mathrm{RT}^{2}$ Profiler PCR Array for Human Tumour Metastasis.

\begin{tabular}{|c|c|c|c|c|}
\hline Position & UniGene & GenBank & Symbol & Description \\
\hline A01 & $\mathrm{Hs.158932}$ & NM_000038 & APC & Adenomatous polyposis coli \\
\hline $\mathrm{A} 02$ & $\mathrm{Hs.100426}$ & NM_015399 & BRMSI & Breast cancer metastasis suppressor 1 \\
\hline A03 & $\mathrm{Hs.251526}$ & NM 006273 & CCL7 & Chemokine (C-C motif) ligand 7 \\
\hline $\mathrm{A} 04$ & $\mathrm{Hs.502328}$ & NM 000610 & $\mathrm{CD} 44$ & CD44 molecule (Indian blood group] \\
\hline A05 & $\mathrm{Hs.527778}$ & NM_002231 & $\mathrm{CD} 82$ & CDB2 molecule \\
\hline A06 & $\mathrm{Hs.461086}$ & NM_004360 & $\mathrm{CDHI}$ & Cadherin 1, type 1, E-cadherin (epithelial) \\
\hline A07 & $\mathrm{Hs.116471}$ & NM_001797 & $\mathrm{CDHII}$ & Codherin 11, type 2, OB-cadherin (osteoblast) \\
\hline A08 & $\mathrm{Hs.171054}$ & NM 004932 & $\mathrm{CDH} 6$ & Cadherin 6, type $2, K$-cadherin (fetal kidney) \\
\hline A09 & $\mathrm{Hs.512599}$ & NM_000077 & CDKN2A & Cyclin-dependent kinose inhibitor $2 \mathrm{~A}$ (melanoma, p16, inhibits CDK4) \\
\hline A10 & $\mathrm{Hs} .162233$ & NM_001273 & $\mathrm{CHD} 4$ & Chromodomain helicase DNA binding protein 4 \\
\hline A11 & $\mathrm{Hs} .508716$ & NM_001846 & COL4A2 & Collogen, type IV, alpha 2 \\
\hline A12 & $\mathrm{Hs.143212}$ & NM 003650 & CST7 & Cystatin F (leukocystatin) \\
\hline 801 & $\mathrm{Hs.208597}$ & NM 001328 & CTBPI & C-terminal binding protein 1 \\
\hline 802 & $\mathrm{Hs.534797}$ & NM_001903 & CTNNAI & Catenin (cadherin-ossociated protein), alpha 1, 102kDa \\
\hline 803 & $\mathrm{Hs.632466}$ & NM_000396 & CTSK & Cathepsin K \\
\hline 804 & $\mathrm{Hs.716407}$ & NM_001912 & CTSLI & Cathepsin LI \\
\hline 805 & Hs.522891 & NM 000609 & $\mathrm{CXCL} 12$ & Chemokine [ $\mathrm{C}-\mathrm{X}-\mathrm{C}$ motif) ligand 12 \\
\hline 806 & $\mathrm{Hs.846}$ & NM_001557 & $\mathrm{CXCR2}$ & Chemokine (C-X.C motif) receptor 2 \\
\hline 807 & $\mathrm{Hs.593413}$ & NM_003467 & CXCR4 & Chemokine (C-X-C motif) receptor 4 \\
\hline 808 & $\mathrm{Hs} .22393$ & NM_003677 & DENR & Density-regulated protein \\
\hline 809 & $\mathrm{Hs.523329}$ & NM 004442 & EPHB2 & EPH receptor B2 \\
\hline 810 & $\mathrm{Hs.434059}$ & NM_001986 & ETV4 & Ets variant 4 \\
\hline 811 & $\mathrm{Hs.374477}$ & NM_005243 & EWSRI & Ewing sarcoma breakpoint region 1 \\
\hline 812 & $\mathrm{Hs.481371}$ & NM_005245 & FATI & FAT fumor suppressor homolog I (Drosophila) \\
\hline $\mathrm{COI}$ & $\mathrm{Hs.165950}$ & NM_002011 & FGFR4 & Fibroblast growth factor receptor 4 \\
\hline $\mathrm{CO} 2$ & $\mathrm{Hs.646917}$ & NM 002020 & FLT4 & Fms-related tyrosine kinose 4 \\
\hline $\mathrm{CO} 3$ & $\mathrm{Hs.203717}$ & NM_002026 & $\mathrm{FN1}$ & Fibronectin 1 \\
\hline $\mathrm{CO} 4$ & $\mathrm{Hs.333418}$ & NM_014164 & FXYD5 & FXYD domain containing ion transport regulator 5 \\
\hline $\cos$ & $\mathrm{Hs.82963}$ & NM_000825 & GNRHI & Gonadatropin-releasing hormone I (luteinizing-releasing hormone) \\
\hline $\mathrm{CO6}$ & $\mathrm{Hs} .396530$ & NM 000601 & HGF & Hepatocyte growth foctor (hepopoietin A; scatter foctor) \\
\hline $\mathrm{CO}$ & $\mathrm{Hs.44227}$ & NM_006665 & HPSE & Heparanase \\
\hline $\operatorname{Cos}$ & $\mathrm{Hs.37003}$ & NM_005343 & HRAS & V-Ha-ras Harvey rat sarcoma viral oncogene homolog \\
\hline $\mathrm{COS}$ & $\mathrm{Hs.90753}$ & NM_006410 & HTATIP2 & HIV-1 Tat interactive protein $2,30 \mathrm{kDa}$ \\
\hline $\mathrm{ClO}$ & $\mathrm{Hs.160562}$ & NM_000618 & IGF] & Insulin-like growth foctor 1 (somatomedin C) \\
\hline $\mathrm{Cll}$ & $\mathrm{Hs.83077}$ & NM 001562 & ILI8 & Interleukin 18 (interferon-gamma-inducing factor) \\
\hline $\mathrm{Cl} 2$ & $\mathrm{Hs.126256}$ & NM_000576 & ILIB & Interleukin 1, beta \\
\hline D01 & $\mathrm{Hs} .524484$ & NM_002206 & ITGA7 & Integrin, alpha 7 \\
\hline D02 & $\mathrm{Hs.218040}$ & NM_000212 & ITG83 & Integrin, beta 3 (platelet glycoprotein lila, antigen CD61) \\
\hline D03 & $\mathrm{H} \varepsilon .95008$ & NM 002256 & KISSI & KiSS-1 metastasis-suppressor \\
\hline D04 & Hs.208229 & NM_032551 & KISSIR & KISSI receptor \\
\hline D05 & $\mathrm{Hs} .505033$ & NM_004985 & KRAS & V-Ki-fas2 Kirsten rat sarcoma viral oncogene homolog \\
\hline D06 & $\mathrm{Hs.599039}$ & NM_006500 & MCAM & Melanoma cell adhesion molecule \\
\hline D07 & $\mathrm{Hs.484551}$ & NM 002392 & MDM2 & Mdm2 p53 binding protein homolog (mouse) \\
\hline D08 & $\mathrm{Hs.132966}$ & NM 000245 & MET & Met proto-oncogene (heoatocyte growth factor receptor) \\
\hline $\mathrm{D} 09$ & $\mathrm{Hs.444986}$ & NM_006838 & METAP2 & Methionyl aminopeptidase 2 \\
\hline
\end{tabular}




\begin{tabular}{|c|c|c|c|c|}
\hline Position & UniGene & GenBank & Symbol & Description \\
\hline D10 & Hs.651869 & NM_002410 & MGAT5 & Mannosyl (alpha-1,6-)-glycoprotein beta-1,6-N-acetyl-glucosaminyltransferase \\
\hline D11 & Hs.2258 & NM_002425 & MMP10 & Matrix metallopeptidase 10 (stromelysin 2) \\
\hline D12 & $\mathrm{Hs.143751}$ & NM_005940 & MMPII & Motrix metallopeptidase 11 (stromelysin 3) \\
\hline E01 & Hs.2936 & NM 002427 & MMP13 & Matrix metallopeptidase 13 (collogenose 3 ) \\
\hline $\mathrm{EO} 2$ & Hs.513617 & NM_004530 & MMP2 & $\begin{array}{c}\text { Matrix metallopeptidase } 2 \text { (gelatinase A, 72kDa gelatinase, } 72 \mathrm{kDa} \text { type IV } \\
\text { collagenase) }\end{array}$ \\
\hline E03 & Hs.375129 & NM_002422 & MMP3 & Matrix metallopeptidase 3 (stromelysin 1, progelatinase) \\
\hline E04 & Hs.2256 & NM_002423 & MMP7 & Matrix metallopeptidase 7 (matrilysin, uterine) \\
\hline EOS & Hs.297413 & NM_004994 & MMP9 & $\begin{array}{l}\text { Matrix metallopeptidase } 9 \text { (gelatinase } B, 92 \mathrm{kDa} \text { gelatinose, } 92 \mathrm{kDa} \text { type IV } \\
\text { collagenase) }\end{array}$ \\
\hline E06 & $\mathrm{Hs} .525629$ & NM_004689 & MTAI & Metastasis associated 1 \\
\hline E07 & $\mathrm{Hs} .700429$ & NM_014751 & MTSS1 & Metastasis suppressor 1 \\
\hline E08 & Hs.202453 & NM 002467 & MYC & V-myc myelocytomatosis viral oncogene homolog (avian) \\
\hline EOS & $\mathrm{Hs.437922}$ & NM_005376 & MYCL1 & $\begin{array}{l}\text { V-myc myelocytomatosis viral oncogene homolog 1, lung carcinoma derived } \\
\text { (avian) }\end{array}$ \\
\hline E10 & Hs.187898 & NM_000268 & NF2 & Neurofibromin $2($ merlin) \\
\hline El1 & Hs.118638 & NM_000269 & NMEI & Non-metostatic cells 1, protein (NM23A) expressed in \\
\hline $\mathrm{E} 12$ & Hs.9235 & NM 005009 & NME4 & Non-metastatic cells 4 , protein expressed in \\
\hline F01 & Hs.279522 & NM_006981 & NR4A3 & Nuclear receptor subfamily 4 , group A, member 3 \\
\hline $\mathrm{F} 02$ & $\mathrm{Hs.466871}$ & NM_002659 & PLAUR & Plasminogen activator, urokinase receptor \\
\hline F03 & $\mathrm{Hs.409965}$ & NM_002687 & PNN & Pinin, desmosome associated protein \\
\hline F04 & $\mathrm{Hs.500466}$ & NM 000314 & PTEN & Phosphatase and tensin homolog \\
\hline F05 & $\mathrm{Hs.408528}$ & NM 000321 & RBI & Retinoblastoma 1 \\
\hline F06 & $\mathrm{Hs.494178}$ & NM_006914 & RORB & RAR-related orphon receptor 8 \\
\hline F07 & $\mathrm{Hs.449909}$ & NM_002295 & RPSA & Ribosomal protein SA \\
\hline F08 & $\mathrm{Hs.414795}$ & NM_000602 & SERPINEI & $\begin{array}{l}\text { Serpin peptidase inhibitor, clade } \mathrm{E} \text { (nexin, plasminogen activator inhibitor type } \\
\qquad 1 \text { ), member } 1\end{array}$ \\
\hline F09 & $\mathrm{Hs.436687}$ & NM_003011 & SET & SET nuclear oncogene \\
\hline $\mathrm{FlO}$ & Hs. 12253 & NM_005901 & SMAD2 & SMAD family member 2 \\
\hline $\mathrm{Fll}$ & Hs.75862 & NM_005359 & SMAD4 & SMAD family member 4 \\
\hline $\mathrm{F} 12$ & Hs. 195659 & NM 005417 & SRC & V-sre sarcoma (Schmidt-Ruppin A-2) viral oncogene homolog (ovian) \\
\hline $\mathrm{G} 01$ & $\mathrm{Hs.514451}$ & NM 001050 & SSTR2 & Somatostatin receptor 2 \\
\hline $\mathrm{GO} 2$ & $\mathrm{Hs.371720}$ & NM_003177 & SYK & Spleen tyrosine kinase \\
\hline $\mathrm{G} 03$ & Hs.475018 & NM_005650 & TCF20 & Transcription factor $20(A R I)$ \\
\hline G04 & $\mathrm{Hs.645227}$ & NM_000660 & TGFB1 & Transforming growth factor, beta I \\
\hline Go5 & $\mathrm{Hs} .633514$ & NM_003255 & TIMP2 & TIMP metallopeptidase inhibitor 2 \\
\hline G06 & $\mathrm{Hs} .644633$ & NM_000362 & TIMP3 & TIMP metallopeptidase inhibitor 3 \\
\hline $\mathrm{G} 07$ & $\mathrm{Hs.591665}$ & NM_003256 & TIMP4 & TIMP metallopeptidase inhibitor 4 \\
\hline G08 & $\mathrm{Hs.478275}$ & NM_003810 & TNFSFI0 & Tumor necrosis factor (ligand) superfarnily, member 10 \\
\hline G09 & $\mathrm{Hs.654481}$ & NM_000546 & TP53 & Tumor protein p53 \\
\hline G10 & Hs. 155942 & $\mathrm{NM} 002420$ & TRPMI & Transient receptor potential cation channel, subfamily $M$, member 1 \\
\hline G11 & Hs.160411 & NM_000369 & TSHR & Thyroid stimulating hormone receptor \\
\hline $\mathrm{G} 12$ & $\mathrm{H} s .73793$ & NM_003376 & VEGFA & Vascular endathelial growth factor A \\
\hline $\mathrm{HOI}$ & $\mathrm{Hs.520640}$ & NM_001101 & ACTB & Actin, beta \\
\hline $\mathrm{HO} 2$ & $\mathrm{Hs.534255}$ & NM 004048 & $\mathrm{~B} 2 \mathrm{M}$ & Beta-2-microglobulin \\
\hline $\mathrm{HO3}$ & $\mathrm{Hs.592355}$ & NM_002046 & GAPDH & Glyceraldehyde-3-phosphate dehydrogenase \\
\hline $\mathrm{HO4}$ & $\mathrm{Hs.412707}$ & NM_000194 & HPRTI & Hypoxanthine phosphoribosyltransferase 1 \\
\hline $\mathrm{HOS}$ & $\mathrm{Hs} .546285$ & NM_001002 & RPLPO & Ribosomal protein, large, $\mathrm{PO}$ \\
\hline $\mathrm{H} 06$ & N/A & SA_00105 & HGDC & Human Genomic DNA Contamination \\
\hline $\mathrm{H} 07$ & N/A & SA 00104 & RTC & Reverse Transcription Control \\
\hline $\mathrm{HOB}$ & N/A & SA_00104 & RTC & Reverse Transcription Control \\
\hline $\mathrm{HOS}$ & $\mathrm{N} / \mathrm{A}$ & SA_00104 & RTC & Reverse Transcription Control \\
\hline $\mathrm{H} 10$ & N/A & SA_00103 & $\mathrm{PPC}$ & Positive PCR Control \\
\hline $\mathrm{HII}$ & N/A & SA_00103 & PPC & Positive PCR Control \\
\hline $\mathrm{H} 12$ & $\mathrm{~N} / \mathrm{A}$ & SA_00103 & $\mathrm{PPC}$ & Positive PCR Control \\
\hline
\end{tabular}




\subsection{Aims \& Objectives}

This chapter will examine the gene-expressional effects of demethylation of colorectal cell lines in vitro by AZA and RRx-001. Firstly, a gene qRT-PCR array will be employed to identify genes of interest, with a focus on those genes important in the EMT and locally invasive processes. Secondly, a more in-depth investigation of differential expression of genes identified by the array will be performed to confirm array results, and to examine for any exposure-expression relationship between identified genes and AZA \& RRx-001. 


\subsection{Methods}

DLD-1 cells were cultured in control conditions or exposed to AZA and RRx-001 according to the protocols described in Sections 2.3 and 4.3.1.2. Treatment arm doses of AZA and RRx001 were $0.50 \mathrm{uM}$. For the purposes of the array, only AZA at $0.50 \mathrm{uM}$ for $72 \mathrm{hrs}$ exposure was used against control, as this provided a baseline for investigation potential differential gene expression in a well characterised compound with a specific mode of demethylation. For subsequent gene-specific qRT-PCR, RRx-001 and an additional dose-exposure of 24hrs AZA were employed. The purpose of this was to examine for any temporal effect of demethylating agent AZA, as it relies on cell proliferation to effect demethylation. This was the only modification.

\subsubsection{RT $\mathrm{RT}^{2}$ Profiler PRC Array Gene Expression}

\subsubsection{RNA Extraction \& Purification}

RNA was extracted from DLD-1 cells by employing the Qiagen RNeasy RNA kit. There was no deviation from the product protocol. The maximum RNA haves from this system is $100 \mu \mathrm{g}$ per sample, and thus a maximum of $5 \times 10^{6}$ cells (as recommended in product literature) were used in each sample to prevent over-saturation of the system. DLD-1 cells were first trypsinised and washed twice with PBS before undergoing RNA extraction. All reagents were prepared as per the kit protocol and RNA extraction was performed under clean laminar-flow conditions using standard precautions to prevent cross-contamination / fouling of reaction.

Firstly, 600uL of Buffer RLT was added to cell pellets in a microcentrifuge eppendorf containing pelleted cells and vortexed thoroughly until no cell clumps were visible and until fully homogenized (approximately 3 minutes). Following homogenization, $600 \mathrm{uL}$ of $70 \%$ ethanol was then added and the suspension mixed thoroughly by pipetting. Of the resulting suspension, $700 \mathrm{uL}$ of was then transferred to spin columns placed in a $2 \mathrm{ml}$ collection tube and centrifuged at 10,000 rpm for 15 seconds. The flow-through was discarded. 700uL of Buffer RW1 was added to the spin column (replaced in the emptied collection tube) and was centrifuged again at 10,000 rpm for 15 seconds. The flow-through was discarded. 500uL of Buffer RPE was then added to the spin column and centrifuged at 10,000rpm for 15 seconds. The flow-through was discarded. A further 500uL of Buffer RPE was added to the spin column but this time centrifugation at 10,000 rpm was for 2 minutes to ensure drying of the RNA collection membrane and full elimination of any residual ethanol. Spin columns were then transferred to a fresh $1.5 \mathrm{ml}$ collection tube and 50uL of nuclease-free water (DEPC-free) was 
added directly to the spin column membrane. Spin columns were centrifuged at 10,000rpm for 1 minute to elute the RNA. Harvested RNA was then quantified and its purity checked by spectrophotometery (Nanodrop) at $260 \mathrm{~nm}$. RNA was stored at $-20^{\circ} \mathrm{C}$.

\subsubsection{2 cDNA Synthesis}

The Qiagen RT $^{2}$ First Strand Kit was used to generate cDNA from mRNA as it is the validated kit for use in conjunction with the $\mathrm{RT}^{2}$ Profiler Array. There was no deviation from the published product protocol. $0.5 \mathrm{ug}$ of total RNA was used for each sample as recommended by the product literature, and thus input of total RNA was standardised based on the quantified RNA harvest as specified above. Reagents were prepared as specified in the protocol.

The gDNA elimination cocktail was made by the combination of 5ug RNA with $2 \mathrm{uL}$ of Buffer GE and a variable amount of nuclease-free water in a clean eppendorf to make a total volume of $10 \mathrm{uL}$. The mixture was mixed by pipetting and then incubated for 5 minutes at $42^{\circ} \mathrm{C}$, and then transferred to ice for 1 minute. $10 \mathrm{uL}$ of reverse-transcription cocktail was then added to each gDNA elimination cocktail and mixed by pipetting. This mix was then incubated at $42^{\circ} \mathrm{C}$ for 15 minutes to facilitate cDNA synthesis and then the reaction stopped by incubation at $95^{\circ} \mathrm{C}$ for 5 minutes. $91 \mathrm{uL}$ nuclease-free water was then added to each sample, mixed, and the sample kept on ice before proceeding directly to the $\mathrm{RT}^{2}$ protocol.

\subsubsection{3 $\mathrm{RT}^{2}$ Profiler Array}

The Qiagen RT ${ }^{2}$ Profiler PCR Array for Human Tumour Metastasis (cat. no. 330231 PAHS028ZA) was employed for expressional analysis. There was no deviation from the published product protocol. Reagents were prepared as specified in the protocol. RT ${ }^{2}$ SYBR Green Mastermix (containing HotStart DNA Taq Polymerase) was employed to ensure accurate results. As the chosen array is a 96-well format plate all volumes were calculated correspondingly.

The PCR components were mixed to a total volume of $2700 \mathrm{uL}$ in a $5 \mathrm{ml}$ tube accordingly; $2 \mathrm{x}$ $\mathrm{RT}^{2}$ SYBR Green Mastermix 1350uL, cDNA product $102 \mathrm{uL}$, nuclease-free water $1248 \mathrm{uL}$. $25 \mathrm{uL}$ of the PCR components was then added to each well of the profiler array using an 8channel pipettor, ensuring fresh tips for each well to avoid cross-contamination of well contents. The array wells were then sealed with the supplies Optical Thin-Wall 8-cap strips. The plate was then centrifuged for 1 minute at 12,000rpm and the plate examined to ensure no 
bubbles were present at the base of the wells, and then placed on ice while the RT-PCR cycler was programmed. As a Bio-Rad (USA) IQ-5 ${ }^{\text {TM }}$ Real-Time PCR System was employed, cycling conditions were set as shown in Table 5.3. Figure 5.3 demonstrates the plate layout for the Qiagen $\mathrm{RT}^{2}$ Profiler PCR Array for Human Tumour Metastasis.

Table 5.3 Cycling conditions for qRT-PCR when employing the $\mathrm{RT}^{2}$ Profiler Array.

\begin{tabular}{|c|c|c|c|}
\hline Cycles & Duration & Temperature & Comment \\
\hline 1 & 10 minutes & $95^{\circ} \mathrm{C}$ & HotStart DNA Taq Polymerase activation \\
\hline \multirow{2}{*}{40} & 15 seconds & $95^{\circ} \mathrm{C}$ & Exponential amplification \& fluorescence data \\
& 1 minute & $60^{\circ} \mathrm{C}$ & collection \\
\hline
\end{tabular}

Figure 5.3 Plate layout for Qiagen $\mathrm{RT}^{2}$ Profiler PCR Array for Human Tumour Metastasis. Please refer to Figure 5.1.4 for corresponding gene information.

\begin{tabular}{|c|c|c|c|c|c|c|c|c|c|c|c|c|}
\hline & $\mathbf{1}$ & $\mathbf{2}$ & $\mathbf{3}$ & $\mathbf{4}$ & $\mathbf{5}$ & $\mathbf{6}$ & $\mathbf{7}$ & $\mathbf{8}$ & $\mathbf{9}$ & $\mathbf{1 0}$ & $\mathbf{1 1}$ & $\mathbf{1 2}$ \\
\hline $\mathbf{A}$ & APC & BRMS1 & CCL7 & CD44 & CD82 & CDH1 & CDH11 & CDH6 & CDKN2A & CHD4 & COL4A2 & CST7 \\
\hline B & CTBP1 & CTNNA1 & CTSK & CTSL1 & CXCL12 & CXCR2 & CXCR4 & DENR & EPHB2 & ETV4 & EWSR1 & FAT1 \\
\hline C & FGFR4 & FLT4 & FN1 & FXYD5 & GNRH1 & HGF & HPSE & HRAS & HTATIP2 & IGF1 & IL18 & IL1B \\
\hline D & ITGA7 & ITGB3 & KISS1 & KISS1R & KRAS & MCAM & MDM2 & MET & METAP2 & MGAT5 & MMP10 & MMP11 \\
\hline E & MMP13 & MMP2 & MMP3 & MMP7 & MMP9 & MTA1 & MTSS1 & MYC & MYCL1 & NF2 & NME1 & NME4 \\
\hline F & NR4A3 & PLAUR & PNN & PTEN & RB1 & RORB & RPSA & SERPINE1 & SET & SMAD2 & SMAD4 & SRC \\
\hline G & SSTR2 & SYK & TCF20 & TGFB1 & TIMP2 & TIMP3 & TIMP4 & TNFSF10 & TP53 & TRPM1 & TSHR & VEGFA \\
\hline H & ACTB & B2M & GAPDH & HPRT1 & RPLP0 & HGDC & RTC & RTC & RTC & PPC & PPC & PPC \\
\hline
\end{tabular}

\subsubsection{Data Analysis}

Baseline was set automatically by the cycler software from cycle 2 through 2 cycles. Threshold $\mathrm{C}_{\mathrm{T}}$ was set manually based upon the log view of the amplification plots, specifically on the lower portion of the linear amplification phase. Raw data was exported to a Microsoft Excel spreadsheet for use with SABioscinces PRC Array Data Analysis Template. A melting (dissociation) curve analysis was performed to ensure single product amplification for each well. 


\subsection{2 qRT-PCR of Metalloproteinases}

Metalloproteinase targets were chosen based upon the results of the Qiagen RT $^{2}$ Profiler PCR Array for Human Tumour Metastasis; for full results see Section 5.4.1. Four sets of qRT-PCR were conducted for each gene target: Control; 24hrs 0.50uM AZA; 72hrs 0.50uM AZA; 72hrs 0.50uM RRx-001. Each culture condition was performed in three biological replicates, each of which were subject to parallel technical triplicate during PCR. mRNA was extracted, purified, and quantified by the same methods described in Section 5.3.1.1. A real-time onestep RT-PCR methodology was applied employing the QuantiFast SYBR Green RT-PCR kit supplied by Qiagen, eliminating a separate cDNA synthesis stage.

Six pairs of cDNA primers were designed for qRT-PCR targeting MMPs and TIMPs: MMP2, MMP9, MMP11, MMP13, TIMP2, \& TIMP4. Primer design employed a dual technique of target sequence verification via the NCBI GenBank open source reference (https://www.ncbi.nlm.nih.gov/genbank/) and then oligo analysis via Beacon Designer, PREMIER Biosoft International (http://www.premierbiosoft.com/qpcr/index.html). Oligos were also cross-referenced using Primer-BLAST open access software (https://www.ncbi.nlm.nih.gov/tools/primer-blast/) ${ }^{529}$. $\beta$-actin was used as a housekeeping gene in order to provide reference for quantitative analysis (sequence supplied and previously validated in-house). Primer sequences are listed in Section 2.2.5. No primer demonstrated dimerization, hairpins, or other significant erroneous pairing events, and each had an efficiency $\geq 90 \%$ and a melting temperature $\mathrm{T}_{\mathrm{m}} \geq 70^{\circ} \mathrm{C}$.

qRT-PCR reactions were set-up individually in a 96-well plate with the following components: 2x QuantiFast SYBR Green Mastermix 12.5uL, Primer A 0.5uL, Primer B 0.5uL, QuantiFast RT Mix $0.25 \mathrm{uL}$, template RNA variable volume to control for $100 \mathrm{ng} /$ reaction, nuclease-free water variable volume to make-up total reaction volume to $25 \mathrm{uL}$. In addition to the experimental wells performed in triplicate, a standard curve was constructed by serial dilution (concentrations 1.0, 0.1, 0.001, 0.0001) for each primer and each plate. Reagents were kept on ice throughout to prevent degradation of mRNA. Plates were sealed with biofilm. The plate was then centrifuged for 1 minute at 12,000rpm and the plate examined to ensure no bubbles were present at the base of the wells, and then placed on ice while the RT-PCR cycler was programmed. As a Bio-Rad (USA) IQ-5 ${ }^{\mathrm{TM}}$ Real-Time PCR System was employed, cycling conditions were set as shown in Table 5.4. 
Table 5.4 Cycling conditions for qRT-PCR when employing the QuantiFast SYBR Green RTPCR kit and gene-specific cDNA primers.

\begin{tabular}{|c|c|c|c|}
\hline Cycles & Duration & Temperature & Comment \\
\hline 1 & 10 minutes & $50^{\circ} \mathrm{C}$ & Reverse transcription \\
\hline 1 & 5 minutes & $95^{\circ} \mathrm{C}$ & HotStart DNA Taq Polymerase activation \\
\hline 35 & 10 seconds & $95^{\circ} \mathrm{C}$ & Denaturation \\
\hline 35 & 30 seconds & $60^{\circ} \mathrm{C}$ & $\begin{array}{c}\text { Exponential amplification \& fluorescence data } \\
\text { collection }\end{array}$ \\
\hline
\end{tabular}

Baseline detection was set automatically by the cycler software from cycle 2 through 2 cycles. A standard curve was calculated based on $\beta$-actin $\&$ target gene serial dilutions. Threshold $\mathrm{C}_{\mathrm{T}}$ was set manually based upon the log view of the amplification plots, specifically on the lower portion of the linear amplification phase. $C_{T}$ values for triplicates not within 1 cycle of each other were removed, provided that there were at least 2 remaining. Raw data was exported to a Microsoft Excel spreadsheet for use with Biorad IQ5 software. A melting (dissociation) curve analysis was performed to ensure single product amplification for each well and anomalies excluded. The $\Delta \Delta \mathrm{CT}$ method was used for relative quantification of PCR products ${ }^{530-532}$. 


\subsection{Results}

\subsubsection{RT ${ }^{2}$ Profiler PRC Array Gene Expression}

No pre-amplification phase was performed. The lower limit of detection was set to $\mathrm{C}_{\mathrm{T}}=20$, which was at the lower end of the Log-linear phase ${ }^{533}$. The average $\mathrm{C}_{\mathrm{T}}$ (PPC) was 23.73 and 23.48 for control and test, respectively. Delta $\mathrm{C}_{\mathrm{T}}$ (Average RTC - Average PPC) was 2.16 and 2.13 for control and test, respectively. There was insignificant genomic DNA contamination. Normalisation (from which $\Delta \Delta \mathrm{C}_{\mathrm{T}}$ will be calculated) was set automatically by the analytical software based on the housekeeping genes (HKG) beta-2-microglobulin (BM2) and ribosomal protein, large, P0 (RPLP0) (Figure 5.4.1). Melt-curve analysis was satisfactory for each well.

Figure 5.4.1. Geometric and average geometric mean $\mathrm{C}_{\mathrm{T}}$ for selected housekeeping genes.

\begin{tabular}{|c|c|c|c|c|c|}
\hline Groups & Samples & B2M & RPLPO & Geometric Mean & $\begin{array}{l}\text { Average Geometric } \\
\text { Mean }\end{array}$ \\
\hline \multirow{3}{*}{ Control Group } & & 26. & 19. & & \\
\hline & & 61628267 & 83350168 & 22.98 & 22.98 \\
\hline & & 4206 & 2052 & & \\
\hline \multirow{3}{*}{ Group 1} & & 26. & 20. & & \\
\hline & & 22507700 & 04349951 & 22.93 & 22.93 \\
\hline & & 7783 & 6228 & & \\
\hline
\end{tabular}

DLD-1 cells were cultured in control conditions or with AZA $0.50 \mathrm{uM}$ applied daily for $72 \mathrm{hrs}$ as described previously. At the end of the treatment period, cells were harvested and counted both by coulter counter and on a haemocytometer to ensure no unexpected toxicity had occurred, but then diluted to a maximum of $5 \times 10^{6}$ cells as per the RNA harvest protocol. Harvested RNA was satisfactorily quantified and its purity ensured by spectrophotometery (Nanodrop) at $260 \mathrm{~nm}$. Extracted RNA was then used to assess the changes in expression of genes associated with invasion using the RT-Profiler. Spare RNA was stored at $-20^{\circ} \mathrm{C}$ for shortterm availability or at $-80^{\circ} \mathrm{C}$ for archive purposes. 
Figure 5.4.2 demonstrates the fold-change results for genes significantly over or under expressed compared to control, where significant fold-change threshold is set to $\pm 2\left[2^{\wedge}\left(-\Delta \mathrm{C}_{\mathrm{T}}\right)\right]$. Values for statistical significance $(p)$ could not be calculated based on only a single repetition of the array; verification will be by targeted qRT-PCR. In order of fold-increase (relative foldincrease in brackets), the genes significantly upregulated were; CKDN2A (18.00), TRPM1 (7.28), ITGB3 (3.11), MMP13 (2.85), SSTR2 (2.63), VEGFA (2.62), TIMP4 (2.58), and MMP11 (2.45). In order of fold-decrease (relative fold-decrease in brackets), the genes significantly downregulated were; IGF1 (-11.93), FN1 (-7.91), MMP2 (-4.10), MET (-3.75), CD44 (-3.68), GAPDH (-3.57), EPHB2 (-3.07), ACTB (-3.00), FGFR4 (-2.97), PNN (-2.73), TIMP2 (-2.68), CDH1 (-2.59), NME1 (-2.54), HPRT1 (-2.40), KISS1 (-2.30), CD82 (-2.55), TNFSF10 (-2.22), CXCR2 (-2.09), TGFB1 (-2.08), FLT4 (-2.06), FXYD5 (-2.06), CST7 (2.02). None of the remaining 66 genes (out of a total of 96 profiled genes) demonstrated significant fold-change. These results are demonstrated graphically on scatter-plot Figure 5.4.3. 
Figure 5.4.2. Fold regulation comparison, where significant fold regulation cut-off is set to \pm 2 .

\begin{tabular}{|c|c|c|c|c|}
\hline Position & Gene Symbol & Fold Regulation & p-Value & Comments \\
\hline $\mathrm{A} 04$ & CD44 & -3.68 & 0.000000 & \\
\hline A05 & CD82 & -2.25 & 0.000000 & \\
\hline A06 & $\mathrm{CDH} 1$ & -2.59 & 0.000000 & \\
\hline $\mathrm{A} 09$ & CDKN2A & 18.00 & 0.000000 & \\
\hline A1 2 & CST7 & -2.02 & 0.000000 & \\
\hline B06 & CXCR2 & -2.09 & 0.000000 & \\
\hline B09 & EPHB2 & -3.07 & 0.000000 & \\
\hline $\mathrm{CO} 1$ & FGFR4 & -2.97 & 0.000000 & \\
\hline $\mathrm{CO} 2$ & FLT4 & -2.06 & 0.000000 & \\
\hline $\mathrm{CO} 3$ & FN1 & -7.91 & 0.000000 & \\
\hline $\mathrm{CO} 4$ & FXYD5 & -2.06 & 0.000000 & \\
\hline $\mathrm{C} 10$ & IGF1 & -11.93 & 0.000000 & \\
\hline D02 & ITGB3 & 3.11 & 0.000000 & \\
\hline D03 & KISS1 & -2.30 & 0.000000 & \\
\hline D08 & MET & -3.75 & 0.000000 & \\
\hline D12 & MMP11 & 2.45 & 0.000000 & \\
\hline E01 & MMP13 & 2.85 & 0.000000 & \\
\hline E02 & MMP2 & -4.10 & 0.000000 & \\
\hline E11 & NME 1 & -2.54 & 0.000000 & \\
\hline F03 & PNN & -2.73 & 0.000000 & \\
\hline G01 & SSTR2 & 2.63 & 0.000000 & \\
\hline G04 & TGFB1 & -2.08 & 0.000000 & \\
\hline G05 & TIMP2 & -2.68 & 0.000000 & \\
\hline G07 & TIMP4 & 2.58 & 0.000000 & \\
\hline G08 & TNFSF 10 & -2.22 & 0.000000 & \\
\hline G10 & TRPM1 & 7.28 & 0.000000 & \\
\hline G12 & VEGFA & 2.62 & 0.000000 & \\
\hline $\mathrm{HO} 1$ & ACTB & -3.00 & 0.000000 & \\
\hline $\mathrm{H} 03$ & GAPDH & -3.57 & 0.000000 & \\
\hline $\mathrm{H} 04$ & HPRTI & -2.40 & 0.000000 & \\
\hline
\end{tabular}


Figure 5.4.3. Scatter-plot of normalised expression for treatment (Group1) versus control, with selected individual genes highlighted.

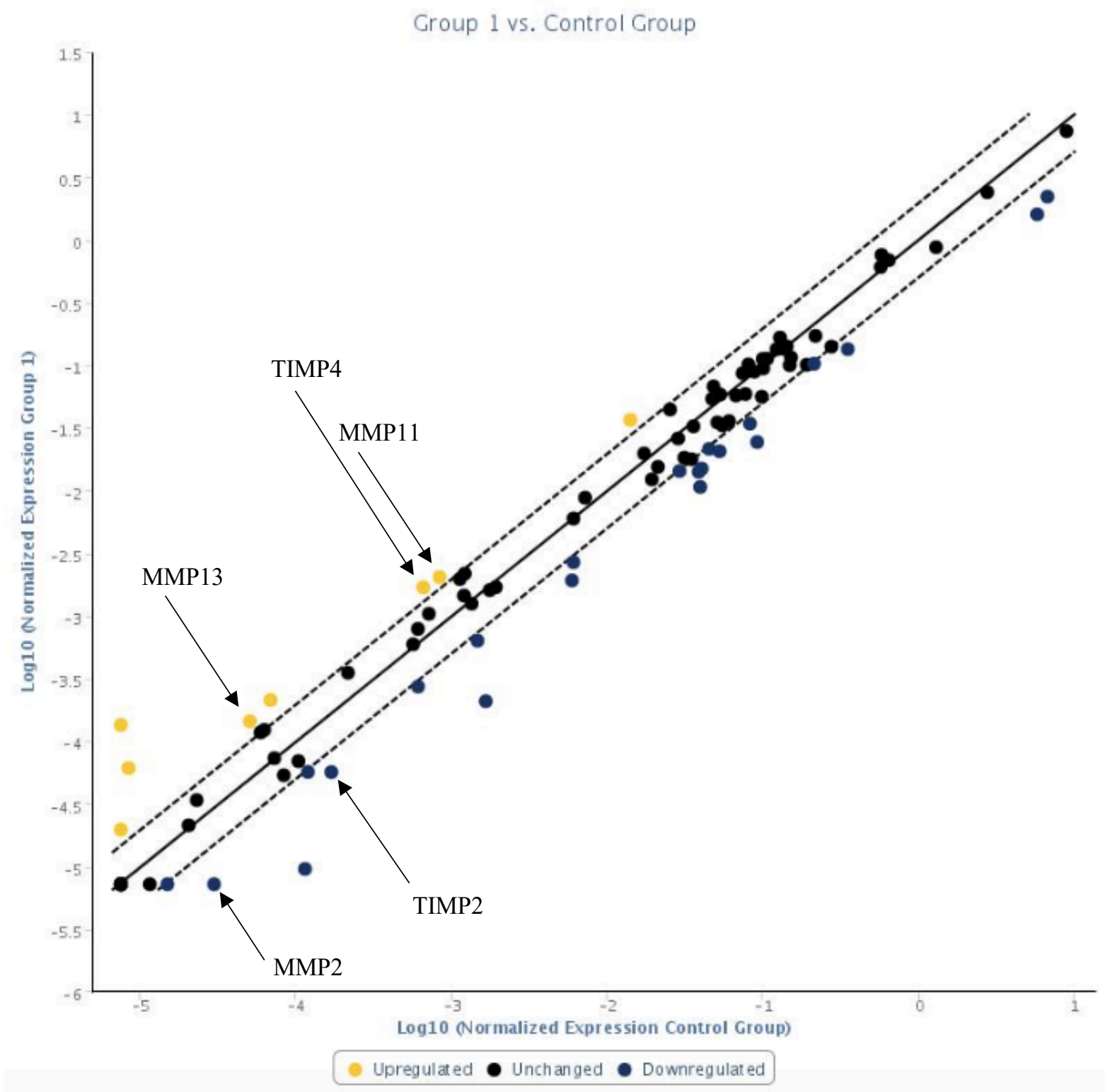




\subsection{2 qPCR of Metalloproteinases}

Candidate genes were selected on the basis of the qPCR array data and conceptualisation of the EMT and EMVI process as pertinent to early invasive rectal cancer. Selected genes were MMP2, MMP9, MMP11, MMP13, TIMP2, and TIMP4. Each of these genes was considerably up- or downregulated in the array when DLD-1 cells were exposed to AZA. MMP9 was also included in this stage of the investigation as it is the partner gelatinase to MMP2. Both a 24hour and 72-hour exposure to $0.50 \mathrm{uM}$ AZA was used during this stage, although only a 72hour exposure to $0.50 \mathrm{uM}$ RRx-001 was employed. Quality control data for mRNA and cDNA are provided in Appendix II.

All results for change in expression are described in terms of relative change in expression in target gene between control and treatment arm, versus change in housekeeping gene (HKG; $\beta$ actin) between the two same arms; $\Delta \Delta \mathrm{C}_{\mathrm{T}}$. Where $\Delta \Delta \mathrm{C}_{\mathrm{T}}=1$, there is no change in expression of target gene versus housekeeping gene, given equal exposure. $\Delta \Delta \mathrm{C}_{\mathrm{T}}<1$ indicates relative downregulation versus HKG, $\Delta \Delta \mathrm{C}_{\mathrm{T}}>1$ indicates relative upregulation versus HKG. $\Delta \Delta \mathrm{C}_{\mathrm{T}}$ cannot be negative, but if the target gene is not expressed at all $\Delta \Delta \mathrm{C}_{\mathrm{T}}$ will be 0 irrespective of HKG expression. Synthesised results are demonstrated in Figure 5.4.4 and raw data provided in Appendix III. For each of the genes other than MMP9, no wells were excluded on the basis of $\mathrm{C}_{\mathrm{T}}$ values in excess of 1 cycle away from each other, or on unfavourable melt curves unless specified.

\subsubsection{MMP2}

Baseline expression of MMP2 was $2.7 \times 10^{-13}$ that of $\beta$-actin. Following $24 \mathrm{hrs}$ treatment with $0.50 \mathrm{uM} A Z A$, relative expression of MMP2 compared to $\beta$-actin was $\Delta \Delta \mathrm{C}_{\mathrm{T}} 0.50(\mathrm{CI} \pm 0.28$, $p>0.05$ ). Following $72 \mathrm{hrs}$ treatment with $0.50 \mathrm{uM}$ AZA $\Delta \Delta \mathrm{C}_{\mathrm{T}}$ was $0.24(\mathrm{CI} \pm 0.01, p \leq 0.001)$. Following $72 \mathrm{hrs}$ treatment with $0.50 \mathrm{uM} R \mathrm{Rx}-001 \Delta \Delta \mathrm{C}_{\mathrm{T}}$ was $4.79(\mathrm{CI} \pm 0.30, p \leq 0.01)$.

\subsubsection{MMP9}

qRT-PCR results for MMP9 were uninterpretable due to numerous anomalous results. Multiple wells provided $\mathrm{C}_{\mathrm{T}}$ values in excess of 1 cycle away from their technical replicates, frequently leading to $2 / 3$ replicates being excluded. Melt curve analysis also highlighted multiple peaks suggesting dimerization and the formation of multiple PCR products. The fact that PCR products were generated suggests that the reactions were not impeded, in which case no amplification would be expected, but that there was a problem with at least one of the primer 
pair. This was confirmed by a complete re-run of the plate which again delivered multiple anomalous results.

\subsubsection{MMP11}

Baseline expression of MMP11 was $3.5 \times 10^{-5}$ that of $\beta$-actin. Following $24 \mathrm{hrs}$ treatment with $0.50 \mathrm{uM}$ AZA, relative expression of MMP11 compared to $\beta$-actin was $\Delta \Delta \mathrm{C}_{\mathrm{T}} 2.25(\mathrm{CI} \pm 1.09$, $p>0.05$ ). Following $72 \mathrm{hrs}$ treatment with $0.50 \mathrm{uM}$ AZA $\Delta \Delta \mathrm{C}_{\mathrm{T}}$ was $3.26(\mathrm{CI} \pm 1.02, p \leq 0.01)$. Following $72 \mathrm{hrs}$ treatment with $0.50 \mathrm{uM} \mathrm{RRx}-001 \Delta \Delta \mathrm{C}_{\mathrm{T}}$ was 1.47 (CI $\left.\pm 0.32, p>0.05\right)$. Three wells were excluded on the basis of $\mathrm{C}_{\mathrm{T}}$ values being in excess of 1 cycle away from each other, but this did not prevent meaningful analysis. There were no unfavourable melt curves resulting in replicate exclusion.

\subsubsection{MMP13}

Baseline expression of MMP13 was $4.0 \times 10^{-4}$ that of $\beta$-actin. Following $24 \mathrm{hrs}$ treatment with $0.50 \mathrm{uM}$ AZA, relative expression of MMP13 compared to $\beta$-actin was $\Delta \Delta \mathrm{C}_{\mathrm{T}} 0.70$ (CI \pm 0.03 , $p \leq 0.05$ ). Following $72 \mathrm{hrs}$ treatment with $0.50 \mathrm{uM}$ AZA $\Delta \Delta \mathrm{C}_{\mathrm{T}}$ was 0.77 (CI $\left.\pm 0.11, p>0.05\right)$. Following $72 \mathrm{hrs}$ treatment with $0.50 \mathrm{uM} \mathrm{RRx}-001 \Delta \Delta \mathrm{C}_{\mathrm{T}}$ was $1.32(\mathrm{CI} \pm 0.42, p>0.05)$.

\subsubsection{TIMP2}

Baseline expression of TIMP2 was 0.062 that of $\beta$-actin. Following $24 \mathrm{hrs}$ treatment with $0.50 \mathrm{uM}$ AZA, relative expression of TIMP2 compared to $\beta$-actin was $\Delta \Delta \mathrm{C}_{\mathrm{T}} 0.87$ (CI \pm 0.69 , $p>0.05$ ). Following $72 \mathrm{hrs}$ treatment with $0.50 \mathrm{uM}$ AZA $\Delta \Delta \mathrm{C}_{\mathrm{T}}$ was $0.37(\mathrm{CI} \pm 0.06, p<0.001)$. Following $72 \mathrm{hrs}$ treatment with $0.50 \mathrm{uM} R \mathrm{Rx}-001 \Delta \Delta \mathrm{C}_{\mathrm{T}}$ was $1.23(\mathrm{CI} \pm 0.31, p>0.05)$.

\subsubsection{TIMP4}

Baseline expression of TIMP4 was $1.25 \times 10^{-7}$ that of $\beta$-actin. Following $24 \mathrm{hrs}$ treatment with $0.50 \mathrm{uM}$ AZA, relative expression of TIMP4 compared to $\beta$-actin was $\Delta \Delta \mathrm{C}_{\mathrm{T}} 1.45(\mathrm{CI} \pm 0.55$, $p>0.05)$. Following $72 \mathrm{hrs}$ treatment with $0.50 \mathrm{uM}$ AZA $\Delta \Delta \mathrm{C}_{\mathrm{T}}$ was $5.89(\mathrm{CI} \pm 1.81, p<0.05)$. Following $72 \mathrm{hrs}$ treatment with $0.50 \mathrm{uM} \mathrm{RRx}-001 \Delta \Delta \mathrm{C}_{\mathrm{T}}$ was $2.94(\mathrm{CI} \pm 0.65, p<0.05)$. 
Figure 5.4.4 qRT-PCR results for $\Delta \Delta \mathrm{C}_{\mathrm{T}}$ of a. MMP2, b. MMP11, c. MMP13, d. TIMP2, and e. TIMP4, following treatment with AZA or RRx-001. [ $\Delta \Delta \mathrm{CT} * \mathrm{p} \leq 0.05$; ** $\mathrm{p} \leq 0.01$; *** $\mathrm{p} \leq 0.001]$
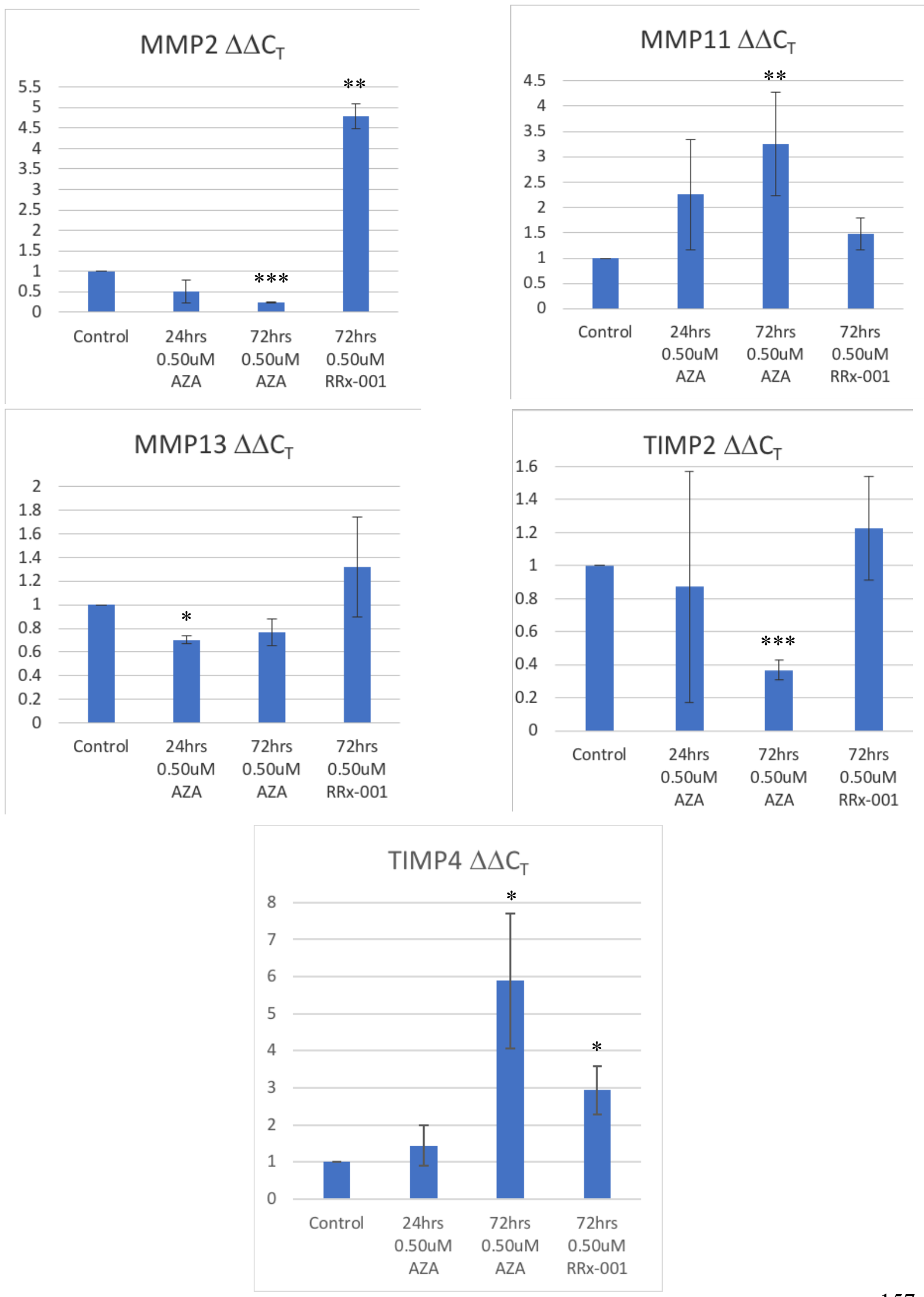


\subsection{Discussion}

The aim of this chapter was to investigate an association between demethylation with agents AZA and RRx-001 and any potential biologic process that may underlie the observed reduced ability of DLD-1 cells to migrate and invade following exposure to each agent. Through this insight any potential association between methylation and the specific genes that underlie the in vivo processes of EMT-like transformation and local invasiveness, such as those which characterises EMVI, may be gained. Results from the $\mathrm{RT}^{2}$ Profiler PRC Array indicated that there was significant dysregulation in the MMP and TIMP genes associated with demethylation with AZA. There was also a significant decrease in cadherin expression (CDH1), but no change in the expression of APC, KRAS, SMADs. Expressional change was confirmed with qRT-PCR for each of the MMP and TIMP target genes of interest when DLD-1 cells were exposed to AZA, but the changes in expression were not consistent when cells were exposed to RRx-001. There was also a degree of temporality to the expressional effects of AZA, where a longer exposure produced a more significant change in expression in some, but not all, genes.

\subsubsection{RT² Profiler PCR Array}

The $\mathrm{RT}^{2}$ Profiler PRC Array demonstrated that $72 \mathrm{hrs}$ of exposure to $0.50 \mathrm{uM}$ of AZA induced significant up- and downregulation (in terms of fold-change) in numerous genes associated with the EMT, locally invasive, and metastatic processes. More genes were downregulated than upregulated, but the family of genes that had undergone widespread expressional change was the metalloproteinases and their respective inhibitors. This group of enzymes are particularly important in the processes of local invasiveness as they define the ability of cells to metabolise the non-cellular components of the basement membrane and stroma ${ }^{506,534}$. This is consistent with the reduced ability of DLD-1 cells to invade the ECM during invasion assays following exposure the AZA, as described in Chapter 4. The specific expression change of each MMP or TIMP will be discussed in greater depth in Section 5.5.2 with corresponding qRT-PCR data. Selected other up- and downregulated genes, and those whose expression did not change, are discussed below. 


\subsubsection{Downregulated Genes}

Contrary to the change in expression theoretically expected with demethylation, based upon the reduced invasive potential of DLD-1 cells after treatment with AZA, was the relative decrease (-2.59) in expression of cadherin-1 (CDH1), the gene coding for E-Cadherin. As previously discussed, E-Cadherin is a vital transmembrane protein that both regulates and effects cell-cell adhesion in epithelial cells. Mutations in CDH1 and subsequent loss of function have previously been strongly associated with epithelial cancers, particularly diffusetype lobular breast and gastric cancers ${ }^{535,536}$. Furthermore, loss of E-Cadherin and reciprocal over-expression of $\mathrm{N}-\mathrm{Cadherin}$ has been associated with colorectal cancer progression ${ }^{490,537}$. However, some authors have reported a complex and dynamic relationship between CDH1 expression and methylation. Graff and colleagues describe how increased methylation of $\mathrm{CpG}$ islands associated with CDH1 led to silencing during early invasion of the ECM (an EMT-like process) as might be expected, but that $\mathrm{CDH} 1$ can be re-expressed when the same cells are cultured in spheroid; suggesting a dynamic pattern of methylation and CDH1 expression. ${ }^{479}$. Re-expression was associated with demethylation and homotypic cell-adhesion in spheroids, and thus the authors therefore suggest that CDH1 methylation is dynamic and at-least partially dependent on stromal interactions and cell-cell relations. Decreased expression of CDH1 coupled with demethylation with AZA and decreased invasion through the ECM is therefore difficult to explain on the basis of a straightforward reciprocal relationship between methylation and expression, although the interaction with the ECM during invasion after AZA exposure may differentially alter $\mathrm{CDH} 1$ expression when compared to exposure during monolayer culture.

Another significantly downregulated (-11.93) gene identified on the array was Type 1 Insulinlike Growth Factor (IGF1). IGF1 has been shown to be associated with many cancer types, including colorectal cancer, and primarily exerts a tumourigenic effect via the MAPK and PIK3AK/Akt pathways ${ }^{538,539}$. Activation of these pathways by IGF1 increases the risk of CRC, as well as increasing cell survival, invasion, and resistance to chemotherapy via a number of downstream mechanisms ${ }^{540,541}$. Differential methylation has already been associated with colorectal cancer and irregular expression of IGF1, and also that aberrant IGF1 methylation is a shared pathway in other cancer types ${ }^{542}$. Downregulation of IGF1 corresponds with the observed propensity of AZA exposed DLD-1 cells to invade, as the tumourigenic effects of downstream signalling would be correspondingly downregulated. 
Fibronectin (FN1) was also significantly downregulated by AZA (-7.91) based on the array data. Fibronectin is a large glycoprotein secreted into the extracellular matrix by fibroblasts and other cells, and has important roles in integrin binding, cell signalling, adhesion, migration, and proliferation ${ }^{543}$. Fibronectin also has an important role in wound healing, where its crossbinding with fibrin is important in clot formation and recruitment of fibroblasts ${ }^{413,544}$. In colorectal cancer, over-expression of FN1 is associated with worse disease stage, and FN1knockdown in vitro results in decreased expression of MMP9 and increased expression of ECadherin, leading to inhibition of cell migration, proliferation, and survival ${ }^{545}$. However, fibronectin has also been demonstrated to induce demethylation-dependent MMP2 expression in breast ductal carcinoma cells in vitro, and that by doing so cell migration and invasion increased $^{546}$. No studies have yet investigated the role of methylation and fibronectin in colorectal cancer, although downregulation and corresponding reduced invasion in vitro would suggest it is pro-invasive as a chemoattractant, although it has been reported that fibronectin may sequester MMP2 by binding the fibronectin-like domain ${ }^{547}$. This hypothesis corresponds with the observation that the addition of fibronectin in the lower chamber of the Boyden chamber assay increased the Percentage Invasion through the ECM (Chapter 4, Figure 4.4.7), although the overall Invasion Index remained equal across all doses.

c-MET is a tyrosine-protein kinase coded by the MET gene that was also downregulated in the array (-3.75). MET has an important role in many cancers via activation of a number of downstream oncogenic signalling pathways including ras, PIK3A, stat, and $\beta$-catenin. MET has been associated with anti-EGFR resistance in colorectal cancer and is a poor prognostic marker for survival ${ }^{548,549}$. The centrality of the c-MET in the development and progression of colorectal and other gastrointestinal cancers had led to much interest in this mechanism and the possibility for novel targeted therapies, although thus far there has been little success in translating benchtop concepts through to successful clinical trials ${ }^{550}$. Part of the reason for these shortcomings is the complexity of the downstream effects of MET signalling, although accurate gene-typing could provide better targeted therapies for individuals who are more likely to benefit; this is the basis for the Framework 7 MErCuRIC programme. Only a limited number of studies have investigated methylation-dependent expression of MET, although methylation events have been associated with pancreatic cancer and insulinomas, all-be-it via aberrant methylation of its regulators rather than the MET epigene itself ${ }^{551}$. 
CD44 is a cell surface glycoprotein that has important roles in cell-cell interaction and relations to the stroma, including in cell migration and adhesion. CD44 was downregulated on the assay (-3.68) following exposure to AZA. CD44 is implicated in the progression and metastasis of cancers by facilitating pro-invasive mechanisms associated with tumour cell migration, angiogenesis, and activation of growth-factor receptors ${ }^{552}$. CD44 has previously been shown to interact with MMPs in cancer (MMP9 and transmembrane-MMP1) to facilitate the progression of prostate cancer, and knockdown of MMP9 has resulted in a change to the CD44 isoform and the failure of cancer cells to produce invadopodia ${ }^{553-555}$. In colorectal cancer, CD44 is upregulated and occurs early in the adenoma-carcinoma sequence; facilitating an EMT-like progression by associated loss of APC/ $\beta$-catenin tumour suppression, as well as by promoting growth-factor binging and inhibition of apoptosis ${ }^{556}$. None of these effects have thus far been associated with aberrant methylation events, although there may be an oblique relationship (via canonical signalling) in gastric cancer ${ }^{557}$. Downregulation of CD44 corresponds with the hypothesis that a degree of reversal in the loss of APC/ $\beta$-catenin tumour suppression may reduce the propensity of colorectal cancer cells to invade in vitro.

\subsubsection{Upregulated Genes}

The most prominently upregulated gene on the array was tumour-suppressor CDKN2A $(+18.0)$. This well characterised gene is important in cell cycle regulation and exerts antitumoural effects by initiating cell-cycle arrest at G2 phase (leading to apoptosis) or G1 into S phase (cell-cycle arrest) via its two coded proteins P16 and P14ARF, respectively 558 . Hypermethylation of $\mathrm{CpG}$ islands associated with $\mathrm{CDKN} 2 \mathrm{~A}$ has been shown to be associated with the progression of colorectal cancer and in poor prognosis, as well as in the pathogenesis of other cancer types (e.g. melanoma \& pancreatic cancers) ${ }^{559,560}$. However, in MSI-high colorectal tumours, methylation of CDKN2A has been demonstrated to occur in association with methylation of hMLH1 in a sub-population of tumours identified as having better overall survival, thus hypermethylation events associated with P16 function are not universally indicators of worse prognosis ${ }^{561}$. It must be noted, however, that this study included a limited number of patients $(n=51)$, and that dual hypermethylation was associated with typically adverse features, such as poor-differentiation. Despite slight inconsistency, abnormal genetic and epigenetic events associated with CDKN2A, including hypermethylation, are generally considered pro-carcinogenic, although via proliferative and anti-apoptotic mechanisms, rather than invasive and migratory. 
TRPM1 was an upregulated (+7.28) gene coding for a transmembrane cation channel found in the retina and associated with depolarisation of the synapse in response to light, particularly in night-sight ${ }^{562}$. TRPM1 is also expressed in melanocytes and is expressed inversely with the aggressiveness (invasiveness and metastatic potential) of melanomas ${ }^{563}$. Calcium $\left(\mathrm{Ca}^{2+}\right)$ remodelling is an observed event in cancers and thought to promote tumour cell migration, encourage proliferation, and support resistance to cell death; although it the role of cation channels is not well characterised in colorectal cancers ${ }^{564-566}$. As calcium is a critical initiator of progression through the cell-cycle as well as being important to multiple other cellular processes, increased cation channel expression might be assumed to promote cell proliferation. However, this is contrary to the observed effect in melanomas, although the upregulation of other TRP-family genes has been shown to occur in association with the development and progression of other cancers ${ }^{567,568}$. There is no published evidence examining a relationship between aberrant methylation events and expression of TRPM1 in cancers. The observation that upregulation of TRPM1 in DLD-1 following exposure to AZA may be associated with a reduction in invasion in vitro corresponds to the inverse relationship of expression and invasion in melanomas.

ITG $\beta 1$ (integrin- $\beta 1$ ) was also upregulated based on array data (+3.11). Integrins are a large group of cell surface receptors that bind a huge array of ECM ligands and help regulate the cellular processes that are required for multicellular organisms to function normally, but also to dysfunction in cancers ${ }^{569}$. ITG $\beta 1$ is one of the many integrin regulators of TGF $\beta 1$ which can, as previously discussed, act as both a tumour suppressor and promotor ${ }^{483,485,570}$. Integrindependent upregulation of TGF $\beta 1$ and its downstream targets has been demonstrated in several cancer types and results in an EMT-like progression, including in colorectal cancer ${ }^{571}$. In breast cancer, Allen demonstrated that integrin-dependent activation of TGF $\beta 1$ was a key indicator of malignant transformation to ductal carcinoma in situ, a process dependent on TGF $\beta 1$ induced upregulation of MMP2 and MMP9 ${ }^{572}$. Integrins also function to prevent anoikis by maintaining contact with the ECM, a regulatory control that may be lost in cancers ${ }^{569}$. Upon loss of integrin binding a number of downstream pathways are activated, including PIK3AK, resulting in caspase- 8 recruitment to the cell membrane and activation by non-liganded integrins ${ }^{573}$. In neuroblastomas this process may be prevented by hypermethylation of caspase8, resulting in silencing, but methylation events are not otherwise well characterised in integrins and cancer ${ }^{574}$. 


\subsubsection{Unchanged Genes}

As discussed, some genes of interest on the array that mechanistically could be important to the EMT-like and EMVI processes have been shown to be up- and downregulated following exposure to AZA. Other genes have however not demonstrated any significant fold-change in expression, some of which might have been important oncogenes in the progression of colorectal cancer. Of particular note were APC, KRAS, and SMADs.

The central role of adenomatous polyposis coli (APC) and KRAS genes in the classical Vogelstein model of colorectal cancer development has already been discussed in Chapter 1.3.1, but it is important to note that expression of these genes was not affected by demethylation with AZA. DLD-1 cells are known to demonstrated APC truncation, although the downstream effects of APC on $\beta$-catenin phosphorylation (and therefore function) are not

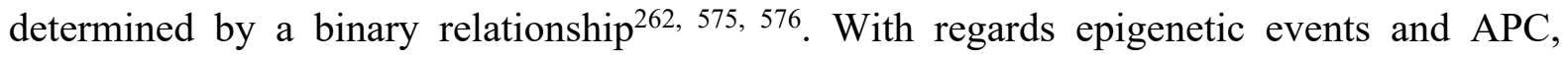
hypermethylation of $\mathrm{CpG}$ islands associated with APC is a frequently observed event in sporadic CRC, with a significant increase in frequency of methylation of APC observed as adenomatous polyps progress to early cancers. Two recent meta-analyses have however demonstrated that APC promoter methylation does not correlate to any pathophysiological feature (including grade and TNM stage) of colorectal cancers, other than being a marker for distinguishing cancer from healthy or adenomatous tissue ${ }^{577,578}$. These results suggest that APC may be important as an early initiator of colorectal cancer by stimulating proliferation and cell survival, but that it does not significantly affect the relationships that govern interactions with and invasion of the $\mathrm{ECM}^{119,120}$.

KRAS expression was also not affected by demethylation with AZA. KRAS mutation in CRC leads to deactivation and subsequent negative-signalling in the MAPK-pathway leading to increased signalling and uncontrolled cellular proliferation ${ }^{111}$. This process is not methylationdependent and DLD-1 cells are known to demonstrate KRAS G13D amino acid substitution as a result of an underlying mutation that is not methylation dependent. This is consistent with KRAS-mutated tumours in vivo, where mutations most commonly occur at codon 12 and 13 and occur as part of the microsatellite-stable adenoma-carcinoma pathway ${ }^{579}$.

Another family of genes not affected by AZA exposure was the SMAD family of intracellular signalling proteins (specifically SMAD2 and SMAD4). As previously discussed in Section 5.1.1, SMADs are important in EMT-like and metastatic processes in colorectal (and other) 
cancers as they act to induce transcription that is a negative control on cellular proliferation and migration in response to TGF $\beta$ interactions with the stroma ${ }^{482}$. The lack of change in expression of these genes despite the marginally significantly decreased expression of TGF $\beta$ $(-2.08)$, suggests that their downstream transcriptional targets may also not be dysregulated by AZA demethylation, although this process is also heavily depended on a number of other cotranscription factors not examined by the array ${ }^{580}$. Furthermore, as SMAD expression is associated with SLUG/SNAIL dependent transcription and subsequent CDH1 (E-cadherin) expression, the observed decreased expression of CHD1 (-2.59) may not be dependent on SMADs.

\subsubsection{Summary}

In addition to the MMP family of genes discussed in the following section, a number of gene important to EMT-like and invasive processes were up- and downregulated following exposure to AZA. Contrary to what might have been expected given the increased ability of DLD-1 cells to invade and migrate following demethylation as demonstrated in Chapter 4, the expression of E-cadherin (CDH1), an important cell-cell adhesion protein, was downregulated. Similarly, genes with important roles in the development of colorectal cancer, including early EMT-like progression, remained unchanged, although the genetic mechanisms linked to aberrant expression of these genes (APC and KRAS) in cancer is not methylation-dependent, although methylation may be observed. A number of canonical intracellular signalling genes associated with cancers were dysregulated (c-MET, ITG $\beta 1$, IGF1) or showed no change (SMADs). These results are difficult to interpret as there are multiple downstream targets of these pathways, many of which act in opposition to each other as a homeostatic mechanism, and some even display pro- and anti-tumoural activity depending on the stromal environment and other factors. However, classical tumour-suppressor gene CDKN2A was significantly up-regulated by demethylation with AZA, as was TRPM1, another gene associated with favourable features in melanoma. Clearly there is a mixed-picture in terms of the balance of up- and downregulated genes and those that may be hypothesised as pro- and anti-tumoural, and thus it is pragmatic to address the specific observations of the effects of AZA on DLD-1 cells when interreacting with and ECM-like substrate such as that encountered during the invasion assay. This might be best evaluated by examining the effects of AZA on the metalloproteinase group of genes on the PRC array. 


\subsubsection{Metalloproteinases \& qRT-PCR}

Based on array data, MMP and TIMP expression was significantly changed across the family (relative fold-change): MMP2 (-4.10); MMP11 (+2.54); MMP13 (+2.85); TIMP2 (-2.86); TIMP4 (+2.58). For each of the genes highlighted by the array, qRT-PCR data individually validated and showed statistical significance for the up- or downregulation observed in the array following exposure to $0.50 \mathrm{uM}$ AZA for $72 \mathrm{hrs}$, except for MMP13 which only showed an inverse but statistically significant $\Delta \Delta \mathrm{C}_{\mathrm{T}}$ after $24 \mathrm{hrs}$ exposure. Exposure to $\mathrm{RRx}-0010.50 \mathrm{uM}$ for $72 \mathrm{hrs}$ universally upregulated every MMP or TIMP in a manner that was inconsistent with the effects of demethylation with AZA, but these effects only reached significance for MMP2 and TIMP4. In the case of MMP2, exposure to RRx-001 upregulated MMP2 in a contrary manner to exposure to AZA ( $\mathrm{p} \leq 0.01)$. Unfortunately, MMP9 could not be included in the analysis due to unreliable qRT-PCR data. These results are summarised in Table 5.5.

Table 5.5 Summary of $\mathrm{RT}^{2}$ Profiler PRC Array and qRT-PCR data for MMPs \& TIMPs.

\begin{tabular}{|c|c|c|c|c|c|c|c|}
\hline \multirow{2}{*}{$\begin{array}{c}\text { fold- } \\
\text { fhange }\end{array}$} & $\Delta \Delta \mathrm{C}_{\mathrm{T}}$ & $p$ & $\Delta \Delta \mathrm{C}_{\mathrm{T}}$ & $p$ & $\Delta \Delta \mathrm{C}_{\mathrm{T}}$ & $p$ \\
\cline { 3 - 8 } & & \multicolumn{2}{|c|}{$24 \mathrm{hrs}$ AZA } & \multicolumn{2}{|c|}{$72 \mathrm{hrs} \mathrm{AZA}$} & \multicolumn{2}{|c|}{$72 \mathrm{hrs} \mathrm{RRx}-001$} \\
\hline MMP2 & -4.10 & 0.50 & $\mathrm{NS}$ & 0.24 & $\mathrm{p} \leq 0.001$ & 4.79 & $\mathrm{p} \leq 0.01$ \\
\hline MMP11 & +2.54 & 2.25 & $\mathrm{NS}$ & 3.26 & $\mathrm{p} \leq 0.01$ & 1.47 & $\mathrm{NS}$ \\
\hline MMP13 & +2.85 & 0.70 & $\mathrm{p} \leq 0.05$ & 0.76 & $\mathrm{NS}$ & 1.31 & $\mathrm{NS}$ \\
\hline TIMP2 & -2.86 & 0.87 & $\mathrm{NS}$ & 0.37 & $\mathrm{p} \leq 0.001$ & 1.22 & $\mathrm{NS}$ \\
\hline TIMP4 & +2.58 & 1.45 & $\mathrm{NS}$ & 5.89 & $\mathrm{p} \leq 0.05$ & 2.94 & $\mathrm{p} \leq 0.05$ \\
\hline
\end{tabular}

These results demonstrate that as well as having the biggest fold-change based on the array data, MMP2 and TIMP2 also had the most statistically significant expressional change following $72 \mathrm{hrs}$ AZA exposure, based on $\Delta \Delta \mathrm{C}_{\mathrm{T}}$ qRT-PCR, although not necessarily the biggest absolute change. The large observed $\Delta \Delta \mathrm{C}_{\mathrm{T}}$ in MMP11 and TIMP4 were less statistically significant due to a relatively high standard deviation of mean $\Delta \Delta \mathrm{C}_{\mathrm{T}}$ for both genes, although they were above the threshold for significance. The results for MMP13 demonstrated borderline significance for change in expression (actual figure $p=0.03$ ) at $24 \mathrm{hrs}$ exposure to AZA, but not at $72 \mathrm{hrs}$, despite a minimal standard deviation from the mean $\Delta \Delta \mathrm{C}_{\mathrm{T}}$ for each exposure time. This result was also contrary to that expected from the array data and suggests 
the likelihood of a weak or insignificant effect of AZA on MMP13 at that dose across those time-points.

The results for RRx-001 were in many ways inconsistent and divergent from those of AZA, although there was an increased expression of each gene examined. Only MMP2 and TIMP4 demonstrated statistically significant $\Delta \Delta \mathrm{C}_{\mathrm{T}}$, but the absolute value for expressional change in each of these genes was high. There was however a high degree of deviation in the mean $\Delta \Delta \mathrm{C}_{\mathrm{T}}$ values for most genes following exposure to $\mathrm{RRx}-001$; even in the genes demonstrating significant expressional change and statistical significance, and thus the effects of this agent on this family of genes is probably highly variable. The variability on results is likely be the result of alkylation and free-radical generation being imprecise modes of action affecting multiple downstream processes, as opposed to AZA's specific epigenetic effect.

The data from this study indicate that MMP2, the primary proteolytic enzyme of type-IV collagen was significantly downregulated by exposure to AZA; corresponding with the observation that DLD-1 cell treated with AZA are less able to invade a type-IV collagen-rich ECM. However, simultaneous upregulation of MMP11 expression, which is itself a proteolytic inhibitor of alpha-1-antitrypsin (another inhibitor of proteases), may have increased overall non-MMP proteolytic activity via a double-negative effect. TIMP2 was also observed to decrease expression following AZA exposure, thus the inhibitory effect of TIMP2 on MMP2 has also been down-regulated, contrary to the observed effect of less-ECM degradation. TIMP4 expression on the other hand increased following exposure to AZA; an unexpected finding as this protein is not normally found in the colon. Although the reduced propensity of DLD-1 cells to invade the ECM correlated with decreased MMP2 expression, the other findings of differential MMP and TIMP expression following AZA exposure do not fit well into a unified theoretical construct of hypermethylation resulting in increased potential for invasion.

However, the relationship between expression and inhibition of MMPs and TIMPs is complex, and separating the homeostatic controls from direct demethylation is challenging ${ }^{581}$. The data from this chapter suggests that AZA significantly reduced the expression of MMP2 but also TIMP2, although in different proportions. However, whether the decreased expression of MMP2 is as a result of direct demethylation with AZA or a homeostatic mechanism in response to reduced expression of TIMP2, or visa versa, or novel expression of TIMP4, is not clear. 


\subsection{Conclusions}

RT-PCR array for gene expression associated with the EMT-like and invasive / metastatic processes associated with EMVI identified a number of genes of interest that may mechanistically account for the decreased propensity of DLD-1 cells to invade following exposure to AZA, and for CIMP-high tumours to demonstrate worse clinicopathological features. The family of genes that showed the most global change in expression were the metalloproteinases and their associated inhibitors. The MMPs and TIMPs are important in the EMT-like and invasive processes of colorectal cancer due to their actions on the components of the $\mathrm{ECM}^{503,504}$. qRT-PCR for these MMPs and TIMPs revealed a complex picture of increased and decreased expression; both temporally and in relation to known and putative demethylating agents, although $72 \mathrm{hrs}$ of exposure to AZA resulted in statistically significant expressional change in metalloproteinases and their inhibitors with low margins of error. Despite the observations of expressional change qRT-PCR does not elucidate the transcriptomal or homeostatic mechanisms responsible for the observed effects in vitro. This, primarily, is due to the complexity of MMP and TIMP regulation, and how epigenetic modification may influence these processes. Chapter 6 will aim to explore these relationships in more detail. 


\section{Chapter 6}

\section{Metalloproteinase Homeostasis and Demethylation}

\subsection{Introduction}

\subsubsection{Metalloproteinase Homeostasis}

The regulation and function of MMPs and TIMPs is complex, occurring at a transcriptomal, post-transcriptomal, and extracellular level ${ }^{581}$. At a transcriptomal level, MMPs are regulated by trans activators such as AP-1, PEA3, Sp-1, etc, in response to a large number of cytokines, growth factors, and integrins; primarily via MAPKs and wnt/ $\beta$-catenin canonical pathways ${ }^{582}$. Snail protein, for instance, has been shown to induce multiple MMPs and reduce E-cadherin expression, based upon downregulation of $\beta$-catenin in a process associated with EMT and differential intermediate filament expression ${ }^{583}$. Despite the consistency of $c i s$ elements within the MMP family, functionally similar MMPs often display different combinations of elements that may be shared between functionally unrelated MMPs, facilitating cis-element grouping (Groups 1-3) ( $^{582}$. The balance and distribution of trans-activators and cis-elements across the MMP family is believed to facilitate homeostasis; a fall in one cis group being compensated by the reciprocal increase in expression of the other as they both express MMPs with a similar class of action. For instance, MMP9 (Gelatinase B) is in cis group 1, whilst MMP2 (Gelatinase A) in in cis group 3. Loss of this reciprocal expression is thought to underlie the development of some cancers ${ }^{584,585}$.

Epigenetic events have also been identified in the transcriptomal regulation of MMPs and TIMPs, with both hyper- and hypomethylation (and histone modification) being implicated in the differential expression of several metalloproteinases ${ }^{586}$. In lymphoma cell lines it has been shown that pre-existing hypermethylation of MMP9 inhibits expression, an effect that can be overcome by demethylation with $\mathrm{AZA}^{587}$. MMP9 is itself important in the progression of tumours but has primarily been identified as a key factor in inducing angiogenesis by VEGF and the metastatic processes associated with MET, rather than EMT, whereas MMP2 is not critical in this function ${ }^{588}$. In Chapter 5, expression of MMP9 was not changed following exposure to AZA based on the array, but unfortunately qRT-PCR for MMP9 failed. In another study, however, knockdown of DNMT1 and DNMT3a by homologous recombination in 
colorectal cancer cell line HCT116 induced MMP3 expression whilst not affecting MMP1 and MMP2 expression significantly, thus the role of methylation, and the manipulation thereof, in inducing and repressing MMPs is complex ${ }^{589}$. As well as promoter methylation, histone modification also has a role in the epigenetic regulation of MMPs and can act in tandem with DNA methylation to control the "inducibility" of MMPs. For instance, methylation-dependent chromatin restructuring, which was itself regulated by activation of the MEK-1/extracellular signal-regulated kinase and NF- $\kappa \mathrm{B}$ signalling pathways, was found to be regulatory in inducible MMP9 expression ${ }^{590}$. The role of methylation and chromatin was also highlighted in the specific case of MMP2 regulation by Vincent, who reported that methylation does not directly regulate MMP14, MMP2, or TIMP2 expression in human placenta, although it may contribute to chromatin remodelling-dependent expression of the MMPs ${ }^{591}$. Similarly, Chernov indicated that DNA demethylation alone was insufficient to modify the expression of MMPs in number of cancer cell types but was important in a "multilayer epigenetic regulation" that also involved chromatin restructuring ${ }^{592}$.

In addition to transcriptomic control, post-transcriptomal regulation also has a role in regulating the expression of gelatinase MMPs. Such activity includes the prolongation of the half-life of MMP2 and MMP9 mRNA as may be observed in activated fibroblasts, or in response to innate cortisol, integrins, or applied therapeutics in the case of other MMPs ${ }^{593-596}$. Translational regulation of MMP \& TIMP proteins in the ribosome may also contribute to the overall phenotypic expression of gelatinase-related activity, and dysregulation at this level has been associated with some forms of autism 597,598 . However, once gene transcription and protein translation has occurred, the primary method of regulating MMP activity is by specific inhibitors secreted into the stroma.

TIMPs are the specific inhibitors of MMPs (as well as some other ECM proteolytic enzymes) and classically are the primary regulators of ECM turnover through their direct inhibition of MMPs; inhibition thus resulting in a homeostatic shift towards EMC component deposition as opposed to degradation ${ }^{581}, 599,600$. TIMPs therefore have an important role in healthy organogenesis and tissue regeneration but have also been associated with conditions characterised by maladaptive ECM turnover such as keloid, lung fibrosis, heart failure, and cancer $^{601,602}$. As Type-IV collagen is the primary component of the basement membrane, much of the focus of research has been directed towards MMP2 and TIMP2, both in cancer and other conditions $^{603,604}$. 
MMP2 is inhibited by the wedge-shaped TIMP2 directly blocking its substrate binding site in a stochastic manner ${ }^{605,606}$. As well as its role as an inhibitor, TIMP2 also auto-antagonistically activates MMP2 from its zymogen by co-association with membrane-type-1 MMP (MMP14) ${ }^{602}$. The regulation of MMP2 activity in the stroma is therefore based upon the concentration of MMP2, activation by MMP14, and the balance of the dual roles of TIMP2: the MMP2/TIMP2/MT1-MMP axis ${ }^{592}$. MMP14 was not assessed on the array or in qRT-PCR in Chapter 5. The role of TIMP2 and membrane-type MMPs in modulating MMP2 remains controversial. Some authors report that the activating/inhibitory relationship is dependent of the induced over-expression of activating membrane-type MMPs by fibroblasts in the stroma adjacent to early tumours, with tumour cell expression of activating factors being a characteristic only of more advanced tumours ${ }^{607}$. The activation and secretion of MMPs has also been suggested as a role of pro-tumoural tumour-associated neutrophils; a mechanism potentially underling the observations that the ratio of neutrophils to lymphocytes can have prognostic implications for colorectal cancers ${ }^{608,609}$.

Like MMPs, most TIMPs have multiple transcriptional control sites and respond to a range of signalling pathways; TIMP2 includes a TATA box at -30 bp, as well as a Sp1, two AP-2 sites, three PEA3s, and an AP- $1^{610}$. Methylation events in the regulation of TIMPs are not well characterised, and the overarching narrative of the MMP/TIMP literature is that TIMPs play a poor second-fiddle to their better studied counterparts. There is, however, some evidence that TIMP2 has dual CpG islands in its promotor region, and that the balance of methylation at these islands has an important regulatory role in transcription ${ }^{592}$.

As well as MMP2 and TIMP2, the array and qRT-PCR data also highlighted significant changes to the expression of TIMP4, an inhibitor of metalloproteinases which may also be important in cancer ${ }^{611}$. TIMP4 is a less well characterised inhibitor than TIMP2 that has also been shown to inhibit MMP2 by interaction with MMP14 ${ }^{612,613}$. However, unlike TIMP2, TIMP4 has no role in activating the MMP2 zymogen ${ }^{614,615}$. TIMP4 has also shown activity against MMP9, and thus is considered as a less-potent but broader-acting inhibitor of Gelatinases, although its normal expression is limited to the heart, brain, and kidneys. One of the reasons for the limited distribution of TIMP4 is the differential expression of initiator sites in the promotor region; which demonstrates sites for myogenin, GATA and Ets binding but lacks the AP1 or AP2 sites characteristic of other TIMPs and MMPs ${ }^{616,617}$. Although elevated 
levels of MMP4 have been identified in a number of different cancers (ovarian, head \& neck, and pancreatic adenocarcinomas), there is little evidence for its dysregulation in colorectal cancers $^{611,618}$.

Clearly the regulation and function of MMPs and TIMPs is complex, occurring at a transcriptomal, post-transcriptomal, and the extracellular level ${ }^{581}$. In many circumstances TIMP and MMP genes are transcribed simultaneously, though the specific subset of MMPs and TIMPs depends on the external stimulus or internal genetic or epigenetic factor promoting or limiting expression. Once secreted into the stroma, the action of MMPs is dependent on the balance of activation and inhibition, although the relationship between MMPs and their corresponding TIMPs is not binary, and in many cases likely to be multifaceted. There is however an established relationship between MMP2, TIMP2, \& MMP14 (MT-1 MMP), and potentially an emerging role for TIMP4 in this axis.

\subsection{2 siRNA Knockdowns}

When investigating the function of a gene and its coded protein a key tool to the investigator is the ability to prevent that gene being expressed, thus the function can be analysed by its absence. This is the principle behind knockdown technology, which can occur at the transcriptomal or post-transcriptomal levels. Pharmacological inhibition of target proteins is fundamentally different in terms of the phenotypic effects as the protein has already been synthesised so it is still present in the cell or microenvironment and its degree of function determined by the pharmacokinetics of the inhibitory agent ${ }^{619}$. At a genetic level, knockdowns are divided by their level of action; either to prevent transcription (as in the cases of CRISPR/Cas9 or TALE/TALEN) or post-transcriptional (such as RNA interference) ${ }^{620}$. However, the degree of knockdown is rarely $100 \%$, meaning that a limited expression is usually apparent despite application of the technology due to a number of factors, including the binding saturations or transfection efficiency of the method used or cellular heteroploidy. The degree of knockdown and its reversibility will determine partly which method is applied, but also the more rudimental considerations such as speed, complexity, local expertise, and cost of each technology ${ }^{620}$. Note that knockout technology (mice and, increasingly, rats) is different in that it describes otherwise fully functioning animals that have had genes completely removed or replaced at a stem cell level prior to embryologic development, and therefore completely lack a particular gene ${ }^{621}$. 
At the post-transcriptomal level, short interfering RNAs (siRNAs) and their cousin the short hairpin RNA (shRNA) are a well-established method of preventing RNA transcription and thus gene knockdown ${ }^{622,}{ }^{623}$. siRNA knockdown describes a process where RNA-targeted RNA oligonucleotides (20-25bp) bind intracellular proteins (following transfection) to form an RNA-induced silencing complex (RISC) that blocks translation of partially-complementary RNA sequences and degrades highly-complementary RNA sequences ${ }^{624,625}$. As translation is prevented no protein is synthesised. siRNAs demonstrate less off-target effect than other knockdown technology, such as phosphorothioate S-DNA knockdowns, whilst being acceptably specific in terms of the sequence-specificity and thus gene of interest targeting. Concerns regarding the activation of the innate immune system by siRNAs is relevant only to their application in experimental systems or therapeutics involving live organisms with an intact immune system ${ }^{626}$.

In an siRNA model dsRNA must be delivered into the target cell, necessitating penetration of the cell membrane. This may be achieved by three methods; transfection, electroporation, or viral-vector. Transfection involves the use of cationic liposomes, or other positively charged microparticles (such as polymer nanoparticles), that interact favourably with the negatively charged cell membrane to deliver siRNAs. This technique has the benefits of being cheap, reproducible, and effective at delivering siRNAs to most cell types, although it has a low efficiency in in vivo models ${ }^{627}$. Electroporation describes a process whereby an electrostatic field is applied to cells which transiently disrupts the cell membrane allowing passage in of siRNAs, but also the passage out of cell contents. This method has some advantages, including the potential for targeted siRNA delivery in vivo, but has a higher risk of toxicity and cell death than transfection ${ }^{628,629}$. Viral-vector methods rely on engineered viruses, typically lentivirus but also retrovirus or adenovirus, to deliver siRNAs intracellularly ${ }^{630}$. Viral vectors have the benefit of being able to be applied to whole-organism systems, and may have a future role in delivering siRNA based therapeutic to hard-to-reach targets, such as the central nervous system in Huntingdon's Disease ${ }^{631}$. As such, siRNA-based technologies are increasingly being regarded as potentially viable therapeutic systems and are established in the in vitro manipulation of cells to investigate the knockdown-phenotype ${ }^{632-634}$. 


\subsubsection{Immunocytochemistry \& Protein Analysis}

In Chapter 5 the expression of genes was investigated by assessment of the relative amounts of mRNA that had been transcribed from DNA, thus demonstrating the relative transcription of the genes of interest (identification and quantification). Other methods of investigating gene expression examine the protein end-products of transcription via protein-specific methods; specifically, immunocytochemistry (ICC) and Western Blotting (WB) for proteins of interest. These techniques have the benefits of relatively rapidly demonstrating quantifiable changes in protein expression and can act as a verification of qRT-PCR.

ICC exploits protein (or polypeptide) specific ligands (primary monoclonal antibodies (mAbs)) that bind the protein of interest and can then be detected by fluorescence microscopy, either directly if they primary antibodies are themselves conjugated to a fluorophore (direct immunofluorescence), or via a secondary antibody that targets the primary and is itself conjugated to a fluorophore (indirect immunofluorescence (IIF) ${ }^{635,636}$. This method allows the detection of specific proteins and is able to facilitate in localising the target within the cell. As techniques in ICC have evolved the sensitivity and specificity of primary antibodies has improved, as has the stability of the fluorophore ${ }^{637}$. The combination of fluorescence techniques with electron microscopy have further refined the level detail available to investigators examining the intracellular distribution of proteins ${ }^{638}$. ICC does, however, have limitations as a process; specifically, that as the cells being studied have to be fixed and made permeable to the mAbs; cells are thus not live during assessment by standard techniques and dynamic changes are therefore more challenging to study. Also, as cells require fixing and washing, ICC is limited in its ability to examine the extracellular distribution of secreted proteins, although it is able to determine intracellular stores of secreted proteins and proteins undergoing synthesis ${ }^{639}$. As with all laboratory investigations, it is important to use appropriate controls in ICC experiments to avoid confounding reults ${ }^{640}$.

Western Blotting is a well-established and widely-used semiquantitative or quantitative technique for examining specific cellular proteins ${ }^{641}$. WB can be applied to samples as limited as a single cell and only a few $\mu \mathrm{g}$ of total protein, but care must be taken when processing samples during this multi-stage process to avoid erroneous results ${ }^{642}$. Like ICC, WB relies on specific mAbs binding target proteins and then a tagged secondary $\mathrm{mAb}$ facilitating visualisation, this time on polyacrylamide gels. Once protein-specific bands can be visualised a semiquantitative assessment of protein abundance can be made by densitometry or be used 
in combination with fluorescence techniques ${ }^{643}$. Despite the multi-step process, WB does has the advantage of being able to assess the quantity of extracellular proteins as well as intracellular proteins, unlike ICC, and therefore offers a more complete examination of the effects of expressional modification ${ }^{644}$.

\subsection{Aims \& Objectives}

This chapter will examine the effects of demethylation with AZA on MMP and TIMP protein expression by two methods; immunocytochemistry and Western Blotting. These investigations will serve not only to aid in understanding the distribution of these proteins, how then interact, and how this is affected by demethylation, but also to cross-validate the results of the qRTPCR. siRNA was also applied to DLD-1 cells in control and in conjunction with AZA exposure and the same assessment of MMP and TIMP proteins made; providing an insight into the homeostatic mechanisms of MMP and TIMP at a protein rather than transcriptomal level. Due to the divergence of the qRT-PCR results between AZA and RRx-001, and the unknown mechanisms of its actions, no further investigation will be conducted with RRx-001. 


\subsection{Methods}

DLD-1 and HFL-1 cells were cultured in control conditions according to the protocols described in Sections 2.3 and 4.3.1.2. Modification in culture protocols for siRNA knockdown (KD) and exposure to AZA are described in the relevant sections. HFL-1 were used as a positive control for the expression of MMPs \& TIMPs.

\subsection{1 siRNA Knockdown \& AZA Exposure}

The siRNA KD protocol employed in this assay indicates that, following a successful transfection, treated cells should be ready to assay 24-72hrs following treatment. However, because the effects of AZA require cell division to become evident, as demonstrated by the $72 \mathrm{hr}$ exposure producing significant effects on qRT-PCR whereas 24hrs exposure did not, the manufacturers protocol was modified to facilitate longer AZA exposure. As transfection occurs in a serum-free medium, the transfection reagent removal and $1 \mathrm{x}$ complete medium replacement method was employed, rather than the addition of $2 \mathrm{x}$ medium to transfection reagents. This was to avoid toxicity associated with transfection and to facilitate AZA exposure. KDs were performed against TIMP2 and TIMP4 and with a scrambled siRNA control.

DLD-1 and HFL-1 cell lines were cultured according to maintenance protocols and prepared in normal $(1 \mathrm{x})$ antibiotic-free growth medium at a concentration of $3 \times 10^{4}$ cells ml ${ }^{-1}$ (coulter counter assessment). Glass coverslips were sterilised in ethanol for 2 minutes and allowed to air dry in the bottom of a 6-well plate; three coverslips per well. Cells were then transferred to the wells containing coverslips allowed to adhere overnight. This technique facilitated both the production of control, AZA, and KD cells for subsequent ICC (cell on coverslips) and for protein analysis by WB (cells in the gaps). Three experimental arms were run: control, KD only, and KD with AZA.

All solutions (A\&B) were prepared in accordance with the manufacturer's protocols for siRNA transfection. $100 \mu \mathrm{L}$ of the siRNA Duplex Solution (Solution A) was added directly to $100 \mu \mathrm{L}$ of the dilute Transection Reagent (Solution B) per well and mixed by pipetting and incubated at room temperature for 30 minutes. Normal growth medium then was removed from the wells by pipette aspiration and the cells washed once with $2 \mathrm{ml}$ of Transfection Medium which was then aspirated. $800 \mu \mathrm{L}$ per well siRNA Transfection Medium was added to the siRNA Transfection Reagent mixture and mixed gently by pipetting. $1 \mathrm{ml}$ total volume per well of 
complete transfection and siRNA KD preparation was then overlaid onto the washed cells. Cells were then incubated for 6 hours in standard incubator conditions.

Following incubation, the transfection and KD preparation was removed and replaced by $1 \mathrm{x}$ antibiotic-free normal growth medium (containing serum) and the cells allowed a recoveryperiod of 2 hours. Following recovery, growth medium was removed and replaced with fresh medium for all wells, with the addition of $0.50 \mathrm{uM}$ AZA for treatment wells (fresh medium only for $\mathrm{KD}$ only) and incubated overnight. The entire process of transfection, recovery, and treatment with AZA or control was repeated for three consecutive days to allow effective demethylation with AZA. Following the final overnight incubation, the cells were assayed by ICC or protein analysis as described below. Figure 6.1 demonstrates a flow chart of treatment protocols for siRNA KD and AZA cell culture.

Figure 6.1 Flow-chart demonstrating treatment algorithms for siRNA KD and AZA cell culture of DLD-1 and HFL-1 cells. NGM = normal growth medium; D1-5 = Day 1-5.

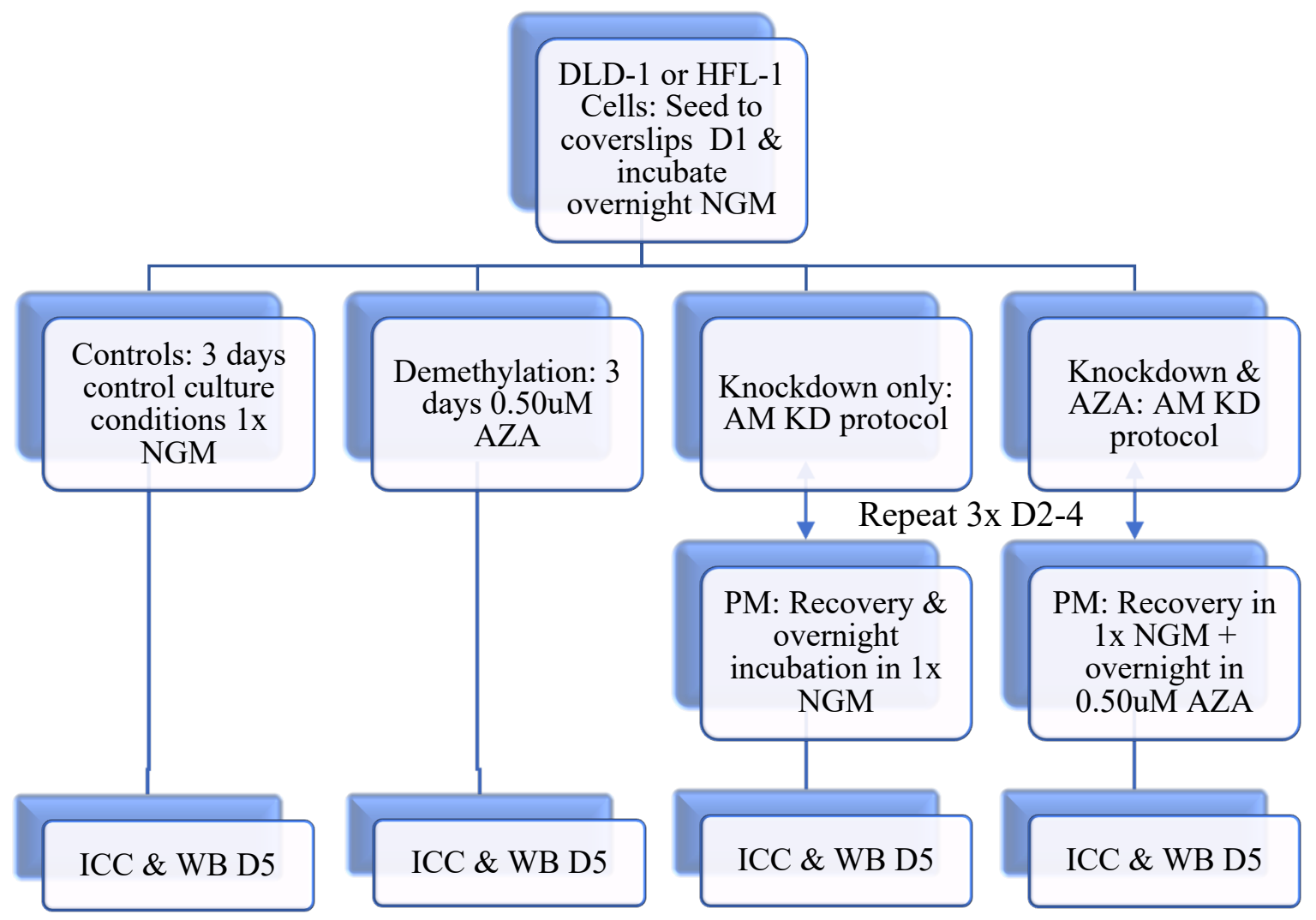




\subsubsection{Immunocytochemistry \& Immunofluorescence}

An indirect immunofluorescence (IIF) method was adopted for all ICC studies. Working concentrations of each primary and secondary mAb was determined by means of a serial dilution and probe test. Serial dilutions of primary antibodies in PBS and 1.5\% normal blocking solution were 1:50, 1:100, 1:200, 1:500; and 1:1000 and 1:2000 for secondary antibodies. Primary and secondary antibodies are listed in Chapter 2, Table 2.4.

Cells (DLD-1 and HFL-1; control, AZA, KDs, \& KDs + AZA) were grown on coverslips according to the appropriate protocols as described in Section 6.3.1. Cell-covered coverslips were then removed from growth medium using sterile forceps and scalpel-blades and then washed twice in PBS by submersion to ensure removal of phenol-red, and then fixed by submersion in $3.7 \%$ paraformaldehyde (PFA) in $\mathrm{PBS}$ at $4^{\circ} \mathrm{C}$ for 10 minutes. This was a deviation from the manufacturers protocol (recommending methanol, acetone, or formalin fixation), but $3.7 \%$ PFA is equivalent to $10 \%$ formalin and is thus considered interchangeable ${ }^{639}$. Following fixation coverslips were kept wet and rinsed in PBS 3 times and then permeabilised by incubation for 3 minutes in a solution of $0.1 \%$ Triton X-100 in PBS. Coverslips were then rinsed 3 times with PBS and then incubated in 10\% normal blocking agent (3\% BSA in PBS) for 30 minutes at room temperature. Following blocking, coverslips were then washed 3 times in PBS by submersion for 5 minutes. Coverslips were then replaced in 24-well plates and the primary antibody applied to completely cover each slide. The plate was sealed with biofilm to prevent drying and the cells incubated overnight at $4^{\circ} \mathrm{C}$.

Following primary antibody incubation, coverslips were washed 3 times in PBS by submersion and then incubated for 60 minutes at room temperature with the appropriate Alexa Fluor ${ }^{\circledR} 488$ conjugated secondary antibody (mouse or rabbit to match the primary $\mathrm{mAb}$ ) diluted in PBS with $1.5 \%$ normal blocking serum. Incubation with the fluorophore-conjugated secondary mAb was conducted in a darkened room with the plate wrapped in tinfoil to prevent degradation of the fluorophore. Coverslips were then washed 3 times in PBS by submersion for 5 minutes and immediately mounted to slides with VECTASHIELD mounting medium with DAPI nuclear stain (Vector Laboratories, Burlingame, CA, USA). Following incubation at $37^{\circ} \mathrm{C}$ for 10 minutes, slides were imaged on the Metafer Automated Slide-Scanning platform (fluorescence intensity assessment: RAPIDSCORE) and Olympus BX51 Fluorescence Microscope (highresolution images for cellular localisation of signal). Microscope settings were controlled for laser power and detector gain. 


\subsubsection{Western Blotting}

Total protein was prepared by gently pipetting excess growth medium off cells cultured in a 12-well plate so that cells remained in a thin film of medium containing their secreted extracellular proteins. Plates were kept on ice throughout protein extraction and an ionic detergent method of cell membrane disruption was employed, exploiting the sodium dodecylsulfate and sodium deoxycholate constituents of RIPA Buffer ${ }^{645}$. Cells were then scraped from the bottom of their wells with a sterile scalpel until fully detached and then resuspend in $200 \mu \mathrm{L}$ ice-cold RIPA Buffer supplemented with $2 \mu \mathrm{L}$ of protease and $2 \mu \mathrm{L}$ of phosphatase inhibitors. The cells and RIPA buffer were mixed thoroughly by pipetting and transfer to pre-chilled microcentrifuge tubes and incubated for 5 minutes at $4^{\circ} \mathrm{C}$. Cells were then lysed by vortexing thoroughly and then centrifuged for 10 minutes at $12,000 \mathrm{rpm}$ in a centrifuge pre-cooled to $4^{\circ} \mathrm{C}$. The supernatant was then transferred to a fresh microcentrifuge tube and stored at $-80^{\circ} \mathrm{C}$ until required for $\mathrm{WB}$.

The Bio-Rad DC Protein assay kit was used to determine the protein quantity in each sample. All reagents were prepared as per kit instructions and all consumables were supplied by BioRad (except Tris buffers and gel ingredients). A protein standard of Bovine Serum Albumin (BSA) was diluted in RIPA buffer at 5 concentrations plus a negative control ( 0 to $2.5 \mathrm{mg} / \mathrm{mL}$ ). Aliquots of $5 \mu \mathrm{L}$ of each protein sample or standard curve was then added to wells of microtiter plate (3 technical replicates). $25 \mu \mathrm{L}$ of working Reagent A was then added to every well plus $200 \mu \mathrm{L}$ of Reagent B and the plate was agitated for 15 minutes. The POLARstar Omega Microplate reader was then used to determine the protein concentration in each well, based on absorbances at $750 \mathrm{~nm}$ relative to the standard curve.

The proteins in the solution were the separated according to their molecular weight using the SDS-PAGE technique ${ }^{646}$. Two densities of gels were prepared for use as a stacking gel (4\%) and a resolving gel (10\%). Gels were hand-cast by the same method described in Section 3.3.6 but with different recipes and the additional step of $0.5 \mathrm{~mL}$ of isopropanol being added to the top of each gel to ensure that it was level. Gel recipes are listed in Table 6.1. The gels were then placed into an electrode assembly kit filled with running buffer. 
Table 6.1 Recipes for stacking (4\%) and resolving (10\%) gels. Listed quantities make 2 gels.

\begin{tabular}{|l|l|l|}
\hline & $\mathbf{4 \%}$ Stacking Gel & $\mathbf{1 0} \%$ Resolving Gel \\
\hline $30 \%$ Acrylamide & $650 \mu \mathrm{l}$ & $5 \mathrm{ml}$ \\
\hline ddH2O & $3 \mathrm{ml}$ & $6 \mathrm{ml}$ \\
\hline $1.5 \mathrm{M}$ Tris & $1.25 \mathrm{ml}$ & $3.75 \mathrm{ml}$ \\
\hline $1.0 \mathrm{M}$ Tris & $50 \mu \mathrm{l}$ & $150 \mu \mathrm{l}$ \\
\hline $10 \%$ SDS & $25 \mu \mathrm{l}$ & $75 \mu \mathrm{l}$ \\
\hline TEMED & $5 \mu \mathrm{l}$ & $15 \mu \mathrm{l}$ \\
\hline
\end{tabular}

Proteins were thawed to room temperature and diluted at a 1:1 ratio with Laemmli buffer and then sonicated and heated at $95^{\circ} \mathrm{C}$ for 5 minutes. The first two wells of the gel were loaded with $8 \mu \mathrm{L}$ of dual-colour standard and biotinylated standard, respectively, and then a total of $40 \mu \mathrm{g}$ of each protein sample was loaded into subsequent individual wells. Gels were the run at 160 volts for 5 minutes (until the samples had reached the end of the stacking gel) and then 120 volts until the dye front approached the base of the resolving gel.

Immunoblotting was then performed to transfer the proteins onto PVDF membranes for antibody-probe analysis. One Immuno-Blot PVDF membrane per gel was activated by immersion in $100 \%$ methanol until translucent. All materials required for the protein transfer cassette, including fibre pads, filter paper, gels and membranes were equilibrated for 10 minutes in transfer buffer pre-cooled to $4^{\circ} \mathrm{C}$. The transfer cassette was then assembled closed tightly to ensure that no air bubbles were present between the gel and the membrane. Electroblotting was performed at $400 \mathrm{~mA}$ for $1 \mathrm{~h}$ at $4^{\circ} \mathrm{C}$. Following transfer, membranes were carefully removed from the transfer cassette using forceps and briefly washed in TBS/T wash buffer.

The PVDF membranes were then blocked for 60 minutes in 5\% BSA at room temperature and then washed with TBS/T. Membranes were then incubated at $4^{\circ} \mathrm{C}$ overnight in $8 \mathrm{~mL}$ of primary antibody solution diluted in blocking buffer to 1:1000. The same set of primary antibodies for MMP2, TIMP2, and TIMP4 employed in IIF were used for WB as they are all recommended for use in both processes by the manufacturer, and are listed in Chapter 2, Table 2.4. Membranes were then washed in TBS/T washing buffer 4 times each for 5 minutes with strong agitation. A second blocking process was then carried out at room temperature for 60 minutes 
and with 1:1000 times diluted horseradish peroxidase conjugated secondary antibody (antimouse or anti-rabbit) for chemiluminescent detection. Membranes were again washed in TBS/T washing buffer 4 times each for 5 minutes with strong agitation.

Visual analysis of the protein quantities was then carried out the ImmunoStar Chemiluminescence Kit (BioRad). A 1:1 ratio of luminol/enhancer and peroxide solution was applied over the membrane and the ChemiDoc XRS (BioRad) system used to assess and quantify the relative band intensities on the membrane. Following imaging, the membrane was then stripped of the bands of interest using a stripping buffer (Thermo Scientific) at room temperature for 10 minutes with strong agitation, followed by washing 4 times for 5 minutes with a gentle agitation in TBS/T solution. A primary antibody for $\beta$-actin was then applied to the membrane (pre-mixed laboratory consumable supplied by a colleague) and the membrane re-incubated overnight at $4^{\circ} \mathrm{C}$ before being washed, blocked, and imaged using the ChemiDoc $\mathrm{XRS}$ as described above. This visualisation using the beta-actin was then used to normalise the absolute protein quantities for MMP2, TIMP2, and TIMP4.

\subsubsection{Statistics and Data Presentation}

Results for change in abundance are described in terms of relative change in target protein abundance from the control based on either change in fluorescence or chemiluminescence. Control abundance will always be 1.0 with no standard deviation. Results of $<1.0$ indicates relative downregulation and $>1.0$ indicates relative upregulation. If absolute values for baseline are 0.0 (no protein detected) then the control abundance will still be 1.0, and subsequent results returning no protein detection will remain as 1.0. In the case of WB, protein abundance will also be controlled against $\beta$-actin abundance to provide internal normalisation of bands using ChemiDoc XRS. Standard descriptive statistics will be employed as appropriate and a Student's-T test used to assess any significance between two data-arrays. Cut-off for significance is set to $p=0.05$. 


\subsection{Results}

Protein abundance was assessed by indirect immunofluorescence (IIF) for MMP2, TIMP2, and TIMP4 following the 4-arm exposure protocol described in Section 6.3.1. These proteins were selected as their corresponding genes were most significantly changed by AZA exposure based on array and qRT-PCR data in Chapter 5, and the MMP2-TIMP2-MMP14 axis is important in regulating degradation of Type-IV collagen rich extracellular components. Unfortunately, some technical and methodological issues limited the results gathered in this section of the investigation, although meaningful results were still recorded. Firstly, although HFL-1 cells were able to be maintained in normal culture medium in control conditions they did not survive the transfection process adequately, meaning that although data could be collected for their baseline MMP2 and TIMP expression, the relative effects of AZA and KD could not be assessed. This technical issue was unexpected as HFL-1 cells are generally easy to culture and have been shown to tolerate siRNA transfection targeting their metalloproteinase activity in a number of pervious studies ${ }^{647,648}$. Specific issues relating to ICC and WB will be mentioned in the relevant results sections and explored further in the Discussion. Raw proteomic data is provided in Appendix IV.

\subsubsection{Immunocytochemistry and Immunofluorescence}

All treated samples underwent successful fixation, permeabilization, and primary and secondary antibody conjugation. There was not an apparent high-degree of cellular dehydration during fixation, consistent with the use of PFA ${ }^{649}$. Control HFL-1 cells demonstrated appropriate fluorescence when probed with each primary mAb an a manner consistent with the Human Protein Atlas (HPA) indicating adequate permeabilization with Triton X-100 and appropriate probe-access to crosslinked proteins ${ }^{650}$. A significant methodological limitation of this element of the experimental design is that MMP2 protein is only normally weakly present inside colonic cells and TIMP2 and TIMP4 are usually undetectable as they are almost exclusively extracellular, although significant upward expression of any protein may be detectable immediately following intracellular synthesis and before secretion, as should any decrease in MMP2. Results are presented graphically in Figure 6.2 and Metafer images illustrating fluorescence are presented in Figure 6.3. 
Figure 6.2. Relative fluorescence RAPIDSCORE for MMP2 and TIMP2 normalised to control fluorescence of 1.0. Error bars are standard deviation from the mean corrected fluorescence. [ $\Delta$ mean fluorescence from control* $p \leq 0.05 ; * * p \leq 0.01 ; * * * p \leq 0.001 . \Delta \Delta$ mean fluorescence between target proteins ${ }^{\circledR} p \leq 0.05 ;{ }^{\circledR @} p \leq 0.01$; $\left.@ @ @ p \leq 0.001\right]$

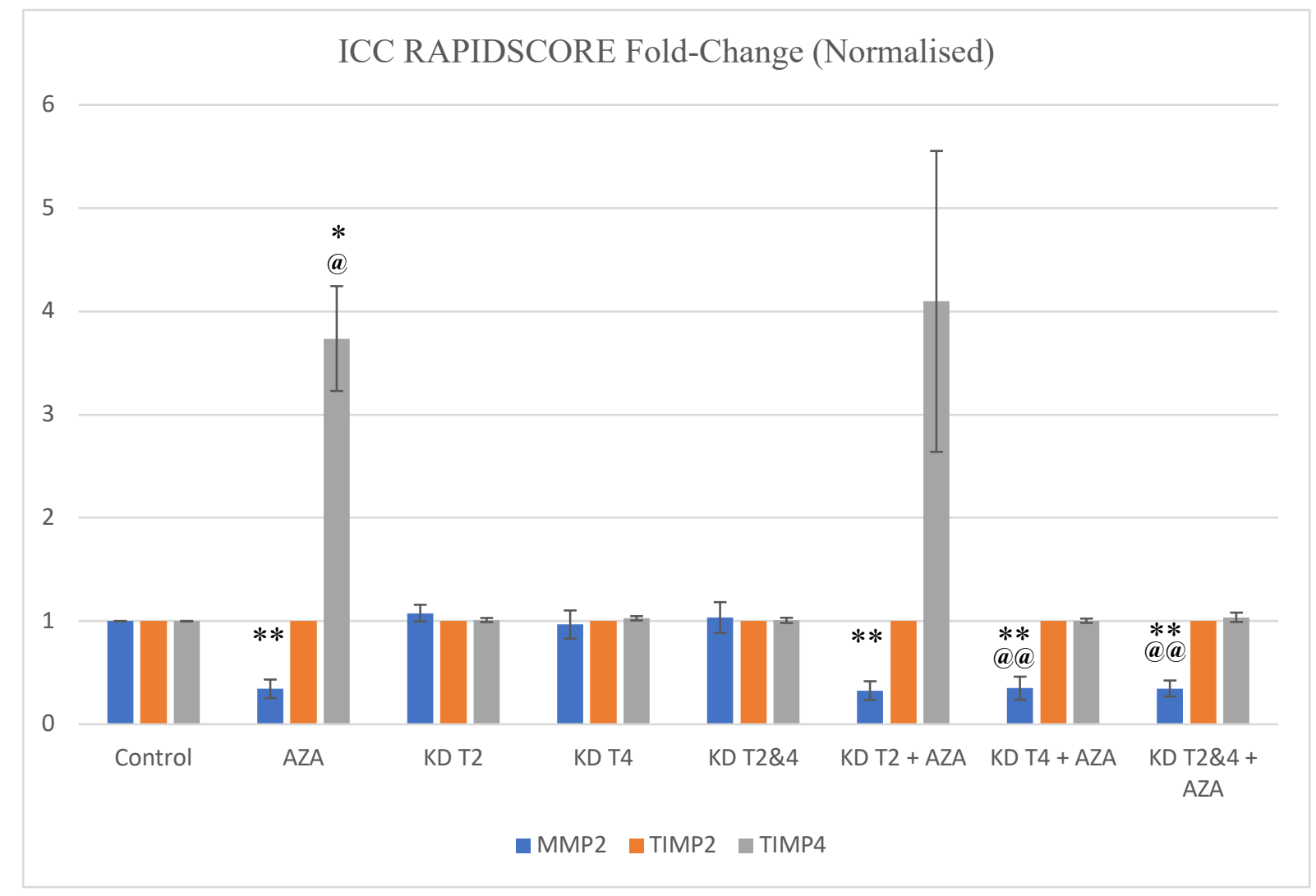

Note that the relative fluorescence is normalised to control and does not represent absolute values, thus TIMP2 and TIMP4 values are controlled to an expression of close to zero and subsequent negative fluorescence will return a result of 1.0 and standard deviation will be low. Demethylation with AZA resulted in a de novo expression of TIMP4 as well as a reduction in MMP2, but that KD of TIMP4 in association with AZA did not prevent the MMP2 response, therefore reduction in MMP2 expression is not dependent of the presence of TIMP4. The lack of significance observed in the response of TIMP4 to KD of TIMP2 and AZA is due to the high degree of deviation in individual TIMP4 RAPIDSCORE values (precise figures for $\Delta$ control and $\triangle \triangle \mathrm{MMP} 2$ were $p=0.067$ and $p=0.052$, respectively, indicating a high degree of trend). 
Figure 6.3 Representative immunofluorescence (IIF) images for MMP2, TIMP2, and TIMP4 in DLD-1 cells following exposure to control, AZA, knockdown, or combination protocols.

MMP2 IIF
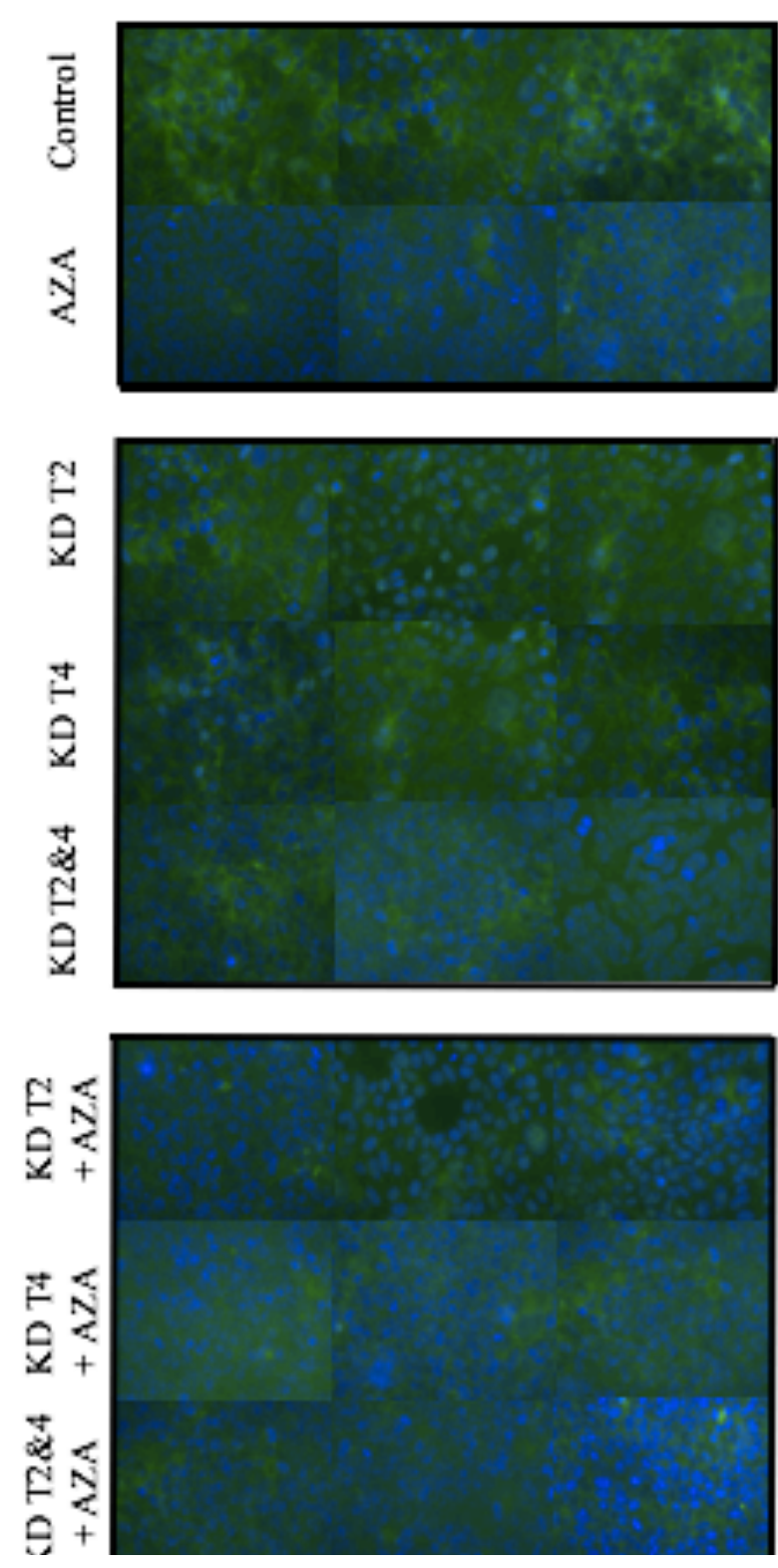

TIMP4 IIF
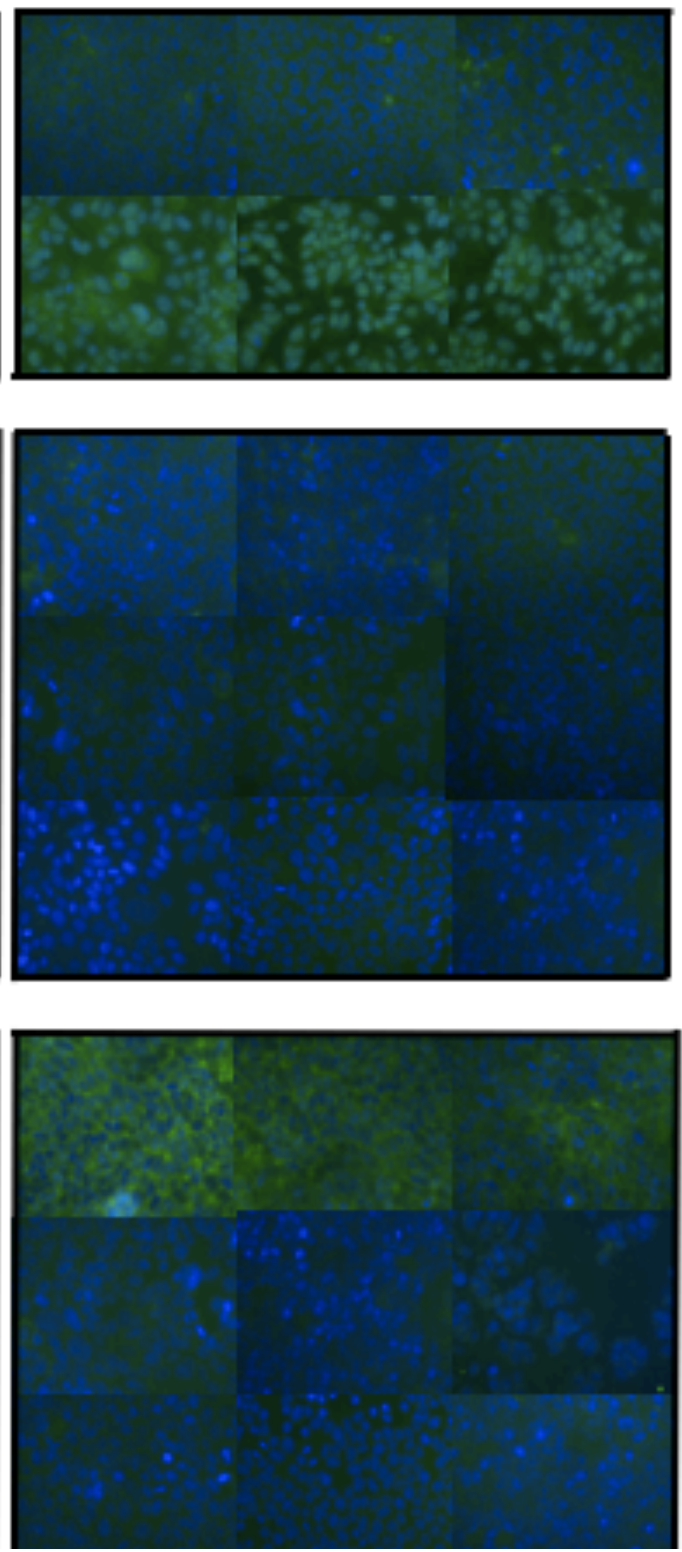

TIMP2 IIF

AZA: $0.50 \mathrm{uMAZA} 72 \mathrm{hrs}$ KD T2: Knockdown TIMP2 KD T4: Knockdown TIMP4 KD T2\&4: Knockdown both TIMP2 \& TIMP4

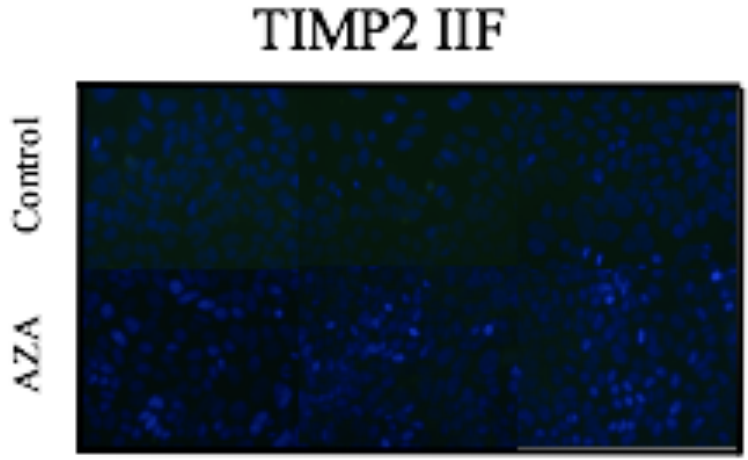


Control ICC for baseline signal from each of MMP2, TIMP2, and TIMP4 was consistent with that expected based upon the HPA; specifically, that MMP2 demonstrated a detectable signal and that TIMP2 and TIMP4 were undetectable. This was due to limited abundance of these TIMPs within the cell. Control ICC for DLD-1 cells demonstrated the presence of intracellular MMP2 but neither TIMP2 nor TIMP4. This was consistent with the predicted result of the HPA which predicted no intracellular TIMPs, but not with the array or qRT-PCR data that suggested that there was a baseline expression of each gene, although this was much higher for MMP2 and TIMP2 than TIMP4.

Following exposure to $0.50 \mathrm{uM}$ AZA for $72 \mathrm{hrs}$, ICC demonstrated a relative decrease in MMP2 expression $(\Delta=0.34 \pm 0.09 ; p=0.006)$, no change in TIMP2 expression, but an increase in TIMP4 expression $(\Delta=3.74 \pm 0.51 ; p=0.011)$. These results are partially consistent with the qRT-PCR data, confirming the relative change in abundance/expression of MMP2 and TIMP4, but not demonstrating any significant change in TIMP2. As baseline TIMP2 was negative, the expected decrease in protein abundance/expression would not be expected to be evident.

Following siRNA KD of TIMP2, TIMP4, or TIMP2 \& TIMP4, DLD-1 cells demonstrated no change in abundance of MMP2 and no change to the lack of signal for TIMP2 and TIMP4. Scramble knockdowns did not result in any change in baseline signal for any target. Validation was provided by Western blotting, see Section 6.4.2.

When KDs were performed in association with treatment with $0.50 \mathrm{uM} \mathrm{AZA,} \mathrm{the} \mathrm{previously}$ observed decrease in MMP2 following AZA exposure alone was preserved in each experimental arm $(\Delta=0.32-0.35 \pm 0.07-0.11 ; p=0.005-0.01)$. The increased expression in TIMP4 following exposure to AZA was also observed when anti-TIMP2 siRNA was employed in combination with AZA but did not reach statistical significance due to a high degree of deviation, although a strong trend was observed $(\Delta=4.10 \pm 1.46 ; p=0.07)$. This effect was lost when TIMP4 was knocked down. These observations confirm the effective KD of TIMP4 and also indicate that the increase in TIMP4 observed with AZA is not dependent on TIMP2 downstream signalling (TIMP2 KD was confirmed by WB). The results also indicate that the reduction in MMP2 abundance is not dependent on TIMP4 protein abundance, as the effect is preserved when TIMP4 is knocked down. 
Fluorescence microscopy images acquired using the Metafer platform are provided in Figure 6.3 to illustrate the differing abundance of detected proteins. The quality of slide preparation and resulting image blurring prevented meaningful evaluation of the cellular distribution of MMP2 and TIMP4 and was thus abandoned.

\subsubsection{Western Blotting}

Protein abundance was observed for MMP2, TIMP2, and TIMP4 following the 4-arm exposure protocol described in Section 6.3.1. Unfortunately, a technical difficulty was encountered in the primary antibody probing or secondary conjugation for TIMP4 as chemiluminescence failed to detect any bands irrespective of experimental arm. Protein extraction and other processes for these samples were however adequate as $\beta$-actin did produce visible bands after stripping and re-probing. All results are illustrated in Figure 6.4.

Baseline expression of MMP2, TIMP2, and TIMP4 in HFL-1 cells was in keeping with the expected phenotype; with both MMP2 and TIMP2 detectable, although probing for TIMP4 failed. This is consistent with typical fibroblast activity and confirms that ICC results for TIMP2 are due to lack of intracellular protein, rather than no transcription or protein synthesis. As stated previously, HFL-1 cells did not tolerate transfection and thus only this baseline assessment produced reliable results. In DLD-1 cells, baseline abundance of MMP2 and TIMP2 was in keeping with that observed in the qRT-PCR data and matched that observed in HFL-1 cells. Detection of MMP4 failed.

Following exposure to AZA, DLD-1 cells demonstrated reduced abundance of both MMP2 $(\Delta=0.35 \pm 0.11 ; p=0.009)$ and TIMP2 $(\Delta=0.36 \pm 0.06 ; p=0.003)$. These results are in keeping with the results of the array and qRT-PCR data. Although TIMP4 was not detectable by WB due to technical failure, increased abundance was previously demonstrated by IIF.

Following knockdown of TIMP2 there was a significant reduction in the abundance of TIMP2, although minimal amounts were still detectable, confirming effective but incomplete KD with $\operatorname{siRNA}(\Delta=0.06 \pm 0.05 ; p=0.001)$. This was important as IIF had shown no change to TIMP2 as it was not detectable due to its extracellular location. KD of TIMP2 did not significantly affect the abundance of MMP2 $(\Delta=1.02 \pm 0.08 ; p=\mathrm{NS})$. KD of TIMP4 did not significantly affect the abundance of MMP2 or TIMP2, although combined KD of TIMP2 and TIMP4 produced a reduction in TIMP2 abundance consistent with TIMP2 KD alone. 
Following exposure to AZA and KD of TIMP2 both a reduction in TIMP2 and MMP2 abundance was observed; the reduction in MMP2 being proportionate to that observed with AZA alone $(\Delta=0.27 \pm 0.04 ; p=0.001)$ and that of TIMP2 being proportionate to siRNA KD alone $(\Delta=0.06 \pm 0.04 ; p=0.001)$. There was also a significant difference between the reduced abundance of MMP2 and TIMP2 $(p=0.009)$. KD of TIMP4 in association with AZA also produced a decreased abundance of both TIMP2 $(\Delta=0.34 \pm 0.06 ; p=0.003)$ and MMP2 $(\Delta=0.32$ $\pm 0.08 ; p=0.005)$ but was proportionate to that observed with AZA alone. Combined KD of TIMP2 \& TIMP4 with AZA exposure resulted in a response identical to TIMP2 KD and AZA.

The most important findings based on WB data is that KD of TIMP2 alone did not significantly affect the abundance of MMP2, and that demethylation with AZA in combination with KD of TIMP2 produced a reduced abundance of MMP2 that was proportional to that of AZA alone. It can be inferred from these results that a reduction in TIMP2 protein, whether by demethylation or $\mathrm{KD}$, is not necessary for the decrease in MMP2 following AZA exposure. Additionally, neither MMP2 or TIMP2 appear to be affected by TIMP4. 
Figure 6.3. Relative protein abundance for MMP2 and TIMP2 normalised to control abundance of 1.0. Error bars are standard deviation from mean corrected abundance. A corresponding blot excerpt is shown to demonstrate abundance for MMP2. [ $\Delta$ mean abundance from control* $p \leq 0.05 ; * * p \leq 0.01 ; * * * p \leq 0.001 . \Delta \Delta$ mean abundance between target proteins ${ }^{\circledR}$ $p \leq 0.05 ; @ @ \leq 0.01 ; @ @ p \leq 0.001]$

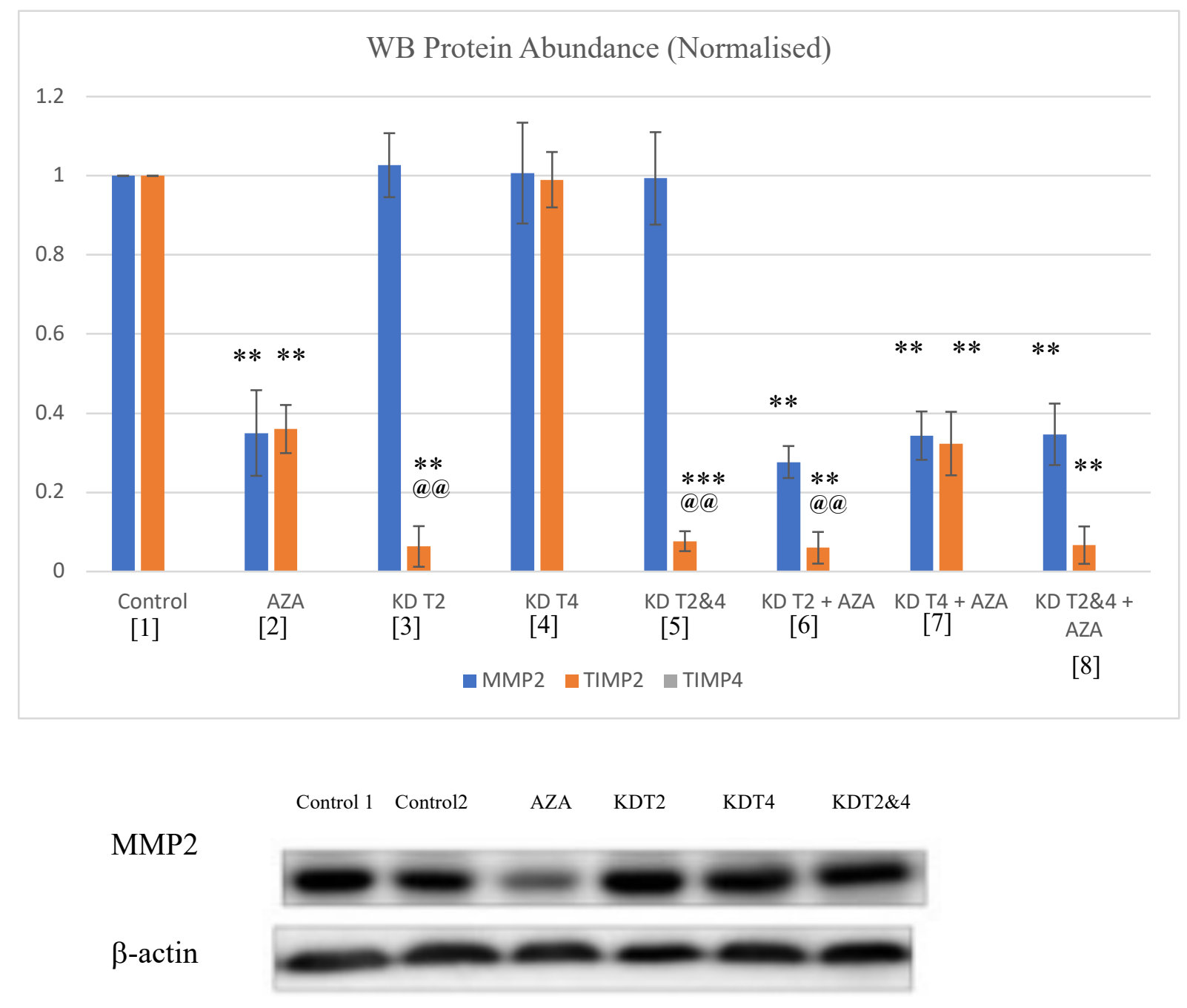

Note the significant reduction in abundance of MMP2 and TIMP2 observed with AZA alone [2] $(p \leq 0.01)$, and that there was an effective siRNA KD of T2 [3] $(p \leq 0.01)$, but that this did not affect MMP2 abundance ( $p=\mathrm{NS}$ ). However, when TIMP2 was KD, MMP2's abundance was significantly reduced by AZA [6], indicating that the reduction in abundance of MMP2 in response to AZA is not dependent on the abundance of TIMP2. KD of TIMP4 had no effect on the abundance of MMP2 or TIMP2 in any treatment [4-5], or KD combination [7-8]. 


\subsection{Discussion}

The aims of this chapter were to assess the effects of demethylation on the protein abundance of MMP2, TIMP2, and TIMP4 by both an immunocytochemistry and Western Blotting methodology. Further to this baseline assessment, the knockdown of TIMPs singularly, in combination, and concurrently with AZA exposure was intended to provide insight into the homeostatic dependency of any relationship between MMP2 and TIMP abundance, and to isolate the effects of AZA at a transcriptomic level.

As previously discussed, the regulation of MMP and TIMP expression is complex and occurs at a transcriptomal, post-transcriptomal, and protein level; the balance of which determines phenotype 581,600 . The results demonstrate that the reduced expression (qRT-PCR) and abundance (IIF \& WB) of MMP2 observed with AZA is independent of the abundance of TIMP2 and TIMP4 and must therefore be assumed to be dependent on a direct demethylating effect at a transcriptomic level, or due to AZA's effect on another regulatory mechanism that is not dependent on these TIMPs. The results also indicate that the increase in expression of TIMP4 observed following exposure to AZA does not independently affect MMP2 or TIMP2 expression. Unfortunately, due to a number of technical and methodological shortcomings, cross-correlation between protein abundance studies was not possible, although the observed effects on MMP2, TIMP2, and TIMP4 were consistent with those observed in Chapter 5.

The expression of MMP2 in relation to methylation status has been reported to be variable across a number of different studies; some indicating that hypomethylation of MMP2 promotor sequences increased expression in breast cancer and glioma cell lines, and was in associated with an increasingly aggressing phenotype ${ }^{546,592,651}$. However, whole-genome pyrosequencing studies have confirmed the frequent occurrence of MMP2 $\mathrm{CpG}$ island hypermethylation in colorectal cancer, a finding which has been supported by clinical datasets ${ }^{652,653}$. The findings in this study are in alignment with the whole-genome pyrosequencing studies. The divergent findings relating to the expression of MMPs and methylation status across tumour types is not unexpected; Couillard having previously shown that expression of MMPs (based on mRNA techniques) in response to demethylation was not only variable between MMPs in colorectal cell lines, but also that often the directly opposite effect was observed between different tumour types $^{589}$. The implication of the acknowledgement that different tumour types express different patterns of MMP and TIMP methylation, and that they respond differently to manipulation of methylation at transcriptomal and phenotypic level, should not undermine the concept of 
hypermethylation-associated upregulation of MMP2 being important in the pathogenesis of colorectal cancer. Breast cancers and gliomas are, after all, different cancer types with different cells-of-origin and a different phenotype; particularly considering the differences in phenotype expected between diffuse-type and solid-type tumours.

With regards to the potentially reciprocal transcriptomal control of MMPs, the findings of this Chapter do not help delineate MMP or TIMP response to demethylation based on promotor motif grouping ${ }^{582}$. The system proposed by Yan and Boyd suggest that MMP2 is a Group 3 MMP characterised by no TATA box and no proximal AP-1, instead demonstrating a number of GC boxes, AP-2, and STAT ${ }^{581,582}$. However, TIMP2 shares GC boxes, STAT, and AP-2 sites with MMP2, but also has a TATA box and AP-1 as well as NF- $\kappa$ B sites. TIMP4 on the other hand, like MMP2, has no TATA box, but does have NF- $\kappa$ B, AP-1, and AP-2, although reduced GS sites. Due to the divergence in transcriptional response to AZA and the lack of clear association between this response and putative grouping based on promotor motifs, or indeed any specific promotor sites, no direct (motif demethylation) or indirect (motif activator effect) action of demethylation effecting MMP and TIMP transcription via one-or-other binding site or group can be identified. Furthermore, the mechanisms by which nuclear hormone receptors interact with MMP promotor motifs is complex and diverse, and do not necessarily correspond to neat binary relationships between co-active or counter-active MMPs and TIMPs, suggesting an additional layer of complexity in constitutive and induced transcriptomal regulation ${ }^{654}$. This is despite previous studies indicating that specific promotor motifs influence the balance between constitutive MMP2 and TIMP2 expression, although admittedly not in relation to methylation but instead to polymorphism ${ }^{585,655,656}$.

As the promotor motifs in the promotor regions in MMP2 and the two TIMPs are highly overlapping but the effects of demethylation not convergent, promotor demethylation affecting transcription is not occurring at the identified activator motifs but may be occurring at another upstream site. $\mathrm{CpG}$ islands and differential methylation in approximation to MMPs and TIMPs have been associated with a variety of diseases, including cancer, stroke, clotting disorders, and placental defects ${ }^{657-659}$. Although epigenetic events have previously been identified in metalloproteinase transcription, including the MMP2/TIMP2/MT1-MMP axis, CpG islands are not well characterised in relation to MMPs and TIMPs, and these genes do not contribute to the classical CIMP classification systems, although some of the ADAM group of proteinases are included in a limited number panels (see Chapter 1, Table 1.2) (592, 660, $661 .^{.}$ 
As well as DNA methylation, transcriptomal regulation of MMP2 and TIMP2 by chromatin restructuring has also been shown to be important in cancers including colorectal cancer, and indirect effects of DNA demethylation affecting chromatin structure cannot be excluded (such as DNA methylation-dependent deacetylase expression) ${ }^{189}$. However, as AZA specifically affects DNMT activity, the effects observed in this study are not due to direct modification of histone methylation as might be observed following dysregulation with enhancer of zeste homolog 2 (EZH2) in breast and ovarian cancers ${ }^{662,663}$.

At the post-translational level, over-expression of TIMP2 has been associated with suppression of wnt/ $\beta$-catenin and subsequent upregulation of proliferative and pro-tumoural cell activity in ovarian cancer mediated via ubiquitination ${ }^{664}$. The effects of low TIMP2 were overcome with application of lithium in this study, which re-activated the previously down-regulated signalling pathway. The results in this chapter are to a degree contrary to this study, as the reduction of MMP2 following AZA exposure was not dependent of the abundance of TIMP2, and therefore not reliant on a post-translational process dependent on TIMP2. This was demonstrated by the persistence of the change in abundance of MMP2 following TIMP2 KD. The independence of MMP2 abundance and TIMP4 abundance also indicates a lack of dependency between these two proteins.

When considering the results of this chapter however, it should be acknowledged that there are several limitations that prohibit the transference of the hypothesis that hypermethylationrelated upregulation of MMP2 drives invasiveness of DLD-1 cell in vitro, an effect that can be reversed by direct demethylation with AZA, to the in vivo colorectal cancer. One limitation is that the investigations in this Chapter were somewhat limited by both the methodological construct (probing for extracellular proteins with intracellular ICC techniques) and technical failures (failure of chemiluminescence for MMP4). There was however consistency across each of the elements of this Chapter and the findings of Chapter 5's qRT-PCR and thus they are, together, confirmatory.

With regards the detection of TIMP2 (or failure thereof) in the intracellular cytoplasm, although primarily an extracellular protein, other authors have reported being able to detect TIMP2 with standard techniques and the product literature supplied with the kit demonstrates intracellular detection in HeLa cells following a standard methanol-fixation technique ${ }^{665,} 666$. 
The use of intracellular transport blockers such as monensin or Brefeldin-A was avoided due to increased complexity and the potential for confounding (monensin has been shown to interfere with wnt signalling in colorectal cancers), especially in the siRNA arms of the experiment ${ }^{667,668}$. The use of novel single-cell dynamic intracellular inducible protein analysis was beyond the remit of this study ${ }^{669}$.

The lack of detectable bands against TIMP4 in the WB analysis was also disappointing. Protein extraction was successful as bands were demonstrated for $\beta$-actin, but no chemiluminescence was detectable when probed for TIMP4. The first possibility is that the primary antibody is not effective in the WB assay, although it worked adequately in ICC and is supported for both of these uses in the product literature ${ }^{670}$. The second possibility is that the conjugation to the secondary antibody was not effective due to a cross-reactivity issue or other fouling. The host for the primary antibody used for TIMP4 was rabbit and thus specific anti-rabbit secondary was acquired for ICC, but the anti-rabbit horseradish peroxidase secondary for WB was not ordered specifically for use with this primary (laboratory stock, different manufacturer), and thus there is a possibility it was not compatible with the primary or was degraded. Unfortunately, due to time and resource constraints, the re-running of the whole assay (including cell culture, siRNA transfection, etc) was not possible, and since results from ICC for TIMP4 were in accordance with the qRT-PCR data, was also deemed unnecessary.

Another consideration is that the experiments conducted in this and the previous Chapter were performed on cells not interacting with an ECM-like environment, as was the case for the invasion assays in Chapter $4^{671}$. When cells of any kind interact with the stroma their phenotype is changes by ligand activation of multiple pathways, and thus the expression of important genes of interest and translation of proteins will be markedly different from cells cultured on plastic or glass. These differences are likely to be augmented when cells are not only interaction with the ligands of the ECM but with other cell types, as would be encountered in the in vivo tumour microenvironment ${ }^{672}$. It should be noted, however, that the initial array and RT-PCR data in Chapter 5 was based on expression following exposure to AZA on cells not cultured in an ECM-like environments (e.g. on Matrigel), thus the effects of demethylation were not confounded by other changes in cellular phenotype in response to ECM ligands. Furthermore, the methods employed in this Chapter do not model the heterogeneity observed in in vitro tumours; the assessment of gene expression by mRNA and protein techniques having 
been performed for total culture extracts. This homogeneity, or apparent homogeneity, is unlikely to be representative of the tumour environment in vivo ${ }^{399,477}$.

Despite these issues an overarching narrative is emerging linking demethylation of DLD-1 cells in vitro, reduced transcription and protein abundance of MMP2 and TIMP2, and a reduced propensity of demethylated cells to migrate and invade. These finding are summarised in Table

\section{2 .}

Table 6.2 Summary of findings relating to expression of genes of interest and the methylation status of DLD-1 cells in vitro. Direction of arrows denotes direction of regulation, $\mathrm{X}$ denoted fouled reaction. Figures denote statistically significant relative fold change ${ }^{\circledR}, \Delta \Delta \mathrm{CT}^{*}$, or relative abundance\$.

\begin{tabular}{|c|c|c|c|c|c|c|c|c|}
\hline \multirow{3}{*}{ MMP2 } & \multirow{2}{*}{\multicolumn{2}{|c|}{$\frac{\text { ARRAY }}{\text { AZA }^{@}}$}} & \multirow{2}{*}{\multicolumn{2}{|c|}{$\frac{\text { qRT-PCR }}{\text { AZA* }^{*}}$}} & \multirow{2}{*}{\multicolumn{2}{|c|}{$\begin{array}{c}\text { IIF } \\
\text { AZA }^{\$}\end{array}$}} & \multirow{2}{*}{\multicolumn{2}{|c|}{$\begin{array}{c}\text { WB } \\
\text { AZA }^{s}\end{array}$}} \\
\hline & & & & & & & & \\
\hline & & -4.10 & & 0.24 & 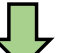 & 0.34 & 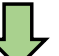 & 0.35 \\
\hline MMP9 & & & X & & - & & - & \\
\hline MMP11 & & +2.45 & & 3.26 & - & & - & \\
\hline MMP13 & & +2.85 & & & - & & - & \\
\hline TIMP2 & & -2.68 & & 0.37 & $X$ & & 5 & 0.36 \\
\hline TIMP4 & & -2.58 & $\widehat{A}$ & 5.89 & 1 & 3.74 & X & \\
\hline
\end{tabular}




\subsection{Conclusions}

The results of this Chapter demonstrate that exposure to AZA reduces the protein abundance of MMP2 \& TIMP2 and increases TIMP4, as demonstrated by ICC and WB methodologies. These findings are in keeping with those observed based on expressional array data and individual qRT-PCR (the results for exposure to RRx-001 were divergent and prone to statistical deviation). AZA has also been shown to decrease the abundance of MMP2 \& TIMP2 protein and increase TIMP4 protein in a manner that was not dependent upon the presence of the counterpart proteins, suggesting AZA is directly affecting the transcription of each gene rather than via a post-translational homeostatic mechanism within the MMP2/TIMP2/MT1MMP axis. Furthermore, as the motifs in the promotor regions of MMP2 and the two TIMPs are highly overlapping but the effects of demethylation not convergent, demethylation affecting these sites is less likely to be responsible for the observed change in expression which is more likely occurring at another upstream site, such as a $\mathrm{CpG}$ island.

MMP2 and TIMP2 are the major gelatinase and respective inhibitor responsible for degradation of Type-IV collagen in the basement membrane. Demethylation with AZA results in a reduced propensity for DLD-1 CRC cells to migrate and invade in vitro. The relative decrease in MMP2 and dysregulation to gelatinase expression and abundance may be able to account for the reduced ability of AZA exposed DLD-1 cells to invade in an EMT-like model of early cancer development and metastasis that is analogous the processes involved in EMVI. These findings do not however directly translate to the in vivo process of early cancer progression and EMVI due to the relative crudity of the in vitro modelling (lack of ECM, stromal cells, microenvironmental hypoxia, etc). Investigating the effects of methylation status on gelatinase expression and tumour histopathological characteristics (including EMVI) will be the overarching aim of Chapter 7. 


\section{Chapter 7}

\section{Extramural Vascular Invasion (EMVI) in Rectal Cancer and the In Vivo Expression of Metalloproteinases \& their Inhibitors.}

\subsection{Introduction}

Chapter 3 introduced the concept of extramural vascular invasion (EMVI) in rectal cancer and how this radiological and histopathological feature of rectal cancers is associated with poor prognosis and the requirement of adjunct therapies. The diagnostic challenges in detecting the presence of EMVI pre-operatively, thus presenting the possibility of neoadjuvant therapies in an effort to improve outcomes, were also discussed. Chapter 3 also examined the association between EMVI and the CpG Island Methylator Phenotype (CIMP), demonstrating a statistically significant association between the epigenetic events characterised by CIMP and the presence of histopathologically demonstrated EMVI, although the presence of the CIMP phenotype did not translate to poorer clinical outcomes ${ }^{258}$.

Subsequent Chapters in this thesis have examined the relationship between the biological effects of methylation on colorectal cancer cell lines in vitro, and how manipulation of methylation can alter the phenotype. Demethylation with 5-azacytidine (AZA) has been shown to reduce the migratory and invasive ability of DLD-1 cells in association with dysregulation of metalloproteinases (MMPs) and their inhibitors (TIMPs) gene expression and protein abundance. Investigation with putative demethylator RRx-001 produced less consistent results. The reduced ability of cancer cells to migrate and invade is important in vivo as these abilities are crucial to the processes defining the epithelial-to-mesenchymal transition (EMT) and metastasis, processes analogous to EMVI and early rectal cancer progression ${ }^{476,490}$.

Novel associations between EMVI, MMP \& TIMP expression, CIMP, and clinicopathological features and outcomes may, therefore, be beneficial in facilitating the diagnosis of EMVI or in risk-stratifying rectal cancers: Can metalloproteinases serve as biomarkers in rectal cancer? 


\subsubsection{Metalloproteinases as Biomarkers}

As already introduced in Chapter 5, MMPs and TIMPs have an established role in the progression of many cancers, specifically relating to their ability to remodel the extracellular matrix as early cancer cells interact with the cellular and acellular components of the stroma ${ }^{505}$, 510. The MMP2/TIMP2/MT1-MMP axis has itself already been identified as a potentially important mechanism for early cancer progression in colorectal cancer and has been associated with a worse Duke's Stage ${ }^{511,512}$. However, the translation of benchtop observations into the manipulation of MMPs in in vivo therapeutics has been limited in success, suggesting additional layers of complexity in the relationship between MMP expression and cancer biology $y^{514}$. This is also reflected in the failure of MMPs and TIMPs to be adopted as diagnostic and predictive biomarkers.

Aside from MMPs' and TIMPs' mechanistic role in cancer progression, their potential as biomarkers has been investigated via a number of methods for over 25 years. In 1991, Levy examined the mRNA expression of MMP2 in 18 colorectal cancers and compared expression to adjacent normal mucosa, demonstrating an increase in expression in 13 cases $(72 \%)^{511}$. Immunohistochemical examination of a further 70 specimens, including 30 adenomas, demonstrated a statistically significant increase in staining for MMP2 (expressed as a percentage of positively stained cells) in Duke's A/B over adenoma, and Duke's C over Duke's A/B (all $p<0.001)$. However, contrary to these findings, Ring (1997) demonstrated that MMP2 and MMP9 were not correlated with worse Duke's stage or to survival, but that TIMP2 was inversely proportional to worse Duke's stage and tumour differentialtion ${ }^{512}$. Interestingly, IHC demonstrated that MMP9 expression in tumour-associated macrophages was significantly associated with poorly differentiated tumours in this study $(\mathrm{p}<0.05)$ and MMP2 demonstrated a trend $(p=0.08)$. In another series of 71 colorectal cancers, Zeng showed that MMP9 expression (assessed by mRNA Northern blot hybridisation) was associated with synchronous distant metastasis $(p=0.004)$ and worse Duke's Stage $(p=0.008)$, and that dichotomisation into MMP9-high and -low groups was a predictor of shorter disease-free and overall-survival $(p<0.0001 \text { and } p<0.0002 \text {, respectively })^{673}$. Circulating concentrations of MMP1 have also been found to be associated with and predictive of metastasis and Duke's C stage by other authors, based on ELISA-like assessments ${ }^{674}$. What this early research best illustrates is that there is huge variability between studies in terms of the association with disease stage and prognostic significance of individual MMPs and TIMPs, and that these associations have been found to be variably demonstrable between different investigations. 
Very few studies have investigated the role of MMPs and TIMPs specifically in rectal cancer. This is largely due to colorectal disease being considered one biological entity in much of the literature despite a growing appreciation of the longitudinal differentiation between molecular subtypes along the colon ${ }^{202}$. In 2007, Schwandner examined 94 rectal cancers by IHC for multiple MMPs and TIMPs, finding that MMP2, TIMP2, and MT1-MMP were associated with the depth of invasion of the tumour, but not with differentiation, overall tumour stage (UICC), pre-operative CEA level, or nodal status ${ }^{675}$. None of the studied MMPs or TIMPs was associated with OS or DFS, although pre-operative CEA was significantly associated with both (both $p<0.01$ ). However, Tohoku found that only MMP9 was associated with worse survival $(p=0.03)$ and nodal involvement in a series of 64 rectal cancers examined by IHC, whereas MMP2, TIMP2, and TIMP3 bore no association ${ }^{676}$. Again, the divergence in finding between studies is highlighted, and note that neither study examined any association between MMPs \& TIMPs and EMVI.

More recent reviews of the utility of MMPs and TIMPs in the diagnosis and staging of colorectal cancer have highlighted the challenges of the multiple different techniques in quantifying transcription, translation, and activity of MMPs; and have attributed the failure of this group of mechanistically important proteins to translate to useful biomarkers on the reliability and variability of different methodologies ${ }^{677}$. Although a variety of methodologies are employed, the assessment of proteases in cancer diagnosis and prognosis has been blighted by inconsistencies in findings, leading to few such genes and associated proteins being used as viable biomarkers ${ }^{604,678}$. These issues are not unique to colorectal cancer and MMPs have also failed to become viable biomarkers in urological, breast, and other cancers despite extensive investigation. There are however ongoing efforts to build consensus that MMPs and TIMPs are potential diagnostic and prognostic biomarkers, with a particular focus on MMP2 and MMP9, although none has yet been adopted ${ }^{682,683}$. Even given promising predictive values of MMPs in symptomatic patients (specifically circulating MMP9 assessed by ELISA), the focus on screening and diagnostic tools aimed at unselected populations has led to the predominance of faecal testing and other risk-stratification tools that direct patients towards colonoscopy (or equivalent) ${ }^{679-681}$. No studies have examined any association between metalloproteinases and EMVI from the perspective of being a potential biomarker that may aid in identifying at-risk patients, and certainly none in the context of DNA hypermethylation events. 


\subsubsection{Tissue Microarrays}

Tissue microarrays (TMA) are a high throughput method of assessing intact tissue at a genomic, transcriptomic, or proteomic level ${ }^{684}$. The fundamental principle of a TMA is the precision micro-sampling of tissue blocks and side-by-side mounting onto a limited number of slides so that multiple sections can be exposed to the same assay and then assessed rapidly $^{685}$.These techniques allow assessment and storage of multiple tissue samples and are an evolution of the pre-existing "sausage" techniques ${ }^{686}$. The primary time and resource advantage of TMAs is that once representative specimens have been selected the process may then be automated and the number of new slides, as well as consumables employed to process them, dramatically reduced. Samples are then mounted sequentially on slides without individual labelling, thus keeping input samples in the same order as the mounting is crucial ${ }^{687}$.

The pairing of TMA slide preparation with modern techniques in IHC facilitates internal control as multiple specimens can be exposed to the same probing and resolving process symaltaneously ${ }^{688}$. The biological principles in IHC are essentially the same as those exploited in ICC and IIF (see Chapter 6) but are employed on an intact tissue sample, thus facilitating an appreciation of the tissue-distribution of staining ${ }^{689}$. This advantage is therefore beneficial in examining potential intra-sample heterogeneity (such as tumour heterogeneity) and also the distribution of targets across different cell types when compared to ICC, although is less powerful than traditional full-slide assessment. TMAs have therefore become a standard and invaluable method of molecular profiling and can be combined with bioinformatic techniques to generate large datasets and meaningful insights into in cancer and other diseases ${ }^{690,691}$.

When assessing TMAs, as in other forms of IHC, it is important to adhere to standards of reporting and interpreting data. Standard methods for ensuring the reliability of data is dual specimen selection and reporting by pathologists, but also techniques in intensity and distribution scoring are important ${ }^{692,}$ 693. As such, the five-step process of masking, examination, lesion parameters, scoring definitions, and interpretation consistency advocated by Gibson-Corley is recommended ${ }^{694}$. By employing these methods, a semi-quantitative output can be achieved that is robust and meaningfully descriptive, avoiding vague descriptions of staining such as "weak" or "strong". 


\subsection{Aims \& Objectives}

This Chapter will investigate any associations between the histopathological and clinical features of resected rectal tumours and metalloproteinase expression and $\mathrm{CpG}$-methylator phenotype (CIMP), with a particular focus on EMVI. The principle source for the histopathological and clinical data will be the database from which similar data was extracted and investigated in Chapter 3. Tissue microarrays will be employed to probe the expression of key MMPs and TIMPs in resected cancers and an inductive semi-quantitative methodology employed to cross-correlate against the clinical dataset and the observed effects of demethylation in vitro. The objective is to close the circle on a potential association between clinical outcomes in rectal cancer, EMVI, hypermethylation, and differential expression of MMPs \& TIMPs. 


\subsection{Methods}

\subsubsection{Patient Selection, Data Collection, \& Storage}

Sixty rectal cancer patients were extracted from the prospectively maintained database as described in section 2.1.1 \& 2.1.2, and in accordance with the ethics laid out in section 2.1.3. The sixty patients were selected to provide an even split between those patients demonstrating EMVI on the histopathology examination of their resected tumours. Patients were in all other ways unselected and otherwise drawn at random from the pool. As previously stated, patients were neoadjuvant therapy naïve, and exclusion criteria also included patients with hereditary or other identifiable predispositions to carcinogenesis, such as inflammatory bowel disease. All patients underwent index surgery between January 2010 and May 2013 at a single centre (ABMU, Morriston Hospital, Swansea, SA6 6NL, UK) by a team of surgeons participating in the Swansea Pelvic Oncology MDT. Tumour characteristics were defined as per the ACPGBI or Royal College of Pathology guidelines with respective relation to pre-operative (mTNM) or postoperative (pTNM) staging and other tumour characteristics (CRM, differentiation, EMVI) ${ }^{35,206}$. Adjuvant chemotherapy was administered based on current guidelines and bestpractice. Patient identifiable data was stored in keeping with the principles of NIHR Good Clinical Practice (GCP) and the UK Policy Framework for Health and Social Care Research ${ }^{364}$. For further specific detail of patient selection, data collection, and storage please refer to Chapter 3, Section 3.3.1.

\subsubsection{Tissue Microarrays \& IHC}

TMAs were constructed by biomedical scientists of the Pathology Department of Singleton Hospital. Tumours were retrieved from storage and assessed by a consultant histopathologist to ensure representative areas of solid tumour were being sampled. Samples were anonymised after retrieval and mounting to TMA by means of dual-coding to remove any patient identifiable data from the data collection phase. Data was then re-coupled to patient clinical, histopathological, and CIMP datasets for analysis.

The Ventana BenchMark ULTRA IHC/ISH System (Roche, Basel, Switzerland) was employed to process TMAs as it fully automates the baking, deparaffinization, cell conditioning and staining, counter stain and titration processes. Each primary antibody was optimised for antibody concentration and incubation time and temperature by the automated BenchMark ULTRA System and checked against positive control (human liver) by biomedical scientists. Details of the primary antibodies are found in Chapter 2 (Table 2.4), but each was 
supplied by Abcam (USA) and chosen based on experimental requirement (for targeting MMP2, MMP9, MMP11, TIMP2, and TIMP4) and the expertise of the biomedical scientists (choice of supplier, variant of mAb, process optimisation, etc). Positive control was also mounted on the TMA to ensure internal validity of the staining process.

Unstained FFPE TMA sections (4um) were mounted onto glass slides according to standard protocol and baked for 60 minutes at $60^{\circ} \mathrm{C}$. The slides were then barcode labelled and placed on the BenchMark ULTRA System. Complimentary Ventana reaction buffer was used throughout the process to rinse slides and all other consumables were drawn from existing histopathology laboratory stock that had previously been optimised for the equipment. Liquid cover slip solution and reaction buffer is applied to the slides before each step and after each wash. Dewaxing is carried out at $72^{\circ} \mathrm{C}$ with EZprep solution. Retrieval with $\mathrm{CC} 1$ (cell conditioner) was then be performed at $98^{\circ} \mathrm{C}$ for between 8 and 48 mins depending on antibody. Pre-peroxidase inhibitor was added. $100 \mu 1$ of diluted (dilution determined during optimization) primary antibodies was titrated onto the slides and incubated for between 4 minutes and 2 hours (depending on the protocol determined during optimization of the antibodies) at either room temp, $40^{\circ} \mathrm{C}, 37^{\circ} \mathrm{C}$ or $42^{\circ} \mathrm{C}$ (depending on the protocol determined during optimization of the antibodies). OV HQ Universal linker containing secondary antibody was then applied and incubated for 8 minutes, followed by OV- HPR multimer for 8 minutes. Sections were then treated with $\mathrm{OV}$ DAB and $\mathrm{H}_{2} \mathrm{O}_{2}$ for 8 minutes before finally being incubated in copper for 4 minutes. An optional final incubation with OV AMP multimer was carried out depending on the antibody for 4-8 minutes. The slides were then be counterstained with Heamatoxylin for 8 minutes.

IHC staining was assessed according to a modified protocol based upon the recommendations set out in the Fedchenko review and with reference to the Gibson-Corley recommendations ${ }^{692}$, ${ }^{694}$. Scoring was carried out by a panel of at least three expert observers comprising at least one consultant histopathologist. The remaining participants were the investigator and academic supervisor (clinical or non-clinical), or biomedical scientist or other parallel Doctorate coinvestigators. Disputes were resolved by the consultant histopathologist who had the deciding opinion. TMAs were observed using light microscopy at 40-100x magnification and a consensus scoring system was adopted. The scoring system is set out in Table 7.1 and the scoring proforma in Appendix V. The overall staining metric Mx was determined by multiplying the score for the proportion of tumour cells stained by the score for the intensity 
of staining; giving a maximum score of 12. Multiplying the scores (as opposed to adding the scores) ensured that uniformly non-staining tissues scored 0 ( $\mathrm{p} 4 \times \mathrm{i} 0=0$, rather than 4$)$, and that the score was powered towards the more objective proportion of stained cells rather than the subjective intensity of staining for those that did score ${ }^{692}$. TMAs were performed in triplicate for each tumour (patient) sampled and the metrics averaged. This method provides semi-quantitative data for non-parametric analysis.

Table 7.1 IHC scoring system for TMAs

\begin{tabular}{|l|l|l|l|}
\hline Proportion of Cells & p-Score & $\begin{array}{l}\text { Intensity of } \\
\text { Staining }\end{array}$ & i-Score \\
\hline None & 0 & None & 0 \\
\hline$<10 \%$ & 1 & Weak & 1 \\
\hline $10 \%-50 \%$ & 2 & Intermediate & 2 \\
\hline $51 \%-90 \%$ & 3 & Strong & 3 \\
\hline$>90 \%$ & 4 & & \\
\hline MAX Multiplication Score $(\mathrm{Mx})=12$ & \\
\hline
\end{tabular}

\subsubsection{Statistical \& Test Analysis}

Multiplication scores (Mx) were for each MMP or TIMP (0-12) in sample triplicates and then averaged to provide the overall score, as described in Section 7.3.2. Scores were then categorised (Cat scores) according to the system set out below. Nominal categorisation was preferred as the data is discrete rather than continuous.

- Category 0 (Cat 0): Mx 0

- Category 1 (Cat 1): Mx 1 to 4

- Category 2 (Cat 1): Mx 5 to 8

- Category 3 (Cat 2): Mx 9 to 12

Categorisation is consistent with the methods set out by other authors in benchmark papers on methods in IHC ${ }^{695-697}$. Following categorisation, global assessment of association to EMVI was made for each MMP and TIMP by logistic regression and Cox proportionate hazard (time-toevent) modelling ${ }^{698}$. A survival analysis by Kaplan-Meyer method (survival-distribution function by Log-rank) was also estimated for each MMP and TIMP. Following global assessment, if association was indicated, test optimisation was performed by Category subanalysis for sensitivity and specificity so that the optimal combination of categories could be constructed into the best test by binarisation. Binarised tests were then re-assessed for proportionate hazard and survival modelling. This process is summarised in Figure 7.1. 
Pearson's correlation matrix was them employed to demonstrate association between CIMP and both MMP2 and EMVI in the sample population.

Data analysis was performed using the Microsoft Office Excel (v.16.12) and the XLStat Addinsoft (v.2017) plug-in (macro). Normalcy of data distribution was determined using a Kolmogorov-Smirnov test. If data were normally distributed, statistical significance was determined using Student's t-test for single comparisons or one-way ANOVA where more than one comparison was made. If data were not normally distributed, Mann-Whitney's test was used to compare unpaired means and Wilcoxon test used for paired data. Log-Rank (MantelCox) tests were used where data was right-side skewed and censored. Comparison of expected frequencies was performed with two-tailed Chi Squared or Fishers exact test if observed events were less than 10. Survival curves were calculated using Kaplan-Meier estimate. The null hypothesis was rejected at the level of $5 \%(p>0.05)$. 
Figure 7.1 Flow diagram of IHC analysis \& outputs for each MMP or TIMP.

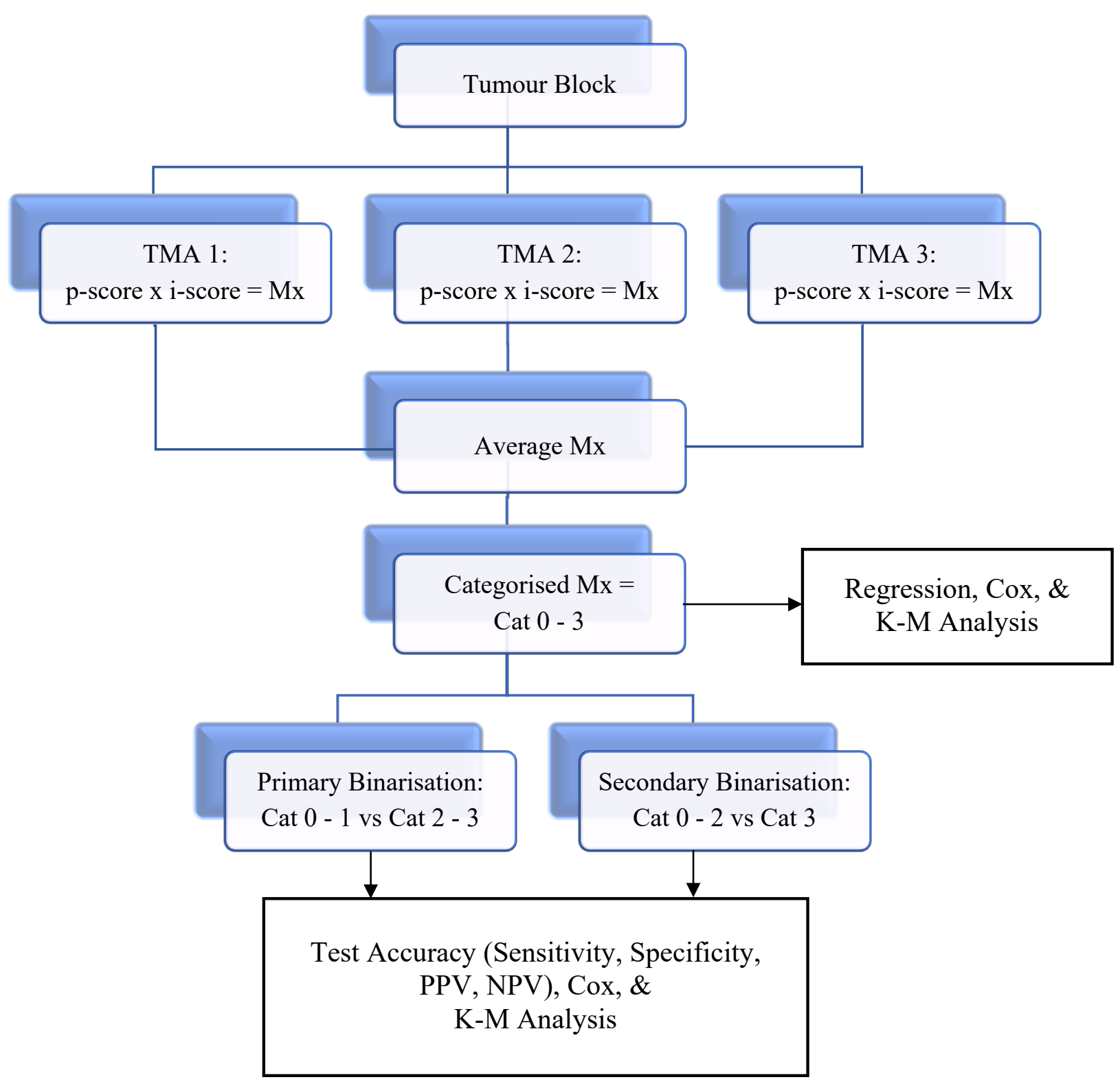




\subsection{Results}

\subsubsection{Patient and Tumour Characteristics}

There were 60 patients included in this study (30 patients with an EMVI-positive tumour and 30 with no evidence of EMVI). Forty-two patients were male (70\%) and males were statistically more likely to demonstrate EMVI than females $(p=0.005)$. The median age was 68 years (range $45-89$ years), and patients were of a similar age in both groups. Nineteen patients had died at the time analysis was undertaken. Median follow up was 56.5 months (IQR $25-$ 71 months). Mean overall DFS and OS were 28 and 33 months, respectively, but DFS was 26 months in the EMVI-positive group and 31 months in the EMVI-negative group $(p=0.20)$. OS between EMVI-positive and -negative groups was 32 and 34 months, respectively $(\mathrm{p}=0.51)$. When differences between both groups were analysed between groups, patients with EMVIpositive tumours had more advanced pathological staging by $\mathrm{pT}, \mathrm{pN}$, and AJCC classifications $(p=0.038, p=<0.0001$, and $p=0.001$, respectively). Specifically, when the $T$-grade was compared against EMVI, pT1 and pT2 were significantly less frequently occurring events than others within the contingency table (each Fisher's exact text $p<0.05$ ). There was a corresponding association between EMVI-positive tumours and the need for adjuvant chemotherapy $(\mathrm{p}=<0.0001)$. There was, however, no statistically significant difference in $\mathrm{CRM}$ positivity, tumour perforation, or tumour differentiation (all $p=\mathrm{NS}$ ). These findings are demonstrated as three-dimensional (3D) contingency tables in Figure 7.2.

\subsubsection{Staining Characteristics and EMVI}

TMAs for 60 tumour specimens were successfully constructed and stained against the targets MMP2, MMP9, MMP11, TIMP2, and TIMP4. All staining was successful and controlled against the human liver positive control except for MMP11 which failed to stain against tumour and control despite multiple attempts at automised and manual optimisation. Following percentage and intensity scoring, the multiplied metric (Mx) was calculated and tumour Categorised scoring (Cat) performed; these results are demonstrated in the frequency histograms in Appendix VI.a. Representative TMAs stained for MMP2 and TIMP2 are demonstrated in Figure 7.3 a \& b. Logistic regression analysis of the Cat scores demonstrated a significant relationship between EMVI and MMP2 and TIMP2 (both $p<0.0001$ ). No relationship was discernible between EMVI for and Cat scores for MMP9 or TIMP4 (both $p=\mathrm{NS}$ ). The receiver operating characteristic (ROC) analysis of global MMP2 Cat scores demonstrated an area-under-curve (AOC) of 0.947 indicting it is a good binary classifier. 
However, ROC analysis for TIMP2 was less favourable (AOC 0.560). These results are also demonstrated graphically in Appendix VI.b.

Figure 7.2 3D contingency tables for EMVI against a. pT; b. pN; c. AJCC classification, and d. adjuvant chemotherapy. $X^{2}$ test for all contingencies $p<0.05$.
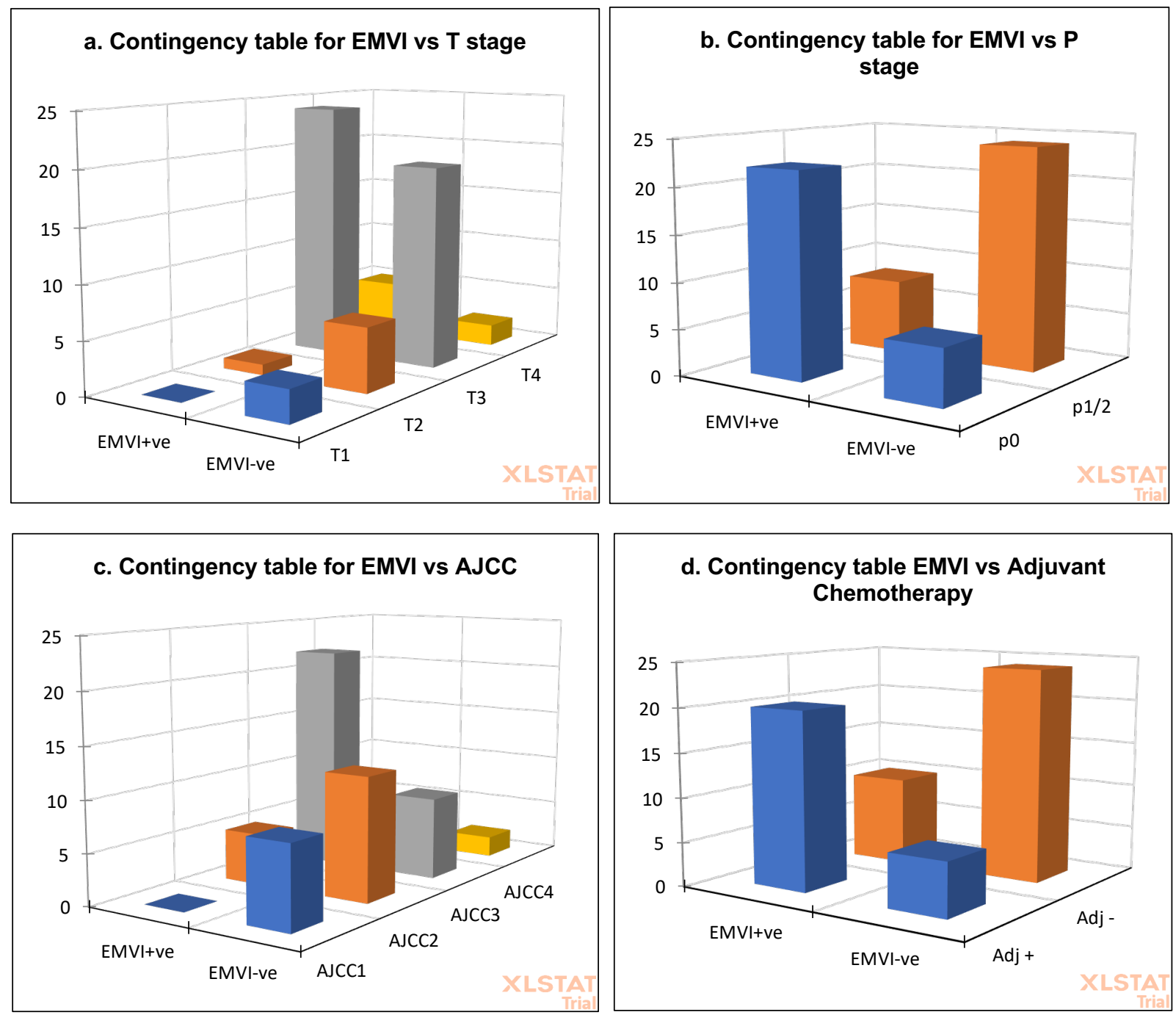
Figure 7.3a $\& \boldsymbol{\&} .3 \mathrm{~b}$. Selected representative light microscopy images at $\mathrm{x} 4$ and $\mathrm{x} 10$ magnification of IHC for MMP2 \& TIMP2 in EMVI-positive and EMVI-negative tumours.

a.

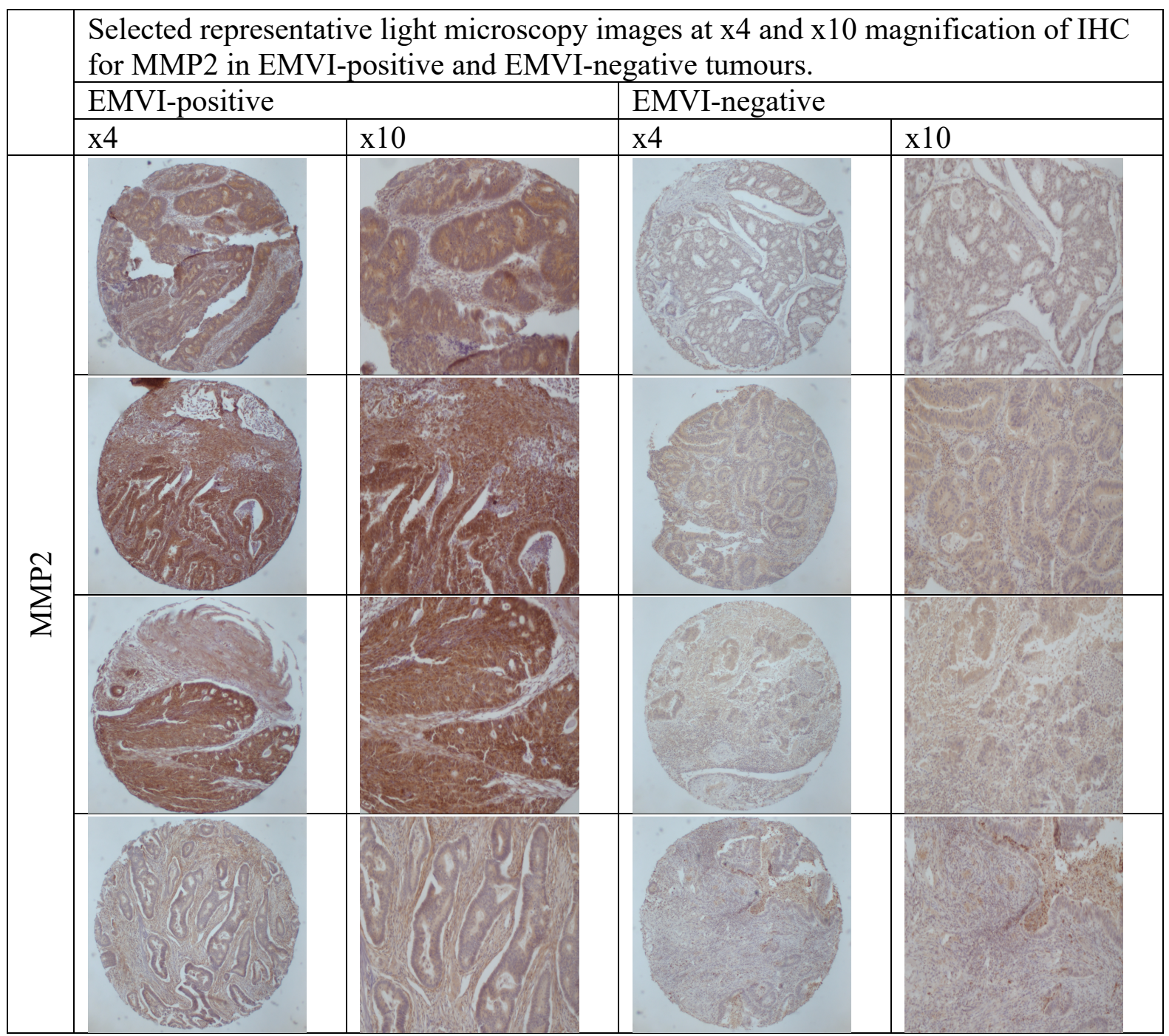

Note the differential staining intensity between EMVI-positive and EMVI-negative tumours in this selected representative series. Following scoring categorisation, this differential staining was demonstrated to be significant $(p<0.0001)$. Binarised scoring was then demonstrated to be predictive of EMVI-status and DFS and OS (see further results in this Chapter). 
b.

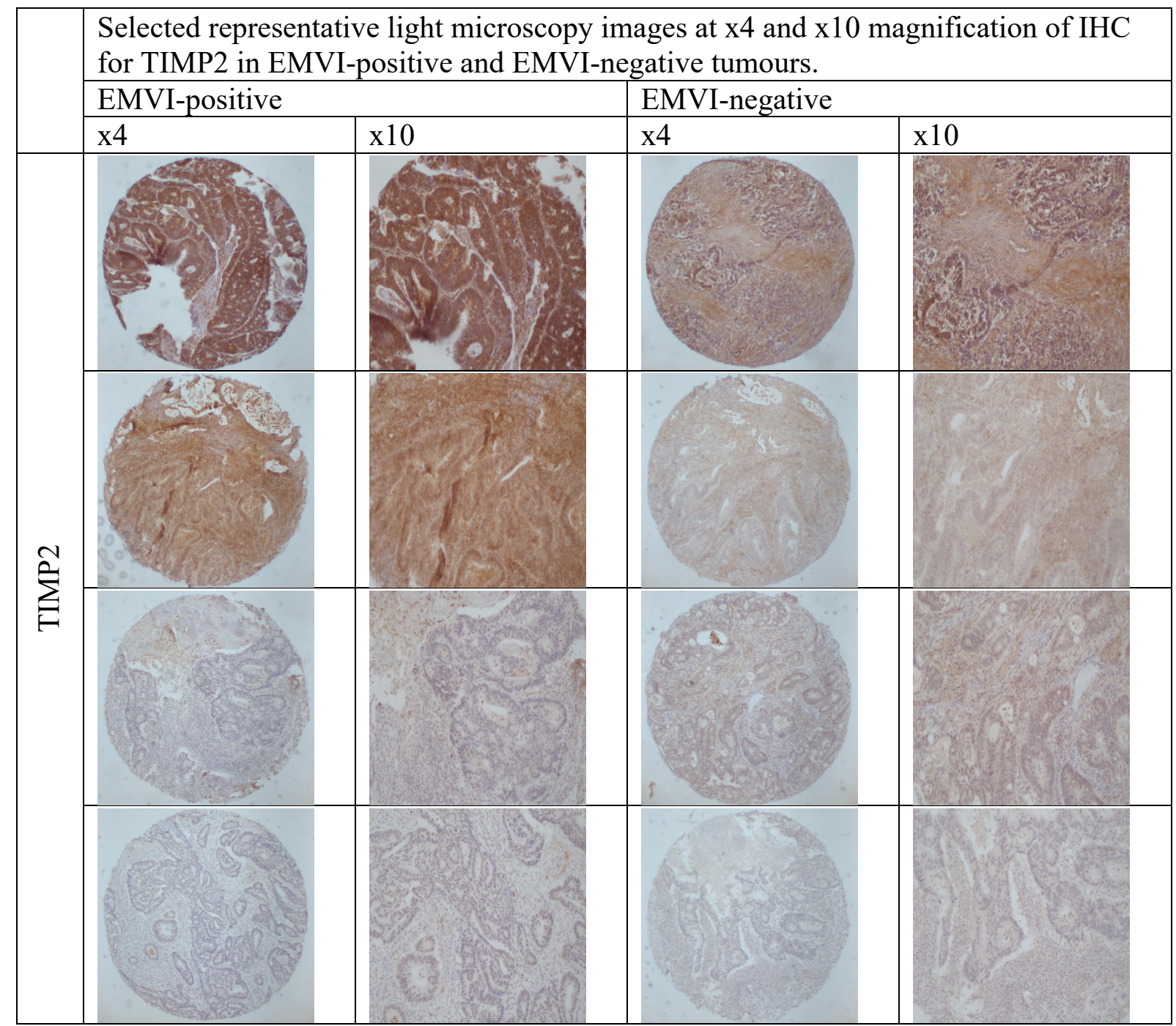

Although staining intensity was associated with EMVI-status in this series $(p<0.0001)$, TIMP2 was not found to be a predictive discriminator for EMVI, as was the case for MMP2. This series has been constructed to also highlight the differential degrees of staining proportion and intensity used to calculate the multiplication score, $\mathrm{Mx}$, in the IHC analysis (top row = high score, bottom row $=$ low score; see Section 7.3.2) $)^{695-697}$. 
7.4.3 Proportional Hazards in relation to EMVI and Staining Characteristics

The Cox Proportionate Hazards Model was used to examine the proportionate risk of poor disease-free (DFS) and overall (OS) survival in relation to EMVI, and Global MMP2 \& TIMP2 Cat scores. Cox modelling demonstrated that EMVI was significantly related to DFS and OS with Hazard Ratios of $3.32(p=0.027)$ and $3.05(p=0.043)$, respectively. The same analysis for MMP2 and TIMP2 demonstrated statistical non-significance for their risk relating to DFS and OS, $(p=0.11$ and $p=0.15$, respectively, for DFS and OS $)$. These results are demonstrated in Appendix VI.c with the accompanying $\log (-\log (\mathrm{SDF}))$ curves demonstrating similar slopes for each test, although there is a degree of left-shift for each and more so for EMVI, indicating that the risk-ratio is divergent, and hence it reaching significance for increased risk in this timepoint model.

\subsubsection{Survival Analysis}

Kaplan-Meyer (KM) analysis was performed for DFS and OS in relation to EMVI, and Global MMP2 \& TIMP2 Cat scores. This analysis showed that EMVI was significantly related to both DFS and OS ( $p=0.029$ and $p=0.046$, respectively). Similar to the results demonstrated in Cox modelling, the KM analysis demonstrated non-significance for both MMP2 and TIMP2 when Cat scores were analysed as a group, $(p=0.15$ and $p=0.20$, respectively, for DFS and OS). These results are demonstrated in Figure 7.4. 
Figure 7.4 Kaplan-Mayer Survival Distribution Functions for Disease-Free (DFS) and Overall Survival (OS) assessed on EMVI and categorised staining (Cat) scores for MMP2 \& TIMP2.

a. EMVI

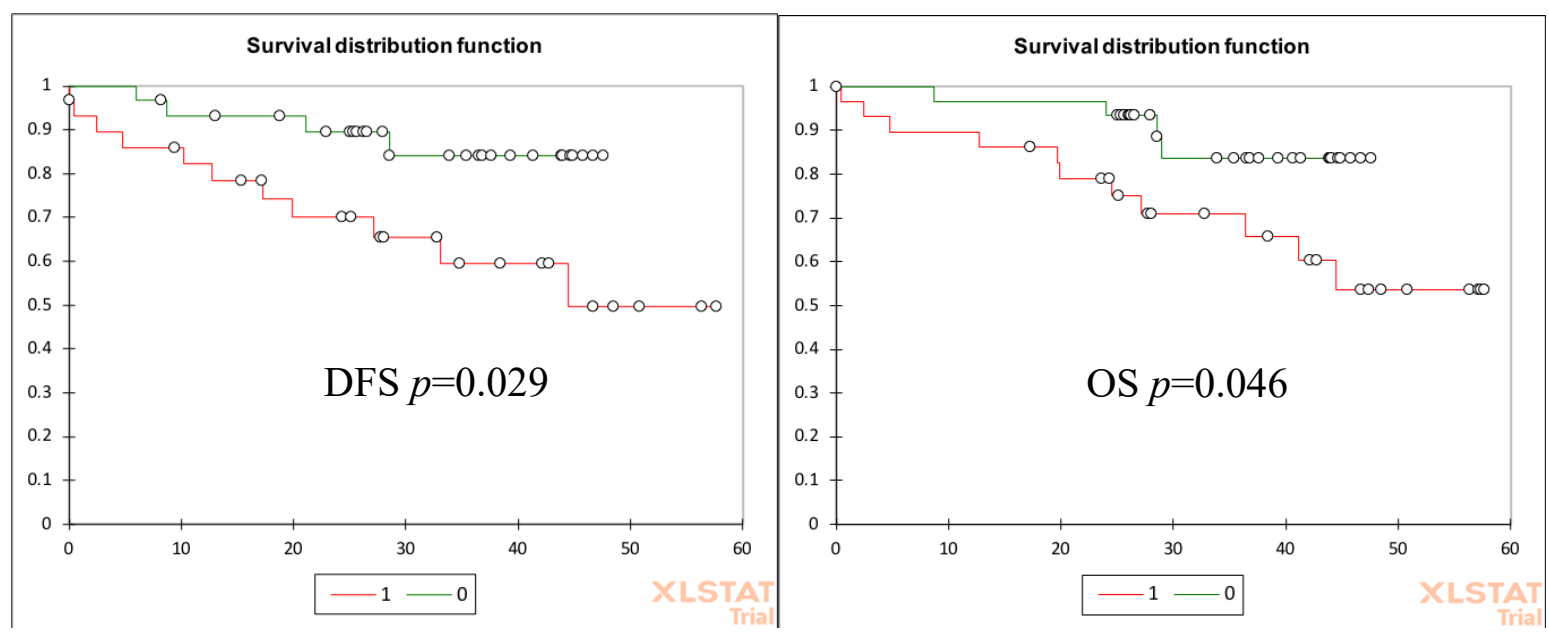

b. Global MMP2 Cat Score.
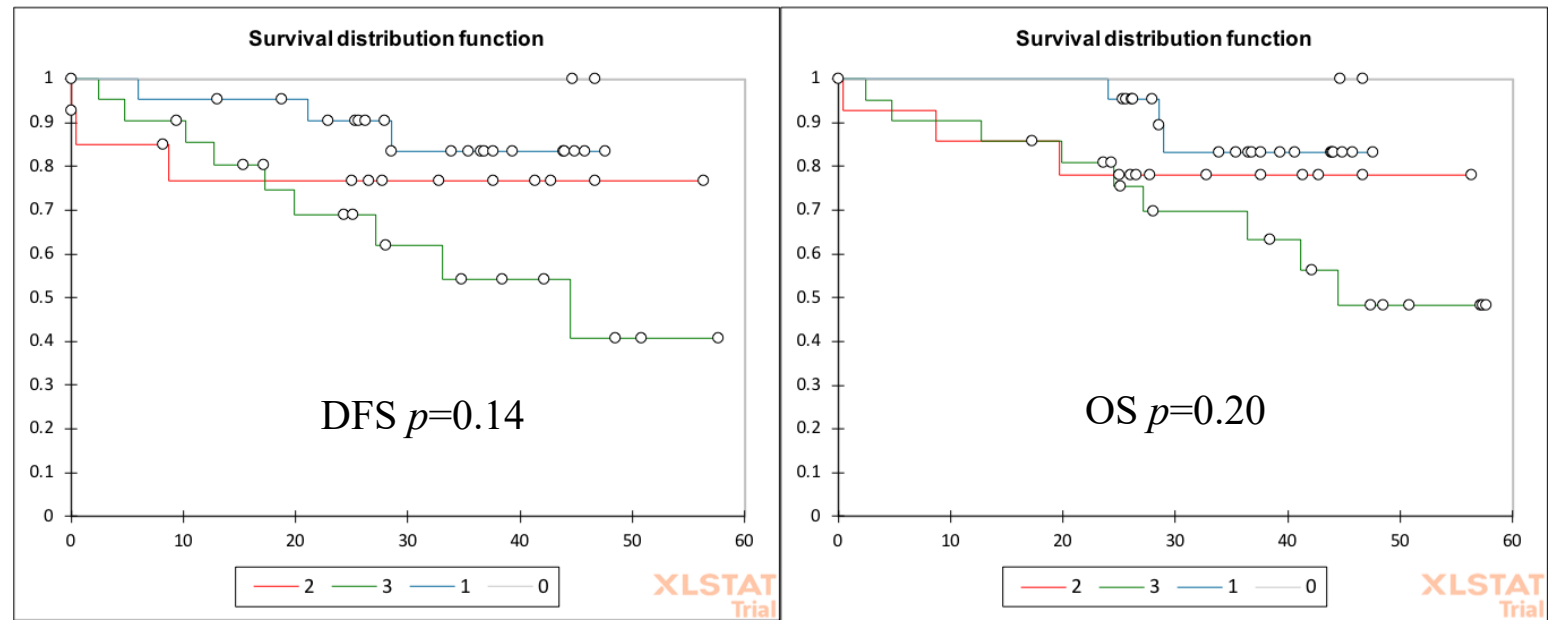

c. Global TIMP2 Cat Score.
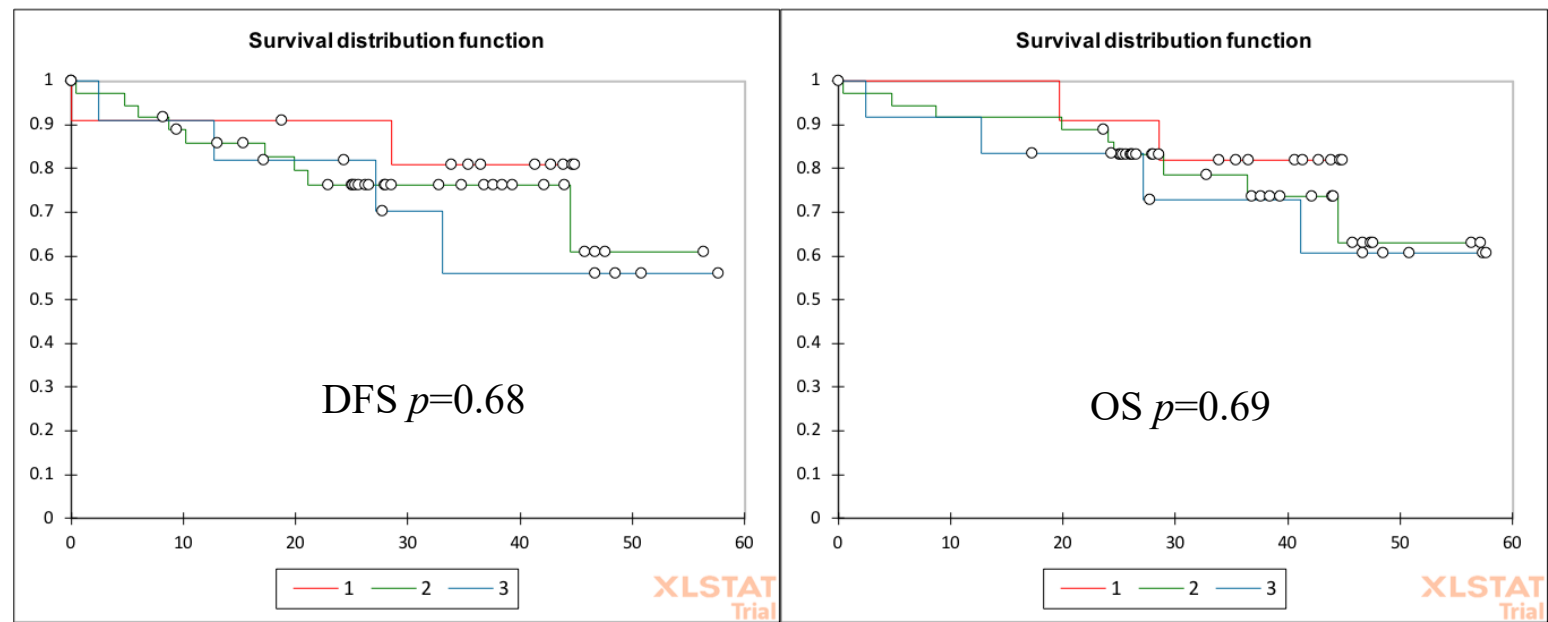


\subsubsection{MMP2 Biomarker Test Optimisation}

Global MMP2 Cat scores have been shown to be associated with EMVI on logistic regression analysis but the sensitivity and specificity thresholds for each Category has not been established. Additionally, sensitivity \& specificity may be improved by test binarisation into MMP2-high or -low (combining Cat scores). Table 7.2 demonstrates Primary and Secondary Binarisation strategies. Optimal binarisation is indicated by the threshold-based diagnostic accuracy analysis (table) and ROC curve in Figure 7.5, demonstrating superior sensitivity, specificity, and AOC for Primary Binarisation over Secondary Binarisation for global MMP2 Cat score (AOC 0.973 vs 0.947). These analyses indicate an optimal binarisation of individual Cat scores was produced by Primary Binarisation (accuracy 0.900).

Table 7.2 MMP2 Category Binarisation

\begin{tabular}{|l|l|l|}
\hline MMP2 & Primary Binarisation & Secondary Binarisation \\
\hline Low & Cat 0 \& Cat 1 & Cat 0 to Cat 2 \\
\hline High & Cat $2 \&$ Cat 3 & Cat 3 \\
\hline
\end{tabular}

Figure 7.5 MMP2 Test Breakdown and Binarisation Data

\begin{tabular}{|r|r|r|rr|rrrr|r|}
\hline MMP2 Cat & Sensitivity & Specificity & PPV & NPV & TP & TN & FP & FN & Accuracy \\
\hline 0.000 & 1.000 & 0.000 & 0.500 & & 30 & 0 & 30 & 0 & 0.500 \\
1.000 & 1.000 & 0.067 & 0.517 & 1.000 & 30 & 2 & 28 & 0 & 0.533 \\
$\mathbf{2 . 0 0 0}$ & $\mathbf{1 . 0 0 0}$ & $\mathbf{0 . 8 0 0}$ & $\mathbf{0 . 8 3 3}$ & $\mathbf{1 . 0 0 0}$ & $\mathbf{3 0}$ & $\mathbf{2 4}$ & $\mathbf{6}$ & $\mathbf{0}$ & $\mathbf{0 . 9 0 0}$ \\
3.000 & 0.733 & 1.000 & 1.000 & 0.789 & 22 & 30 & 0 & 8 & 0.867 \\
\hline
\end{tabular}

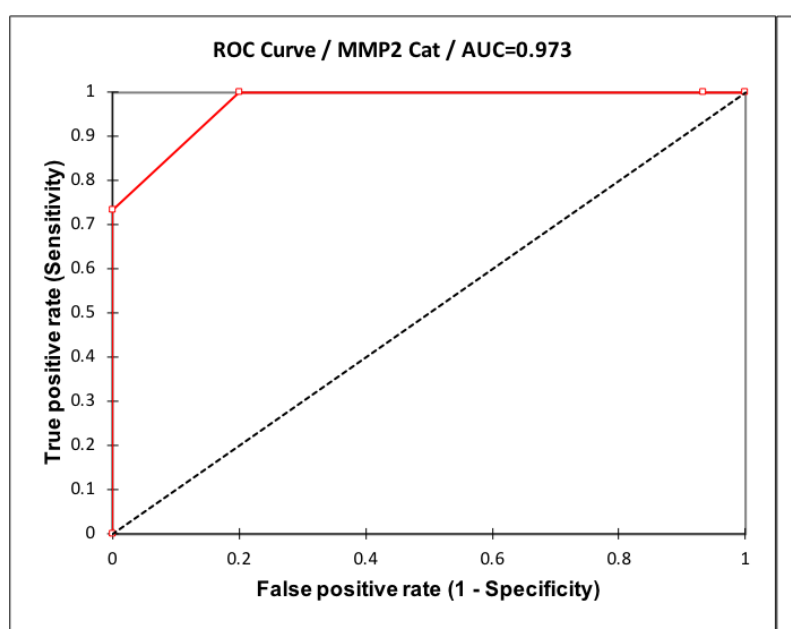

True positive, True negative, False positive, False negative / MMP2 Cat

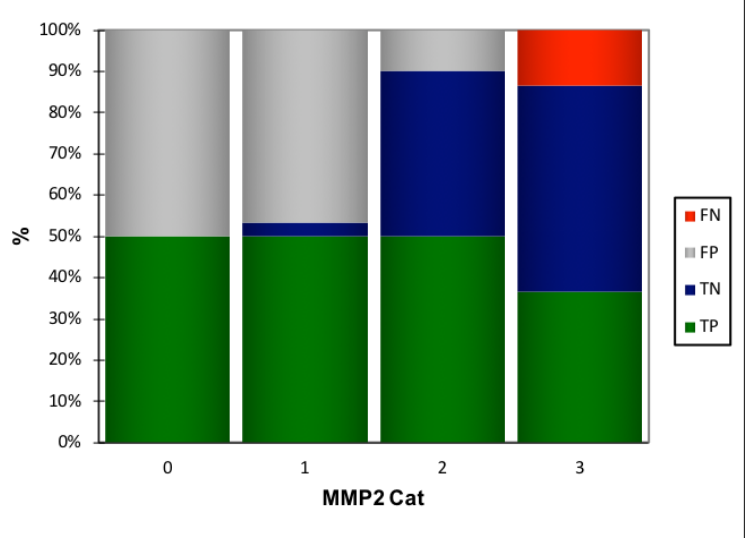


When binarisation (Primary or Secondary) is applied and Cox and KM modelling re-applied the hazard estimations indicate that Primary Binarisation is a better indicator of early adverse event than Secondary Binarisation, as demonstrated in Table 7.3. However, for DFS and OS based on KM analysis, Secondary Binarization of Cat scores was found to be significantly ( $p=0.030 \& p=0.049)$ associated with MMP2 and outperformed Primary Binarisation $(p=0.041$ $\& p=0.057$ [NS], respectively). These results are demonstrated in Figure 7.6.

Table 7.3 Cox Proportionate Hazard modelling for binarized MMP2 scores.

\begin{tabular}{|l|l|l|l|l|}
\hline \multirow{2}{*}{ MMP2 } & \multicolumn{2}{|l|}{ Disease-free survival } & \multicolumn{2}{l|}{ Overall Survival } \\
\cline { 2 - 5 } & Hazard Ratio & $p$-value & Hazard Ratio & $p$-value \\
\hline Primary Binarisation & 3.45 & 0.033 & 3.21 & 0.047 \\
\hline Secondary Binarisation & 2.97 & 0.037 & - & $0.055[\mathrm{NS}]$ \\
\hline
\end{tabular}

Figure 7.6 Kaplan-Mayer Survival Distribution Functions for Disease-Free (DFS) and Overall Survival (OS) assessed on a. Primary or b. Secondary binarized MMP2 scores.
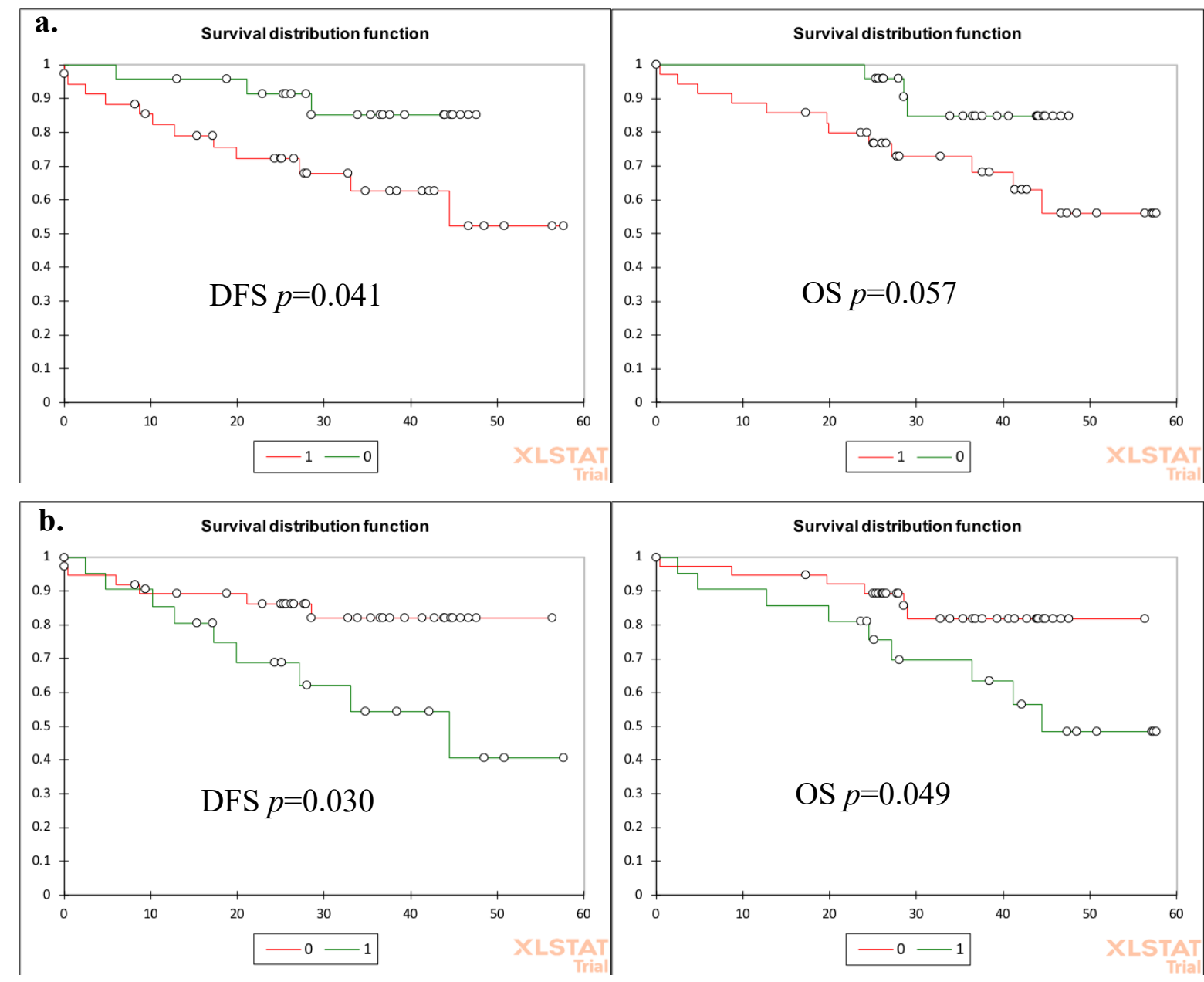


\subsubsection{CIMP, MMP2, \& EMVI}

When correlation between CIMP, MMP2 binarised score, and EMVI was examined, $X^{2}$-based contingency tables illustrated an association between CIMP and both MMP2 and EMVI in the sample population ( $p=0.004$ and $p<0.001$, respectively). However, correlation matrix (Pearson) indicated a stronger and more significant association between EMVI positivity and MMP2 (Person=0.816, $p<0.0001$ ), than between CIMP and any other factor. These results are demonstrated in Table 7.4 and Appendix VI.d.

Table 7.4 Pearson correlation matrix for CIMP, EMVI, and MMP2.

\begin{tabular}{|c|c|c|c|}
\hline Variable & CIMP & EMVI & MMP2 \\
\hline CIMP & 1 & $\begin{array}{l}\mathbf{0 . 4 1 5} p=0.001 \\
\text { CI } 0.108-0.605\end{array}$ & $\begin{array}{l}\mathbf{0 . 3 6 7} p=0.004 \\
\text { CI } 0.125-0.568\end{array}$ \\
\hline EMVI & $\begin{array}{l}\mathbf{0 . 4 1 5} p=0.001 \\
\text { CI } 0.108-0.605\end{array}$ & 1 & $\begin{array}{l}\mathbf{0 . 8 1 6} p<0.0001 \\
\text { CI } 0.710-0.887\end{array}$ \\
\hline MMP2 & $\begin{array}{l}\mathbf{0 . 3 6 7} p=0.004 \\
\text { CI } 0.125-0.568\end{array}$ & $\begin{array}{l}\mathbf{0 . 8 1 6} \quad p<0.0001 \\
\text { CI } 0.710-0.887\end{array}$ & 1 \\
\hline
\end{tabular}




\subsection{Discussion}

The aims of this Chapter were to investigate any associations between the histopathological and clinical features of resected rectal tumours, metalloproteinase expression in these tumours, and CpG-methylator phenotype (CIMP); with a particular focus on EMVI. The data from this Chapter clearly demonstrate that, in this selected trial population, there was an association between tumours strongly expressing MMP2 and those demonstrating EMVI. This was demonstrated by global MMP2 scoring based on logistic regression modelling and the 4-teir system as described but was further improved by binarisation of the scoring system (AUC 0.947 vs 0.973 ). There was also an association between TIMP2 and EMVI, but TIMP2 was not a good discriminator between EMVI positive and negative tumours. No other marker demonstrated association with EMVI (MMP9 and TIMP4) and staining with MMP11 was unsuccessful.

Primary Binarised MMP2 scores demonstrated a sensitivity and specificity for EMVI positivity of $100 \%$ and $80 \%$, respectively, with overall accuracy of $90 \%$ and a positive-predictive value of $88.3 \%$. When this model of binarisation was tested against hazard (RR; Cox) and survival modelling (KM), relative risk for adverse DFS and OS was estimated at 3.45 and 3.21 in MMP2-positive tumours, respectively (both $p<0.05$ ), although this only translated into significant observed difference in DFS $(p=0.041)$ based on Kaplan-Meyer analysis (OS was not significantly affected but was nearing significance $(p=0.057)$ ). Overall survival was however observed to be worse for those patients demonstrating higher MMP2 staining when a Secondary Binarisation protocol was employed, although the Cox hazard ratios were not as significant. Based on these assessments, the Primary Binarisation strategy produced the better accuracy when compared to Secondary Binarisation, produced significant RR and KM survival analysis, and was thus deemed to be a better test than Secondary Binarisation. Given the limited difference in significance for DFS and OS between the binarisation strategies, and the findings that insignificant $p$ values were approaching threshold $(p<0.05)$, there was only a minor degree of discrepancy between each of the two binarised scoring systems. However, care must be taken when indicating "trend", as this can often be misleading when assessing small populations $^{699}$.

When assessed as a factor associated with EMVI, MMP2 was more strongly associated with EMVI than CIMP, as demonstrated by superior Pearson correlation ( 0.816 vs 0.415$)$, although both were significantly associated $(p<0.0001$ and $p=0.001$, respectively). This finding 
correlates with the findings in Chapter 3; where although CIMP was associated with EMVI it did not translate into a predictor of survival. In the analysis in this Chapter, MMP2 Primary Binarised score was a predictor of EMVI and was associated with adverse survival. These findings have not however been validated in an unselected or external cohort, which is vital for the complete evaluation of a novel biomarker, although the findings do indicate its potential ${ }^{700 \text {, }}$ 701.

Following the discovery of an association between Duke's stage and MMP2 abundance, the role of MMPs in colorectal cancer has been studied for some decades from both a mechanistic and biomarker persective ${ }^{511}$. Much of the recent interest in MMPs as biomarkers has fallen on circulating (serum) MMP9 and TIMP1, as these potential markers have been most powerfully associated with diagnosis. The quality of evidence for the association ranges from evidence derived from several small series where the identification and prognosis of colorectal cancers was found to correlate to circulating MMP9 activity (assessed by zymography) to much larger population based studies ${ }^{702-704}$. For instance, in a series of 748 asymptomatic patients, Wilson identified that serum MMP9 has a sensitivity and specificity of $79 \%$ and $70 \%$, respectively, and thus might serve as a potential tool for rationalising screening colonoscopy when employed as part of a larger panel of genetic biomarkers ${ }^{705}$. However, more recent studies from the UK have indicated that MMP9 may be more useful as an adjunct to FOB testing in symptomatic patients entered into the screening programme, as the diagnostic accuracy is improved within the symptomatic population and augments faecal testing 679,705 . A further study of 4509 symptomatic patients from Scandinavia indicated that serum TIMP1, in combination with CEA, was also a good diagnostic biomarker for colorectal cancers, but that its diagnostic accuracy was superior specifically for colonic tumours than for rectal tumours or the cohort as a whole ${ }^{706}$. When considering these studies, it must be noted that they are each examining serum levels of their respective biomarkers in either asymptomatic or symptomatic patient populations who then go on to have colonoscopy as a means of validating the test. This focus on diagnostic biomarkers has been driven by the prioritisation of preventative medicine and the high cost and resource burden associated with colonoscopy, especially given the high falsepositive rates associated FOB and symptomatic screening, and thus the need for better discriminators to reduce "unnecessary" endoscopy ${ }^{8,328}$. The patients in this study, by contrast, all have had rectal cancers resected with curative intent, and the test hypothesis was directed towards the detection of EMVI and thus is dissimilar to the screening population. However, 
the investigation of staging and prognostic biomarkers in colorectal cancer, including the MMP family, is still a valid exploit as it may aid in delivering precision medicine ${ }^{683,707}$.

With regards prognosis, MMP9 and TIMP1 have previously shown some promise as potential biomarkers, and have been associated with worse DFS and OS, especially in compound metrics with pre-operative $\mathrm{CEA}^{708,709}$. One study identified TIMP1 as a factor in predicting colorectal cancer liver metastasis and their recurrence, although VEGF was a better discriminator in this study $^{710}$. The findings in this Chapter indicated that MMP2 but no other marker was associated with survival in terms of DFS and OS. This association between MMP2, EMVI, and survival is constructive, but the lack of association with MMP9 and TIMP2 is also important, especially since MMP9 has previously been found to be potentially useful as a screening tool or as a prognostic biomarker. Again, this study in comparison to the screening-oriented studies is highly selective, thus the results are unlikely to be representative of the whole population of colorectal cancers. However, this study's divergent findings may fit with the hypothesis that MSI-high hypermethylated tumours of the rectum form an under-recognised cohort of cancers with an atypical phenotype, including MMP expression, and a poorer prognosis.

Beyond survival data and metastasis, there has been some evidence to indicate that some MMPs may be valuable biomarkers in stratifying the response of cancers to chemotherapy, as has been found in gastric cancer ${ }^{711,712}$. However, in colorectal cancer the data has been more inconsistent; two studies from Denmark, for instance, found that TIMP1 levels correlated to response in patients receiving combination irinotecan, 5-fluorouracil, and folinic acid, but not in patients receiving combination capecitabine and oxaliplatin, although baseline TIMP1 was a predictor of eventual progression-free survival ${ }^{713,714}$. The findings in this study do not aid in predicting response to adjuvant therapy, although this would be an interesting avenue for investigation, as if MMP2 is a good predictor of EMVI and thus increase the number of patients considered for neoadjuvant therapy, knowing that those patients would have a significant response would valuable. Currently, only a limited number of studies have investigated MMP2 as a biomarker in predicting response to adjuvant chemotherapy. One found that an elevated ratio of MMP2 to MMP9 in osteosarcoma was associated with poor response, although another study in inflammatory breast cancer found exactly the opposite ${ }^{715,716}$.

Although associations between MMP2 staining, EMVI, and survival have been demonstrated in this chapter, and that MMP2 is a good predictor of EMVI, there are some limitations to its 
application. Firstly, as already mentioned, the methodology employed in this Chapter relied upon the construction of TMAs form resected cancers, thus the ability to identify EMVI in these tumours is more than a little post hoc. For this method to be clinically useful it would need to be applied to biopsies taken at the point of diagnosis and initial staging so that if high MMP2 was identified, and therefore EMVI predicted, neoadjuvant therapies may be considered irrespective of MRI findings. Furthermore, as this trial population was highly selected, MMP2 as a predictor of EMVI would need to be validated against a large, unselected, external cohort.

\subsection{Conclusions}

In a selected trial population, MMP2 was a predictor of EMVI based upon the Primary Binarised score for staining. Higher MMP2 was also demonstrated to have a significant hazard ratio for DFS and OS and was significantly associated with poor DFS on Kaplan-Mayer analysis. OS for MMP2 did not quite reach significance. MMP2 score demonstrated better correlation to EMVI than did CIMP status. MMP2 has the potential to be useful as a diagnostic biomarker for EMVI in rectal cancers, and may also act as a prognostic biomarker, although further validation is required. 


\section{Chapter 8}

\section{General Discussion}

\subsection{Introduction}

The overarching aim of this thesis was to investigate the relationship between methylation and EMVI in rectal cancers, its implications for prognosis, and seek to illuminate the biological processes that underlie any relationship. Three primary strands of enquiry were followed in order to achieve these aims; the investigation of the methylation status of resected rectal cancers and correlations to clinical outcomes; the in vitro manipulation of methylation to assess biologic effects on colorectal cell lines; and the investigation of putative epigenetic processes that may underlie the in vitro and in vivo observations. The investigation of potential biomarkers associated with EMVI was an additional objective, as it may serve to facilitate the staging and management of progressing rectal cancers. Each of these features is discussed in the context of consensus molecular subtyping in colorectal cancer and the potential for precision medicine ${ }^{118,201}$.

Throughout this Chapter the findings of this research are discussed and the overarching narrative pieced together. The limitations of each stage of the enquiry will be highlighted and complementary or tangential investigation suggested. The first comment in this vein, however, is the acknowledgement of the limitations of the principle investigator and author: As a clinician, stepping into the laboratory has been a challenging and rewarding process; methodologies and techniques that are the bread-and-butter of the undergraduate, graduate, and doctoral scientists have had to be learned from scratch and on-the-fly. The concepts of the scientific enquiry into cellular physiology and epi-/genetic investigation have all been novel and will inform my future clinical and academic insight, but as a novice there were hard barriers to overcome and thus the primary limitation in this investigation is my own limited expertise. On the other hand, my experience as a clinician and surgeon has greatly facilitated by insight into the translational aspects of this research and I hope that I have been able to support colleagues in the laboratory with clinical aspects of their own research, as they have supported me with the science. 


\subsection{The CpG Methylator Phenotype and Extramural Vascular Invasion}

As highlighted in Chapter $1 \&$ 3, the molecular and genetic aberrations that underlie CRC carcinogenesis are complex and not fully understood, although there is a consensus that there are divergent processes responsible for tumour development at different sites throughout the colon and rectum ${ }^{118}$. DNA methylation is one epigenetic process implicated in CRC, as well as other cancers, and $\mathrm{CpG}$ island methylator phenotype (CIMP) has drawn interest as a potential mechanism underlying both carcinogenesis and as a potential biomarker ${ }^{148,} 717$. CIMP, however, has primarily been associated with carcinogenesis in the right colon that is characterised by hypermethylation and microsatellite instability (MSI) (the serrated pathway), rather than the traditional chromosomal instability pattern typical of other sites in the colon and

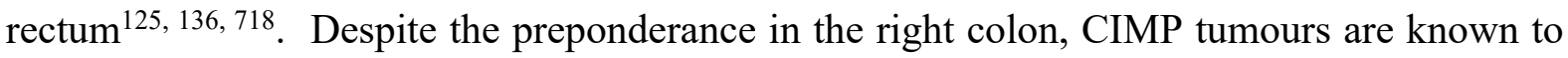
occur in the rectum, although the clinical significance of this molecular tumour type occurring at this site is poorly understood, although some authors have suggested they represent a poorprognostic subgroup ${ }^{149,719}$. Many of the studies that have examined the role of methylation in rectal cancer or indeed colorectal cancer as a whole have focused on a single gene locus or have relied upon small cohorts, making outcomes interpretation challenging ${ }^{353,720}$.

The primary findings of Chapter $\mathbf{3}$ were that a clear association was demonstrated between the presence of pEMVI and male sex, T and N stage, AJCC classification, and the requirement for adjuvant chemotherapy ${ }^{258}$. This study confirms the association of EMVI with worse clinicopathological features and that these translated into a statistically significant survival disadvantage, in keeping with the findings of other authors ${ }^{280}$. Although a significant relationship was demonstrated between CIMP-intermediate status and EMVI positivity $(p=<0.001)$, this was not translated in to a disease-free or overall survival disadvantage for CIMP-intermediate patients (Log-rank $p=0.66$ and $p=0.46$, respectively). This was despite EMVI-positivity being an independent risk-factor for disease recurrence (Cox Proportional Hazard $\mathrm{r}=5.98(1.10-32.50), \mathrm{p}=0.038)$.

In order to gain a fuller understanding of the role of CIMP in EMVI in rectal cancer, a metaanalysis including the data gathered in Chapter 3 was undertaken ${ }^{721}$. Briefly, a systematic search was performed of PubMed, Embase, MEDLINE, PubMed Central, and Cochrane electronic databases for articles pertaining to CIMP and rectal cancer. Articles were analysed and data extracted according to PRISMA standards - see Appendix VI for further detail. Six studies including 1529 patients were included in analysis ${ }^{149}$, 204, 258, 318, 366, 719 . Following 
dichotomisation, the prevalence of CIMP-positive tumours was $10 \%$ to $57 \%$, with a median of $12.5 \%$. Meta-analysis demonstrated the pooled odds ratio for all-cause death for CIMP-positive tumours vs CIMP-negative tumours was 1.24 (95\% CI 0.88-1.74). Z-test for overall effect was $1.21(p=0.23)$, therefore no significant association between CIMP and poor outcomes in rectal cancer was demonstrated (Figure 8.1). Heterogeneity between the studies was low $\left(X^{2} 5.96\right.$, d.f. $5, \mathrm{p}=0.31, I^{2}=16 \%$ ) but total of 15 different loci were used for assessing CIMP across the studies, with a median of 6.5 loci (range 5-8). Another major finding was that rectal cancer datasets were frequently not extractable from larger colorectal cohorts, limiting analysis.

Figure 8.1 Pooled analysis on CIMP and overall survival (adapted from Kokelaar) ${ }^{721}$.

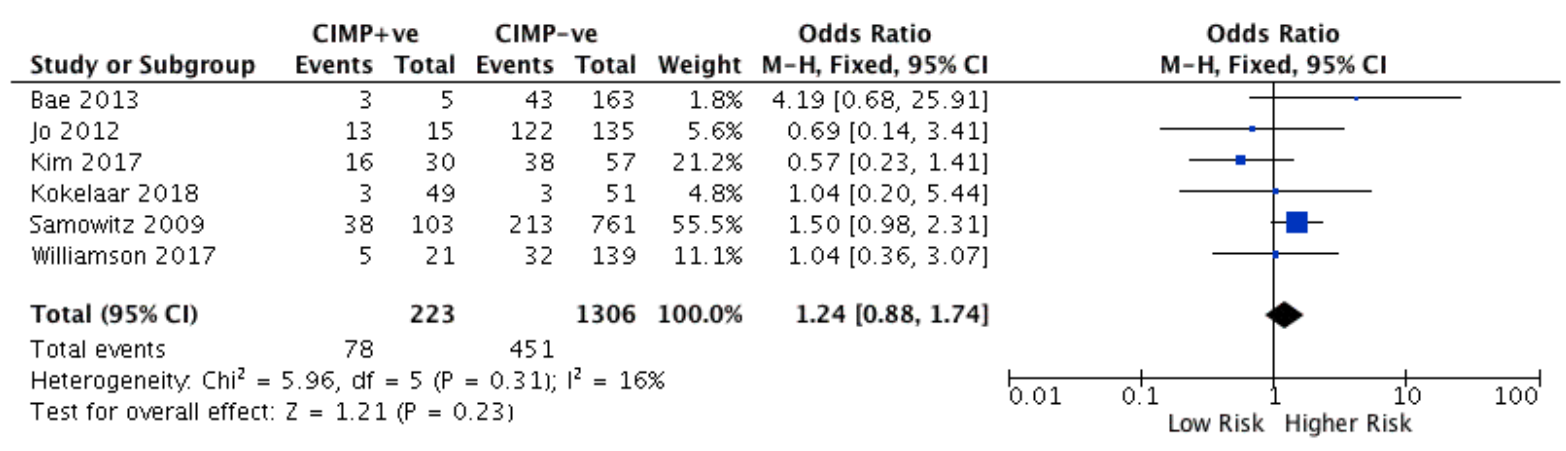

From the investigation in Chapter $\mathbf{3}$ and subsequent meta-analysis, there does not appear to be a significant relationship between CIMP and poor DFS or OS, despite the association between CIMP and EMVI. The differentiation in outcomes suggests an additional layer of complexity beyond a binary relationship between EMVI and CIMP. The significant limitation of all CIMP studies in all cancer types is the heterogeneity in assessing CIMP, a concern highlighted by many authors ${ }^{148,361}$. With regards consensus molecular subtyping (CMS) of colorectal cancers, the original CMS study identified the frequency of CMS1 in the rectum as $3 \%$, whereas this study of 100 consecutive patients with rectal cancers, given exclusions, demonstrated a higher than expected proportion of CIMP-intermediate and -high tumours (49\%) which may be considered as analogous to CMS1 tumours. These findings must, however, be considered in the context of the methodology chosen to determine CIMP as other less discriminatory methods may have returned a lower frequency ${ }^{185}$, 186 . The limited extractability of rectal datasets is also a constraint to analysis. Due to these limitations, the incidence of CIMP-high tumours in the rectum is probably under-reported. 


\subsection{Demethylating Agents in Colorectal Cell Lines}

Chapter 4 investigated the biologic effects of demethylation on the colorectal cancer cell line DLD-1. The well characterised DNA-methyl transferase inhibitor azacytidine (AZA) was employed as the primary controlled variable to effect demethylation, but the putative demethylator RRx-001 was also investigated. These chemicals were first screen and optimised against standard tests for cytotoxicity and inhibition of cellular proliferation to ensure that the effects of biologic assays for invasion and migration were as a result of demethylation. Both agents demonstrated tolerable cytotoxicity at biologically effective doses. This was demonstrated by effective locus-specific and pan-DNA demethylation (msPCR and ELISAlike assays) at doses that were not cytotoxic but did reduce propensity of cells to migrate and invade.

The implications of the findings in this Chapter are twofold; firstly, they serve as a useful link to the findings in Chapter 3, as the same CIMP markers employed in assessing resected tumour methylation were employed, thus linking in vivo observations to potential in vitro processes. The second implication is that the findings serve to confirm the demethylating activity of the two investigated compounds and associate them to changes in cell behaviours (phenotype), thus allowing investigation of underlying mechanisms to be linked to both demethylation, cellular phenotype, and potentially tumour behaviour.

There were however limitations in each stage of this part of the investigation, although attempts at mitigation were made. With regards to the baseline investigation of cytotoxicity, only reasonably basic assays (RPD and trypan blue) were undertaken, although they were done in multiple biologic and technical replicates. Other more sophisticated methods of assessing cytotoxicity and the effects of potentially genotoxic agents are possible, although the methods employed in this study are well-established and accepted as adequate $439,440,722$. There was perhaps an additional element of concern in this assessment, however, when considering the investigation of RRx-001, which is not well characterised, and its modes of biologic action not well understood ${ }^{235,239}$. The full investigation of new compounds in biologic processes, especially in humans with a view to potential therapeutic applications, is the basis of the pharmaceutical industry, and thus a more global and in-depth investigation of RRx-001 beyond the remit of this research. The addition of RRx-001 to the experimental construct was however insightful and will be pursued by the compound's proprietor, EpicentRx. 
The assessment of DLD-1 cell propensity to migrate and invade based on scratch and Transwell assays was successful, although to some degree limited as biological models. The assessment by time-to-convergence was a simple and precise method of assessing cell activity and did not depend on more operator-dependent metrics that might have been more prone to confounding. Similarly, the Transwell system was effective at demonstrating the change in cell activity following demethylation and provided a link to EMT-like processes of early cancer progression due to the addition of an extracellular matrix component. These assays were, however, limited in their sophistication by the use of monoculture and monolayer techniques which do not model the tumour microenvironment well ${ }^{447}$. The addition of, for instance, tumour-associated macrophages or fibroblasts, could provide additional insight into the biologic activity of colorectal cancer cells in vitro and exposure to demethylating agents, as discussed in Chapter 5 \& 6 when considering EMT and metalloproteinase activity. Both scratch and Transwell systems are however widely used and accepted as robust methodologies for cellular investigation and the results gathered valid.

Finally, the demethylating effects of AZA and RRx-001 on DLD-1 cells was demonstrated by locus-specific and whole-DNA assays. These techniques showed that although both agents caused demethylation, it was to different degrees and at different rates; AZA causing a more profound demethylation at both levels, whereas RRx-001 demonstrating a slightly more dosedependent whole-DNA effect and less effective locus-specific effect. The results for AZA are not unexpected as its mode of action is well characterised ${ }^{216,220}$. Although identified as an alkylating agent, the demethylating action of RRx-001 is not understood, although it is apparently effective. However, the reduced ability of RRx-001 to demethylate at more locusspecific sites that AZA suggests that it's action as a demethylator is dependent on other genetic, epigenetic, or cellular events. One culprit in this effect may be chromatin restructuring, which has been shown to be important in regulating DNA methylation in metalloproteinases and other genes $^{592,723}$. 


\subsection{Mechanisms Underlying the Response to Demethylation}

Chapters 5 \& 6 were designed to investigate the potential cellular mechanisms underlying the observed biologic effects of demethylation with AZA and RRx-001. The first stage in this process was identifying genes of interest that could then be investigated further, hence the initial approach was by a RT-PCR profiler array. As the array was only intended as a screening tool and to be controlled for demethylation, it was only performed once and only against AZA, as this was deemed to provide the best benchmarking for investigating the biologic effects. The $\mathrm{RT}^{2}$ Profiler PCR Array - Human Tumour Metastasis (QIAGEN, Germany) was selected as it ties together the biologic effects observed in vitro (reduced migration and invasion) with the putative processes involved in EMVI in vivo. Arguably other arrays could have been chosen but the array selected provided coverage not only of genes associated with the EMT process, which is characterised by proliferative as well as early invasive changes, but also genes associated with metastasis. As the process of EMVI is not well understood as an individual biological process, the coverage of genes possibly associated locally invasive and locally metastatic processes is appropriate. Less focus was given to proliferative (primary tumour growth) and angiogenic (distantly metastatic) genes as they were deemed less likely to be important in the EMVI process. The array highlighted a number of up- and down-regulated genes, as well as many that did not change significantly, but the family of genes most that demonstrated the broadest and greatest magnitude in change was the metalloproteinases (MMPs) and their inhibitors (TIMPs).

As discussed in the respective Chapters, the MMPs and TIMPs are responsible for degrading and regulating the protein components of the ECM and have been implicated in a broad range of diseases, including colorectal cancer ${ }^{683}$. Due to the significant dysregulation demonstrated in the array, the preferred substrate of Type-IV collagen, and previous association with colorectal cancer drawn from the literature, the MMP2/TIMP2/MTP1-MMP axis was primarily investigated as a target for further expressional investigation by qRT-PCR. Complementary MMPs and TIMPs were also included for further depth of analysis. Following exposure to AZA, the qRT-PCR demonstrated a robust change in expression of MMP2, TIMP2, and TIMP4. Response in MMP11 and MMP13 was less pronounced and MMP9 uninterpretable due to reaction fouling. As MMP2 is the primary protease responsible for the degradation of collagen in the basement membrane, it's reduced expression in DLD-1 cells following demethylation may be causative in the reduced propensity of these cells to migrate and invade. The results obtained from RRX-001 were divergent from those of AZA, thus, due to this 
inconsistency and previous discrepancy in demethylating effect, no further investigations were conducted with RRx-001.

The regulation and homeostasis of MMPs is complex, occurring at a transcriptomal, posttranscriptomal, and post-translational level. This was perhaps reflected in the finding that as well as the downregulation of MMP2, its primary inhibitor TIMP2 was also downregulated; challenging the notion that demethylation simply swings that balance of homeostasis towards anti-MMP2 activity. To discern between AZA having a direct transcriptional effect on MMP2 expression and one mediated via TIMP2 or TIMP4 in a homeostatic feedback mechanism, knockdown (KD) experiments and cross-correlation with PCR data was performed utilising proteomic techniques (immunocytochemistry/immunofluorescence and Western blotting). These experiments, however, proved challenging to interpret and suffered somewhat from fouling, although they did confirm the decrease of MMP2 protein abundance in response to AZA exposure. One limitation encountered in this phase of investigation was the inability to detect TIMP2 with ICC techniques, largely due to the protein predominantly being expressed extracellularly. This was despite other authors having success in detecting TIMP2 intracellularly under similar experimental conditions and the product literature suggesting it was possible. ICC was however successful for MMP2 and TIMP4 and corroborated the findings of PCR. Western blotting for MMP2, TIMP2, and TIMP4 was also undertaken, but this time was somewhat limited by a failure of the conjugation of the TIMP4 and secondary antibody in this protocol, the same primary antibody having probed adequately during ICC. Despite this there was sufficient data from WB to support the findings of the PCR for MMP2 and TIMP2, therefore providing proteomic cross-validation for each of the genes against PCR by one or other of the techniques.

When the proteomic data was considered in combination with the quantitative PCR, exposure to AZA was found to decrease MMP2 expression independently of TIMP2 and TIMP4, and thus could be assumed to be occurring via a direct effect of AZA on MMP2 transcription rather than via a homeostatic mechanism dependent on the expression of the counter-acting proteins. A greater understanding of the specific mechanism by which AZA prevents MMP2 expression could be addressed in further research, perhaps by focused study of the methylation of promotor sequences and transcriptomal binding sites by techniques such as methylationspecific pyrosequencing, but limited time and resources prevented this element (which would have required out-sourcing) being included in this study ${ }^{724}$. 


\subsection{Metalloproteinases in Rectal Cancers \& EMVI}

Chapter 7 aimed to close the loop on the presence of EMVI in rectal cancers, the incidence of methylation assessed by CIMP-status, and the potential mechanisms underlying the relationship by examination of metalloproteinases and their inhibitors. The metalloproteinases were also investigated as potential biomarkers for the diagnosis of EMVI and as prognostic indicators. In the highly selected trial population, MMP2 was a predictor of EMVI based upon the Primary Binarised score. Higher MMP2 was also demonstrated to have a significant Cox hazard ratio for DFS and OS and was significantly associated with poor DFS on Kaplan-Mayer analysis. OS for MMP2 did not quite reach significance based upon Primary Binarisation but did on Secondary Binarisation. MMP2 score demonstrated better correlation to EMVI than CIMP status. These assessments were made on the basis of the scoring of immunohistochemically stained tissue microarrays where MMP2, MMP9, MMP11, TIMP2, and TIMP4 were targeted. MMP2 was the only factor identified that was a significant biomarker for diagnosis of EMVI and prognosis, although TIMP2 did demonstrate association with EMVI but was not a discriminator.

Although MMP2 has previously been associated with advancing Duke's stage in colorectal cancer, no previous study has identified MMP2 as a prognostic biomarker in rectal cancer, nor as a diagnostic biomarker in the identification of EMVI ${ }^{511}$. However, the method employed to assess MMP2 during this part of the research process is limited in that the trial population was highly selected, both in terms of the prevalence of EMVI (50\% incidence of EMVI versus $44 \%$ in the unselected population in Chapter 3 and 17-70\% in published series), and in the fact that all patients included in this research were those treated by surgery with curative intent and neoadjuvant naïve ${ }^{271-273}$. Its utility as a biomarker must therefore be tempered by this selectivity, and the need for large-scale validation in external cohorts. Furthermore, if MMP2 is to be considered as a viable biomarker, it would need to be examined in a test that can be applied at the time of diagnosis and staging such as blood test or IHC of biopsy specimens. Biopsies taken during the endoscopic diagnosis of rectal cancer are notorious for not being adequately diagnostic or representative of the tumour as a whole; partly due to tumour heterogeneity, but also due to the limitations of superficial sampling ${ }^{477,725,726}$. Serum detection of MMPs is feasible, but the specificity of MMP2 to rectal cancer (avoidance of confounding with comorbidities) and thresholds would need to be established and validated from scratch ${ }^{683}$, 704. 


\subsection{Concluding Remarks}

The overarching aim of this body of research was to investigate epigenetic events in rectal cancer and their relationship, if any, to EMVI. The initial hypothesis was that hypermethylation, as defined by the $\mathrm{CpG}$ island phenotype (CIMP), was associated with EMVI and this was demonstrated to be the case. However, CIMP status did not correspond to survival as EMVI does and does not in itself elucidate how methylation status may be mechanistically associated with EMVI. By use of demethylating agents, primarily 5-azacytidine (AZA), invasive colorectal cancer cells were rendered less invasive and at the same time dysregulation in metalloproteinase (MMP) expression was observed. The association between demethylation and MMP dysregulation was confirmed by means of knockdown and observation of biologic effect by both immunocytochemistry and Western blotting. As MMPs are vital in the epithelialto-mesenchymal transition and to metastasis, due largely to their role in remodelling the extracellular matrix, they constitute a good mechanism for the observed in vivo effects of demethylation. This is particularly the case for MMP2, which is the primary protease responsible for degradation of type-IV collagen in the basement membrane. On assessment of resected tumours, MMP2 was found to be expressed significantly more strongly in tumours exhibiting EMVI those that did not, and MMP2 demonstrated good diagnostic accuracy in discriminating tumours with and without EMVI. MMP2 was also shown to be associated with survival and is a hazard for early poor outcomes in rectal cancer. By these means MMP2 may serve as a potential biomarker for the diagnosis of EMVI and in the prognosis of rectal cancers and serves as a means of understanding the fundamental biological processed underlying EMVI in rectal cancer. 


\section{Bibliography}

1. MacFarlane JK, Ryall RD, Heald RJ. Mesorectal excision for rectal cancer. Lancet (London, England) 1993;341(8843): 457-460.

2. Chen J, Pitmon E, Wang K. Microbiome, inflammation and colorectal cancer. Seminars in immunology 2017;32: 43-53.

3. Kang M, Martin A. Microbiome and colorectal cancer: Unraveling host-microbiota interactions in colitis-associated colorectal cancer development. Seminars in immunology 2017;32(Supplement C): 3-13

4. American Cancer Society - Key Statistics. https://www.cancer.org/cancer/colonrectal-cancer/about/key-statistics.html.

5. Cancer Research UK - Bowel Cancer Statistics.

https://www.cancerresearchuk.org/health-professional/cancer-statistics/statistics-bycancer-type/bowel-cancer.

6. Schreuders EH, Ruco A, Rabeneck L, Schoen RE, Sung JJY, Young GP, Kuipers EJ. Colorectal cancer screening: a global overview of existing programmes. Gut 2015;64(10): 1637-1649.

7. Center MM, Jemal A, Smith RA, Ward E. Worldwide variations in colorectal cancer. CA: a cancer journal for clinicians 2009;59(6): 366-378.

8. Rees CJ, Bevan R. The National Health Service Bowel Cancer Screening Program: the early years. Expert review of gastroenterology \& hepatology 2013;7(5): 421-437.

9. Hwang MJ, Evans T, Lawrence G, Karandikar S. Impact of bowel cancer screening on the management of colorectal cancer. Colorectal Dis 2014;16(6): 450-458.

10. Brenner H, Kloor M, Pox CP. Colorectal cancer. Lancet (London, England) 2014;383(9927): 1490-1502.

11. Jasperson KW, Tuohy TM, Neklason DW, Burt RW. Hereditary and Familial Colon Cancer. Gastroenterology 2010;138(6): 2044-2058.

12. Vasen HF, Tomlinson I, Castells A. Clinical management of hereditary colorectal cancer syndromes. Nature reviews Gastroenterology \& hepatology 2015;12(2): 88-97.

13. Grady WM. Genetic testing for high-risk colon cancer patients. Gastroenterology 2003;124(6): 1574-1594.

14. Kim ER, Chang DK. Colorectal cancer in inflammatory bowel disease: the risk, pathogenesis, prevention and diagnosis. World journal of gastroenterology 2014;20(29): 9872-9881.

15. Eaden JA, Abrams KR, Mayberry JF. The risk of colorectal cancer in ulcerative colitis: a meta-analysis. Gut 2001;48(4): 526-535.

16. Canavan C, Abrams KR, Mayberry J. Meta-analysis: colorectal and small bowel cancer risk in patients with Crohn's disease. Alimentary pharmacology \& therapeutics 2006;23(8): 1097-1104.

17. Brown KF, Rumgay H, Dunlop C, Ryan M, Quartly F, Cox A, Deas A, Elliss-Brookes L, Gavin A, Hounsome L, Huws D, Ormiston-Smith N, Shelton J, White C, Parkin DM. The fraction of cancer attributable to modifiable risk factors in England, Wales, Scotland, Northern Ireland, and the United Kingdom in 2015. Br J Cancer 2018;118(8): 1130-1141.

18. Fedirko V, Tramacere I, Bagnardi V, Rota M, Scotti L, Islami F, Negri E, Straif K, Romieu I, La Vecchia C, Boffetta P, Jenab M. Alcohol drinking and colorectal cancer risk: an 
overall and dose-response meta-analysis of published studies. Annals of oncology : official journal of the European Society for Medical Oncology / ESMO 2011;22(9): 1958-1972.

19. Tsoi KK, Pau CY, Wu WK, Chan FK, Griffiths S, Sung JJ. Cigarette smoking and the risk of colorectal cancer: a meta-analysis of prospective cohort studies. Clinical gastroenterology and hepatology : the official clinical practice journal of the American Gastroenterological Association 2009;7(6): 682-688.e681-685.

20. Walter V, Jansen L, Hoffmeister M, Brenner H. Smoking and survival of colorectal cancer patients: systematic review and meta-analysis. Annals of oncology : official journal of the European Society for Medical Oncology / ESMO 2014;25(8): 1517-1525.

21. Bardou M, Barkun AN, Martel M. Obesity and colorectal cancer. Gut 2013;62(6): 933947.

22. Peeters PJ, Bazelier MT, Leufkens HG, de Vries F, De Bruin ML. The risk of colorectal cancer in patients with type 2 diabetes: associations with treatment stage and obesity.

Diabetes care 2015;38(3): 495-502.

23. Diet, Nutrition, Physical Activity and Cancer: a Global Perspective. https://www.wcrf.org/dietandcancer.

24. Aune D, Chan DS, Lau R, Vieira R, Greenwood DC, Kampman E, Norat T. Dietary fibre, whole grains, and risk of colorectal cancer: systematic review and dose-response metaanalysis of prospective studies. Bmj 2011;343: d6617.

25. Alexander DD, Weed DL, Miller PE, Mohamed MA. Red Meat and Colorectal Cancer: A Quantitative Update on the State of the Epidemiologic Science. Journal of the American College of Nutrition 2015;34(6): 521-543.

26. Bernstein AM, Song $M$, Zhang X, Pan A, Wang M, Fuchs CS, Le N, Chan AT, Willett WC, Ogino S, Giovannucci EL, Wu K. Processed and Unprocessed Red Meat and Risk of Colorectal Cancer: Analysis by Tumor Location and Modification by Time. PLoS One 2015;10(8): e0135959.

27. Chiavarini M, Bertarelli G, Minelli L, Fabiani R. Dietary Intake of Meat CookingRelated Mutagens (HCAs) and Risk of Colorectal Adenoma and Cancer: A Systematic Review and Meta-Analysis. Nutrients 2017;9(5).

28. Ollberding NJ, Wilkens LR, Henderson BE, Kolonel LN, Le Marchand L. Meat consumption, heterocyclic amines and colorectal cancer risk: the Multiethnic Cohort Study. Int J Cancer 2012;131(7): E1125-1133.

29. Weiser MR. AJCC 8th Edition: Colorectal Cancer. Annals of Surgical Oncology 2018;25(6): 1454-1455.

30. AJCC. American Joint Committee on Cancer-Staging Manual (8th edn), 2018. 31. Langman G, Loughrey M, Shepherd N, Quirke P. Association of Coloproctology of Great Britain \& Ireland (ACPGBI): Guidelines for the Management of Cancer of the Colon, Rectum and Anus (2017) - Pathology Standards and Datasets. Colorectal Disease 2017;19: 74-81.

32. Dukes CE. The classification of cancer of the rectum. The Journal of Pathology and Bacteriology 1932;35(3): 323-332.

33. Haq Al, Schneeweiss J, Kalsi V, Arya M. The Dukes staging system: a cornerstone in the clinical management of colorectal cancer. The Lancet Oncology 2009;10(11): 1128.

34. Moran B, Karandikar S, Geh I. Association of Coloproctology of Great Britain \& Ireland (ACPGBI): Guidelines for the Management of Cancer of the Colon, Rectum and Anus (2017) - Introduction. Colorectal Disease 2017;19: 6-8. 
35. Standards and Datasets for ReportingCcancers - Dataset for histopathological reporting of colorectal cancer. https://www.rcpath.org/profession/publications/cancerdatasets.html.

36. Ryan R, Gibbons D, Hyland JM, Treanor D, White A, Mulcahy HE, O'Donoghue DP, Moriarty M, Fennelly $D$, Sheahan K. Pathological response following long-course neoadjuvant chemoradiotherapy for locally advanced rectal cancer. Histopathology 2005;47(2): 141-146.

37. NICE. Molecular testing strategies for Lynch syndrome in people with colorectal cancer. 2017; Diagnostics guidance [DG27].

38. Chan DLH, Segelov E, Wong RS, Smith A, Herbertson RA, Li BT, Tebbutt N, Price T, Pavlakis N. Epidermal growth factor receptor (EGFR) inhibitors for metastatic colorectal cancer. The Cochrane database of systematic reviews 2017;6: Cd007047.

39. Qureshi A, Verma A, Ross P, Landau D. Colorectal cancer treatment. BMJ Clinical Evidence 2010;2010.

40. Colorectal Cancer Survival Rates: Americal Cancer Society.

https://www.cancer.org/cancer/colon-rectal-cancer/detection-diagnosis-staging/survivalrates.html.

41. CCCG. Surgery for colorectal cancer in elderly patients: a systematic review. Colorectal Cancer Collaborative Group. Lancet (London, England) 2000;356(9234): 968-974.

42. Wilhelmsen $M$, Kring $T$, Jorgensen $L N$, Madsen MR, Jess $P$, Bulut $O$, Nielsen KT, Andersen $\mathrm{CL}$, Nielsen HJ. Determinants of recurrence after intended curative resection for colorectal cancer. Scandinavian journal of gastroenterology 2014;49(12): 1399-1408.

43. Leong K, Hartley J, Karandikar S. Association of Coloproctology of Great Britain \& Ireland (ACPGBI): Guidelines for the Management of Cancer of the Colon, Rectum and Anus (2017) - Follow Up, Lifestyle and Survivorship. Colorectal Disease 2017;19: 67-70.

44. Primrose JN, Perera R, Gray A, Rose P, Fuller A, Corkhill A, George S, Mant D. Effect of 3 to 5 years of scheduled CEA and CT follow-up to detect recurrence of colorectal cancer: the FACS randomized clinical trial. Jama 2014;311(3): 263-270.

45. Mokhles S, Macbeth F, Farewell V, Fiorentino F, Williams NR, Younes RN, Takkenberg $\mathrm{JJ}$, Treasure T. Meta-analysis of colorectal cancer follow-up after potentially curative resection. Br J Surg 2016;103(10): 1259-1268.

46. Macmillan Impact Briefs. Cancer Clinical Nurse Specialist. http://www.macmillan.org.uk/documents/aboutus/research/impactbriefs/impactbriefsclinicalnursespecialists2014.pdf.

47. Nygren J, Thacker J, Carli F, Fearon KC, Norderval S, Lobo DN, Ljungqvist O, Soop M, Ramirez J. Guidelines for perioperative care in elective rectal/pelvic surgery: Enhanced Recovery After Surgery (ERAS(R)) Society recommendations. Clinical nutrition (Edinburgh, Scotland) 2012;31(6): 801-816.

48. Noblett SE, Watson DS, Huong H, Davison B, Hainsworth PJ, Horgan AF. Preoperative oral carbohydrate loading in colorectal surgery: a randomized controlled trial. Colorectal Dis 2006;8(7): 563-569.

49. Bretagnol F, Panis Y, Rullier E, Rouanet P, Berdah S, Dousset B, Portier G, Benoist S, Chipponi J, Vicaut E. Rectal cancer surgery with or without bowel preparation: the French GRECCAR III multicenter single-blinded randomized trial. Ann Surg 2010;252: 863-868.

50. Guenaga KF, Matos D, Wille-Jorgensen P. Mechanical bowel preparation for elective colorectal surgery. The Cochrane database of systematic reviews 2011(9): Cd001544.

51. Bachmann R, Leonard D, Delzenne N, Kartheuser A, Cani PD. Novel insight into the role of microbiota in colorectal surgery. Gut 2017;66(4): 738-749. 
52. Blanco-Colino R, Espin-Basany E. Intraoperative use of ICG fluorescence imaging to reduce the risk of anastomotic leakage in colorectal surgery: a systematic review and metaanalysis. Tech Coloproctol 2018;22(1): 15-23.

53. Moran B, Cunningham C, Singh T, Sagar P, Bradbury J, Geh I, Karandikar S. Association of Coloproctology of Great Britain \& Ireland (ACPGBI): Guidelines for the Management of Cancer of the Colon, Rectum and Anus (2017) - Surgical Management. Colorectal Disease 2017;19: 18-36.

54. Stevenson AR, Solomon MJ, Lumley JW, Hewett P, Clouston AD, Gebski VJ, Davies L, Wilson K, Hague W, Simes J. Effect of Laparoscopic-Assisted Resection vs Open Resection on Pathological Outcomes in Rectal Cancer: The ALaCaRT Randomized Clinical Trial. Jama 2015;314(13): 1356-1363.

55. Fleshman J, Branda M, Sargent DJ, Boller AM, George V, Abbas M, Peters WR, Jr., Maun D, Chang G, Herline A, Fichera A, Mutch M, Wexner S, Whiteford M, Marks J, Birnbaum E, Margolin D, Larson D, Marcello P, Posner M, Read T, Monson J, Wren SM, Pisters PW, Nelson H. Effect of Laparoscopic-Assisted Resection vs Open Resection of Stage II or III Rectal Cancer on Pathologic Outcomes: The ACOSOG Z6051 Randomized Clinical Trial. Jama 2015;314(13): 1346-1355.

56. Jayne D, Pigazzi A, Marshall H, Croft J, Corrigan N, Copeland J, Quirke P, West N, Rautio T, Thomassen N, Tilney H, Gudgeon M, Bianchi PP, Edlin R, Hulme C, Brown J. Effect of Robotic-Assisted vs Conventional Laparoscopic Surgery on Risk of Conversion to Open Laparotomy Among Patients Undergoing Resection for Rectal Cancer: The ROLARR Randomized Clinical Trial. Jama 2017;318(16): 1569-1580.

57. Clancy C, Burke JP, Albert MR, O'Connell PR, Winter DC. Transanal endoscopic microsurgery versus standard transanal excision for the removal of rectal neoplasms: a systematic review and meta-analysis. Diseases of the colon and rectum 2015;58(2): 254-261.

58. Martin-Perez B, Andrade-Ribeiro GD, Hunter L, Atallah S. A systematic review of transanal minimally invasive surgery (TAMIS) from 2010 to 2013. Tech Coloproctol 2014;18(9): 775-788.

59. Melin AA, Kalaskar S, Taylor L, Thompson JS, Ternent C, Langenfeld SJ. Transanal endoscopic microsurgery and transanal minimally invasive surgery: is one technique superior? Am J Surg 2016;212(6): 1063-1067.

60. Hong KD, Kang S, Urn JW, Lee SI. Transanal Minimally Invasive Surgery (TAMIS) for Rectal Lesions: A Systematic Review. Hepato-gastroenterology 2015;62(140): 863-867.

61. Simillis $C$, Hompes R, Penna M, Rasheed S, Tekkis PP. A systematic review of transanal total mesorectal excision: is this the future of rectal cancer surgery? Colorectal Dis 2016;18(1): 19-36.

62. Motson RW, Whiteford MH, Hompes R, Albert M, Miles WF. Current status of transanal total mesorectal excision (TaTME) following the Second International Consensus Conference. Colorectal Dis 2016;18(1): 13-18.

63. NICE. Transanal total mesorectal excision of the rectum. [IPG514]. 2015(IPG514). 64. International TaTME Educational Collaborative. https://www.tatme.surgery/?page id=460.

65. Kokelaar RF, Evans MD, Davies M, Harris DA, Beynon J. Locally advanced rectal cancer: management challenges. Onco Targets Therapy 2016;9: 6265-6272.

66. Beyond TMEC. Consensus statement on the multidisciplinary management of patients with recurrent and primary rectal cancer beyond total mesorectal excision planes. Br J Surg 2013;100(8): 1009-1014. 
67. Brown KGM, Solomon MJ, Koh CE. Pelvic Exenteration Surgery: The Evolution of Radical Surgical Techniques for Advanced and Recurrent Pelvic Malignancy. Diseases of the colon and rectum 2017;60(7): 745-754.

68. Koh CE, Solomon MJ, Brown KG, Austin K, Byrne CM, Lee P, Young JM. The Evolution of Pelvic Exenteration Practice at a Single Center: Lessons Learned from over 500 Cases.

Diseases of the colon and rectum 2017;60(6): 627-635.

69. The PelvEx Collaborative: International Registry of Pelvic Exenterative Surgery. https://www.pelvex.org.

70. Habr-Gama A, Perez RO, Nadalin W, Sabbaga J, Ribeiro U, Jr., Silva e Sousa AH, Jr., Campos FG, Kiss DR, Gama-Rodrigues J. Operative versus nonoperative treatment for stage 0 distal rectal cancer following chemoradiation therapy: long-term results. Ann Surg 2004;240(4): 711-717; discussion 717-718.

71. Garcia-Aguilar J, Renfro LA, Chow OS, Shi Q, Carrero XW, Lynn PB, Thomas CR, Jr., Chan E, Cataldo PA, Marcet JE, Medich DS, Johnson CS, Oommen SC, Wolff BG, Pigazzi A, McNevin SM, Pons RK, Bleday R. Organ preservation for clinical T2NO distal rectal cancer using neoadjuvant chemoradiotherapy and local excision (ACOSOG Z6041): results of an open-label, single-arm, multi-institutional, phase 2 trial. The Lancet Oncology 2015;16(15): 1537-1546.

72. Sao Juliao GP, Habr-Gama A, Vailati BB, Araujo SEA, Fernandez LM, Perez RO. New Strategies in Rectal Cancer. The Surgical clinics of North America 2017;97(3): 587-604.

73. Habr-Gama A, Sao Juliao GP, Vailati BB, Castro I, Raffaele D. Management of the Complete Clinical Response. Clinics in colon and rectal surgery 2017;30(5): 387-394.

74. Bernier L, Balyasnikova S, Tait D, Brown G. Watch-and-Wait as a Therapeutic Strategy in Rectal Cancer. Current Colorectal Cancer Reports 2018;14(2): 37-55.

75. Wolthuis AM, Penninckx F, Haustermans K, De Hertogh G, Fieuws S, Van Cutsem E, D'Hoore A. Impact of interval between neoadjuvant chemoradiotherapy and TME for locally advanced rectal cancer on pathologic response and oncologic outcome. Ann Surg Oncol 2012;19(9): 2833-2841.

76. Das P, Skibber JM, Rodriguez-Bigas MA, Feig BW, Chang GJ, Wolff RA, Eng C, Krishnan $\mathrm{S}$, Janjan NA, Crane $\mathrm{CH}$. Predictors of tumor response and downstaging in patients who receive preoperative chemoradiation for rectal cancer. Cancer 2007;109(9): 1750-1755.

77. Al-Sukhni E, Attwood K, Mattson DM, Gabriel E, Nurkin SJ. Predictors of Pathologic Complete Response Following Neoadjuvant Chemoradiotherapy for Rectal Cancer. Ann Surg Oncol 2016;23(4): 1177-1186.

78. Sauer R, Becker H, Hohenberger W, Rodel C, Wittekind C, Fietkau R, Martus P, Tschmelitsch J, Hager E, Hess CF, Karstens JH, Liersch T, Schmidberger H, Raab R. Preoperative versus postoperative chemoradiotherapy for rectal cancer. The New England journal of medicine 2004;351(17): 1731-1740.

79. Gerard JP, Conroy T, Bonnetain F, Bouche O, Chapet O, Closon-Dejardin MT, Untereiner M, Leduc B, Francois E, Maurel J, Seitz JF, Buecher B, Mackiewicz R, Ducreux M, Bedenne L. Preoperative radiotherapy with or without concurrent fluorouracil and leucovorin in T3-4 rectal cancers: results of FFCD 9203. Journal of clinical oncology : official journal of the American Society of Clinical Oncology 2006;24(28): 4620-4625.

80. Bosset JF, Collette L, Calais G, Mineur L, Maingon P, Radosevic-Jelic L, Daban A, Bardet $E$, Beny A, Ollier JC. Chemotherapy with preoperative radiotherapy in rectal cancer. The New England journal of medicine 2006;355(11): 1114-1123.

81. Roh MS, Colangelo LH, O'Connell MJ, Yothers G, Deutsch M, Allegra CJ, Kahlenberg MS, Baez-Diaz L, Ursiny CS, Petrelli NJ, Wolmark N. Preoperative multimodality therapy 
improves disease-free survival in patients with carcinoma of the rectum: NSABP R-03. Journal of clinical oncology : official journal of the American Society of Clinical Oncology 2009;27(31): 5124-5130.

82. Li Y, Wang J, Ma X, Tan L, Yan Y, Xue C, Hui B, Liu R, Ma H, Ren J. A Review of Neoadjuvant Chemoradiotherapy for Locally Advanced Rectal Cancer. International Journal of Biological Sciences 2016;12(8): 1022-1031.

83. Bosset JF, Calais G, Mineur L, Maingon P, Stojanovic-Rundic S, Bensadoun RJ, Bardet E, Beny A, Ollier JC, Bolla M, Marchal D, Van Laethem JL, Klein V, Giralt J, Clavere P, Glanzmann C, Cellier P, Collette L. Fluorouracil-based adjuvant chemotherapy after preoperative chemoradiotherapy in rectal cancer: long-term results of the EORTC 22921 randomised study. The Lancet Oncology 2014;15(2): 184-190.

84. Foxtrot Collaborative G. Feasibility of preoperative chemotherapy for locally advanced, operable colon cancer: the pilot phase of a randomised controlled trial. The Lancet Oncology 2012;13(11): 1152-1160.

85. Colorectal cancer: diagnosis and management. [CG131].

https://www.nice.org.uk/guidance/CG131.

86. NICE. Capecitabine and oxaliplatin in the adjuvant treatment of stage III (Dukes' C) colon cancer. [TA100]. 2006.

87. Twelves C, Scheithauer W, McKendrick J, Seitz JF, Van Hazel G, Wong A, Diaz-Rubio E, Gilberg F, Cassidy J. Capecitabine versus 5-fluorouracil/folinic acid as adjuvant therapy for stage III colon cancer: final results from the X-ACT trial with analysis by age and preliminary evidence of a pharmacodynamic marker of efficacy. Annals of oncology : official journal of the European Society for Medical Oncology / ESMO 2012;23(5): 1190-1197.

88. Andre T, Boni C, Mounedji-Boudiaf L, Navarro M, Tabernero J, Hickish T, Topham C, Zaninelli M, Clingan P, Bridgewater J, Tabah-Fisch I, de Gramont A. Oxaliplatin, fluorouracil, and leucovorin as adjuvant treatment for colon cancer. The New England journal of medicine 2004;350(23): 2343-2351.

89. Kuebler JP, Wieand HS, O'Connell MJ, Smith RE, Colangelo LH, Yothers G, Petrelli NJ, Findlay MP, Seay TE, Atkins JN, Zapas JL, Goodwin JW, Fehrenbacher L, Ramanathan RK, Conley BA, Flynn PJ, Soori G, Colman LK, Levine EA, Lanier KS, Wolmark N. Oxaliplatin combined with weekly bolus fluorouracil and leucovorin as surgical adjuvant chemotherapy for stage II and III colon cancer: results from NSABP C-07. Journal of clinical oncology: official journal of the American Society of Clinical Oncology 2007;25(16): 2198-2204.

90. Liu Y, Wu W, Hong W, Sun X, Wu J, Huang Q. Raltitrexed-based chemotherapy for advanced colorectal cancer. Clinics and research in hepatology and gastroenterology 2014;38(2): 219-225.

91. NICE. Cetuximab and panitumumab for previously untreated metastatic colorectal cancer [TA439]. 2017.

92. Bokemeyer C, Bondarenko I, Hartmann JT, de Braud F, Schuch G, Zubel A, Celik I, Schlichting M, Koralewski P. Efficacy according to biomarker status of cetuximab plus FOLFOX-4 as first-line treatment for metastatic colorectal cancer: the OPUS study. Annals of oncology : official journal of the European Society for Medical Oncology / ESMO 2011;22(7): 1535-1546.

93. Douillard JY, Siena S, Cassidy J, Tabernero J, Burkes R, Barugel M, Humblet Y, Bodoky G, Cunningham D, Jassem J, Rivera F, Kocakova I, Ruff P, Blasinska-Morawiec M, Smakal M, Canon JL, Rother M, Oliner KS, Tian Y, Xu F, Sidhu R. Final results from PRIME: randomized phase III study of panitumumab with FOLFOX4 for first-line treatment of metastatic 
colorectal cancer. Annals of oncology : official journal of the European Society for Medical Oncology / ESMO 2014;25(7): 1346-1355.

94. Feng QY, Wei Y, Chen JW, Chang WJ, Ye LC, Zhu DX, Xu JM. Anti-EGFR and anti-VEGF agents: important targeted therapies of colorectal liver metastases. World journal of gastroenterology 2014;20(15): 4263-4275.

95. Heinemann V, von Weikersthal LF, Decker T, Kiani A, Vehling-Kaiser U, Al-Batran SE, Heintges T, Lerchenmuller C, Kahl C, Seipelt G, Kullmann F, Stauch M, Scheithauer W, Hielscher J, Scholz M, Muller S, Link H, Niederle N, Rost A, Hoffkes HG, Moehler M, Lindig RU, Modest DP, Rossius L, Kirchner T, Jung A, Stintzing S. FOLFIRI plus cetuximab versus FOLFIRI plus bevacizumab as first-line treatment for patients with metastatic colorectal cancer (FIRE-3): a randomised, open-label, phase 3 trial. The Lancet Oncology 2014;15(10): 1065-1075.

96. NICE. Bevacizumab in combination with oxaliplatin and either fluorouracil plus folinic acid or capecitabine for the treatment of metastatic colorectal cancer. [TA212]. 2010.

97. Van Cutsem E, Joulain F, Hoff PM, Mitchell E, Ruff P, Lakomy R, Prausova J, Moiseyenko VM, van Hazel G, Cunningham D, Arnold D, Schmoll HJ, Ten Tije AJ, McKendrick J, Kroning $\mathrm{H}$, Humblet $\mathrm{Y}$, Gravalos $\mathrm{C}$, Le-Guennec $\mathrm{S}$, Andria $\mathrm{M}$, Dochy $\mathrm{E}$, Vishwanath $\mathrm{RL}$, Macarulla T, Tabernero J. Aflibercept Plus FOLFIRI vs. Placebo Plus FOLFIRI in Second-Line Metastatic Colorectal Cancer: a Post Hoc Analysis of Survival from the Phase III VELOUR Study Subsequent to Exclusion of Patients who had Recurrence During or Within 6 Months of Completing Adjuvant Oxaliplatin-Based Therapy. Targeted oncology 2016;11(3): 383-400. 98. NICE. Aflibercept in combination with irinotecan and fluorouracil-based therapy for treating metastatic colorectal cancer that has progressed following prior oxaliplatin-based chemotherapy. [TA307]. 2014.

99. Bosset JF, Calais G, Mineur L, Maingon P, Radosevic-Jelic L, Daban A, Bardet E, Beny A, Briffaux A, Collette L. Enhanced tumorocidal effect of chemotherapy with preoperative radiotherapy for rectal cancer: preliminary results--EORTC 22921. Journal of clinical oncology : official journal of the American Society of Clinical Oncology 2005;23(24): 56205627.

100. Vuong T, Belliveau PJ, Michel RP, Moftah BA, Parent J, Trudel JL, Reinhold C, Souhami L. Conformal preoperative endorectal brachytherapy treatment for locally advanced rectal cancer: early results of a phase I/II study. Diseases of the colon and rectum 2002;45(11): 1486-1493; discussion 1493-1485.

101. Kusters $M$, Valentini V, Calvo FA, Krempien R, Nieuwenhuijzen GA, Martijn $H$, Doglietto GB, Del Valle E, Roeder F, Buchler MW, van de Velde CJ, Rutten HJ. Results of European pooled analysis of IORT-containing multimodality treatment for locally advanced rectal cancer: adjuvant chemotherapy prevents local recurrence rather than distant metastases. Annals of oncology : official journal of the European Society for Medical Oncology / ESMO 2010;21(6): 1279-1284.

102. Mirnezami R, Chang GJ, Das P, Chandrakumaran K, Tekkis P, Darzi A, Mirnezami AH. Intraoperative radiotherapy in colorectal cancer: systematic review and meta-analysis of techniques, long-term outcomes, and complications. Surgical oncology 2013;22(1): 22-35. 103. Glynne-Jones R, Hall M. Radiotherapy and locally advanced rectal cancer. Br J Surg 2015;102(12): 1443-1445.

104. Bossé D, Mercer J, Raissouni S, Dennis K, Goodwin R, Jiang D, Powell E, Kumar A, LeeYing R, Price-Hiller J, Heng DYC, Tang PA, MacLean A, Cheung WY, Vickers MM. PROSPECT Eligibility and Clinical Outcomes: Results From the Pan-Canadian Rectal Cancer Consortium. Clinical colorectal cancer. 
105. Glynne-Jones R, Hava N, Goh V, Bosompem S, Bridgewater J, Chau I, Gaya A, Wasan H, Moran B, Melcher L, MacDonald A, Osborne M, Beare S, Jitlal M, Lopes A, Hall M, West N, Quirke $\mathrm{P}$, Wong WL, Harrison M. Bevacizumab and Combination Chemotherapy in rectal cancer Until Surgery (BACCHUS): a phase II, multicentre, open-label, randomised study of neoadjuvant chemotherapy alone in patients with high-risk cancer of the rectum. $B M C$ Cancer 2015;15: 764.

106. Gelsomino F, Bertolini F, Luppi G, Spallanzani A, Pettorelli E, Reggiani Bonetti L, Meduri B, Manco G, Conte P, Cascinu S. A Dose-finding and Biomarker Evaluation Phase Ib

Study of Everolimus in Association With 5-Fluorouracil and Pelvic Radiotherapy as Neoadjuvant Treatment of Locally Advanced Rectal Cancer (E-LARC Study). Clinical colorectal cancer 2017;16(4): 410-415.e411.

107. Hill J, Kay C, Morton DG. Randomised phase III study of stenting as a bridge to surgery in obstructing colorectal cancer-Results of the UK ColoRectal Endoscopic Stenting Trial (CREST). Journal of clinical oncology : official journal of the American Society of Clinical Oncology 2016;34: suppl; abstr 3507.

108. Powell SM, Zilz N, Beazer-Barclay Y, Bryan TM, Hamilton SR, Thibodeau SN, Vogelstein B, Kinzler KW. APC mutations occur early during colorectal tumorigenesis. Nature 1992;359(6392): 235-237.

109. Vogelstein B, Fearon ER, Hamilton SR, Kern SE, Preisinger AC, Leppert M, Nakamura Y, White R, Smits AM, Bos JL. Genetic alterations during colorectal-tumor development. The New England journal of medicine 1988;319(9): 525-532.

110. Thorstensen L, Lind GE, Lovig T, Diep CB, Meling GI, Rognum TO, Lothe RA. Genetic and epigenetic changes of components affecting the WNT pathway in colorectal carcinomas stratified by microsatellite instability. Neoplasia (New York, NY) 2005;7(2): 99-108.

111. Hartman DJ, Davison JM, Foxwell TJ, Nikiforova MN, Chiosea SI. Mutant allelespecific imbalance modulates prognostic impact of KRAS mutations in colorectal adenocarcinoma and is associated with worse overall survival. Int J Cancer 2012;131(8): 1810-1817.

112. Russo A, Bazan V, lacopetta B, Kerr D, Soussi T, Gebbia N. The TP53 colorectal cancer international collaborative study on the prognostic and predictive significance of p53 mutation: influence of tumor site, type of mutation, and adjuvant treatment. Journal of clinical oncology : official journal of the American Society of Clinical Oncology 2005;23(30): 7518-7528.

113. Wang JJ, Zheng Y, Sun L, Wang L, Yu PB, Dong JH, Zhang L, Xu J, Shi W, Ren YC. TP53 codon 72 polymorphism and colorectal cancer susceptibility: a meta-analysis. Molecular biology reports 2011;38(8): 4847-4853.

114. Leslie A, Carey FA, Pratt NR, Steele RJ. The colorectal adenoma-carcinoma sequence. Br J Surg 2002;89(7): 845-860.

115. Pino MS, Chung DC. The chromosomal instability pathway in colon cancer. Gastroenterology 2010;138(6): 2059-2072.

116. Grady WM, Carethers JM. Genomic and epigenetic instability in colorectal cancer pathogenesis. Gastroenterology 2008;135(4): 1079-1099.

117. Grady WM, Markowitz S. Genomic instability and colorectal cancer. Current opinion in gastroenterology 2000;16(1): 62-67.

118. Dienstmann R, Vermeulen L, Guinney J, Kopetz S, Tejpar S, Tabernero J. Consensus molecular subtypes and the evolution of precision medicine in colorectal cancer. Nat Rev Cancer 2017;17(2): 79-92. 
119. Rubinfeld B, Albert I, Porfiri E, Fiol C, Munemitsu S, Polakis P. Binding of GSK3beta to the APC-beta-catenin complex and regulation of complex assembly. Science 1996;272(5264): 1023-1026.

120. Mann B, Gelos M, Siedow A, Hanski ML, Gratchev A, llyas M, Bodmer WF, Moyer MP, Riecken EO, Buhr HJ, Hanski C. Target genes of beta-catenin-T cell-factor/lymphoidenhancer-factor signaling in human colorectal carcinomas. Proc Natl Acad Sci U S A 1999;96(4): 1603-1608.

121. Lipsick J. A History of Cancer Research: Tyrosine Kinases. Cold Spring Harbor perspectives in biology 2019;11(2).

122. Shiovitz S, Grady WM. Molecular markers predictive of chemotherapy response in colorectal cancer. Current gastroenterology reports 2015;17(2): 431.

123. Sinicrope FA, Okamoto K, Kasi PM, Kawakami H. Molecular Biomarkers in the Personalized Treatment of Colorectal Cancer. Clinical gastroenterology and hepatology : the official clinical practice journal of the American Gastroenterological Association 2016;14(5): 651-658.

124. Yamaoka T, Ohba M, Ohmori T. Molecular-Targeted Therapies for Epidermal Growth Factor Receptor and Its Resistance Mechanisms. International journal of molecular sciences 2017;18(11).

125. Yamauchi M, Morikawa T, Kuchiba A, Imamura Y, Qian ZR, Nishihara R, Liao X, Waldron L, Hoshida Y, Huttenhower C, Chan AT, Giovannucci E, Fuchs C, Ogino S. Assessment of colorectal cancer molecular features along bowel subsites challenges the conception of distinct dichotomy of proximal versus distal colorectum. Gut 2012;61(6): 847854.

126. Rosty C, Young JP, Walsh MD, Clendenning M, Walters RJ, Pearson S, Pavluk E, Nagler B, Pakenas D, Jass JR, Jenkins MA, Win AK, Southey MC, Parry S, Hopper JL, Giles GG, Williamson E, English DR, Buchanan DD. Colorectal carcinomas with KRAS mutation are associated with distinctive morphological and molecular features. Modern Pathology 2013;26: 825.

127. Peyssonnaux C, Eychene A. The Raf/MEK/ERK pathway: new concepts of activation. Biology of the cell 2001;93(1-2): 53-62.

128. Davies H, Bignell GR, Cox C, Stephens P, Edkins S, Clegg S, Teague J, Woffendin H, Garnett MJ, Bottomley W, Davis N, Dicks E, Ewing R, Floyd Y, Gray K, Hall S, Hawes R, Hughes

J, Kosmidou V, Menzies A, Mould C, Parker A, Stevens C, Watt S, Hooper S, Wilson R, Jayatilake H, Gusterson BA, Cooper C, Shipley J, Hargrave D, Pritchard-Jones K, Maitland N, Chenevix-Trench G, Riggins GJ, Bigner DD, Palmieri G, Cossu A, Flanagan A, Nicholson A, Ho JWC, Leung SY, Yuen ST, Weber BL, Seigler HF, Darrow TL, Paterson H, Marais R, Marshall CJ, Wooster R, Stratton MR, Futreal PA. Mutations of the BRAF gene in human cancer. Nature 2002;417: 949.

129. Chen D, Huang JF, Liu K, Zhang LQ, Yang Z, Chuai ZR, Wang YX, Shi DC, Huang Q, Fu WL. BRAFV600E mutation and its association with clinicopathological features of colorectal cancer: a systematic review and meta-analysis. PLoS One 2014;9(3): e90607.

130. Chouhan H, Sammour T, Thomas ML, Moore JW. The interaction between BRAF mutation and microsatellite instability (MSI) status in determining survival outcomes after adjuvant 5FU based chemotherapy in stage III colon cancer. J Surg Oncol 2018;118(8): 13111317.

131. Luke JJ, Flaherty KT, Ribas A, Long GV. Targeted agents and immunotherapies: optimizing outcomes in melanoma. Nat Rev Clin Oncol 2017;14(8): 463-482. 
132. Cohen R, Cervera P, Svrcek M, Pellat A, Dreyer C, de Gramont A, Andre T. BRAFMutated Colorectal Cancer: What Is the Optimal Strategy for Treatment? Current treatment options in oncology 2017;18(2): 9.

133. Sanz-Garcia E, Argiles G, Elez E, Tabernero J. BRAF mutant colorectal cancer: prognosis, treatment, and new perspectives. Annals of oncology : official journal of the European Society for Medical Oncology / ESMO 2017;28(11): 2648-2657.

134. Lodish H, Berk A, Zipursky S, Matsudaira P, Baltimore D, Darnell J. Molecular Cell Biology (4th Edition edn). W. H. Freeman: New York, 2000.

135. Vogelstein B, Lane D, Levine AJ. Surfing the p53 network. Nature 2000;408(6810): 307-310.

136. Fearon ER, Vogelstein B. A genetic model for colorectal tumorigenesis. Cell 1990;61(5): 759-767.

137. Cho KR, Oliner JD, Simons JW, Hedrick L, Fearon ER, Preisinger AC, Hedge P, Silverman GA, Vogelstein B. The DCC gene: structural analysis and mutations in colorectal carcinomas. Genomics 1994;19(3): 525-531.

138. Takagi $Y$, Koumura H, Futamura M, Aoki S, Ymaguchi K, Kida H, Tanemura H, Shimokawa K, Saji S. Somatic alterations of the SMAD-2 gene in human colorectal cancers. British journal of cancer 1998;78(9): 1152-1155.

139. Park DY, Sakamoto H, Kirley SD, Ogino S, Kawasaki T, Kwon E, Mino-Kenudson M, Lauwers GY, Chung DC, Rueda BR, Zukerberg LR. The Cables gene on chromosome 18q is silenced by promoter hypermethylation and allelic loss in human colorectal cancer. Am J Pathol 2007;171(5): 1509-1519.

140. Boland CR, Goel A. Microsatellite instability in colorectal cancer. Gastroenterology 2010;138(6): 2073-2087.e2073.

141. Korol AB, Nevo E, Fahima T, Li Y-C. Microsatellites Within Genes: Structure, Function, and Evolution. Molecular Biology and Evolution 2004;21(6): 991-1007.

142. Boland CR, Thibodeau SN, Hamilton SR, Sidransky D, Eshleman JR, Burt RW, Meltzer SJ, Rodriguez-Bigas MA, Fodde R, Ranzani GN, Srivastava S. A National Cancer Institute Workshop on Microsatellite Instability for cancer detection and familial predisposition: development of international criteria for the determination of microsatellite instability in colorectal cancer. Cancer Res 1998;58(22): 5248-5257.

143. Cancer Genome Atlas N. Comprehensive molecular characterization of human colon and rectal cancer. Nature 2012;487(7407): 330-337.

144. Kloor M, Staffa L, Ahadova A, von Knebel Doeberitz M. Clinical significance of microsatellite instability in colorectal cancer. Langenbecks Arch Surg 2014;399(1): 23-31.

145. Møller P, Seppälä TT, Bernstein I, Holinski-Feder E, Sala P, Gareth Evans D, Lindblom A, Macrae F, Blanco I, Sijmons RH, Jeffries J, Vasen HFA, Burn J, Nakken S, Hovig E, Rødland EA, Tharmaratnam K, de Vos tot Nederveen Cappel WH, Hill J, Wijnen JT, Jenkins MA, Green K, Lalloo F, Sunde L, Mints M, Bertario L, Pineda M, Navarro M, Morak M, Renkonen-Sinisalo $L$, Valentin MD, Frayling IM, Plazzer J-P, Pylvanainen K, Genuardi M, Mecklin J-P, Moeslein G, Sampson JR, Capella G. Cancer risk and survival in <em>path_MMR</em> carriers by gene and gender up to 75 years of age: a report from the Prospective Lynch Syndrome Database.

Gut 2018;67(7): 1306-1316.

146. Vilar E, Tabernero J. Molecular dissection of microsatellite instable colorectal cancer. Cancer discovery 2013;3(5): 502-511.

147. Missiaglia E, Jacobs B, D'Ario G, Di Narzo AF, Soneson C, Budinska E, Popovici V, Vecchione L, Gerster S, Yan P, Roth AD, Klingbiel D, Bosman FT, Delorenzi M, Tejpar S. Distal and proximal colon cancers differ in terms of molecular, pathological, and clinical features. 
Annals of oncology : official journal of the European Society for Medical Oncology / ESMO 2014;25(10): 1995-2001.

148. Williamson JS, Harris DA, Beynon J, Jenkins GJ. Review of the development of DNA methylation as a marker of response to neoadjuvant therapy and outcomes in rectal cancer.

Clin Epigenetics 2015;7(1): 70.

149. Jo P, Jung K, Grade M, Conradi LC, Wolff HA, Kitz J, Becker H, Ruschoff J, Hartmann A,

Beissbarth T, Muller-Dornieden A, Ghadimi M, Schneider-Stock R, Gaedcke J. CpG island methylator phenotype infers a poor disease-free survival in locally advanced rectal cancer.

Surgery 2012;151(4): 564-570.

150. Setaffy L, Langner C. Microsatellite instability in colorectal cancer: clinicopathological significance. Polish journal of pathology : official journal of the Polish Society of Pathologists 2015;66(3): 203-218.

151. Heng HH, Bremer SW, Stevens JB, Horne SD, Liu G, Abdallah BY, Ye KJ, Ye CJ. Chromosomal instability (CIN): what it is and why it is crucial to cancer evolution. Cancer metastasis reviews 2013;32(3-4): 325-340.

152. Duijf PH, Benezra R. The cancer biology of whole-chromosome instability. Oncogene 2013;32(40): 4727-4736.

153. Rowan A, Halford S, Gaasenbeek M, Kemp Z, Sieber O, Volikos E, Douglas E, Fiegler $\mathrm{H}$, Carter N, Talbot I, Silver A, Tomlinson I. Refining molecular analysis in the pathways of colorectal carcinogenesis. Clinical gastroenterology and hepatology : the official clinical practice journal of the American Gastroenterological Association 2005;3(11): 1115-1123.

154. Weber JC, Meyer N, Pencreach E, Schneider A, Guerin E, Neuville A, Stemmer C, Brigand C, Bachellier P, Rohr S, Kedinger M, Meyer C, Guenot D, Oudet P, Jaeck D, Gaub MP. Allelotyping analyses of synchronous primary and metastasis $\mathrm{CIN}$ colon cancers identified different subtypes. Int J Cancer 2007;120(3): 524-532.

155. Sinicrope FA, Rego RL, Halling KC, Foster N, Sargent DJ, La Plant B, French AJ, Laurie JA, Goldberg RM, Thibodeau SN, Witzig TE. Prognostic impact of microsatellite instability and DNA ploidy in human colon carcinoma patients. Gastroenterology 2006;131(3): 729-

737.

156. Walther A, Houlston R, Tomlinson I. Association between chromosomal instability and prognosis in colorectal cancer: a meta-analysis. Gut 2008;57(7): 941-950.

157. Loeb LA, Loeb KR, Anderson JP. Multiple mutations and cancer. Proc Natl Acad Sci U $S$ A 2003;100(3): 776-781.

158. Fodde R, Kuipers J, Rosenberg C, Smits R, Kielman M, Gaspar C, van Es JH, Breukel C, Wiegant J, Giles RH, Clevers H. Mutations in the APC tumour suppressor gene cause chromosomal instability. Nature cell biology 2001;3(4): 433-438.

159. Kaplan KB, Burds AA, Swedlow JR, Bekir SS, Sorger PK, Nathke IS. A role for the Adenomatous Polyposis Coli protein in chromosome segregation. Nature cell biology 2001;3(4): 429-432.

160. Walther A, Johnstone E, Swanton C, Midgley R, Tomlinson I, Kerr D. Genetic prognostic and predictive markers in colorectal cancer. Nat Rev Cancer 2009;9(7): 489-499.

161. Jass JR. Classification of colorectal cancer based on correlation of clinical, morphological and molecular features. Histopathology 2007;50(1): 113-130.

162. Ogino S, Cantor M, Kawasaki T, Brahmandam M, Kirkner GJ, Weisenberger DJ, Campan M, Laird PW, Loda M, Fuchs CS. CpG island methylator phenotype (CIMP) of colorectal cancer is best characterised by quantitative DNA methylation analysis and prospective cohort studies. Gut 2006;55(7): 1000-1006. 
163. Saini A, Mastana S, Myers F, Lewis MP. 'From death, lead me to immortality' mantra of ageing skeletal muscle. Current genomics 2013;14(4): 256-267.

164. Smith SS, Kaplan BE, Sowers LC, Newman EM. Mechanism of human methyl-directed DNA methyltransferase and the fidelity of cytosine methylation. Proc Natl Acad Sci U S A 1992;89(10): 4744-4748.

165. Curtin K, Slattery ML, Samowitz WS. CpG island methylation in colorectal cancer: past, present and future. Pathology research international 2011;2011: 902674.

166. Laird PW, Jackson-Grusby L, Fazeli A, Dickinson SL, Jung WE, Li E, Weinberg RA, Jaenisch R. Suppression of intestinal neoplasia by DNA hypomethylation. Cell 1995;81(2): 197-205.

167. Yamada $Y$, Jackson-Grusby L, Linhart H, Meissner A, Eden A, Lin H, Jaenisch R. Opposing effects of DNA hypomethylation on intestinal and liver carcinogenesis. Proc Natl Acad Sci U S A 2005;102(38): 13580-13585.

168. Eden A, Gaudet F, Waghmare A, Jaenisch R. Chromosomal instability and tumors promoted by DNA hypomethylation. Science 2003;300(5618): 455.

169. Yang AS, Estecio MR, Garcia-Manero G, Kantarjian HM, Issa JP. Comment on "Chromosomal instability and tumors promoted by DNA hypomethylation" and "Induction of tumors in nice by genomic hypomethylation". Science 2003;302(5648): 1153; author reply 1153.

170. Tsai HC, Baylin SB. Cancer epigenetics: linking basic biology to clinical medicine. Cell research 2011;21(3): 502-517.

171. Wade PA. Methyl CpG binding proteins: coupling chromatin architecture to gene regulation. Oncogene 2001;20(24): 3166-3173.

172. Ashktorab H, Daremipouran M, Goel A, Varma S, Leavitt R, Sun X, Brim H. DNA methylome profiling identifies novel methylated genes in African American patients with colorectal neoplasia. Epigenetics 2014;9(4): 503-512.

173. Ashktorab H, Rahi H, Wansley D, Varma S, Shokrani B, Lee E, Daremipouran M, Laiyemo A, Goel A, Carethers JM, Brim H. Toward a comprehensive and systematic methylome signature in colorectal cancers. Epigenetics 2013;8(8): 807-815.

174. Gonzalo V, Lozano JJ, Munoz J, Balaguer F, Pellise M, Rodriguez de Miguel C, Andreu M, Jover R, Llor X, Giraldez MD, Ocana T, Serradesanferm A, Alonso-Espinaco V, Jimeno $M$, Cuatrecasas M, Sendino O, Castellvi-Bel S, Castells A. Aberrant gene promoter methylation associated with sporadic multiple colorectal cancer. PLoS One 2010;5(1): e8777.

175. Samowitz WS, Albertsen H, Herrick J, Levin TR, Sweeney C, Murtaugh MA, Wolff RK, Slattery ML. Evaluation of a large, population-based sample supports a CpG island methylator phenotype in colon cancer. Gastroenterology 2005;129(3): 837-845.

176. Park SJ, Rashid A, Lee JH, Kim SG, Hamilton SR, Wu TT. Frequent CpG island methylation in serrated adenomas of the colorectum. Am J Pathol 2003;162(3): 815-822.

177. Toyota M, Ahuja N, Ohe-Toyota M, Herman JG, Baylin SB, Issa JPJ. CpG island methylator phenotype in colorectal cancer. Proceedings of the National Academy of Sciences of the United States of America 1999;96(15): 8681-8686.

178. Weisenberger DJ, Siegmund KD, Campan M, Young J, Long TI, Faasse MA, Kang GH, Widschwendter M, Weener D, Buchanan D, Koh H, Simms L, Barker M, Leggett B, Levine J, Kim M, French AJ, Thibodeau SN, Jass J, Haile R, Laird PW. CpG island methylator phenotype underlies sporadic microsatellite instability and is tightly associated with BRAF mutation in colorectal cancer. Nature genetics 2006;38(7): 787-793. 
179. Ogino S, Kawasaki T, Kirkner GJ, Kraft P, Loda M, Fuchs CS. Evaluation of markers for CpG island methylator phenotype (CIMP) in colorectal cancer by a large population-based sample. The Journal of molecular diagnostics : JMD 2007;9(3): 305-314.

180. Ogino S, Kawasaki T, Kirkner GJ, Loda M, Fuchs CS. CpG island methylator phenotype-low (CIMP-low) in colorectal cancer: possible associations with male sex and

KRAS mutations. The Journal of molecular diagnostics : JMD 2006;8(5): 582-588.

181. Sandoval J, Heyn H, Moran S, Serra-Musach J, Pujana MA, Bibikova M, Esteller M. Validation of a DNA methylation microarray for $450,000 \mathrm{CpG}$ sites in the human genome.

Epigenetics 2011;6(6): 692-702.

182. Shen L, Toyota M, Kondo Y, Lin E, Zhang L, Guo Y, Hernandez NS, Chen X, Ahmed S, Konishi K, Hamilton SR, Issa JP. Integrated genetic and epigenetic analysis identifies three different subclasses of colon cancer. Proc Natl Acad Sci U S A 2007;104(47): 18654-18659.

183. Tanaka N, Huttenhower C, Nosho K, Baba Y, Shima K, Quackenbush J, Haigis KM, Giovannucci E, Fuchs CS, Ogino S. Novel application of structural equation modeling to correlation structure analysis of $\mathrm{CpG}$ island methylation in colorectal cancer. Am J Pathol 2010;177(6): 2731-2740.

184. Ang PW, Loh M, Liem N, Lim PL, Grieu F, Vaithilingam A, Platell C, Yong WP, lacopetta B, Soong R. Comprehensive profiling of DNA methylation in colorectal cancer reveals subgroups with distinct clinicopathological and molecular features. BMC Cancer 2010;10: 227.

185. Kaneda A, Yagi K. Two groups of DNA methylation markers to classify colorectal cancer into three epigenotypes. Cancer science 2011;102(1): 18-24.

186. Yagi K, Akagi K, Hayashi H, Nagae G, Tsuji S, Isagawa T, Midorikawa Y, Nishimura Y, Sakamoto H, Seto Y, Aburatani H, Kaneda A. Three DNA methylation epigenotypes in human colorectal cancer. Clin Cancer Res 2010;16(1): 21-33.

187. Ptashne M. On the use of the word epigenetic. Current Biology 2007;17(7): R233R236.

188. Jablonka E, Lachmann M, Lamb MJ. Evidence, mechanisms and models for the inheritance of acquired characters. Journal of Theoretical Biology 1992;158(2): 245-268.

189. Huang T, Lin C, Zhong LL, Zhao L, Zhang G, Lu A, Wu J, Bian Z. Targeting histone methylation for colorectal cancer. Therapeutic advances in gastroenterology 2017;10(1):

114-131.

190. Morera L, Lubbert M, Jung M. Targeting histone methyltransferases and demethylases in clinical trials for cancer therapy. Clin Epigenetics 2016;8: 57.

191. McCabe MT, Mohammad HP, Barbash O, Kruger RG. Targeting Histone Methylation in Cancer. Cancer journal (Sudbury, Mass) 2017;23(5): 292-301.

192. Zlobec I, Bihl MP, Foerster A, Rufle A, Terracciano L, Lugli A. Stratification and Prognostic Relevance of Jass's Molecular Classification of Colorectal Cancer. Front Oncol 2012;2: 7.

193. Jass JR. Towards a molecular classification of colorectal cancer. Int J Colorectal Dis 1999;14(4-5): 194-200.

194. Budinska E, Popovici V, Tejpar S, D'Ario G, Lapique N, Sikora KO, Di Narzo AF, Yan P, Hodgson JG, Weinrich S, Bosman F, Roth A, Delorenzi M. Gene expression patterns unveil a new level of molecular heterogeneity in colorectal cancer. The Journal of pathology

2013;231(1): 63-76.

195. De Sousa EMF, Wang X, Jansen M, Fessler E, Trinh A, de Rooij LP, de Jong JH, de Boer OJ, van Leersum $R$, Bijlsma MF, Rodermond $H$, van der Heijden $M$, van Noesel CJ, Tuynman JB, Dekker E, Markowetz F, Medema JP, Vermeulen L. Poor-prognosis colon cancer is 
defined by a molecularly distinct subtype and develops from serrated precursor lesions.

Nature medicine 2013;19(5): 614-618.

196. Marisa L, de Reynies A, Duval A, Selves J, Gaub MP, Vescovo L, Etienne-Grimaldi MC, Schiappa R, Guenot D, Ayadi M, Kirzin S, Chazal M, Flejou JF, Benchimol D, Berger A, Lagarde A, Pencreach E, Piard F, Elias D, Parc Y, Olschwang S, Milano G, Laurent-Puig P, Boige V. Gene expression classification of colon cancer into molecular subtypes: characterization, validation, and prognostic value. PLoS medicine 2013;10(5): e1001453.

197. Perez-Villamil B, Romera-Lopez A, Hernandez-Prieto S, Lopez-Campos G, Calles A, Lopez-Asenjo JA, Sanz-Ortega J, Fernandez-Perez C, Sastre J, Alfonso R, Caldes T, MartinSanchez F, Diaz-Rubio E. Colon cancer molecular subtypes identified by expression profiling and associated to stroma, mucinous type and different clinical behavior. BMC Cancer 2012;12: 260.

198. Roepman P, Schlicker A, Tabernero J, Majewski I, Tian S, Moreno V, Snel MH, Chresta CM, Rosenberg R, Nitsche U, Macarulla T, Capella G, Salazar R, Orphanides G, Wessels LF, Bernards $R$, Simon IM. Colorectal cancer intrinsic subtypes predict chemotherapy benefit, deficient mismatch repair and epithelial-to-mesenchymal transition. Int J Cancer 2014;134(3): 552-562.

199. Sadanandam A, Lyssiotis CA, Homicsko K, Collisson EA, Gibb WJ, Wullschleger S, Ostos LC, Lannon WA, Grotzinger C, Del Rio M, Lhermitte B, Olshen AB, Wiedenmann B, Cantley LC, Gray JW, Hanahan D. A colorectal cancer classification system that associates cellular phenotype and responses to therapy. Nature medicine 2013;19(5): 619-625.

200. Schlicker A, Beran G, Chresta CM, McWalter G, Pritchard A, Weston S, Runswick S, Davenport S, Heathcote K, Castro DA, Orphanides G, French T, Wessels LF. Subtypes of primary colorectal tumors correlate with response to targeted treatment in colorectal cell lines. BMC medical genomics 2012;5: 66.

201. Guinney J, Dienstmann R, Wang X, de Reynies A, Schlicker A, Soneson C, Marisa L, Roepman P, Nyamundanda G, Angelino P, Bot BM, Morris JS, Simon IM, Gerster S, Fessler E, De Sousa EMF, Missiaglia E, Ramay H, Barras D, Homicsko K, Maru D, Manyam GC, Broom B, Boige V, Perez-Villamil B, Laderas T, Salazar R, Gray JW, Hanahan D, Tabernero J, Bernards R, Friend SH, Laurent-Puig P, Medema JP, Sadanandam A, Wessels L, Delorenzi M, Kopetz S, Vermeulen L, Tejpar S. The consensus molecular subtypes of colorectal cancer. Nature medicine 2015;21(11): 1350-1356.

202. Lee MS, Menter DG, Kopetz S. Right Versus Left Colon Cancer Biology: Integrating the Consensus Molecular Subtypes. Journal of the National Comprehensive Cancer Network: JNCCN 2017;15(3): 411-419.

203. Gunal A, Hui P, Kilic S, Xu R, Jain D, Mitchell K, Robert M, Kenney B. KRAS mutations are associated with specific morphologic features in colon cancer. Journal of clinical gastroenterology 2013;47(6): 509-514.

204. Williamson JS, Jones HG, Williams N, Griffiths AP, Jenkins G, Beynon J, Harris DA. Extramural vascular invasion and response to neoadjuvant chemoradiotherapy in rectal cancer: Influence of the CpG island methylator phenotype. World Journal of Gastrointestinal Oncology 2017;9(5): 209-217.

205. Schneider BJ, Shah MA, Klute K, Ocean A, Popa E, Altorki N, Lieberman M, Schreiner A, Yantiss R, Christos PJ, Palmer R, You D, Viale A, Kermani P, Scandura JM. Phase I Study of Epigenetic Priming with Azacitidine Prior to Standard Neoadjuvant Chemotherapy for Patients with Resectable Gastric and Esophageal Adenocarcinoma: Evidence of Tumor Hypomethylation as an Indicator of Major Histopathologic Response. Clin Cancer Res 2017;23(11): 2673-2680. 
206. Cunningham C, Leong K, Clark S, Plumb A, Taylor S, Geh I, Karandikar S, Moran B. Association of Coloproctology of Great Britain \& Ireland (ACPGBI): Guidelines for the Management of Cancer of the Colon, Rectum and Anus (2017) - Diagnosis, Investigations and Screening. Colorectal Disease 2017;19: 9-17.

207. Loughrey MB, Quirke P, Shepherd NA. G049: Standards and datasets for reporting cancers dataset for colorectal cancer histopathology reports. In: Pathologists RCo, editor. London; 2014.

208. Good Clinical Practice (GCP). https://www.nihr.ac.uk/health-and-careprofessionals/learning-and-support/good-clinical-practice.htm.

209. Jones PA. Altering gene expression with 5-azacytidine. Cell 1985;40(3): 485-486.

210. Glover AB, Leyland-Jones B. Biochemistry of azacitidine: a review. Cancer treatment reports 1987;71(10): 959-964.

211. Zhou X, Zhuang Z, Wang W, He L, Wu H, Cao Y, Pan F, Zhao J, Hu Z, Sekhar C, Guo Z. OGG1 is essential in oxidative stress induced DNA demethylation. Cellular signalling 2016;28(9): 1163-1171.

212. Silverman LR, Demakos EP, Peterson BL, Kornblith AB, Holland JC, Odchimar-Reissig $\mathrm{R}$, Stone RM, Nelson D, Powell BL, DeCastro CM, Ellerton J, Larson RA, Schiffer CA, Holland JF. Randomized controlled trial of azacitidine in patients with the myelodysplastic syndrome: a study of the cancer and leukemia group B. Journal of clinical oncology : official journal of the American Society of Clinical Oncology 2002;20(10): 2429-2440.

213. Juttermann R, Li E, Jaenisch R. Toxicity of 5-aza-2'-deoxycytidine to mammalian cells is mediated primarily by covalent trapping of DNA methyltransferase rather than DNA demethylation. Proc Natl Acad Sci U S A 1994;91(25): 11797-11801.

214. Taylor SM, Jones PA. Multiple new phenotypes induced in 10T1/2 and 3T3 cells treated with 5-azacytidine. Cell 1979;17(4): 771-779.

215. Mohandas T, Sparkes RS, Shapiro $L$. Reactivation of an inactive human X chromosome: evidence for X inactivation by DNA methylation. Science 1981;211(4480): 393396.

216. 5-Azacytidine. Substance Database: SID $=24278211$.

https://pubchem.ncbi.nlm.nih.gov/substance/24278211.

217. Wong N, Lam WC, Lai PB, Pang E, Lau WY, Johnson PJ. Hypomethylation of chromosome 1 heterochromatin DNA correlates with q-arm copy gain in human hepatocellular carcinoma. Am J Pathol 2001;159(2): 465-471.

218. Schulz WA, Elo JP, Florl AR, Pennanen S, Santourlidis S, Engers R, Buchardt M, Seifert $\mathrm{HH}$, Visakorpi T. Genomewide DNA hypomethylation is associated with alterations on chromosome 8 in prostate carcinoma. Genes, chromosomes \& cancer 2002;35(1): 58-65.

219. Agrawal S, Hofmann WK, Tidow N, Ehrich M, van den Boom D, Koschmieder S, Berdel WE, Serve $H$, Muller-Tidow $C$. The C/EBPdelta tumor suppressor is silenced by hypermethylation in acute myeloid leukemia. Blood 2007;109(9): 3895-3905.

220. Kaminskas E, Farrell AT, Wang YC, Sridhara R, Pazdur R. FDA drug approval summary: azacitidine (5-azacytidine, Vidaza) for injectable suspension. Oncologist 2005;10(3): 176-

182.

221. Silverman LR. Azacitidine (Aza C) in myelodysplastic syndromes (MDS), CALGB studies 8421 and 8921 [abstract]. Ann Hematol 1994;68: A12.

222. Kantarjian HM, Thomas XG, Dmoszynska A, Wierzbowska A, Mazur G, Mayer J, Gau JP, Chou W-C, Buckstein R, Cermak J, Kuo C-Y, Oriol A, Ravandi F, Faderl S, Delaunay J, Lysák

$D$, Minden $M$, Arthur C. Multicenter, randomized, open-label, phase III trial of decitabine versus patient choice, with physician advice, of either supportive care or low-dose 
cytarabine for the treatment of older patients with newly diagnosed acute myeloid leukemia. Journal of clinical oncology : official journal of the American Society of Clinical Oncology 2012;30(21): 2670-2677.

223. Cowan LA, Talwar S, Yang AS. Will DNA methylation inhibitors work in solid tumors? A review of the clinical experience with azacitidine and decitabine in solid tumors. Epigenomics 2010;2(1): 71-86.

224. Brueckner B, Kuck D, Lyko F. DNA methyltransferase inhibitors for cancer therapy. Cancer journal (Sudbury, Mass) 2007;13(1): 17-22.

225. Lin J, Gilbert J, Rudek MA, Zwiebel JA, Gore S, Jiemjit A, Zhao M, Baker SD, Ambinder RF, Herman JG, Donehower RC, Carducci MA. A phase I dose-finding study of 5-azacytidine in combination with sodium phenylbutyrate in patients with refractory solid tumors. Clin Cancer Res 2009;15(19): 6241-6249.

226. Ogino S, Meyerhardt JA, Kawasaki T, Clark JW, Ryan DP, Kulke MH, Enzinger PC, Wolpin BM, Loda M, Fuchs CS. CpG island methylation, response to combination chemotherapy, and patient survival in advanced microsatellite stable colorectal carcinoma. Virchows Archiv : an international journal of pathology 2007;450(5): 529-537.

227. Wei SH, Chen CM, Strathdee G, Harnsomburana J, Shyu CR, Rahmatpanah F, Shi H, Ng SW, Yan PS, Nephew KP, Brown R, Huang TH. Methylation microarray analysis of latestage ovarian carcinomas distinguishes progression-free survival in patients and identifies candidate epigenetic markers. Clin Cancer Res 2002;8(7): 2246-2252.

228. Kanda T, Tada M, Imazeki F, Yokosuka O, Nagao K, Saisho H. 5-aza-2'-deoxycytidine sensitizes hepatoma and pancreatic cancer cell lines. Oncology reports 2005;14(4): 975-979.

229. Cheetham S, Tang MJ, Mesak F, Kennecke H, Owen D, Tai IT. SPARC promoter hypermethylation in colorectal cancers can be reversed by 5-Aza-2'deoxycytidine to increase SPARC expression and improve therapy response. Br J Cancer 2008;98(11): 18101819.

230. Juergens RA, Wrangle J, Vendetti FP, Murphy SC, Zhao M, Coleman B, Sebree R, Rodgers K, Hooker CM, Franco N, Lee B, Tsai S, Delgado IE, Rudek MA, Belinsky SA, Herman JG, Baylin SB, Brock MV, Rudin CM. Combination epigenetic therapy has efficacy in patients with refractory advanced non-small cell lung cancer. Cancer discovery 2011;1(7): 598-607.

231. Fu S, Hu W, lyer R, Kavanagh JJ, Coleman RL, Levenback CF, Sood AK, Wolf JK, Gershenson DM, Markman M, Hennessy BT, Kurzrock R, Bast RC, Jr. Phase 1b-2a study to reverse platinum resistance through use of a hypomethylating agent, azacitidine, in patients with platinum-resistant or platinum-refractory epithelial ovarian cancer. Cancer 2011;117(8): 1661-1669.

232. Overman MJ, Morris V, Moinova H, Manyam G, Ensor J, Lee MS, Eng C, Kee B, Fogelman D, Shroff RT, LaFramboise T, Mazard T, Feng T, Hamilton S, Broom B, Lutterbaugh J, Issa J-P, Markowitz SD, Kopetz S. Phase I/II study of azacitidine and capecitabine/oxaliplatin (CAPOX) in refractory CIMP-high metastatic colorectal cancer: evaluation of circulating methylated vimentin. Oncotarget 2016;7(41): 67495-67506.

233. Azad NS, El-Khoueiry A, Yin J, Oberg AL, Flynn P, Adkins D, Sharma A, Weisenberger DJ, Brown T, Medvari P, Jones PA, Easwaran H, Kamel I, Bahary N, Kim G, Picus J, Pitot HC, Erlichman C, Donehower R, Shen H, Laird PW, Piekarz R, Baylin S, Ahuja N. Combination epigenetic therapy in metastatic colorectal cancer ( $\mathrm{mCRC}$ ) with subcutaneous 5 -azacitidine and entinostat: a phase 2 consortium/stand up 2 cancer study. Oncotarget 2017;8(21): 35326-35338. 
234. Gerwing M, Herrmann K, Helfen A, Schliemann C, Berdel WE, Eisenblatter M, Wildgruber $M$. The beginning of the end for conventional RECIST - novel therapies require novel imaging approaches. Nat Rev Clin Oncol 2019.

235. Scicinski J, Oronsky B, Taylor M, Luo G, Musick T, Marini J, Adams CM, Fitch WL. Preclinical evaluation of the metabolism and disposition of RRx-001, a novel investigative anticancer agent. Drug Metab Dispos 2012;40(9): 1810-1816.

236. Reid T, Dad S, Korn R, Oronsky B, Knox S, Scicinski J. Two Case Reports of

Resensitization to Previous Chemotherapy with the Novel Hypoxia-Activated Hypomethylating Anticancer Agent RRx-001 in Metastatic Colorectal Cancer Patients. Case Rep Oncol 2014;7(1): 79-85.

237. Ning S, Bednarski M, Oronsky B, Scicinski J, Saul G, Knox SJ. Dinitroazetidines are a novel class of anticancer agents and hypoxia-activated radiation sensitizers developed from highly energetic materials. Cancer Res 2012;72(10): 2600-2608.

238. Oronsky BT, Reid T, Knox SJ, Scicinski JJ. The Scarlet Letter of Alkylation: A Mini

Review of Selective Alkylating Agents. Translational Oncology 2012;5(4): 226-229.

239. Scicinski J, Oronsky B, Cooper V, Taylor M, Alexander M, Hadar R, Cosford R, Fleischmann T, Fitch WL. Development of methods for the bioanalysis of RRx-001 and metabolites. Bioanalysis 2014;6(7): 947-956.

240. Grothey A, Van Cutsem E, Sobrero A, Siena S, Falcone A, Ychou M, Humblet $Y$, Bouche O, Mineur L, Barone C, Adenis A, Tabernero J, Yoshino T, Lenz HJ, Goldberg RM, Sargent DJ, Cihon F, Cupit L, Wagner A, Laurent D. Regorafenib monotherapy for previously treated metastatic colorectal cancer (CORRECT): an international, multicentre, randomised,

placebo-controlled, phase 3 trial. Lancet (London, England) 2013;381(9863): 303-312.

241. Oronsky B, Oronsky N, Knox S, Fanger G, Scicinski J. Episensitization: therapeutic tumor resensitization by epigenetic agents: a review and reassessment. Anti-cancer agents in medicinal chemistry 2014;14(8): 1121-1127.

242. Oronsky B, Oronsky N, Scicinski J, Fanger G, Lybeck M, Reid T. Rewriting the epigenetic code for tumor resensitization: a review. Trans/ Oncol 2014;7(5): 626-631. 243. Zhao H, Ning S, Scicinski J, Oronsky B, Knox SJ, Peehl DM. Epigenetic effects of RRx001: a possible unifying mechanism of anticancer activity. Oncotarget 2015;6(41): 4317243181.

244. Reid T, Oronsky B, Scicinski J, Scribner CL, Knox SJ, Ning S, Peehl DM, Korn R, Stirn M, Carter CA, Oronsky A, Taylor MJ, Fitch WL, Cabrales P, Kim MM, Burris HA, 3rd, Lao CD, Abrouk NE, Fanger GR, Infante JR. Safety and activity of RRx-001 in patients with advanced cancer: a first-in-human, open-label, dose-escalation phase 1 study. The Lancet Oncology 2015;16(9): 1133-1142.

245. Fens MH, Cabrales P, Scicinski J, Larkin SK, Suh JH, Kuypers FA, Oronsky N, Lybeck M, Oronsky A, Oronsky B. Targeting tumor hypoxia with the epigenetic anticancer agent, RRx001: a superagonist of nitric oxide generation. Medical oncology (Northwood, London, England) 2016;33(8): 85.

246. Brzezniak C, Oronsky B, Trepel J, Summers TA, Jr., Cabrales P, Lee MJ, Day R, Jha S, Caroen S, Zeman K, Ferry L, Harmer C, Oronsky N, Lybeck M, Lybeck HE, Brown JF, Reid TR, Carter CA. RRx-001 Priming of PD-1 Inhibition in the Treatment of Small Cell Carcinoma of the Vagina: A Rare Gynecological Tumor. Case Rep Oncol 2017;10(1): 276-280.

247. Carter CA, Oronsky B, Caroen S, Scicinski J, Cabrales P, Degesys A, Brzezniak C. Partial response to carboplatin in an RRx-001 pretreated patient with EGFR-inhibitor-resistance and T790M-negative NSCLC. Respiratory medicine case reports 2016;18: 62-65. 
248. Carter CA, Oronsky B, Caroen S, Scicinski J, Degesys A, Cabrales P, Reid TR, Brzezniak

C. Partial Response in an RRx-001-Primed Patient with Refractory Small-Cell Lung Cancer after a Third Introduction of Platinum Doublets. Case Rep Oncol 2016;9(2): 285-289.

249. Carter CA, Oronsky BT, Caroen SZ, Scicinski JJ, Cabrales P, Reid T, Degesys A, Jenkins J, Brzezniak C. Partial Response to Platinum Doublets in Refractory EGFR-Positive Non-Small

Cell Lung Cancer Patients after RRx-001: Evidence of Episensitization. Case Rep Oncol 2016;9(1): 62-67.

250. Kim MM, Parmar H, Cao Y, Pramanik P, Schipper M, Hayman J, Junck L, Mammoser A, Heth J, Carter CA, Oronsky A, Knox SJ, Caroen S, Oronsky B, Scicinski J, Lawrence TS, Lao

CD. Whole Brain Radiotherapy and RRx-001: Two Partial Responses in Radioresistant Melanoma Brain Metastases from a Phase I/II Clinical Trial: A TITE-CRM Phase I/II Clinical Trial. Trans/ Oncol 2016;9(2): 108-113.

251. Ojemuyiwa M, Zeman K, Spira A, Oronsky B, Ray C, Trepel JB, Lee MJ, Onyiuke I, Brzezniak C. Complete metabolic response of metastatic castration-resistant neuroendocrine carcinoma of the prostate after treatment with RRx-001 and reintroduced platinum doublets. Clinical case reports 2018;6(12): 2478-2481.

252. RRx-001 Prior to a Platinum Doublet or a Platinum Doublet Alone in Patients With Small Cell Carcinoma (REPLATINUM). https://clinicaltrials.gov/ct2/show/NCT03699956. 253. Cabrales P, Caroen S, Oronsky A, Carter C, Trepel J, Summers T, Reid T, Oronsky N, Lybeck M, Oronsky B. The macrophage stimulating anti-cancer agent, RRx-001, protects against ischemia-reperfusion injury. Expert review of hematology 2017;10(6): 575-582.

254. Carter CA, Schmitz B, Peterson PG, Quinn M, Degesys A, Jenkins J, Oronsky B, Scicinski J, Caroen S, Reid TR, Cabrales P, Brzezniak C. Immune Reactivity and Pseudoprogression or Tumor Flare in a Serially Biopsied Neuroendocrine Patient Treated with the Epigenetic Agent RRx-001. Case Rep Oncol 2016;9(1): 164-170.

255. Oronsky B, Paulmurugan R, Foygel K, Scicinski J, Knox SJ, Peehl D, Zhao H, Ning S, Cabrales P, Summers TA, Jr., Reid TR, Fitch WL, Kim MM, Trepel JB, Lee MJ, Kesari S, Abrouk ND, Day RM, Oronsky A, Ray CM, Carter CA. RRx-001: a systemically non-toxic M2-to-M1 macrophage stimulating and prosensitizing agent in Phase II clinical trials. Expert opinion on investigational drugs 2017;26(1): 109-119.

256. Cabrales P. RRx-001 Acts as a Dual Small Molecule Checkpoint Inhibitor by Downregulating CD47 on Cancer Cells and SIRP-alpha on Monocytes/Macrophages. Trans/ Oncol 2019;12(4): 626-632.

257. Zhao H, Ning S, Nolley R, Scicinski J, Oronsky B, Knox SJ, Peehl DM. The immunomodulatory anticancer agent, RRx-001, induces an interferon response through epigenetic induction of viral mimicry. Clin Epigenetics 2017;9: 4.

258. Kokelaar RF, Jones HG, Williamson J, Williams N, Griffiths AP, Beynon J, Jenkins GJ, Harris DA. DNA hypermethylation as a predictor of extramural vascular invasion (EMVI) in rectal cancer. Cancer Biology \& Therapy 2017: 00-00.

259. DLD-1 Datasheet - Public Health England. https://www.pheculturecollections.org.uk/products/celllines/generalcell/detail.jsp?refld=90102540\&collecti on=ecacc gc.

260. Vermeulen SJ, Chen TR, Speleman F, Nollet F, Van Roy FM, Mareel MM. Did the four human cancer cell lines DLD-1, HCT-15, HCT-8, and HRT-18 originate from one and the same patient? Cancer genetics and cytogenetics 1998;107(1): 76-79.

261. Ahmed D, Eide PW, Eilertsen IA, Danielsen SA, Eknas M, Hektoen M, Lind GE, Lothe RA. Epigenetic and genetic features of 24 colon cancer cell lines. Oncogenesis 2013;2: e71. 
262. Ahmed D, Eide PW, Eilertsen IA, Danielsen SA, Eknæs M, Hektoen M, Lind GE, Lothe

RA. Epigenetic and genetic features of 24 colon cancer cell lines. Oncogenesis 2013;2(9):

e71-e71.

263. HFL-1 Datasheet - Public Health England. https://www.pheculturecollections.org.uk/products/celllines/generalcell/detail.jsp?refld=89071902\&collecti on=ecacc gc\#medDoc.

264. Edwards BK, Ward E, Kohler BA, Eheman C, Zauber AG, Anderson RN, Jemal A, Schymura MJ, Lansdorp-Vogelaar I, Seeff LC, van Ballegooijen M, Goede SL, Ries LA. Annual report to the nation on the status of cancer, 1975-2006, featuring colorectal cancer trends and impact of interventions (risk factors, screening, and treatment) to reduce future rates.

Cancer 2010;116(3): 544-573.

265. Antelo M, Balaguer F, Shia J, Shen Y, Hur K, Moreira L, Cuatrecasas M, Bujanda L, Giraldez M, Takahashi M, Cabanne A, Barugel M, Arnold M, Roca E, Andreu M, Castellvi-Bel S, Llor X, Jover R, Castells A, Boland C, Goel A. A high degree of LINE-1 hypomethylation is a unique feature of early-onset colorectal cancer. PloS one 2012;7(9).

266. Eisenberg B, Decosse JJ, Harford F, Michalek J. Carcinoma of the colon and rectum: the natural history reviewed in 1704 patients. Cancer 1982;49(6): 1131-1134.

267. Lee YC, Lee YL, Chuang JP, Lee JC. Differences in survival between colon and rectal cancer from SEER data. PLoS One 2013;8(11): e78709.

268. National Bowel Cancer Audit Annual Report 2018.

https://www.nboca.org.uk/reports/annual-report-2018/.

269. Benitez Majano S, Di Girolamo C, Rachet B, Maringe C, Guren MG, Glimelius B, Iversen LH, Schnell EA, Lundqvist K, Christensen J, Morris M, Coleman MP, Walters S. Surgical treatment and survival from colorectal cancer in Denmark, England, Norway, and

Sweden: a population-based study. The Lancet Oncology 2019;20(1): 74-87.

270. Platt E, Dovell G, Smolarek S. Systematic review of outcomes following pelvic exenteration for the treatment of primary and recurrent locally advanced rectal cancer.

Tech Coloproctol 2018;22(11): 835-845.

271. Talbot IC, Ritchie S, Leighton M, Hughes AO, Bussey HJ, Morson BC. Invasion of veins

by carcinoma of rectum: method of detection, histological features and significance. Histopathology 1981;5(2): 141-163.

272. Talbot IC, Ritchie S, Leighton MH, Hughes AO, Bussey HJ, Morson BC. Spread of rectal cancer within veins. Histologic features and clinical significance. Am J Surg 1981;141(1): 15-

17.

273. Talbot IC, Ritchie S, Leighton MH, Hughes AO, Bussey HJ, Morson BC. The clinical significance of invasion of veins by rectal cancer. Br J Surg 1980;67(6): 439-442.

274. Messenger DE, Driman DK, Kirsch R. Developments in the assessment of venous invasion in colorectal cancer: implications for future practice and patient outcome. Human pathology 2012;43(7): 965-973.

275. Brown CF. Visceral metastasis from rectal carcinoma. Surg Gynecol Obstet 1938;66: 611-621.

276. Krasna MJ, Flancbaum L, Cody RP, Shneibaum S, Ben Ari G. Vascular and neural invasion in colorectal carcinoma. Incidence and prognostic significance. Cancer 1988;61(5): 1018-1023.

277. Rich T, Gunderson LL, Lew R, Galdibini JJ, Cohen AM, Donaldson G. Patterns of recurrence of rectal cancer after potentially curative surgery. Cancer 1983;52(7): 1317-1329. 
278. Ouchi K, Sugawara T, Ono H, Fujiya T, Kamiyama Y, Kakugawa Y, Mikuni J, Tateno H. Histologic features and clinical significance of venous invasion in colorectal carcinoma with hepatic metastasis. Cancer 1996;78(11): 2313-2317.

279. Taylor F, Mangat N, Swift IR, Brown G. Proforma-based reporting in rectal cancer. Cancer Imaging 2010.

280. Smith NJ, Barbachano Y, Norman AR, Swift RI, Abulafi AM, Brown G. Prognostic significance of magnetic resonance imaging-detected extramural vascular invasion in rectal cancer. Br J Surg 2008;95(2): 229-236.

281. MERCURY SG. Diagnostic accuracy of preoperative magnetic resonance imaging in predicting curative resection of rectal cancer: prospective observational study. $B M J$ 2006;333(7572): 779.

282. Taylor FG, Quirke P, Heald RJ, Moran B, Blomqvist L, Swift I, Sebag-Montefiore DJ, Tekkis $P$, Brown G. Preoperative high-resolution magnetic resonance imaging can identify good prognosis stage I, II, and III rectal cancer best managed by surgery alone: a prospective, multicenter, European study. Ann Surg 2011;253(4): 711-719.

283. Chand M, Swift RI, Tekkis PP, Chau I, Brown G. Extramural venous invasion is a potential imaging predictive biomarker of neoadjuvant treatment in rectal cancer. British journal of cancer 2014;110(1): 19-25.

284. Brown G, Radcliffe AG, Newcombe RG, Dallimore NS, Bourne MW, Williams GT.

Preoperative assessment of prognostic factors in rectal cancer using high-resolution magnetic resonance imaging. British Journal of Surgery 2003;90(3): 355-364.

285. Battersby NJ, How P, Moran B, Stelzner S, West NP, Branagan G, Strassburg J, Quirke P, Tekkis P, Pedersen BG, Gudgeon M, Heald B, Brown G. Prospective Validation of a Low Rectal Cancer Magnetic Resonance Imaging Staging System and Development of a Local Recurrence Risk Stratification Model: The MERCURY II Study. Ann Surg 2016;263(4): 751760.

286. Bugg WG, Andreou AK, Biswas D, Toms AP, Williams SM. The prognostic significance of MRI-detected extramural venous invasion in rectal carcinoma. Clinical radiology 2014;69(6): 619-623.

287. Siddiqui MRS, Simillis C, Hunter C, Chand M, Bhoday J, Garant A, Vuong T, Artho G, Rasheed S, Tekkis P, Abulafi AM, Brown G. A meta-analysis comparing the risk of metastases in patients with rectal cancer and MRI-detected extramural vascular invasion (mrEMVI) vs mrEMVI-negative cases. Br J Cancer 2017;116(12): 1513-1519.

288. Al-Sukhni E, Milot L, Fruitman M, Beyene J, Victor JC, Schmocker S, Brown G, McLeod $R$, Kennedy E. Diagnostic accuracy of MRI for assessment of T category, lymph node metastases, and circumferential resection margin involvement in patients with rectal cancer: a systematic review and meta-analysis. Ann Surg Oncol 2012;19(7): 2212-2223.

289. Taylor F, Mangat N, Swift IR, Brown G. Proforma-based reporting in rectal cancer. Cancer imaging : the official publication of the International Cancer Imaging Society 2010;10

Spec no A(1a): S142-150.

290. KSAR. Essential Items for Structured Reporting of Rectal Cancer MRI: 2016 Consensus Recommendation from the Korean Society of Abdominal Radiology. Korean journal of radiology 2017;18(1): 132-151.

291. Glynne-Jones R, Wyrwicz L, Tiret E, Brown G, Rödel C, Cervantes A, Arnold D. Rectal cancer: ESMO Clinical Practice Guidelines for diagnosis, treatment and follow-up. Annals of Oncology 2017;28(suppl_4): iv22-iv40. 
292. Recommendations for cross-sectional imaging in cancer management. 2nd Edition.

https://www.rcr.ac.uk/publication/recommendations-cross-sectional-imaging-cancer-

management-second-edition.

293. Gollins S, Moran B, Adams R, Cunningham C, Bach S, Myint AS, Renehan A, Karandikar S, Goh V, Prezzi D, Langman G, Ahmedzai S, Geh I. Association of Coloproctology of Great Britain \& Ireland (ACPGBI): Guidelines for the Management of Cancer of the Colon, Rectum and Anus (2017) - Multidisciplinary Management. Colorectal Dis 2017;19 Suppl 1: 37-66.

294. Smith N, Brown G. Preoperative staging of rectal cancer. Acta oncologica (Stockholm, Sweden) 2008;47(1): 20-31.

295. Kapiteijn E, Marijnen CA, Nagtegaal ID, Putter H, Steup WH, Wiggers T, Rutten HJ,

Pahlman L, Glimelius B, van Krieken JH, Leer JW, van de Velde CJ. Preoperative radiotherapy combined with total mesorectal excision for resectable rectal cancer. The New England journal of medicine 2001;345(9): 638-646.

296. Peeters KC, Marijnen CA, Nagtegaal ID, Kranenbarg EK, Putter H, Wiggers T, Rutten

H, Pahlman L, Glimelius B, Leer JW, van de Velde CJ. The TME trial after a median follow-up of 6 years: increased local control but no survival benefit in irradiated patients with resectable rectal carcinoma. Ann Surg 2007;246(5): 693-701.

297. Sebag-Montefiore D, Stephens RJ, Steele R, Monson J, Grieve R, Khanna S, Quirke P, Couture J, de Metz C, Myint AS, Bessell E, Griffiths G, Thompson LC, Parmar M. Preoperative radiotherapy versus selective postoperative chemoradiotherapy in patients with rectal cancer (MRC CRO7 and NCIC-CTG C016): a multicentre, randomised trial. Lancet (London, England) 2009;373(9666): 811-820.

298. Sauer R, Liersch T, Merkel S, Fietkau R, Hohenberger W, Hess C, Becker H, Raab HR, Villanueva MT, Witzigmann $\mathrm{H}$, Wittekind $\mathrm{C}$, Beissbarth T, Rodel C. Preoperative versus postoperative chemoradiotherapy for locally advanced rectal cancer: results of the German CAO/ARO/AIO-94 randomized phase III trial after a median follow-up of 11 years. Journal of clinical oncology : official journal of the American Society of Clinical Oncology 2012;30(16): 1926-1933.

299. Bujko K, Nowacki MP, Nasierowska-Guttmejer A, Michalski W, Bebenek M, Pudelko M, Kryj M, Oledzki J, Szmeja J, Sluszniak J, Serkies K, Kladny J, Pamucka M, Kukolowicz P. Sphincter preservation following preoperative radiotherapy for rectal cancer: report of a randomised trial comparing short-term radiotherapy vs. conventionally fractionated radiochemotherapy. Radiotherapy and oncology : journal of the European Society for Therapeutic Radiology and Oncology 2004;72(1): 15-24.

300. Bujko K, Nowacki MP, Nasierowska-Guttmejer A, Michalski W, Bebenek M, Kryj M. Long-term results of a randomized trial comparing preoperative short-course radiotherapy with preoperative conventionally fractionated chemoradiation for rectal cancer. Br J Surg 2006;93(10): 1215-1223.

301. Ngan SY, Burmeister B, Fisher RJ, Solomon M, Goldstein D, Joseph D, Ackland SP, Schache D, McClure B, McLachlan SA, McKendrick J, Leong T, Hartopeanu C, Zalcberg J, Mackay J. Randomized trial of short-course radiotherapy versus long-course chemoradiation comparing rates of local recurrence in patients with T3 rectal cancer: Trans-Tasman Radiation Oncology Group trial 01.04. Journal of clinical oncology : official journal of the American Society of Clinical Oncology 2012;30(31): 3827-3833.

302. Feinberg AP, Vogelstein B. Hypomethylation distinguishes genes of some human cancers from their normal counterparts. Nature 1983;301(5895): 89-92. 
303. Feinberg AP, Vogelstein B. Hypomethylation of ras oncogenes in primary human cancers. Biochemical and biophysical research communications 1983;111(1): 47-54.

304. Xu L, Czito BG, Willett CG. Epigenetic markers in rectal cancer. Clin Cancer Res 2010;16(10): 2699-2701.

305. Heyn H, Esteller M. DNA methylation profiling in the clinic: applications and challenges. Nat Rev Genet 2012;13(10): 679-692.

306. Coppedè F, Lopomo A, Spisni R, Migliore L. Genetic and epigenetic biomarkers for diagnosis, prognosis and treatment of colorectal cancer. World journal of gastroenterology 2014;20(4): 943-956.

307. Lao VV, Grady WM. Epigenetics and colorectal cancer. Nature reviews Gastroenterology \& hepatology 2011;8(12): 686-700.

308. Coppede F. Epigenetic biomarkers of colorectal cancer: Focus on DNA methylation. Cancer Lett 2014;342(2): 238-247.

309. Goel A, Boland CR. Epigenetics of colorectal cancer. Gastroenterology 2012;143(6): 1442-1460.e1441.

310. Esteller M, Garcia-Foncillas J, Andion E, Goodman SN, Hidalgo OF, Vanaclocha V, Baylin SB, Herman JG. Inactivation of the DNA-repair gene MGMT and the clinical response of gliomas to alkylating agents. The New England journal of medicine 2000;343(19): 13501354.

311. Esteller M, Corn PG, Urena JM, Gabrielson E, Baylin SB, Herman JG. Inactivation of glutathione S-transferase P1 gene by promoter hypermethylation in human neoplasia. Cancer Res 1998;58(20): 4515-4518.

312. Hegi ME, Liu L, Herman JG, Stupp R, Wick W, Weller M, Mehta MP, Gilbert MR. Correlation of 06-methylguanine methyltransferase (MGMT) promoter methylation with clinical outcomes in glioblastoma and clinical strategies to modulate MGMT activity. Journal of clinical oncology : official journal of the American Society of Clinical Oncology 2008;26(25): 4189-4199.

313. Esteller M. Epigenetic gene silencing in cancer: the DNA hypermethylome. Human molecular genetics 2007;16 Spec No 1: R50-59.

314. Adams D, Altucci L, Antonarakis SE, Ballesteros J, Beck S, Bird A, Bock C, Boehm B, Campo E, Caricasole A, Dahl F, Dermitzakis ET, Enver T, Esteller M, Estivill X, Ferguson-Smith A, Fitzgibbon J, Flicek P, Giehl C, Graf T, Grosveld F, Guigo R, Gut I, Helin K, Jarvius J, Kuppers $\mathrm{R}$, Lehrach $\mathrm{H}$, Lengauer $\mathrm{T}$, Lernmark $\mathrm{A}$, Leslie $\mathrm{D}$, Loeffler $\mathrm{M}$, Macintyre $E$, Mai A, Martens JH, Minucci S, Ouwehand WH, Pelicci PG, Pendeville H, Porse B, Rakyan V, Reik W, Schrappe M,

Schubeler D, Seifert M, Siebert R, Simmons D, Soranzo N, Spicuglia S, Stratton M, Stunnenberg HG, Tanay A, Torrents D, Valencia A, Vellenga E, Vingron M, Walter J, Willcocks S. BLUEPRINT to decode the epigenetic signature written in blood. Nature biotechnology 2012;30(3): 224-226.

315. Bernstein BE, Stamatoyannopoulos JA, Costello JF, Ren B, Milosavljevic A, Meissner A, Kellis M, Marra MA, Beaudet AL, Ecker JR, Farnham PJ, Hirst M, Lander ES, Mikkelsen TS, Thomson JA. The NIH Roadmap Epigenomics Mapping Consortium. Nature biotechnology 2010;28(10): 1045-1048.

316. Ahlquist DA, Zou H, Domanico M, Mahoney DW, Yab TC, Taylor WR, Butz ML, Thibodeau SN, Rabeneck L, Paszat LF, Kinzler KW, Vogelstein B, Bjerregaard NC, Laurberg S, Sorensen HT, Berger BM, Lidgard GP. Next-generation stool DNA test accurately detects colorectal cancer and large adenomas. Gastroenterology 2012;142(2): 248-256; quiz e225246. 
317. Jia M, Gao X, Zhang Y, Hoffmeister M, Brenner H. Different definitions of CpG island methylator phenotype and outcomes of colorectal cancer: a systematic review. Clin Epigenetics 2016;8: 25.

318. Samowitz WS, Curtin K, Wolff RK, Tripp SR, Caan BJ, Slattery ML. Microsatellite instability and survival in rectal cancer. Cancer causes \& control : CCC 2009;20(9): 17631768.

319. Lange CP, Laird PW. Clinical applications of DNA methylation biomarkers in colorectal cancer. Epigenomics 2013;5(2): 105-108.

320. Lamb YN, Dhillon S. Epi proColon((R)) 2.0 CE: A Blood-Based Screening Test for Colorectal Cancer. Molecular diagnosis \& therapy 2017;21(2): 225-232.

321. Grützmann R, Molnar B, Pilarsky C, Habermann JK, Schlag PM, Saeger HD, Miehlke S,

Stolz T, Model F, Roblick UJ, Bruch H-P, Koch R, Liebenberg V, Devos T, Song X, Day RH, Sledziewski AZ, Lofton-Day C. Sensitive detection of colorectal cancer in peripheral blood by septin 9 DNA methylation assay. PloS one 2008;3(11): e3759-e3759.

322. Summers T, Langan RC, Nissan A, Brücher BLDM, Bilchik AJ, Protic M, Daumer M, Avital I, Stojadinovic A. Serum-based DNA methylation biomarkers in colorectal cancer: potential for screening and early detection. Journal of Cancer 2013;4(3): 210-216.

323. deVos T, Tetzner R, Model F, Weiss G, Schuster M, Distler J, Steiger KV, Grützmann R, Pilarsky C, Habermann JK, Fleshner PR, Oubre BM, Day R, Sledziewski AZ, Lofton-Day C. Circulating Methylated <em>SEPT9</em> DNA in Plasma Is a Biomarker for Colorectal Cancer. Clinical chemistry 2009;55(7): 1337-1346.

324. Potter NT, Hurban P, White MN, Whitlock KD, Lofton-Day CE, Tetzner R, Koenig T, Quigley NB, Weiss G. Validation of a real-time PCR-based qualitative assay for the detection of methylated SEPT9 DNA in human plasma. Clinical chemistry 2014;60(9): 1183-1191. 325. Song L, Jia J, Peng X, Xiao W, Li Y. The performance of the SEPT9 gene methylation assay and a comparison with other CRC screening tests: A meta-analysis. Scientific reports 2017;7(1): 3032-3032.

326. Wang Y, Chen P-M, Liu R-B. Advance in plasma SEPT9 gene methylation assay for colorectal cancer early detection. World Journal of Gastrointestinal Oncology 2018;10(1): 15-22.

327. Church TR, Wandell M, Lofton-Day C, Mongin SJ, Burger M, Payne SR, Castaños-Vélez E, Blumenstein BA, Rösch T, Osborn N, Snover D, Day RW, Ransohoff DF, Presept Clinical Study Steering Committee I, Study T. Prospective evaluation of methylated SEPT9 in plasma for detection of asymptomatic colorectal cancer. Gut 2014;63(2): 317-325.

328. Rex DK, Boland CR, Dominitz JA, Giardiello FM, Johnson DA, Kaltenbach T, Levin TR, Lieberman D, Robertson DJ. Colorectal Cancer Screening: Recommendations for Physicians and Patients from the U.S. Multi-Society Task Force on Colorectal Cancer. The American journal of gastroenterology 2017;112(7): 1016-1030.

329. Tang D, Liu J, Wang DR, Yu HF, Li YK, Zhang JQ. Diagnostic and prognostic value of the methylation status of secreted frizzled-related protein 2 in colorectal cancer. Clinical and investigative medicine Medecine clinique et experimentale 2011;34(2): E88-95.

330. Lange $\mathrm{CP}$, Campan M, Hinoue $T$, Schmitz RF, van der Meulen-de Jong AE, Slingerland H, Kok PJ, van Dijk CM, Weisenberger DJ, Shen H, Tollenaar RA, Laird PW. Genome-scale discovery of DNA-methylation biomarkers for blood-based detection of colorectal cancer. PLoS One 2012;7(11): e50266.

331. Lee BB, Lee EJ, Jung EH, Chun HK, Chang DK, Song SY, Park J, Kim DH. Aberrant methylation of APC, MGMT, RASSF2A, and Wif-1 genes in plasma as a biomarker for early detection of colorectal cancer. Clin Cancer Res 2009;15(19): 6185-6191. 
332. Lofton-Day C, Model F, Devos T, Tetzner R, Distler J, Schuster M, Song X, Lesche R, Liebenberg V, Ebert M, Molnar B, Grutzmann R, Pilarsky C, Sledziewski A. DNA methylation biomarkers for blood-based colorectal cancer screening. Clinical chemistry 2008;54(2): 414423.

333. Glockner SC, Dhir M, Yi JM, McGarvey KE, Van Neste L, Louwagie J, Chan TA, Kleeberger W, de Bruine AP, Smits KM, Khalid-de Bakker CA, Jonkers DM, Stockbrugger RW, Meijer GA, Oort FA, lacobuzio-Donahue C, Bierau K, Herman JG, Baylin SB, Van Engeland M, Schuebel KE, Ahuja N. Methylation of TFPI2 in stool DNA: a potential novel biomarker for the detection of colorectal cancer. Cancer Res 2009;69(11): 4691-4699.

334. Warren JD, Xiong W, Bunker AM, Vaughn CP, Furtado LV, Roberts WL, Fang JC, Samowitz WS, Heichman KA. Septin 9 methylated DNA is a sensitive and specific blood test for colorectal cancer. BMC medicine 2011;9: 133.

335. Itzkowitz S, Brand R, Jandorf L, Durkee K, Millholland J, Rabeneck L, Schroy PC, 3rd, Sontag S, Johnson D, Markowitz S, Paszat L, Berger BM. A simplified, noninvasive stool DNA test for colorectal cancer detection. The American journal of gastroenterology 2008;103(11): 2862-2870.

336. Luo YX, Chen DK, Song SX, Wang L, Wang JP. Aberrant methylation of genes in stool samples as diagnostic biomarkers for colorectal cancer or adenomas: a meta-analysis. International journal of clinical practice 2011;65(12): 1313-1320.

337. Yang $H$, Xia BQ, Jiang B, Wang G, Yang YP, Chen H, Li BS, Xu AG, Huang YB, Wang XY. Diagnostic value of stool DNA testing for multiple markers of colorectal cancer and advanced adenoma: a meta-analysis. Canadian journal of gastroenterology = Journal canadien de gastroenterologie 2013;27(8): 467-475.

338. Navarro M, Nicolas A, Ferrandez A, Lanas A. Colorectal cancer population screening programs worldwide in 2016: An update. World journal of gastroenterology 2017;23(20): 3632-3642.

339. Wei J, Li G, Zhang J, Zhou Y, Dang S, Chen H, Wu Q, Liu M. Integrated analysis of genome-wide DNA methylation and gene expression profiles identifies potential novel biomarkers of rectal cancer. Oncotarget 2016;7(38): 62547-62558.

340. Rashid A, Shen L, Morris JS, Issa JP, Hamilton SR. CpG island methylation in colorectal adenomas. Am J Pathol 2001;159(3): 1129-1135.

341. Chan AO, Broaddus RR, Houlihan PS, Issa JP, Hamilton SR, Rashid A. CpG island methylation in aberrant crypt foci of the colorectum. Am J Pathol 2002;160(5): 1823-1830. 342. Li H, Myeroff L, Smiraglia D, Romero MF, Pretlow TP, Kasturi L, Lutterbaugh J, Rerko RM, Casey G, Issa JP, Willis J, Willson JK, Plass C, Markowitz SD. SLC5A8, a sodium transporter, is a tumor suppressor gene silenced by methylation in human colon aberrant

crypt foci and cancers. Proc Natl Acad Sci U S A 2003;100(14): 8412-8417.

343. Kim YH, Petko Z, Dzieciatkowski S, Lin L, Ghiassi M, Stain S, Chapman WC, Washington MK, Willis J, Markowitz SD, Grady WM. CpG island methylation of genes accumulates during the adenoma progression step of the multistep pathogenesis of colorectal cancer. Genes, chromosomes \& cancer 2006;45(8): 781-789.

344. Oster B, Thorsen K, Lamy P, Wojdacz TK, Hansen LL, Birkenkamp-Demtroder K, Sorensen KD, Laurberg S, Orntoft TF, Andersen CL. Identification and validation of highly frequent $\mathrm{CpG}$ island hypermethylation in colorectal adenomas and carcinomas. Int J Cancer 2011;129(12): 2855-2866.

345. Sunami E, de Maat M, Vu A, Turner RR, Hoon DS. LINE-1 hypomethylation during primary colon cancer progression. PLoS One 2011;6(4): e18884. 
346. Yano $Y$, Konishi K, Yamochi T, Katagiri A, Nozawa H, Suzuki H, Toyota M, Kubota $Y$, Muramoto T, Kobayashi Y, Tojo M, Konda K, Makino R, Kaneko K, Yoshikawa N, Ota H,

Imawari M. Clinicopathological and molecular features of colorectal serrated neoplasias with different mucosal crypt patterns. The American journal of gastroenterology 2011;106(7): 1351-1358.

347. Kim MS, Lee J, Sidransky D. DNA methylation markers in colorectal cancer. Cancer metastasis reviews 2010;29(1): 181-206.

348. Nilsson TK, Lof-Ohlin ZM, Sun XF. DNA methylation of the p14ARF, RASSF1A and APC1A genes as an independent prognostic factor in colorectal cancer patients. International journal of oncology 2013;42(1): 127-133.

349. Jensen LH, Rasmussen AA, Byriel L, Kuramochi H, Cruger DG, Lindebjerg J, Danenberg PV, Jakobsen A, Danenberg K. Regulation of MLH1 mRNA and protein expression by promoter methylation in primary colorectal cancer: a descriptive and prognostic cancer marker study. Cellular oncology (Dordrecht) 2013;36(5): 411-419.

350. Xing X, Cai W, Shi H, Wang Y, Li M, Jiao J, Chen M. The prognostic value of CDKN2A hypermethylation in colorectal cancer: a meta-analysis. Br J Cancer 2013;108(12): 25422548.

351. Katoh H, Yamashita K, Waraya M, Margalit O, Ooki A, Tamaki H, Sakagami H, Kokubo $\mathrm{K}$, Sidransky D, Watanabe M. Epigenetic silencing of HOPX promotes cancer progression in colorectal cancer. Neoplasia (New York, NY) 2012;14(7): 559-571.

352. Dahlin AM, Palmqvist R, Henriksson ML, Jacobsson M, Eklof V, Rutegard J, Oberg A, Van Guelpen BR. The role of the CpG island methylator phenotype in colorectal cancer prognosis depends on microsatellite instability screening status. Clin Cancer Res 2010;16(6): 1845-1855.

353. Juo YY, Johnston FM, Zhang DY, Juo HH, Wang H, Pappou EP, Yu T, Easwaran H, Baylin S, van Engeland M, Ahuja N. Prognostic value of CpG island methylator phenotype among colorectal cancer patients: a systematic review and meta-analysis. Annals of oncology : official journal of the European Society for Medical Oncology / ESMO 2014;25(12): 2314-2327.

354. Samowitz WS, Albertsen H, Sweeney C, Herrick J, Caan BJ, Anderson KE, Wolff RK, Slattery ML. Association of smoking, CpG island methylator phenotype, and V600E BRAF mutations in colon cancer. Journal of the National Cancer Institute 2006;98(23): 1731-1738.

355. Amatu A, Sartore-Bianchi A, Moutinho C, Belotti A, Bencardino K, Chirico G, Cassingena A, Rusconi F, Esposito A, Nichelatti M, Esteller M, Siena S. Promoter CpG island hypermethylation of the DNA repair enzyme MGMT predicts clinical response to dacarbazine in a phase II study for metastatic colorectal cancer. Clin Cancer Res 2013;19(8): 2265-2272.

356. Sawada T, Yamamoto E, Suzuki H, Nojima M, Maruyama R, Shioi Y, Akasaka R, Kamimae S, Harada T, Ashida M, Kai M, Adachi Y, Yamamoto H, Imai K, Toyota M, Itoh F, Sugai T. Association between genomic alterations and metastatic behavior of colorectal cancer identified by array-based comparative genomic hybridization. Genes, chromosomes \& cancer 2013;52(2): 140-149.

357. Sun W, Sun Y, Zhu M, Wang Z, Zhang H, Xin Y, Jiang G, Guo X, Zhang Z, Liu Y. The role of plasma cell-free DNA detection in predicting preoperative chemoradiotherapy response in rectal cancer patients. Oncology reports 2014;31(3): 1466-1472.

358. Molinari C, Casadio V, Foca F, Zingaretti C, Giannini M, Avanzolini A, Lucci E, Saragoni

L, Passardi A, Amadori D, Calistri D, Zoli W. Gene methylation in rectal cancer: predictive marker of response to chemoradiotherapy? J Cell Physiol 2013;228(12): 2343-2349. 
359. Kawakami K, Matsunoki A, Kaneko M, Saito K, Watanabe G, Minamoto T. Long interspersed nuclear element-1 hypomethylation is a potential biomarker for the prediction of response to oral fluoropyrimidines in microsatellite stable and CpG island methylator phenotype-negative colorectal cancer. Cancer science 2011;102(1): 166-174.

360. Ebert MP, Tanzer M, Balluff B, Burgermeister E, Kretzschmar AK, Hughes DJ, Tetzner R, Lofton-Day C, Rosenberg R, Reinacher-Schick AC, Schulmann K, Tannapfel A, Hofheinz R, Rocken C, Keller G, Langer R, Specht K, Porschen R, Stohlmacher-Williams J, Schuster T, Strobel P, Schmid RM. TFAP2E-DKK4 and chemoresistance in colorectal cancer. The New England journal of medicine 2012;366(1): 44-53.

361. Powell A, Soul S, Christian A, Lewis WG. Meta-analysis of the prognostic value of CpG island methylator phenotype in gastric cancer. Br J Surg 2018;105(2): e61-e68.

362. Wang H, Yan W, Zhang S, Gu Y, Wang Y, Wei Y, Liu H, Wang F, Wu Q, Zhang Y. Survival differences of CIMP subtypes integrated with CNA information in human breast cancer. Oncotarget 2017;8(30): 48807-48819.

363. Hughes LA, Melotte V, de Schrijver J, de Maat M, Smit VT, Bovee JV, French PJ, van den Brandt PA, Schouten LJ, de Meyer T, van Criekinge W, Ahuja N, Herman JG, Weijenberg $\mathrm{MP}$, van Engeland M. The CpG island methylator phenotype: what's in a name? Cancer Res 2013;73(19): 5858-5868.

364. Good Clinical Practice - UK Policy Framework for Health and Social Care Research. https://www.hra.nhs.uk/planning-and-improving-research/policies-standards-

legislation/uk-policy-framework-health-social-care-research/.

365. Herman JG, Graff JR, Myohanen S, Nelkin BD, Baylin SB. Methylation-specific PCR: a novel PCR assay for methylation status of CpG islands. Proc Natl Acad Sci U S A 1996;93(18): 9821-9826.

366. Kim CH, Huh JW, Kim HR, Kim YJ. CpG island methylator phenotype is an independent predictor of survival after curative resection for colorectal cancer: A prospective cohort study. Journal of gastroenterology and hepatology 2017.

367. Cha Y, Kim KJ, Han SW, Rhee YY, Bae JM, Wen X, Cho NY, Lee DW, Lee KH, Kim TY, Oh DY, Im SA, Bang YJ, Jeong SY, Park KJ, Kang GH, Kim TY. Adverse prognostic impact of the CpG island methylator phenotype in metastatic colorectal cancer. Br J Cancer 2016;115(2): 164-171.

368. Gallois C, Laurent-Puig P, Taieb J. Methylator phenotype in colorectal cancer: A prognostic factor or not? Critical reviews in oncology/hematology 2016;99: 74-80.

369. Tsang JS, Vencken S, Sharaf O, Leen E, Kay EW, McNamara DA, Deasy J, Mulligan ED. Global DNA methylation is altered by neoadjuvant chemoradiotherapy in rectal cancer and may predict response to treatment - A pilot study. European journal of surgical oncology : the journal of the European Society of Surgical Oncology and the British Association of Surgical Oncology 2014;40(11): 1459-1466.

370. McCarthy K, Pearson K, Fulton R, Hewitt J. Pre-operative chemoradiation for nonmetastatic locally advanced rectal cancer. The Cochrane database of systematic reviews 2012;12: Cd008368.

371. Reggiani Bonetti L, Lionti S, Domati F, Barresi V. Do pathological variables have prognostic significance in rectal adenocarcinoma treated with neoadjuvant chemoradiotherapy and surgery? World journal of gastroenterology 2017;23(8): 1412-1423. 372. Sinclair RCF, Sumpter K, Griffin SM. Fitness after chemotherapy. BJA: British Journal of Anaesthesia 2016;116(1): 140-140.

373. Kretschmer C, Sterner-Kock A, Siedentopf F, Schoenegg W, Schlag PM, Kemmner W. Identification of early molecular markers for breast cancer. Mol Cancer 2011;10(1): 15. 
374. Wang M, Gu H, Wang S, Qian H, Zhu W, Zhang L, Zhao C, Tao Y, Xu W. Circulating miR-17-5p and miR-20a: molecular markers for gastric cancer. Molecular medicine reports 2012;5(6): 1514-1520.

375. Hartmann C, Hentschel B, Tatagiba M, Schramm J, Schnell O, Seidel C, Stein R, Reifenberger G, Pietsch T, von Deimling A. Molecular markers in low-grade gliomas: predictive or prognostic? Clinical Cancer Research 2011;17(13): 4588-4599.

376. Amado RG, Wolf M, Peeters M, Van Cutsem E, Siena S, Freeman DJ, Juan T, Sikorski $\mathrm{R}$, Suggs $\mathrm{S}$, Radinsky R. Wild-type KRAS is required for panitumumab efficacy in patients with metastatic colorectal cancer. Journal of Clinical Oncology 2008;26(10): 1626-1634.

377. Yokoi K, Yamashita K, Ishii S, Tanaka T, Nishizawa N, Tsutsui A, Miura H, Katoh H, Yamanashi T, Naito M, Sato T, Nakamura T, Watanabe M. Comprehensive molecular exploration identified promoter DNA methylation of the CRBP1 gene as a determinant of radiation sensitivity in rectal cancer. Br J Cancer 2017.

378. Shiovitz S, Bertagnolli MM, Renfro LA, Nam E, Foster NR, Dzieciatkowski S, Luo Y, Lao VV, Monnat Jr RJ, Emond MJ, Maizels N, Niedzwiecki D, Goldberg RM, Saltz LB, Venook A, Warren RS, Grady WM. CpG island methylator phenotype is associated with response to adjuvant irinotecan-based therapy for stage III colon cancer. Gastroenterology 2014;147(3): 637-645.

379. Donada M, Bonin S, Barbazza R, Pettirosso D, Stanta G. Management of stage li colon cancer - the use of molecular biomarkers for adjuvant therapy decision. BMC gastroenterology 2013: 36.

380. Jover R, Nguyen T, Prezcarbonell L, Zapater P, Pay A, Alenda C, Rojas E, Cubiella J,

Balaguer F, Morillas JD, Clofent J, Bujanda L, Re JM, Bessa X, Xicola RM, Nicolsprez D, Castells A, Andreu M, Llor X, Boland CR, Goel A. 5-fluorouracil adjuvant chemotherapy does not increase survival in patients with $\mathrm{CpG}$ island methylator phenotype colorectal cancer. Gastroenterology 2011;140(4): 1174-1181.

381. Harada H, Miyamoto K, Yamashita Y, Taniyama K, Ohdan H, Okada M. Methylated DLX4 Predicts Response to Pathologic Stage I Non-Small Cell Lung Cancer Resection. Ann Thorac Surg 2015;99(5): 1746-1754.

382. Wu F, Lu M, Qu L, Li DQ, Hu CH. DNA methylation of hMLH1 correlates with the clinical response to cisplatin after a surgical resection in Non-small cell lung cancer. International journal of clinical and experimental pathology 2015;8(5): 5457-5463.

383. Montenegro MF, Gonzalez-Guerrero R, Sanchez-del-Campo L, Pinero-Madrona A, Cabezas-Herrera J, Rodriguez-Lopez JN. Targeting the epigenetics of the DNA damage response in breast cancer. Cell death \& disease 2016;7: e2180.

384. Larijani L, Madjd Z, Samadikuchaksaraei A, Younespour S, Zham H, Rakhshan A, Mohammadi F, Rahbari A, Moradi A. Methylation of O6-methyl guanine methyltransferase gene promoter in meningiomas--comparison between tumor grades I, II, and III. Asian Pacific journal of cancer prevention : APJCP 2014;15(1): 33-38.

385. van den Bent MJ, Erdem-Eraslan L, Idbaih A, de Rooi J, Eilers PH, Spliet WG, den Dunnen WF, Tijssen C, Wesseling P, Sillevis Smitt PA, Kros JM, Gorlia T, French PJ. MGMT-

STP27 methylation status as predictive marker for response to PCV in anaplastic Oligodendrogliomas and Oligoastrocytomas. A report from EORTC study 26951. Clin Cancer Res 2013;19(19): 5513-5522.

386. Andrade AF, Borges KS, Castro-Gamero AM, Silveira VS, Suazo VK, Oliveira JC, Moreno DA, de Paula Queiroz RG, Scrideli CA, Tone LG. Zebularine induces chemosensitization to methotrexate and efficiently decreases AhR gene methylation in childhood acute lymphoblastic leukemia cells. Anti-cancer drugs 2014;25(1): 72-81. 
387. Chand M, Evans J, Swift RI, Tekkis PP, West NP, Stamp G, Heald RJ, Brown G. The prognostic significance of postchemoradiotherapy high-resolution MRI and histopathology detected extramural venous invasion in rectal cancer. Ann Surg 2015;261(3): 473-479.

388. Baylin SB, Herman JG. DNA hypermethylation in tumorigenesis: epigenetics joins genetics. Trends in genetics : TIG 2000;16(4): 168-174.

389. Feinberg AP, Tycko B. The history of cancer epigenetics. Nat Rev Cancer 2004;4(2): 143-153.

390. Lorenz E, Stewart HL. Intestinal Carcinoma and Other Lesions in Mice Following Oral Administration of 1,2,5,6-Dibenzanthracene and 20-Methylcholanthrene1. JNCl: Journal of the National Cancer Institute 1940;1(1): 17-40.

391. Banerjee A, Quirke P. Experimental models of colorectal cancer. Diseases of the Colon \& Rectum 1998;41(4): 490-505.

392. Berwald Y, Sachs LEO. In Vitro Cell Transformation with Chemical Carcinogens. Nature 1963;200(4912): 1182-1184.

393. LaMont JT, O'Gorman TA. Experimental colon cancer. Gastroenterology 1978;75(6): 1157-1169.

394. Hawks A, Magee PN. The Alkylation of Nucleic Acids of Rat and Mouse In Vivo by the Carcinogen 1,2-Dimethylhydrazine. British Journal of Cancer 1974;30(5): 440-447.

395. Willson JK, Bittner GN. In vitro models of human colonic adenomatous polyps. Progress in clinical and biological research 1988;279: 347-356.

396. Willson JK, Bittner GN, Oberley TD, Meisner LF, Weese JL. Cell culture of human colon adenomas and carcinomas. Cancer Res 1987;47(10): 2704-2713.

397. Park JG, Gazdar AF. Biology of colorectal and gastric cancer cell lines. Journal of cellular biochemistry Supplement 1996;24: 131-141.

398. Shoemaker $\mathrm{RH}$. The $\mathrm{NCl} 60$ human tumour cell line anticancer drug screen. Nat Rev Cancer 2006;6(10): 813-823.

399. Fisher R, Pusztai L, Swanton C. Cancer heterogeneity: implications for targeted therapeutics. Br J Cancer 2013;108(3): 479-485.

400. Golovko D, Kedrin D, Yilmaz OH, Roper J. Colorectal cancer models for novel drug discovery. Expert opinion on drug discovery 2015;10(11): 1217-1229.

401. Carvalho MR, Lima D, Reis RL, Oliveira JM, Correlo VM. Anti-Cancer Drug Validation: the Contribution of Tissue Engineered Models. Stem cell reviews 2017;13(3): 347-363.

402. Sensi F, D'Angelo E, D'Aronco S, Molinaro R, Agostini M. Preclinical threedimensional colorectal cancer model: The next generation of in vitro drug efficacy evaluation. J Cell Physiol 2018;234(1): 181-191.

403. Olejniczak A, Szarynska M, Kmiec Z. In vitro characterization of spheres derived from colorectal cancer cell lines. International journal of oncology 2018;52(2): 599-612.

404. Gil-Bazo I. Novel translational strategies in colorectal cancer research. World journal of gastroenterology 2007;13(44): 5902-5910.

405. Lu W, Kang Y. Epithelial-Mesenchymal Plasticity in Cancer Progression and Metastasis. Developmental cell 2019;49(3): 361-374.

406. Davies JA. Mesenchyme to Epithelium Transition during Development of the Mammalian Kidney Tubule. Cells Tissues Organs 1996;156(3): 187-201.

407. Thiery JP, Acloque H, Huang RYJ, Nieto MA. Epithelial-Mesenchymal Transitions in Development and Disease. Cell 2009;139(5): 871-890.

408. Haensel D, Dai X. Epithelial-to-mesenchymal transition in cutaneous wound healing: Where we are and where we are heading. Developmental dynamics : an official publication of the American Association of Anatomists 2018;247(3): 473-480. 
409. Li L, Li W. Epithelial-mesenchymal transition in human cancer: Comprehensive reprogramming of metabolism, epigenetics, and differentiation. Pharmacology \& Therapeutics 2015;150: 33-46.

410. Bosman FT. The borderline: basement membranes and the transition from premalignant to malignant neoplasia. Microscopy research and technique 1994;28(3): 216225.

411. Yang J, Weinberg RA. Epithelial-Mesenchymal Transition: At the Crossroads of Development and Tumor Metastasis. Developmental cell 2008;14(6): 818-829.

412. Kramer N, Walzl A, Unger C, Rosner M, Krupitza G, Hengstschläger M, Dolznig H. In vitro cell migration and invasion assays. Mutation Research/Reviews in Mutation Research 2013;752(1): 10-24.

413. Grinnell F. Fibroblast biology in three-dimensional collagen matrices. Trends in Cell Biology 2003;13(5): 264-269.

414. Gadea G, Sanz-Moreno V, Self A, Godi A, Marshall CJ. DOCK10-Mediated Cdc42 Activation Is Necessary for Amoeboid Invasion of Melanoma Cells. Current Biology 2008;18(19): 1456-1465.

415. Rodriguez LG, Wu X, Guan J-L. Wound-Healing Assay. In: Cell Migration: Developmental Methods and Protocols, Guan J-L (ed). Humana Press: Totowa, NJ, 2005; 2329.

416. Lo C-M, Keese CR, Giaever I. Monitoring Motion of Confluent Cells in Tissue Culture. Experimental cell research 1993;204(1): 102-109.

417. Lo CM, Keese CR, Giaever I. Impedance analysis of MDCK cells measured by electric cell-substrate impedance sensing. Biophysical Journal 1995;69(6): 2800-2807.

418. Poujade M, Grasland-Mongrain E, Hertzog A, Jouanneau J, Chavrier P, Ladoux B, Buguin A, Silberzan P. Collective migration of an epithelial monolayer in response to a model wound. Proceedings of the National Academy of Sciences 2007;104(41): 15988-15993.

419. Culture-Insert 2 Well. https://ibidi.com/removable-chambers/24-culture-insert-2well.html [Last accessed January 2019.

420. Boyden S. The chemotactic effect of mixtures of antibody and antigen on polymorphonuclear leucocytes. . The Journal of Experimental Medicine 1962;115(3): 453466.

421. Albini A, Iwamoto Y, Kleinman HK, Martin GR, Aaronson SA, Kozlowski JM, McEwan RN. A Rapid in Vitro Assay for Quantitating the Invasive Potential of Tumor Cells. Cancer Research 1987;47(12): 3239-3245.

422. Albini A, Benelli R. The chemoinvasion assay: a method to assess tumor and endothelial cell invasion and its modulation. Nature Protocols 2007;2: 504.

423. Marshall J. Transwell ${ }^{\circledR}$ Invasion Assays. In: Cell Migration: Developmental Methods and Protocols, Wells CM, Parsons M (eds). Humana Press: Totowa, NJ, 2011; 97-110.

424. Corning Matrigel Matrix. https://www.corning.com/emea/en/products/lifesciences/products/surfaces/matrigel-matrix.html [Last accessed January 19.

425. Kleinman HK, Martin GR. Matrigel: basement membrane matrix with biological activity. Seminars in cancer biology 2005;15(5): 378-386.

426. Hughes CS, Postovit LM, Lajoie GA. Matrigel: a complex protein mixture required for optimal growth of cell culture. Proteomics 2010;10(9): 1886-1890.

427. Benton G, Arnaoutova I, George J, Kleinman HK, Koblinski J. Matrigel: from discovery and ECM mimicry to assays and models for cancer research. Advanced drug delivery reviews 2014;79-80: 3-18. 
428. Ljubimov AV, Bartek J, Couchman JR, Kapuller LL, Veselov VV, Kovarik J, Perevoshchikov AG, Krutovskikh VA. Distribution of individual components of basement membrane in human colon polyps and adenocarcinomas as revealed by monoclonal antibodies. Int J Cancer 1992;50(4): 562-566.

429. Amenta PS, Briggs K, Xu K, Gamboa E, Jukkola AF, Li D, Myers JC. Type XV collagen in human colonic adenocarcinomas has a different distribution than other basement membrane zone proteins. Human pathology 2000;31(3): 359-366.

430. Bae JM, Kim JH, Kang GH. Epigenetic alterations in colorectal cancer: the CpG island methylator phenotype. Histology and histopathology 2013;28(5): 585-595.

431. Mossman D, Kim KT, Scott RJ. Demethylation by 5-aza-2'-deoxycytidine in colorectal cancer cells targets genomic DNA whilst promoter $\mathrm{CpG}$ island methylation persists. BMC Cancer 2010;10: 366.

432. Hagemann S, Heil O, Lyko F, Brueckner B. Azacytidine and decitabine induce genespecific and non-random DNA demethylation in human cancer cell lines. PLoS One 2011;6(3): e17388.

433. Hu YH, Chen Q, Lu YX, Zhang JM, Lin C, Zhang F, Zhang WJ, Li XM, Zhang W, Li XN. Hypermethylation of NDN promotes cell proliferation by activating the Wnt signaling pathway in colorectal cancer. Oncotarget 2017;8(28): 46191-46203.

434. Flis S, Gnyszka A, Flis K. DNA methyltransferase inhibitors improve the effect of chemotherapeutic agents in SW48 and HT-29 colorectal cancer cells. PloS one 2014;9(3): e92305-e92305.

435. Sharma A, Vatapalli R, Abdelfatah E, Wyatt McMahon K, Kerner Z, A AG, Singh J, Zahnow C, S BB, Yerram S, Hu Y, Azad N, Ahuja N. Hypomethylating agents synergize with irinotecan to improve response to chemotherapy in colorectal cancer cells. PLoS One 2017;12(4): e0176139.

436. Blainey P, Krzywinski M, Altman N. Replication. Nature Methods 2014;11: 879. 437. Hynes L. Comparison of different methods for an accurate assessment of cytotoxicity in the in vitro micronucleus test without cytokinesis block. Mutation research 2010;702(2): 181-188.

438. OECD GUIDELINE FOR THE TESTING OF CHEMICALS Proposal for updating Test Guideline 487 In Vitro Mammalian Cell Micronucleus Test.

http://www.oecd.org/env/ehs/testing/TG487\%200ct\%202012\%20updated\%2029oct.pdf.

439. Fellows MD, O'Donovan MR, Lorge E, Kirkland D. Comparison of different methods for an accurate assessment of cytotoxicity in the in vitro micronucleus test. II: Practical aspects with toxic agents. Mutation research 2008;655(1-2): 4-21.

440. Lorge $\mathrm{E}$, Hayashi M, Albertini S, Kirkland D. Comparison of different methods for an accurate assessment of cytotoxicity in the in vitro micronucleus test. I. Theoretical aspects. Mutation research 2008;655(1-2): 1-3.

441. Strober W. Trypan blue exclusion test of cell viability. Current protocols in immunology 2001;Appendix 3: Appendix 3B.

442. Trepat X, Chen Z, Jacobson K. Cell migration. Comprehensive Physiology 2012;2(4): 2369-2392.

443. Grada A, Otero-Vinas M, Prieto-Castrillo F, Obagi Z, Falanga V. Research Techniques Made Simple: Analysis of Collective Cell Migration Using the Wound Healing Assay. Journal of Investigative Dermatology 2017;137(2): e11-e16.

444. Cory G, Wells C, Parsons M. Scratch-Wound Assay. Humana Press, 2011.

445. Schwarzbauer JE, Sechler JL. Fibronectin fibrillogenesis: a paradigm for extracellular matrix assembly. Current opinion in cell biology 1999;11(5): 622-627. 
446. Winters BS, Raj BK, Robinson EE, Foty RA, Corbett SA. Three-dimensional culture regulates Raf-1 expression to modulate fibronectin matrix assembly. Molecular biology of the cell 2006;17(8): 3386-3396.

447. Iwai S, Kishimoto S, Amano Y, Nishiguchi A, Matsusaki M, Takeshita A, Akashi M. Three-dimensional cultured tissue constructs that imitate human living tissue organization for analysis of tumor cell invasion. Journal of biomedical materials research Part $A$ 2019;107(2): 292-300.

448. Maiuri AR, Peng M, Podicheti R, Sriramkumar S, Kamplain CM, Rusch DB, DeStefano Shields CE, Sears CL, O'Hagan HM. Mismatch Repair Proteins Initiate Epigenetic Alterations during Inflammation-Driven Tumorigenesis. Cancer Res 2017;77(13): 3467-3478.

449. Manzardo AM, Butler MG. Examination of Global Methylation and Targeted Imprinted Genes in Prader-Willi Syndrome. Journal of clinical epigenetics 2016;2(3). 450. Fowler P, Smith R, Smith K, Young J, Jeffrey L, Kirkland D, Pfuhler S, Carmichael P. Reduction of misleading ("false") positive results in mammalian cell genotoxicity assays. II. Importance of accurate toxicity measurement. Mutation research 2012;747(1): 104-117. 451. Greenwood SK, Hill RB, Sun JT, Armstrong MJ, Johnson TE, Gara JP, Galloway SM. Population doubling: a simple and more accurate estimation of cell growth suppression in the in vitro assay for chromosomal aberrations that reduces irrelevant positive results.

Environmental and molecular mutagenesis 2004;43(1): 36-44.

452. Kang XC, Chen ML, Yang F, Gao BQ, Yang QH, Zheng WW, Hao S. Promoter methylation and expression of SOCS-1 affect clinical outcome and epithelial-mesenchymal transition in colorectal cancer. Biomedicine \& pharmacotherapy = Biomedecine \& pharmacotherapie 2016;80: 23-29.

453. Shin TH, Paterson AJ, Grant JH, 3rd, Meluch AA, Kudlow JE. 5-Azacytidine treatment of HA-A melanoma cells induces Sp1 activity and concomitant transforming growth factor alpha expression. Molecular and cellular biology 1992;12(9): 3998-4006.

454. Scicinski J, Oronsky B, Ning S, Knox S, Peehl D, Kim MM, Langecker P, Fanger G. NO to cancer: The complex and multifaceted role of nitric oxide and the epigenetic nitric oxide donor, RRx-001. Redox biology 2015;6: 1-8.

455. Tanaka S, Hosokawa M, Ueda K, Iwakawa S. Effects of Decitabine on Invasion and Exosomal Expression of miR-200c and miR-141 in Oxaliplatin-Resistant Colorectal Cancer Cells. Biological \& pharmaceutical bulletin 2015;38(9): 1272-1279.

456. Ahrens TD, Timme S, Hoeppner J, Ostendorp J, Hembach S, Follo M, Hopt UT, Werner M, Busch H, Boerries M, Lassmann S. Selective inhibition of esophageal cancer cells by combination of HDAC inhibitors and Azacytidine. Epigenetics 2015;10(5): 431-445.

457. Xi W, Chen X, Sun J, Wang W, Huo Y, Zheng G, Wu J, Li Y, Yang A, Wang T. Combined Treatment with Valproic Acid and 5-Aza-2'-Deoxycytidine Synergistically Inhibits Human

Clear Cell Renal Cell Carcinoma Growth and Migration. Medical science monitor : international medical journal of experimental and clinical research 2018;24: 1034-1043.

458. Zuo Y, Lv Y, Qian X, Wang S, Chen Z, Jiang Q, Cao C, Song Y. Inhibition of HHIP Promoter Methylation Suppresses Human Gastric Cancer Cell Proliferation and Migration.

Cellular physiology and biochemistry : international journal of experimental cellular physiology, biochemistry, and pharmacology 2018;45(5): 1840-1850.

459. Liu ZH, Liu JJ, Li SS, Yang XM. Association of RUNX3 Methylation with Clinical Outcome and Cell Migration/Invasion in Laryngeal Squamous Cell Carcinoma. Cancer investigation 2016;34(2): 105-113.

460. Tost J. DNA Methylation: An Introduction to the Biology and the Disease-Associated Changes of a Promising Biomarker. Molecular Biotechnology 2010;44(1): 71-81. 
461. Bird A. The essentials of DNA methylation. Cell 1992;70(1): 5-8.

462. Donoghue MT, Fort A, Clifton R, Zhang X, McKeown PC, Voigt-Zielinksi ML, Borevitz JO, Spillane $C$. $C(m) C G G$ methylation-independent parent-of-origin effects on genome-wide transcript levels in isogenic reciprocal F1 triploid plants. DNA research : an international journal for rapid publication of reports on genes and genomes 2014;21(2): 141-151. 463. Hansen KD, Timp W, Bravo HC, Sabunciyan S, Langmead B, McDonald OG, Wen B, Wu H, Liu Y, Diep D, Briem E, Zhang K, Irizarry RA, Feinberg AP. Increased methylation variation in epigenetic domains across cancer types. Nature genetics 2011;43(8): 768-775. 464. Lee EJ, Luo J, Wilson JM, Shi H. Analyzing the cancer methylome through targeted bisulfite sequencing. Cancer Lett 2013;340(2): 171-178.

465. Xiong L, He L, Luo L, Li Y, Liao L, Huang R, Zhu Z, Wang Y. Global and Complement Gene-Specific DNA Methylation in Grass Carp after Grass Carp Reovirus (GCRV) Infection. International journal of molecular sciences 2018;19(4).

466. Kalluri R, Weinberg RA. The basics of epithelial-mesenchymal transition. The Journal of clinical investigation 2009;119(6): 1420-1428.

467. Nieto MA, Huang RY, Jackson RA, Thiery JP. EMT: 2016. Cell 2016;166(1): 21-45.

468. Stone RC, Pastar I, Ojeh N, Chen V, Liu S, Garzon KI, Tomic-Canic M. Epithelialmesenchymal transition in tissue repair and fibrosis. Cell and tissue research 2016;365(3): 495-506.

469. Pei D, Shu X, Gassama-Diagne A, Thiery JP. Mesenchymal-epithelial transition in development and reprogramming. Nature cell biology 2019;21(1): 44-53.

470. Pastushenko I, Brisebarre A, Sifrim A, Fioramonti M, Revenco T, Boumahdi S, Van Keymeulen A, Brown D, Moers V, Lemaire S, De Clercq S, Minguijon E, Balsat C, Sokolow $Y$, Dubois C, De Cock F, Scozzaro S, Sopena F, Lanas A, D'Haene N, Salmon I, Marine JC, Voet T, Sotiropoulou PA, Blanpain C. Identification of the tumour transition states occurring during EMT. Nature 2018;556(7702): 463-468.

471. Greenburg G, Hay ED. Cytodifferentiation and tissue phenotype change during transformation of embryonic lens epithelium to mesenchyme-like cells in vitro.

Developmental Biology 1986;115(2): 363-379.

472. Guarino M, Rubino B, Ballabio $G$. The role of epithelial-mesenchymal transition in cancer pathology. Pathology 2007;39(3): 305-318.

473. Mareel M, Leroy A. Clinical, cellular, and molecular aspects of cancer invasion. Physiological reviews 2003;83(2): 337-376.

474. Klymkowsky MW, Savagner P. Epithelial-Mesenchymal Transition: A Cancer Researcher's Conceptual Friend and Foe. The American Journal of Pathology 2009;174(5): 1588-1593.

475. Meacham CE, Morrison SJ. Tumour heterogeneity and cancer cell plasticity. Nature 2013;501: 328.

476. Brabletz T, Hlubek F, Spaderna S, Schmalhofer O, Hiendlmeyer E, Jung A, Kirchner T. Invasion and metastasis in colorectal cancer: epithelial-mesenchymal transition, mesenchymal-epithelial transition, stem cells and beta-catenin. Cells Tissues Organs 2005;179(1-2): 56-65.

477. Jones HG, Jenkins G, Williams N, Griffiths P, Chambers P, Beynon J, Harris D. Genetic and Epigenetic Intra-tumour Heterogeneity in Colorectal Cancer. World Journal of Surgery 2017;41(5): 1375-1383.

478. Boyer B, Valles AM, Edme N. Induction and regulation of epithelial-mesenchymal transitions. Biochemical pharmacology 2000;60(8): 1091-1099. 
479. Graff JR, Gabrielson E, Fujii H, Baylin SB, Herman JG. Methylation patterns of the Ecadherin $5^{\prime} \mathrm{CpG}$ island are unstable and reflect the dynamic, heterogeneous loss of $\mathrm{E}$ cadherin expression during metastatic progression. The Journal of biological chemistry 2000;275(4): 2727-2732.

480. Vigneri PG, Tirro E, Pennisi MS, Massimino M, Stella S, Romano C, Manzella L. The Insulin/IGF System in Colorectal Cancer Development and Resistance to Therapy. Front Oncol 2015;5: 230.

481. Morali OG, Delmas V, Moore R, Jeanney C, Thiery JP, Larue L. IGF-II induces rapid beta-catenin relocation to the nucleus during epithelium to mesenchyme transition.

Oncogene 2001;20(36): 4942-4950.

482. Jung B, Staudacher JJ, Beauchamp D. Transforming Growth Factor beta Superfamily

Signaling in Development of Colorectal Cancer. Gastroenterology 2017;152(1): 36-52.

483. Inman GJ. Switching TGFbeta from a tumor suppressor to a tumor promoter. Current opinion in genetics \& development 2011;21(1): 93-99.

484. Caja F, Vannucci L. TGFbeta: A player on multiple fronts in the tumor microenvironment. Journal of immunotoxicology 2015;12(3): 300-307.

485. Yang L, Moses HL. Transforming growth factor beta: tumor suppressor or promoter? Are host immune cells the answer? Cancer Res 2008;68(22): 9107-9111.

486. Barak V, Goike H, Panaretakis KW, Einarsson R. Clinical utility of cytokeratins as tumor markers. Clinical Biochemistry 2004;37(7): 529-540.

487. Moll R, Divo M, Langbein L. The human keratins: biology and pathology. Histochemistry and cell biology 2008;129(6): 705-733.

488. Lane EB, Alexander CM. Use of keratin antibodies in tumor diagnosis. Seminars in cancer biology 1990;1(3): 165-179.

489. Satelli A, Li S. Vimentin in cancer and its potential as a molecular target for cancer therapy. Cellular and molecular life sciences : CMLS 2011;68(18): 3033-3046.

490. Vu T, Datta PK. Regulation of EMT in Colorectal Cancer: A Culprit in Metastasis. Cancers 2017;9(12).

491. Von Kleist $\mathrm{S}$, Hesse $\mathrm{Y}$, Kananeeh $\mathrm{H}$. Comparative evaluation of four tumor markers, CA 242, CA 19/9, TPA, and CEA in carcinomas of the colon. Anticancer Research 1996;16/4 B): $2325-2331$.

492. Carpelan-Holmström M, Haglund C, Lundin J, Alfthan H, Stenman UH, Roberts PJ. Independent prognostic value of preoperative serum markers CA 242, specific tissue polypeptide antigen and human chorionic gonadotrophin beta, but not of carcinoembryonic antigen or tissue polypeptide antigen in colorectal cancer. British Journal of Cancer 1996;74(6): 925-929.

493. Dow LE, Humbert PO. Polarity Regulators and the Control of Epithelial Architecture, Cell Migration, and Tumorigenesis. In: International Review of Cytology, vol 262. Academic Press, 2007; 253-302.

494. Greenburg G, Hay ED. Epithelia suspended in collagen gels can lose polarity and express characteristics of migrating mesenchymal cells. The Journal of Cell Biology 1982;95(1): 333-339.

495. Weaver VM, Petersen OW, Wang F, Larabell CA, Briand P, Damsky C, Bissell MJ. Reversion of the malignant phenotype of human breast cells in three- dimensional culture and in vivo by integrin blocking antibodies. Journal of Cell Biology 1997;137(1): 231-245.

496. Murray NR, Jamieson L, Yu W, Zhang J, Gökmen-Polar Y, Sier D, Anastasiadis P, Gatalica Z, Thompson EA, Fields AP. Protein kinase $C_{1}$ is required for Ras transformation and colon carcinogenesis in vivo. Journal of Cell Biology 2004;164(6): 797-802. 
497. Archibald A, Al-Masri M, Liew-Spilger A, McCaffrey L. Atypical protein kinase C induces cell transformation by disrupting Hippo/Yap signaling. Molecular biology of the cell 2015;26(20): 3578-3595.

498. Aranda V, Haire T, Nolan ME, Calarco JP, Rosenberg AZ, Fawcett JP, Pawson T, Muthuswamy SK. Par6-aPKC uncouples ErbB2 induced disruption of polarized epithelial

organization from proliferation control. Nature cell biology 2006;8(11): 1235-1245.

499. Takegawa N, Yonesaka K. HER2 as an Emerging Oncotarget for Colorectal Cancer

Treatment After Failure of Anti-Epidermal Growth Factor Receptor Therapy. Clinical colorectal cancer 2017;16(4): 247-251.

500. Diepenbruck M, Christofori G. Epithelial-mesenchymal transition (EMT) and metastasis: yes, no, maybe? Current opinion in cell biology 2016;43: 7-13.

501. Chambers AF, Naumov GN, Varghese HJ, Nadkarni KV, MacDonald IC, Groom AC. Critical steps in hematogenous metastasis: an overview. Surgical oncology clinics of North America 2001;10(2): 243-255, vii.

502. Chambers AF, Groom AC, MacDonald IC. Dissemination and growth of cancer cells in metastatic sites. Nat Rev Cancer 2002;2(8): 563-572.

503. Ala-aho R, Kähäri V-M. Collagenases in cancer. Biochimie 2005;87(3): 273-286.

504. Kahari VM, Saarialho-Kere U. Matrix metalloproteinases and their inhibitors in tumour growth and invasion. Annals of medicine 1999;31(1): 34-45.

505. Stamenkovic I. Matrix metalloproteinases in tumor invasion and metastasis. Seminars in cancer biology 2000;10(6): 415-433.

506. Nagase H, Woessner JF, Jr. Matrix metalloproteinases. The Journal of biological chemistry 1999;274(31): 21491-21494.

507. Hooper.N.M. Zinc Metalloproteases In Health And Disease. CRC Press: London, 1996.

508. Shofuda K, Yasumitsu H, Nishihashi A, Miki K, Miyazaki K. Expression of three membrane-type matrix metalloproteinases (MT-MMPs) in rat vascular smooth muscle cells and characterization of MT3-MMPs with and without transmembrane domain. The Journal of biological chemistry 1997;272(15): 9749-9754.

509. Parks WC, Mecham RP. Matrix Metalloproteinases. In, Parks WC, Mecham RP (eds). Academic Press: San Diego, 1998; xi-xii.

510. Björklund M, Koivunen E. Gelatinase-mediated migration and invasion of cancer cells. Biochimica et Biophysica Acta (BBA) - Reviews on Cancer 2005;1755(1): 37-69.

511. Levy AT, Cioce V, Sobel ME, Garbisa S, Grigioni WF, Liotta LA, Stetler-Stevenson WG. Increased expression of the $\mathrm{Mr} 72,000$ type IV collagenase in human colonic adenocarcinoma. Cancer Res 1991;51(1): 439-444.

512. Ring $P$, Johansson $K$, Hoyhtya $M$, Rubin $K$, Lindmark $G$. Expression of tissue inhibitor of metalloproteinases TIMP-2 in human colorectal cancer--a predictor of tumour stage. $\mathrm{Br} J$ Cancer 1997;76(6): 805-811.

513. Turpeenniemi-Hujanen T. Gelatinases (MMP-2 and -9) and their natural inhibitors as prognostic indicators in solid cancers. Biochimie 2005;87(3): 287-297.

514. Mook ORF, Frederiks WM, Van Noorden CJF. The role of gelatinases in colorectal cancer progression and metastasis. Biochimica et biophysica acta 2004;1705(2): 69-89.

515. Vandenbroucke RE, Libert C. Is there new hope for therapeutic matrix metalloproteinase inhibition? Nat Rev Drug Discov 2014;13(12): 904-927.

516. Moon JW, Choi JH, Lee SK, Lee YW, Lee JO, Kim N, Lee HJ, Seo JS, Kim J, Kim HS, Kim GJ, Park SH. Promoter hypermethylation of membrane type 3 matrix metalloproteinase is associated with cell migration in colorectal adenocarcinoma. Cancer genetics 2015;208(5):

261-270. 
517. Lin HF, Hsi E, Huang LC, Liao YC, Juo SH, Lin RT. Methylation in the matrix metalloproteinase-2 gene is associated with cerebral ischemic stroke. Journal of investigative medicine : the official publication of the American Federation for Clinical Research 2017;65(4): 794-799.

518. Kowluru RA, Mishra M. Regulation of Matrix Metalloproteinase in the Pathogenesis of Diabetic Retinopathy. Progress in molecular biology and translational science 2017;148: 67-85.

519. Araki Y, Mimura T. Matrix Metalloproteinase Gene Activation Resulting from Disordred Epigenetic Mechanisms in Rheumatoid Arthritis. International journal of molecular sciences 2017;18(5).

520. Hanahan D, Weinberg RA. The hallmarks of cancer. Cell 2000;100(1): 57-70. 521. Burczynski ME, McMillian M, Ciervo J, Li L, Parker JB, Dunn RT, 2nd, Hicken S, Farr S, Johnson MD. Toxicogenomics-based discrimination of toxic mechanism in HepG2 human hepatoma cells. Toxicological sciences : an official journal of the Society of Toxicology 2000;58(2): 399-415.

522. Scherf U, Ross DT, Waltham M, Smith LH, Lee JK, Tanabe L, Kohn KW, Reinhold WC, Myers TG, Andrews DT, Scudiero DA, Eisen MB, Sausville EA, Pommier Y, Botstein D, Brown $\mathrm{PO}$, Weinstein JN. A gene expression database for the molecular pharmacology of cancer. Nature genetics 2000;24(3): 236-244.

523. Eisen MB, Spellman PT, Brown PO, Botstein D. Cluster analysis and display of genome-wide expression patterns. Proc Natl Acad Sci U S A 1998;95(25): 14863-14868.

524. Rampal.J.B. Microarrays. Humana Press, 2007.

525. Quackenbush J. Computational approaches to analysis of DNA microarray data. Yearbook of medical informatics 2006: 91-103.

526. Deepak S, Kottapalli K, Rakwal R, Oros G, Rangappa K, Iwahashi H, Masuo Y, Agrawal G. Real-Time PCR: Revolutionizing Detection and Expression Analysis of Genes. Current genomics 2007;8(4): 234-251.

527. Arikawa.E, Quellhorst.G, Han.Y, Pan.H, Yang.J. RT2 Profiler PCR Arrays: Pathwayfocused gene expression profiling with qRT-PCR. In: Qiagen; 2017.

528. Rajeevan MS, Vernon SD, Taysavang N, Unger ER. Validation of array-based gene expression profiles by real-time (kinetic) RT-PCR. The Journal of molecular diagnostics : JMD 2001;3(1): 26-31.

529. Ye J, Coulouris G, Zaretskaya I, Cutcutache I, Rozen S, Madden TL. Primer-BLAST: a tool to design target-specific primers for polymerase chain reaction. BMC bioinformatics 2012;13: 134-134.

530. Bustin SA, Benes V, Nolan T, Pfaffl MW. Quantitative real-time RT-PCR--a perspective. Journal of molecular endocrinology 2005;34(3): 597-601.

531. Bustin SA. Quantification of mRNA using real-time reverse transcription PCR (RT-

PCR): trends and problems. Journal of molecular endocrinology 2002;29(1): 23-39.

532. Reue K. mRNA Quantitation Techniques: Considerations for Experimental Design and Application. The Journal of Nutrition 1998;128(11): 2038-2044.

533. Caraguel CGB, Stryhn H, Gagné N, Dohoo IR, Hammell KL. Selection of a Cutoff Value for Real-Time Polymerase Chain Reaction Results to Fit a Diagnostic Purpose: Analytical and Epidemiologic Approaches. Journal of Veterinary Diagnostic Investigation 2011;23(1): 2-15.

534. Nagase $H$, Visse R, Murphy G. Structure and function of matrix metalloproteinases and TIMPs. Cardiovasc Res 2006;69.

535. Corso G, Intra M, Trentin C, Veronesi P, Galimberti V. CDH1 germline mutations and hereditary lobular breast cancer. Familial cancer 2016;15(2): 215-219. 
536. van der Post RS, Vogelaar IP, Carneiro F, Guilford P, Huntsman D, Hoogerbrugge N, Caldas C, Schreiber KE, Hardwick RH, Ausems MG, Bardram L, Benusiglio PR, Bisseling TM,

Blair V, Bleiker E, Boussioutas A, Cats A, Coit D, DeGregorio L, Figueiredo J, Ford JM, Heijkoop E, Hermens R, Humar B, Kaurah P, Keller G, Lai J, Ligtenberg MJ, O'Donovan M, Oliveira C, Pinheiro H, Ragunath K, Rasenberg E, Richardson S, Roviello F, Schackert $H$, Seruca R, Taylor A, Ter Huurne A, Tischkowitz M, Joe ST, van Dijck B, van Grieken NC, van Hillegersberg R, van Sandick JW, Vehof R, van Krieken JH, Fitzgerald RC. Hereditary diffuse gastric cancer: updated clinical guidelines with an emphasis on germline $\mathrm{CDH} 1$ mutation carriers. Journal of medical genetics 2015;52(6): 361-374.

537. Kim SA, Inamura K, Yamauchi M, Nishihara R, Mima K, Sukawa Y, Li T, Yasunari M, Morikawa T, Fitzgerald KC, Fuchs CS, Wu K, Chan AT, Zhang X, Ogino S, Qian ZR. Loss of $\mathrm{CDH} 1$ (E-cadherin) expression is associated with infiltrative tumour growth and lymph node metastasis. Br J Cancer 2016;114(2): 199-206.

538. Baserga R, Peruzzi F, Reiss K. The IGF-1 receptor in cancer biology. Int J Cancer 2003;107(6): 873-877.

539. Furstenberger $\mathrm{G}$, Senn HJ. Insulin-like growth factors and cancer. The Lancet Oncology 2002;3(5): 298-302.

540. Reinmuth N, Liu W, Fan F, Jung YD, Ahmad SA, Stoeltzing O, Bucana CD, Radinsky R,

Ellis LM. Blockade of insulin-like growth factor I receptor function inhibits growth and angiogenesis of colon cancer. Clin Cancer Res 2002;8(10): 3259-3269.

541. Sekharam M, Zhao H, Sun M, Fang Q, Zhang Q, Yuan Z, Dan HC, Boulware D, Cheng

$J Q$, Coppola D. Insulin-like growth factor 1 receptor enhances invasion and induces resistance to apoptosis of colon cancer cells through the Akt/Bcl-x $(\mathrm{L})$ pathway. Cancer Res 2003;63(22): 7708-7716.

542. Ozer B, Sezerman U. An integrative study on the impact of highly differentially methylated genes on expression and cancer etiology. PloS one 2017;12(2): e0171694e0171694.

543. Pankov R, Yamada KM. Fibronectin at a glance. Journal of Cell Science 2002;115(20): 3861-3863.

544. Grinnell F, Billingham RE, Burgess L. Distribution of Fibronectin During Wound Healing in Vivo. Journal of Investigative Dermatology 1981;76(3): 181-189.

545. Cai X, Liu C, Zhang TN, Zhu YW, Dong X, Xue P. Down-regulation of FN1 inhibits colorectal carcinogenesis by suppressing proliferation, migration, and invasion. Journal of cellular biochemistry 2018;119(6): 4717-4728.

546. Pereira IT, Ramos EA, Costa ET, Camargo AA, Manica GC, Klassen LM, Chequin A, Braun-Prado K, Pedrosa Fde O, Souza EM, Costa FF, Klassen G. Fibronectin affects transient MMP2 gene expression through DNA demethylation changes in non-invasive breast cancer cell lines. PLoS One 2014;9(9): e105806.

547. Allan JA, Docherty AJ, Barker PJ, Huskisson NS, Reynolds JJ, Murphy G. Binding of gelatinases $A$ and $B$ to type-I collagen and other matrix components. The Biochemical journal 1995;309 ( Pt 1)(Pt 1): 299-306.

548. Bardelli A, Corso S, Bertotti A, Hobor S, Valtorta E, Siravegna G, Sartore-Bianchi A, Scala E, Cassingena A, Zecchin D, Apicella M, Migliardi G, Galimi F, Lauricella C, Zanon C, Perera T, Veronese S, Corti G, Amatu A, Gambacorta M, Diaz LA, Sausen M, Velculescu VE, Comoglio P, Trusolino L, Di Nicolantonio F, Giordano S, Siena S. Amplification of the $<$ em $>\mathrm{MET}</$ em $>$ Receptor Drives Resistance to Anti-EGFR Therapies in Colorectal Cancer. Cancer discovery 2013;3(6): 658-673. 
549. Lee SJ, Lee J, Park SH, Park JO, Lim HY, Kang WK, Park YS, Kim ST. c-MET

Overexpression in Colorectal Cancer: A Poor Prognostic Factor for Survival. Clinical colorectal cancer 2018;17(3): 165-169.

550. Bradley CA, Salto-Tellez M, Laurent-Puig P, Bardelli A, Rolfo C, Tabernero J, Khawaja HA, Lawler M, Johnston PG, Van Schaeybroeck S. Targeting c-MET in gastrointestinal tumours: rationale, opportunities and challenges. Nat Rev Clin Oncol 2017;14(9): 562-576.

551. Zhang J, Babic A. Regulation of the MET oncogene: molecular mechanisms. Carcinogenesis 2016;37(4): 345-355.

552. Morath I, Hartmann TN, Orian-Rousseau V. CD44: More than a mere stem cell marker. The international journal of biochemistry \& cell biology 2016;81(Pt A): 166-173.

553. Desai B, Rogers MJ, Chellaiah MA. Mechanisms of osteopontin and CD44 as metastatic principles in prostate cancer cells. Molecular cancer 2007;6: 18.

554. Yu Q, Stamenkovic I. Localization of matrix metalloproteinase 9 to the cell surface provides a mechanism for CD44-mediated tumor invasion. Genes \& development 1999;13(1): 35-48.

555. Gupta A, Cao W, Sadashivaiah K, Chen W, Schneider A, Chellaiah MA. Promising noninvasive cellular phenotype in prostate cancer cells knockdown of matrix metalloproteinase 9. TheScientificWorldJournal 2013;2013: 493689.

556. Wielenga VJM, van der Neut R, Offerhaus GJA, Pals ST. CD44 Glycoproteins in Colorectal Cancer: Expression, Function, and Prognostic Value. In: Advances in Cancer Research, Vande Woude GF, Klein G (eds), vol 77. Academic Press, 1999; 169-187.

557. Qu Y, Dang S, Hou P. Gene methylation in gastric cancer. Clinica Chimica Acta 2013;424: 53-65.

558. Nabel EG. CDKs and CKIs: molecular targets for tissue remodelling. Nat Rev Drug Discov 2002;1(8): 587-598.

559. Kim JC, Choi JS, Roh SA, Cho DH, Kim TW, Kim YS. Promoter methylation of specific genes is associated with the phenotype and progression of colorectal adenocarcinomas. Ann Surg Oncol 2010;17(7): 1767-1776.

560. Zhao R, Choi BY, Lee M-H, Bode AM, Dong Z. Implications of Genetic and Epigenetic Alterations of CDKN2A (p16(INK4a)) in Cancer. EBioMedicine 2016;8: 30-39.

561. Veganzones S, Maestro ML, Rafael S, de la Orden V, Vidaurreta M, Mediero B, Espantaleon M, Cerdan J, Diaz-Rubio E. Combined methylation of p16 and hMLH1 (CMETH2) discriminates a subpopulation with better prognosis in colorectal cancer patients with microsatellite instability tumors. Tumour Biology 2015;36(5): 3853-3861.

562. Morgans CW, Zhang J, Jeffrey BG, Nelson SM, Burke NS, Duvoisin RM, Brown RL. TRPM1 is required for the depolarizing light response in retinal ON-bipolar cells. Proceedings of the National Academy of Sciences 2009;106(45): 19174-19178.

563. Guo H, Carlson JA, Slominski A. Role of TRPM in melanocytes and melanoma. Exp Dermatol 2012;21(9): 650-654.

564. Prevarskaya N, Ouadid-Ahidouch H, Skryma R, Shuba Y. Remodelling of Ca2+ transport in cancer: how it contributes to cancer hallmarks? Philosophical transactions of

the Royal Society of London Series B, Biological sciences 2014;369(1638): 20130097.

565. Stewart TA, Yapa KT, Monteith GR. Altered calcium signaling in cancer cells. Biochim Biophys Acta 2015;1848(10 Pt B): 2502-2511.

566. Pérez-Riesgo E, Gutiérrez LG, Ubierna D, Acedo A, Moyer MP, Núñez L, Villalobos C. Transcriptomic Analysis of Calcium Remodeling in Colorectal Cancer. International journal of molecular sciences 2017;18(5): 922. 
567. Monteith GR, Davis FM, Roberts-Thomson SJ. Calcium channels and pumps in cancer: changes and consequences. The Journal of biological chemistry 2012;287(38):

\section{6-31673.}

568. Bodding M. TRP proteins and cancer. Cellular signalling 2007;19(3): 617-624.

569. Bianconi D, Unseld M, Prager GW. Integrins in the Spotlight of Cancer. International journal of molecular sciences 2016;17(12): 2037.

570. Khan Z, Marshall JF. The role of integrins in TGF $\beta$ activation in the tumour stroma. Cell and tissue research 2016;365(3): 657-673.

571. Bates RC, Bellovin DI, Brown C, Maynard E, Wu B, Kawakatsu H, Sheppard D, Oettgen

$P$, Mercurio AM. Transcriptional activation of integrin beta 6 during the epithelialmesenchymal transition defines a novel prognostic indicator of aggressive colon carcinoma. J Clin Invest 2005;115(2): 339-347.

572. Allen MD, Thomas GJ, Clark S, Dawoud MM, Vallath S, Payne SJ, Gomm JJ, Dreger SA, Dickinson S, Edwards DR, Pennington CJ, Sestak I, Cuzick J, Marshall JF, Hart IR, Jones JL. Altered microenvironment promotes progression of preinvasive breast cancer: myoepithelial expression of alphavbeta 6 integrin in DCIS identifies high-risk patients and predicts recurrence. Clin Cancer Res 2014;20(2): 344-357.

573. Frisch SM, Ruoslahti E. Integrins and anoikis. Current opinion in cell biology 1997;9(5): 701-706.

574. Teitz T, Wei T, Valentine MB, Vanin EF, Grenet J, Valentine VA, Behm FG, Look AT, Lahti JM, Kidd VJ. Caspase 8 is deleted or silenced preferentially in childhood neuroblastomas with amplification of MYCN. Nature medicine 2000;6(5): 529-535.

575. Yang J, Zhang W, Evans PM, Chen X, He X, Liu C. Adenomatous Polyposis Coli (APC) Differentially Regulates $\beta$-Catenin Phosphorylation and Ubiquitination in Colon Cancer Cells. Journal of Biological Chemistry 2006;281(26): 17751-17757.

576. Barua D, Hlavacek WS. Modeling the effect of APC truncation on destruction complex function in colorectal cancer cells. PLoS computational biology 2013;9(9): e1003217.

577. Liang T-J, Wang H-X, Zheng Y-Y, Cao Y-Q, Wu X, Zhou X, Dong S-X. APC hypermethylation for early diagnosis of colorectal cancer: a meta-analysis and literature review. Oncotarget 2017;8(28): 46468-46479.

578. Ding Z, Jiang T, Piao Y, Han T, Han Y, Xie X. Meta-analysis of the association between APC promoter methylation and colorectal cancer. OncoTargets and therapy 2015;8: 211222.

579. Rimbert J, Tachon G, Junca A, Villalva C, Karayan-Tapon L, Tougeron D. Association between clinicopathological characteristics and RAS mutation in colorectal cancer. Modern pathology : an official journal of the United States and Canadian Academy of Pathology, Inc 2018;31(3): 517-526.

580. Massague J, Wotton D. Transcriptional control by the TGF-beta/Smad signaling system. The EMBO journal 2000;19(8): 1745-1754.

581. Clark IM, Swingler TE, Sampieri CL, Edwards DR. The regulation of matrix metalloproteinases and their inhibitors. The international journal of biochemistry \& cell biology 2008;40(6): 1362-1378.

582. Yan C, Boyd DD. Regulation of matrix metalloproteinase gene expression. J Cell Physiol 2007;211(1): 19-26.

583. Abdul-Muneer PM, Conte AA, Haldar D, Long M, Patel RK, Santhakumar V, Overall $\mathrm{CM}$, Pfister BJ. Traumatic brain injury induced matrix metalloproteinase2 cleaves 
CXCL12alpha (stromal cell derived factor 1alpha) and causes neurodegeneration. Brain, behavior, and immunity 2017;59: 190-199.

584. Sato H, Kita M, Seiki M. v-Src activates the expression of 92-kDa type IV collagenase gene through the AP-1 site and the GT box homologous to retinoblastoma control elements.

A mechanism regulating gene expression independent of that by inflammatory cytokines.

The Journal of biological chemistry 1993;268(31): 23460-23468.

585. Qin H, Sun Y, Benveniste EN. The transcription factors Sp1, Sp3, and AP-2 are required for constitutive matrix metalloproteinase-2 gene expression in astroglioma cells.

The Journal of biological chemistry 1999;274(41): 29130-29137.

586. Fuks F. DNA methylation and histone modifications: teaming up to silence genes.

Current opinion in genetics \& development 2005;15(5): 490-495.

587. Chicoine E, Esteve PO, Robledo O, Van Themsche C, Potworowski EF, St-Pierre Y. Evidence for the role of promoter methylation in the regulation of MMP-9 gene expression. Biochemical and biophysical research communications 2002;297(4): 765-772.

588. Bergers G, Brekken R, McMahon G, Vu TH, Itoh T, Tamaki K, Tanzawa K, Thorpe P, Itohara S, Werb Z, Hanahan D. Matrix metalloproteinase-9 triggers the angiogenic switch during carcinogenesis. Nature cell biology 2000;2(10): 737-744.

589. Couillard J, Demers M, Lavoie G, St-Pierre Y. The role of DNA hypomethylation in the control of stromelysin gene expression. Biochemical and biophysical research communications 2006;342(4): 1233-1239.

590. Ma Z, Shah RC, Chang MJ, Benveniste EN. Coordination of cell signaling, chromatin remodeling, histone modifications, and regulator recruitment in human matrix metalloproteinase 9 gene transcription. Molecular and cellular biology 2004;24(12): 54965509.

591. Vincent ZL, Mitchell MD, Ponnampalam AP. Regulation of MT1-MMP/MMP-2/TIMP2 axis in human placenta. J Inflamm Res 2015;8: 193-200.

592. Chernov AV, Sounni NE, Remacle AG, Strongin AY. Epigenetic control of the invasionpromoting MT1-MMP/MMP-2/TIMP-2 axis in cancer cells. The Journal of biological chemistry 2009;284(19): 12727-12734.

593. Overall CM, Wrana JL, Sodek J. Transcriptional and post-transcriptional regulation of 72-kDa gelatinase/type IV collagenase by transforming growth factor-beta 1 in human fibroblasts. Comparisons with collagenase and tissue inhibitor of matrix metalloproteinase gene expression. The Journal of biological chemistry 1991;266(21): 14064-14071.

594. Delany AM, Jeffrey JJ, Rydziel S, Canalis E. Cortisol increases interstitial collagenase expression in osteoblasts by post-transcriptional mechanisms. The Journal of biological chemistry 1995;270(44): 26607-26612.

595. Iyer V, Pumiglia K, DiPersio CM. Alpha3beta1 integrin regulates MMP-9 mRNA stability in immortalized keratinocytes: a novel mechanism of integrin-mediated MMP gene expression. J Cell Sci 2005;118(Pt 6): 1185-1195.

596. Liu J, Xiong W, Baca-Regen L, Nagase H, Baxter BT. Mechanism of inhibition of matrix metalloproteinase-2 expression by doxycycline in human aortic smooth muscle cells. Journal of vascular surgery 2003;38(6): 1376-1383.

597. Cui N, Hu M, Khalil RA. Biochemical and Biological Attributes of Matrix Metalloproteinases. Progress in molecular biology and translational science 2017;147: 1-73. 598. Gkogkas CG, Khoutorsky A, Cao R, Jafarnejad SM, Prager-Khoutorsky M, Giannakas N, Kaminari A, Fragkouli A, Nader K, Price TJ, Konicek BW, Graff JR, Tzinia AK, Lacaille J-C, Sonenberg N. Pharmacogenetic inhibition of elF4E-dependent Mmp9 mRNA translation reverses fragile X syndrome-like phenotypes. Cell Rep 2014;9(5): 1742-1755. 
599. Arpino V, Brock M, Gill SE. The role of TIMPs in regulation of extracellular matrix proteolysis. Matrix Biology 2015;44-46: 247-254.

600. Young DA, Barter MJ, Wilkinson DJ. Recent advances in understanding the regulation of metalloproteinases. F1000Research 2019;8.

601. Selman M, Ruiz V, Cabrera S, Segura L, Ramirez R, Barrios R, Pardo A. TIMP-1, -2, -3, and -4 in idiopathic pulmonary fibrosis. A prevailing nondegradative lung microenvironment? American journal of physiology Lung cellular and molecular physiology 2000;279(3): L562-574.

602. Jezierska A, Motyl T. Matrix metalloproteinase-2 involvement in breast cancer progression: a mini-review. Medical science monitor : international medical journal of experimental and clinical research 2009;15(2): Ra32-40.

603. Yoshizaki T, Sato H, Furukawa M. Recent advances in the regulation of matrix metalloproteinase 2 activation: from basic research to clinical implication (Review). Oncology reports 2002;9(3): 607-611.

604. Duffy MJ, McCarthy K. Matrix metalloproteinases in cancer: prognostic markers and targets for therapy (review). International journal of oncology 1998;12(6): 1343-1348.

605. Fernandez-Catalan C, Bode W, Huber R, Turk D, Calvete JJ, Lichte A, Tschesche H, Maskos K. Crystal structure of the complex formed by the membrane type 1-matrix metalloproteinase with the tissue inhibitor of metalloproteinases-2, the soluble progelatinase A receptor. The EMBO journal 1998;17(17): 5238-5248.

606. Visse R, Nagase $\mathrm{H}$. Matrix metalloproteinases and tissue inhibitors of metalloproteinases: structure, function, and biochemistry. Circulation research 2003;92(8): 827-839.

607. Polette $\mathrm{M}$, Birembaut $\mathrm{P}$. Membrane-type metalloproteinases in tumor invasion. The international journal of biochemistry \& cell biology 1998;30(11): 1195-1202.

608. Mizuno R, Kawada K, Itatani Y, Ogawa R, Kiyasu Y, Sakai Y. The Role of TumorAssociated Neutrophils in Colorectal Cancer. International journal of molecular sciences 2019;20(3).

609. Halazun KJ, Aldoori A, Malik HZ, Al-Mukhtar A, Prasad KR, Toogood GJ, Lodge JP. Elevated preoperative neutrophil to lymphocyte ratio predicts survival following hepatic resection for colorectal liver metastases. European journal of surgical oncology : the journal of the European Society of Surgical Oncology and the British Association of Surgical Oncology 2008;34(1): 55-60.

610. Hammani K, Blakis A, Morsette D, Bowcock AM, Schmutte C, Henriet P, DeClerck YA. Structure and characterization of the human tissue inhibitor of metalloproteinases- 2 gene.

The Journal of biological chemistry 1996;271(41): 25498-25505.

611. Melendez-Zajgla J, Del Pozo L, Ceballos G, Maldonado V. Tissue inhibitor of metalloproteinases-4. The road less traveled. Molecular cancer 2008;7: 85-85.

612. English JL, Kassiri Z, Koskivirta I, Atkinson SJ, Di Grappa M, Soloway PD, Nagase H, Vuorio E, Murphy G, Khokha R. Individual Timp deficiencies differentially impact pro-MMP-2 activation. The Journal of biological chemistry 2006;281(15): 10337-10346.

613. Hernandez-Barrantes S, Shimura Y, Soloway PD, Sang QA, Fridman R. Differential roles of TIMP-4 and TIMP-2 in pro-MMP-2 activation by MT1-MMP. Biochemical and biophysical research communications 2001;281(1): 126-130.

614. Bigg HF, Morrison CJ, Butler GS, Bogoyevitch MA, Wang Z, Soloway PD, Overall CM. Tissue inhibitor of metalloproteinases- 4 inhibits but does not support the activation of gelatinase A via efficient inhibition of membrane type 1-matrix metalloproteinase. Cancer Res 2001;61(9): 3610-3618. 
615. Bigg HF, Shi YE, Liu YE, Steffensen B, Overall CM. Specific, high affinity binding of tissue inhibitor of metalloproteinases-4 (TIMP-4) to the $\mathrm{COOH}$-terminal hemopexin-like domain of human gelatinase A. TIMP-4 binds progelatinase $\mathrm{A}$ and the $\mathrm{COOH}$-terminal domain in a similar manner to TIMP-2. The Journal of biological chemistry 1997;272(24): 15496-15500.

616. Chaturvedi P, Tyagi SC. Epigenetic silencing of TIMP4 in heart failure. Journal of Cellular and Molecular Medicine 2016;20(11): 2089-2101.

617. Young DA, Phillips BW, Lundy C, Nuttall RK, Hogan A, Schultz GA, Leco KJ, Clark IM, Edwards DR. Identification of an initiator-like element essential for the expression of the tissue inhibitor of metalloproteinases-4 (Timp-4) gene. The Biochemical journal 2002;364(Pt 1): 89-99.

618. Rhodes DR, Yu J, Shanker K, Deshpande N, Varambally R, Ghosh D, Barrette T, Pandey A, Chinnaiyan AM. ONCOMINE: a cancer microarray database and integrated datamining platform. Neoplasia (New York, NY) 2004;6(1): 1-6.

619. Weiss WA, Taylor SS, Shokat KM. Recognizing and exploiting differences between RNAi and small-molecule inhibitors. Nat Chem Biol 2007;3(12): 739-744.

620. Boettcher M, McManus MT. Choosing the Right Tool for the Job: RNAi, TALEN, or CRISPR. Mol Cell 2015;58(4): 575-585.

621. Pilcher HR. It's a knockout. Nature 2003.

622. Summerton JE. Morpholino, siRNA, and S-DNA compared: impact of structure and mechanism of action on off-target effects and sequence specificity. Current topics in medicinal chemistry 2007;7(7): 651-660.

623. Fellmann C, Lowe SW. Stable RNA interference rules for silencing. Nature cell biology 2014;16(1): 10-18.

624. Saxena S, Jonsson ZO, Dutta A. Small RNAs with imperfect match to endogenous mRNA repress translation. Implications for off-target activity of small inhibitory RNA in mammalian cells. The Journal of biological chemistry 2003;278(45): 44312-44319.

625. Zeng Y, Yi R, Cullen BR. MicroRNAs and small interfering RNAs can inhibit mRNA expression by similar mechanisms. Proc Natl Acad Sci U S A 2003;100(17): 9779-9784.

626. Whitehead KA, Dahlman JE, Langer RS, Anderson DG. Silencing or Stimulation? siRNA Delivery and the Immune System. Annual Review of Chemical and Biomolecular Engineering 2011;2(1): 77-96.

627. Transfection: In Vitro Transfection. http://transfection.ws [Last accessed 12/04/2019.

628. Jensen K, Anderson JA, Glass EJ. Comparison of small interfering RNA (siRNA) delivery into bovine monocyte-derived macrophages by transfection and electroporation. Veterinary immunology and immunopathology 2014;158(3-4): 224-232.

629. Takei Y. Electroporation-Mediated siRNA Delivery into Tumors. In: Electroporation Protocols: Preclinical and Clinical Gene Medicine, Li S, Cutrera J, Heller R, Teissie J (eds).

Springer New York: New York, NY, 2014; 131-138.

630. Morris KV, Rossi JJ. Lentiviral-mediated delivery of siRNAs for antiviral therapy. Gene Therapy 2006;13(6): 553-558.

631. Cambon K, Déglon N. Lentiviral-Mediated Gene Transfer of siRNAs for the Treatment of Huntington's Disease. In: Trinucleotide Repeat Protocols, Kohwi Y, McMurray CT (eds). Humana Press: Totowa, NJ, 2013; 95-109.

632. Schütze N. siRNA technology. Molecular and Cellular Endocrinology 2004;213(2): 115-119. 
633. Wittrup A, Lieberman J. Knocking down disease: a progress report on siRNA therapeutics. Nat Rev Genet 2015;16(9): 543-552.

634. Cejka D, Losert D, Wacheck V. Short interfering RNA (siRNA): tool or therapeutic? Clinical science (London, England : 1979) 2006;110(1): 47-58.

635. Goding JW. 12 - Immunofluorescence. In: Monoclonal Antibodies (Third Edition), Goding JW (ed). Academic Press: London, 1996; 352-399.

636. Goding JW. 13 - Immunohistology. In: Monoclonal Antibodies (Third Edition), Goding JW (ed). Academic Press: London, 1996; 400-423.

637. Davidoff MS. Immunocytochemistry--possibilities for detection of different tissue antigens and establishment of the functional role of cells. Acta histochemica Supplementband 1986;33: 175-193.

638. Webster P, Schwarz H, Griffiths G. Chapter 3 Preparation of Cells and Tissues for Immuno EM. In: Methods in Cell Biology, vol 88. Academic Press, 2008; 45-58.

639. Immunocytochemistry (ICC) Handbook.

https://images.novusbio.com/design/BR_ICCguide.pdf 2019].

640. Burry RW. Controls for immunocytochemistry: an update. J Histochem Cytochem 2011;59(1): 6-12.

641. Vallejo-Illarramendi A, Marciano DK, Reichardt LF. A novel method that improves sensitivity of protein detection in PAGE and Western blot. Electrophoresis 2013;34(8): 11481150 .

642. Murphy RM, Lamb GD. Important considerations for protein analyses using antibody based techniques: down-sizing Western blotting up-sizes outcomes. The Journal of physiology 2013;591(23): 5823-5831.

643. Hagner-McWhirter A, Soderquist K, Grimsby S, Winkvist M. New Approaches to Quantitative Western Blotting. J Biomol Tech 2011;22(Suppl): S51-S51.

644. Bass JJ, Wilkinson DJ, Rankin D, Phillips BE, Szewczyk NJ, Smith K, Atherton PJ. An overview of technical considerations for Western blotting applications to physiological research. Scand J Med Sci Sports 2017;27(1): 4-25.

645. Western Blotting Sample Preparation.

https://www.sigmaaldrich.com/content/dam/sigma-aldrich/docs/SigmaAldrich/General Information/1/ge-western-blotting.pdf 2017].

646. Brunelle JL, Green R. Chapter Twelve - One-dimensional SDS-Polyacrylamide Gel Electrophoresis (1D SDS-PAGE). In: Methods in Enzymology, Lorsch J (ed), vol 541. Academic Press, 2014; 151-159.

647. Ning W, Dong Y, Sun J, Li C, Matthay MA, Feghali-Bostwick CA, Choi AMK. Cigarette smoke stimulates matrix metalloproteinase-2 activity via EGR-1 in human lung fibroblasts. Am J Respir Cell Mol Biol 2007;36(4): 480-490.

648. Li Z, Guo Y, Jiang H, Zhang T, Jin C, Young CYF, Yuan H. Differential regulation of MMPs by E2F1, Sp1 and NF-kappa B controls the small cell lung cancer invasive phenotype.

BMC cancer 2014;14: 276-276.

649. Stadler $C$, Skogs $M$, Brismar $H$, Uhlén $M$, Lundberg E. A single fixation protocol for proteome-wide immunofluorescence localization studies. Journal of Proteomics 2010;73(6): 1067-1078.

650. The Human Protein Atlas. https://www.proteinatlas.org [last Accessed September 2019.

651. Chik F, Szyf M. Effects of specific DNMT gene depletion on cancer cell transformation and breast cancer cell invasion; toward selective DNMT inhibitors. Carcinogenesis 2011;32(2): 224-232. 
652. Mokarram P, Kumar K, Brim H, Naghibalhossaini F, Saberi-firoozi M, Nouraie M, Green R, Lee E, Smoot DT, Ashktorab H. Distinct high-profile methylated genes in colorectal cancer. PLoS One 2009;4(9): e7012.

653. Vidal E, Sayols S, Moran S, Guillaumet-Adkins A, Schroeder MP, Royo R, Orozco M, Gut M, Gut I, Lopez-Bigas N, Heyn H, Esteller M. A DNA methylation map of human cancer at single base-pair resolution. Oncogene 2017;36(40): 5648-5657.

654. Schroen DJ, Brinckerhoff CE. Nuclear hormone receptors inhibit matrix metalloproteinase (MMP) gene expression through diverse mechanisms. Gene expression 1996;6(4): 197-207.

655. Benbow $U$, Brinckerhoff CE. The AP-1 site and MMP gene regulation: what is all the fuss about? Matrix Biol 1997;15(8-9): 519-526.

656. Zhang C, Li C, Zhu M, Zhang Q, Xie Z, Niu G, Song X, Jin L, Li G, Zheng H. MetaAnalysis of MMP2, MMP3, and MMP9 Promoter Polymorphisms and Head and Neck Cancer Risk. PLOS ONE 2013;8(4): e62023.

657. Noroozi-Aghideh A, Kashani khatib Z, Naderi M, Dorgalaleh A, Yaghmaie M, Paryan $\mathrm{M}$, Alizadeh S. Expression and $\mathrm{CpG}$ island methylation pattern of MMP-2 and MMP-9 genes in patients with congenital factor XIII deficiency and intracranial hemorrhage. Hematology 2019;24(1): 601-605.

658. Hussain Z, Khan MI, Shahid M, Almajhdi FN. S-adenosylmethionine, a methyl donor, up regulates tissue inhibitor of metalloproteinase- 2 in colorectal cancer. Genetics and molecular research : GMR 2013;12(2): 1106-1118.

659. Sundrani D, Narang A, Mehendale S, Joshi S, Chavan-Gautam P. Investigating the expression of MMPs and TIMPs in preterm placenta and role of CpG methylation in regulating MMP-9 expression. IUBMB life 2017;69(12): 985-993.

660. Moncada-Pazos A, Obaya AJ, Fraga MF, Viloria CG, Capellá G, Gausachs M, Esteller M, López-Otín C, Cal S. The ADAMTS12 metalloprotease gene is epigenetically silenced in tumor cells and transcriptionally activated in the stroma during progression of colon cancer. Journal of Cell Science 2009;122(16): 2906-2913.

661. Brocker CN, Vasiliou V, Nebert DW. Evolutionary divergence and functions of the ADAM and ADAMTSgene families. Human genomics 2009;4(1): 43.

662. Chien Y-C, Liu L-C, Ye H-Y, Wu J-Y, Yu Y-L. EZH2 promotes migration and invasion of triple-negative breast cancer cells via regulating TIMP2-MMP-2/-9 pathway. American journal of cancer research 2018;8(3): 422-434.

663. Yi X, Guo J, Guo J, Sun S, Yang P, Wang J, Li Y, Xie L, Cai J, Wang Z. EZH2-mediated epigenetic silencing of TIMP2 promotes ovarian cancer migration and invasion. Scientific reports $2017 ; 7(1): 3568-3568$.

664. Xia Y, Wu S. Tissue inhibitor of metalloproteinase 2 inhibits activation of the $\beta$ catenin signaling in melanoma cells. Cell cycle (Georgetown, Tex) 2015;14(11): 1666-1674.

665. TIMP-2 Antibody (B-12). https://www.scbt.com/p/timp-2-antibody-b-12 [2018.

666. Ukaji T, Sasazawa Y, Umezawa K, Simizu S. Involvement of conserved tryptophan residues for secretion of TIMP-2. Oncology letters 2014;7(3): 631-634.

667. Barzilay E, Ben-Califa N, Hirschberg K, Neumann D. Uncoupling of Brefeldin AMediated Coatomer Protein Complex-I Dissociation from Golgi Redistribution. Traffic 2005;6(9): 794-802.

668. Tumova L, Pombinho AR, Vojtechova M, Stancikova J, Gradl D, Krausova M, Sloncova E, Horazna M, Kriz V, Machonova O, Jindrich J, Zdrahal Z, Bartunek P, Korinek V. Monensin Inhibits Canonical Wnt Signaling in Human Colorectal Cancer Cells and Suppresses Tumor 
Growth in Multiple Intestinal Neoplasia Mice. Molecular Cancer Therapeutics 2014;13(4):

812-822.

669. Aymoz D, Wosika V, Durandau E, Pelet S. Real-time quantification of protein expression at the single-cell level via dynamic protein synthesis translocation reporters.

Nature communications 2016;7(1): 11304.

670. TIMP4 Polyclonal Antibody.

https://www.thermofisher.com/antibody/product/TIMP4-Antibody-Polyclonal/PA5-30228 2018].

671. Walker C, Mojares E, Del Río Hernández A. Role of Extracellular Matrix in

Development and Cancer Progression. International journal of molecular sciences 2018;19(10): 3028.

672. Bussard KM, Mutkus L, Stumpf K, Gomez-Manzano C, Marini FC. Tumor-associated stromal cells as key contributors to the tumor microenvironment. Breast cancer research : BCR 2016;18(1): 84-84.

673. Zeng ZS, Huang Y, Cohen AM, Guillem JG. Prediction of colorectal cancer relapse and survival via tissue RNA levels of matrix metalloproteinase-9. Journal of clinical oncology:

official journal of the American Society of Clinical Oncology 1996;14(12): 3133-3140.

674. Yukawa N, Yoshikawa T, Akaike M, Sugimasa Y, Takemiya S, Yanoma S, Noguchi Y, Takanashi Y. Plasma concentration of tissue inhibitor of matrix metalloproteinase 1 in patients with colorectal carcinoma. Br J Surg 2001;88(12): 1596-1601.

675. Schwandner O, Schlamp A, Broll R, Bruch HP. Clinicopathologic and prognostic significance of matrix metalloproteinases in rectal cancer. Int J Colorectal Dis 2007;22(2):

127-136.

676. Svagzdys S, Lesauskaite V, Pangonyte D, Saladzinskas Z, Tamelis A, Pavalkis D. Matrix metalloproteinase-9 is a prognostic marker to predict survival of patients who underwent surgery due to rectal carcinoma. The Tohoku journal of experimental medicine 2011;223(1): 67-73.

677. Hadler-Olsen E, Winberg J-O, Uhlin-Hansen L. Matrix metalloproteinases in cancer: their value as diagnostic and prognostic markers and therapeutic targets. Tumor Biology 2013;34(4): 2041-2051.

678. Pokorny RM, Hunt L, Galandiuk S. What's new with tumor markers for colorectal cancer? Digestive surgery 2000;17(3): 209-215.

679. Hurst NG, Stocken DD, Wilson S, Keh C, Wakelam MJ, Ismail T. Elevated serum matrix metalloproteinase 9 (MMP-9) concentration predicts the presence of colorectal neoplasia in symptomatic patients. Br J Cancer 2007;97(7): 971-977.

680. Fernandez-Banares F, Cleries R, Boadas J, Ribes J, Oliva JC, Alsius A, Sanz X, MartinezBauer E, Galter S, Pujals M, Pujol M, Del Pozo P, Campo R. Prediction of advanced colonic neoplasm in symptomatic patients: a scoring system to prioritize colonoscopy (COLONOFIT study). BMC Cancer 2019;19(1): 734.

681. Williams TG, Cubiella J, Griffin SJ, Walter FM, Usher-Smith JA. Risk prediction models for colorectal cancer in people with symptoms: a systematic review. BMC gastroenterology 2016;16(1): 63.

682. Herszenyi L, Barabas L, Hritz I, Istvan G, Tulassay Z. Impact of proteolytic enzymes in colorectal cancer development and progression. World journal of gastroenterology 2014;20(37): 13246-13257.

683. Herszenyi L, Hritz I, Lakatos G, Varga MZ, Tulassay Z. The behavior of matrix metalloproteinases and their inhibitors in colorectal cancer. International journal of molecular sciences 2012;13(10): 13240-13263. 
684. Packeisen J, Korsching E, Herbst H, Boecker W, Buerger H. Demystified...tissue microarray technology. Mol Pathol 2003;56(4): 198-204.

685. Kononen J, Bubendorf L, Kallioniemi A, Barlund M, Schraml P, Leighton S, Torhorst J, Mihatsch MJ, Sauter G, Kallioniemi OP. Tissue microarrays for high-throughput molecular profiling of tumor specimens. Nature medicine 1998;4(7): 844-847.

686. Battifora $\mathrm{H}$. The multitumor (sausage) tissue block: novel method for immunohistochemical antibody testing. Laboratory investigation; a journal of technical methods and pathology 1986;55(2): 244-248.

687. Bubendorf L, Nocito A, Moch H, Sauter G. Tissue microarray (TMA) technology: miniaturized pathology archives for high-throughput in situ studies. The Journal of pathology 2001;195(1): 72-79.

688. Hsu FD, Nielsen TO, Alkushi A, Dupuis B, Huntsman D, Liu CL, van de Rijn M, Gilks CB.

Tissue microarrays are an effective quality assurance tool for diagnostic immunohistochemistry. Modern pathology : an official journal of the United States and Canadian Academy of Pathology, Inc 2002;15(12): 1374-1380.

689. Kim SW, Roh J, Park CS. Immunohistochemistry for Pathologists: Protocols, Pitfalls, and Tips. Journal of pathology and translational medicine 2016;50(6): 411-418.

690. Simon R. Applications of tissue microarray technology. Methods in molecular biology (Clifton, NJ) 2010;664: 1-16.

691. Kallioniemi OP, Wagner U, Kononen J, Sauter G. Tissue microarray technology for high-throughput molecular profiling of cancer. Human molecular genetics 2001;10(7): 657662.

692. Fedchenko N, Reifenrath J. Different approaches for interpretation and reporting of immunohistochemistry analysis results in the bone tissue - a review. Diagnostic pathology 2014;9.

693. Cross SS. Grading and scoring in histopathology. Histopathology 1998;33(2): 99-106.

694. Gibson-Corley KN, Olivier AK, Meyerholz DK. Principles for valid histopathologic scoring in research. Veterinary pathology 2013;50(6): 1007-1015.

695. Elledge RM, Clark GM, Fuqua SA, Yu YY, Allred DC. p53 protein accumulation detected by five different antibodies: relationship to prognosis and heat shock protein 70 in breast cancer. Cancer Res 1994;54(14): 3752-3757.

696. Remmele W, Stegner HE. [Recommendation for uniform definition of an immunoreactive score (IRS) for immunohistochemical estrogen receptor detection (ER-ICA) in breast cancer tissue]. Der Pathologe 1987;8(3): 138-140.

697. Detre S, Saclani Jotti G, Dowsett M. A "quickscore" method for immunohistochemical semiquantitation: validation for oestrogen receptor in breast carcinomas. J Clin Pathol 1995;48(9): 876-878.

698. Spruance SL, Reid JE, Grace M, Samore M. Hazard ratio in clinical trials. Antimicrob Agents Chemother 2004;48(8): 2787-2792.

699. Wood J, Freemantle N, King M, Nazareth I. Trap of trends to statistical significance: likelihood of near significant $\mathrm{P}$ value becoming more significant with extra data. BMJ : British Medical Journal 2014;348: g2215.

700. Kraus VB. Biomarkers as drug development tools: discovery, validation, qualification and use. Nature reviews Rheumatology 2018;14(6): 354-362.

701. Allinson JL. Clinical biomarker validation. Bioanalysis 2018;10(12): 957-968.

702. Dragutinovic VV, Radonjic NV, Petronijevic ND, Tatic SB, Dimitrijevic IB, Radovanovic NS, Krivokapic ZV. Matrix metalloproteinase-2 (MMP-2) and -9 (MMP-9) in preoperative 
serum as independent prognostic markers in patients with colorectal cancer. Molecular and cellular biochemistry 2011;355(1-2): 173-178.

703. Emara M, Cheung PY, Grabowski K, Sawicki G, Wozniak M. Serum levels of matrix metalloproteinase-2 and -9 and conventional tumor markers (CEA and CA 19-9) in patients with colorectal and gastric cancers. Clinical chemistry and laboratory medicine 2009;47(8): 993-1000.

704. Groblewska M, Mroczko B, Gryko M, Pryczynicz A, Guzińska-Ustymowicz K, Kędra B, Kemona A, Szmitkowski M. Serum levels and tissue expression of matrix metalloproteinase 2 (MMP-2) and tissue inhibitor of metalloproteinases 2 (TIMP-2) in colorectal cancer patients. Tumour Biology 2014;35(4): 3793-3802.

705. Wilson S, Damery S, Stocken DD, Dowswell G, Holder R, Ward ST, Redman V, Wakelam MJ, James J, Hobbs FD, Ismail T. Serum matrix metalloproteinase 9 and colorectal neoplasia: a community-based evaluation of a potential diagnostic test. Br J Cancer 2012;106(8): 1431-1438.

706. Nielsen HJ, Brunner N, Jorgensen LN, Olsen J, Rahr HB, Thygesen K, Hoyer U, Laurberg S, Stieber P, Blankenstein MA, Davis G, Dowell BL, Christensen IJ. Plasma TIMP-1 and CEA in detection of primary colorectal cancer: a prospective, population based study of 4509 high-risk individuals. Scandinavian journal of gastroenterology 2011;46(1): 60-69. 707. Das V, Kalita J, Pal M. Predictive and prognostic biomarkers in colorectal cancer: A systematic review of recent advances and challenges. Biomedicine $\&$ pharmacotherapy $=$ Biomedecine \& pharmacotherapie 2017;87: 8-19.

708. Nielsen HJ, Christensen IJ, Brunner N. A novel prognostic index in colorectal cancer defined by serum carcinoembryonic antigen and plasma tissue inhibitor of

metalloproteinases-1. Scandinavian journal of gastroenterology 2010;45(2): 200-207.

709. Birgisson H, Nielsen HJ, Christensen IJ, Glimelius B, Brunner N. Preoperative plasma TIMP-1 is an independent prognostic indicator in patients with primary colorectal cancer: a prospective validation study. European journal of cancer (Oxford, England : 1990) 2010;46(18): 3323-3331.

710. Min BS, Kim NK, Jeong HC, Chung HC. High levels of serum VEGF and TIMP-1 are correlated with colon cancer liver metastasis and intrahepatic recurrence after liver resection. Oncology letters 2012;4(1): 123-130.

711. Wu CY, Wu MS, Chiang EP, Chen YJ, Chen CJ, Chi NH, Shih YT, Chen GH, Lin JT. Plasma matrix metalloproteinase-9 level is better than serum matrix metalloproteinase-9 level to predict gastric cancer evolution. Clin Cancer Res 2007;13(7): 2054-2060.

712. Shen $\mathrm{W}, \mathrm{Xi} \mathrm{H}$, Wei B, Chen L. The prognostic role of matrix metalloproteinase 2 in gastric cancer: a systematic review with meta-analysis. Journal of cancer research and clinical oncology 2014;140(6): 1003-1009.

713. Frederiksen C, Qvortrup C, Christensen IJ, Glimelius B, Berglund A, Jensen BV, Nielsen SE, Keldsen N, Nielsen HJ, Brunner N, Pfeiffer P. Plasma TIMP-1 levels and treatment outcome in patients treated with XELOX for metastatic colorectal cancer. Annals of oncology : official journal of the European Society for Medical Oncology / ESMO 2011;22(2): 369-375.

714. Sorensen NM, Bystrom P, Christensen IJ, Berglund A, Nielsen HJ, Brunner N,

Glimelius B. TIMP-1 is significantly associated with objective response and survival in metastatic colorectal cancer patients receiving combination of irinotecan, 5-fluorouracil, and folinic acid. Clin Cancer Res 2007;13(14): 4117-4122.

715. Kunz P, Sähr H, Lehner B, Fischer C, Seebach E, Fellenberg J. Elevated ratio of MMP2/MMP9 activity is associated with poor response to chemotherapy in osteosarcoma. BMC Cancer 2016;16(1): 223. 
716. Tabouret E, Goncalves A, Bertucci F, Pierga J-Y, Petit T, Levy C, Ferrero J-M, Campone M, Gligorov J, Lerebours F, Roche HH, Bachelot TD, Toiron Y, Borg J-P, Chinot OL, Viens P. Effect of high MMP2 and low MMP9 baseline serum levels on outcome in patients with HER2-positive inflammatory breast cancer (IBC) treated with bevacizumab (BEV)- and trastuzumab (TRA)-based neoadjuvant chemotherapy (NAC) in the BEVERLY 2 study. Journal of Clinical Oncology 2015;33(15_suppl): 600-600.

717. Issa JP. CpG island methylator phenotype in cancer. Nat Rev Cancer 2004;4(12): 988993.

718. Bae JM, Kim JH, Kang GH. Molecular Subtypes of Colorectal Cancer and Their Clinicopathologic Features, With an Emphasis on the Serrated Neoplasia Pathway. Archives of pathology \& laboratory medicine 2016;140(5): 406-412.

719. Bae JM, Kim JH, Cho NY, Kim TY, Kang GH. Prognostic implication of the CpG island methylator phenotype in colorectal cancers depends on tumour location. Br J Cancer 2013;109(4): 1004-1012.

720. Draht MXG, Goudkade D, Koch A, Grabsch HI, Weijenberg MP, van Engeland M, Melotte V, Smits KM. Prognostic DNA methylation markers for sporadic colorectal cancer: a systematic review. Clin Epigenetics 2018;10: 35.

721. Kokelaar RF, Jones H, Beynon J, Evans ME, Harris DA. Meta-analysis of the prognostic value of $\mathrm{CPG}$ island methylator phenotype in rectal cancer. International Journal of Colorectal Disease 2018;33(8): 995-1000.

722. Kirkland D, Pfuhler S, Tweats D, Aardema M, Corvi R, Darroudi F, Elhajouji A, Glatt H, Hastwell P, Hayashi M, Kasper P, Kirchner S, Lynch A, Marzin D, Maurici D, Meunier JR, Muller L, Nohynek G, Parry J, Parry E, Thybaud V, Tice R, van Benthem J, Vanparys P, White $P$. How to reduce false positive results when undertaking in vitro genotoxicity testing and thus avoid unnecessary follow-up animal tests: Report of an ECVAM Workshop. Mutation research 2007;628(1): 31-55.

723. Cameron EE, Bachman KE, Myohanen S, Herman JG, Baylin SB. Synergy of demethylation and histone deacetylase inhibition in the re-expression of genes silenced in cancer. Nature genetics 1999;21(1): 103-107.

724. Delaney C, Garg SK, Yung R. Analysis of DNA Methylation by Pyrosequencing. Methods in molecular biology (Clifton, NJ) 2015;1343: 249-264.

725. Petrelli NJ, Letourneau R, Weber T, Nava ME, Rodriguez-Bigas M. Accuracy of biopsy and cytology for the preoperative diagnosis of colorectal adenocarcinoma. J Surg Oncol 1999;71(1): 46-49.

726. Tang JH, An X, Lin X, Gao YH, Liu GC, Kong LH, Pan ZZ, Ding PR. The value of forceps biopsy and core needle biopsy in prediction of pathologic complete remission in locally advanced rectal cancer treated with neoadjuvant chemoradiotherapy. Oncotarget 2015;6(32): 33919-33925. 


\section{Appendices}

\section{Index:}

I. Primer Sequences \& Reaction Conditions $\quad \ldots \ldots \ldots \ldots \ldots \ldots \ldots \ldots \ldots . \ldots 272$

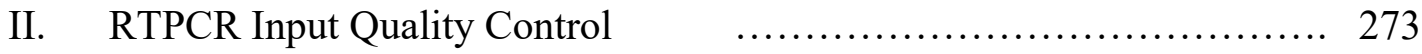

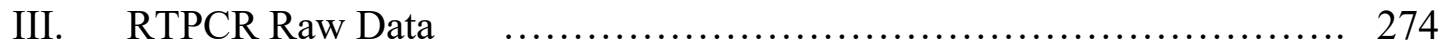

IV. Proteomics Raw Data ............................................. 279

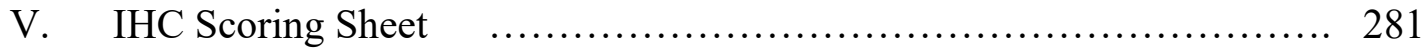

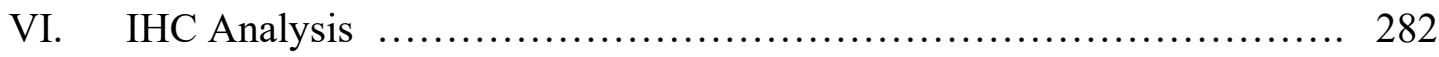

a. Frequency histograms for Mx \& Cat scores for each MMP \& TIMP

b. Regression for EMVI, Cat scores, and MMP2 \& TIMP2

c. Cox DFS \& OS assessed on EMVI and Cat Scores

d. Pearson's Correlations EMVI, CIMP, MMP2

VII. Meta-Analysis

Search Strategy, Consort Diagram, \& Characteristics of Included Studies 


\section{msPCR Primer Sequences \& Reaction Conditions}

\begin{tabular}{|c|c|c|c|c|c|c|}
\hline $\begin{array}{l}\text { Primer } \\
\text { Name }\end{array}$ & $\begin{array}{l}\text { Forward Primer Sequence (5'- } \\
\text { 3') }\end{array}$ & Reverse Primer Sequence $\left(5^{\prime}-3^{\prime}\right)$ & $\begin{array}{l}\text { Anneali } \\
\text { ng } \\
\text { Tempera } \\
\text { ture }\left({ }^{\circ} \mathrm{C}\right)\end{array}$ & $\begin{array}{l}\text { Anneal } \\
\text { ing } \\
\text { Time } \\
\text { (Secs) } \\
\end{array}$ & $\begin{array}{l}\text { Cycl } \\
\mathrm{e} \\
\text { num } \\
\text { ber } \\
\end{array}$ & $\begin{array}{l}\text { Product } \\
\text { Length } \\
\text { (bp) }\end{array}$ \\
\hline hMLH U & $\begin{array}{l}\text { AGAGTGGARAGTGATTTT } \\
\text { TAATGT }\end{array}$ & ACTCTATAAATTACTAAATCTCTTCA & 52.5 & 35 & 37 & 93 \\
\hline hMLH M & GATAGCGATTTTTAACGC & TCTATAAATTACTAAATCTCTTCG & 52.5 & 35 & 37 & $\approx 100$ \\
\hline MINT1 U & $\begin{array}{l}\text { AATTTTTTTATATATATTT } \\
\text { TTGAAGTGT }\end{array}$ & AACAAAAAACCTCAACCCCACA & 55 & 35 & 37 & $\approx 100$ \\
\hline MINT1 M & $\begin{array}{l}\text { AATTTTTTTATATATATTT } \\
\text { TCGAAGC }\end{array}$ & AAAAACCTCAACCCCGCG & 55 & 35 & 37 & 100 \\
\hline SOCS1 U & $\begin{array}{l}\text { TTTTTTGGTGTTGTTTGGA } \\
\text { GGTTGGATTTT }\end{array}$ & $\begin{array}{l}\text { AAAACAAAACAATAAACTAAAACA } \\
\text { CTACAAAACCA }\end{array}$ & 50 & 35 & 37 & 243 \\
\hline SOCS1 M & $\begin{array}{l}\text { TTGTTCGGAGGTCGGATT } \\
\mathrm{T}\end{array}$ & ACTAAAACGCTACGAAACCG & 50 & 35 & 37 & 218 \\
\hline HAND1 U & $\begin{array}{l}\text { AATAGTTTAGGGTGTTGG } \\
\text { TT }\end{array}$ & AAATTTTACACTCAACCCA & 55 & 30 & 35 & 184 \\
\hline HAND1 M & $\begin{array}{l}\text { AATAGTTTAGGGCGTTGG } \\
\text { TC }\end{array}$ & AATTTTACGCTCAACCCG & 53 & 30 & 35 & 184 \\
\hline $\begin{array}{l}\text { ADAMTS1 } \\
\mathrm{U}\end{array}$ & $\begin{array}{l}\text { GTGAGTAATATTGTAGTT } \\
\text { AAGGTGG }\end{array}$ & AAAACAAAAAACACTCTAAAACACC & 58 & 30 & 35 & 101 \\
\hline $\begin{array}{l}\text { ADAMTS1 } \\
\text { M }\end{array}$ & $\begin{array}{l}\text { GTGAGTAATATCGTAGTT } \\
\text { AAGGCGG }\end{array}$ & CTAAAACAAAAAACGCTCTAAAACG & 61 & 30 & 35 & 103 \\
\hline $\begin{array}{l}\text { NEUROG1 } \\
\text { U }\end{array}$ & $\begin{array}{l}\text { TTGTTGGTTAATTGGTGG } \\
\text { TGTTGT }\end{array}$ & CATTACCTCAACCACTAATCACCCA & 59 & 30 & 35 & 119 \\
\hline $\begin{array}{l}\text { NEUROG1 } \\
\mathrm{M}\end{array}$ & $\begin{array}{l}\text { AATTTATGTTCGCGGGAG } \\
\text { GTC }\end{array}$ & ACCAACTTAACCCGAACCGA & 59 & 30 & 35 & 118 \\
\hline IGFBP3 U & $\begin{array}{l}\text { TTATTTTGGTTTTTATATA } \\
\text { GTGGTT }\end{array}$ & AACAAAAAACAACTAATCCTCAACA & 51 & 35 & 35 & $\approx 100$ \\
\hline IGFBP3 M & $\begin{array}{l}\text { TTTCGGTTTTTATATAGCG } \\
\text { GTC }\end{array}$ & AAAAAACGACTAATCCTCAACG & 54 & 35 & 35 & $\approx 100$ \\
\hline THBD U & $\begin{array}{l}\text { ATGTGTTTGTTTTTATTTG } \\
\text { GTGTT }\end{array}$ & CATAACTAACCAAAAACCCACA & 57 & 30 & 35 & 158 \\
\hline THBD M & $\begin{array}{l}\text { CGTTCGTTTTTATTCGGCG } \\
\text { TC }\end{array}$ & GCCAAACCCCATCTCATCG & 58 & 30 & 35 & 118 \\
\hline
\end{tabular}




\section{RTPCR Input Quality Control}

\section{NanoDrop RNA assessment}

\begin{tabular}{|c|c|c|c|c|c|c|}
\hline RNA-40 & 260 & 280 & $260 / 280$ & & & \\
\hline \multicolumn{7}{|c|}{ Blanks OK } \\
\hline Control & 7.468 & 3.503 & & 2.13 & 1.50 & 298.7 \\
\hline $\mathrm{vC} 1$ & 8.168 & 3.849 & & 2.12 & 0.98 & 326.7 \\
\hline $\mathrm{vC} 2$ & 6.736 & 3.171 & & 2.12 & 0.68 & 269.4 \\
\hline $\mathrm{vC} 3$ & 6.989 & 3.275 & & 2.13 & 0.98 & 279.6 \\
\hline $1 \mathrm{dA} 1$ & 7.599 & 3.537 & & 2.15 & 0.62 & 303.9 \\
\hline $1 \mathrm{dA} 2$ & 6.604 & 3.107 & & 2.13 & 1.16 & 264.2 \\
\hline $1 \mathrm{dA} 3$ & 6.953 & 3.229 & & 2.15 & 1.84 & 278.1 \\
\hline $3 \mathrm{dA} 1$ & 3.326 & 1.529 & & 2.18 & 0.29 & 133.0 \\
\hline $3 \mathrm{dA} 2$ & 2.930 & 1.382 & & 2.12 & 0.23 & 117.2 \\
\hline $3 \mathrm{dA} 3$ & 3.273 & 1.538 & & 2.13 & 1.88 & 130.9 \\
\hline $3 \mathrm{dR} 1$ & 6.081 & 2.844 & & 2.14 & 0.91 & 243.3 \\
\hline $3 \mathrm{dR} 2$ & 6.304 & 2.955 & & 2.13 & 1.34 & 252.2 \\
\hline $3 \mathrm{dR} 3$ & 5.864 & 2.727 & & 2.15 & 1.19 & 234.6 \\
\hline
\end{tabular}

NanoDrop cDNA quantification \& normalisation

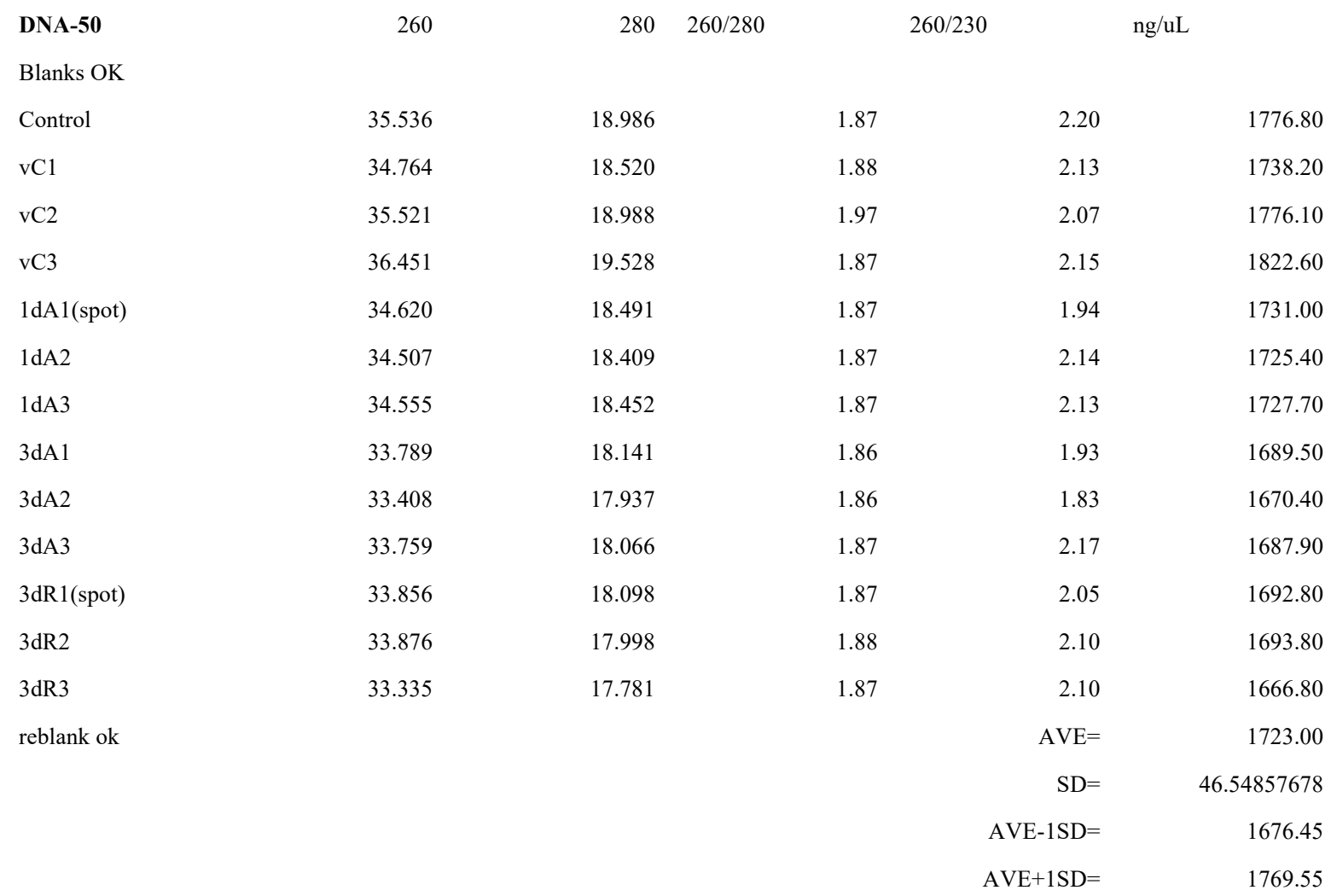


MMP2

\begin{tabular}{|c|c|c|c|c|}
\hline & MMP2 & $\mathrm{bACT}$ & Realtive to HK & Relative to Control \\
\hline vCont & $\begin{array}{r}0.0000043306969 \\
642637\end{array}$ & $\begin{array}{r}16290909.1402 \\
8050\end{array}$ & $\begin{array}{r}0.00000000000026 \\
6\end{array}$ & 1.0000 \\
\hline 1D AZA & $\begin{array}{r}0.0000030538663 \\
755296\end{array}$ & $\begin{array}{r}11967462.3485 \\
5990\end{array}$ & $\begin{array}{r}0.00000000000025 \\
5\end{array}$ & 0.9599 \\
\hline 3D AZA & $\begin{array}{r}0.0000000844196 \\
074400\end{array}$ & $\begin{array}{r}1455086.64121 \\
182\end{array}$ & $\begin{array}{r}0.00000000000005 \\
8\end{array}$ & 0.2182 \\
\hline 3D RRX & $\begin{array}{r}0.0000063927427 \\
907786\end{array}$ & $\begin{array}{r}5645616.89086 \\
577\end{array}$ & $\begin{array}{r}0.00000000000113 \\
2\end{array}$ & 4.2595 \\
\hline
\end{tabular}

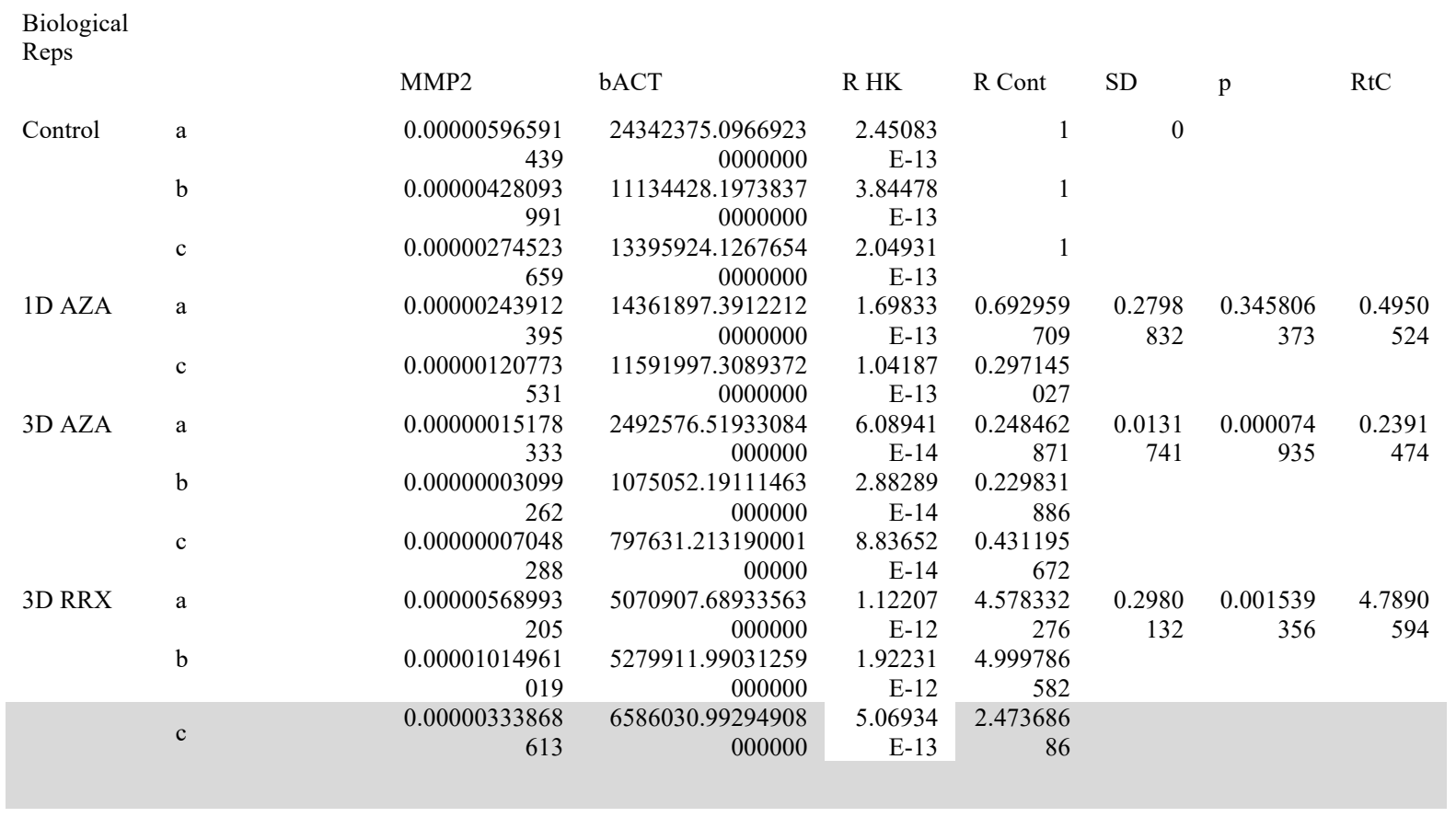

Relative Fold- SD Change

Control

24hrs $0.50 \mathrm{uM}$

AZA

72hrs 0.50uM

AZA

$72 \mathrm{hrs} 0.50 \mathrm{uM}$

RRx-001

1
0.495052368
0.239147378
4.789059429

0.00000000000

02782

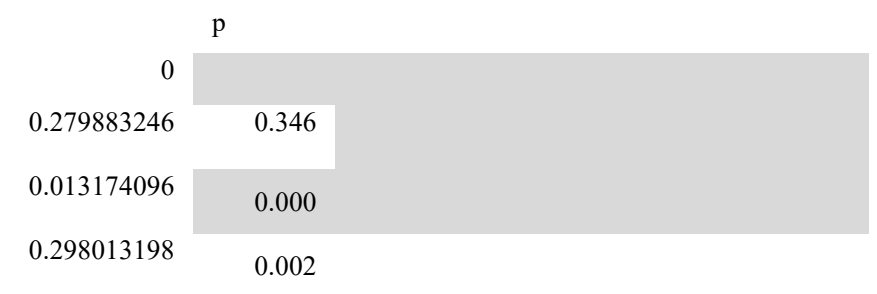


MMP11

$\begin{array}{lrrrr} & \text { MMP11 } & \text { bACT } & \text { Relative to HK } & \begin{array}{l}\text { Relative to } \\ \text { control }\end{array} \\ \text { Contr } & 0.0106527275 & 295.654631644 & 0.000036030984 & 1.000 \\ \text { ol } & & 5060 & 8 & \\ \text { 1D } & 0.0182501810 & 251.909384483 & 0.000072447404 & 011 \\ \text { AZA } & & 8590 & 0 & 3.016 \\ \text { 3D } & 0.0079434563 & 73.1002334957 & 0.000108665265 & 1.429 \\ \text { AZA } & & 253 & 9 & \\ \text { 3D } & 0.0091971004 & 178.681916015 & 0.000051471915 & \\ \text { RRX } & & 5820 & & \\ & & & & \end{array}$

Biological Reps

\begin{tabular}{|c|c|c|c|c|c|c|c|c|}
\hline & & MMP9 & $\mathrm{bACT}$ & R HK & R Cont & SD & $\mathrm{p}$ & $\mathrm{RtC}$ \\
\hline \multirow{6}{*}{$\begin{array}{l}\text { Contr } \\
\text { ol }\end{array}$} & $\mathrm{a}$ & 0.01524224035 & 358.2947105577 & $4.25411 \mathrm{E}-05$ & 1 & 0 & & 1 \\
\hline & & 251 & 9300 & & & & & \\
\hline & $\mathrm{b}$ & 0.00926991186 & 310.8859554725 & $2.98177 \mathrm{E}-05$ & 1 & & & \\
\hline & & 852 & 9600 & & & & & \\
\hline & $\mathrm{c}$ & 0.00744603041 & 217.7832289031 & $3.41901 \mathrm{E}-05$ & 1 & & & \\
\hline & & 909 & 2800 & & & & & \\
\hline \multirow{6}{*}{$\begin{array}{l}\text { 1D } \\
\text { AZA }\end{array}$} & $\mathrm{a}$ & 0.01588036996 & 298.4622001558 & 5.32073E-05 & 1.250727 & 1.091597 & 0.05893 & 2.25217 \\
\hline & & 453 & 2800 & & 996 & 06 & 75 & 7 \\
\hline & $\mathrm{b}$ & 0.02075984569 & 203.8260168419 & 0.000101851 & 3.415780 & & & \\
\hline & & 560 & 8800 & & 864 & & & \\
\hline & $\mathrm{c}$ & 0.01811032719 & 253.4399364537 & $7.14581 \mathrm{E}-05$ & 2.090022 & & & \\
\hline & & 764 & 6000 & & 07 & & & \\
\hline \multirow{6}{*}{$\begin{array}{l}\text { 3D } \\
\mathrm{AZA}\end{array}$} & $\mathrm{a}$ & 0.00967191705 & 101.8263416066 & 9.49844E-05 & 2.232770 & 1.021140 & 0.00928 & 3.26001 \\
\hline & & 643 & 8300 & & 131 & 969 & 18 & 97 \\
\hline & $\mathrm{b}$ & 0.00604818754 & 61.98565656622 & $9.7574 \mathrm{E}-05$ & 3.272348 & & & \\
\hline & & 562 & 050 & & 442 & & & \\
\hline & $\mathrm{c}$ & 0.00811026433 & 55.48870231427 & 0.000146161 & 4.274940 & & & \\
\hline & & 037 & 250 & & 427 & & & \\
\hline \multirow{6}{*}{$\begin{array}{l}\text { 3D } \\
\text { RRX }\end{array}$} & $\mathrm{a}$ & 0.01131417954 & 206.0509917530 & $5.49096 \mathrm{E}-05$ & 1.290743 & 0.316234 & 0.03051 & 1.47186 \\
\hline & & 840 & 1300 & & 505 & 88 & 93 & 88 \\
\hline & $\mathrm{b}$ & 0.00890627501 & 162.5950639816 & $5.47758 \mathrm{E}-05$ & 1.837021 & & & \\
\hline & & 853 & 9700 & & 512 & & & \\
\hline & & 0.00737084664 & 167.3996923120 & 4.40314E-05 & 1.287841 & & & \\
\hline & $\mathrm{c}$ & 046 & 3600 & & 293 & & & \\
\hline
\end{tabular}

0.00003551629

RtC SD

$\begin{array}{lrr}\text { Control } & 1 & 0 \\ \text { 24hrs 0.50uM } & 2.252176977 & 1.09159706 \\ \text { AZA } & & \\ \text { 72hrs 0.50uM } & 3.260019667 & 1.021140969 \\ \text { AZA } & & \\ \text { 72hrs 0.50uM } & 1.47186877 & 0.31623488 \\ \text { RRx-001 } & & \end{array}$


MMP13

\begin{tabular}{|c|c|c|c|c|}
\hline & MMP13 & $\mathrm{bACT}$ & $\mathrm{R}$ to $\mathrm{HK}$ & $\begin{array}{l}\mathrm{R} \text { to } \\
\text { Control }\end{array}$ \\
\hline \multirow{2}{*}{ Control } & 0.0134142290925 & 33.4046747916767 & 0.0004015674206 & \multirow{2}{*}{1.00} \\
\hline & 012000000 & 000000000 & 127450000 & \\
\hline \multirow{2}{*}{ 1D AZA } & 0.0064268553975 & 27.7857306427657 & 0.0002313005722 & \multirow{2}{*}{0.58} \\
\hline & 820300000 & 000000000 & 329400000 & \\
\hline \multirow{2}{*}{ 3D AZA } & 0.0047488056649 & 15.1938504831490 & 0.0003125478739 & \multirow{2}{*}{0.78} \\
\hline & 093300000 & 000000000 & 030670000 & \\
\hline \multirow{2}{*}{ 3D RRX } & 0.0109125832195 & 25.5770542941965 & 0.0004266552001 & \multirow{2}{*}{1.06} \\
\hline & 946000000 & 000000000 & 678590000 & \\
\hline
\end{tabular}

Biological Reps

\begin{tabular}{|c|c|c|c|c|c|c|c|c|}
\hline & & MMP13 & $\mathrm{bACT}$ & R HK & R Cont & SD & & $\mathrm{RtC}$ \\
\hline \multirow[t]{6}{*}{ Control } & $\mathrm{a}$ & 0.01781333265517 & 38.285717183925 & 0.00046 & 1 & 0 & & 1 \\
\hline & & & 40 & 5274 & & & & \\
\hline & $\mathrm{b}$ & 0.01052692850921 & 30.708864164429 & 0.00034 & 1 & & & \\
\hline & & & 70 & 2798 & & & & \\
\hline & $\mathrm{c}$ & 0.01190242611312 & 31.219443026674 & 0.00038 & 1 & & & \\
\hline & & & 90 & 125 & & & & \\
\hline \multirow{6}{*}{$\begin{array}{l}24 \mathrm{hrs} 0.50 \mathrm{uM} \\
\text { AZA }\end{array}$} & $\mathrm{a}$ & 0.00633897503081 & 32.264378668951 & 0.00019 & 0.42226 & 0.03299 & 0.024 & 0.701 \\
\hline & & & 40 & 647 & 7195 & 6996 & 1661 & 3985 \\
\hline & $\mathrm{b}$ & 0.00657188287138 & 26.453012553003 & 0.00024 & 0.72473 & & & \\
\hline & & & 20 & 8436 & 0863 & & & \\
\hline & $\mathrm{c}$ & 0.00636970829055 & 24.639800706342 & 0.00025 & 0.67806 & & & \\
\hline & & & 30 & 8513 & 6064 & & & \\
\hline \multirow{6}{*}{$\begin{array}{l}72 \mathrm{hrs} 0.50 \mathrm{uM} \\
\text { AZA }\end{array}$} & $\mathrm{a}$ & 0.00545618696941 & 17.011899287845 & 0.00032 & 0.68933 & 0.11178 & 0.306 & 0.768 \\
\hline & & & 30 & 0728 & 1381 & 8527 & 5912 & 3778 \\
\hline & $\mathrm{b}$ & 0.00443191229175 & 15.079785196726 & 0.00029 & 0.00034 & & & \\
\hline & & & 70 & 3898 & 2798 & & & \\
\hline & $\mathrm{c}$ & 0.00435831773357 & 13.489866964875 & 0.00032 & 0.84742 & & & \\
\hline & & & 10 & 3081 & 4231 & & & \\
\hline \multirow{6}{*}{$\begin{array}{l}\text { 72hrs } 0.50 \mathrm{uM} \\
\text { RRx-001 }\end{array}$} & $\mathrm{a}$ & 0.01179629192676 & 24.873852086158 & 0.00047 & 1.01928 & 0.42179 & 0.125 & 1.317 \\
\hline & & & 40 & 4245 & 1338 & 519 & 3035 & 5356 \\
\hline & $\mathrm{b}$ & 0.01432146426390 & 25.856196341203 & 0.00055 & 1.61578 & & & \\
\hline & & & 90 & 3889 & 9816 & & & \\
\hline & c & 0.00661999346813 & 26.001114455227 & 0.00025 & 0.66781 & & & \\
\hline & $c$ & & 30 & 4604 & 3593 & & & \\
\hline
\end{tabular}

RtC

Control

24hrs $0.50 \mathrm{uM}$

AZA

72hrs $0.50 \mathrm{uM}$

AZA

72hrs $0.50 \mathrm{uM}$

RRx-001
0.0003964405754

SD

\section{9}

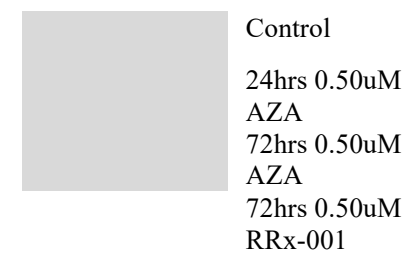

1

0.701398464

0.768377806

1.317535577

$$
0
$$

0.032996996

0.111788527

0.42179519 
TIMP2

\begin{tabular}{llrrr} 
& TIMP2 & bACT & Relative to HK & Relative to Control \\
\hline Contro & 0.2411600769635 & 3.82013046750 & 0.063128754113 & 1.0000 \\
1 & & 78 & 2 & \\
1D & 0.2678045305019 & 3.84917626301 & 0.069574504310 & 1.1021 \\
AZA & & 56 & 2 & \\
3D & 0.0395042563544 & 1.75781634072 & 0.022473483400 & 0.3560 \\
AZA & 7.06038923031 & 0.075088499253 & 1.1895 \\
3D & 0.2298000344371 & 82 & 8 & \\
RRX & & & & \\
& & & &
\end{tabular}

Biological Reps

\begin{tabular}{|c|c|c|c|c|c|c|c|c|}
\hline & & TIMP2 & bACT & R HK & R Cont & SD & $\mathrm{p}$ & $\mathrm{RtC}$ \\
\hline Contro & $\mathrm{a}$ & 0.33204094096 & 4.722750646712 & 0.0703066 & 1 & 0 & & 1 \\
\hline \multirow[t]{5}{*}{1} & & 041 & 54 & 85 & & & & \\
\hline & $\mathrm{b}$ & 0.21108259988 & 3.635877572788 & 0.0580554 & 1 & & & \\
\hline & & 091 & 01 & 75 & & & & \\
\hline & $\mathrm{c}$ & 0.18035669004 & 3.101763183022 & 0.0581465 & 1 & & & \\
\hline & & 905 & 70 & 06 & & & & \\
\hline \multirow{6}{*}{$\begin{array}{l}\text { 1D } \\
\text { AZA }\end{array}$} & $\mathrm{a}$ & 0.29722828860 & 4.660676028794 & 0.0637736 & 0.0703066 & 0.69868 & 0.3828016 & 0.87127 \\
\hline & & 285 & 05 & 43 & 85 & 45 & 75 & 02 \\
\hline & $\mathrm{b}$ & 0.25650767421 & 3.718913322745 & 0.0689738 & 1.1880675 & & & \\
\hline & & 782 & 07 & 24 & 2 & & & \\
\hline & c & 0.24967762868 & 3.167939437507 & 0.0788138 & 1.3554363 & & & \\
\hline & & 495 & 78 & 89 & 89 & & & \\
\hline \multirow{6}{*}{$\begin{array}{l}3 \mathrm{D} \\
\mathrm{AZA}\end{array}$} & $\mathrm{a}$ & 0.04354987832 & 2.027726609101 & 0.0214771 & 0.3054786 & 0.06038 & 0.0000272 & 0.36781 \\
\hline & & 252 & 60 & 94 & 94 & 65 & 0 & 48 \\
\hline & $\mathrm{b}$ & 0.03694993526 & 1.711261840933 & 0.0215922 & 0.3719238 & & & \\
\hline & & 837 & 22 & 16 & 53 & & & \\
\hline & c & 0.03801295547 & 1.534460572147 & 0.0247728 & 0.4260418 & & & \\
\hline & & 243 & 06 & 46 & 71 & & & \\
\hline \multirow{6}{*}{$\begin{array}{l}\text { 3D } \\
\text { RRX }\end{array}$} & $\mathrm{a}$ & 0.23524654348 & 3.085687670167 & 0.0762379 & 1.0843629 & 0.31413 & 0.1394538 & 1.22699 \\
\hline & & 059 & 91 & 63 & 39 & 03 & 56 & 77 \\
\hline & $\mathrm{b}$ & 0.26548180295 & 2.881220995270 & 0.0921421 & 1.5871391 & & & \\
\hline & & 319 & 59 & 17 & 49 & & & \\
\hline & & 0.18867175687 & 3.214259025516 & 0.0586983 & 1.0094908 & & & \\
\hline & $\mathrm{c}$ & 737 & 07 & 67 & 85 & & & \\
\hline
\end{tabular}

RtC

0.062169555129

SD

Control

24hrs 0.50uM

AZA

$72 \mathrm{hrs} 0.50 \mathrm{uM}$

AZA

$72 \mathrm{hrs} 0.50 \mathrm{uM}$

RRx-001

$\begin{array}{rr}1 & 0 \\ 0.871270198 & 0.698684476 \\ 0.367814806 & 0.060386531 \\ 1.226997657 & 0.314130349\end{array}$


TIMP4

\begin{tabular}{|c|c|c|c|c|}
\hline & $\begin{array}{l}\text { TIMP } \\
4\end{array}$ & $\mathrm{bACT}$ & $\begin{array}{l}\text { Relative to } \\
\text { HK }\end{array}$ & $\begin{array}{l}\text { Relative to } \\
\text { Control }\end{array}$ \\
\hline Control & $\begin{array}{r}0.0011 \\
3\end{array}$ & 9573.92110 & $1.17886 \mathrm{E}-07$ & 1 \\
\hline 1D & 0.0014 & 6261.27349 & $2.35669 \mathrm{E}-07$ & 1.999128973 \\
\hline AZA & 8 & & & \\
\hline 3D & 0.0004 & 1124.13516 & $3.92426 \mathrm{E}-07$ & 3.328864832 \\
\hline AZA & 4 & & & \\
\hline 3D & 0.0006 & 3806.41091 & $1.6823 \mathrm{E}-07$ & 1.427054421 \\
\hline
\end{tabular}

Biological Reps

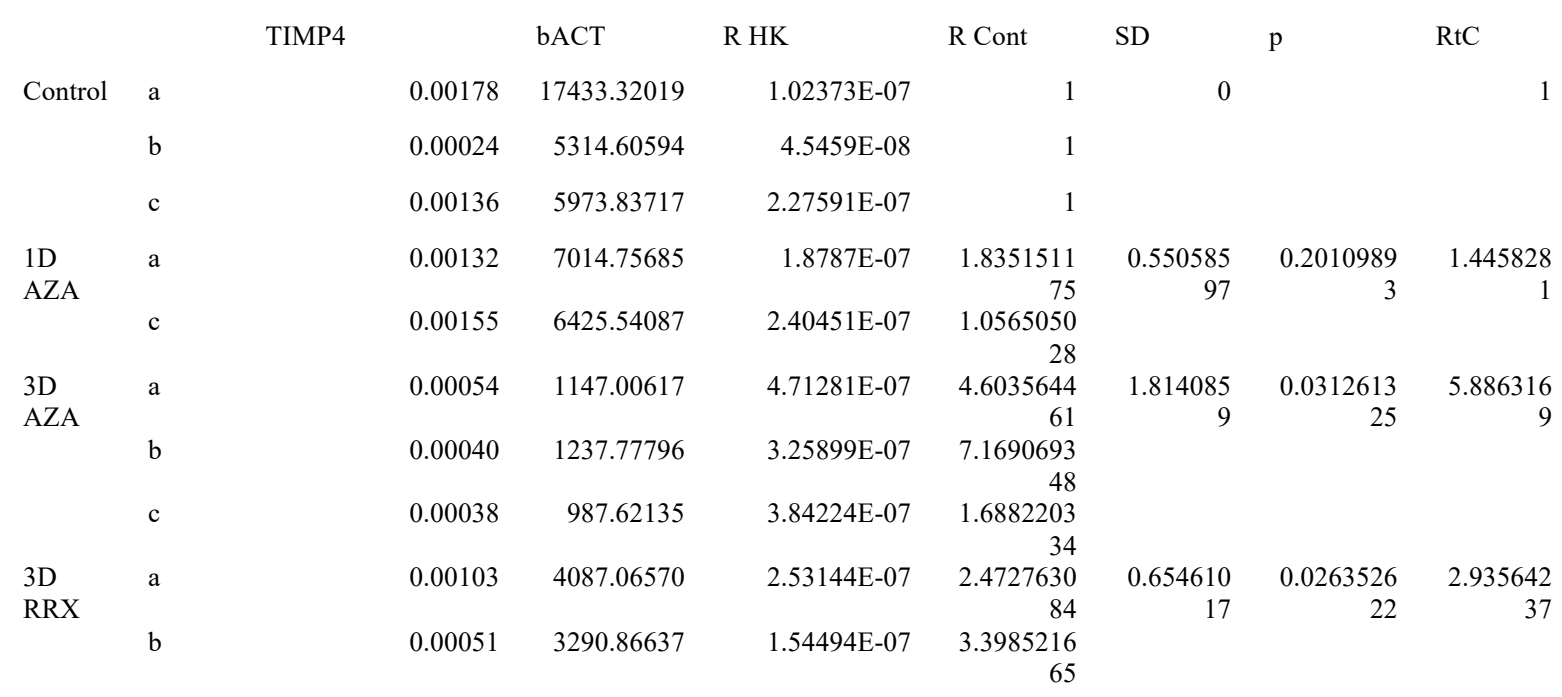

RtC $\quad$ SD

$\begin{array}{lrr}\text { Control } & 1 & 0 \\ \text { 24hrs 0.50uM AZA } & 1.445828101 & 0.550585971 \\ \text { 72hrs 0.50uM AZA } & 5.886316904 & 1.814085903 \\ \text { 72hrs 0.50uM RRx- } & 2.935642374 & 0.65461017\end{array}$

1.25141E-

07 


\section{Proteomics Raw Data}

\section{Western Blot (Normalised) Data}

\begin{tabular}{|c|c|c|}
\hline Prot Fold Change & MMP2 & TIMP2 \\
\hline Control 1 & 1 & 1 \\
\hline Control 2 & 1 & 1 \\
\hline Control 3 & 1 & 1 \\
\hline AZA 1 & 0.26 & 0.29 \\
\hline AZA 2 & 0.47 & 0.39 \\
\hline AZA 3 & 0.32 & 0.4 \\
\hline KD T2 1 & 0.94 & 0.02 \\
\hline KD T2 2 & 1.1 & 0.05 \\
\hline KD T2 3 & 1.04 & 0.12 \\
\hline KD T4 1 & 1.07 & 0.92 \\
\hline KD T4 2 & 0.86 & 0.99 \\
\hline KD T4 3 & 1.09 & 1.06 \\
\hline KD T2\&4 1 & 1.12 & 0.05 \\
\hline KD T2\&4 2 & 0.89 & 0.1 \\
\hline KD T2\&4 3 & 0.97 & 0.08 \\
\hline $\mathrm{KD}$ T2 + AZA 1 & 0.32 & 0.1 \\
\hline $\mathrm{KD} \mathrm{T} 2+\mathrm{AZA} 2$ & 0.27 & 0.02 \\
\hline $\mathrm{KD}$ T $2+\mathrm{AZA} 3$ & 0.24 & 0.06 \\
\hline KD T4 + AZA 1 & 0.33 & 0.24 \\
\hline $\mathrm{KD}$ T4 + AZA 2 & 0.41 & 0.33 \\
\hline $\mathrm{KD}$ T4 + AZA 3 & 0.29 & 0.4 \\
\hline $\mathrm{KD}$ T2\&4 + AZA 1 & 0.41 & 0.03 \\
\hline $\mathrm{KD}$ T $2 \& 4+\mathrm{AZA} 2$ & 0.37 & 0.05 \\
\hline KD T2\&4+AZA 3 & 0.26 & 0.12 \\
\hline
\end{tabular}

TIMP4

$\mathrm{x}$

$\mathrm{X}$

$\mathrm{x}$

$05 x$

$2 \mathrm{x}$

$\mathrm{X}$

$05 \mathrm{x}$

$1 \mathrm{x}$

$08 \mathrm{x}$

$1 \quad \mathrm{x}$

$02 \mathrm{x}$

$06 x$

$\mathrm{x}$

$4 \mathrm{x}$

$03 x$

$.12 \mathrm{x}$

\begin{tabular}{|c|c|c|c|}
\hline & MMP2 & TIMP2 & TIMP4 \\
\hline Control & 1 & 1 & $\mathrm{x}$ \\
\hline $\mathrm{AZA}$ & 0.35 & 0.36 & $\mathrm{x}$ \\
\hline KD T2 & 1.026666667 & 0.063333333 & $\mathrm{x}$ \\
\hline KD T4 & 1.006666667 & 0.99 & $\mathrm{x}$ \\
\hline KD T2\&4 & 0.993333333 & 0.076666667 & $\mathrm{x}$ \\
\hline $\mathrm{KD} \mathrm{T} 2+\mathrm{AZA}$ & 0.276666667 & 0.06 & $\mathrm{x}$ \\
\hline $\mathrm{KD} \mathrm{T} 4+\mathrm{AZA}$ & 0.343333333 & 0.323333333 & $\mathrm{x}$ \\
\hline $\mathrm{KD}$ T2\&4 + AZA & 0.3466666667 & 0.066666667 & $\mathrm{x}$ \\
\hline & MMP2 & TIMP2 & \\
\hline Control SD & 0 & 0 & \\
\hline AZA SD & 0.108166538 & 0.060827625 & \\
\hline KD T2 SD & 0.080829038 & 0.051316014 & \\
\hline KD T4 SD & 0.127410099 & 0.07 & \\
\hline KD T2\&4 SD & 0.116761866 & 0.025166115 & \\
\hline KD T2 + AZA SD & 0.040414519 & 0.04 & \\
\hline KD T4 + AZA SD & 0.061101009 & 0.080208063 & \\
\hline $\mathrm{KD}$ T2\& $4+$ AZA SD & 0.077674535 & 0.047258156 & \\
\hline & MMP2 & TIMP2 & M vs T SdT \\
\hline Control SdT & - & - & \\
\hline AZA SdT & 0.009 & 0.003 & 0.852 \\
\hline KD T2 SdT & 0.625 & 0.001 & 0.002 \\
\hline KD T4 SdT & 0.936 & 0.828 & 0.856 \\
\hline KD T2\&4 SdT & 0.930 & 0.000 & 0.008 \\
\hline KD T2 + AZA SdT & 0.001 & 0.001 & 0.009 \\
\hline KD T4 + AZA SdT & 0.003 & 0.005 & 0.788 \\
\hline $\mathrm{D} T 2 \& 4+\mathrm{AZA} \mathrm{SdT}$ & 0.005 & 0.001 & 0.060 \\
\hline
\end{tabular}


Immunocytochemistry RapidScore (Normalised) Data

\begin{tabular}{|c|c|c|c|c|c|c|c|c|}
\hline RapidScore & MMP2 & TIMP2 & TIMP4 & & MMP2 & TIMP2 & TIMP4 & \\
\hline Control 1 & 1 & 1 & 1 & Control & 1 & 1 & 1 & \\
\hline Control 2 & 1 & 1 & 1 & AZA & 0.343333333 & 1 & 3.736666667 & \\
\hline Control 3 & 1 & 1 & 1 & $\mathrm{KD}$ T2 & 1.076666667 & 1 & 1.01 & \\
\hline AZA 1 & 0.25 & $\mathrm{x}$ & 4.21 & KD T4 & 0.966666667 & 1 & 1.026666667 & \\
\hline AZA 2 & 0.43 & $\mathrm{x}$ & 3.2 & KD T2\&4 & 1.033333333 & 1 & 1.006666667 & \\
\hline AZA 3 & 0.35 & $\mathrm{x}$ & 3.8 & $\mathrm{KD} \mathrm{T} 2+\mathrm{AZA}$ & 0.326666667 & 1 & 4.096666667 & \\
\hline KD T2 1 & 0.99 & $\mathrm{x}$ & 1.01 & $\mathrm{KD} \mathrm{T} 4+\mathrm{AZA}$ & 0.35 & 1 & 1.003333333 & \\
\hline KD T2 2 & 1.09 & $\mathrm{x}$ & 1.03 & $\mathrm{KD}$ T2\& $4+\mathrm{AZA}$ & 0.346666667 & 1 & 1.036666667 & \\
\hline KD T2 3 & 1.15 & $\mathrm{x}$ & 0.99 & & & & & \\
\hline KD T4 1 & 1.12 & $\mathrm{x}$ & 1.01 & & & & & \\
\hline KD T4 2 & 0.86 & $\mathrm{x}$ & 1.05 & & MMP2 & & TIMP4 & \\
\hline KD T4 3 & 0.92 & $\mathrm{x}$ & 1.02 & Control SD & 0 & 0 & 0 & \\
\hline KD T2\&4 1 & 1.2 & $\mathrm{x}$ & 1.03 & AZA SD & 0.090184995 & 0 & 0.507969815 & \\
\hline $\mathrm{KD} T 2 \& 42$ & 0.91 & $\mathrm{x}$ & 1.01 & KD T2 SD & 0.080829038 & 0 & 0.02 & \\
\hline $\mathrm{KD}$ T2\&4 3 & 0.99 & $\mathrm{x}$ & 0.98 & KD T4 SD & 0.136137186 & 0 & 0.02081666 & \\
\hline $\mathrm{KD} \mathrm{T} 2+\mathrm{AZA} 1$ & 0.32 & $\mathrm{x}$ & 3.67 & KD T2\& 4 SD & 0.149777613 & 0 & 0.025166115 & \\
\hline $\mathrm{KD}$ T2 + AZA 2 & 0.24 & $\mathrm{x}$ & 5.72 & $\mathrm{KD}$ T2 + AZA SD & 0.090184995 & 0 & 1.457612203 & \\
\hline $\mathrm{KD}$ T2 + AZA 3 & 0.42 & $\mathrm{x}$ & 2.9 & KD T4 + AZA SD & 0.111355287 & 0 & 0.02081666 & \\
\hline $\mathrm{KD}$ T4 + AZA 1 & 0.33 & $\mathrm{x}$ & 1.01 & $\mathrm{KD} T 2 \& 4+\mathrm{AZA} \mathrm{SD}$ & 0.077674535 & 0 & 0.045092498 & \\
\hline KD T4 + AZA 2 & 0.47 & $\mathrm{x}$ & 0.98 & & & & & \\
\hline KD T4 + AZA 3 & 0.25 & $\mathrm{x}$ & 1.02 & & MMP2 & & TIMP4 & $\mathrm{M}$ vs $\mathrm{T}$ SdT \\
\hline $\mathrm{KD}$ T $2 \& 4+\mathrm{AZA} 1$ & 0.41 & $\mathrm{x}$ & 1.08 & Control SdT & - & & - & \\
\hline $\mathrm{KD}$ T $2 \& 4+\mathrm{AZA} 2$ & 0.37 & $\mathrm{x}$ & 1.04 & AZA SdT & 0.006 & & 0.011 & 0.010 \\
\hline \multirow[t]{6}{*}{$\mathrm{KD}$ T $2 \& 4+\mathrm{AZA} 3$} & 0.26 & $\mathrm{x}$ & 0.99 & KD T2 SdT & 0.242 & & 0.478 & 0.329 \\
\hline & & & & KD T4 SdT & 0.713 & & 0.157 & 0.569 \\
\hline & & & & $\mathrm{KD}$ T2\&4 SdT & 0.737 & & 0.691 & 0.766 \\
\hline & & & & $\mathrm{KD}$ T2 + AZA SdT & 0.006 & & 0.067 & 0.052 \\
\hline & & & & KD T4 + AZA SdT & 0.010 & & 0.808 & 0.013 \\
\hline & & & & KD T2\&4+ AZA SdT & 0.005 & & 0.294 & 0.001 \\
\hline
\end{tabular}


Immunohistochemistry Scoring Sheet

Scoring system for IHC

(with reference to Fedchenko and Reifenrath, 2014)

\begin{tabular}{|l|l|l|l|}
\hline Proportion of Cells & p-Score & $\begin{array}{l}\text { Intensity of } \\
\text { Staining }\end{array}$ & i-Score \\
\hline None & 0 & None & 0 \\
\hline$<\mathbf{1 0} \%$ & 1 & Weak & 1 \\
\hline $\mathbf{1 0 \%}-\mathbf{5 0 \%}$ & 2 & Intermediate & 2 \\
\hline $\mathbf{5 1 \%} \mathbf{- 9 0 \%}$ & 3 & Strong & 3 \\
\hline $\mathbf{> 9 0 \%}$ & 4 & & \\
\hline & & MAX SCORE & $\mathbf{7}$ \\
\hline
\end{tabular}

$\begin{array}{lllll}\text { MMP2 } & \text { MMP9 } & \text { MMP11 } & \text { TIMP2 } & \text { TIMP4 }\end{array}$

\begin{tabular}{|l|l|l|l|l|l|l|}
\hline $\begin{array}{l}\text { Path } \\
\text { Number }\end{array}$ & p-score & i-score & p-score & i-score & p-score & i-score \\
\hline Rep... & A & & B & & C & \\
\hline SH1015214 & & & & & & \\
\hline MH127050 & & & & & & \\
\hline SH1001950 & & & & & & \\
\hline SH1003461 & & & & & & \\
\hline SH1014485 & & & & & & \\
\hline SH1016041 & & & & & & \\
\hline SH1204044 & & & & & & \\
\hline SH1115961 & & & & & & \\
\hline SH1301209 & & & & & & \\
\hline SH1125646 & & & & & & \\
\hline SH105224 & & & & & & \\
\hline SH1101201 & & & & & & \\
\hline SH1242267 & & & & & & \\
\hline SH1006849 & & & & & & \\
\hline SH1211596 & & & & & & \\
\hline MH1100593 & & & & & & \\
\hline SH1300417 & & & & & & \\
\hline SH1005494 & & & & & & \\
\hline SH1014844 & & & & & & \\
\hline MH1204971 & & & & & & \\
\hline SH1209984 & & & & & & \\
\hline etc... & & & & & \\
\hline etc... & & & & & \\
\hline
\end{tabular}


Frequency histograms demonstrating multiplied metric $(\mathrm{Mx})$ and Categorised staining scores for a. MMP2; b. MMP9; c. TIMP2; and d. TIMP4.

a.
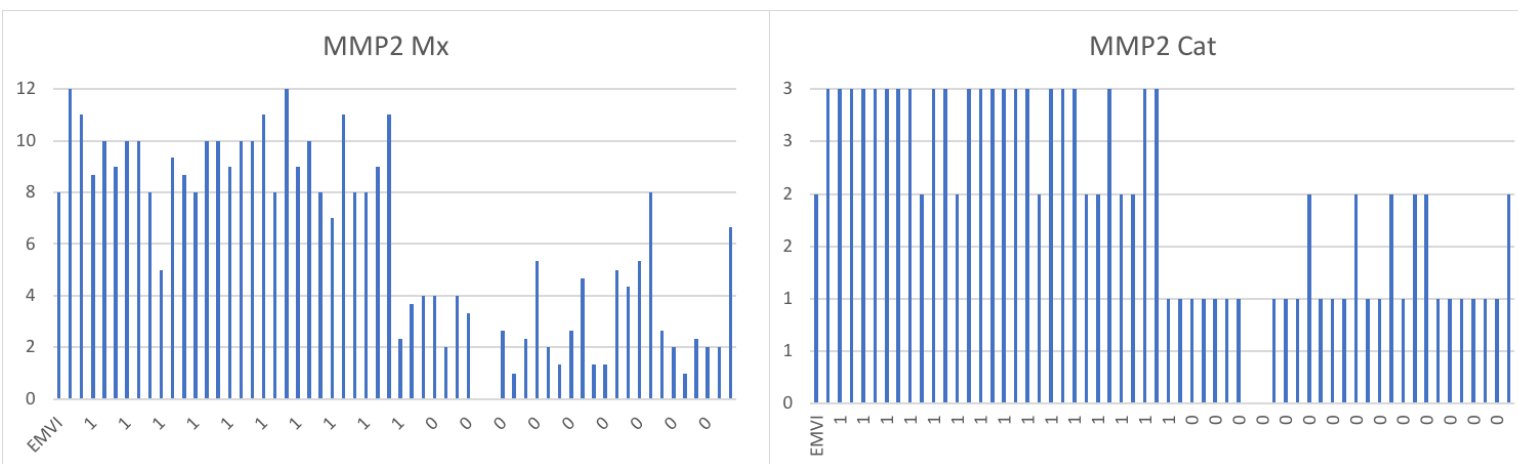

b.
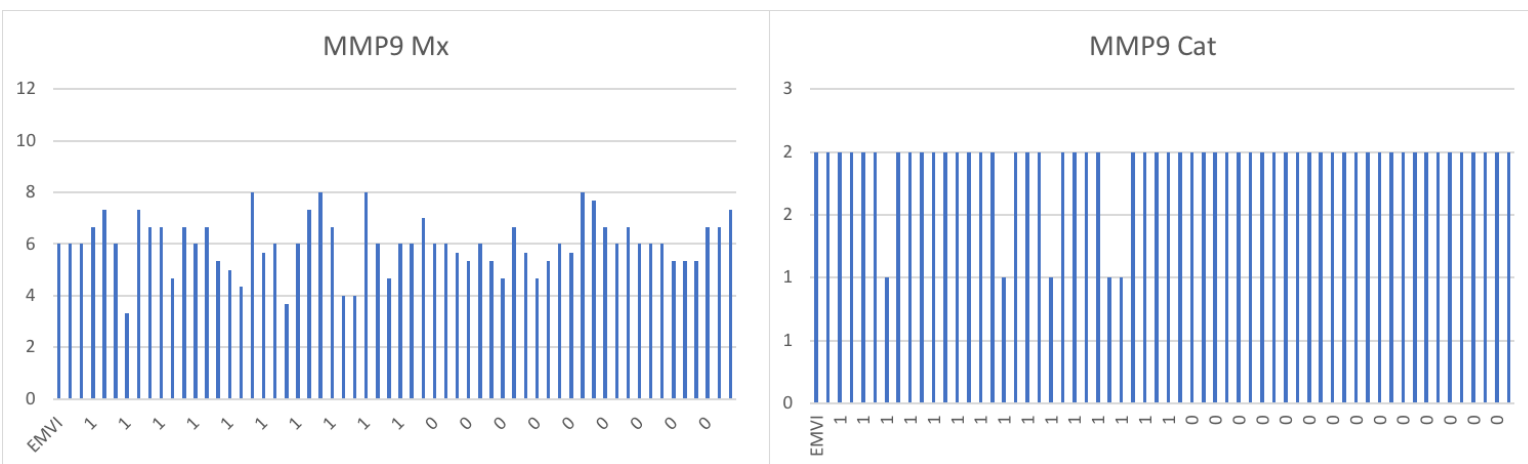

c.
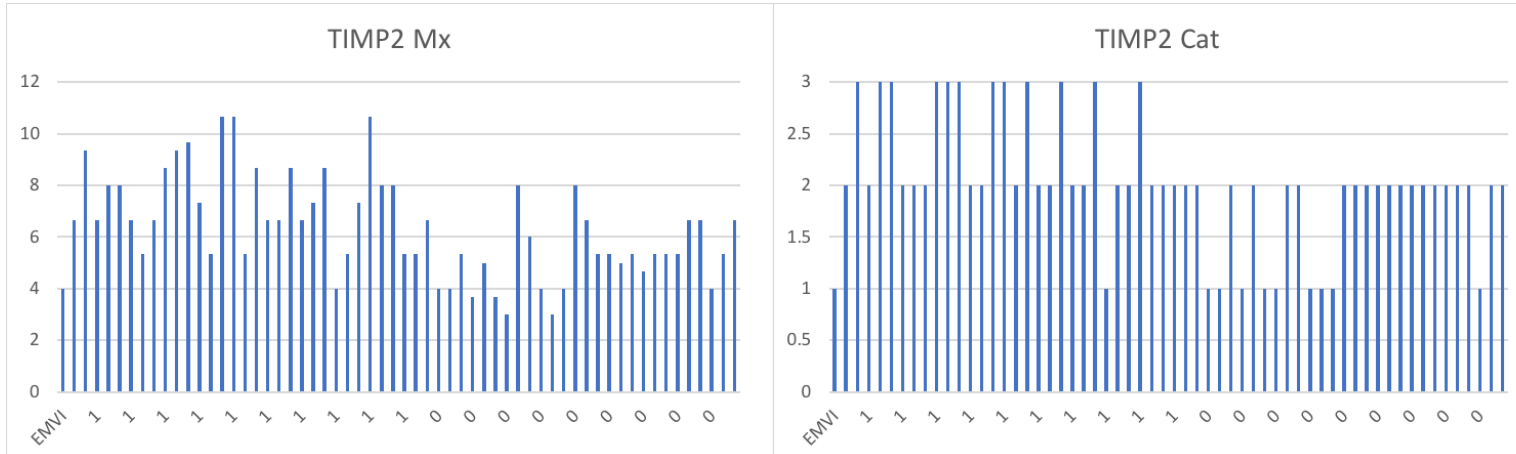

d.
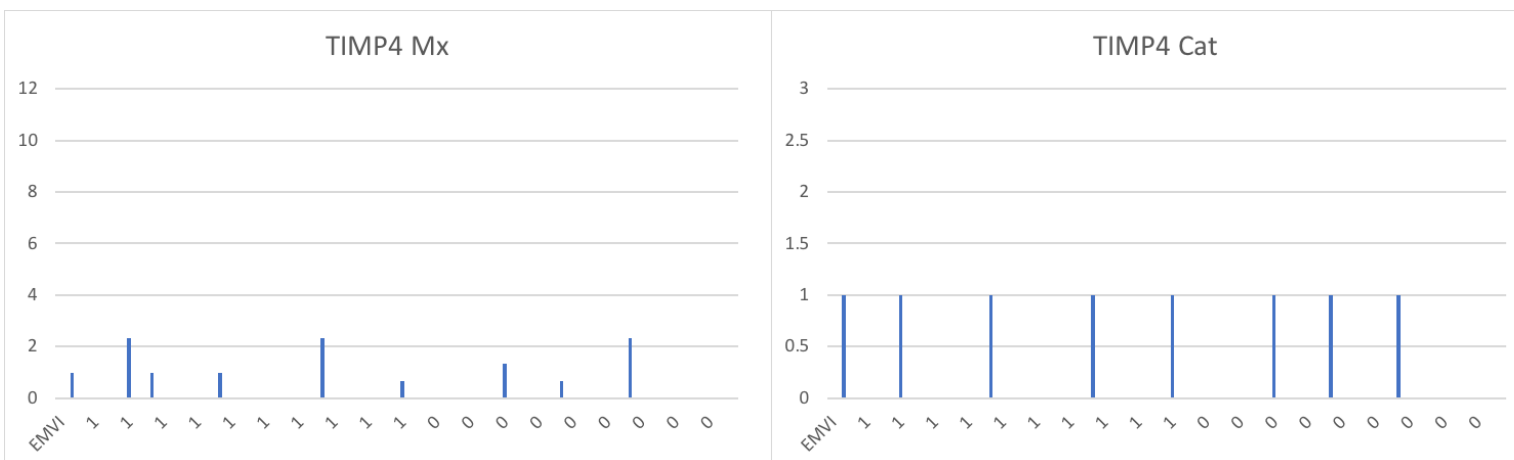
Logistic regression modelling for EMVI in relation to nominal categorised (Cat) scores for $\mathbf{a}$. MMP2 and b. TIMP2

a. MMP2 Summary Statistics \& Test of the Null Hypothesis H0: Y=0.5.

\begin{tabular}{|l|l|l|l|l|l|l|r|r|}
\hline Variable & Categories & Frequencies & Percentage & Statistic & DF & $\boldsymbol{X}^{2}$ & PR $>\boldsymbol{X}^{2}$ \\
\hline EMVI & 1 & 30 & $50 \%$ & -2 Log & 3 & 64.467 & $<0.0001$ \\
\hline & 0 & 30 & $50 \%$ & Score & 3 & 46.286 & $<0.0001$ \\
\hline Cat Score & 0 & & & Wald & 3 & 14.477 & 0.002 \\
\hline & 1 & 2 & $3.3 \%$ & & & & & \\
\hline & 2 & 14 & $36.7 \%$ & & & & & \\
\hline & 3 & 22 & $23 \%$ & & & & & \\
\hline
\end{tabular}

Standardised Coefficient Model \& ROC Curve.
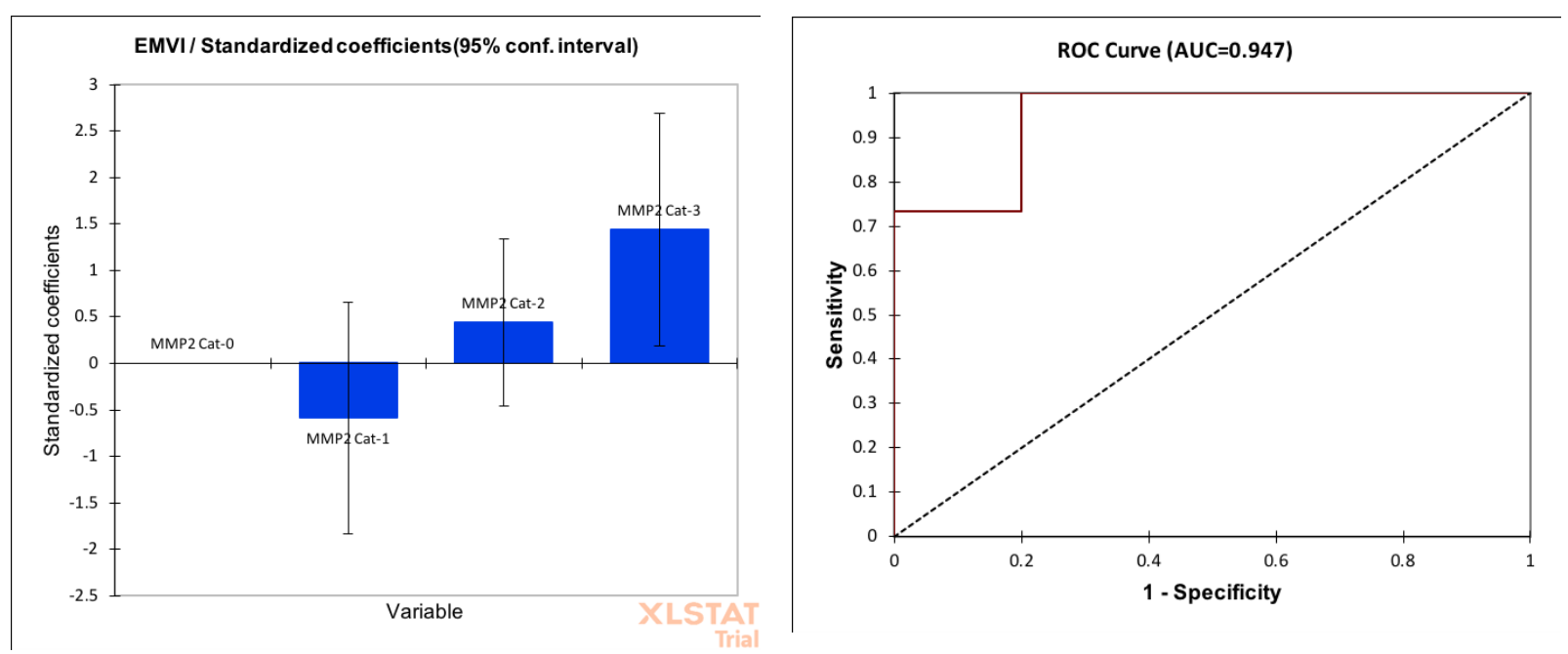

Classification Table for the training sample.

\begin{tabular}{|l|l|l|l|l|}
\hline from / to & $\mathbf{0}$ & $\mathbf{1}$ & Total & \% correct \\
\hline $\mathbf{0}$ & 24 & 6 & 30 & $80.0 \%$ \\
\hline $\mathbf{1}$ & 0 & 30 & 30 & $100.0 \%$ \\
\hline Total & 24 & 36 & 60 & $90.0 \%$ \\
\hline
\end{tabular}


b. TIMP2 Summary Statistics \& Test of the Null Hypothesis H0: Y=0.5.

\begin{tabular}{|l|l|l|l|l|l|l|r|r|}
\hline Variable & Categories & Frequencies & Percentage & Statistic & DF & $\boldsymbol{X}^{2}$ & \multicolumn{1}{|c|}{ PR $>\boldsymbol{X}^{2}$} \\
\hline EMVI & 1 & 30 & $50 \%$ & -2 Log & 2 & 26.406 & $<0.0001$ \\
\hline & 0 & 30 & $50 \%$ & Score & 2 & 17.130 & 0.0002 \\
\hline & & & & Wald & 2 & 7.450 & 0.024 \\
\hline Cat Score & 1 & 11 & $18.3 \%$ & & & & & \\
\hline & 2 & 37 & $61.7 \%$ & & & & & \\
\hline & 3 & 12 & $20.0 \%$ & & & & & \\
\hline
\end{tabular}

Standardised Coefficient Model \& ROC Curve.
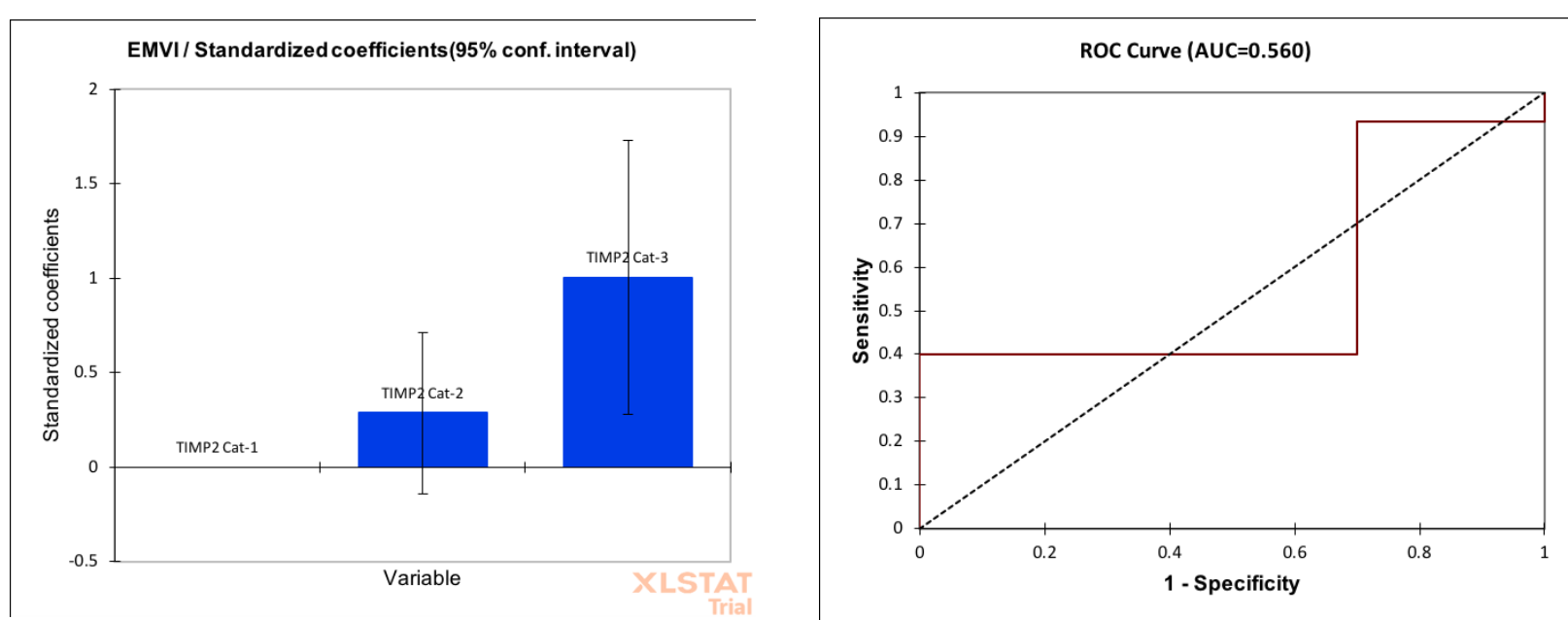

Classification Table for the training sample.

\begin{tabular}{|l|l|l|l|l|}
\hline from $/$ to & $\mathbf{0}$ & $\mathbf{1}$ & Total & \% correct \\
\hline $\mathbf{0}$ & 30 & 0 & 30 & $100.0 \%$ \\
\hline $\mathbf{1}$ & 18 & 12 & 30 & $40.0 \%$ \\
\hline Total & 48 & 12 & 60 & $70.0 \%$ \\
\hline
\end{tabular}


Cox Proportionate Hazard modelling for Disease-Free (DFS) and Overall Survival (OS) assessed on EMVI and categorised staining (Cat) scores for MMP2 \& TIMP2.

(HR, Hazard Ratio; T, Statistical Trend; NS, Statistical Non-Significance)

a. EMVI. (DFS HR 3.32, $p=0.027$; OS HR 3.05, $p=0.043$ )

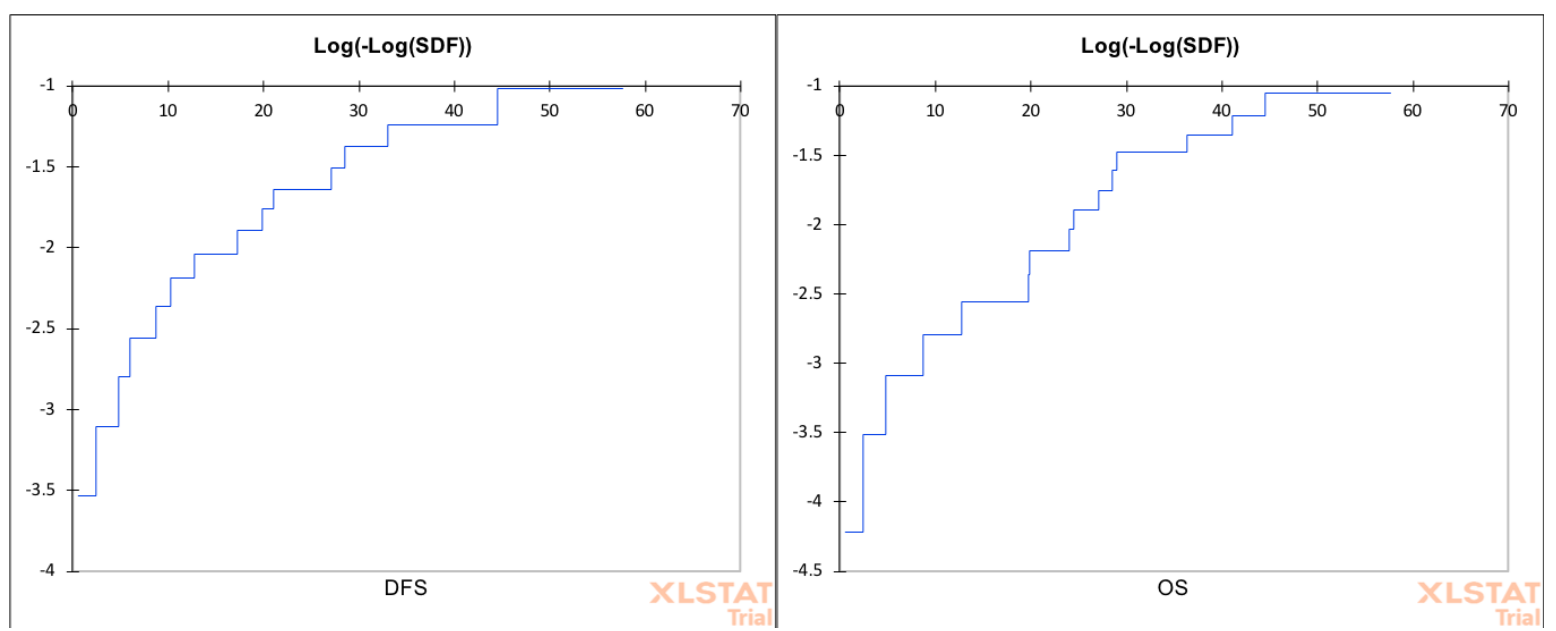

b. MMP2. (DFS $p=0.11, \mathrm{OS} p=0.15)$

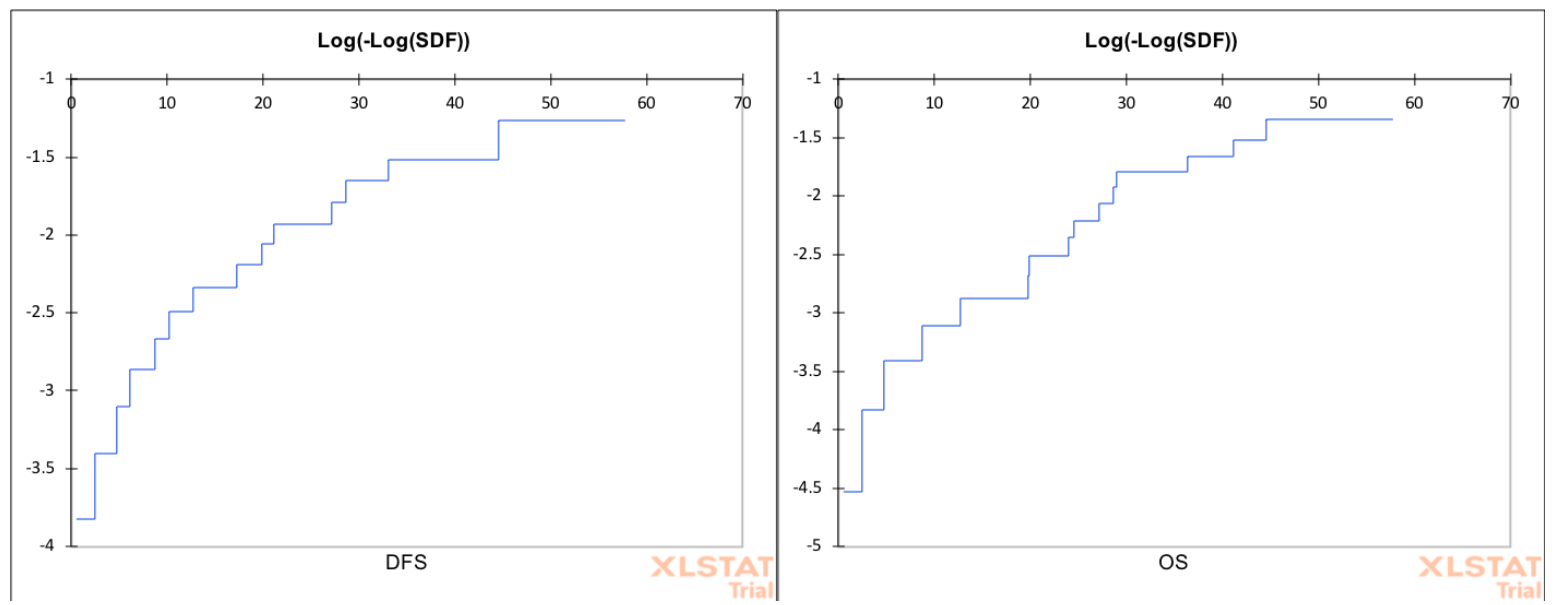

c. TIMP2. (DFS $p=0.66$, OS $p=0.67)$

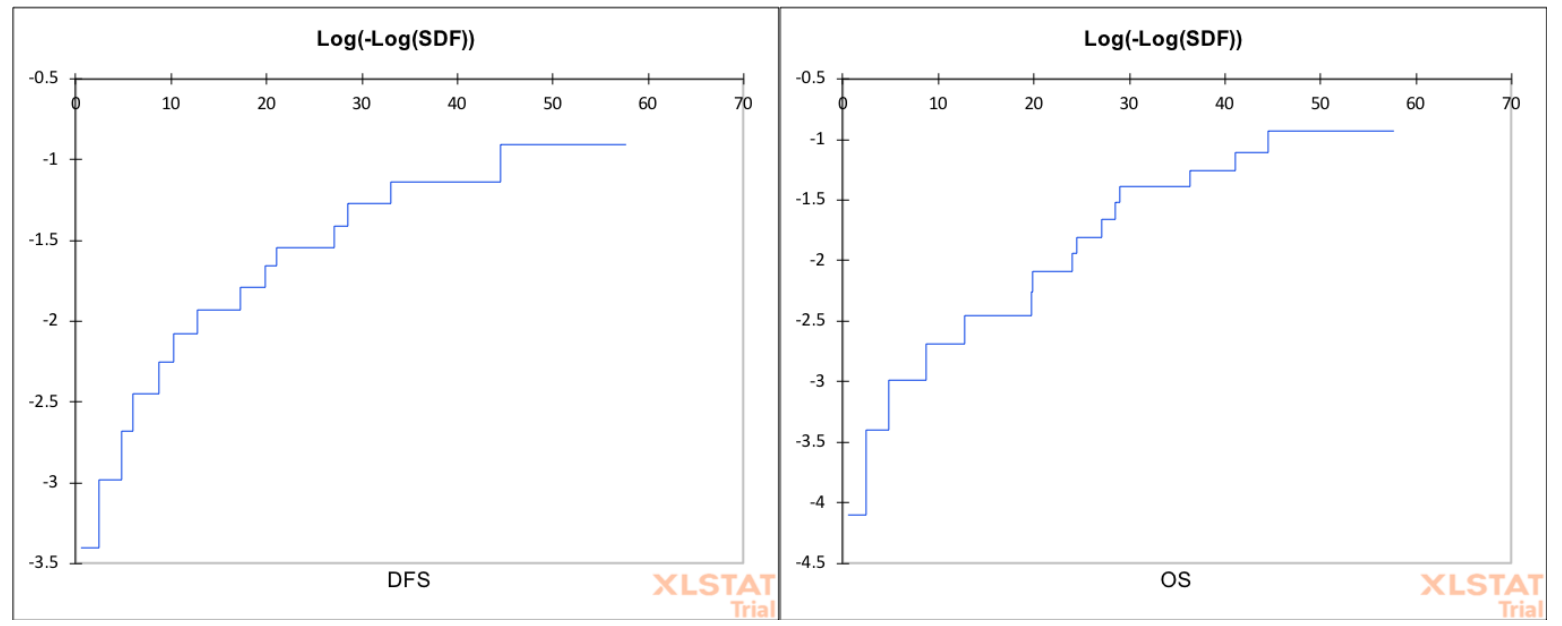


Heat-Map and Scatter-Plots of Pearson's Correlations between CIMP, EMVI, and MMP2.

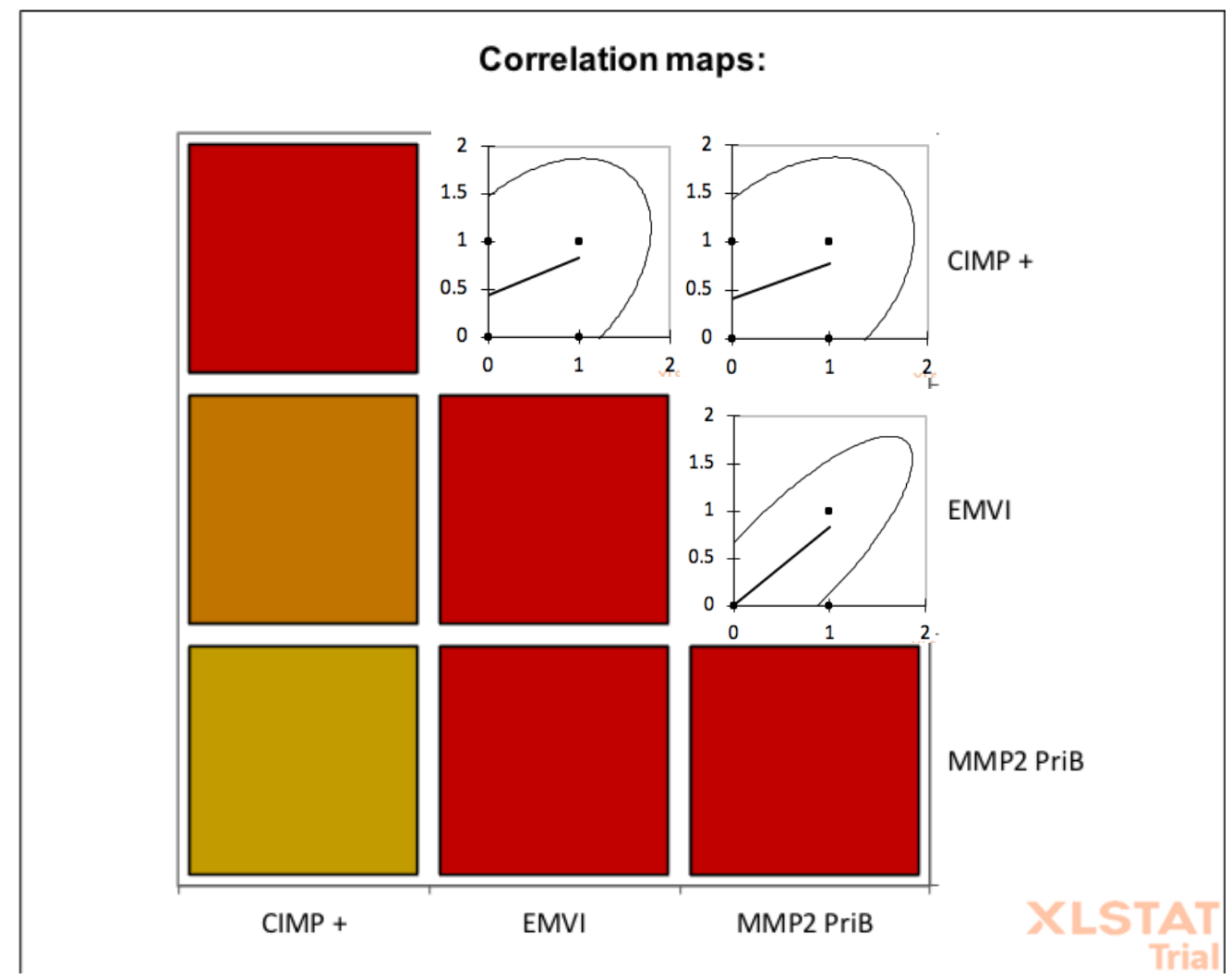




\section{Meta-analysis Search Strategy \& Consort Diagram}

Aim: Meta-analysis of CIMP in rectal tumours and relationship to outcomes (DFS/OS).

Boolean Search Term: CpG island methylator phenotype AND (cancer OR carcinoma OR adenocarcinoma OR tumor OR tumour) AND (colorectal OR rectal) AND (prognosis OR outcome)

PubMed: 223

Cochrane: 8

Medline: 10

After duplications removed: 203

Excluded based on title/abstract: 160

Excluded based on title/abstract: 160

Not colorectal cancer - 2

Non-CpG methylation focus of analysis -89

Single gene methylation only 3

Not related to prognosis -59

Not colorectal cancer - 2

Not related to prognosis -59

Review articles - 7

Screened for eligibility: 43

Excluded on full text assessment:

Colon assessed as single cohort -13

Rectum not separated from left / distal

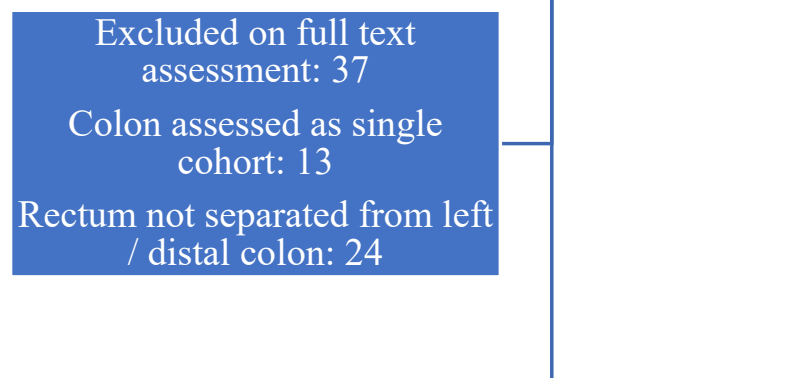

$$
\text { colon }-24
$$

Analysis: 6 
Characteristics of Included Studies

\begin{tabular}{|l|l|l|l|l|l|l|l|}
\hline Reference & \# Patients & $\begin{array}{l}\text { Study } \\
\text { Interval }\end{array}$ & $\begin{array}{l}\text { Age Range } \\
\text { (Mean) }\end{array}$ & $\begin{array}{l}\text { Men } \\
(\%)\end{array}$ & $\begin{array}{l}\text { AJC } \\
\text { C }\end{array}$ & $\begin{array}{l}\text { nCR } \\
\text { T }\end{array}$ & $\begin{array}{l}\text { N-O } \\
\text { Score }\end{array}$ \\
\hline Samowitz et.al. 2009 & 864 & $\begin{array}{l}1997- \\
2001\end{array}$ & $30-79(\mathrm{nr})$ & $\mathrm{nr}$ & I-IV & $\mathrm{nr}$ & 6 \\
\hline Jo et.al. 2011 & 150 & $\begin{array}{l}2004- \\
2006\end{array}$ & $\mathrm{nr}(61)$ & 71 & II-IV & NO & 6 \\
\hline Bae et.al. 2013 & 168 & $\begin{array}{l}2004- \\
2006\end{array}$ & $36-87(62)$ & 67 & I-IV & NO & 7 \\
\hline Williamson et.al. 2017 & 160 & $\begin{array}{l}2002- \\
2011\end{array}$ & $\mathrm{nr}(65)$ & 71 & II-IV & YES & 7 \\
\hline Kim et.al. 2017 & 87 & $\begin{array}{l}2006- \\
2007\end{array}$ & $31-88(65)$ & 59 & I-IV & $\mathrm{nr}$ & 7 \\
\hline Kokelaar et.al. 2018 & 100 & $\begin{array}{l}2010- \\
2013\end{array}$ & $24-89(71)$ & 70 & I-IV & NO & 7 \\
\hline
\end{tabular}

